\title{
On the variability of squeezing behaviour in tunnelling
}

Monograph

Author(s):

Mezger, Florence

Publication date:

2020-04

Permanent link:

https://doi.org/10.3929/ethz-b-000424080

Rights / license:

In Copyright - Non-Commercial Use Permitted

Originally published in:

Veröffentlichungen des Instituts für Geotechnik (IGT) der ETH Zürich 254 


\section{On the variability of squeezing behaviour in tunnelling}

Florence Mezger 
Weitere aktuelle vdf-Publikationen finden Sie in unserem Webshop:

\section{vdf.ch}

, Bauwesen

, Naturwissenschaften, Umwelt und Technik

, Informatik, Wirtschaftsinformatik und Mathematik
, Wirtschaft

, Geistes- und Sozialwissenschaften, Interdisziplinäres, Militärwissenschaft, Politik, Recht

\section{Gerne informieren wir Sie regelmässig per E-Mail über unsere Neuerscheinungen.}

\section{Newsletter abonnieren}

Anmeldung auf vdf.ch 


\section{On the variability of squeezing behaviour in tunnelling}

Florence Mezger

Veröffentlichungen des Instituts für Geotechnik (IGT) der ETH Zürich

Band 254, April 2020 
ETH-Dissertation Nr. 25638

(C) 2020, vdf Hochschulverlag AG an der ETH Zürich

ISBN: 978-3-7281-4005-0 (Printversion)

ISBN: 978-3-7281-4006-7 (E-Book)

Bibliografische Information der Deutschen Nationalbibliothek Die Deutsche Nationalbibliothek verzeichnet diese Publikation in der Deutschen Nationalbibliografie; detaillierte bibliografische Daten sind im Internet über http://dnb.d-nb.de abrufbar. 


\section{Vorwort}

Bei Tunnelvortrieben durch druckhaftes Gebirge stellt man oft eine wechselhafte Intensität der Gebirgsverformungen fest und dies trotz gleichbleibender Ausbruchsicherung, gleicher Überlagerungshöhe und vordergründig gleichbleibender Gebirgsbeschaffenheit. Die Variabilität der Druckhaftigkeit erschwert Voraussagen über das Gebirgsverhalten während des Vortriebs. Sie ist eine der Hauptursachen für Rückschläge, die selbst bei erfahrenen Ingenieuren vorkommen können.

Bei gegebener Überlagerungshöhe und Ausbruchsicherung hängen die Gebirgsverformungen bekanntlich von den mechanischen Eigenschaften des Gebirges, von der Raumstellung allfälliger Schichtungs- oder Schieferungsebenen sowie von der Grösse des Porenwasserdrucks ab. Es ist daher naheliegend, dass die Gründe einer Variabilität der Druckhaftigkeit während des Vortriebs in einer Wechselhaftigkeit dieser Einflussfaktoren längs des Tunnels liegen müssen. Allerdings ist nicht bekannt, in welchem Masse diese Faktoren das Gebirgsverhalten beeinflussen und wie sie bei tunnelstatischen Berechnungen berücksichtigt werden können, wenn sie sich innert kurzen Abständen längs des Tunnels ändern, wie dies bei einer Wechsellagerung von schwächeren und festeren Gesteinen oder bei einem intensiv gefalteten Gebirge der Fall ist. Ferner muss ein heterogener Gebirgsaufbau nicht zwangsläufig zu einer wechselhaften Intensität der Gebirgsverformungen führen. Eine im Vergleich zum Tunnelradius kleinmassstäbliche Variation der Gesteinseigenschaften dürfte sich kaum durch wechselhafte Konvergenzen beim Vortrieb bemerkbar machen; dabei ist allerdings unbekannt, (a), wie eng die Wechsellagerung von weichen und harten Gesteinen sein muss, damit das Gebirgsverhalten makroskopisch homogen erscheint und, (b), wie man repräsentative, für tunnelstatische Berechnungen adäquate Gebirgsparameter anhand der Eigenschaften und der Anteile der verschiedenen Gesteine bestimmen soll.

Mit diesen wissenschaftlich anspruchsvollen und für die Tunnelbaupraxis relevanten Fragen setzt sich diese Dissertationsschrift auseinander. Frau Mezger hat die Erfahrungen aus den druckhaften Strecken der drei Alptransit-Basistunnel ausgewertet; eine Reihe von geotechnisch-geologischen Situationen quantitativ untersucht; beim Schildvortrieb (der bekanntlich sehr empfindlich auf wechselhafte Gebirgsverformungen bzw. Gebirgsdrücke reagiert) verschiedene Ausbauoptionen systematisch analysiert und basierend auf ihren Untersuchungen nützliche Hilfsmittel und Hinweise für die Projektierung ausgearbeitet.

Prof. Dr. G. Anagnostou 



\section{Acknowledgements}

I wish to express my sincerest gratitude to my supervisor Prof. Dr. Georg Anagnostou (ETH Zurich) for his guidance, encouragement and support during the last years and thank him for the opportunity to work together with him on challenging research and consulting projects.

I am also deeply grateful to Prof. Dr. Frédéric L. Pellet (MINES ParisTech) for agreeing to be co-examiner of this doctoral thesis. His constructive comments were of great value.

This doctoral thesis evolved within the framework of the research projects "On the variability of squeezing behaviour in tunnelling" and "Einsatzgrenzen von Tübbingausbauten in druckhaftem Gebirge", respectively. The support given by the Federal Road Office of Switzerland (FEDRO) as well as the Commission for Technology and Innovation (a promotional agency of the Swiss confederation) is greatly appreciated.

Furthermore, I would like to acknowledge the AlpTransit Gotthard AG (Switzerland) and BLS AlpTransit AG (Switzerland) for their permission to use data from the construction of the Gotthard, Ceneri and Lötschberg Base Tunnel for this doctoral thesis. Special thanks go to all those involved, who allowed access to the documents and monitoring data needed for this research, particularly to Stefano Morandi (Pini Swiss Engineers), Reto Wagner (Kellerhals + Haefeli AG), Hans-Ulrich Riesen (ristag Ingenieure AG), Dr. Stefan Irngartinger and Peter Teuscher (BLS Netz AG - Alptransit, Switzerland). I would also like to express my gratitude to Werner Burger (Herrenknecht AG) for his comments and suggestions concerning TBM technology and construction process, Flavio Chiaverio (Aegerter \& Bosshardt AG) for his ideas about a double shell lining with an inner ring made of cast in-situ concrete, Prof. Fritz Grübl (Hochschule für Technik Stuttgart, PSP Consulting Engineers $\mathrm{GmbH}$ ) for his inputs on high or ultra-high performance concretes and Dr. Eike Klingsch (ETH Zurich) for discussions about the fire resistance of high or ultra-high performance concretes.

Furthermore, I express my warmest thanks to all my colleagues I have collaborated with during the years at the ETH Zurich for all the interesting discussions, the support and especially the pleasant working atmosphere.

Last but not least, I owe my deepest thanks to my family and friends - especially to Stefan, to my sister Isabelle and to my parents Anne-Chantal and Anton Christian - for their continuous understanding, support and encouragement. 



\section{Summary}

The magnitude of squeezing deformations in tunnelling often varies over short distances, even if there is no obvious change in the construction method, in the depth of cover, in the lithology or rock structure. As long as the reasons for the variability are not known, the tunnelling-induced convergences cannot be predicted with sufficient reliability. Reliable predictions, however, are important for determining the temporary support or the excavation diameter. Otherwise, large-scale tunnel repairs may be necessary, which can cause delay and additional costs due to remedial actions as well as due to the enforced interruption of other operations in progress at the same time.

The analysis of different case studies concerning the AlpTransit project shows that the squeezing variability can be traced back to the heterogeneities in the rock mass at different scales as well as the variation of the orientation of the planes of anisotropy (bedding or schistosity) to the tunnel axis.

In order to improve safety and economy of tunnel construction in squeezing ground, the influence of these factors on the convergences was determined quantitatively (by means of analytical solutions or numerical modelling), so that they can be used as indicators during construction for the timely identification and prediction of the squeezing behaviour. Particular attention was paid on factors whose variation, even if small, may affect the convergences sensitively. Based on these quantitative investigations, design aids were developed, which should help the project engineer to estimate and better understand the variability of the squeezing intensity.

The squeezing variability also concerns TBM tunnelling: In contrast to conventional tunnelling, shielded TBMs are particularly vulnerable to squeezing due to the very limited space available. Therefore, in order to better deal with the squeezing variability in TBM tunnelling, different (stiff as well as deformable) lining options were discussed.

Keywords: tunnelling; squeezing; schistosity; heterogeneity; analytical solution; numerical modelling; segmental lining. 



\section{Zusammenfassung}

Die Intensität der Gebirgsverformung bei einer druckhaften Strecke ist in der Regel stark veränderlich. Trotz gleichbleibender Ausbruchsicherung, gleicher Überlagerungshöhe und derselben lithologischen Einheit lassen sich häufig sprunghafte Änderungen der Konvergenzen auf kurzer Distanz feststellen. So lange die Gründe für diese Variabilität nicht bekannt sind, sind zuverlässige Voraussagen des Gebirgsverhaltens nach dem Tunnelausbruch schwierig. Zuverlässige Voraussagen sind aber wichtig, um die Sicherungsmittel und den Ausbruchsquerschnitt zu bestimmen und damit kostspielige und aufwändige Nachprofilierungsarbeiten zu vermeiden.

Die Analyse von ausgewählten Vortrieben des AlpTransit-Projektes zeigt, dass die Variabilität der Druckhaftigkeit auf die Heterogenität des Gebirges in verschiedenen Massstäben sowie auf die variierende Orientierung der Trennflächen (Schieferung, Schichtung) in Bezug zur Tunnelachse zurückgeführt werden kann.

Um die Sicherheit und Wirtschaftlichkeit beim Tunnelbau in druckhaftem Gebirge zu steigern, wurde der Einfluss dieser Faktoren auf die Konvergenzen quantitativ (mittels analytischer Lösungen oder numerischer Berechnungen) ermittelt, so dass diese als Indikatoren für die rechtzeitige Identifikation und Vorhersage des druckhaften Verhaltens genützt werden können. Besondere Beachtung wurden Faktoren geschenkt, deren kleine Veränderung einen starken Einfluss auf die Intensität der Konvergenzen haben. Basierend auf diesen quantitativen Untersuchungen wurden Entscheidungshilfen entwickelt, welche dem projektierenden Ingenieur helfen sollen, die Variabilität der Druckhaftigkeit zu beurteilen und besser zu erfassen.

Die Variabilität der Druckhaftigkeit betrifft auch den Vortrieb mit einer TBM: Im Gegensatz zum konventionellen Vortrieb, sind Einfachschild-TBMs besonders anfällig für Druckhaftigkeit. Um die Variabilität der Druckhaftigkeit bei einem TBM-Vortrieb besser beherrschen zu können, wurden deshalb verschiedene (steife wie auch verformbare) Ausbausysteme diskutiert.

Stichwörter: Tunnelbau; Druckhaftigkeit; Schieferung; Heterogenität; analytische Lösung; numerische Modellierung; Tübbingsysteme. 



\section{Contents}

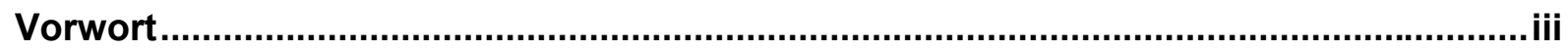

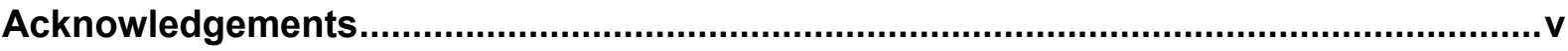

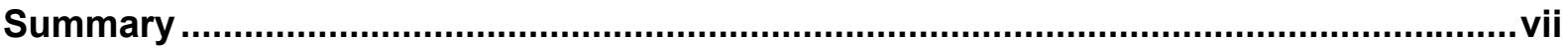

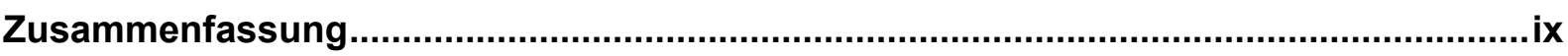

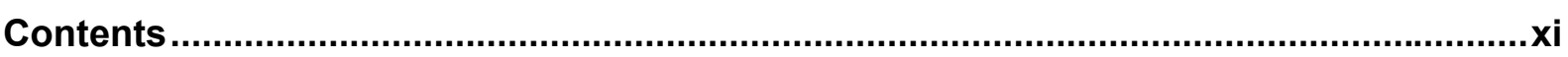

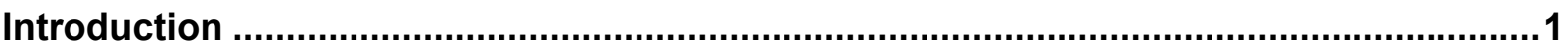

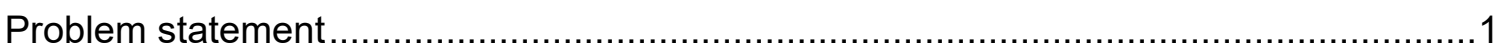

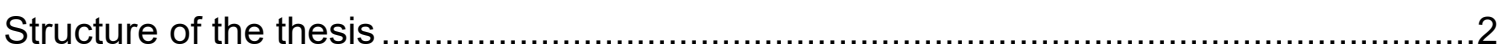

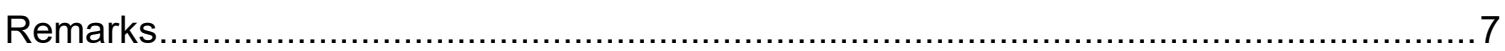

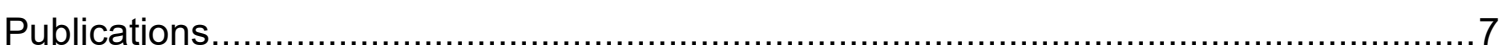

PART I: ANALYSIS OF CASE HISTORIES

1 Sedrun Section of the Gotthard Base Tunnel.....................................................11

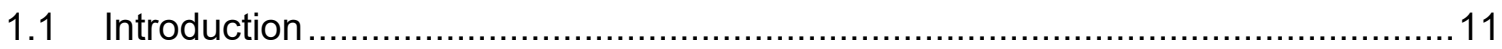

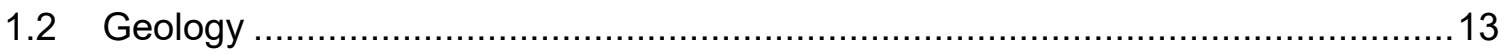

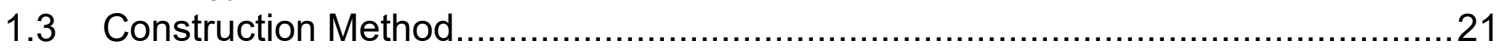

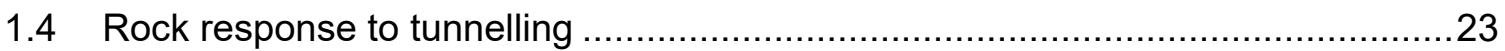

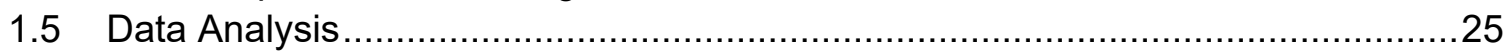

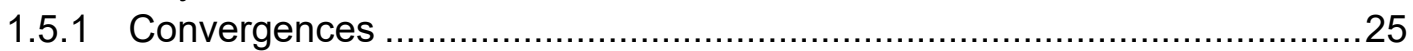

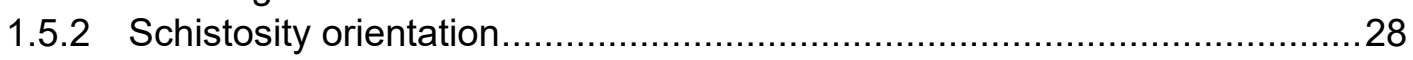

1.5.3 Combined effect of schistosity orientation and shearing degree ................30

1.5.4 Influence of adjacent weaker or stronger zones .......................................31

1.5.5 The longitudinal component of displacement ………..............................33

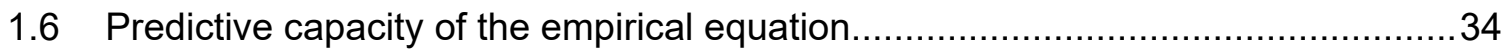

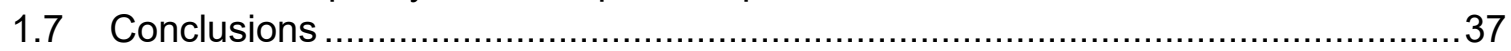

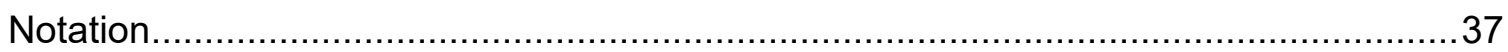

2 Giumello Gneiss Section of the Ceneri Base Tunnel.............................................39

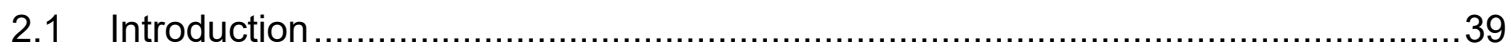

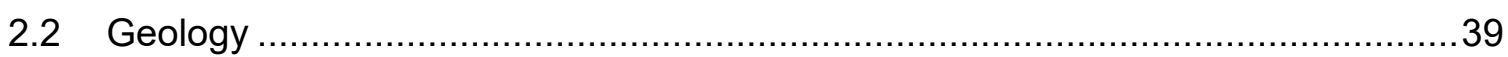

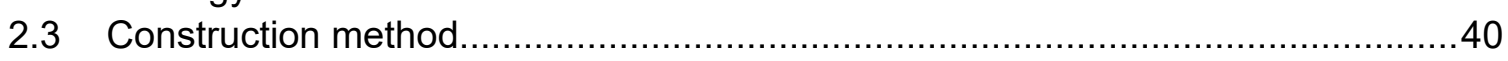

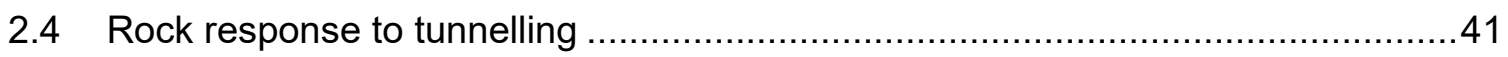

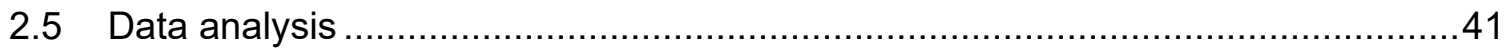

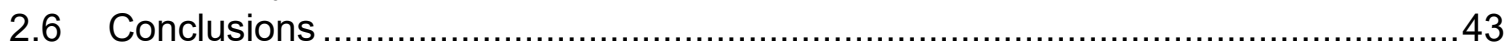

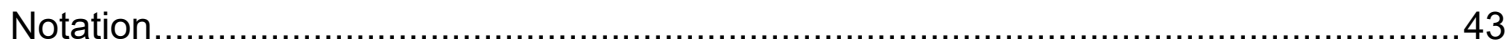




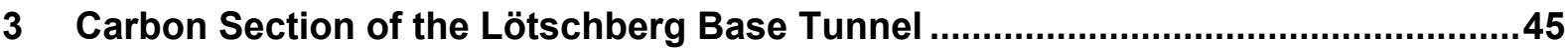

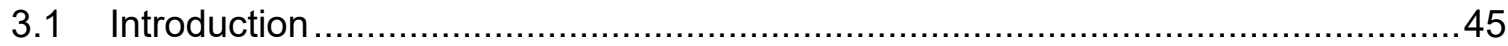

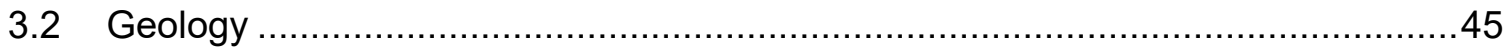

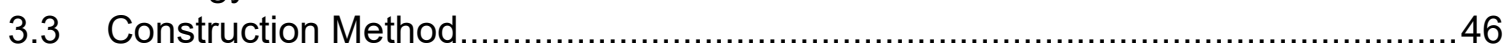

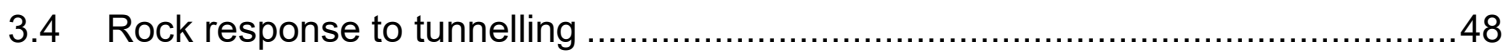

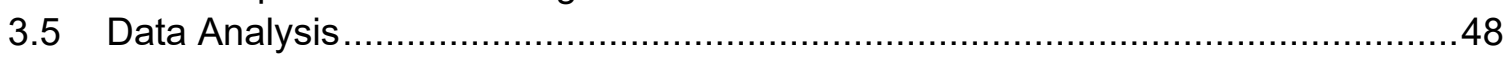

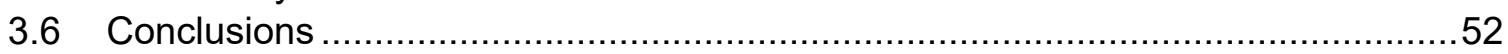

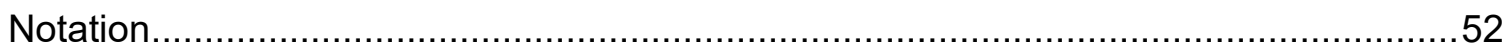

PART II: TUNNELLING PERPENDICULARLY TO ALTERNATING WEAK AND

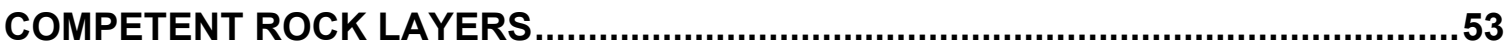

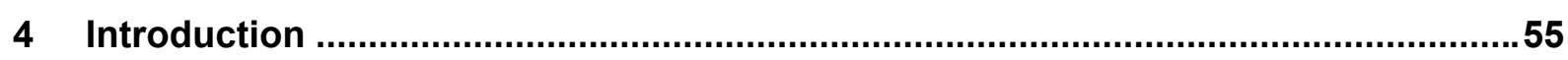

5 Closed-form solution for the ground response curve in tunnelling perpendicularly to thinly alternating weak and competent rocks ..........................57

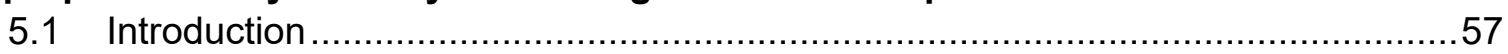

5.2 Perfectly plastic behaviour disregarding out-of-plane plastic flow ......................58

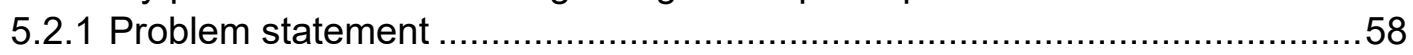

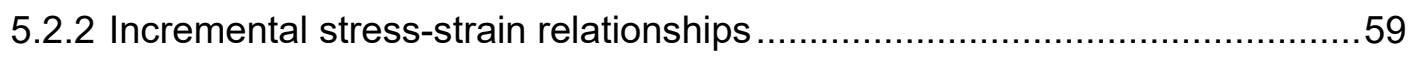

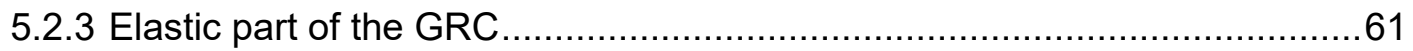

5.2.4 GRC part for elasto-plastic behaviour either of the weak or the hard layers

5.2.5 GRC part for elasto-plastic behaviour both of the weak and of the

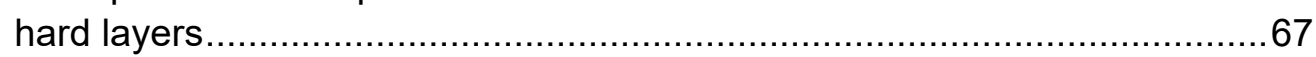

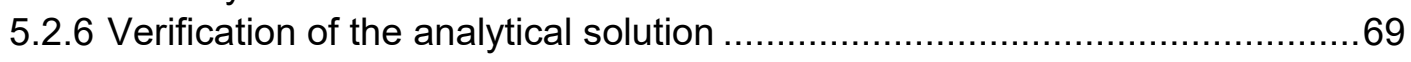

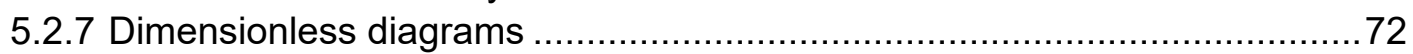

5.3 Extension of the closed-form solution for brittle behaviour of the hard

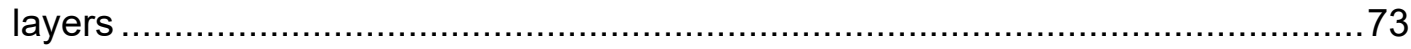

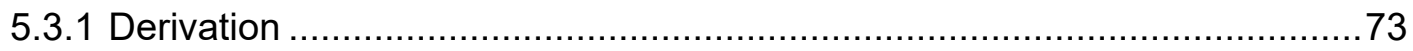

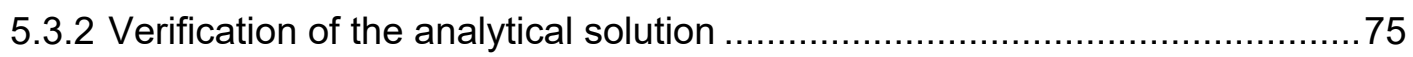

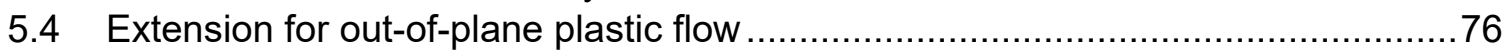

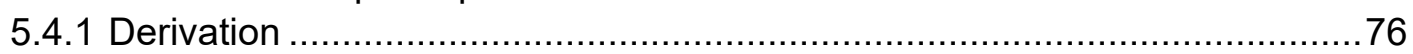

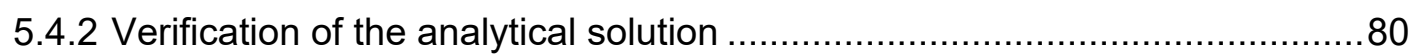

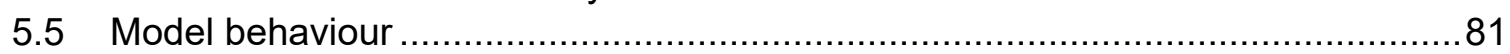

5.5.1 Perfectly plastic behaviour of the hard layers......................................... 81

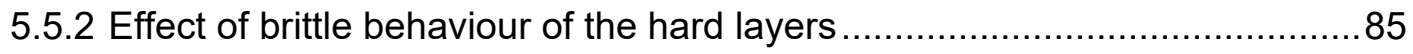

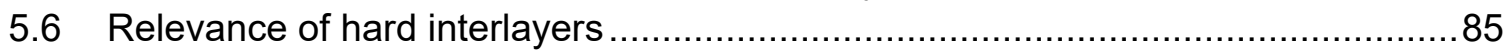

5.7 Remarks concerning the adequacy of the homogenised model...........................86

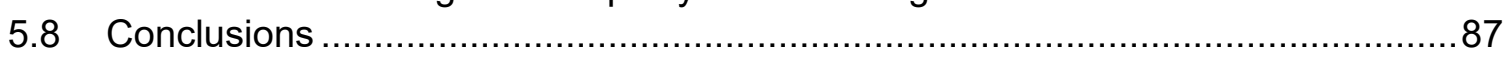

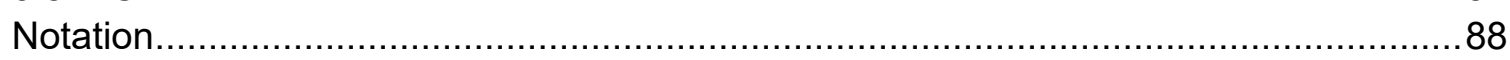

6 Determination of equivalent parameters for a rock mass consisting of thinly alternating weak and competent rocks..........................................................91

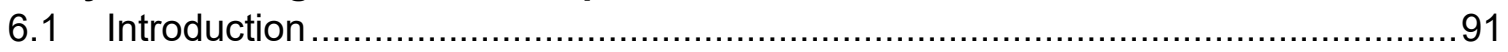

6.2 Determination of the parameters of the equivalent isotropic medium....................91

6.3 Relationships between the material constants of the equivalent isotropic medium and those of the transversely isotropic medium ......................93

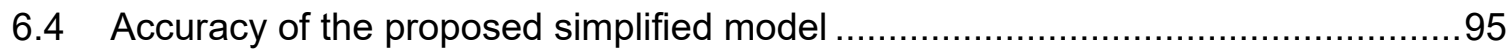

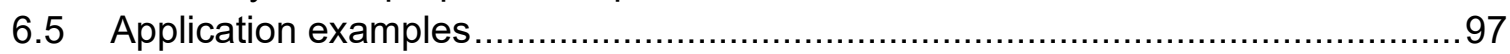

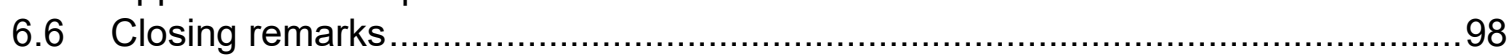

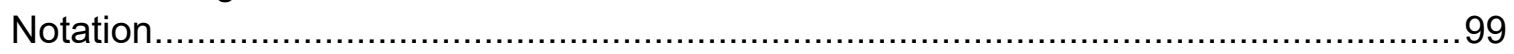


7 Influence of the heterogeneity scale on the squeezing variability along

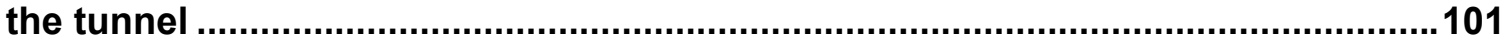

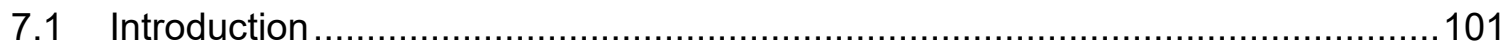

7.2 Longitudinal distribution of the displacements .................................................. 101

7.3 A simple equation for the displacements in a weak zone considering a

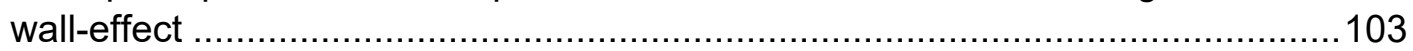

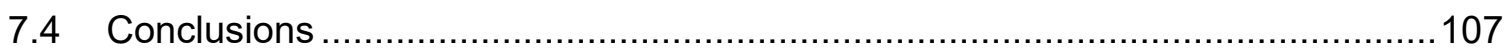

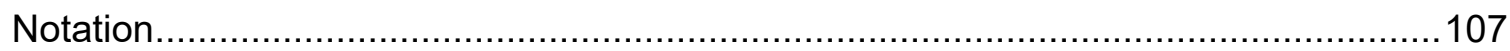

PART III: TUNNELLING PARALLEL TO ALTERNATING WEAK AND

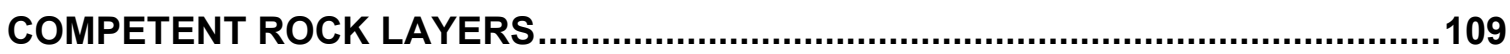

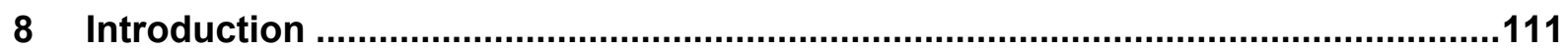

9 Response of a thinly stratified rock mass, striking parallel to tunnel axis

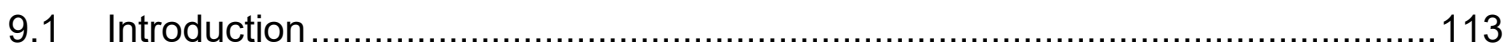

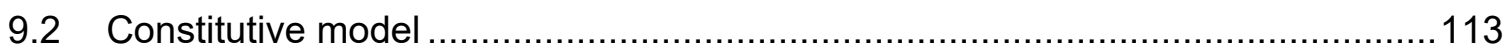

9.2.1 Formulation of the constitutive model...................................................113

9.2.2 Rock element behaviour.................................................................... 114

9.3 Basic aspects of the bedded rock response to excavation..............................117

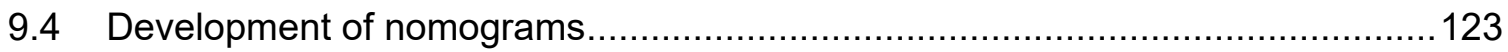

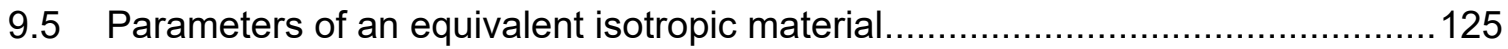

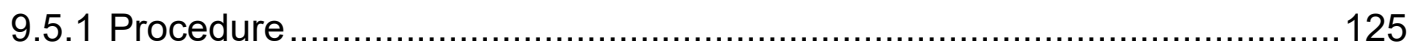

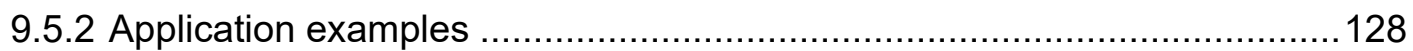

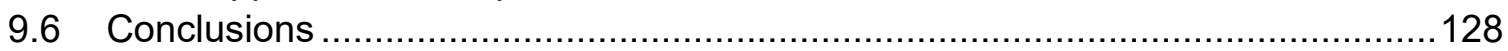

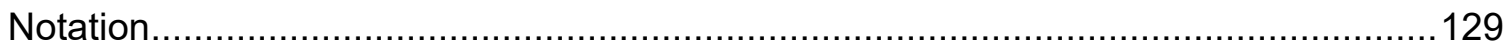

10 Influence of the heterogeneity scale on the distribution of the ground

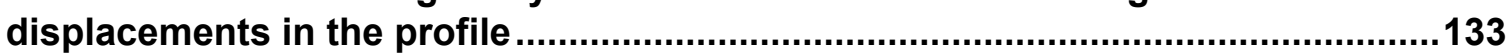

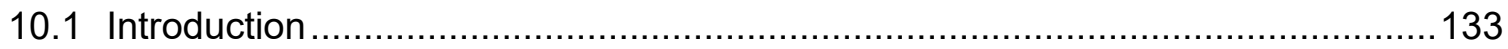

10.2 Adequacy of the homogenised model ........................................................ 133

10.3 Tunnelling parallel to the interface between a weak and a competent

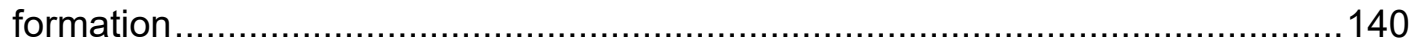

10.4 Alternating weak and competent layers of medium thickness ...........................142

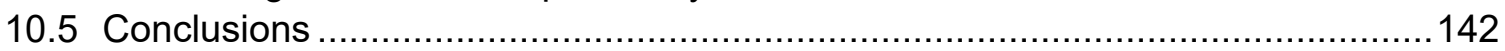

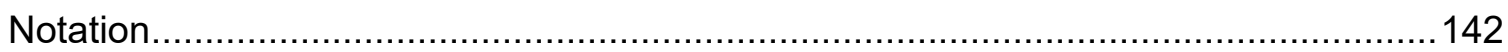

11 Response of a schistous rock mass striking parallel to the tunnel axis ..............145

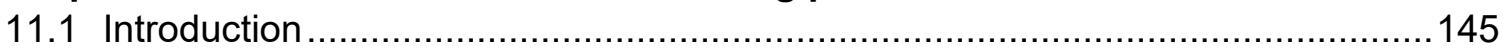

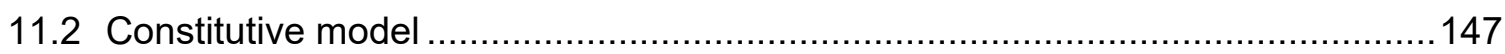

11.2.1 Formulation of the constitutive model .............................................. 147

11.2.2 Rock element behaviour ............................................................ 149

11.3 Basic aspects of the schistous rock response to excavation ..............................150

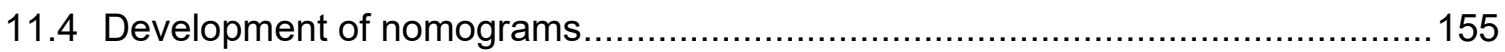

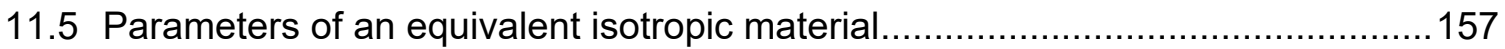

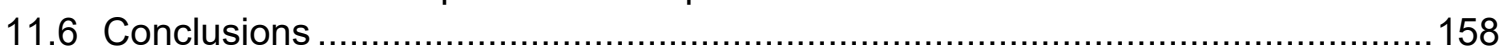

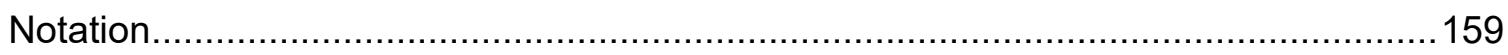

PART IV: TUNNELLING WITH AN ARBITRARY ANGLE TO THE SCHISTOSITY OR BEDDING PLANES

12 Influence of the orientation of the anisotropy planes on the squeezing deformations.

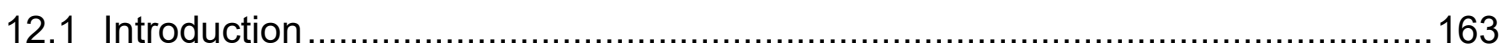

12.2 Tunnelling in schistous rock, perpendicular to the strike direction.......................165 
12.2.1 Influence of the dip angle on the displacements ...............................165

12.2.2 Influence of the dip angle on the pre-deformations...........................169

12.2.3 Influence of the dip angle on the convergences .................................170

12.3 Tunnelling in thinly stratified rock, perpendicular to the strike direction................172

12.3.1 Influence of the dip angle on the displacements ............................... 172

12.3.2 Influence of the dip angle on the pre-deformations............................172

12.3.3 Influence of the dip angle on the convergences ................................175

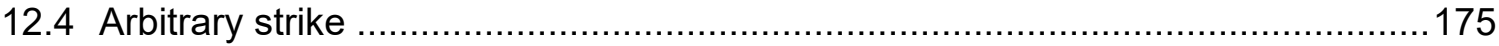

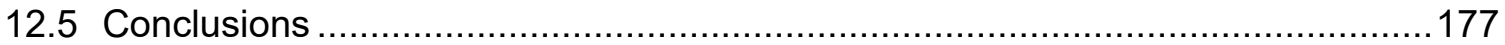

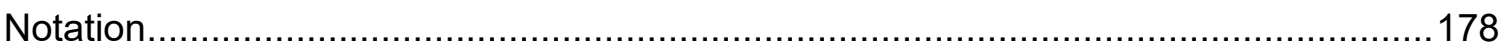

13 Variability of squeezing deformations in folded rocks ......................................181

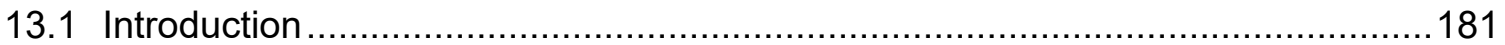

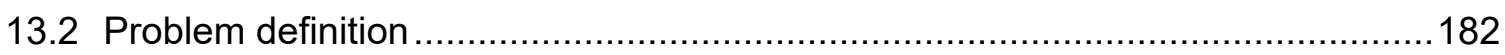

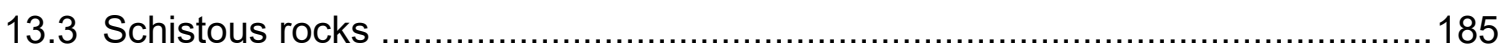

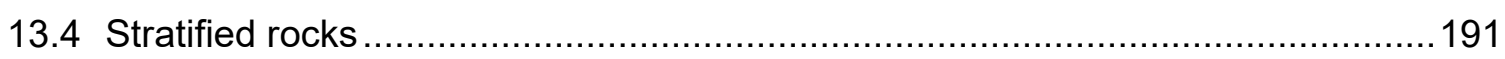

13.5 Case history of the Sedrun section of the Gotthard Base Tunnel........................ 194

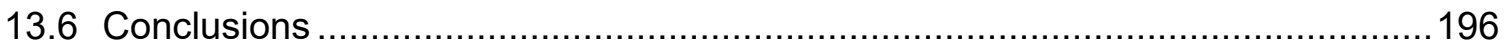

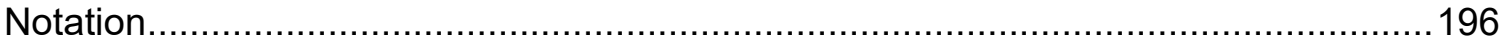

PART V: DEALING WITH THE SQUEEZING VARIABILITY IN SHIELDED TBM

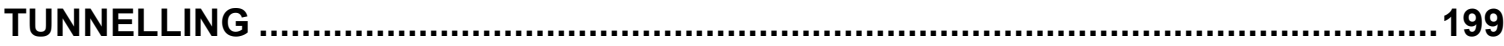

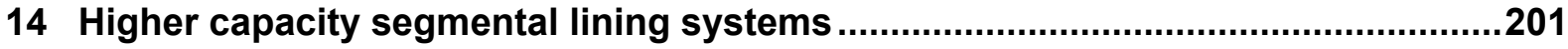

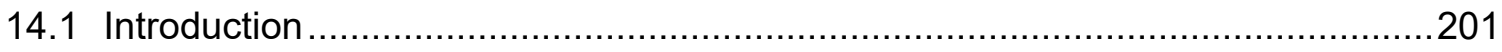

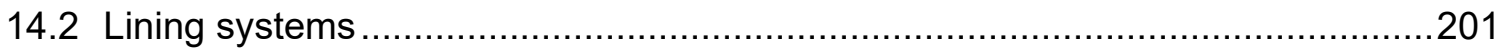

14.3 Single shell segmental lining made of normal-strength concrete ......................204

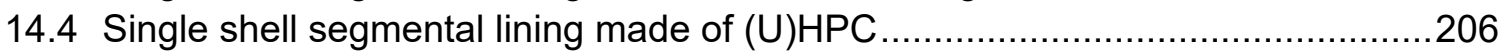

14.4.1 Principle and manufacture ……............................................2. 206

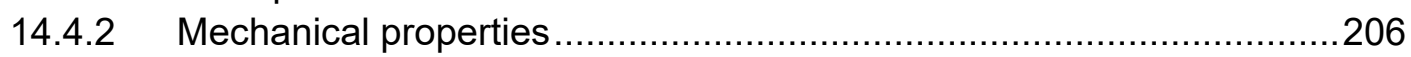

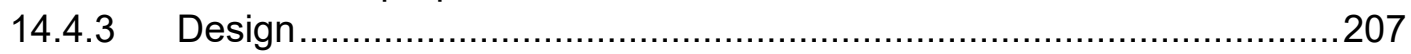

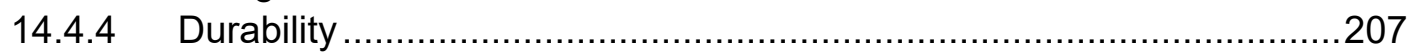

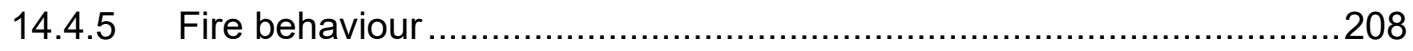

14.4.6 The use of (U)HPC for segmental linings .........................................209

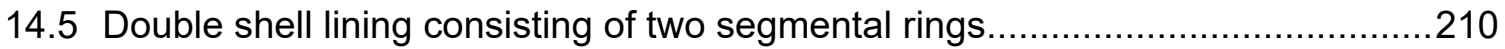

14.6 Double shell lining with inner ring of cast in-situ concrete .................................211

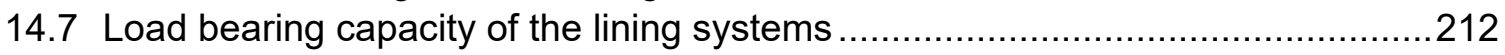

14.7.1 Single or double shell systems of uniform quality concrete .................212

14.7.2 Double shells of different quality concretes .....................................213

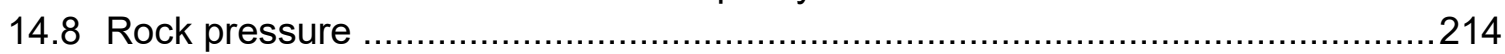

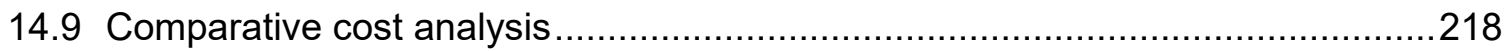

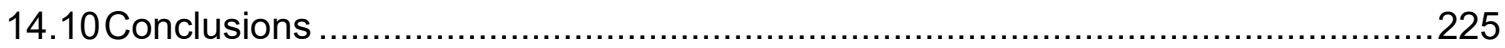

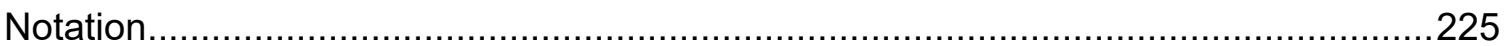

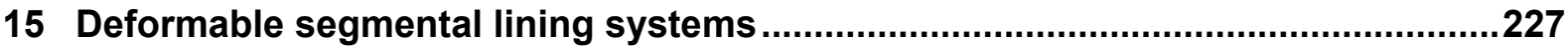

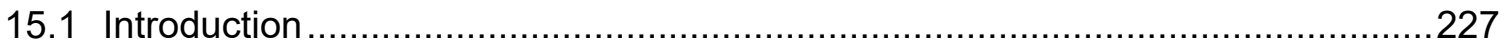

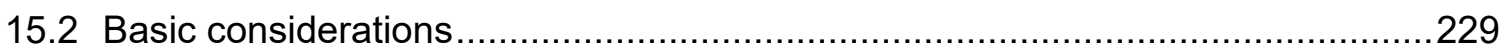

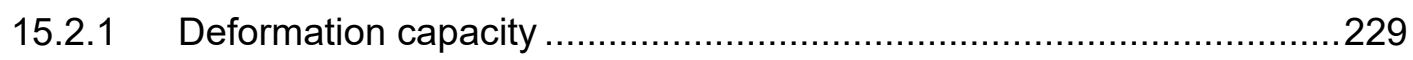

15.2.2 Load-displacement and stress-strain behaviour ...............................230

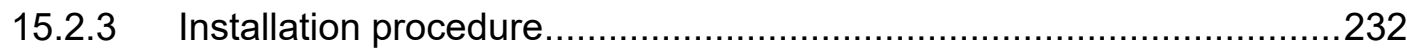

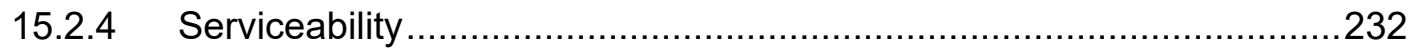

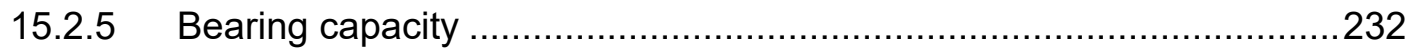




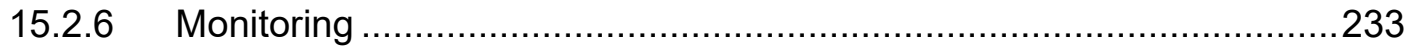

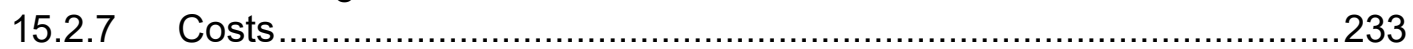

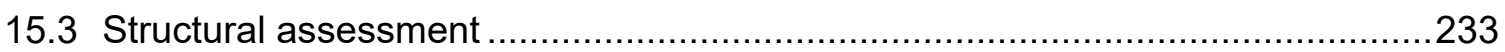

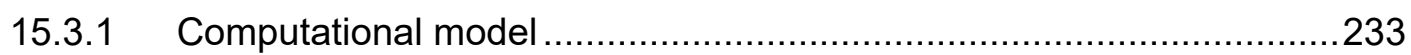

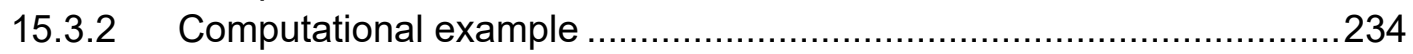

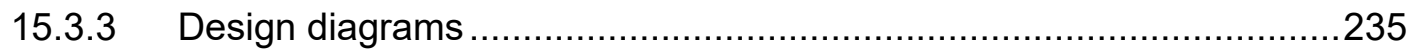

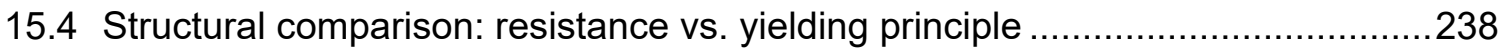

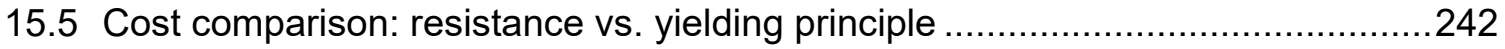

15.5.1 Tunnel costs for a specific geotechnical situation ...............................242

15.5.2 Tunnel costs considering squeezing distribution ................................24

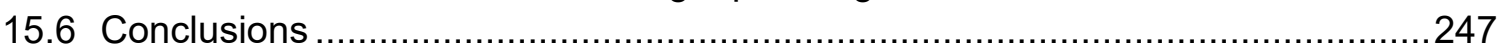

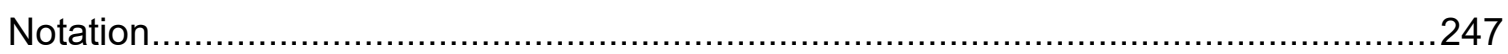

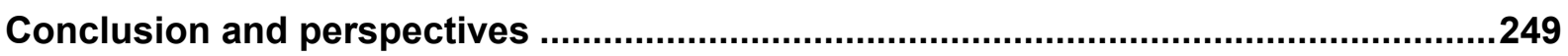

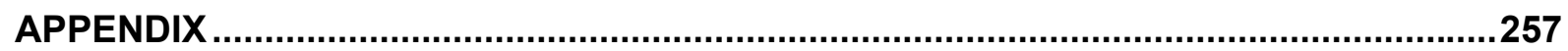

Appendix A: Tunnelling perpendicularly to a thinly stratified rock mass -

Coefficients of the incremental stress strain relationships ..................................259

A.1 Case $i$. Both the weak and the hard layers behave elastically ...........................259

A.2 Case ii. Weak layers yield in-plane, hard layers behave elastically.....................260

A.3 Case iii. Hard layers yield in-plane, weak layers behave elastically ...................261

A.4 Case $i v$. Both the hard and the weak layers yield in-plane .................................263

A.5 Case ii-o. Weak layers yield out-of-plane in triaxial extension,

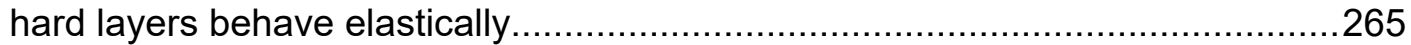

A.6 Case iii-o. Hard layers yield out-of-plane in triaxial extension, weak layers behave elastically

A.7 Case iv-01. Weak layers yield out-of-plane in triaxial extension, hard layers yield in-plane...

A.8 Case iv-o2. Weak layers yield in-plane, hard layers yield out-of-plane in triaxial extension

A.9 Case iv-o3. Both the weak and the hard layers yield out-of-plane in triaxial extension

A.10 Case iv-04. Weak and hard layers yield out-of-plane in triaxial compression and extension, respectively...

A.11 Case iv-05. Weak and hard layers yield out-of-plane in triaxial extension and compression, respectively....

Appendix B: Tunnelling perpendicularly to a thinly stratified rock mass Dimensionless diagrams for the displacements

Appendix C: Tunnelling perpendicularly to a thinly stratified rock mass Flow chart of the Matlab-code for calculating the GRC.

Appendix D: Stratified rock mass - Implementation and validation of the constitutive model.

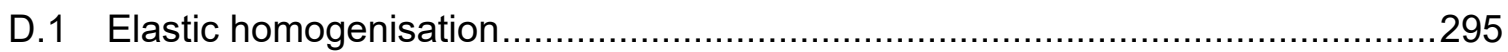

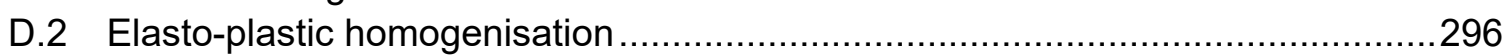

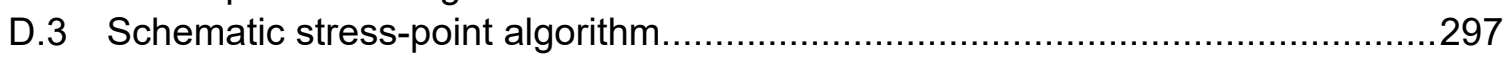

D.4 Validation of the constitutive model analogously to Lourenço (1995)..................297

D.5 Validation of the constitutive model considering the problem of tunnelling perpendicular to the layers of a thinly stratified rock mass. 
Appendix E: Tunnelling parallel to the bedding of a thinly stratified rock

mass - Dimensionless diagrams for the displacements

Appendix F: Schistous rock mass - Implementation and validation of the constitutive model.

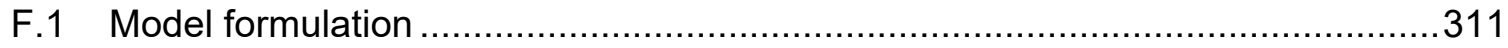

F.2 Schematic stress-point algorithm for the 2D case .......................................... 313

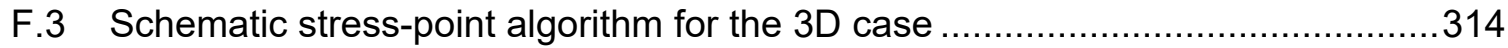

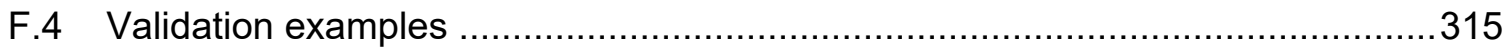

Appendix G: Tunnelling parallel to the schistosity plane - Dimensionless

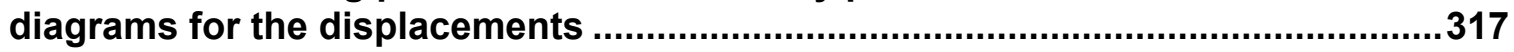

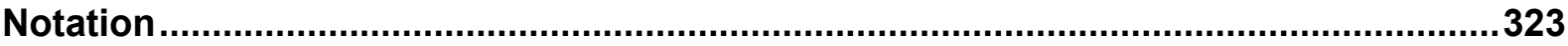

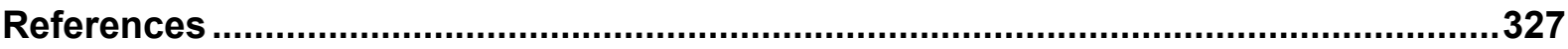


INTRODUCTION 



\section{Introduction}

\section{Problem statement}

"Squeezing ground" is characterised by large time-dependent deformations that develop when tunnelling through weak rocks. The magnitude of squeezing deformations in tunnelling often varies over short distances even where there is no obvious change in the construction method, in the depth of cover, in the lithology or rock structure. As long as the reasons for the variability are not known, the tunnelling-induced convergences cannot be predicted with sufficient reliability. Reliable predictions, however, are important for determining the temporary support or the excavation diameter. Otherwise, large-scale tunnel repairs may be necessary, which can cause delay and additional costs due to remedial actions as well as due to the enforced interruption of other operations in progress at the same time. The variability of squeezing intensity is one main cause of setbacks that even highly qualified engineers may experience in some cases (Kovári 1998).

For a given overburden and construction method, it is known that the rock deformations depend on the mechanical properties of the rock, on the spatial orientation of the stratification or schistosity, on the initial stress state and on the pore water pressure. It is, therefore, obvious that the reasons for the squeezing variability during the advance must be the variability of these influencing factors along the tunnel. In spite of the intensive research of the last years on the problem of squeezing ground, it is not known to what extent these factors influence the rock behaviour and how they can be taken into account during design, particularly if they change within short distances along the tunnel. This is for example the case when tunnelling through alternating weak and hard layers (Fig. 1a) or through folded rocks (Fig. 1b).
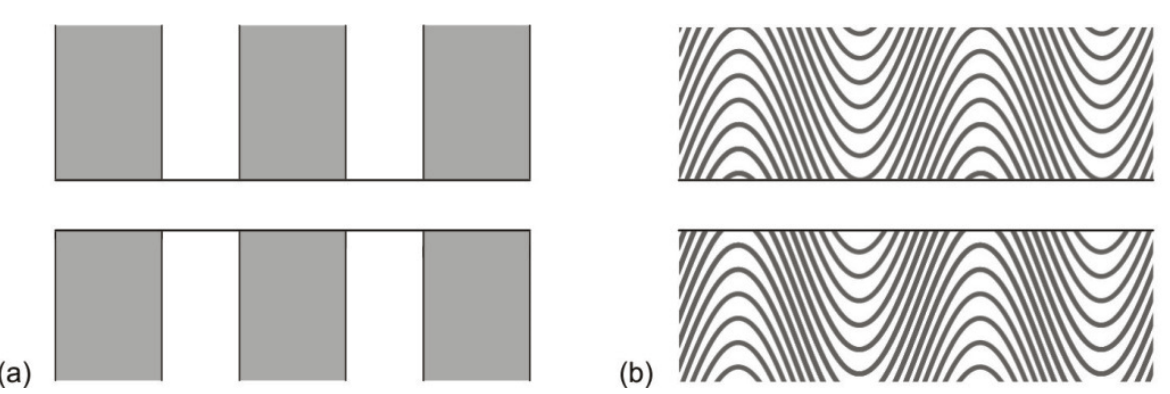

(b)

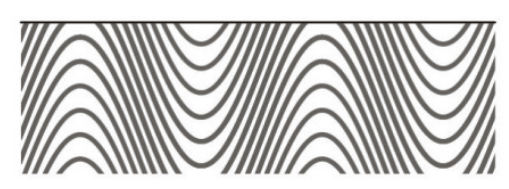

Figure 1. Tunnel drive through, (a), alternating weak and hard layers, (b), through folded rocks.

The goals of the present doctoral thesis are thus to improve safety and economic efficiency of tunnel constructions in squeezing ground, to improve the understanding of the squeezing variability and to make the related experiences from the AlpTransit project available to the 
engineering community. The main objectives serving these goals are: (i) the identification of the factors, which are responsible for the squeezing variability, and (ii) the quantification of the influence of these factors, in order to use them as indicators during construction for the timely identification and prediction of the squeezing behaviour. To achieve these objectives, the data from the Gotthard, Ceneri and Lötschberg Base Tunnels are analysed qualitativelyempirically, numerical calculations are performed to investigate quantitatively the influence of the factors and decision aids for the planning, design and construction of tunnels are developed. Particular attention is paid to factors that influence convergences sensitively, i.e., whose variations - even if relatively small - may cause a significant variability in the macroscopic behaviour. Therefore, this thesis focuses on the squeezing variability due to the heterogeneity of the ground with respect to its mechanical characteristics at different scales as well as the variation of the orientation of the anisotropy planes (bedding, schistosity). In this thesis, the time-dependence of the rock behaviour (due to creep or consolidation) will not be considered. Furthermore, it is known that the initial stress state may influence the squeezing intensity: If the initial stress state varies along the tunnel, as it may be the case in intensively folded rocks or in fault zones, the intensity of squeezing may be variable. The effect of in situ stress variations will not be studied in this thesis.

\section{Structure of the thesis}

The thesis is structured in five parts, where some of the parts have already been published in scientific papers.

Part I analyses the observations of the construction of the three AlpTransit Base Tunnels. Specifically, it gives a concise overview of the available data concerning the geology, the excavation and support and the rock response to tunnelling for different sections of the Gotthard (Chapter 1), Ceneri (Chapter 2) and Lötschberg Base Tunnels (Chapter 3) and identifies the factors that are responsible for the squeezing intensity and variability. Furthermore, the influence of these factors is discussed and empirical correlations are established that allow to improve the predictions of the tunnelling-induced convergences. The observations of the construction of the AlpTransit tunnels could show that the squeezing variability can be traced back to variations of the structure of the ground, such as the variation of the mechanical properties of the rock (for example when tunnelling through alternating weak and competent rocks) or the variation of the orientation of the schistosity to the tunnel axis due to folding.

The theoretical background of these observations is provided in Parts // to IV of this thesis, which investigate the squeezing intensity in tunnelling through alternating weak and competent rocks (Fig. 1a) or through folded rocks (Fig. 1b) by means of analytical methods or numerical computations. For the analysis of tunnelling through alternating weak and competent layers, three cases are distinguished with respect to the orientation of the layers (cf. Fig. 2): a sequence of alternating weak and hard rocks lying (a) perpendicular, (b) parallel or (c) with an arbitrary orientation to the tunnel axis. In the first case, the squeezing intensity may be very variable depending on the thickness of the layers with respect to the tunnel radius, i.e., on the scale of the mechanical heterogeneity of the ground. In the second case (cf. Fig. 2b), the squeezing intensity is constant along the tunnel axis, but the deformations are not uniform along the tunnel profile. As was observed during the construction of the Gotthard and the Ceneri Base Tunnels, these two geotechnical situations can effectively occur in reality and are thus of particular practical importance. Of course, in reality, such a sequence of hard and weak layers may be characterised by transition zones. 
Nevertheless, for simplicity reasons, this thesis focuses on an advance through a sequence of only one hard and one weak material.

(a)

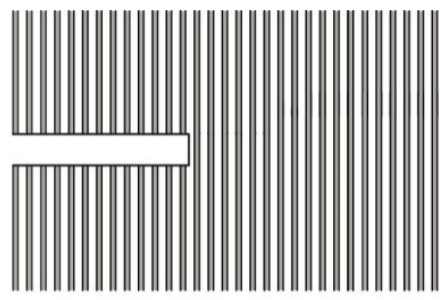

(b)

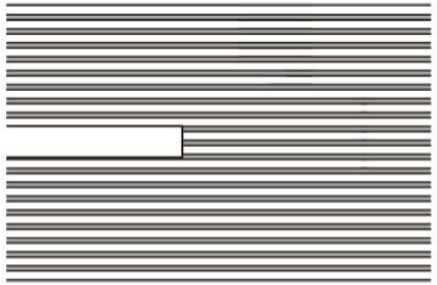

(c)

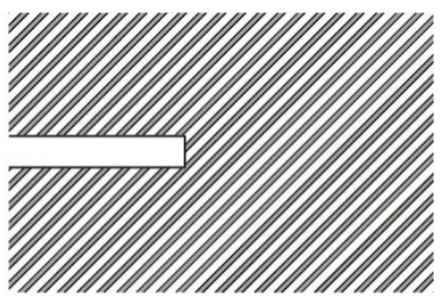

$\square$ weak layer $\square$ hard layer

Figure 2. Investigated orientations of the layers to the tunnel axis for a sequence of alternating weak and hard rocks.

Parts II and III focus on the displacements of the ground in cross-sections far behind the tunnel face. These are greater than the convergences of the excavated profile because they include the deformations that occur ahead of the face (so-called "pre-deformations"). As shown in Parts II and III, the pre-deformations (and thus also the convergences of the excavated profile) can be obtained with the known methods for isotropic elasto-plastic materials.

Part I/ investigates the excavation-induced displacements in tunnelling perpendicular to the layers (cf. Fig. 2a). If the alternating zones are thick relative to the tunnel diameter (Fig. 3a), the response of such formations to the tunnel excavation may exhibit a great variability, as was observed for instance in the Sedrun Section of the Gotthard Base Tunnel. If, however, the formation consists of very thin alternating weak and competent rock layers, the deformation distribution along the tunnel axis will be practically uniform (Fig. 3c). This means that rather than considering a heterogeneous model and modelling the individual layers numerically, which would be demanding in terms of spatial discretisation and computation time, the rock structure can be taken into account by considering a homogeneous, but nevertheless transversely isotropic model (Fig. 3i). For this special case, Chapter 5 develops a closed-form solution for the ground response curve (GRC; i.e., the relationship between the radial displacement at the tunnel boundary and the support pressure; Panet and Guellec 1974), using the homogenisation technique (see, e.g., Salamon 1968) and assuming rotational symmetry, plane strain conditions, perfectly plastic behaviour for the weak layers and either perfectly plastic or brittle behaviour (with post-failure decrease in strength) for the hard layers.

The response of the considered homogenised material to tunnelling is isotropic (since the excavation boundary experiences a uniform radial displacement). This suggests that it may be possible to consider the rock mass as an isotropic and homogeneous material (Fig. 3ii) with mechanical parameters, which depend on the parameters and fractions of the weak and hard layers. Such an approach would be useful in practice because it would allow using common calculation methods and programs to solve problems that do not meet the conditions of rotational symmetry or plane strain and this even for thinly stratified rocks. Chapter 6 investigates this idea into more depth and determines the equivalent parameters (Young's modulus $E_{e q}$, Poisson's ratio $v_{e q}$, friction angle $\varphi_{\text {eq }}$, cohesion $c_{e q}$ and dilatancy angle $\psi_{\text {eq }}$, Fig. 3ii) of an isotropic homogeneous rock mass as a function of the properties and fractions of the weak and the hard layers. 
(a)

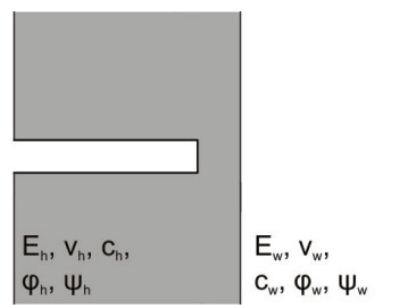

(b)
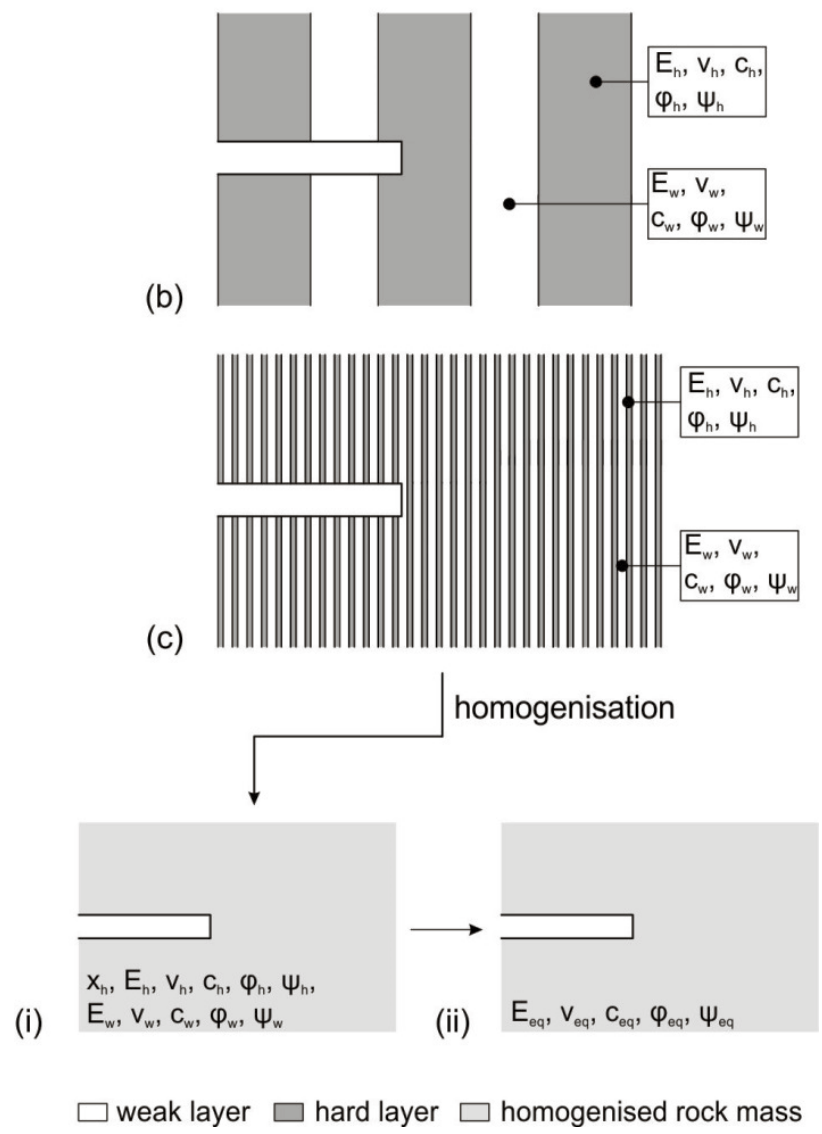

Figure 3. Problem statement for alternating weak and hard layers lying perpendicular to the tunnel axis.

The results of Chapters 5 and 6 apply to alternating weak and hard layers that are so thin (relative to the tunnel radius) that the rock mass can be considered as homogeneous at the scale of the tunnel cross-section. Otherwise, if the layers are thicker and thus the assumption of a homogenised model is not valid, at the current state of research numerical calculations have to be performed, where the weak and the hard layers have to be modelled discretely. However, Chapter 7 shows that the displacements in weak zones can be estimated by means of a simple equation which takes into account the stabilising influence of the adjacent hard layers.

Part III investigates the excavation-induced tunnel displacements in stratified rock mass, consisting of alternating weak and hard layers that are oriented parallel to the tunnel axis (Fig. 2b). Obviously, if the layers are very thick and their interface lies at a great distance to the tunnel (Fig. 4a), then the displacements of the tunnel profile will be practically uniform and thus the heterogeneity of the ground can be neglected. Otherwise (Fig. 4b), the squeezing deformations along the tunnel profile will not be uniform, even if the layers are very thin (Fig. 4c). 


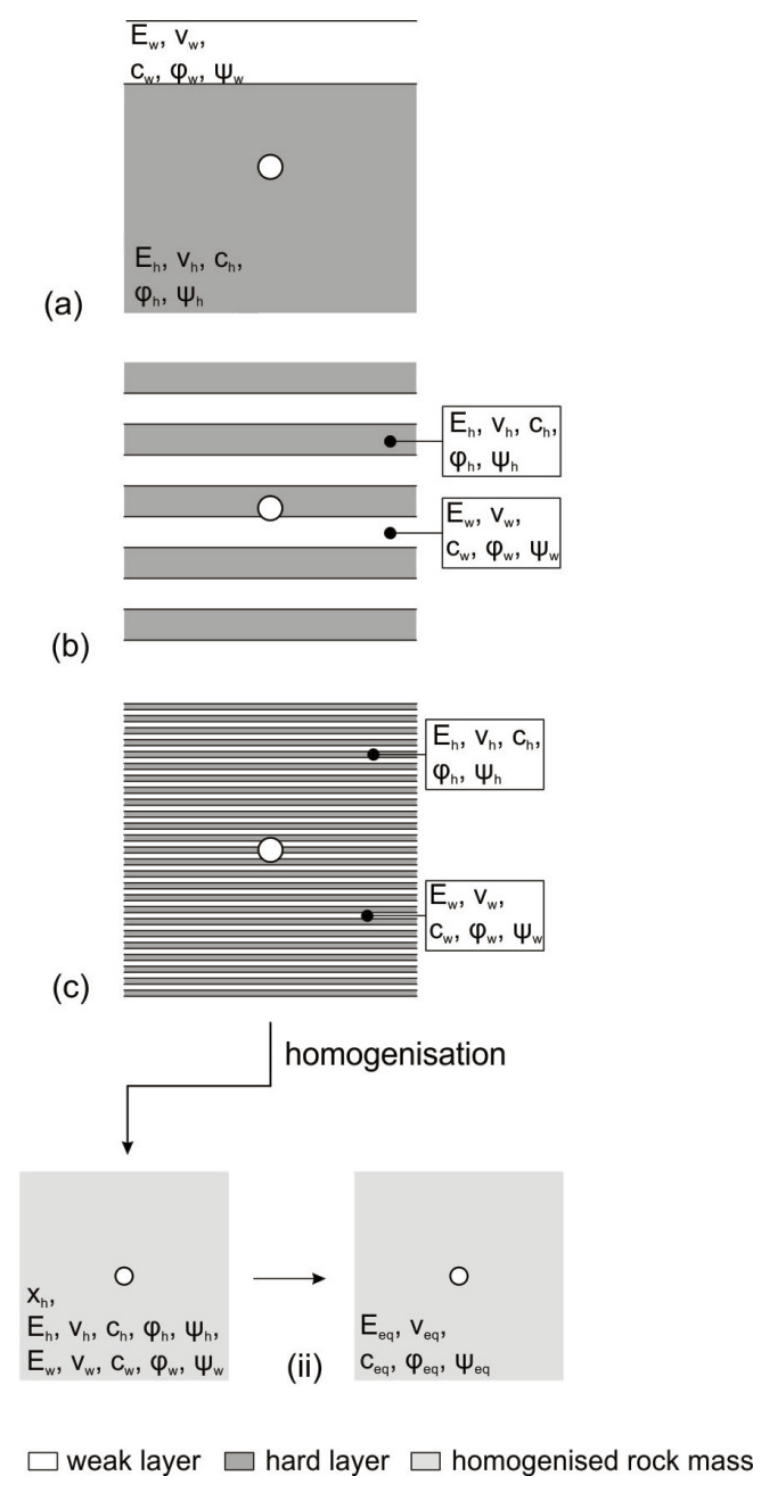

Figure 4. Problem statement for alternating weak and hard layers lying parallel to the tunnel axis.

Tunnelling through thinly alternating weak and hard layers that strike parallel to the tunnel axis can be analysed by considering, analogously to Chapter 5 , a homogeneous and transversely isotropic medium (Fig. $4 \mathrm{i}$; Chapter 9). However, in contrast to Chapter 5 , the conditions of rotational symmetry are not fulfilled anymore and, consequently, this boundary value problem has to be solved numerically. Therefore, the constitutive model of Chapter 5 was formulated for general 3D stress- and strain-states (using the homogenisation technique of Lourenço 1995) and implemented in Abaqus. The material constants of this equivalent homogeneous model consist of the thickness fractions and mechanical parameters of the alternating layers. Using this model, a comprehensive parametric study was carried out covering a wide range of geotechnical parameters. The results are presented in the form of dimensionless design diagrams that allow for a quick estimation of the maximum and minimum displacements of the tunnel profile. Even if the response of the ground is anisotropic in this case, these diagrams can also be used, analogously to Chapter 6, to determine the parameters for an isotropic homogeneous rock mass (Fig. 4ii), which is equivalent to the isotropic rock mass in the sense that its tunnelling-induced displacements are equal either to the maximum or to the minimum displacements of the anisotropic model. As shown in Section 9.5, using this equivalent isotropic model makes it possible to find an 
upper and a lower bound of the displacements in more complex problems (that do not meet the condition of plane strain).

Finally, Chapter 10 examines the adequacy and limits of the homogenisation of a stratified rock mass and the influence of the layer thickness.

Chapter 11 concerns the case of tunnelling through schistous rocks striking parallel to the tunnel axis. The reason for considering schistosity in Part III (which actually deals with stratified rock masses) is that the response of a schistous rock mass exhibits certain similarities to that of a stratified rock mass: A schistous rock mass can be conceived, from the mechanical point of view, as a borderline case of a thinly stratified rock mass. Schistosity is irrelevant for tunnelling perpendicular to the layers (Fig. 3c), but has a pronounced influence on the displacements if the schistosity plane strikes parallel to the tunnel axis.

Since the constitutive model that was implemented for thinly stratified rocks in Chapter 9 is computationally inefficient for the borderline case of schistosity, Chapter 11 starts with the formulation of an optimised constitutive model specifically for schistous rock, continues with the investigation into the effect of schistosity on the displacements and closes with working out design diagrams that allow a quick estimation of the tunnel displacements in schistous rocks (assuming again plane strain conditions) for a wide parameter range.

Part IV considers tunnelling through thinly stratified or schistous rock masses with an arbitrary orientation of the anisotropy planes with respect to the tunnel axis (cf. Fig. 2c). First, in Chapter 12, the relatively simple case of constant orientation of the anisotropy planes along the tunnel is considered, while paying attention to the effect of the dip angle and strike of the anisotropy planes relative to the tunnel axis on the pre-deformations and thus on the deformations of the excavated tunnel profile ("convergences"). The pre-deformations depend essentially on the orientation of the anisotropy planes and, as shown in Section 12.2.2, may be considerably higher than those estimated with the known methods which were developed for isotropic materials. Chapter 12 shows a simplified method for estimating the tunnel convergences.

Subsequently, Chapter 13 analyses numerically the case of tunnelling through folded rock formations (Fig. 1b), where the orientation of the anisotropy planes and consequently the squeezing intensity vary along the tunnel axis. A simplified folded rock structure is considered with a sinusoidal form of the bedding or schistosity surface. Folding is taken into account numerically in the constitutive models by considering that the normal vector to the anisotropy surface is position-dependent. The numerical results show that the variability of the convergences depends essentially on the amplitude and period of the anisotropy surface. Finally, Chapter 13 revisits the case history of the Sedrun section of the Gotthard Base Tunnel, providing the theoretical background of the schistosity factor introduced in Chapter 1 .

The last part of the thesis investigates the aspect of squeezing variability from a completely different perspective: Part $V$ studies technological possibilities for dealing with squeezing variability in the very important case of mechanised tunnelling with shielded TBMs, since they are particularly vulnerable to squeezing: TBM offer only a very limited space for deformations to occur and, consequently, even relatively small convergences may lead to considerable difficulties such as shield jamming or damage to the segmental lining. Depending on the design, the decision-making during construction may have to address the boring diameter (to provide more space for deformations), the lining type or even the lining thickness, whereby the timely implementation of such measures is a demanding task if 
squeezing intensity changes frequently along the tunnel drive. The thesis focuses on the problem of overstressing of the segmental lining (the most serious hazard of shield tunnelling in squeezing rock), and investigates, whether higher bearing or deformation capacity lining systems (Chapters 14 and 15), respectively, could widen the application range of shielded TBMs. Furthermore, the chapters discuss the possibility of installing linings of variable thickness or type (e.g., switching from normal to higher strength segments or from a stiff to a deformable lining of the same thickness). The structural assessment of the various lining types is based on numerical analyses which consider the interaction between the advancing shield, the ground and the lining, whereby the rock mass is taken as homogeneous and isotropic. This assessment can also be adopted for stratified or schistous rocks considering equivalent parameters based on the guidelines formulated in the Chapters 6, 9 and 11.

Concerning the possibilities for increasing the bearing capacity of stiff lining systems (Chapter 14), besides the obvious option of increasing segment thickness, an investigation is made into the technical and economical feasibility of double shell solutions as well as that of high performance or ultra-high performance concretes from the standpoints of structural engineering, TBM technology, process engineering, material technology and construction cost.

Deformable lining systems (Chapter 15) exploit a basic feature of squeezing ground: the rock pressure decreases when deformations are allowed to occur. Two basic options are investigated: radially deformable linings, where rock deformations occur outside the lining extrados; and tangentially deformable linings which can accommodate a reduction in their circumference. Finally, the deformable linings are compared to the stiff linings and the geological conditions are investigated for which the use of deformable lining systems is structurally adequate and economical.

\section{Remarks}

In this thesis, as usual in geotechnics, compression will be taken as positive for the stresses and the strains.

Each chapter of this thesis is presented as an autonomous entity with its notation inserted at its end, while all references are given together at the end of the thesis.

\section{Publications}

Chapter 1 was published in Mezger et al. (2013a) and presented in Mezger et al. (2013b), whereby the contribution of Dr. Hans-Jakob Ziegler concerned geological aspects. Chapter 2 was presented in Mezger and Anagnostou (2015). Chapters 14 and 15 were published in Mezger et al. (2017) and Mezger et al. (2018), respectively, under consideration of the comments and suggestions of Dr. Marco Ramoni, who helped to improve the papers (on the basis of his own doctoral thesis). These chapters have also been presented on several occasions (Mezger et al. 2015b, Mezger et al. 2015a, Mezger et al. 2016). 



\section{PART I: ANALYSIS OF CASE HISTORIES}

This part of the thesis analyses the observations of the construction of the three AlpTransit Base Tunnels. Specifically, it gives a concise overview of the available data concerning the geology, the excavation and support and the rock response to tunnelling of various sections of the Gotthard, Ceneri and Lötschberg Base Tunnel and identifies the factors that are responsible for the squeezing intensity and variability. Furthermore, the influence of the factors are analysed qualitatively - empirically, in order to establish correlations that can be used for making more reliable predictions of the tunnelling-induced convergences. It is shown that the squeezing deformations in a specific tunnel cross-section are mainly affected by the lithology, the orientation of the schistosity planes and the existence of nearby weaker or stronger zones. 



\section{Sedrun Section of the Gotthard Base Tunnel ${ }^{1}$}

\subsection{Introduction}

The magnitude of squeezing deformations occurring in tunnelling often varies over short distances even where there is no obvious change in the excavation method, depth of the cover and lithology. The variability of the ground response to excavation is one of the causes of the setbacks observed sometimes in tunnelling through squeezing rock (Kovári 1998). As long as the reasons for the variability are not identified and understood, the tunnellinginduced convergences cannot be predicted with sufficient reliability. Reliable predictions, however, are important for determining the temporary support or the excavation diameter. Otherwise, large-scale tunnel repairs may be necessary, which can cause, as may be seen for example in the southern section of the Gotthard Base Tunnel (Bachmann and Vicenzi 2008), delay and extra costs due to remedial actions as well as due to the enforced interruption of other operations in progress at the same time. For reviews on the problem and the mechanics of squeezing in tunnelling see Kovári (1998) and Barla (2002).

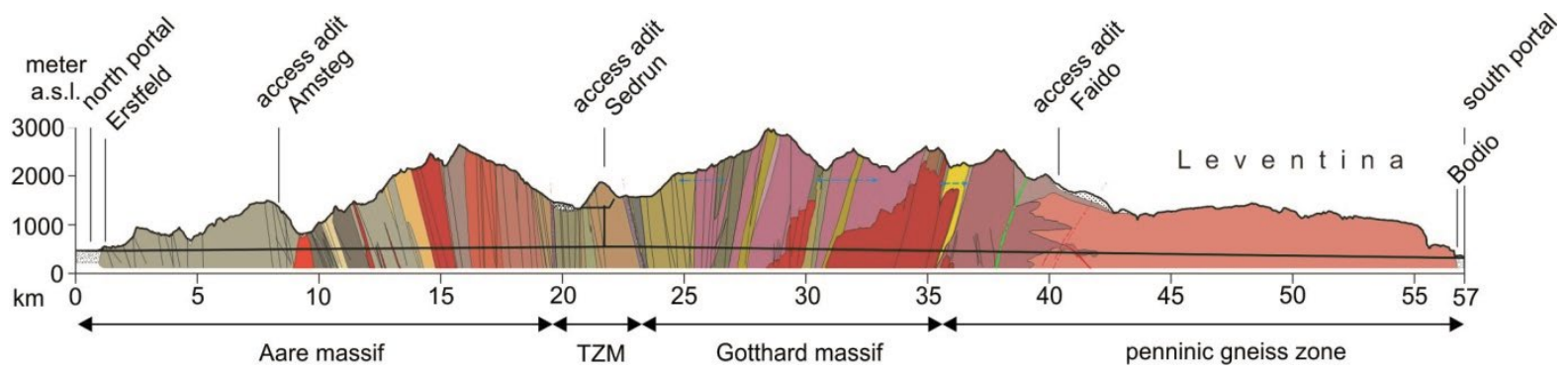

Figure 1.1. Longitudinal geological profile of the Gotthard Base Tunnel (from SKH Geologen AG 2011).

The $57 \mathrm{~km}$ long Gotthard Base Tunnel is the core of the AlpTransit project (Kovári et al. 1999). The project offers the possibility of moving the majority of the goods traffic crossing the Alps from road to rail and guarantees the connection of Switzerland to the European high-speed railway network for passenger traffic. The tunnel crosses the Aare massif, the Tavetsch-Massif, the Gotthard massif and the penninic gneiss zone (Fig. 1.1) from north to south. These tectonic units consist predominantly of granites, gneisses and schists (Kovári et al. 1999). The present chapter focuses on the Clavaniev Zone (abbreviated to CZ, Fig. 1.2) and on the Intermediate Tavetsch-Massif (abbreviated to TZM, Fig. 1.2), where heavily squeezing conditions were expected in the planning phase and also encountered during construction.

1 This chapter has been published in: Mezger, F., Anagnostou, G., Ziegler, H. J. (2013). The excavationinduced convergences in the Sedrun section of the Gotthard Base Tunnel. Tunnelling and Underground Space Technology, Vol. 38: 447-463. 


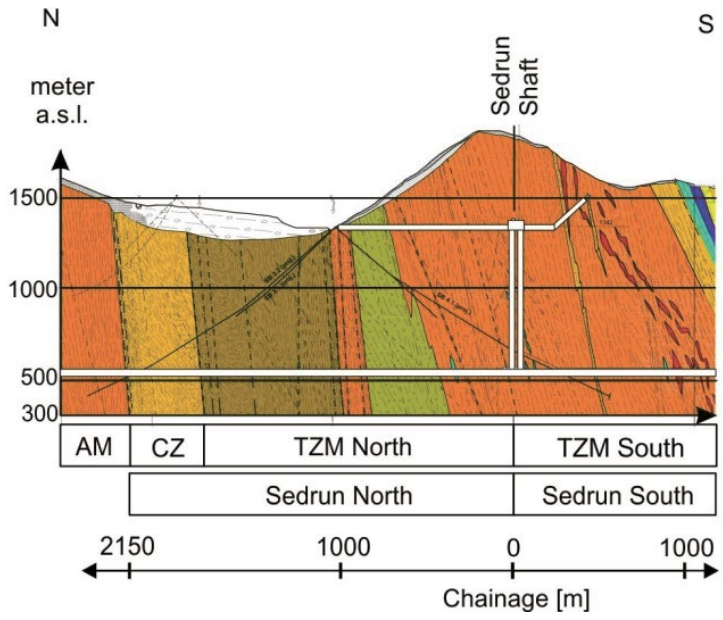

Figure 1.2. Detail of the geological profile of the Gotthard Base Tunnel (from Guntli and Weber 2009).

The aim of this chapter is to identify factors that have a significant influence on the convergences and might be used as indicators during construction for the timely identification of squeezing conditions. The chapter starts with a concise overview of the available data concerning the geology, the excavation and support and the rock response to tunnelling (Sections 1.2 to 1.4) and then seeks for empirical correlations between the deformations observed during construction and the lithological and structural features of the rock mass (Section 1.5). The analysed tunnel section crosses the northern TZM and the Clavaniev Zone, hereafter referred to as "Sedrun North" (Fig. 1.2), and includes both the north-western and north-eastern tubes. The two tubes are separated by a centreline distance of 50 to $70 \mathrm{~m}$. Section 1.5 demonstrates that the observed convergence correlates reasonably well with the degree of shearing and the schistosity orientation of the rock. In addition, Section 1.5 discusses the effect of nearby zones of more or less competent rock (Kovári and Anagnostou 1995, Cantieni and Anagnostou 2007) as well as the usefulness of the displacement vector orientation for predictions (Steindorfer 1998). Finally, Section 1.6 checks the predictive capability of the empirical correlations obtained in Section 1.5 by calibrating them, based on the observations in a part of the tunnel, applying them to the remaining stretch of tunnel and comparing the empirical predictions with the measured deformations. Section 1.6 shows that the comparison is satisfactory and concludes that the empirical relationships in combination with advance probing are in fact very useful for estimating the squeezing intensity ahead of the tunnel face.

The present chapter evolved within the framework of a long-term research programme at the ETH Zurich on squeezing ground. It is closely related in particular to the work of Cantieni et al. (2011), which examined the possibility of predicting ground response to tunnelling on the basis of the axial extrusion of the core ahead of the face. Cantieni et al. (2011) also analysed the monitoring data from the construction of the western tube of the Gotthard Base Tunnel. However, they could not find a clear correlation that would allow them to predict convergences with sufficient reliability on the basis of extrusion monitoring alone and proposed evaluating extrusion data in combination with other information, such as advance probing. 


\subsection{Geology}

The Gotthard Base Tunnel crosses the Clavaniev Zone and the northern TZM over a length of $285 \mathrm{~m}$ and $793 \mathrm{~m}$, respectively. The depth of cover is about $800 \mathrm{~m}$. The tectonic units consist of different rock types: Gneisses alternate with steeply inclined layers composed of soft phyllites and schists, which have a thickness in the range of decimetres to decametres (Kovári et al. 1999). The major part of these units consists of so-called kakiritic rocks, i.e. rocks that are systematically interspersed with shear planes filled with rock fragments (fault breccia) or more finely ground material (fault gouge). In general, the term "kakirite" denotes "a broken or intensively sheared rock, which has lost a large part of its original strength" (Schneider 1997). Vogelhuber (2007) and Anagnostou et al. (2008) performed a total of 112 consolidated drained and undrained triaxial tests to obtain the strength parameters of the kakiritic rocks in the Sedrun section. Depending on the development of the failure surface, a distinction is made between anisotropic and isotropic failure to evaluate the strength parameters. Anisotropic failure occurs when the failure surface develops through the existing discontinuity, e.g. through a plane of schistosity. Figure 1.3 shows the strength parameters determined from triaxial tests. The friction angles are between 25 and $30^{\circ}$ and the cohesionvalues between 200 and $600 \mathrm{kPa}$. Figure 1.3 considers only samples with isotropic failure. In the case of anisotropic failure, the friction angle was about $25^{\circ}$ and the cohesion-values were mostly below $200 \mathrm{kPa}$.

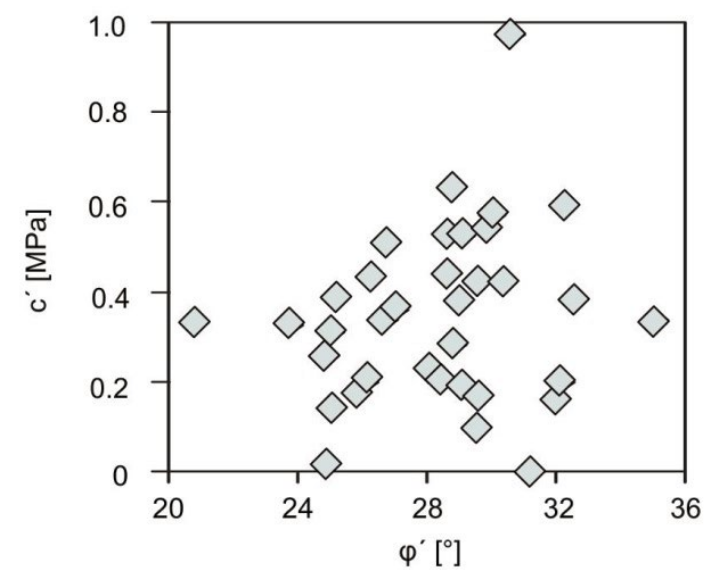

Figure 1.3. Cohesion $c$ and angle of internal friction $\varphi$ of samples with isotropic failure (after Anagnostou et al. 2008).

The Clavaniev Zone is located at the southern boundary of the Aare massif and was intensively sheared and strongly deformed tectonically during the alpine orogeny (Schneider 1997). The degree of kakiritization is variable. About $67 \%$ of the rocks in the northern TZM and over $95 \%$ of the rocks in the Clavaniev Zone may be designated as kakirites. The rest of the gneisses and slates are at least interspersed with irregular hairline cracks. The weak, kakiritic rocks in the encountered section of the tunnel are saturated but have a very low permeability $\left(k=10^{-8} \mathrm{~m} / \mathrm{s}\right.$ to $10^{-10} \mathrm{~m} / \mathrm{s}$ according to Vogelhuber 2007$)$.

The following section provides an overview of the available information on the encountered geology based on the data in the integrated web platform of the Gotthard Base Tunnel project (SISO 2012) and the synthesis report by Guntli and Weber (2009). Some lithologic 
and structural characteristics of the rock (lithological type, degree of shearing and schistosity), which were deemed to be important for its response to tunnelling, have been codified using project-specific classifications, which are presented in Figures 1.4 to 1.8 and are discussed below.

Table 1.1. Rock mass classification on the basis of the degree of shearing $F$ (after Guntli and Weber 2009).

\begin{tabular}{|c|c|}
\hline $\begin{array}{l}\text { Degree of shearing } \\
\text { F }\end{array}$ & Description \\
\hline 1 & Competent \\
\hline 2 & Sporadic shear fractures, slickensides \\
\hline 3 & Schistous and laminated rocks, mylonites, phyllites \\
\hline 4 & $\begin{array}{l}\text { Sheared, fractured rocks (portion of rock flour }<10 \% \text {, disturbed over }<25 \% \text { of the tunnel } \\
\text { face surface) }\end{array}$ \\
\hline 5 & $\begin{array}{l}\text { Sheared, crumbly, friable rocks (portion of rock flour } 10-30 \% \text {, disturbed over }>25 \% \text { of } \\
\text { the tunnel face surface) }\end{array}$ \\
\hline 6 & $\begin{array}{l}\text { Rocks with a portion of rock flour }>30 \% \text { and plastic consistency. It can be deformed by } \\
\text { hand and disturbed over the majority of the tunnel face surface. }\end{array}$ \\
\hline
\end{tabular}

During advance, the ground was classified into rock types based upon both the lithology and the degree of shearing $F$, which was introduced as a project-specific measure of the tectonic disturbance of the rock mass. Six classes for the degree of shearing were defined (Table 1.1) according to the fraction of rock powder, resulting from the failure of the rocks during their tectonic overstressing in the geologic past. The lithological types $T$ are presented in Table 1.2. The quality of the intact rock (on the scale of a specimen) decreases from lithological type 1 (which includes the strongest units, such as amphibolites or quartzites) to lithological type 9 (completely kakiritized, fine grained material). The last two types in Table 1.2 do not represent lithological types in the narrow sense, but have been included because heavily sheared rocks on account of their nature (almost engineering soil) can be seen as another lithological type.

Table 1.2. Rock mass classification on the basis of the lithology (after Guntli and Weber 2009).

\begin{tabular}{ll}
\hline Lithological type & Description \\
\hline$T$ & Pegmatites, amphibolites, quartzites \\
2 & Quartz- and feldspar-rich gneisses, migmatites \\
3 & Striped gneisses \\
4 & Gneisses with a high content of mica, dolomites \\
5 & Gneisses with a high content of schists \\
6 & Schists \\
7 & Phyllites \\
8 & Kakirites (fault gouge) \\
9 & Kakirites with high plasticity and high percentage of fines \\
\hline
\end{tabular}


NE tube

(a)

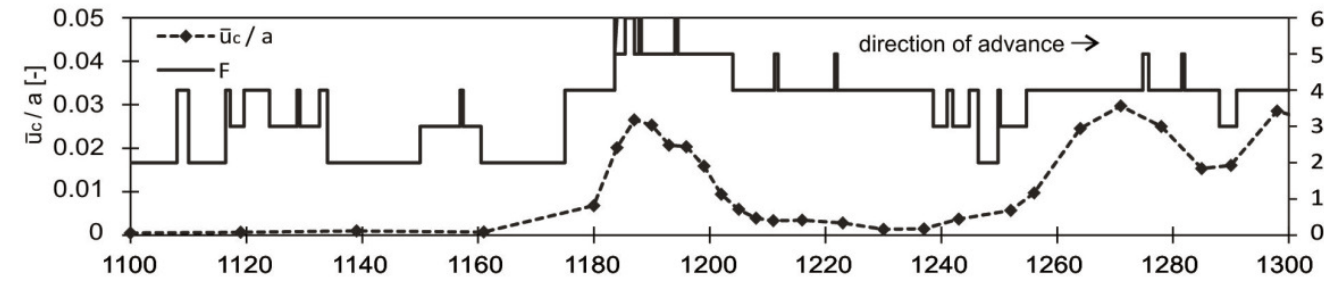

(b)

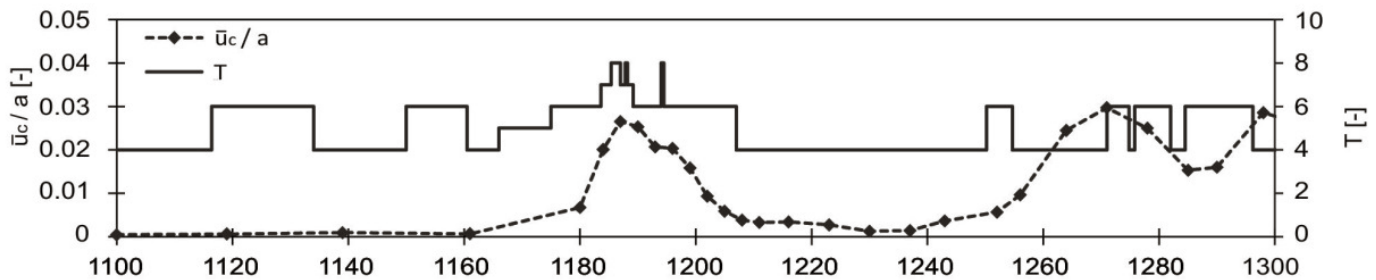

(c)

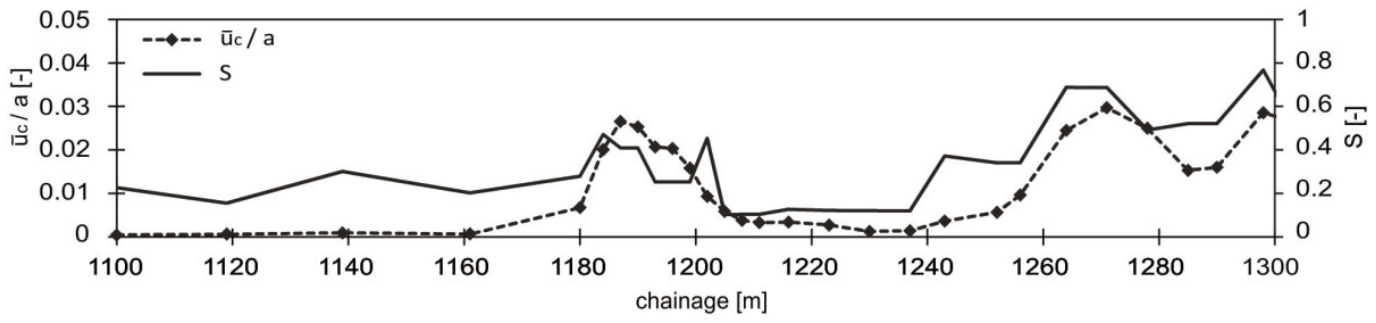

NW tube

(a)

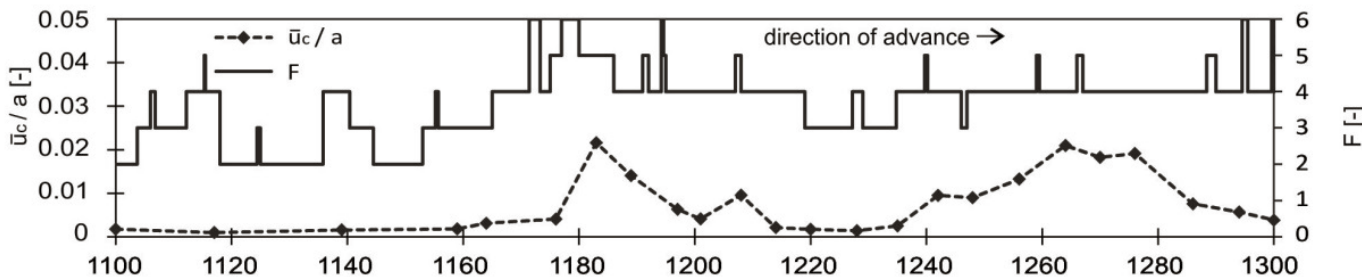

(b)

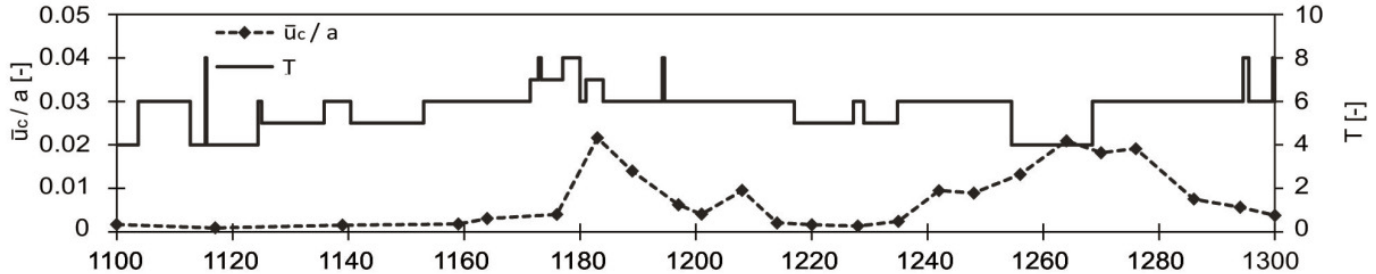

(c)

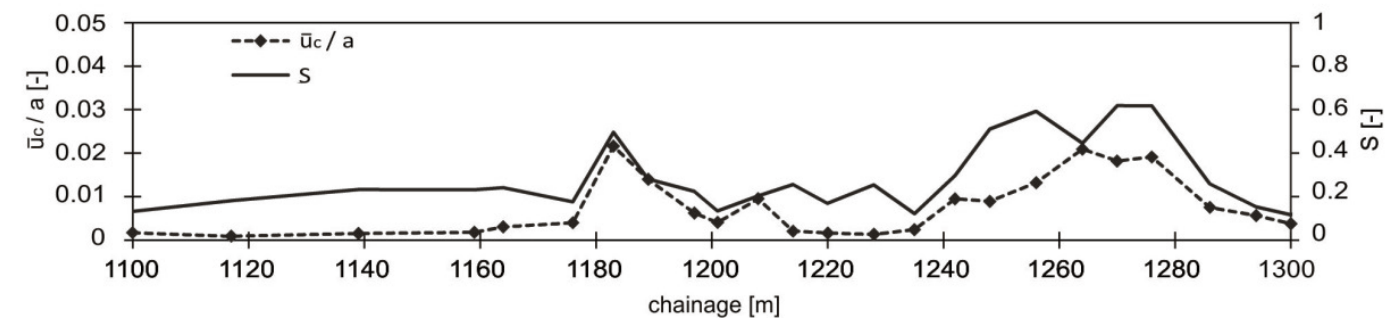

Figure 1.4. Data for chainage 1100 to $1300 \mathrm{~m}$. Average magnitude of the projections of the displacement vectors in the cross-sectional plane of the tunnel normalised by the tunnel radius $\left(\bar{u}_{c} / a\right)$ along the tunnel as well as, (a), degree of shearing $F,(b)$, lithological type $T$ and, (c), schistosity influence factor $S$ (evaluation based upon the data from SISO 2012 and Guntli and Weber 2009). 
NE tube

(a)

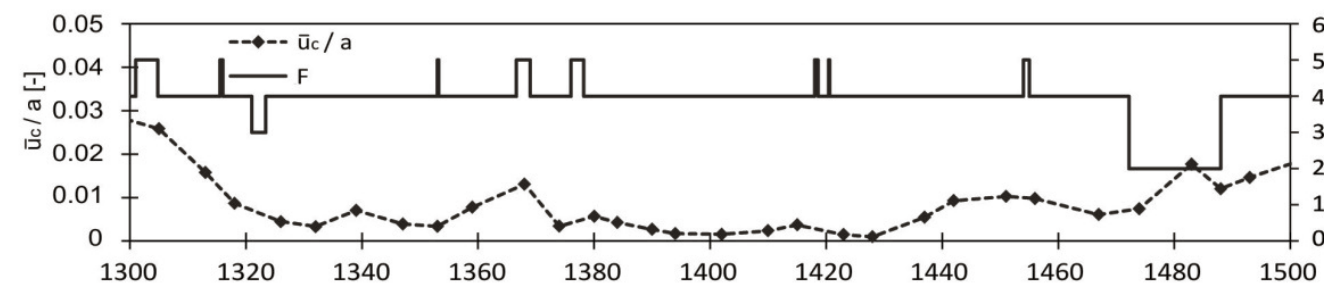

(b)

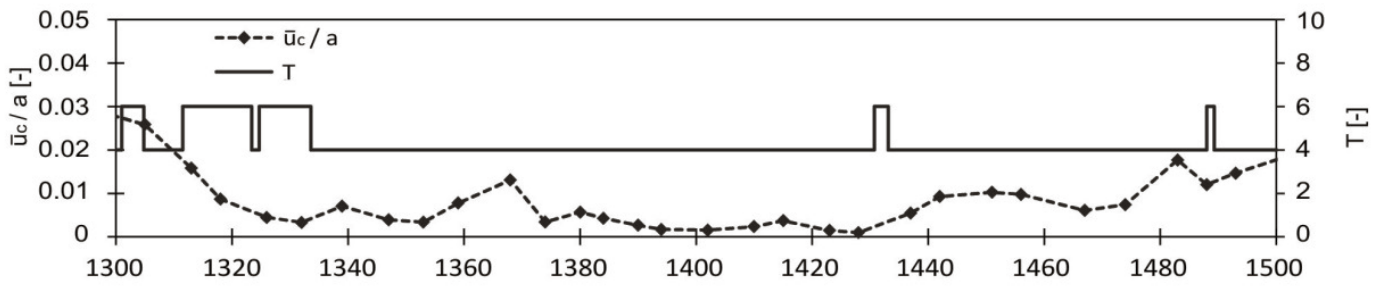

(c)

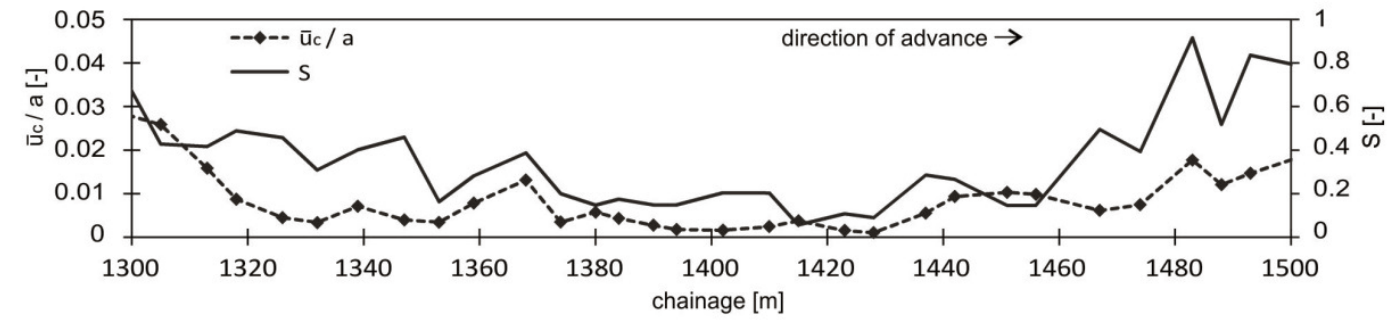

NW tube

(a)

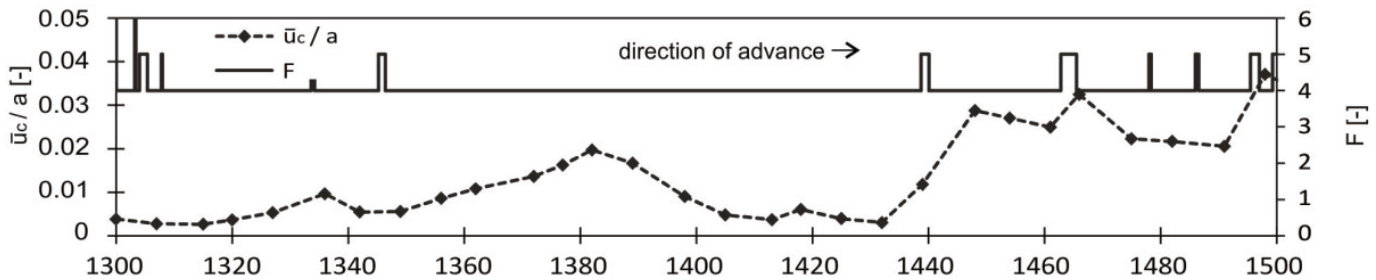

(b)

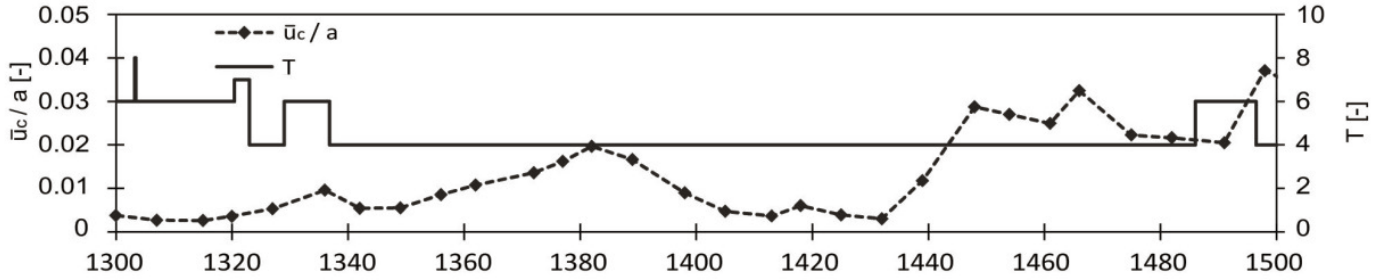

(c)

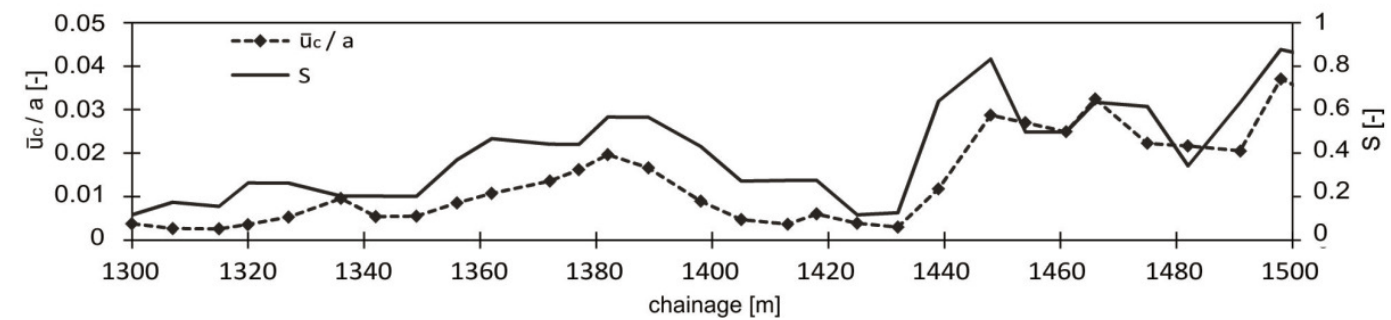

Figure 1.5. Data for chainage 1300 to $1500 \mathrm{~m}$. Average magnitude of the projections of the displacement vectors in the cross-sectional plane of the tunnel normalised by the tunnel radius $\left(\bar{u}_{c} / a\right)$ along the tunnel as well as, (a), degree of shearing $F,(b)$, lithological type $T$ and, (c), schistosity influence factor $S$ (evaluation based upon the data from SISO 2012 and Guntli and Weber 2009). 
NE tube

(a)

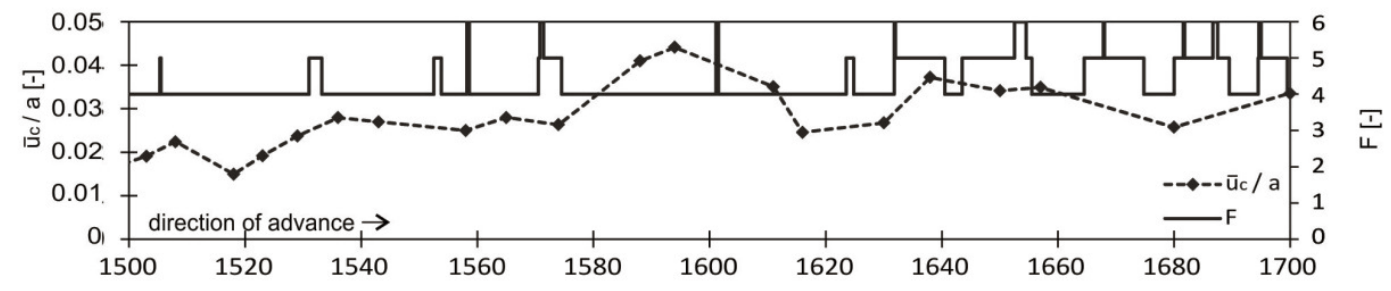

(b)

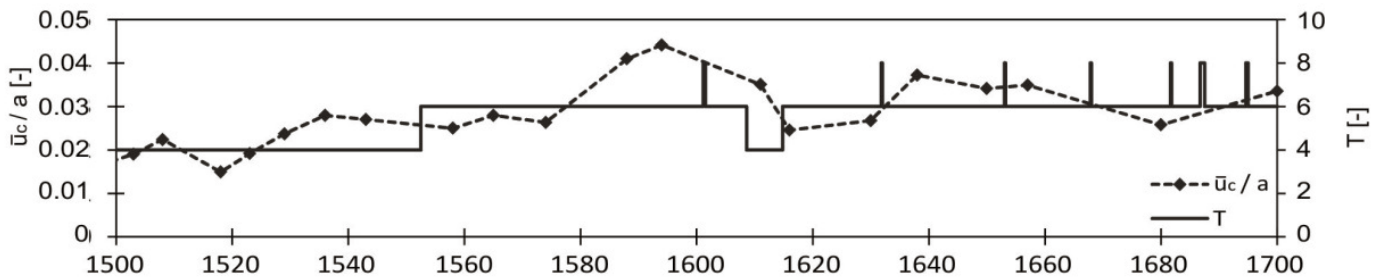

(c)

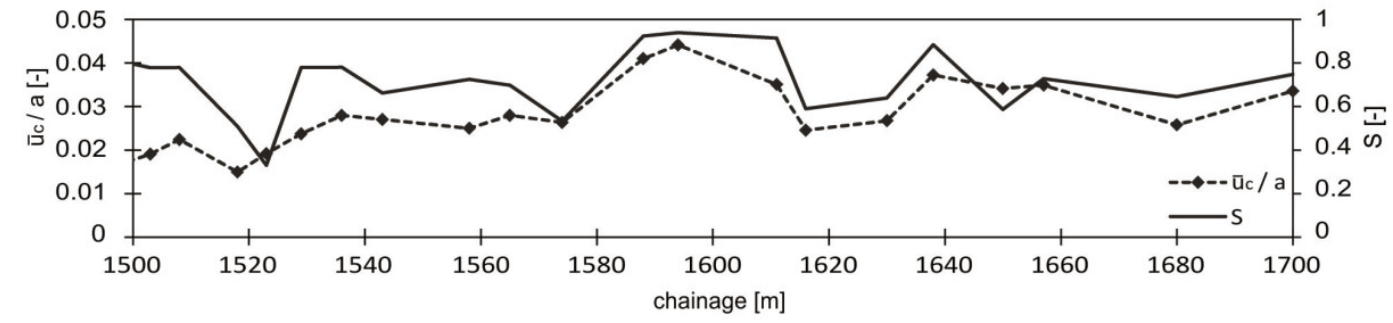

NW tube

(a)

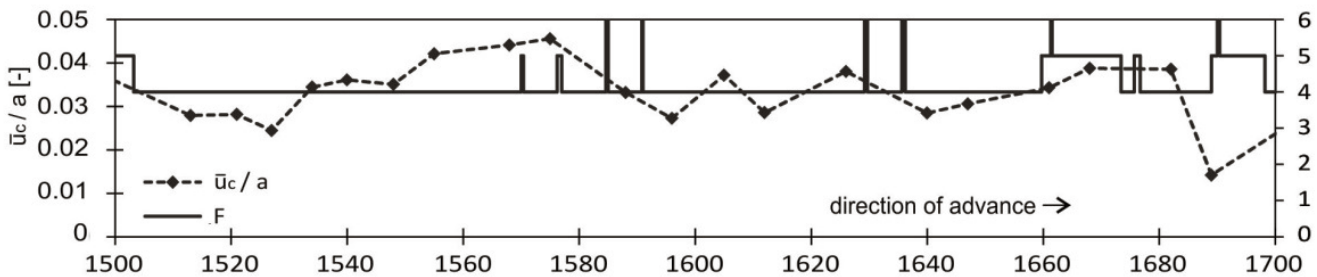

(b)

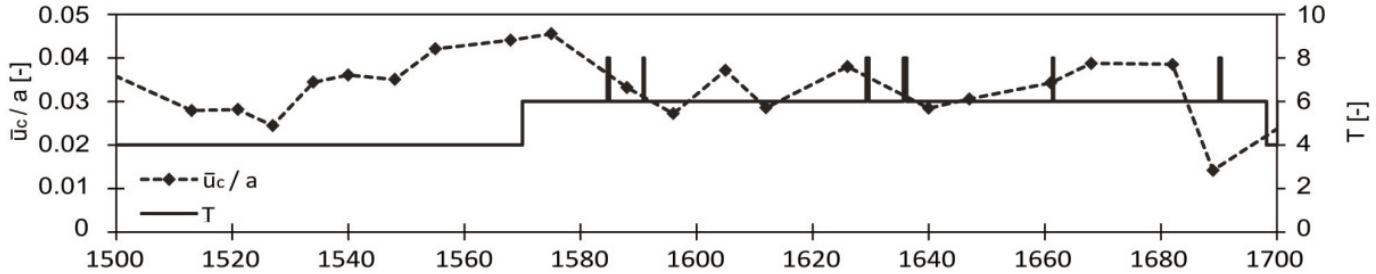

(c)

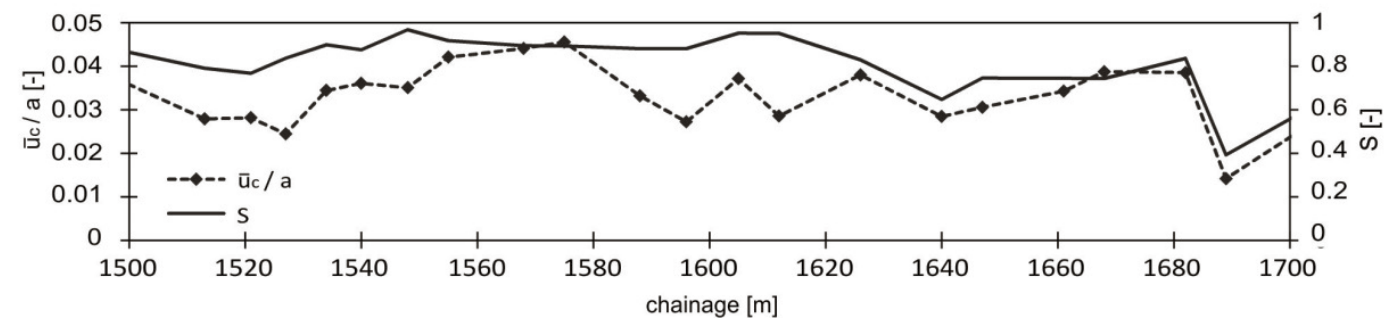

Figure 1.6. Data for chainage 1500 to $1700 \mathrm{~m}$. Average magnitude of the projections of the displacement vectors in the cross-sectional plane of the tunnel normalised by the tunnel radius $\left(\bar{u}_{c} / a\right)$ along the tunnel as well as, (a), degree of shearing $F,(b)$, lithological type $T$ and, (c), schistosity influence factor $S$ (evaluation based upon the data from SISO 2012 and Guntli and Weber 2009). 
NE tube

(a)

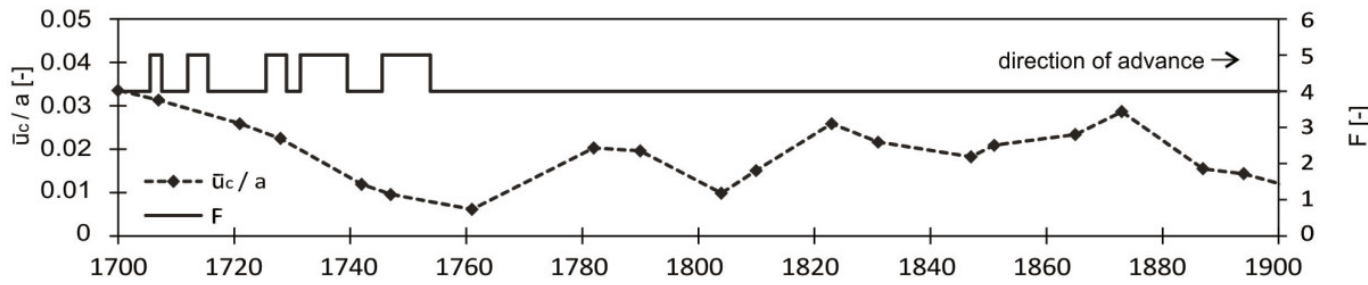

(b)

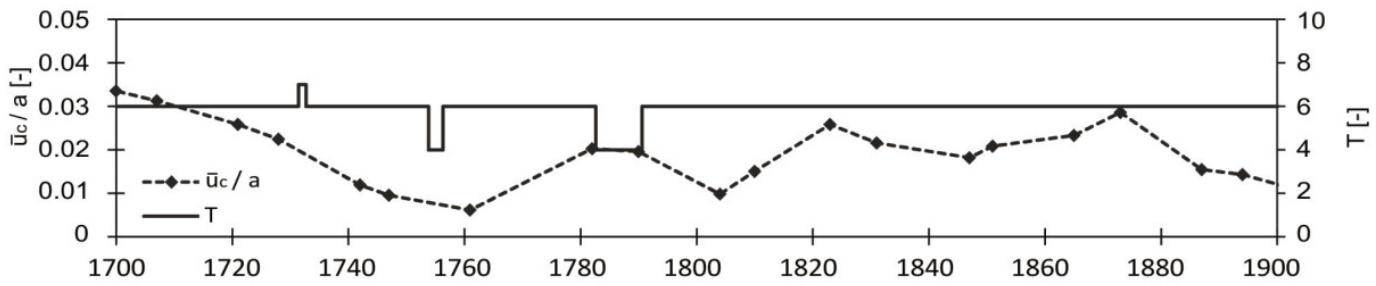

(c)

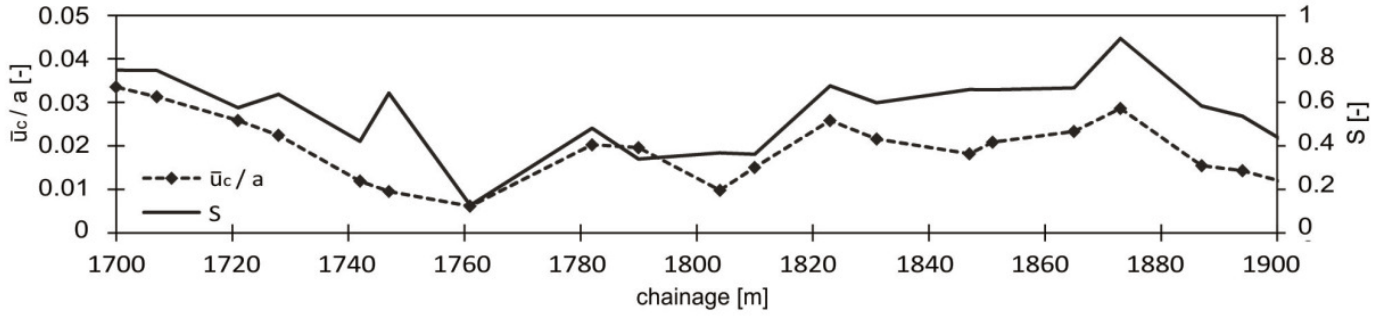

NW tube

(a)

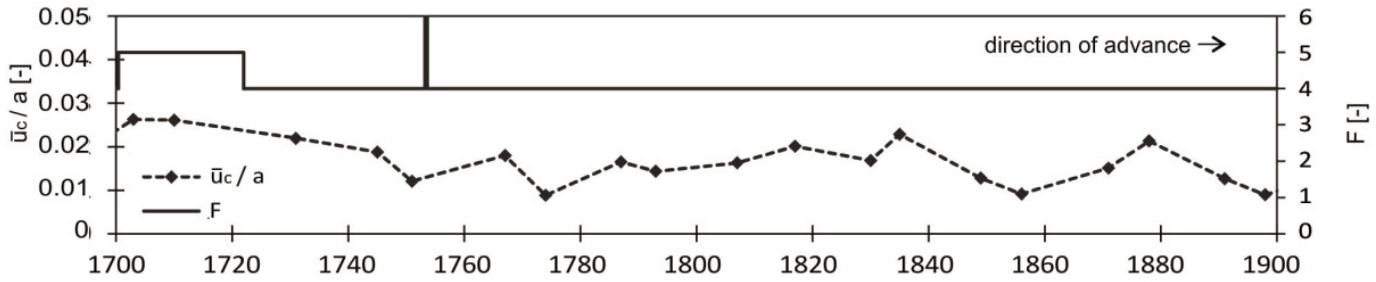

(b)

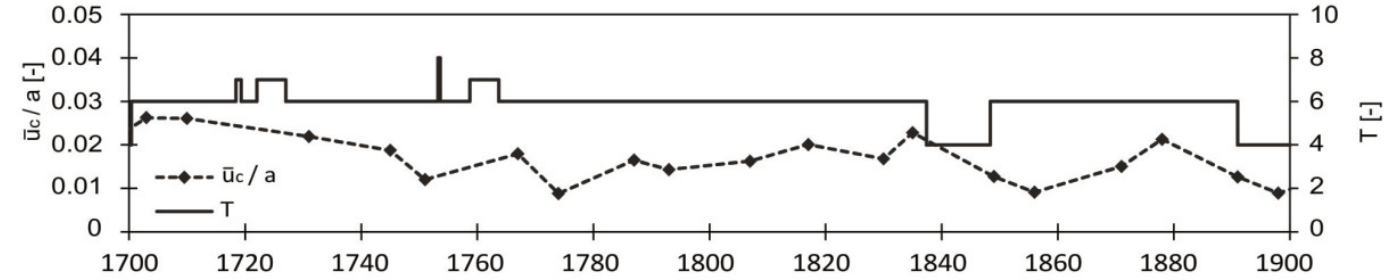

(c)

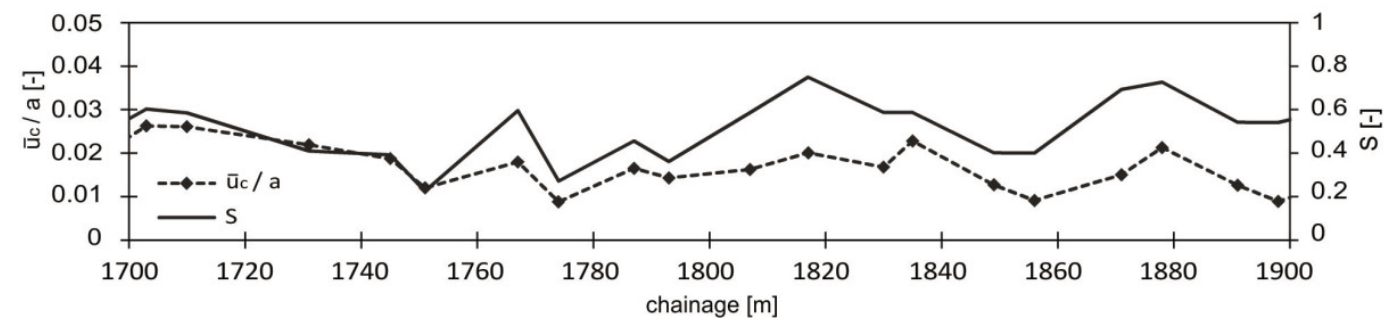

Figure 1.7. Data for chainage 1700 to $1900 \mathrm{~m}$. Average magnitude of the projections of the displacement vectors in the cross-sectional plane of the tunnel normalised by the tunnel radius $\left(\bar{u}_{c} / a\right)$ along the tunnel as well as, (a), degree of shearing $F,(b)$, lithological type $T$ and, (c), schistosity influence factor $S$ (evaluation based upon the data from SISO 2012 and Guntli and Weber 2009). 
NE tube

(a)

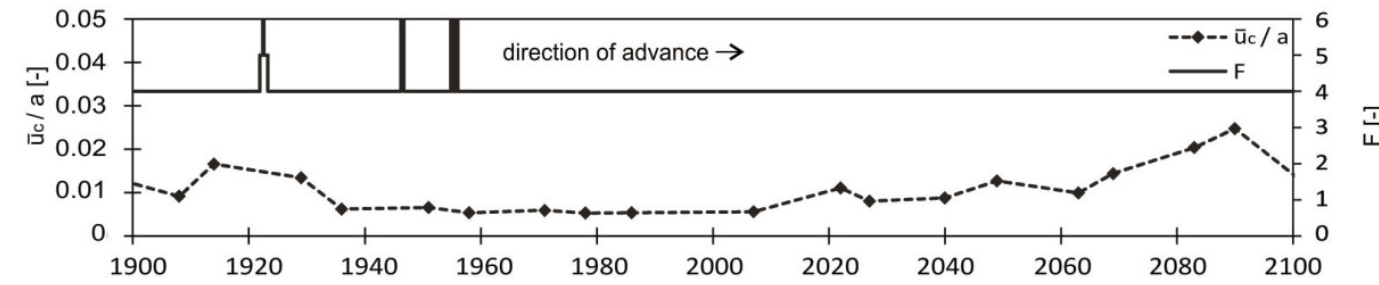

(b)

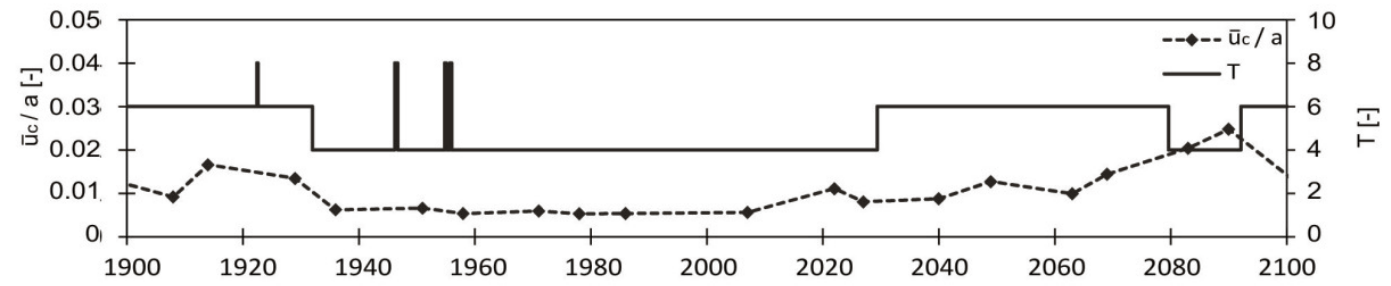

(c)

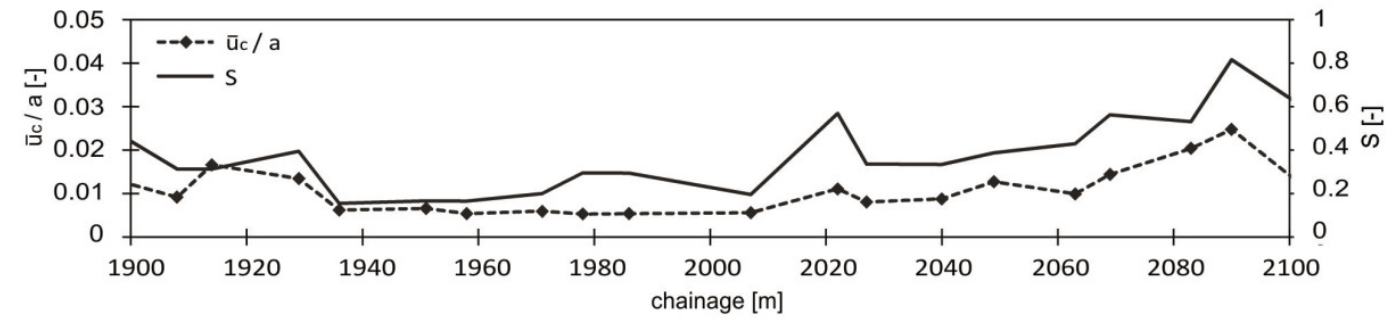

NW tube

(a)

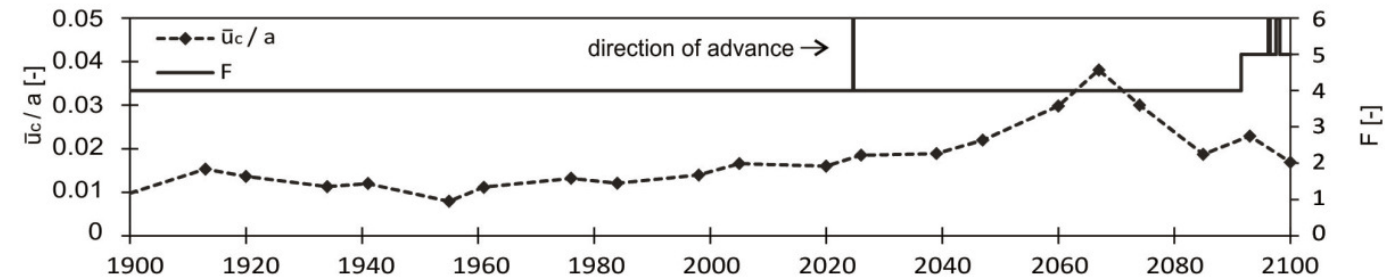

(b)

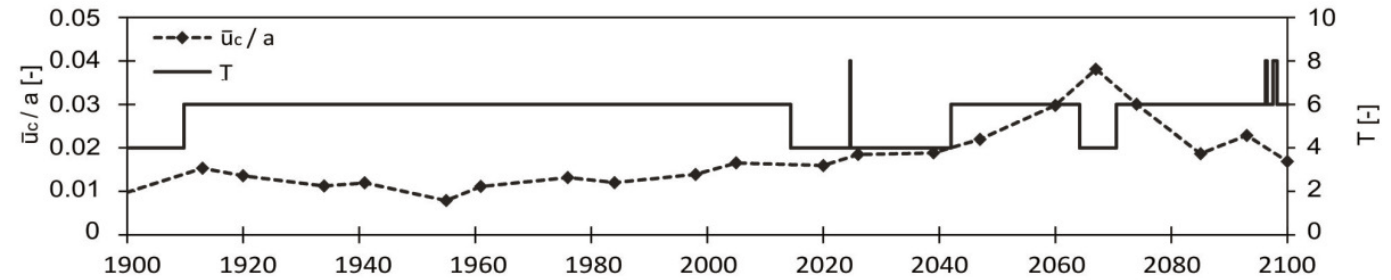

(c)

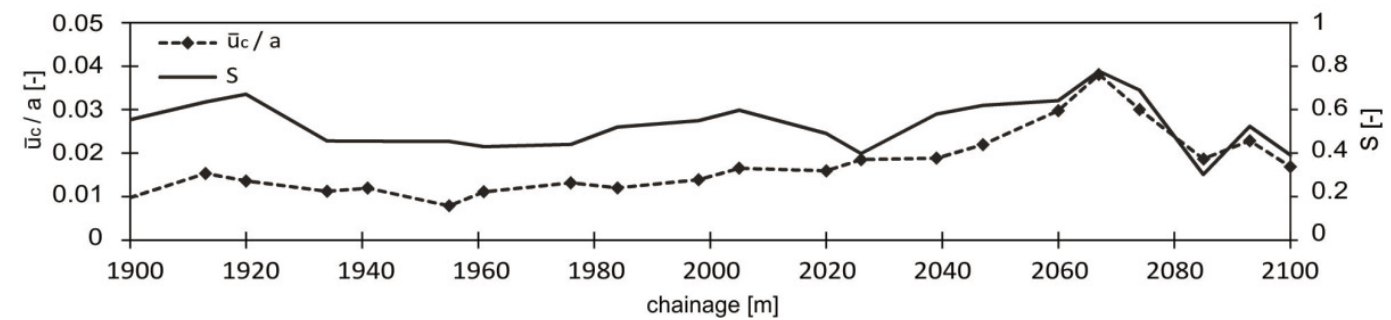

Figure 1.8. Data for chainage 1900 to $2100 \mathrm{~m}$. Average magnitude of the projections of the displacement vectors in the cross-sectional plane of the tunnel normalised by the tunnel radius $\left(\bar{u}_{c} / a\right)$ along the tunnel as well as, (a), degree of shearing $F,(b)$, lithological type $T$ and, (c), schistosity influence factor $S$ (evaluation based upon the data from SISO 2012 and Guntli and Weber 2009). 
The discontinuities of the rock were recorded during tunnel advance both with respect to the surfaces of schistosity and to the jointing. During advance it became evident that the degree of shearing $F$ and the lithological type $T$ are somehow connected with one other: in general, the higher the rock quality on the specimen scale, the smaller the degree of shearing.

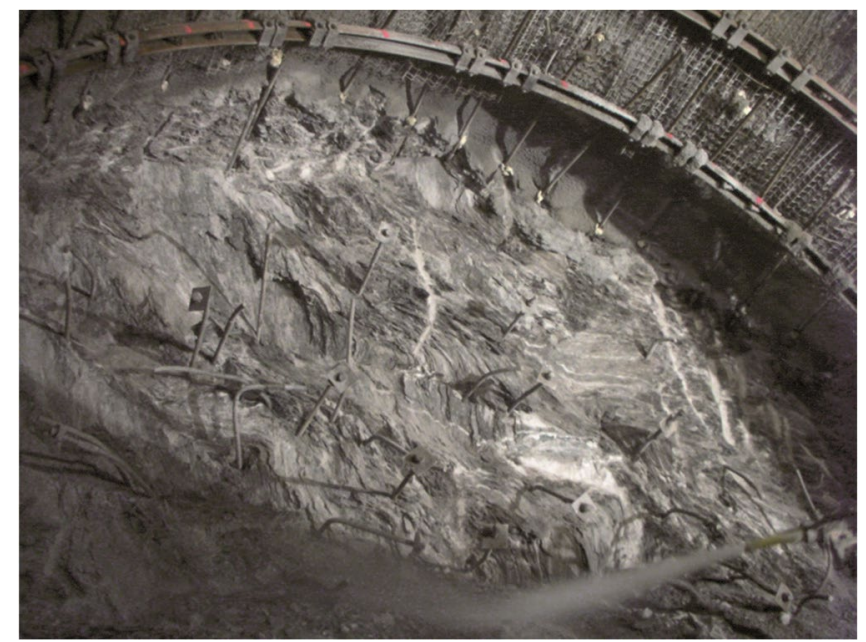

Figure 1.9. Photograph of the tunnel face at a location with about $3 \%$ convergence and degree of shearing 4 (chainage $1300 \mathrm{~m}$ of the NE tube, from SISO 2012).

The alpine schistosity is clearly recognizable over major portions of Sedrun North and this even in strongly kakiritic reaches (Guntli and Weber 2009). In general, the strata dip steeply towards the north but are strongly disturbed by more recent shearing deformations. In fault zones, the intensive shearing (kakiritization) governs the behaviour of the rock mass.

(a)
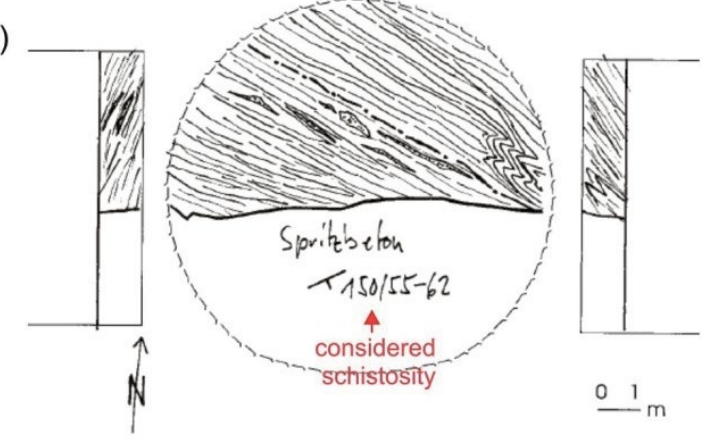

(b)

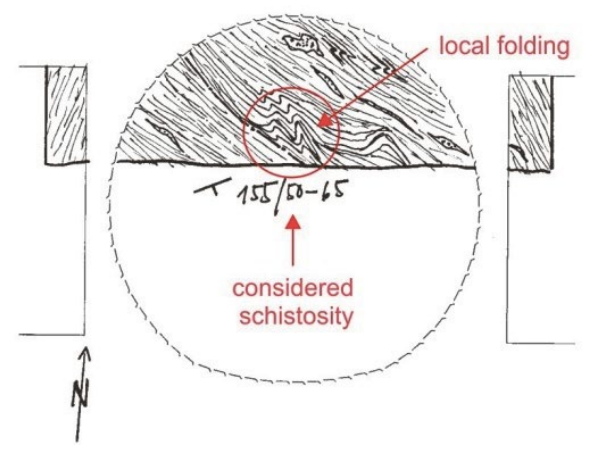

Figure 1.10. Geological mappings of the tunnel face with traces of the schistosity planes. (a) NW tube, chainage $1535 \mathrm{~m}$; (b) NE tube, chainage $1202 \mathrm{~m}$ (after Guntli and Weber 2009).

However, since this shearing did not lead to a complete homogenisation of the rock mass, the older rock structure between these shear zones has been preserved (Guntli and Weber 
2009). Thus, schistosity is still an important structural feature of the rock in the present case (Figs. 1.9 and 1.10). The so-called "schistosity influence factor" presented in Figures $1.4 \mathrm{c}$ to $1.8 \mathrm{c}$ is introduced in Section 1.5.2. It accounts for the orientation of the schistosity planes and combines their dip angle and dip direction in a single number.

Depending on the thickness of the beds, which were developed as a result of the schistosity, the rock mass was classified into classes from "schistous to phyllitic" (thickness $<0.5 \mathrm{~cm}$ ) to "not bedded" (thickness $>100 \mathrm{~cm}$ ). Over $40 \%$ to $50 \%$ of the tunnel in the northern TZM and the Clavaniev Zone was assigned to the class "schistous to phyllitic" (Guntli and Weber 2009).

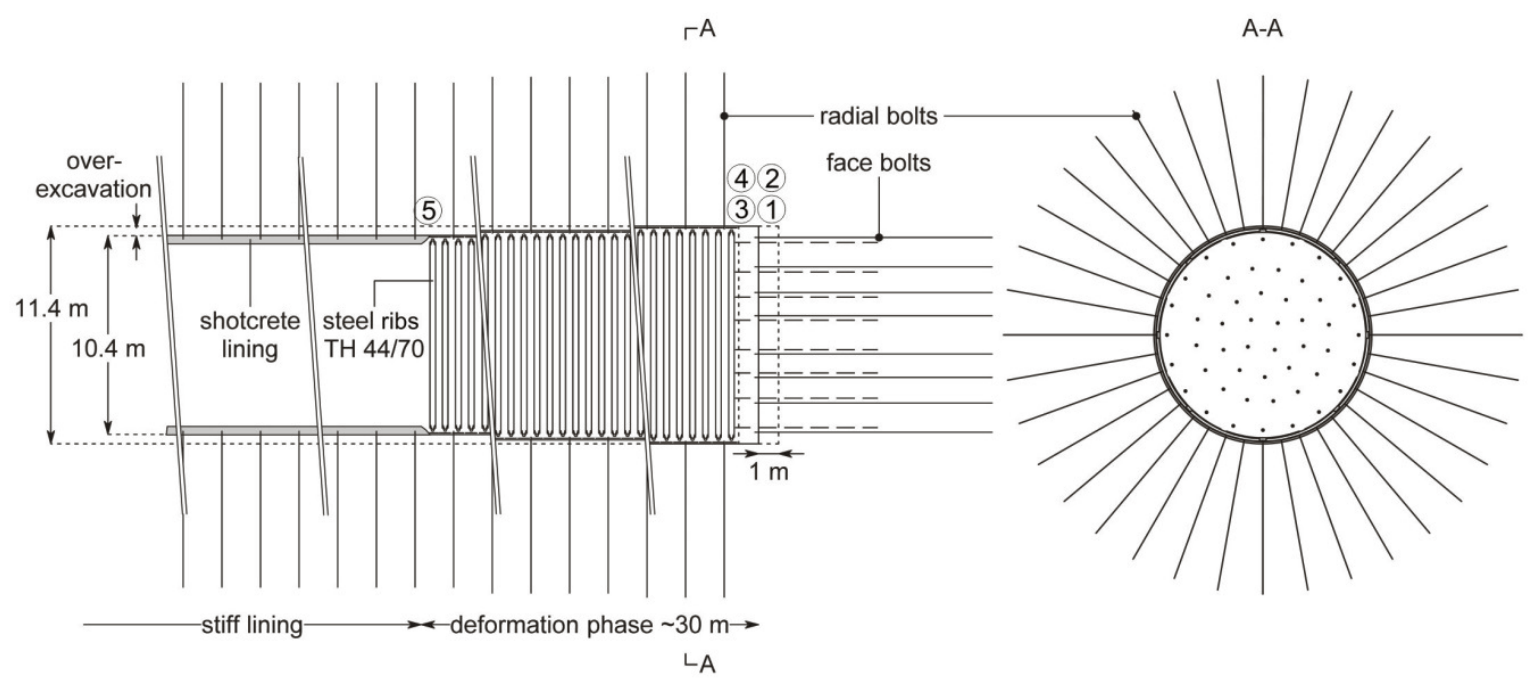

Figure 1.11. Longitudinal section and cross section of the yielding support system (after Ehrbar and Pfenninger 1999) and sequence of applying the support: 1: Excavation, 2: Sealing of the working area, 3: Installation of the steel ribs, 4: Installation of the radial bolts, 5: Application of the shotcrete ring.

As a consequence of the kakiritization of the rock and of the schistosity, the development of jointing was small. Only in the weakly kakiritized rock were small joints or hairline cracks present. The jointing in the TZM and the Clavaniev Zone was described as small for more than $72 \%$ of the tunnel length (Guntli and Weber 2009).

\subsection{Construction Method}

Due to the known presence and poor mechanical characteristics of kakiritic rocks, heavily squeezing conditions were expected for Sedrun North. Therefore, a circular tunnel crosssection in combination with full-face excavation and yielding support was chosen (Figs. 1.11 and 1.12a). The basic idea behind this concept has been explained by Kovári (1998): Fullface excavation makes it possible to have a statically favourable profile right from the start. The yielding support, which consists of sliding steel ribs connected by friction loops (Fig. 1.12b), reduces the rock pressure to a manageable level (Kovári et al. 2000). With this method, deformations could occur, while providing continuous support of the rock. An overexcavation of 0.1 to $0.7 \mathrm{~m}$ (in radius) was foreseen in order to accommodate the convergences without impairing the necessary clearance profile. The steel ribs used were $\mathrm{TH} 44 / 70$. In heavily squeezing rock, the steel ribs were spaced at $0.33-0.66 \mathrm{~m}$, which leads to a steel quantity of up to 9.4 tons per linear metre (Kovári et al. 1999). Additionally, fully grouted bolts with a length of 8 to $12 \mathrm{~m}$ were installed (Fig. 1.11). 
(a)

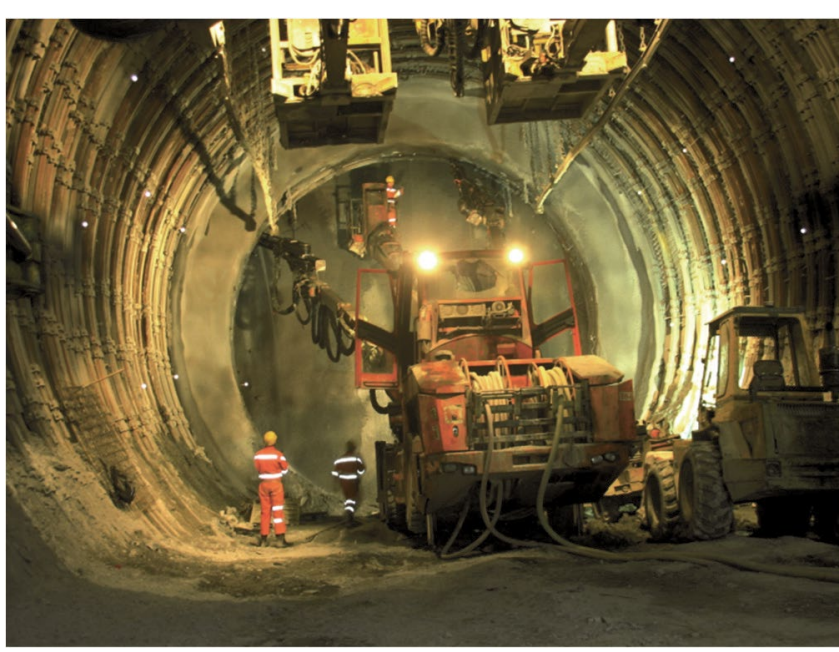

(b)

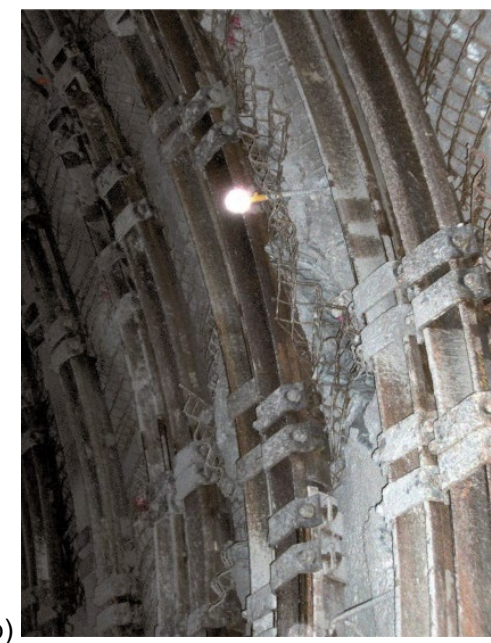

Figure 1.12. (a) Tunnelling works at chainage $2155.5 \mathrm{~m}$ (from SISO 2012); (b) Support detail with steel ribs and friction loops (from SISO 2012).

After the rate of convergence slowed down a 0.3 to $0.6 \mathrm{~m}$ thick shotcrete ring was applied. This was usually at a distance of about $30 \mathrm{~m}$ behind the tunnel face (approximately 1 month after excavation). In less squeezing ground, a stiff support was installed right from the start according to the so-called resistance principle (Kovári 1998).

NE tube

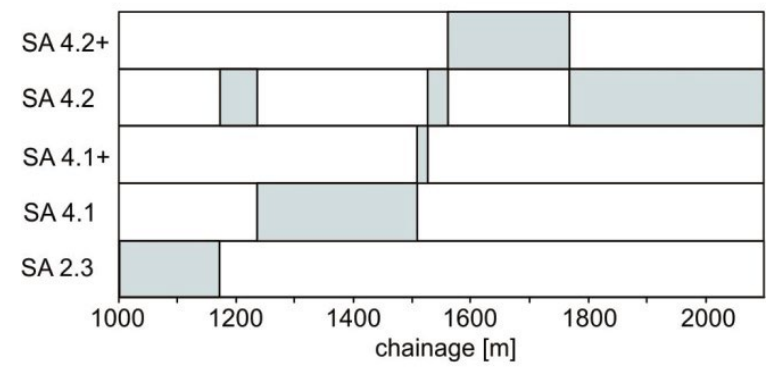

NW tube

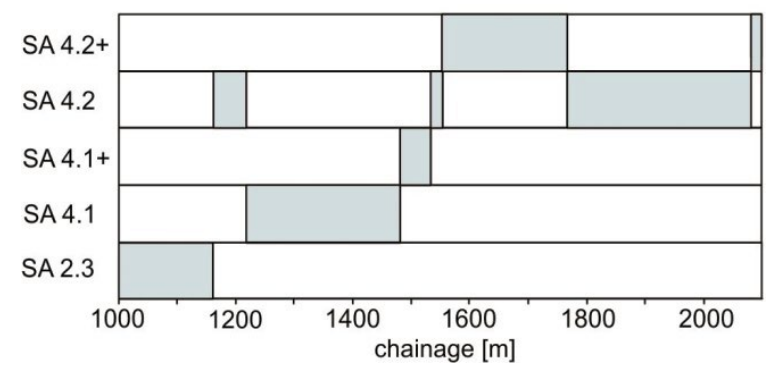

Figure 1.13. Distribution of the applied support classes along the tunnel (after Guntli and Weber 2009).

To ensure stability of the tunnel face the ground ahead of the tunnel face was reinforced using 40 to $6012 \mathrm{~m}$-long steel bolts and steel fibre-reinforced shotcrete was applied to the face immediately after each excavation step. Additionally, systematic forepoling was used to prevent rock loosening and rock fall.

Table 1.3 and Figure 1.13 show the definition of the applied support classes, their distribution along the tunnel as well as the sequence of applying the support. The determination of the temporary support and of the excavation diameter during construction was based on the 
results of design calculations, the experience gained with the rock and support behaviour from the already excavated tunnel section and the findings from advance core borings. More specifically, 28 horizontal advance core borings of lengths between $31 \mathrm{~m}$ to $196 \mathrm{~m}$ were carried out during tunnel construction in order to explore the prevailing rock conditions ahead of the tunnel face and to obtain rock samples for triaxial testing (Anagnostou et al. 2008). Figure 1.14 shows, for example, the estimated degree of kakiritization based on the obtained core (bottom of Fig. 1.14) as well as the encountered degree of shearing $F$ after excavation (top of Fig. 1.14). Figure 1.14 shows that the shearing degree can be estimated on the basis of advance core drilling but is still subject to some uncertainty.

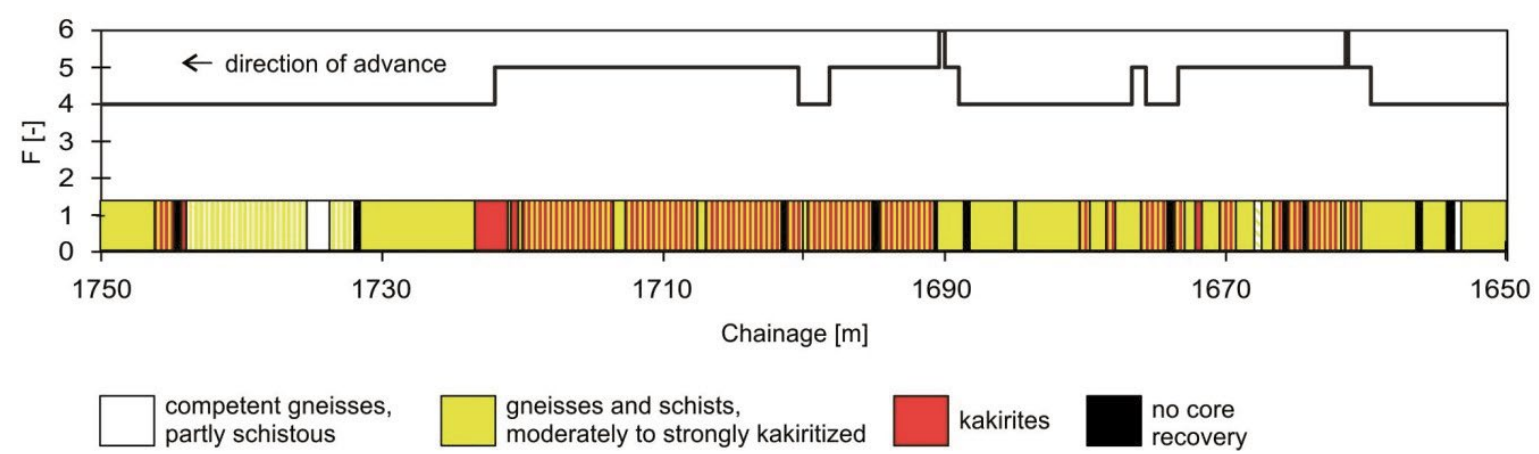

Figure 1.14. Comparison of the borehole findings (degree of kakiritization) with the encountered geology (degree of shearing $F$ ) of the chainage $1650 \mathrm{~m}$ to $1750 \mathrm{~m}$ of the NW tube (evaluation based upon the data from SISO 2012 and Guntli and Weber 2009).

Table 1.3. Definition of the support classes applied in Sedrun North (after Guntli and Weber 2009).

\begin{tabular}{|c|c|c|c|c|c|}
\hline Support class & SA 2.3 & SA 4.1 & SA 4.1+ & SA 4.2 & SA $4.2+$ \\
\hline Excavated radius $[\mathrm{m}]$ & 4.70 & 5.14 & 5.69 & 5.69 & 6.24 \\
\hline Over-excavation [cm] & 10 & 30 & 50 & 50 & 70 \\
\hline Length of round $[\mathrm{m}]$ & $1-2$ & $1-1.5$ & $1-1.34$ & 1.34 & 1 \\
\hline Type of steel ribs [-] & \multicolumn{3}{|c|}{ TH 29/70 } & \multicolumn{2}{|c|}{ TH 44/70 } \\
\hline Sliding resistance $[\mathrm{kN}]$ & \multicolumn{5}{|c|}{4 friction loops $\times 100 \mathrm{kN}=400 \mathrm{kN}$ per connection } \\
\hline Spacing of steel ribs $[\mathrm{m}]$ & $1.0-2.0$ & $1-1.5$ & $0.67-1.34$ & 0.67 & $\begin{array}{c}0.33 / 0.67- \\
1.0\end{array}$ \\
\hline Radial bolts, type [-] & \multicolumn{5}{|c|}{$\varnothing 25 \mathrm{~mm}, 320 \mathrm{kN}, \mathrm{S} 500$} \\
\hline Radial bolts, length [m] & 6 & $6-8$ & 8 & $8-12$ & $8-12$ \\
\hline Radial bolts, quantity [-] & $13-14$ & $17-28$ & $17-28$ & $11-25$ & 28 \\
\hline Face bolts, length [m] & 12 & 12 & $12-18$ & 12 & $12-18$ \\
\hline Face bolts, quantity [-] & 40 & 40 & 50 & 50 & 60 \\
\hline Forepoling, length [m] & 8 & 6 & $6-8$ & 6 & 6 \\
\hline Forepoling, quantity [-] & $0-25$ & $25-30$ & $0-51$ & If required & If required \\
\hline Thickness of the shotcrete ring $[\mathrm{cm}]$ & $20-25$ & $25-30$ & $25-30$ & $25-30$ & $25-60$ \\
\hline Thickness of the concrete lining $[\mathrm{cm}]$ & 30 & 30 & 30 & 60 & 60 \\
\hline
\end{tabular}

\subsection{Rock response to tunnelling}

In order to observe the behaviour of the rock and check the effectiveness of the tunnel support, a monitoring system with 3D optical measurements, radial extensometers, reverse- 
head-extensometers (RH-extensometers, Thut et al. 2006) and measuring anchors was implemented.

- The convergences of the tunnel boundary during tunnel advance were monitored optically at monitoring stations spaced every $5 \mathrm{~m}$ to $20 \mathrm{~m}$. Each monitoring station had 5 or 7 measuring points (Fig. 1.15). The displacements were measured at 165 monitoring stations in the NE tube and in 163 monitoring stations in the NW tube.

- 5 monitoring stations were instrumented with up to 5 radial extensometers of length 4-25 $\mathrm{m}$ to determine the extent of the rock zone around the tunnel affected by the excavation and thus the underlying rock deformation mechanism of the observed convergences. In some cases the measuring head was destroyed due to the large rock deformations so that no measurements were possible.

- To determine the load on the radial anchors, $4 \mathrm{~m}$ long measuring anchors were installed in 2 monitoring stations.

- The RH-extensometers served to observe the extrusion of the tunnel face as well as the axial deformation of the core ahead of the face.

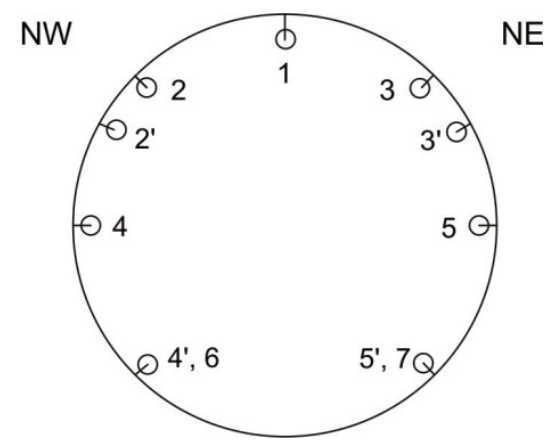

Figure 1.15. Monitoring station with 5 (numbers with apostrophes) or 7 measuring points (numbers without apostrophes).

The optical measurements were the most important means of observing the behaviour of the tunnel since they were installed frequently and systematically in contrast to the other measurements. For this reason, the present chapter analyses only the optical measurements (Sections 1.5 and 1.6). The results of the $\mathrm{RH}$-extensometers have already been discussed by Cantieni et al. (2011).

Figure 1.16a illustrates the typical development of the convergence. The diagram shows the vertical displacement $u_{r, 1}$ of the crown (normalised by its value $u_{r, 1, \infty}$ far behind the face) as a function of the distance to the tunnel face $d_{f}$. Each curve of the diagram corresponds to a different monitoring station. It is readily seen that the biggest portion of the convergence took place within two tunnel diameters behind the face and that significant long-term deformations (which were initially feared) did not occur (Kovári and Ehrbar 2008). The figure also shows that the shotcrete ring (which was applied at a distance of about $30 \mathrm{~m}$ behind the face) almost stopped the displacements.

Water inflows occurred only in the gneiss and weakly kakiritized sections of the formations, where hairline cracks allowed some water circulation. In the core boreholes small water quantities between $<0.1 \mathrm{l} / \mathrm{s}$ (dripping water) and $0.3 \mathrm{l} / \mathrm{s}$ were measured (Guntli and Weber 2009). 
The main phenomenon observed in Sedrun North was squeezing (Guntli and Weber 2009). Rock falls, which could also occur due to the presence of schistosity and kakiritization, hardly occurred at all.

(a)

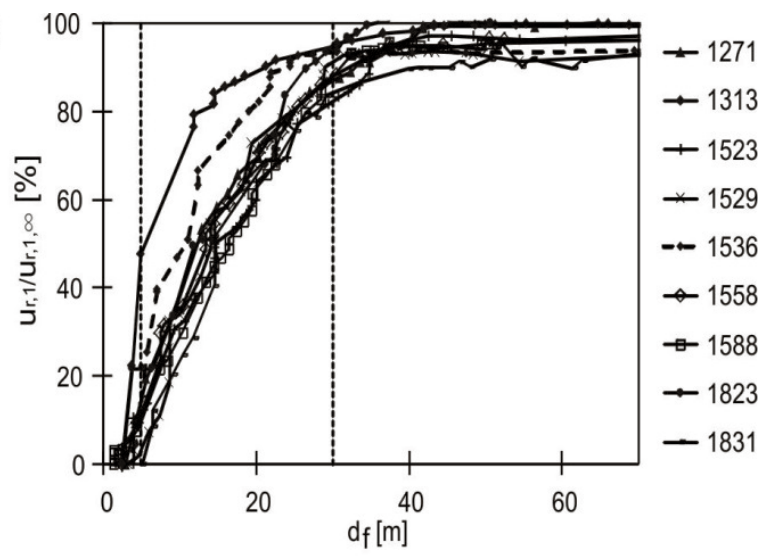

(b)

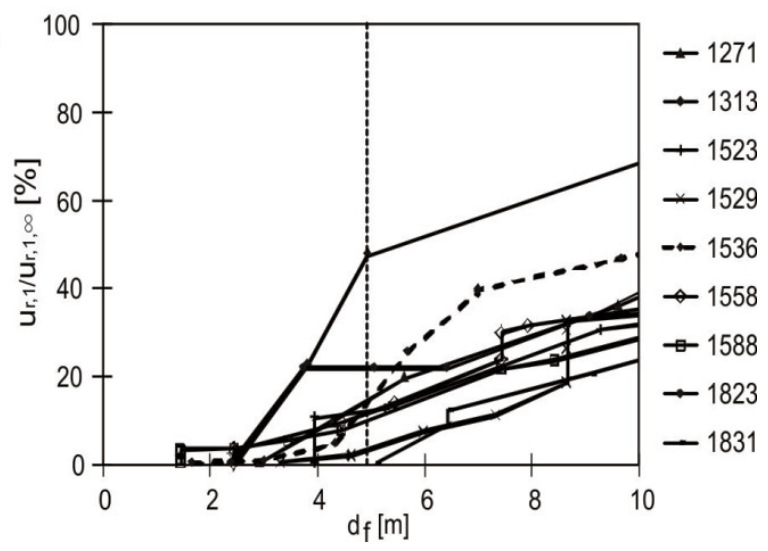

Figure 1.16. Vertical displacement $u_{r, 1}$ of the crown (measuring point 1 of Fig. 1.15) normalised by its value $u_{r, 1, \infty}$ far behind the face as a function of the distance to the face $d_{f}$ for different monitoring stations of the NE tube, (a), large scale, (b), detail of the first $10 \mathrm{~m}$ of $d_{f}$.

During tunnel advance of the Sedrun section no mutual influence between the two tubes regarding deformations could be detected (Guntli and Weber 2009). The reason seems to be that the two tubes were excavated almost simultaneously.

\subsection{Data Analysis}

\subsubsection{Convergences}

Following Cantieni et al. (2011), the present analysis of the monitoring data always takes into account the same portion of the measured displacement at each monitoring station: In order to ensure comparability among the monitoring stations, only the displacement that develops as the face moves from a distance of $5 \mathrm{~m}$ to a distance of $30 \mathrm{~m}$ ahead of the monitoring station, is considered. Cantieni et al. (2011) decided to consider this interval because the zero readings of the monitoring stations were made at various locations, but latest $5 \mathrm{~m}$ behind the face (see Fig. 1.16b), while the shotcrete ring (which practically stops deformations) was applied at a distance of about $30 \mathrm{~m}$ from the face. Due to the choice of this interval, a certain fraction of each displacement cannot be considered for evaluation, especially in the first $5 \mathrm{~m}$ ahead of the monitoring station. 
It should be noted that in general the displacements were not distributed uniformly over the cross-section (see examples of Fig. 1.17). The non-uniformity is partially due to the overall anisotropy of the rock mass (cf. Goricki et al. 2005) which is based on local structural rock features such as quartz inclusions or local schistosity orientation changes.

(a)

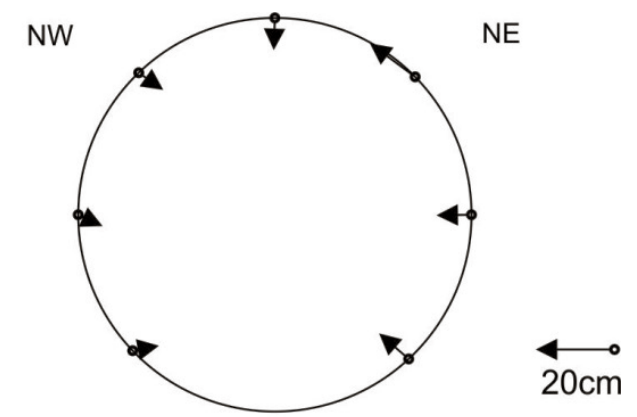

(b)

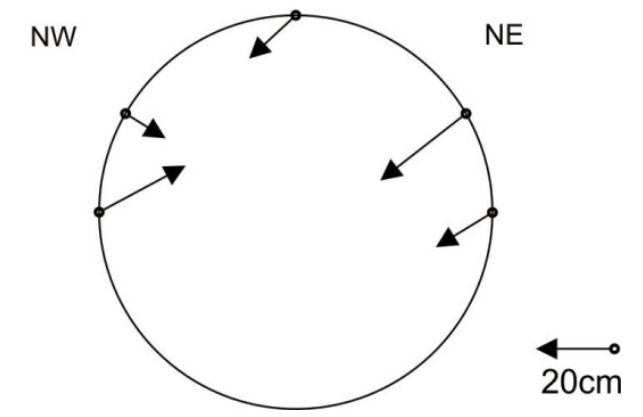

Figure 1.17. Distribution of the displacements over the cross-section, (a), for the NW tube, chainage $1535 \mathrm{~m}$ and, (b), for the NE tube, chainage $1202 \mathrm{~m}$ (based upon the data from SISO 2012 and Guntli and Weber 2009).

In order to reduce these effects, the following analysis of the monitoring data considers the average values of the measuring points of each monitoring station rather than the magnitude of single displacement vectors. Hereafter an overscore (e.g., $\bar{u}$ ) is used to denote the average value of $u$ over all the measuring points of a monitoring station.

Figure 1.18 shows the distribution along the tunnel of the magnitude of the displacement vector $\bar{u}$, the magnitude of the projection $\bar{u}_{c}$ of the displacement vector in the crosssectional plane of the tunnel as well as of the radial displacement $\bar{u}_{r}$ (averaged over each monitoring station as mentioned above). The difference between all these displacement values is small, which indicates that the main component of the displacement vector is the radial one. In the following analysis only the average magnitude of the displacement vector in the cross-sectional plane of the tunnel $\bar{u}_{c}$, which is referred to hereafter as "the average displacement", is considered.

For dimensional reasons, the displacements of a structure increase linearly with its size, all other parameters being constant. In the present case the excavated cross-sectional area varies from $69.4 \mathrm{~m}^{2}$ to $122.3 \mathrm{~m}^{2}$. In order to eliminate the effect of opening size in the evaluation of the monitoring data, the displacements will be normalised by the radius of the relevant monitoring station (4.7 to $6.24 \mathrm{~m}$ ).

To determine the factors influencing the convergences, only factors that are variable over the length of the examined section of the tunnel can be analysed. The monitoring stations were located mostly in tunnel sections with support classes 4.1, 4.2 and variations thereof (marked with a "+" in Table 1.3 and Fig. 1.13). 
Support differences can be neglected in the evaluation of the convergences for the following reason: The deformations occur practically only during the yielding phase of the support, i.e. up to the application of the shotcrete lining. The support pressure during the yielding phase is provided by the frictional resistance of the sliding connections and is very low (in relation to the initial stress) for all support classes in this section of the Gotthard Base Tunnel. Thus the differences between the support classes are small during the yielding phase. Closely spaced steel ribs, however, provide a higher safety against rock loosening. The support classes are also different with respect to the over-excavation. This difference can easily be taken into account by normalizing the displacements.
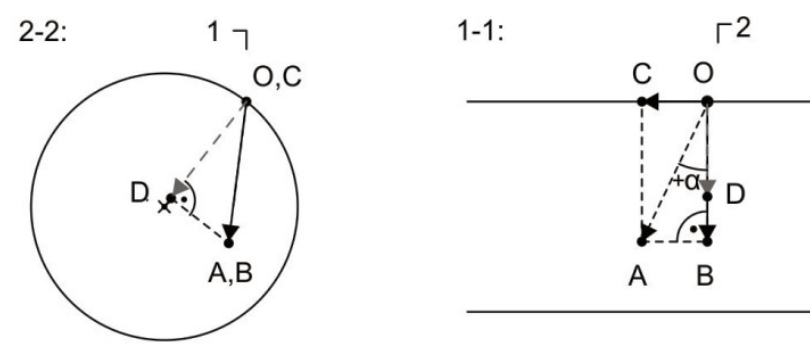

1 -

\begin{tabular}{|llll|}
\hline OA: & u & OD: & ur \\
OB: & uc & OC: & uz \\
\hline
\end{tabular}

NE tube

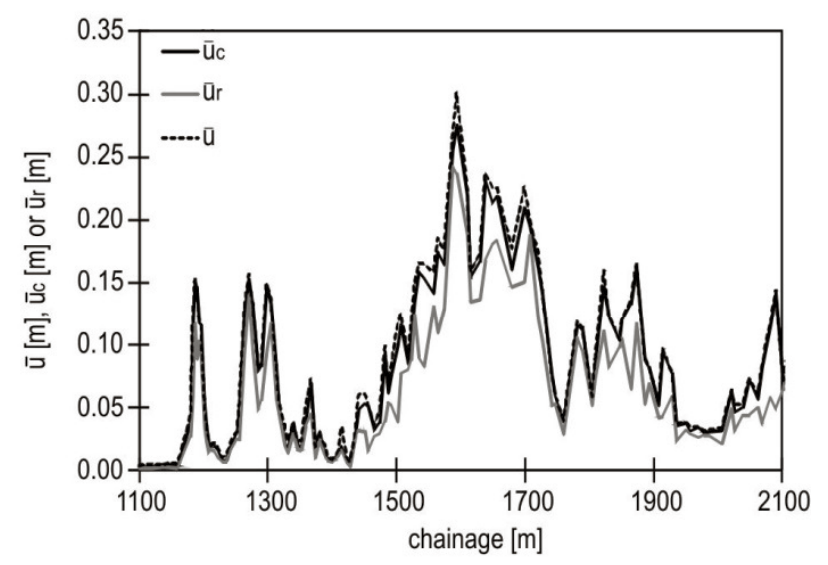

NW tube

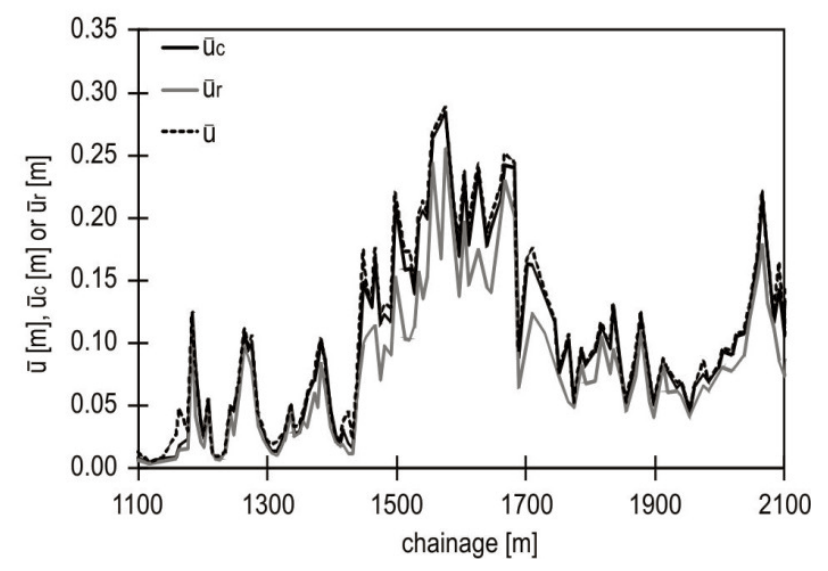

Figure 1.18. Definition of the displacement components and the magnitude of the displacement vector, of its projection in the cross-sectional plane and of the radial displacement along the tunnel (values averaged over every monitoring station). 
As mentioned above, the tectonic units in Sedrun North consist of alternating layers of different permeability. The hard rock is often fractured and has a higher permeability than the weak rock, which was sheared and therefore consists largely of rock powder. In a saturated rock the permeability governs the rate of the deformations associated with the dissipation of excess pore pressures. Permeability variations may therefore lead to variable squeezing intensities (Cantieni et al. 2011). However, the effect of pore water pressure was not considered in the data evaluation because it was not possible to measure the pore pressure in situ (the permeability of the ground was very low) and the macroscopic observations did not indicate the existence of significant differences along the considered tunnel section with respect to water.

During construction in Sedrun North it was observed that both the degree of shearing of the rock and the schistosity orientation had a strong effect on the convergences. Due to the kakiritization, the jointing and the thickness of the beds are only of secondary importance for the development of the convergences. As the degree of shearing and the lithological type are related, only the degree of shearing is used for the evaluation of the data. The following two sections therefore deal with the effects of schistosity orientation and degree of shearing.

\subsubsection{Schistosity orientation}

The effect of schistosity orientation on convergences is well known from the literature (e.g., Steiner 1996, Goricki et al. 2005, Barla and Barla 2009). Planes of weakness may affect rock behaviour adversely, particularly if their strike direction forms a small angle with (or is parallel to) the tunnel axis (Fig. 1.19, cases B, C and D). The anisotropy due to bedding or schistosity may also cause asymmetric deformations of the profile. The anisotropy is irrelevant if the tunnel crosses the schistosity or bedding planes perpendicularly (Fig. 1.19, case A). The orientation of the schistosity, i.e. its angle $\theta_{s}$ to the tunnel axis as well as its dip angle $\omega_{s}$ are thus potentially important factors for the deformations.

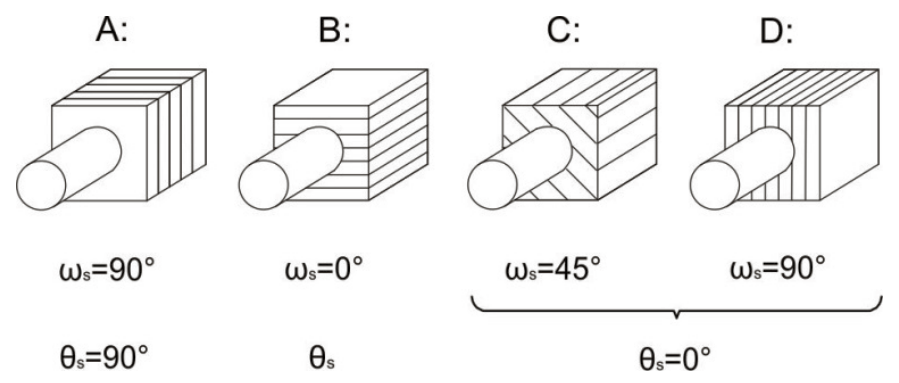

Figure 1.19. Typical cases of schistosity dip angle $\omega_{s}$ and the angle $\theta_{s}$ between strike direction and tunnel axis.

In the present case, the angles $\theta_{s}$ and $\omega_{s}$ have been determined at all monitoring stations based upon the geological mappings (e.g., Fig. 1.10a) of Guntli and Weber (2009). Local folding, as in Figure 1.10b, was not taken into account. Figure 1.20 shows the average displacements as a function of the dip $\omega_{s}$ and of the orientation of the schistosity to the tunnel axis $\theta_{s}$. In order to eliminate the effects of other factors (lithology, degree of shearing, etc.), Figure 1.20 includes only the data from monitoring stations in rock with a degree of shearing $F$ of 4 . Figure 1.20 illustrates clearly that a small dip angle or a small angle between the schistosity strike direction and the tunnel axis is associated with larger deformations (cf. cases $B$ to $D$ in Fig. 1.19), while a large dip angle and a large angle between the strike direction and the tunnel axis leads to smaller deformations (cf. case $A$ in Fig. 1.19). 


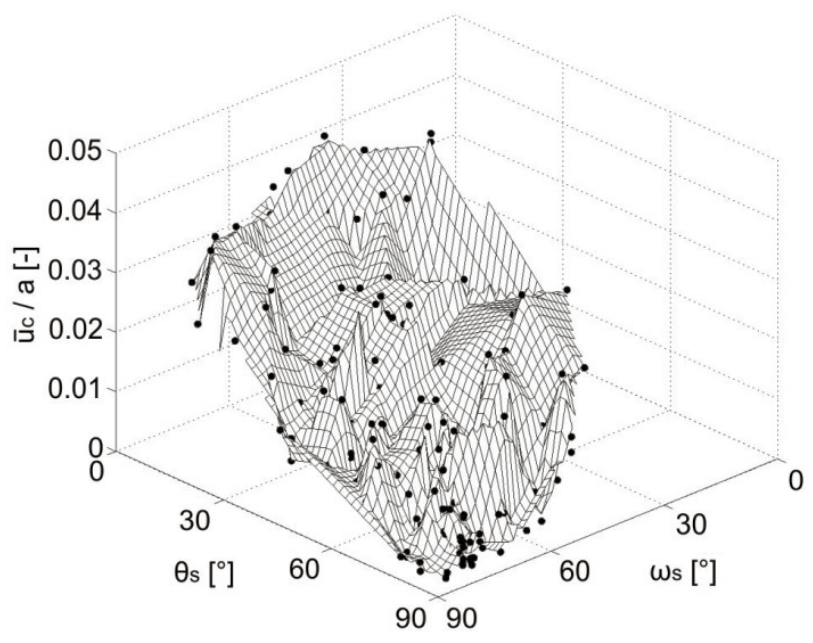

Figure 1.20. Average normalised displacement $\bar{u}_{c} / a$ as a function of the dip angle $\omega_{s}$ and the angle $\theta_{s}$ between the schistosity strike direction and tunnel axis.

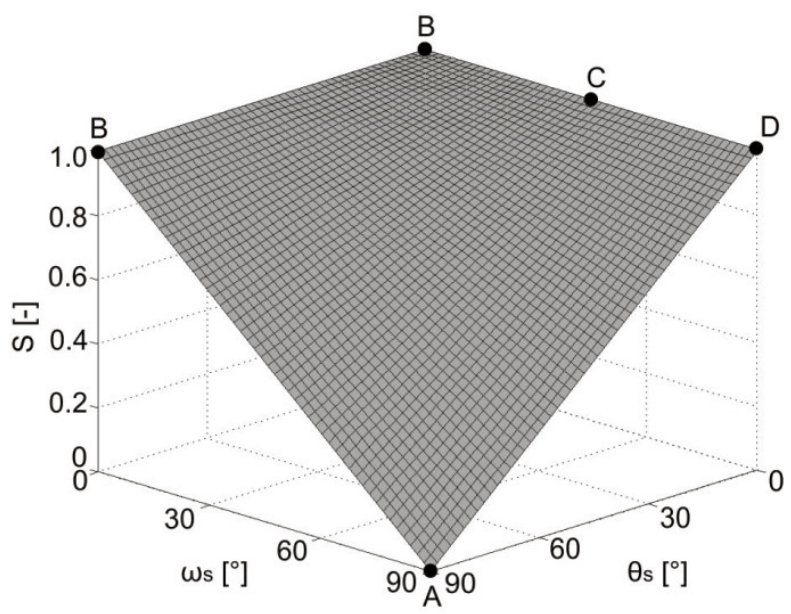

Figure 1.21. Schistosity influence factor $S$ as a function of the schistosity dip angle $\omega_{s}$ and the angle $\theta_{s}$ between strike direction and tunnel axis (points marked by A, B, C and D: see Fig. 1.19).

These two angles, which determine schistosity orientation, are important for the convergences. They can be combined to obtain a "schistosity influence factor $S$ ", that is defined as follows:

$$
S=1-\frac{\omega_{s}}{90} \frac{\theta_{s}}{90} \in[0,1]
$$

The idea behind this definition is that the influence factor $S$ is zero, if the schistosity influence disappears (i.e. when the schistosity planes are perpendicular to the tunnel axis), and is 1 , if the schistosity influence is maximum (i.e. when the schistosity planes are horizontal or strike parallel to the tunnel axis). The 3D diagram in Figure 1.21 shows the schistosity influence factor $S$ as a function of the two angles. A similarity can be recognised between the $S$ versus $\left(\theta_{s}, \omega_{s}\right)$ diagram (Fig. 1.21) and the measured displacement versus $\left(\theta_{s}, \omega_{s}\right)$ diagram (Fig. 1.20).

The diagrams (c) in Figures 1.4 to 1.8 show the schistosity influence factor determined in this way along the tunnel. The diagrams also include the average displacements for the purpose of comparison. In general, the larger the schistosity influence factor $S$, the larger the displacements. This is particularly evident from Figure 1.7 (diagrams for NE tube) for a tunnel 
reach with a constant degree of shearing: The large variability of the convergences in this case is solely due to the change of the schistosity orientation, which is adequately expressed by the schistosity influence factor $S$.

The notion of the schistosity influence factor will be revisited in Chapter 12, where its theoretical background will be shown, and in Section 13.5, which investigates if the measured displacements can also be reproduced by means of numerical calculations.

\subsubsection{Combined effect of schistosity orientation and shearing degree}

Figures 1.4 to 1.8 indicate that besides an unfavourable orientation of the schistosity (characterised by high values of the factor $S$ ) a higher degree of shearing also leads in general to larger displacements. The combined effect of rock shearing and schistosity orientation can be expressed by the product of the schistosity orientation factor $S$ with the degree of shearing $F$ (normalised by the maximum degree of shearing $F$ of 6 in order to obtain a factor between 0 and 1 ):

$$
I_{R}=S \frac{F}{6} \in[0,1]
$$

This product will hereafter be referred to as "influence factor of the rock". Figure 1.22 shows the average displacements as a function of this factor for all monitoring stations. A linear regression model was fitted using the least squares approach with the aim of quantifying the quality of the relationship between the rock influence factor $I_{R}$ and the normalised average displacements and, in the case of a satisfactory relationship, to fit an empirical equation that could be used to predict convergence. According to the regression analysis,

$$
\frac{\bar{u}_{c}}{a}=\beta I_{R},
$$

in which the proportionality constant $\beta=0.052$, while the R-squared coefficient of determination is 0.75 indicating an acceptable fit of the regression.

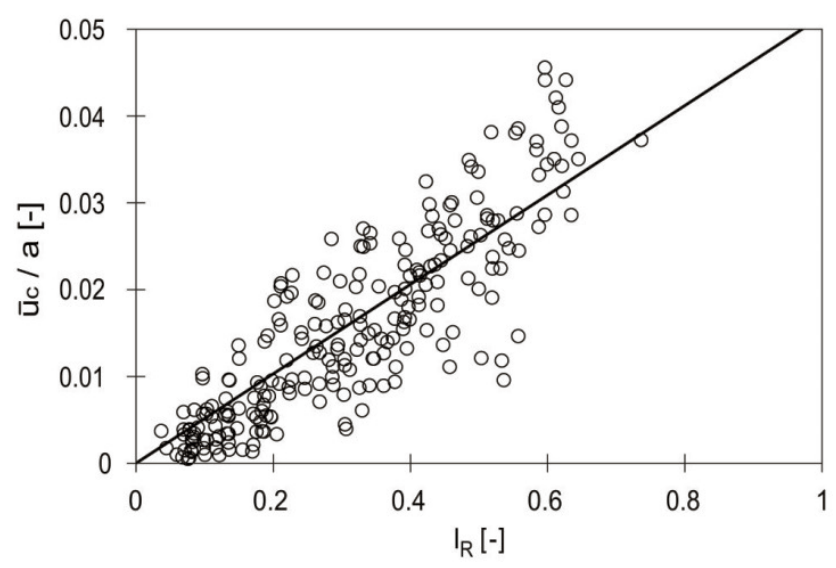

Figure 1.22. Normalised displacement $\bar{u}_{c}$ /a as a function of the influence factor of the rock $I_{R}$.

Figure 1.23 shows the measured average displacements (dashed lines) as well as the calculated average displacements according to Equation (1.3) over the entire length of the two tubes. The average difference between measured and calculated average displacements 
amounts to only $2.4 \mathrm{~cm}$ and the standard deviation to $2.0 \mathrm{~cm}$. Thus the agreement between observed and fitted data is satisfactory.

NE tube

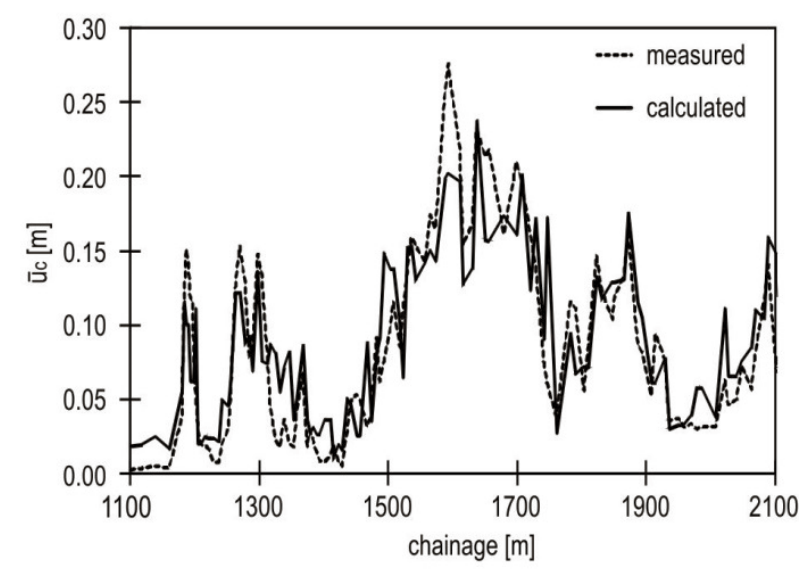

NW tube

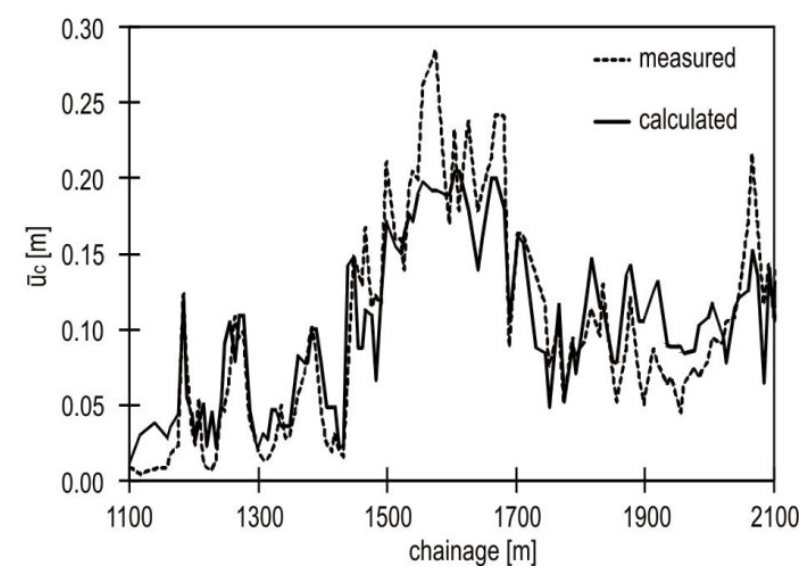

Figure 1.23. Calculated and measured displacement $\bar{u}_{c}$ along the tunnel.

\subsubsection{Influence of adjacent weaker or stronger zones}

The Sedrun North formations consist predominately of rock zones that have a degree of shearing $F$ of 4 and are interrupted by shorter zones with more or less competent rock. Based on theoretical considerations, the deformations when tunnelling through heterogeneous ground depend not only on the quality of the ground at each specific location, but also on the quality of the ground in its vicinity (Kovári and Anagnostou 1995). More specifically, a longitudinal arching effect develops, which is favourable for the weak zones, but leads to an additional loading of the competent rock layers and therefore to higher displacements (Cantieni and Anagnostou 2007). The influence of adjacent competent rocks will further be analysed (analytically and numerically) in Part II.

In order to check the validity of these theoretical considerations, the following "vicinity influence factor $I_{V}$ " is introduced as a measure of the effect of adjacent weaker or stronger zones:

$$
I_{V}=\frac{F_{V}-F}{F} \frac{L_{V}}{d}
$$


in which $F_{v}, L_{v}$ and $d$ denote the degree of shearing, the thickness and the distance of the closest zone to the considered monitoring station (Fig. 1.24), while $F$ is the degree of shearing at the considered monitoring station. This definition assumes that only the closest zone has an influence (Fig. 1.24). The structure of Equation (1.4) is based on plausibility considerations: The thicker this nearby zone and the closer it is to the considered monitoring station, the higher is the absolute value of the vicinity influence factor. The quotient $\left(F_{v}-F\right) / F$ represents a measure of the relative difference of the rock quality between the considered monitoring station and the nearby zone. If the nearby zone exhibits a higher degree of shearing, this quotient (and consequently also the vicinity influence factor $I_{V}$ ) will be positive, otherwise they will be negative. In the first case (positive $I V$ ), one would expect that the convergences would be higher than without the nearby zone. In the second case (negative $I V$ ), one would expect that the nearby more competent zone would have a stabilising effect (reduction of the convergence in the monitoring station).

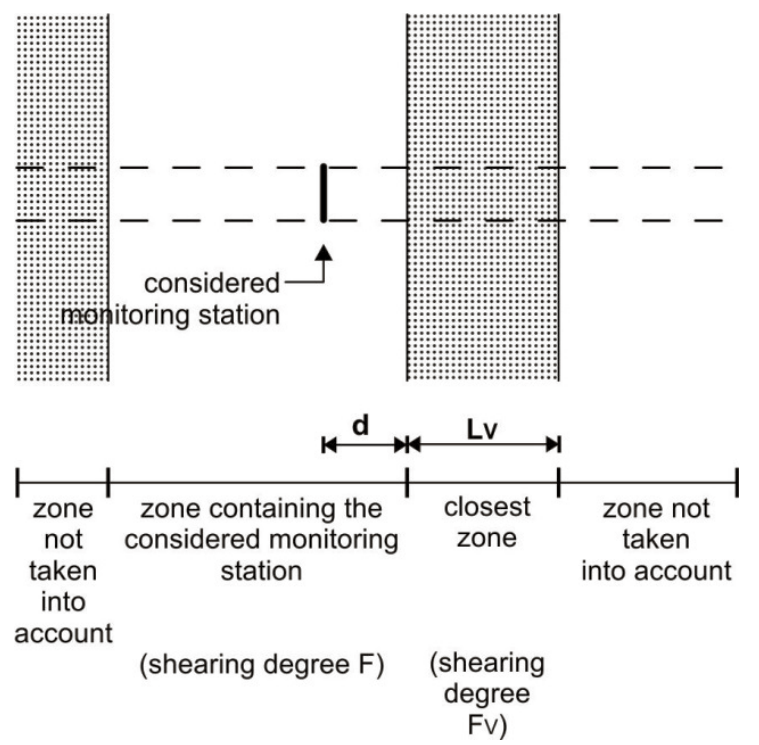

Figure 1.24. Longitudinal section with parameters considered in the definition of the vicinity influence factor.

Figure 1.25 shows the normalised displacements as a function of the influence factor of the rock $I_{R}$ for negative and positive values of the vicinity influence factor $I_{v}$. The black circles apply to $I_{V}<0$, i.e. to monitoring stations that are placed close to more competent rock zones. The black circles are located mainly at the lower part of the cluster of points, which means that the convergences at these monitoring stations are in general lower than average.

Figure 1.26 considers only the monitoring stations with positive vicinity influence factors, i.e. monitoring stations which are close to weaker zones. As mentioned above, one would expect in this case that the nearby weak zone has an unfavourable effect, i.e. it leads to higher convergences; the higher the vicinity influence factor, the more pronounced this effect should be. In fact, the data shows just this tendency: The white circles, which correspond to higher vicinity factors, are located in the upper region of the cluster of points.

In conclusion, Figures 1.25 and 1.26 support qualitatively the hypothesis that weaker or stronger zones in the vicinity have a significant effect on convergence at a specific monitoring station. Nevertheless, the large scatter does not allow one to formulate a reasonable quantitative relationship. A consideration of the schistosity in the closest zone might lead to better results, but was not considered in this chapter due to the difficulty of 
defining the zone boundaries when considering the schistosity. For these reasons, the empirical equation (1.3) was not developed further. As can be seen from Figure 1.23, Equation (1.3), which considers the degree of shearing and the orientation of the schistosity at each specific location, still provides a reasonably good approximation of the convergences.

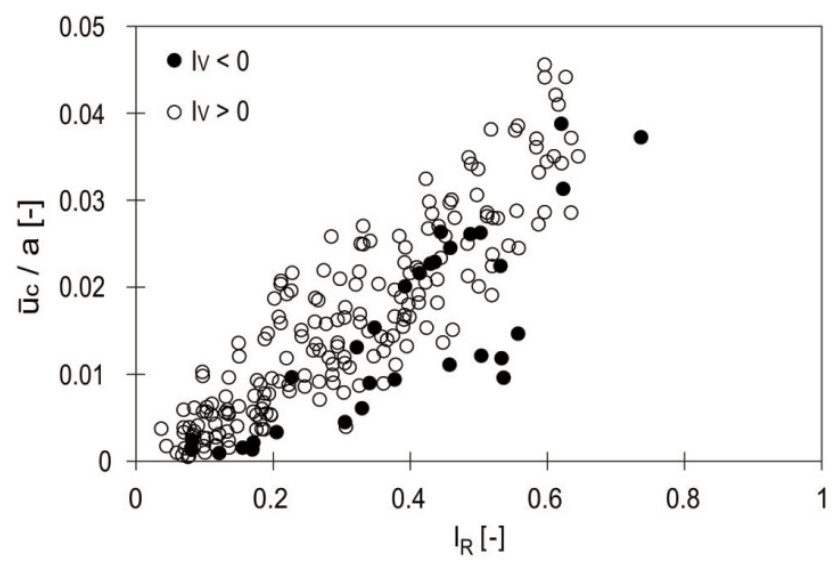

Figure 1.25. Normalised displacement $\bar{u}_{c} / a$ as a function of the influence factor of the rock $I_{R}$ for negative and positive vicinity influence factors $I v$.

A point that should be borne in mind, when evaluating the monitoring results, concerns the spatial resolution of the measured displacements. According to the theoretical results of Cantieni and Anagnostou (2007), the convergences may vary significantly even over very short distances (on the order of the spacing of the monitoring stations), if the geology consists of alternating layers of weak and hard rock. As a result of this variability the displacements may not be completely monitored when the distance between the monitoring stations is large; the displacements at a monitoring station may be representative only for the close vicinity of the monitoring station.

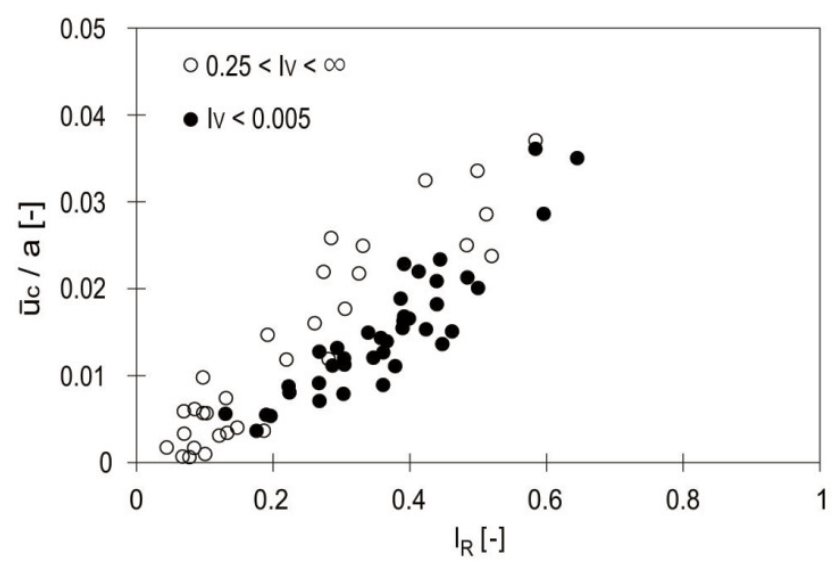

Figure 1.26. Normalised displacement $\bar{u}_{c} / a$ as a function of the influence factor of the rock $I_{R}$ for low and high positive vicinity influence factors $I V$.

\subsubsection{The longitudinal component of displacement}

According to Steindorfer (1998), the direction of the longitudinal component of the displacement vector, i.e. the sign of the angle $\alpha$ (see top of Fig. 1.18) changes when the tunnel face approaches a weaker or a stronger rock zone. More specifically, when the excavation approaches a "stiffer" zone the displacement vectors tend to point in the direction 
of excavation $(\alpha<0)$. Shortly after the excavation enters the "stiffer" rock, the vector orientation shows the opposite tendency $(\alpha>0)$, i.e. an increasing trend against the direction of excavation (Steindorfer 1998). These observations suggest that one might be able to predict the rock behaviour ahead of the tunnel face by evaluating the changes of the displacement vector orientation.
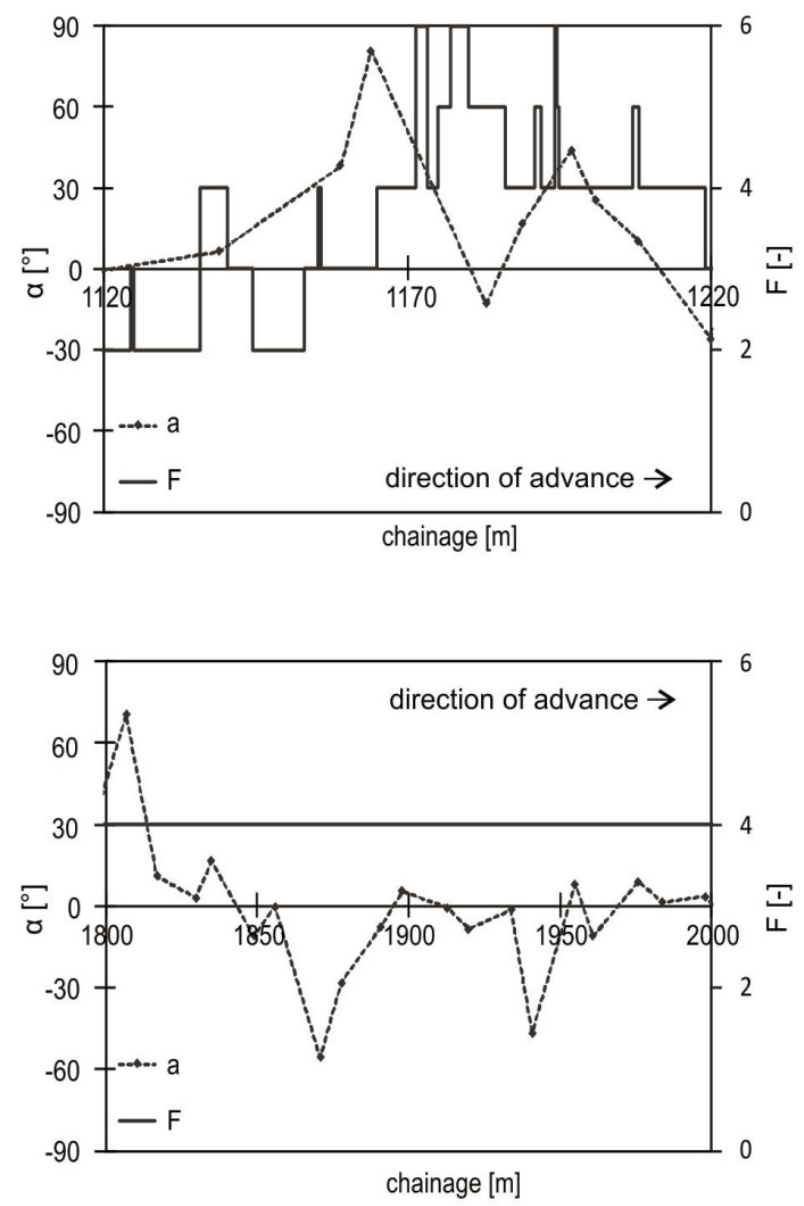

Figure 1.27. Angle $\alpha$ and degree of shearing $F$ along two reaches of the NW tube.

The data from Sedrun North supports, to some extent and under certain conditions, Steindorfer's hypothesis. Figure 1.27 shows the angle $\alpha$ of the crown displacement (i.e., measuring point 1 in Fig. 1.15) and the degree of shearing $F$ along two reaches. Only the displacements were considered that developed as the distance between the tunnel face and monitoring station increased from $5 \mathrm{~m}$ to $10 \mathrm{~m}$ (i.e., one tunnel diameter). In the first reach, the displacement orientation changes correlate with the actual rock mass quality changes. In the second reach, however, the displacement vector orientation varies although the degree of shearing of the rock mass is constant. The reason for this behaviour seems to be the schistosity orientation, which is almost constant in the first case (cf. Fig. 1.4), but varies considerably in the second case (Figs. 1.7 and 1.8) and influences the orientation of the displacement vectors. Therefore, longitudinal displacements alone do not permit a reliable prediction of the conditions ahead of the face. 


\subsection{Predictive capacity of the empirical equation}

This section investigates whether the empirical equation (1.3) derived in the last section could be used to predict the convergences. As mentioned above, Equation (1.3) accounts for the degree of shearing and the schistosity orientation. It is clear that this equation can be used for predictions only if all other possible influence factors, which were not considered for developing this equation, are identical.

NE tube

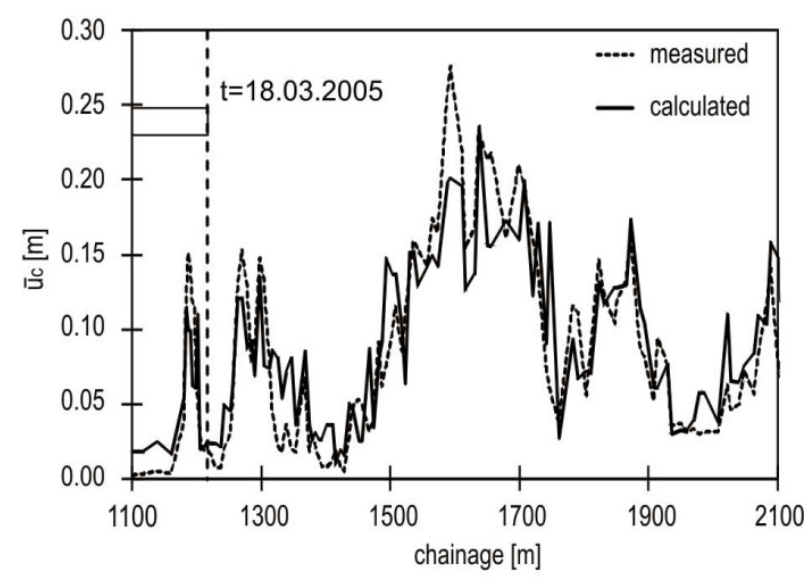

NW tube

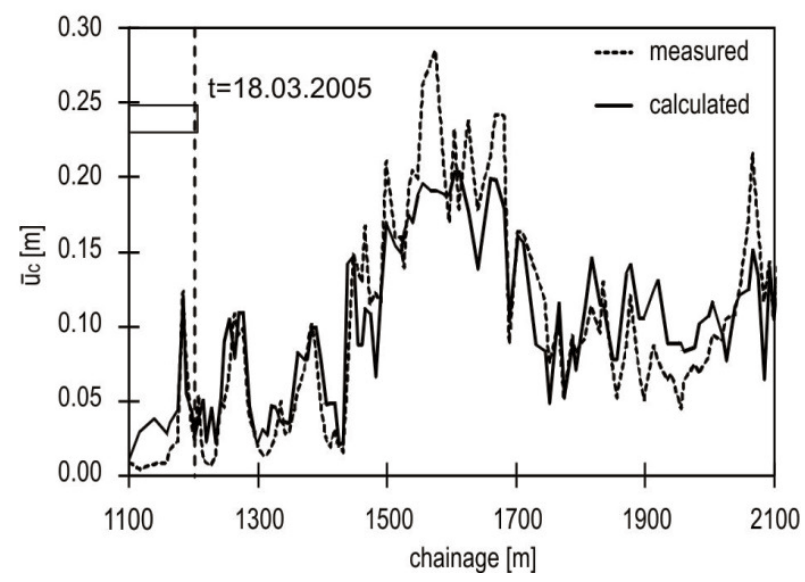

Figure 1.28. Displacement prediction for Sedrun North by means of the empirical equation (1.3), calibrated based upon the monitoring data from the first 100 tunnel metres.

Furthermore, the use of Equation (1.3) presupposes that the input parameters (degree of shearing and the schistosity orientation) can be identified in advance. This information can be obtained by means of advance probing. In fact, during the construction of the Gotthard Base Tunnel the degree of kakiritization was estimated by means of the optical inspection of the cores (cf., e.g., Fig. 1.14). An optical borehole scanner can be used to detect the structures at the borehole walls. Thereby the dip angle and the orientation of the schistosity relative to the tunnel axis can be determined with an accuracy of about half a degree. The only difficulty could be the estimation of the orientation of the schistosity in the case of an intense orientation variation in the cross-section. The optical borehole scanner can only be used if the borehole is sufficiently stable. Otherwise, the determination of the orientation of the schistosity is obtained from borehole findings and the orientation of the drill axis of the boring connected with a reorientation during excavation. 
Finally, the use of Equation (1.3) presupposes calibration of this equation on the basis of project-specific information. For this purpose two tests were carried out.

In the first test, Equation (1.3) was calibrated based on the observations made up to the 18th of March 2005, i.e. during the first $100 \mathrm{~m}$ of advance in Sedrun North, and used the calibrated equation in order to predict the deformations in the remaining part of Sedrun North. The calibration over the first $100 \mathrm{~m}$ gave a correlation factor $\beta$ of 0.051 , which is almost equal to the correlation factor for the entire data set $(0.052$, see Section 1.5.3). As a result, the predicted convergence agrees well with the measured values (Fig. 1.28), which means that such an empirical approach would be useful in the present case. The agreement between predicted and actual convergence would probably be poorer in reality due to the uncertainties that exist with respect to the estimation of the input values (degree of shearing, schistosity orientation) on the basis of advance core drilling.

SE tube

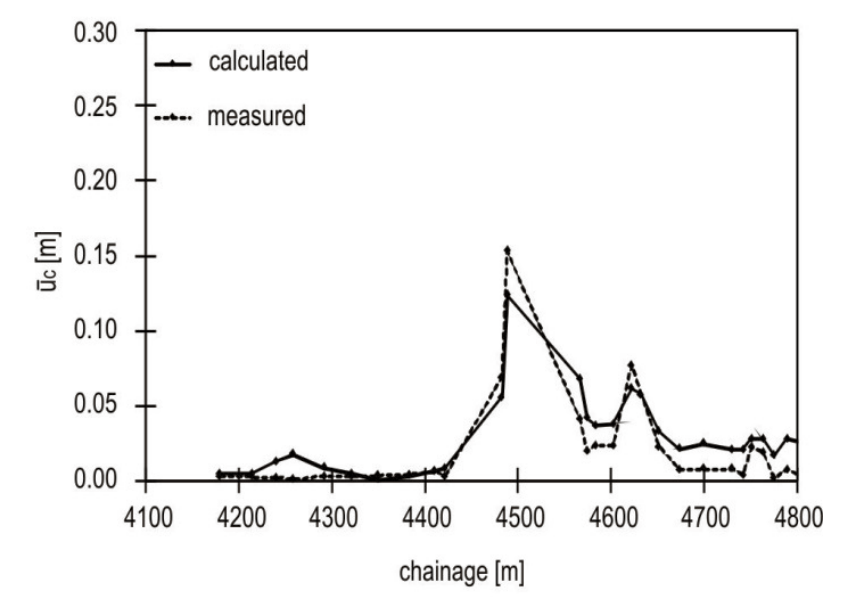

SW tube

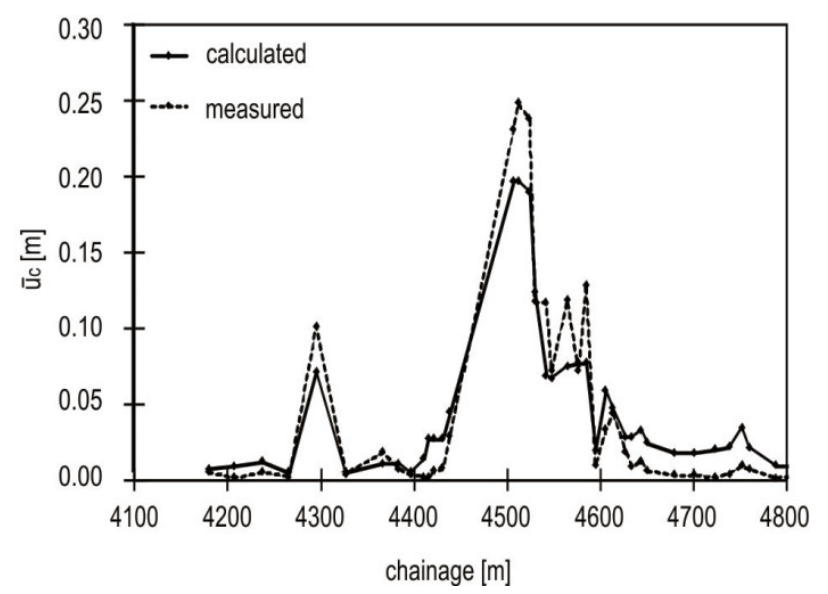

Figure 1.29. Displacement prediction for Sedrun South by means of the empirical equation (1.3), calibrated based upon the monitoring data from Sedrun North.

In the second test, the convergences in Sedrun South (Fig. 1.20) are predicted by using Equation (1.3). The only difference between Sedrun North and South is the overburden, which is about twice as large in Sedrun South (1650 m compared to about $800 \mathrm{~m}$ in Sedrun North). For the prediction of the displacements in Sedrun South Equation (1.3) was calibrated based upon the data from Sedrun North and applied the calibrated equation to predict the convergences in Sedrun South (Fig. 1.20). The difference in overburden is not 
taken into account. Figure 1.29 shows the predicted and the actual displacement. Their average and maximum difference amount to $1.5 \mathrm{~cm}$ and $5 \mathrm{~cm}$, respectively. The comparison with Figure 1.29 shows that the empirical equation from Sedrun North slightly underestimates the convergence, particularly in the weaker zones around chainage 4300 and $4500 \mathrm{~m}$ (perhaps due to the higher overburden). However, the equation is still reliable for estimating the order of magnitude of the convergences.

\subsection{Conclusions}

The case history of the Gotthard Base Tunnel shows that the frequently observed phenomenon of squeezing variability can be traced back to different degrees of shearing, different schistosity orientations and the effect of nearby weaker or stronger zones. A simple empirical equation can map the effect of shearing and schistosity reasonably well and provides, in combination with advance core drilling, reliable indications of the squeezing intensity. The longitudinal displacement of the tunnel boundary (Steindorfer 1998) or the extrusion of the core ahead of the tunnel face (Cantieni et al. 2011) may provide additional indications under certain conditions (constant orientation of the schistosity, no significant creep, respectively).

\section{Notation}

a

$c$

$d$

$d_{f}$

$F$

$F_{V}$

$I_{R}$

IV

$L_{V}$

$S$

$T$

$\bar{u}$

$\bar{u}_{c}$

$\bar{u}_{r}$

$u_{r, 1}$

$u_{r, 1, \infty}$

$u_{z, 1}$

$\alpha$

$\beta$

$\varphi$

$\omega_{s}$

$\theta_{s}$ tunnel radius

cohesion of the ground

distance between the monitoring station and the closest boundary of the rock zone

distance between the tunnel face and the monitoring station

degree of shearing of the rock mass in the monitoring station

degree of shearing of the rock mass in the adjacent rock zone

influence factor of the rock mass at the monitoring station

influence factor of the adjacent rock zone

extent of the adjacent rock zone

influence factor of the schistosity

lithological type at the monitoring station

average magnitude of the displacement vectors

average magnitude of the projections of the displacement vectors in the cross-sectional plane of the tunnel

average radial component of the displacement vectors

radial displacement of the tunnel crown

radial displacement of the tunnel crown far behind the face

longitudinal displacement of the tunnel crown

angle between the cross-sectional plane of the tunnel and the displacement vector at the tunnel crown

empirical curve-fitting parameter

friction angle of the ground

dip angle of the prevalent schistosity plane

angle between the tunnel axis and the strike direction of the prevalent schistosity plane 



\section{Giumello Gneiss Section of the Ceneri Base Tunnel²}

\subsection{Introduction}

In Chapter 1 , the reasons for the variability of rock deformations observed during construction in the Sedrun section of the Gotthard Base Tunnel, where heavy squeezing conditions were encountered, were investigated. It was shown that the variability of the squeezing intensity along the tunnel could be traced back to a variable degree of tectonic shearing, variable schistosity orientation with respect to the tunnel axis and the effect of nearby weaker or stronger zones.

The present chapter analyses data from the construction of the Ceneri Base Tunnel (a $15.4 \mathrm{~km}$ long twin tunnel, which also belongs to the Swiss AlpTransit project) in the Giumello Gneiss formation (hereafter referred to as "GGium"), which was crossed over a length of $1000 \mathrm{~m}$ in the eastern tube and $600 \mathrm{~m}$ in the western tube. Due to the poor rock quality and the high depth of cover (about $650 \mathrm{~m}$ ), squeezing was expected from the planning phase. The deformations in the GGium were, however, higher than those predicted at the project stage, resulting in localised damage in the shotcrete shell (Pini and Skaanes 2006, Merlini and Falanesca 2013). After a brief overview of the available data on the geology (Section 2.2), excavation and support (Section 2.3) and rock response to the tunnelling (Section 2.4), an attempt is made to find empirical correlations between rock structure and observed deformations (Section 2.5). In contrast to the Sedrun section of the Gotthard Base Tunnel, where the alignment crossed the tectonic units almost perpendicularly, in the GGium formation the schistosity and fault zones lay parallel to the tunnel axis (a so-called "parallelismo zone"). Squeezing phenomena were therefore less variable along the tunnel. Nevertheless, squeezing was characterised by a very non-uniform deformation of the tunnel cross-section. As rock anisotropy is known to lead to non-uniformly distributed deformations of the profile (cf., e.g., Steiner 1996, Goricki et al. 2005, Leitner et al. 2006, Barla and Barla 2009 and Part III of the present thesis), particular attention is paid here to the examination of the influence of schistosity on the convergences.

\subsection{Geology}

The Ceneri Base Tunnel is situated in the crystalline bedrock of the Southern Alps (Merlini and Falanesca 2013). The GGium is heterogeneous, consisting of different geological rock layers (Pini and Skaanes 2006). Although the rock formations have retained their original

2 This chapter has been published in: Mezger, F., Anagnostou, G. (2015). On the non-uniformity of squeezing deformations in the Ceneri Base Tunnel. Schubert, W. \& Kluckner, A. (Eds.), ISRM Regional Symposium EUROCK 2015 \& 64th Geomechanics Colloquium, Salzburg, Oesterreich, pp. 113-118. 
pre-alpine structure, they were affected by the orogeny, thus forming disturbed areas of cataclastic and kakiritic fault zones (Stocker 2006). The GGium consists of gneisses with a high content of mesocratic schist (comprising 40 to $60 \%$ biotite). The rocks also contain quartz veins a few centimetres thick and calcium silicates on a scale of decimetres (ITC 2014). During advance, the rock was classified lithologically as follows: Gneisses (with a high content of schist) with medium grains; gneisses (with a high content of schist) with medium to fine grains; mica-schists with medium grains; and mica-schists with medium to fine grains (with the rock quality decreasing from the first to the last-mentioned lithological type, ITC 2014).

(a)

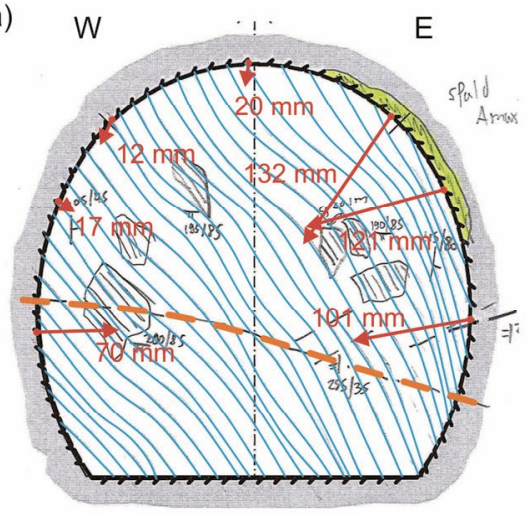

(b)

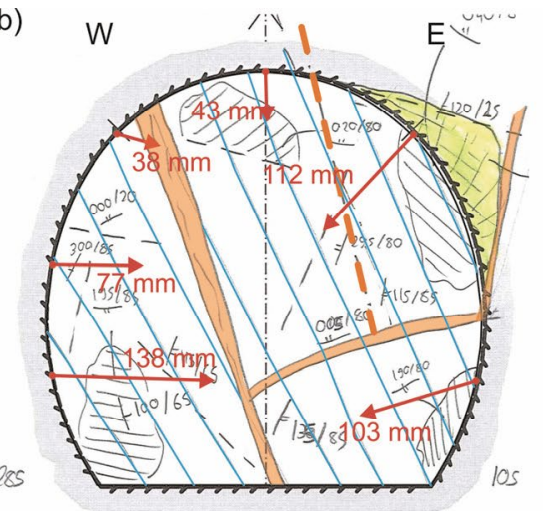

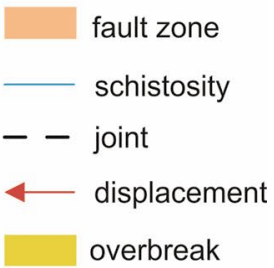

overbreak

Figure 2.1. Examples of geological mappings of the tunnel face showing traces of the schistosity planes and fault zones and measured displacements (see Section 2.5, after ITC 2014): (a), NE tube, chainage 668; (b), NE tube, chainage 614.

Discontinuities in the rock were recorded with respect to schistosity, jointing and fault zones (Fig. 2.1). The fault zones were 5 to $100 \mathrm{~cm}$ thick and ran subparallel to the direction of excavation (Merlini and Falanesca 2013). The fault gouge generally exhibited a low cohesion (ITC 2014). The fault zones were classified according to their degree of shearing into the following project-specific classes: a degree of shearing $Z$ of 1 denotes a superficial, slight shear zone; 2 mylonites; 3 a cataclastic fault zone; 4 a cataclastic-kakiritic fault zone; and 5 a kakiritic fault zone. Kakirites are intensively sheared rocks.

The main element in the anisotropy of the rock mass was the schistosity. Due to the direction of advance (ca. $194^{\circ}$ ), the schistosity planes (oriented in E-ESE) lay parallel to the tunnel axis with variable dip (ITC 2014, Fig. 2.1). The schistosity was apparent even in the fault zones, but tended to be locally distorted in the vicinity. The major part of the GGium was classified with respect to the thickness of the schist beds as "laminated" $(0.5-5 \mathrm{~cm})$ or "thinly bedded" (5-15 cm, ITC 2014). Due to the small thickness of the beds compared to the tunnel size, bed thickness had no observable influence on the convergences. The rock mass was only weakly jointed. Consequently, the jointing is deemed to be of only minor importance compared to the fault zones and the schistosity. No water inflows occurred (ITC 2014).

\subsection{Construction method}

For the excavation of the Ceneri Base Tunnel, a total of ten support classes (abbreviated to SPV) were developed. In the GGium only SPVs 3 to 6 were applied. SPV 3 and 4 consist of radial bolts and fibre-reinforced shotcrete and have no invert arch; SPV 5 and 6 have an invert arch, radial and face bolts, mesh or fibre-reinforced shotcrete and steel sets (Fig. 2.2, Merlini and Falanesca 2013). SPV 3 and SPV 5 differ from SPV 4 and SPV 6, respectively, 
only in the bolt length and spacing (ITC 2014). SPV 3 contains $4 \mathrm{~m}$ long bolts spaced at $1 \mathrm{~m}$ x $1.5 \mathrm{~m}$; SPV 4 also has longer bolts (up to $6 \mathrm{~m}$ ) spaced at $1.2 \mathrm{~m} \times 1.2 \mathrm{~m}$ (ITC 2014, Fontana 2012).

(a)

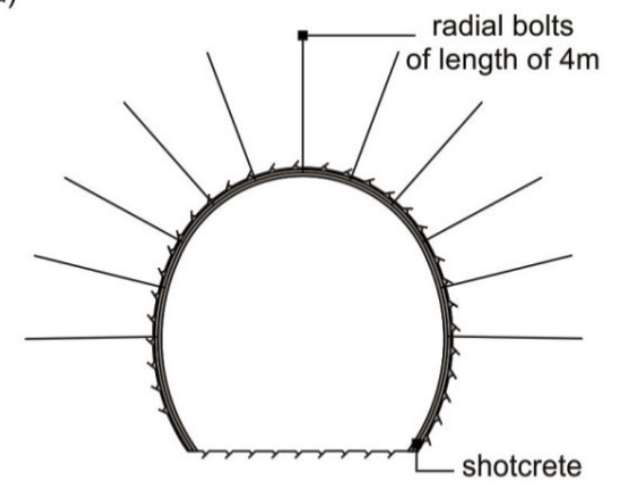

(b)

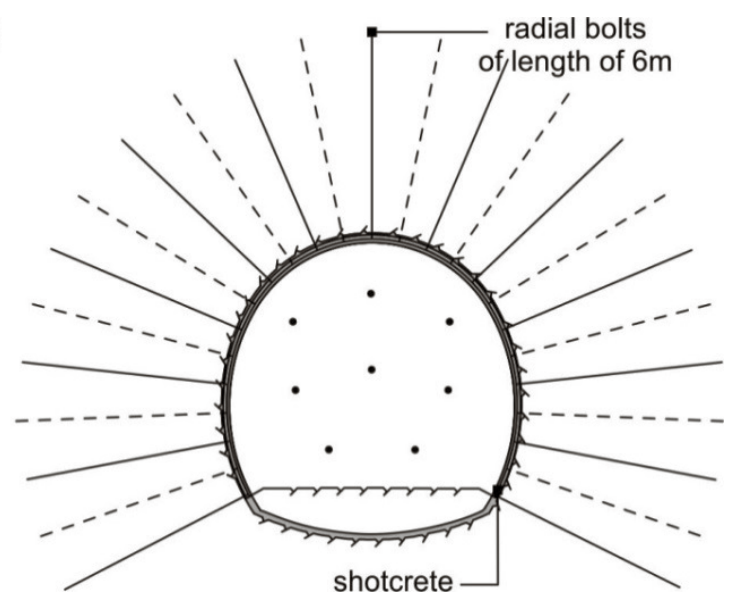

Figure 2.2. Cross-section and longitudinal section of the support classes (after ITC 2014): (a), SPV 3; (b), SPV 6.

The centreline distance of the two tubes is equal to $40 \mathrm{~m}$. The tubes were excavated asynchronously. The NW tube was excavated first. No mutual influence was observed between the two tubes (ITC 2014).

\subsection{Rock response to tunnelling}

The behaviour of the rock mass and the tunnel support was monitored by 3D optical measurements (ITC 2014). In the GGium, 46 monitoring stations were installed, spaced at 2-110 m and each containing up to 7 measuring points (i.e. about 240 measuring points in total). The deformations reached values of up to $40 \mathrm{~cm}$. During excavation through the GGium, the rock response was found to be clearly affected by the faults and shear zones and by the schistosity, so that the displacements were distributed non-uniformly over the cross-section (Fig. 2.1, Merlini and Falanesca 2013). Due to the discontinuities, loosening and rock falls also occurred, mostly in the eastern part of the tunnel roof (overbreak in Fig. 2.1) immediately after blasting above the unsupported span.

\subsection{Data analysis}

In the following, the magnitude of the projection of the displacement vector is considered in the cross-sectional plane of the tunnel at every measuring point (and hereafter referred to as "the displacement"). Measuring points lying in the intersection of the tunnel and the crosspassages are not taken into account as they are influenced by the complex geometry of the excavation. In order to ensure comparability between the measuring points, the same portion of the displacement measured at each measurement point (more specifically, the difference between the final displacement and the displacement that develops when the face is located $5 \mathrm{~m}$ ahead of the measuring point) is considered. For dimensional reasons, the displacements in a structure increase linearly with its size, all other parameters being constant. In this section of the Ceneri Base tunnel, the excavated width $b$ of the tunnel is $9.06-9.36 \mathrm{~m}$. In order to eliminate this effect, the displacements are normalised by $b / 2$. 
In order to investigate the effect of schistosity orientation, the angle $\beta$ is considered between the direction of the maximum principal stress (i.e. the tangential stress at the excavation boundary) and the schistosity planes (hereafter referred to as "the schistosity angle", Fig. 2.3a). Figure 2.3b shows the normalised displacement as a function of the schistosity angle $\beta$ for different lithological types. In order to eliminate the effects of other factors (support class and fault zones), Figure 2.3b includes only the data from measuring stations with support classes SPV 3 and 4 (no invert arch) and measuring points that do not lie inside a fault zone. A distinction between SPV 3 and 4 is not necessary because the difference in support pressures is small in relation to the initial stress.

The Figure $2.3 \mathrm{~b}$ shows that the smaller the schistosity angle $\beta$, the higher are the displacements (for a given lithological type). The biggest displacements occur at locations where the schistosity is almost parallel to the excavation boundary, i.e. to the direction of the maximum principal stress. The schistosity is consequently at least partly responsible for the non-uniformity of the deformations in the cross-section.

Nevertheless, in addition to schistosity, the lithology also has a remarkable influence on deformation (compare the lithological types A and D in Fig. 2.3b).

(a)

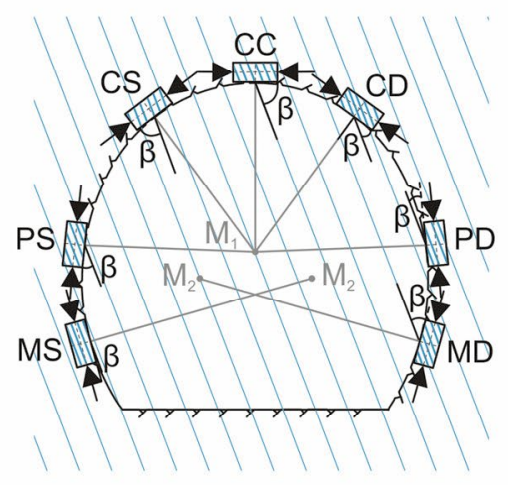

(b)

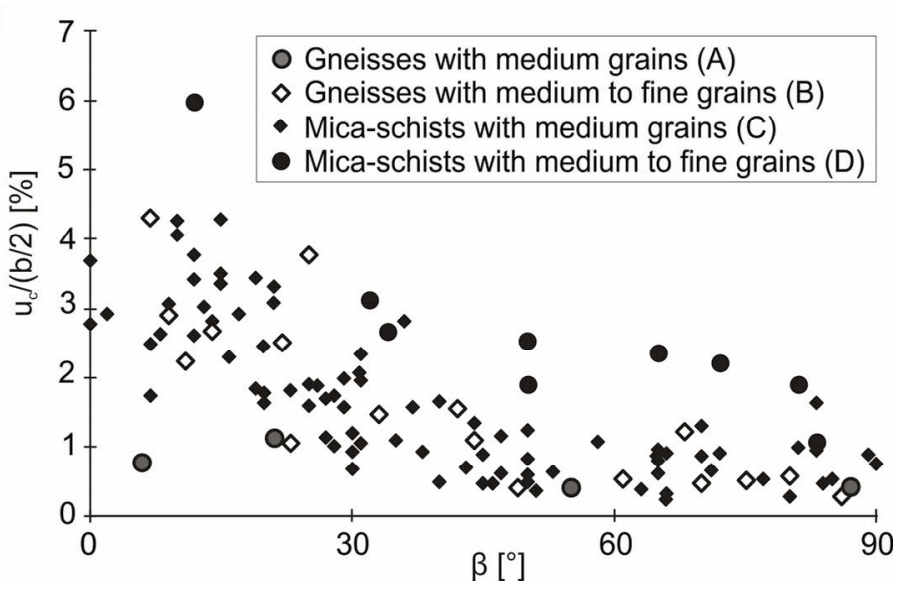

Figure 2.3. (a) Typical cases of the schistosity angle $\beta$ in the cross-sectional plane of the tunnel; (b), normalised displacement $u_{c} /(b / 2)$ as a function of the schistosity angle $\beta$ (data only from the support classes SPV 3 and 4 and measuring points not lying inside a fault zone).

Another potential influence is associated with the heterogeneity of the rock mass. As shown in Chapter 1 based upon monitoring results from the Sedrun section of the Gotthard Base Tunnel, the excavation-induced deformations in heterogeneous ground depend not only on the quality of the ground at each specific location, but also on the quality of the ground in the vicinity of the measuring points. The closer a measuring point is to a weak zone and the weaker and thicker this zone is, the greater will be the displacement. This hypothesis can be checked empirically by considering the following factor as a measure of the effect of adjacent fault zones:

$$
I_{s}=\frac{Z \cdot t}{d}
$$

where $Z, t$ and $d$ denote the degree of shearing in the fault zone (see Section 2.2), the thickness of the fault zone and the distance from the measuring point to the fault zone (see Fig. 2.4a), respectively (see Chapter 1). 
(a)

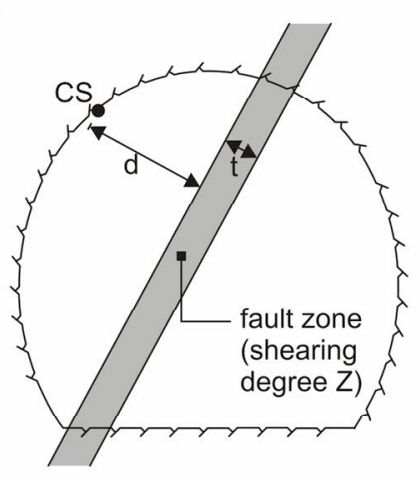

(b)

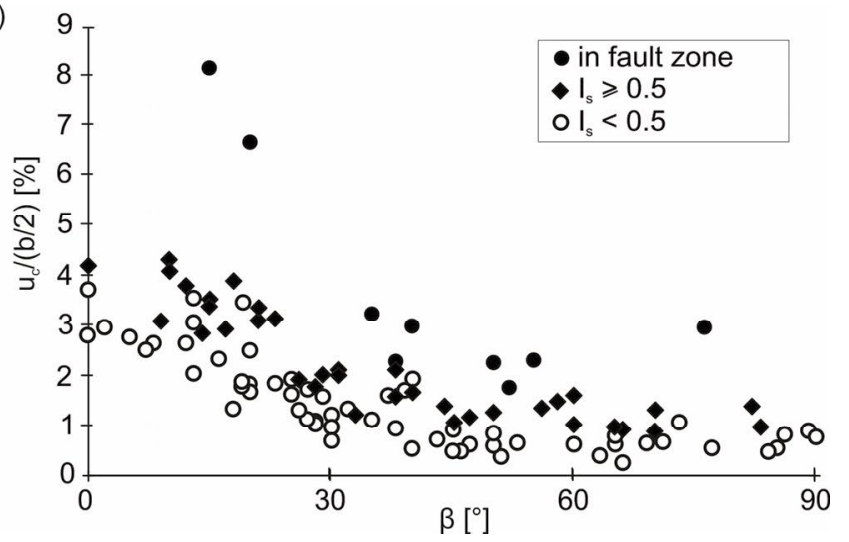

Figure 2.4. (a) Cross-section of the tunnel with the parameters considered for defining the influence of the fault zone; (b), normalised displacement $u_{c} /(b / 2)$ as a function of the schistosity angle $\beta$ for measuring points lying in the fault zone and for measuring points with low and high factors of the fault zone $I_{s}$ (data only from the support classes SPV 3 and 4 and rock consisting of Mica-schists with medium grains).

Figure $2.4 \mathrm{~b}$ shows the normalised displacements as a function of the schistosity angle $\beta$, grouped according to the $I_{s}$ factor (measuring points lying inside fault zones are marked separately). If several fault zones lie in the vicinity of a measuring point, the fault zone with the highest influence factor $I_{s}$ was considered for the data evaluation. In order to eliminate other effects, only cross-sections with the same lithology (mica-schists with medium grains) and support types SPV 3 or 4 were considered. The black circles, which correspond to displacements measured at points inside fault zone, are located in the upper region of the cluster. The white circles, which correspond to small $I_{s}$ factors, are located in the lower region. Higher influence factors $I_{s}$ correlate with higher convergences.

\subsection{Conclusions}

The case history of the Ceneri Base Tunnel shows that schistosity has a considerable influence on deformation and is the main reason for the observed non-uniformity in the profile deformations. In addition to schistosity, the lithological variations and the fault zones also contributed to the non-uniformity in the deformations.

\section{Notation}

$b$

$d$

$I_{s}$

Z

$t$

$u_{c}$

$\beta$ width of the tunnel

distance between the measuring point and the fault zone

influence factor of the adjacent fault zone

shearing degree of the fault zone

thickness of the fault zone

magnitude of the projection of the displacement vector in the crosssectional plane of the tunnel

angle between the direction of the maximum principal stress and the schistosity planes 



\section{Carbon Section of the Lötschberg Base Tunnel}

\subsection{Introduction}

From the viewpoint of the geology, the case histories of the Chapters 1 and 2 were completely different: In contrast to the Sedrun section of the Gotthard Base Tunnel, where the alignment crossed the tectonic units almost perpendicularly, in the GGium, the schistosity and the fault zones were oriented parallel to the tunnel axis. In the Sedrun section, squeezing intensity varied remarkably along the alignment, while in the GGium, squeezing was characterised by a very non-uniform deformation of the tunnel cross-section. Nevertheless, both case histories showed that the squeezing deformations were mainly affected by the lithology, the schistosity and the effect of nearby weaker or stronger zones.

The goal of this chapter is to confirm these findings based on a third case history: the carbon section of the Lötschberg Base Tunnel, a $34.5 \mathrm{~km}$ long twin tunnel (whereupon the western tube only serves as rescue tunnel) of the Swiss AlpTransit project. During excavation within the Aar massif at a depth of cover of ca. $1400 \mathrm{~m}$, a sedimentary cover consisting of schistous carbon rocks was encountered unexpectedly over a length of $300 \mathrm{~m}$. Over a length of $200 \mathrm{~m}$, the rocks turned out to be heavily squeezing, leading to convergences of up to $70 \mathrm{~cm}$. They were distributed non-uniformly over the tunnel cross-section, varied strongly along the tunnel, have led to damage to the support and necessitated considerable tunnel repairs (Keller 2005). Even after a considerable distance to the tunnel face, large long-term deformations occurred, which could be partially traced back to creep (Gimmel 2018). As in this thesis, the time-dependence of the rock behaviour (due to creep or consolidation) will not be considered, only the short-term deformations (predominantly associated with the excavationinduced stress re-distributions that occur around the advancing heading) will be investigated in this chapter. After a brief overview of the available data on the geological units, the excavation and support method as well as rock response to tunnelling (Sections 3.2 to 3.4), an attempt is made in Section 3.5 to identify the decisive factors, which are responsible for the squeezing variability.

\subsection{Geology}

The carbon rocks consist of a sequence of alternating layers of sandstones, siltstones, clay slates and layers of anthracite (Keller 2005, IG-LBT 2009, Ziegler and Isler 2013). One third of the carbon rocks contains thick-bedded sand- and siltstones, while the remaining two thirds mainly consist of schistous rocks with up to $1 \mathrm{~m}$ thick anthracite layers (Keller 2005, IG-LBT 2009). The carbon rocks dip at about $30^{\circ}$ in direction of advance (Keller 2005). The quartz sandstones (containing $40-60 \%$ of quartz) are hard, have medium to coarse grains and form banks of a few centimetres to metres (Ziegler and Isler 2013). In some parts of the 
tunnel, the siltstones can hardly be distinguished from the sandstones and the schists (Ziegler and Isler 2013). The schists consist of foliated and graphitic clay slates with organic compounds and with smoothly polished schistosity planes (Stech et al. 2005, Ziegler and Isler 2013). The up to $1 \mathrm{~m}$ thick anthracite layers have high clay contents and are always associated with the schists (Geological Group of the Lötschberg Base Tunnel 2008, Ziegler and Isler 2013). In the present chapter, the schistous rocks with high anthracite content will be referred to anthracite schists (see also Geological Group of the Lötschberg Base Tunnel 2008).

In the carbon rocks, the schistosity dips mainly to SE (thus lying subparallel to the tunnel axis), but can be folded locally (Geological Group of the Lötschberg Base Tunnel 2008, Ziegler and Isler 2013).

km 30410

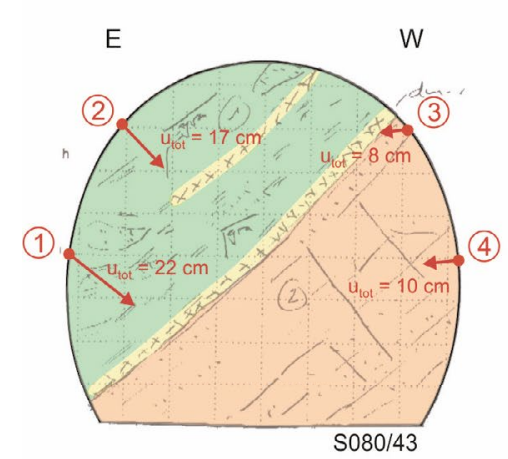

anthracite layers sandstones schists km 30430

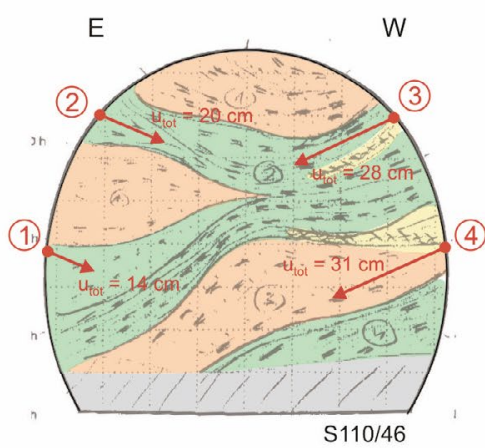

siltstones, partially schistous shotcrete km 30480

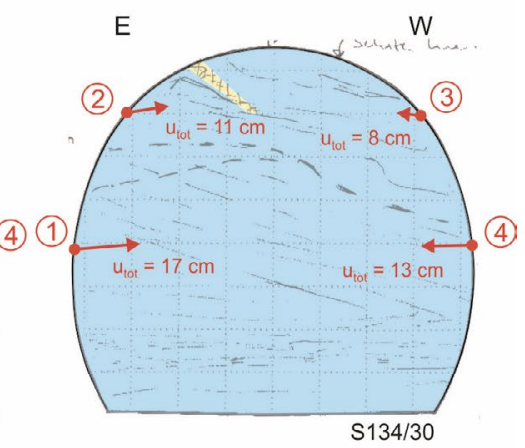

- discontinuities

4 displacement

Figure 3.1. Examples of geological mappings of the tunnel face of the eastern tube showing the lithology, the traces of the discontinuities and measured displacements $U_{\text {tot }}$ (after IG-LBT 2009).

During tunnel advance, the lithology, the schistosity and the jointing of the tunnel face were recorded (see Fig. 3.1) and then summarised in a longitudinal map (with less detailed information). As the tunnel section was already excavated in 2004 and the storing of a huge amount of data is expensive, some of the geological mappings of the tunnel face are missing. This makes the analysis of the tunnel difficult, given that the exact location of the anthracite layers or the orientation change of the schistosity are unknown in some specific measuring points.

Apart from occasional small water inflows, in the whole carbon section, the drive was dry (Stech et al. 2005, Ziegler and Isler 2013).

\subsection{Construction Method}

The carbon section was encountered unexpectedly, but - as squeezing conditions were anticipated for some other sections of the Lötschberg Base Tunnel - the design documents included an appropriate support class foreseeing an invert arch in combination with a fullface excavation and a yielding support (support class ES6A in Figs. 3.2 and 3.3). The yielding support, which consists of sliding steel ribs and 6 longitudinal joints in the shotcrete shell, reduces the rock pressure to a manageable level (Ziegler and Isler 2013). With these 
joints, convergences of up to $1 \mathrm{~m}$ could be theoretically accommodated without damage to the shotcrete shell (Keller 2005).
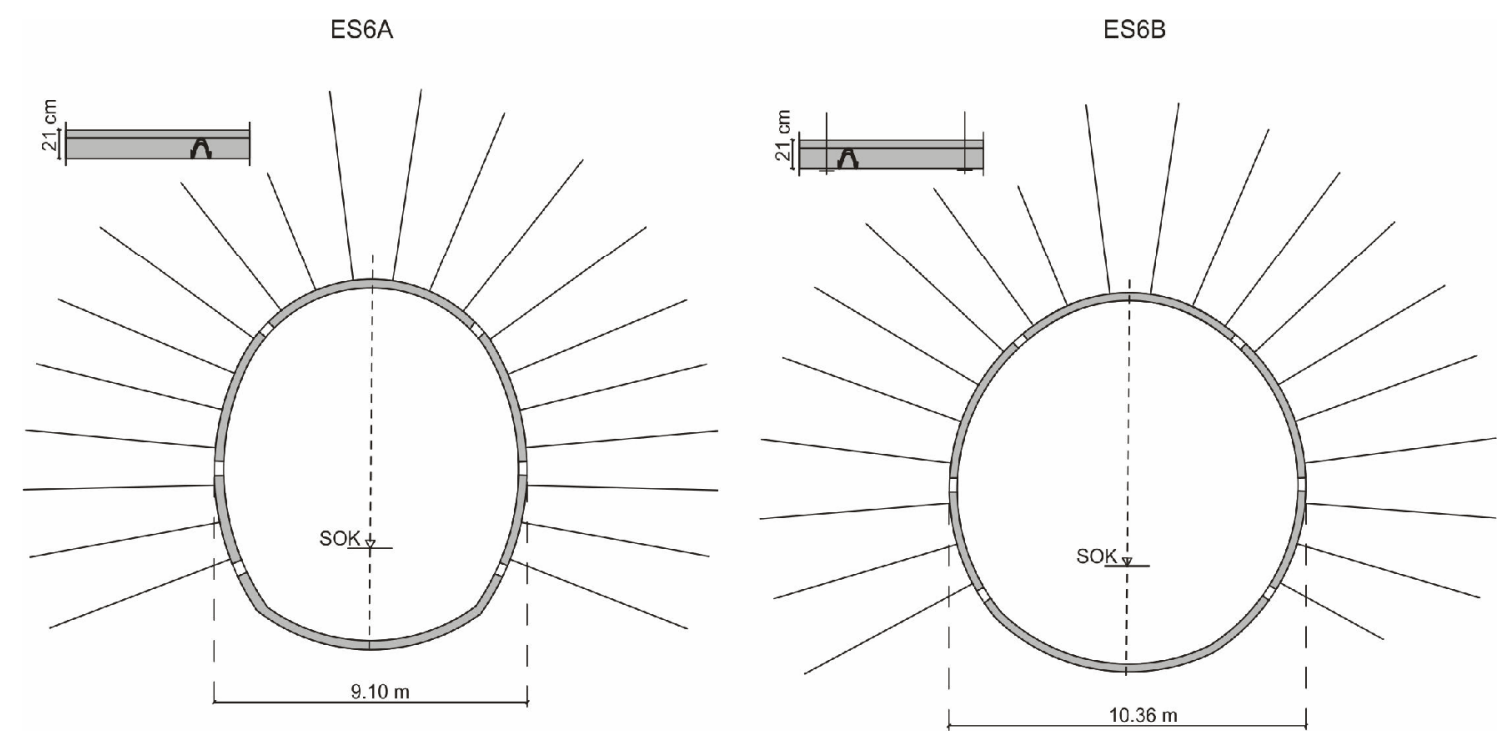

Figure 3.2. Support classes for squeezing conditions (Keller 2005).

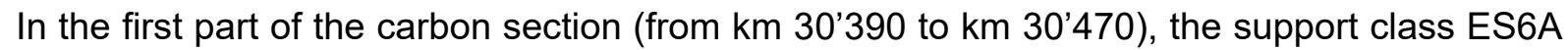
was applied in both tubes. However, several months after excavation, the support was already damaged, so that considerable tunnel repairs became necessary (Keller 2005). As an initial measure, additional radial bolts with a length of $12 \mathrm{~m}$ were installed. However, this measure failed to reduce the deformation rate (Keller 2005). Therefore, a part of the carbon section had to be repaired, using the support class ES6B (Keller 2005) with a nearly circular tunnel cross-section (Fig. 3.2). Furthermore, the support resistance was increased by installing compressible elements in the longitudinal joints in the shotcrete shell. In the eastern tube the so-called hiDCon elements (Kovári 2005) were applied, in the western tube the LSC elements (Schubert 1996). During the repair works, the support pressure was reduced temporarily, leading thus to an acceleration of the convergences (see, e.g., increase in convergences $u_{\text {tot }}$ at distance $d_{f}=53 \mathrm{~m}$ of the tunnel face in Fig. 3.4c, Keller 2005). After these tunnel repairs, the deformations stopped temporarily (during ca. 6 months), but continued to increase again in the same way, leading once again to damage and tunnel repairs. More specifically, the ribs of the tunnel roof had to be set in a higher position, in order to guarantee the necessary clearance profile (Keller 2005).

Based on these experiences, the engineers decided to apply the support class ES6B right

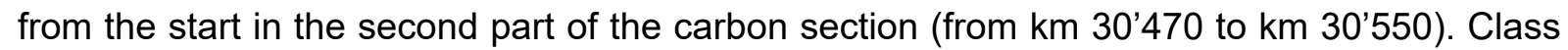
ES6B offers a $50-100 \%$ higher support pressure (Keller 2005). However, damage was observed in some parts of the tunnel after several months, so that tunnel repairs had to be performed again (Keller 2005).

Unfortunately, the compressible elements installed in the joints did not behave as expected in some parts of the tunnel: Due to the heterogeneous rock structure, the deformations were distributed non-uniformly over the tunnel cross-section, so that some elements already reached their deformation capacity, while other elements were still uncompressed (Keller 2005). Particularly, the use of the LSC elements was not successful, as the steel cylinders tipped over and did not buckle (Keller 2005). 

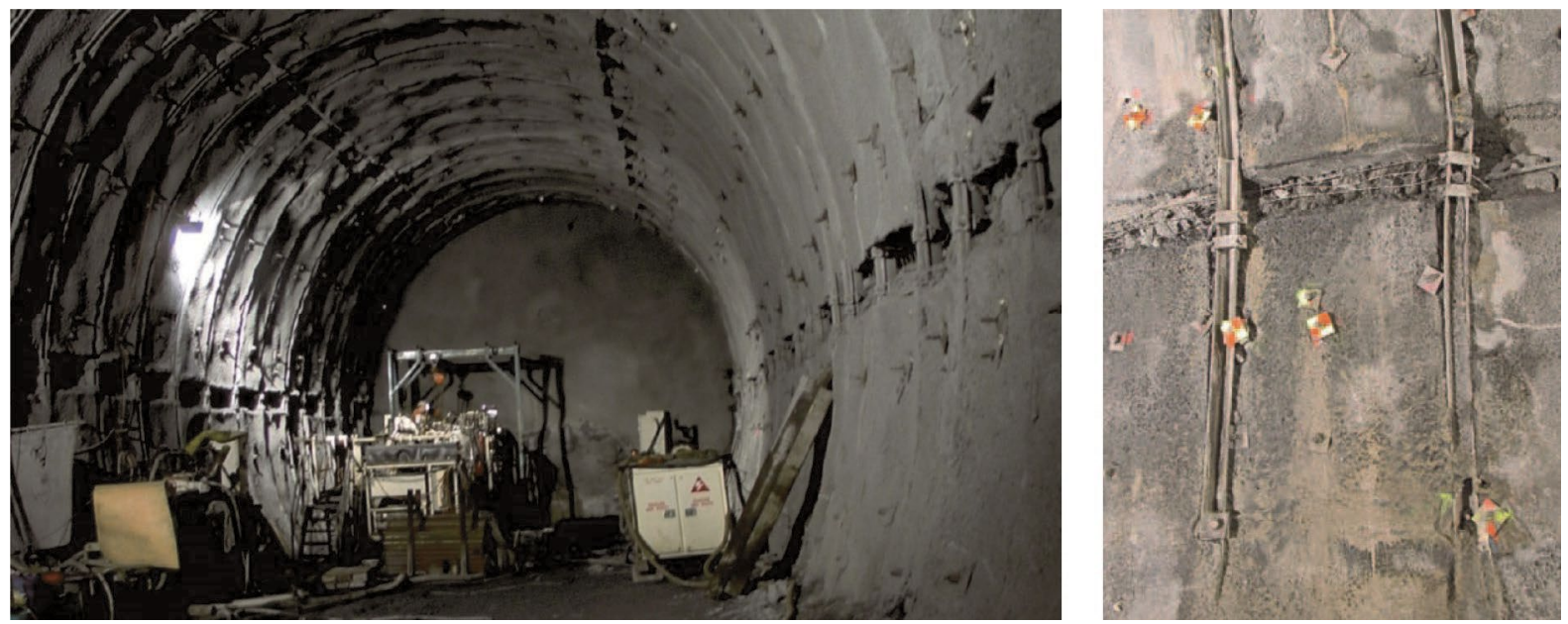

Figure 3.3. Left: Support class ES6A of the Lötschberg Base Tunnel (eastern tube at km 28'759; Geological Group of the Lötschberg Base Tunnel 2008); right: buckled steel ribs (eastern tube at km 30'421; IG-LBT 2009).

The centreline distance of the two tubes is equal to $40 \mathrm{~m}$. The tubes were excavated asynchronously, whereas the western tube was excavated first. No mutual influence was observed between the two tubes (as no increase of the convergences was observed in the western tube due to the excavation of the eastern tube, see, e.g., Fig. 3.4d).

\subsection{Rock response to tunnelling}

The behaviour of the rock mass and the tunnel support was monitored by 3D optical measurements and extensometers. In the carbon section only one monitoring station was instrumented with radial extensometers (Geological Group of the Lötschberg Base Tunnel 2008). However, as this monitoring station was installed at a distance of about $60 \mathrm{~m}$ behind the tunnel face, the extensometer data cannot be used for the purpose of this chapter. The displacements of the tunnel boundary during tunnel advance were monitored optically at stations spaced at about $10 \mathrm{~m}$, each usually containing 4 measuring points (cf. Fig. 3.1). The convergences reached locally about $70 \mathrm{~cm}$. The displacements were clearly affected by the lithology and the discontinuities (i.e., schistosity planes), so that the displacements varied strongly both over the cross-section and along the tunnel (Ziegler and Isler 2013). One could clearly observe that large deformations correlated with high anthracite contents of the schists (Ziegler and Isler 2013).

Due to the discontinuities and the anthracite layers, loosening also occurred in the tunnel roof (particularly in the unsupported span), however, the main phenomenon observed in the carbon section was squeezing.

\subsection{Data Analysis}

Contrary to the Sedrun Section of the Gotthard Base Tunnel (Chapter 1) and the GGium of the Ceneri Base Tunnel (Chapter 2), the zero readings of many monitoring stations were taken only $20 \mathrm{~m}$ behind the tunnel face. These displacement measurements could of course not be considered for the data analysis, as a big fraction of the displacement is missing: Only 
monitoring stations with zero readings within $6 \mathrm{~m}$ from the tunnel face were considered for the data analysis.

(a)

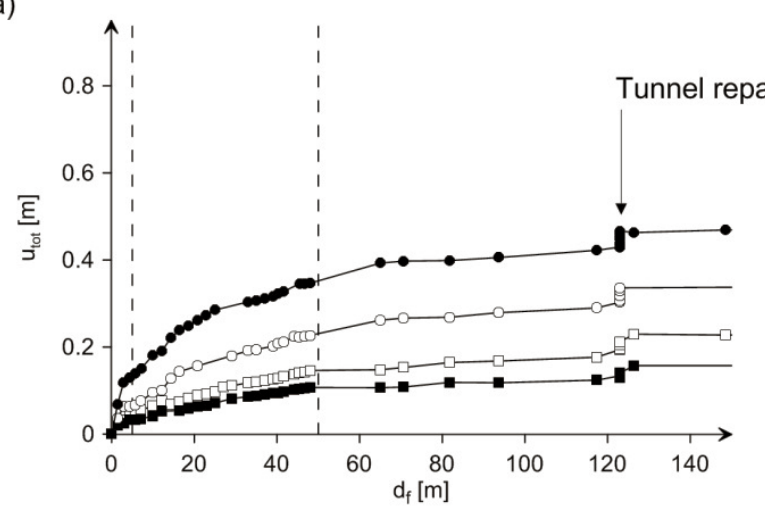

(c)

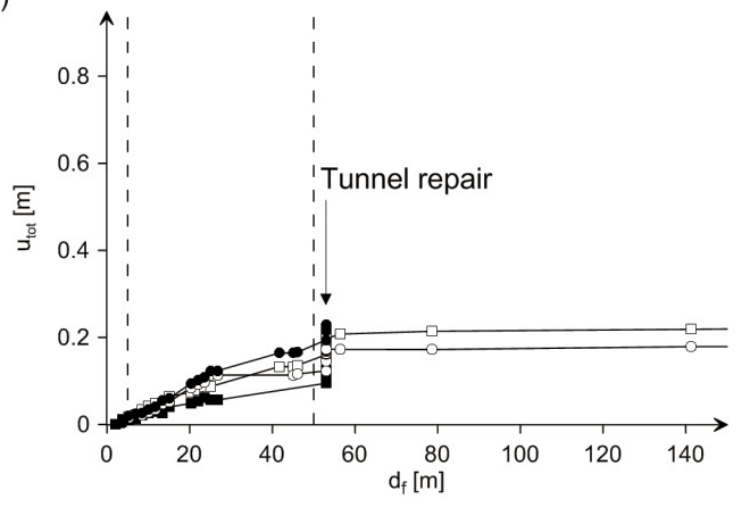

(b)

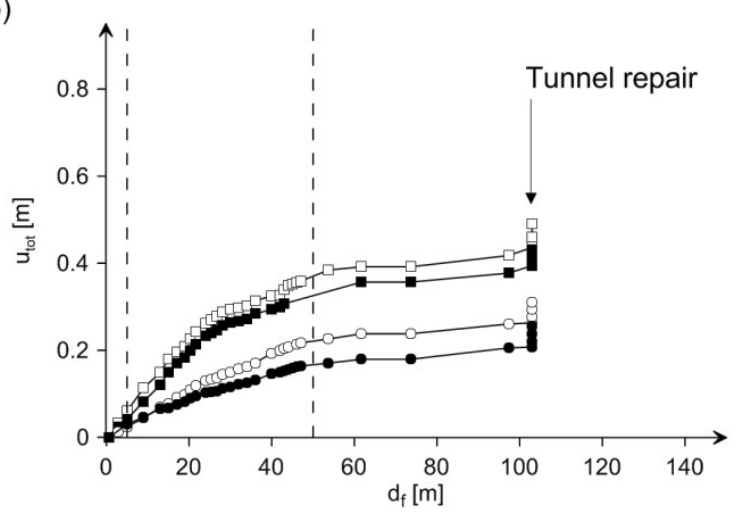

(d)

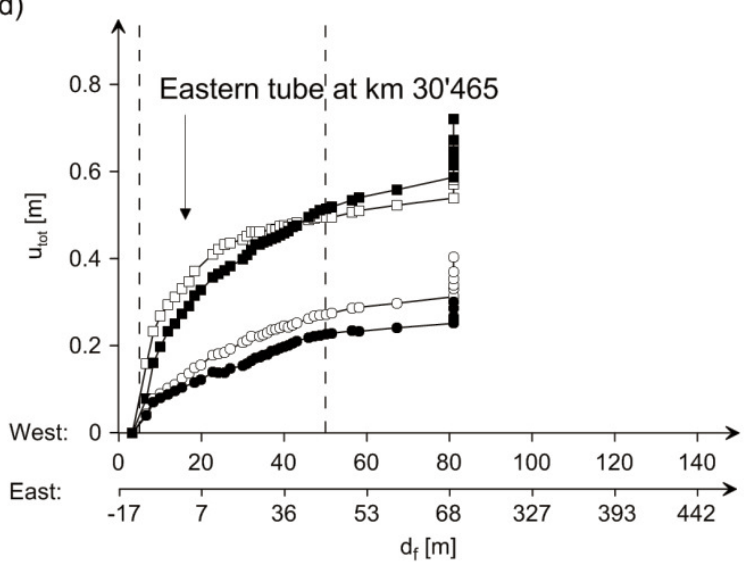

measuring points: $\quad \rightarrow \quad 1 \quad-\circ 2 \quad \bullet \quad 3 \quad \neg-4$

Figure 3.4. Magnitude $u_{t o t}$ of the displacement vector as a function of the distance to the face $d_{f}$ (for the positions of the measuring points in the profile: see Fig. 3.1): (a) eastern tube at km 30'410; (b) eastern tube at km 30'430; (c) eastern tube at km 30'480; (d) western tube at km 30'465.

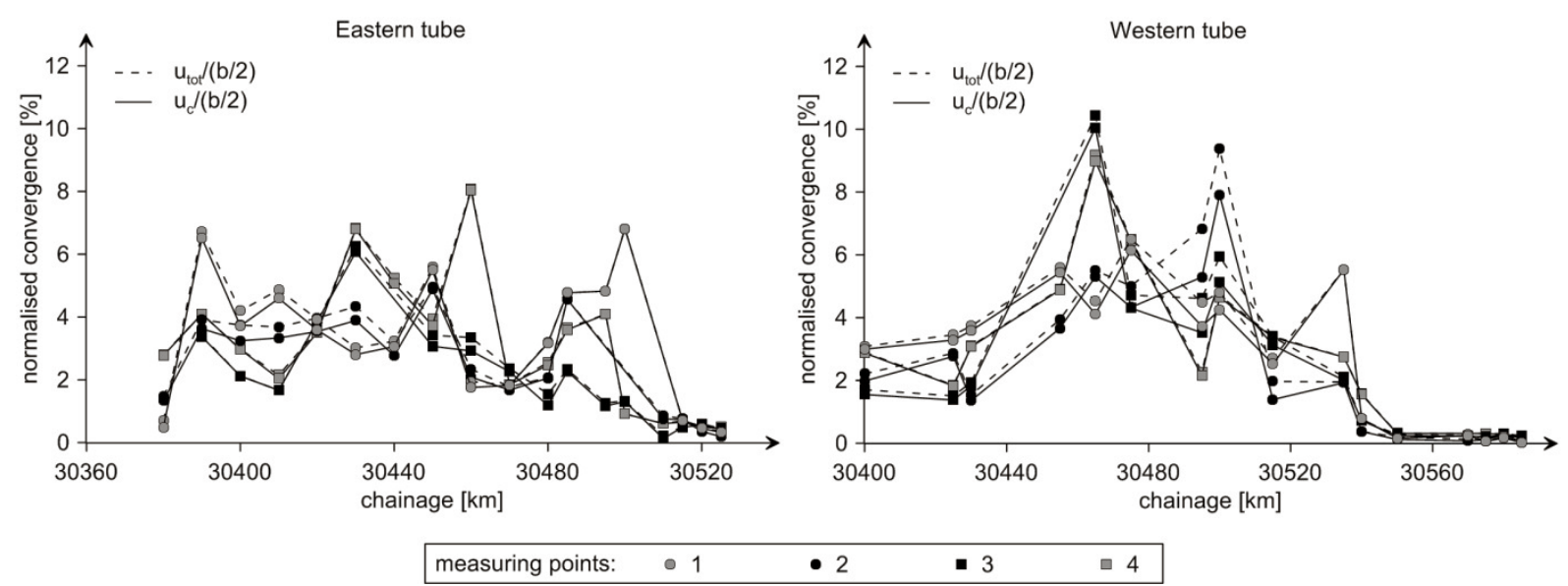

Figure 3.5. Normalised magnitude of the displacement vector $u_{\text {tot }}$ and of its projection in the crosssectional plane $u_{c}$ along the tunnel.

In order to ensure comparability between the measuring points, a fixed interval of the monitoring period is considered. More specifically, only the displacement that develops as the face moves from a distance of $5 \mathrm{~m}$ to a distance of $50 \mathrm{~m}$ ahead of the monitoring station is considered. This interval was considered because the zero readings of the useable 
measuring points were usually made at maximum $5 \mathrm{~m}$ behind the face (see Fig. 3.4), while the tunnel repair works took place at a distance of minimum $50 \mathrm{~m}$ from the face.

For dimensional reasons, the displacements in a structure increase linearly with its size, while all other parameters remain constant. In this section of the Lötschberg Base tunnel, the excavated width $b$ of the tunnel varies from 9.10 to $10.36 \mathrm{~m}$. In order to eliminate this effect, all the displacements are normalised by $b / 2$.

Figure 3.5 shows the longitudinal distribution of the magnitude of the displacement vector $u_{\text {tot }}$ and of the magnitude of the projection of the displacement vector in the cross-sectional plane of the tunnel $u_{c}$ for the measuring points of the different monitoring stations (for the definition, see Chapter 1). The difference between these values is small (the dashed lines almost coincide with the solid lines), which means that the longitudinal displacement component is small compared to the radial and the tangential ones. Subsequently only the magnitude of the displacement vector $u_{\text {tot }}$ will be considered.

A:

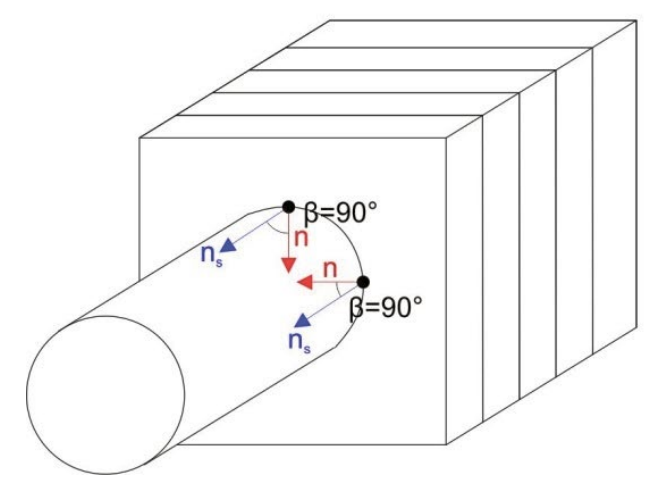

B:

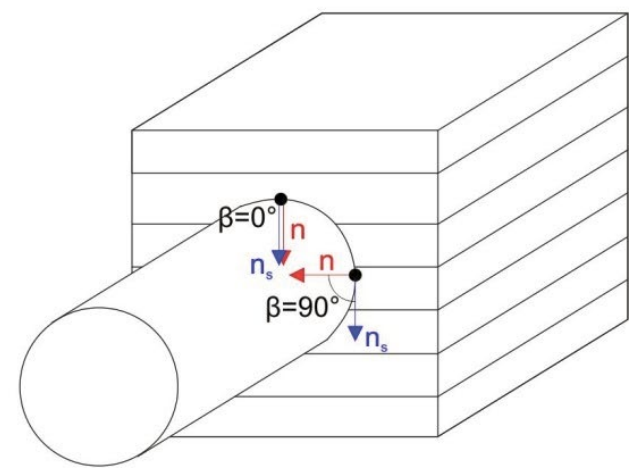

Figure 3.6. Schistosity angle $\beta$ at different points of the tunnel boundary: angle between the normal vector of the schistosity planes $n_{s}$ and the radial direction $n$.

As the tunnel does not cross the tectonic units perpendicularly, the measuring points of every monitoring station lie partially in different lithological units. In order to investigate the effect of the schistosity orientation, analogously to Chapter 2 , a schistosity angle $\beta$ is introduced, which is defined as the angle between the normal vector of the schistosity planes and the perpendicular to the direction of the maximum principal stress (angle between $n_{s}$ and $n$ in Fig. 3.6; the normal vector of the schistosity planes for a given dip and dip direction can be determined, e.g., after Schneider 2002).

From the literature (cf. Goricki et al. 2005, Button et al. 2006), it is principally known that the largest displacements occur at locations where the schistosity is almost parallel to the excavation boundary (i.e., $\beta \approx 0^{\circ}$; cf. Fig. 3.6), the smallest displacement where the schistosity is almost perpendicular to the excavation boundary (i.e., $\beta \approx 90^{\circ}$; cf. Fig. 3.6). Therefore, the smaller the schistosity angle, the higher the displacement should be (for a given lithological type and support class).

Figure 3.7 shows the normalised displacement as a function of the schistosity angle $\beta$ for the various lithological units and for the two support classes ES6A (installed in the first part of carbon) and ES6B (installed in the second part of the carbon). An influence of the schistosity angle can be observed only for the anthracite schists in the first part of the carbon (I.h.s. diagram of Fig. 3.7). For the sand- and siltstones this influence is less pronounced. This is 
presumably because the sand- and siltstones are solely partly schistous (Geological Group of the Lötschberg Base Tunnel 2008).

Furthermore, it must be noted that the schistosity mainly dips towards SE and does not vary strongly along the alignment. As the displacements are always measured at the same location of the cross-section (see Fig. 3.1), the range of the analysed schistosity angles is limited (i.e., mainly between 60 and $90^{\circ}$ ). Therefore, the influence of the schistosity is less visible than, e.g., in the GGium (Chapter 2). Furthermore, local schistosity changes, which might also affect the deformations, were not recorded and could not be considered in the evaluation of the data. In addition, it can be seen in Chapter 12 that, besides the schistosity angle, the dip angle of the schistosity influences the deformations, which was not taken into account in the data analysis.

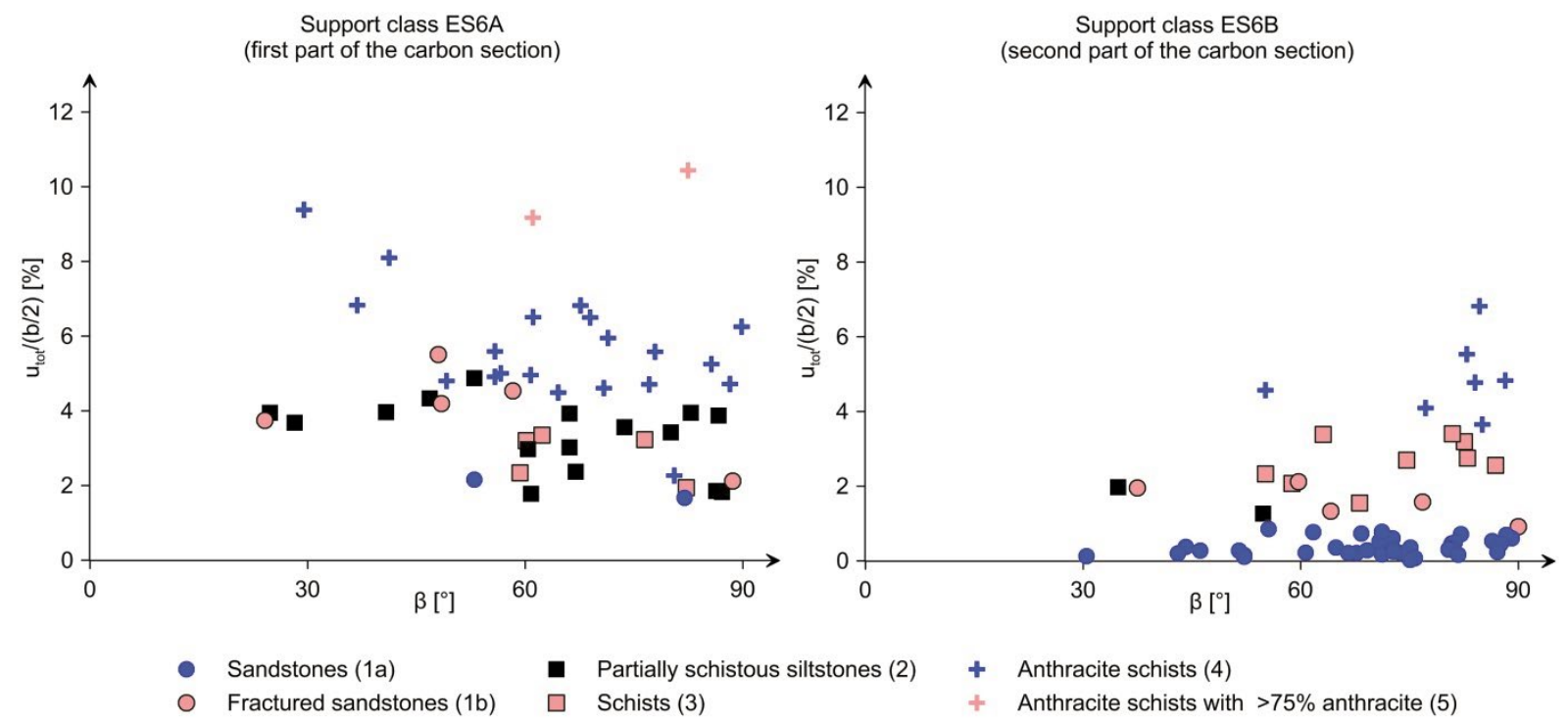

Figure 3.7. Normalised displacement $u_{t o t} /(b / 2)$ as a function of the schistosity angle $\beta$.

The effect of the lithology on the deformations is remarkable (compare, e.g., the lithological types $1 \mathrm{a}$ with 5 in Fig. 3.7):

The schists (lithological type 3 ) and the partly schistous siltstones (lithological type 2) seem to have similar mechanical properties, given that the displacements of these two lithologies are nearly the same. However, the range of the displacements of these two lithologies is large, which results probably from the spacing and the persistence of the schistosity that were not recorded during tunnel advance. An additional source of interpretation uncertainty is that the siltstones could hardly be distinguished from the sandstones and the schists during tunnel construction (see Section 3.2).

The anthracite content of the schists seems to affect the convergences significantly (i.e., compare the lithological types 4 and 5). Unfortunately, the anthracite content was not recorded systematically. A more detailed data analysis is therefore not possible.

According to the Geological Group of the Lötschberg Base Tunnel (2008), the lithologies in the first and second part of the tunnel were not distinguishable. Therefore, a reduction of the convergences in the second part of the tunnel can solely be traced back to the installation of the heavier support class ES6B. 


\subsection{Conclusions}

As for the case histories of the GGium and the Sedrun section, the squeezing deformations in the carbon section were mainly affected by the lithology and partly by the schistosity. However, the correlations found in this chapter are much weaker than for the other two case histories, which is to some extent due to the quantity and quality of the data.

\section{Notation}

$b$

$d_{f}$

$n$

$n_{s}$

$u_{c}$

$u_{\text {tot }}$

$\beta$ width of the tunnel

distance between the tunnel face and the monitoring station

radial direction

normal vector of the schistosity planes

magnitude of the projections of the displacement vectors in the crosssectional plane of the tunnel

magnitude of the displacement vectors

angle between the direction of the maximum principal stress and the schistosity planes 


\section{PART II: TUNNELLING PERPENDICULARLY TO ALTERNATING WEAK AND COMPETENT ROCK LAYERS}

This part of the thesis investigates the excavation-induced displacements in tunnelling perpendicular to alternating weak and hard rocks. If the alternating zones are thick relatively to the tunnel diameter, the response of such formations to tunnel excavation may exhibit a great variability, as was observed for instance in the Sedrun Section of the Gotthard Base Tunnel. If, however, the formation consists of very thin alternating weak and competent rock layers, the deformation distribution along the tunnel axis will be practically uniform. This means that rather than modelling the individual layers, which would be demanding in terms of spatial discretisation and computation time, the rock mass can be taken as homogeneous. In this chapter, the effect of these rock structures on squeezing deformations is analysed paying attention to the heterogeneity scale, either by modelling the individual layers or by considering a homogenised model. 



\section{Introduction}

Rock mass heterogeneity may lead - depending on the heterogeneity scale (Anagnostou et al. 2014) - to significant variations in the squeezing intensity during tunnelling. A specific case of heterogeneous rock mass is that of frequently alternating weak and competent zones (Fig. 4.1). Theoretical considerations (Kovári and Anagnostou 1995, Cantieni and Anagnostou 2007) and field observations (cf. Part I) show that there is a mutual influence between the weaker and the stronger rock mass components. The latter reduce the deformations of the adjacent weak rock (via shear stresses that develop along the zone interfaces), but may - due to the influence of the weak interlayers - become overstressed. The rock mass response to tunnelling depends thus on thickness, strength and deformability both of the hard and the weak zones and obviously also on their orientation relatively to the tunnel axis.

(a)

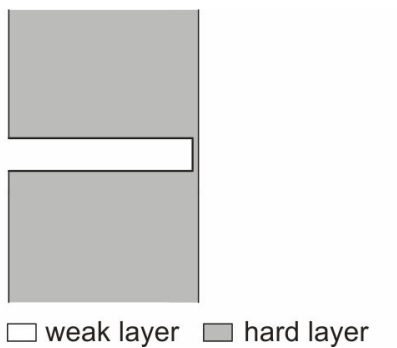

(b)

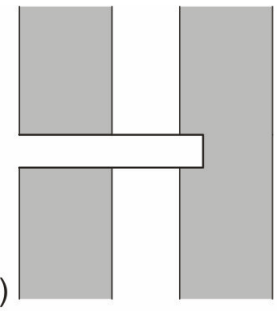

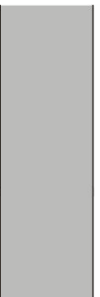

(c)

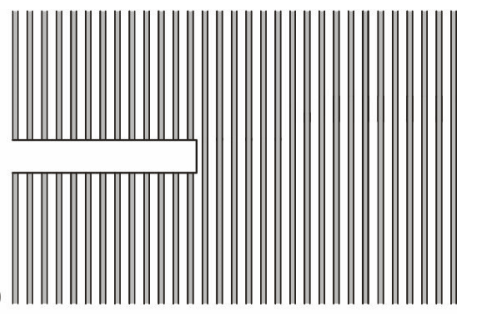

Figure 4.1. Tunnelling through a sequence of, (a), very thick weak and competent rock zones, (b), alternating weak and competent layers of medium thickness, (c), thinly alternating weak and competent layers.

Part II investigates the squeezing behaviour during tunnelling perpendicular to a sequence of alternating weak and hard rock layers (Fig. 4.1). Depending on the heterogeneity scale, the following three cases can be distinguished:

- If a weak formation is very thick relatively to the tunnel diameter (Fig. 4.1a), then the stabilising effect of adjacent competent rock is limited to the zone close to the formation interface and can be neglected in design.

- If, on the other hand, the alternating weak and competent rock layers are very thin relatively to the tunnel radius (Fig. 4.1c), then the deformation distribution along the tunnel axis will be practically uniform. This means that rather than modelling the individual layers, which would be demanding in terms of spatial discretisation and computation time, rock mass can be conceived as a homogeneous, but, in view of the rock structure transversely isotropic material, the mechanical behaviour of which depends on the behaviour of the individual layers and on the ratio of their thicknesses. For this special case, a closed-form solution (assuming rotational symmetry and plane strain 
conditions) is developed and the adequacy of a homogeneous model is investigated in Chapter 5.

- In a medium thickness weak zone (Fig. 4.1b), the excavation-induced displacements vary frequently along the tunnel. The stabilising effect of the hard interlayers can be taken into account computationally by numerical methods, whereby, due to the non-uniformity of squeezing intensity along the tunnel, the alternating weak and competent zones have to be modelled discretely (Kovári and Anagnostou 1995, Cantieni and Anagnostou 2007).

The response of the considered homogenised material to tunnelling is isotropic. This suggests that the rock mass could be considered as an isotropic and homogeneous elastoplastic material, with mechanical parameters that depend on those of the weak and hard components and on the fractions of the latter. Chapter 6 shows how the parameters of the equivalent homogeneous and isotropic material can be determined. This is very valuable for design practice, as it makes it possible to analyse problems, which do not fulfil the conditions of rotational symmetry or plane strain, also for the case of tunnelling perpendicular to thinly alternating weak and hard layers.

Chapter 7 investigates how the heterogeneity scale (represented by the thickness of the weak layers normalised by the tunnel radius) affects the longitudinal distribution of the displacements in tunnelling perpendicularly to a sequence of weak and hard layers of finite thickness. Furthermore, this section introduces a simple equation which allows estimating the displacements in weak zones (considering the stabilising effect of the hard layers) analytically, rendering numerical modelling unnecessary at least in the preliminary design stage. 


\section{Closed-form solution for the ground response curve in tunnelling perpendicularly to thinly alternating weak and competent rocks}

\subsection{Introduction}

For the formulation of appropriate constitutive models accounting for the thickness fractions and mechanical properties of the rock layers, homogenisation techniques have been proposed in the literature (e.g., Suquet 1987), initially only focussing on the linearly elastic response of the stratified rock mass (cf., e.g., Salamon 1968, Gerrard 1982) and later on also investigating some aspects of failure of a stratified rock mass (e.g., Taliercio and Sacchi Landriani 1988, Lai et al. 1999, Tien and Kuo 2001, Lydzba et al. 2003, Triantafyllidis and Gerolymatou 2014).

Closed-form solutions exist for estimating the displacements and the stresses of tunnels excavated in transversely isotropic rocks, considering either elastic or elasto-plastic behaviour (e.g., Hefny and Lo 1999, Bobet 2011, Zhang and Sun 2011, Tran Manh et al. 2015), whereby these methods do not account explicitly for the mechanical parameters of the individual layers. At the current state of research, there is no closed-form solution that allows determining the response of a homogenised rock mass consisting of alternating weak and hard layers due to tunnelling. Such a solution would, however, be of great importance, as numerical modelling of thinly alternating rock structures is very demanding in terms of spatial discretisation and computation time.

Section 5.2 derives analytically the ground response curve (GRC) for tunnelling perpendicularly to thinly alternating weak and hard layers, considering the rock mass as homogeneous, transversely isotropic material, assuming perfectly plastic behaviour for both rock constituents and disregarding, for simplicity, out-of-plane plastic flow. Sections 5.3 and 5.4 extend the closed-form solution considering additionally a brittle behaviour of the hard layers with a sudden post-failure decrease in strength (Park and Kim 2006 considered only a isotropic elastic, brittle-plastic rock mass) and out-of-plane plastic flow of the individual layers, respectively.

Section 5.5 discusses some aspects of the model behaviour by means of computational examples and, finally, Sections 5.6 and 5.7 investigate:

- under which conditions it is indispensable to consider both the weak and hard layers (or, in contrary, one can disregard the hard interlayers); and

- under which conditions it is adequate to consider the stratified rock mass as a homogeneous material. 


\subsection{Perfectly plastic behaviour disregarding out-of-plane plastic flow}

\subsubsection{Problem statement}

A deep, cylindrical tunnel supported by an uniform and radial pressure $\sigma_{a}$ is considered (Fig. 5.1). The initial stress field is taken as isotropic and uniform with a magnitude $\sigma_{0}$. Under these conditions, the problem obeys rotational symmetry. For the derivation of the GRC, plane strain conditions are considered (which is admissible because the tunnel axis is perpendicular to the layers, see further remarks in Section 12.1) and cylindrical coordinates $(r, t, z)$ are used with the axes origin being placed at the centre of the tunnel.

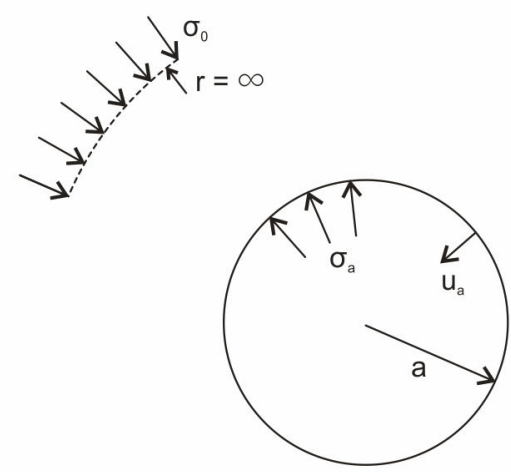

Figure 5.1. Problem statement for the determination of the GRC.

The unknown variables of the problem (i.e., the radial displacement $u(r)$, the radial strain $\varepsilon_{r}(r)$, the tangential strain $\varepsilon_{t}(r)$, the radial stress $\sigma_{r}(r)$ and the tangential stress $\left.\sigma_{t}(r)\right)$ are interconnected by the kinematic equations (using the infinitesimal strain formulation),

$$
\varepsilon_{r}=\frac{d u}{d r} \text { and } \varepsilon_{t}=\frac{u}{r}
$$

the stress equilibrium condition in the radial direction,

$$
\frac{d \sigma_{r}}{d r}=\frac{\sigma_{t}-\sigma_{r}}{r},
$$

as well as the incremental stress-strain relations of the homogenised material, which will be formulated in the next section.

For the determination of the GRC, the support pressure $\sigma_{a}$ decreases gradually, starting from its initial value $\sigma_{0}$, whereas the stresses in the far field remain unaltered by the excavation:

$$
\begin{gathered}
\sigma_{r}(\infty)=\sigma_{0}, \\
\sigma_{t}(\infty)=\sigma_{0}, \\
\sigma_{r}(a)=\sigma_{a} .
\end{gathered}
$$




\subsubsection{Incremental stress-strain relationships}

The constitutive equations of the homogeneous continuum are derived by using the homogenisation technique, based on Salamon (1968) and disregarding relative displacements at the layer interfaces. For that, a representative elementary volume (REV) is considered, which consists of the alternating thin hard and weak layers, whereby the z-axis is perpendicular to the layers (Fig. 5.2).

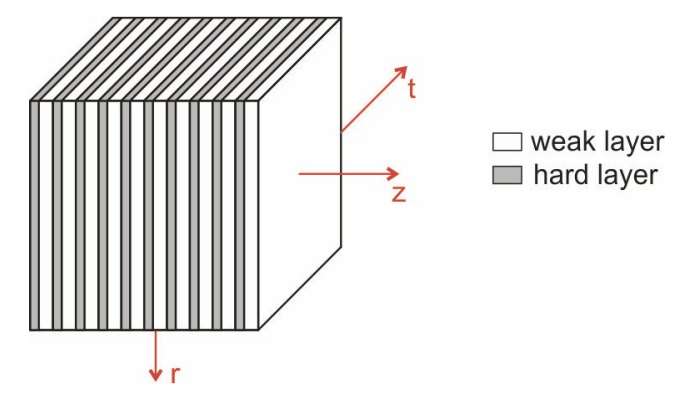

Figure 5.2. REV consisting of a sequence of hard and weak layers.

By definition, the variation of stresses and strains across the REV can be neglected, meaning that the resulting strains and stresses of the homogeneous equivalent element are the volumetric averages of the strain and stress components in the different layers (see also Salamon 1968):

$$
\begin{gathered}
d \varepsilon_{r}=d \varepsilon_{r, h}=d \varepsilon_{r, w}, \\
d \varepsilon_{t}=d \varepsilon_{t, h}=d \varepsilon_{t, w}, \\
d \varepsilon_{z}=d \varepsilon_{z, h} \cdot x_{h}+d \varepsilon_{z, w} \cdot x_{w}, \\
d \sigma_{r}=d \sigma_{r, h} \cdot x_{h}+d \sigma_{r, w} \cdot x_{w}, \\
d \sigma_{t}=d \sigma_{t, h} \cdot x_{h}+d \sigma_{t, w} \cdot x_{w}, \\
d \sigma_{z}=d \sigma_{z, h}=d \sigma_{z, w},
\end{gathered}
$$

where $x_{h}$ and $x_{w}$ denote the thickness fractions of the hard and weak layers, respectively $\left(x_{h}+x_{w}=1\right)$. It should be noted that the plane strain condition,

$$
d \varepsilon_{\mathrm{z}}=0
$$

applies to the REV, but not to the individual layers. For the formulation of the constitutive equations of the REV, both the weak and hard layers are considered as homogeneous, isotropic, linearly elastic and perfectly plastic materials obeying Mohr-Coulomb failure criterion (in general, with a non-associated flow rule).

Hooke's Law can be written in incremental form as follows:

$$
\begin{aligned}
& d \sigma_{r, w}=2 \cdot \mu_{w} \cdot d \varepsilon_{r, w}^{E I}+\lambda_{w} \cdot\left(d \varepsilon_{r, w}^{E I}+d \varepsilon_{t, w}^{E I}+d \varepsilon_{z, w}^{E I}\right) \\
& d \sigma_{t, w}=2 \cdot \mu_{w} \cdot d \varepsilon_{t, w}^{E I}+\lambda_{w} \cdot\left(d \varepsilon_{r, w}^{E I}+d \varepsilon_{t, w}^{E I}+d \varepsilon_{z, w}^{E I}\right) \text { and } \\
& d \sigma_{z, w}=2 \cdot \mu_{w} \cdot d \varepsilon_{z, w}+\lambda_{w} \cdot\left(d \varepsilon_{r, w}^{E I}+d \varepsilon_{t, w}^{E I}+d \varepsilon_{z, w}^{E I}\right)
\end{aligned}
$$




$$
\begin{aligned}
& d \sigma_{r, h}=2 \cdot \mu_{h} \cdot d \varepsilon_{r, h}^{E I}+\lambda_{h} \cdot\left(d \varepsilon_{r, h}^{E I}+d \varepsilon_{t, h}^{E I}+d \varepsilon_{z, h}^{E I}\right) \\
& d \sigma_{t, h}=2 \cdot \mu_{h} \cdot d \varepsilon_{t, h}^{E I}+\lambda_{h} \cdot\left(d \varepsilon_{r, h}^{E I}+d \varepsilon_{t, h}^{E I}+d \varepsilon_{z, h}^{E I}\right), \\
& d \sigma_{z, h}=2 \cdot \mu_{h} \cdot d \varepsilon_{z, h}+\lambda_{h} \cdot\left(d \varepsilon_{r, h}^{E I}+d \varepsilon_{t, h}^{E I}+d \varepsilon_{z, h}^{E I}\right)
\end{aligned}
$$

where $\lambda$ und $\mu$ are the Lamé constants and the subscripts $w$ and $h$ denote the weak and the hard layers, respectively ${ }^{3}$.

Considering that the principal stresses act in $t-, r$ - and $z$-direction and assuming that the maximum and minimum principal stresses are in $t$ - and $r$-direction (i.e., the axial stress is the intermediate principal stress, which is not always true; see Section 5.2.6), the Mohr-Coulomb failure criteria for the weak and the hard layers are:

$$
\begin{gathered}
\sigma_{t, w}=m_{w} \cdot \sigma_{r, w}+\sigma_{d, w} \text { and } \\
\sigma_{t, h}=m_{h} \cdot \sigma_{r, h}+\sigma_{d, h},
\end{gathered}
$$

respectively, or, in incremental form,

$$
\begin{gathered}
d \sigma_{t, w}=m_{w} \cdot d \sigma_{r, w} \text { and } \\
d \sigma_{t, h}=m_{h} \cdot d \sigma_{r, h},
\end{gathered}
$$

where $m_{w}=\frac{1+\sin \varphi_{w}}{1-\sin \varphi_{w}}, m_{h}=\frac{1+\sin \varphi_{h}}{1-\sin \varphi_{h}}, \sigma_{d, w}=\frac{2 \cdot c_{w} \cdot \cos \varphi_{w}}{1-\sin \varphi_{w}}, \sigma_{d, h}=\frac{2 \cdot c_{h} \cdot \cos \varphi_{h}}{1-\sin \varphi_{h}}$; and $\varphi_{w}$ and $\varphi_{h}$ denote the friction angles and $c_{w}$ and $c_{h}$ denote the cohesions.

During yielding, the plastic strain increments are interconnected via the flow rule:

$$
\begin{gathered}
d \varepsilon_{r, w}-d \varepsilon_{r, w}^{\boxminus}=-\kappa_{w} \cdot\left(d \varepsilon_{t, w}-d \varepsilon_{t, w}^{\boxminus}\right), \\
d \varepsilon_{r, h}-d \varepsilon_{r, h}^{\boxminus}=-\kappa_{h} \cdot\left(d \varepsilon_{t, h}-d \varepsilon_{t, h}^{\boxminus}\right),
\end{gathered}
$$

where $\kappa_{w}=\frac{1+\sin \psi_{w}}{1-\sin \psi_{w}}, \kappa_{h}=\frac{1+\sin \psi_{h}}{1-\sin \psi_{h}}$; and $\psi_{w}$ and $\psi_{h}$ denote the dilatancy angles, while only elastic strains occur in z-direction. (Out-of-plane plastic flow renders the mathematical treatment more cumbersome and will be considered later in Section 5.4.)

${ }^{3}$ The Lamé constants are interconnected with the Young's modulus $E$ and the Poisson's ratio $v$ by the following equations:

$$
\lambda=\frac{E \cdot v}{(1+v) \cdot(1-2 v)}, \mu=\frac{E}{2 \cdot(1+v)}, E=\frac{3 \mu \cdot(\lambda+2 \cdot \mu / 3)}{(\lambda+\mu)} \text { and } v=\frac{\lambda}{2 \cdot(\lambda+\mu)} .
$$


The incremental stress-strain relationships

$$
\left(\begin{array}{l}
d \sigma_{r} \\
d \sigma_{t} \\
d \sigma_{z} \\
d \sigma_{r, h} \\
d \sigma_{t, h} \\
d \sigma_{r, w} \\
d \sigma_{t, w}
\end{array}\right)=\left(\begin{array}{cc}
A_{1} & -A_{2} \\
A_{3} & -A_{4} \\
B_{1} & B_{2} \\
K_{1} & K_{2} \\
K_{3} & K_{4} \\
K_{5} & K_{6} \\
K_{7} & K_{8}
\end{array}\right) \cdot\left(\begin{array}{l}
d \varepsilon_{r} \\
d \varepsilon_{t}
\end{array}\right),
$$

of the REV (first three rows), of the hard layers (fourth and fifth row) and of the weak layers (last two rows) can be obtained from Equations (5.6) to (5.12), Hooke's law (Eqs. 5.13 and 5.14 ) and, in the case of yielding, additional equations according to Table 5.1, where four cases are distinguished depending on whether the weak and/or the hard layers yield. The coefficients in the r.h.s. of Equation (5.21) are case-specific (cf. Table 5.1) and can be found in Sections A.1 to A.4 of Appendix A. The incremental stress-strain relationships of the hard and of the weak layers are important for checking, whether the weak or the hard layers reach failure, and thus to choose the applicable case-specific coefficients.

Table 5.1. Cases to be considered in the incremental stress-strain relationships.

\begin{tabular}{|c|c|c|}
\hline Case & & Applicable equations \\
\hline $\mathrm{i}$ & $\begin{array}{l}\text { Both the weak and the hard layers behave } \\
\text { elastically }{ }^{(1)}\end{array}$ & $\begin{array}{l}d \varepsilon_{r, w}=d \varepsilon_{r, w}^{\boxminus I}, d \varepsilon_{t, w}=d \varepsilon_{t, w}^{E I} \\
d \varepsilon_{r, h}=d \varepsilon_{r, h}^{E I}, d \varepsilon_{t, h}=d \varepsilon_{t, h}^{E I}\end{array}$ \\
\hline ii & $\begin{array}{l}\text { weak layers are at failure; } \\
\text { hard layers still behave elastically }\end{array}$ & $\begin{array}{l}d \varepsilon_{r, h}=d \varepsilon_{r, h}^{E l}, d \varepsilon_{t, h}=d \varepsilon_{t, h}^{\boxminus}, \\
\text { Mohr-Coulomb failure criterion (Eq. 5.17) and } \\
\text { flow rule (Eq. 5.19) for the weak layers }\end{array}$ \\
\hline iii & $\begin{array}{l}\text { hard layers are at failure; } \\
\text { weak layers still behave elastically }\end{array}$ & $\begin{array}{l}d \varepsilon_{r, w}=d \varepsilon_{r, w}^{\boxminus I}, d \varepsilon_{t, w}=d \varepsilon_{t, w}^{E l}, \\
\text { Mohr-Coulomb failure criterion (Eq. 5.18) and } \\
\text { flow rule (Eq. 5.20) for the hard layers }\end{array}$ \\
\hline iv & both the weak and hard layers are at failure & $\begin{array}{l}\text { Mohr-Coulomb failure criterion and flow rule } \\
\text { both for the hard and the weak layers (Eqs. } \\
5.17 \text { to } 5.20 \text { ), }\end{array}$ \\
\hline
\end{tabular}

\subsubsection{Elastic part of the GRC}

During the decrease in support pressure, the ground behaves initially elastically (Fig. 5.3). By integrating the incremental stress-strain relationship of the REV for case $\mathrm{i}$ of Table 5.1 (Eq. 5.21), considering the initial value of the stresses, the following equation can be obtained:

$$
\left(\begin{array}{c}
\sigma_{r}-\sigma_{0} \\
\sigma_{t}-\sigma_{0}
\end{array}\right)=\left(\begin{array}{cc}
2 \cdot \mu^{*}+\lambda^{*} & \lambda^{*} \\
\lambda^{*} & 2 \cdot \mu^{*}+\lambda^{*}
\end{array}\right) \cdot\left(\begin{array}{l}
\varepsilon_{r} \\
\varepsilon_{t}
\end{array}\right) .
$$




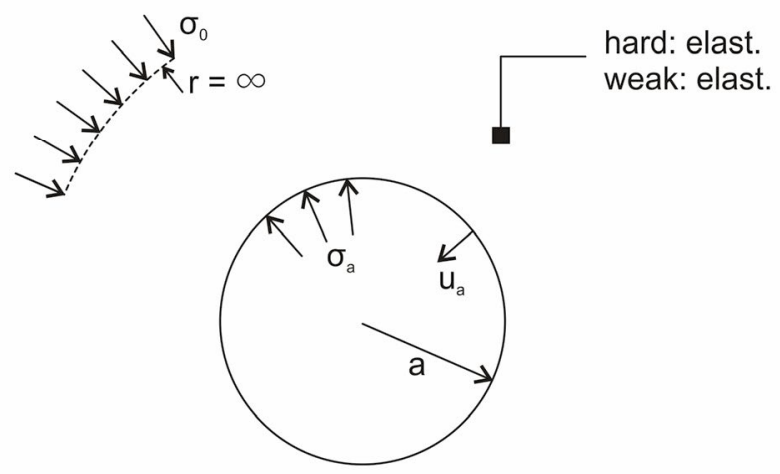

Figure 5.3. Purely elastic response.

This equation in combination with the kinematic relationships (cf. Eq. 5.1) and the equilibrium condition (Eq. 5.2) leads to a second order differential equation for the displacement $u(r)$ :

$$
\frac{\left(2 \cdot \mu^{*}+\lambda^{*}\right) \cdot\left(u(r)-r \cdot\left(u^{\prime}(r)+r \cdot u^{\prime \prime}(r)\right)\right)}{r^{2}}=0
$$

the solution of which provides the displacement field and thus (cf. Eqs. 5.1 and 5.22) the stress- and strain-fields as a function of two integration constants. With the boundary conditions (Eqs. 5.3 and 5.5), the two integration constants and the following stress-, strainand displacement fields are obtained:

$$
\begin{gathered}
u(r)=\frac{1}{2 \cdot \mu^{*}} \cdot\left(\sigma_{0}-\sigma_{a}\right) \cdot \frac{a^{2}}{r}, \\
\sigma_{r}(r)=\sigma_{0}-\left(\sigma_{0}-\sigma_{a}\right) \cdot\left(\frac{a}{r}\right)^{2}, \\
\sigma_{t}(r)=\sigma_{0}+\left(\sigma_{0}-\sigma_{a}\right) \cdot\left(\frac{a}{r}\right)^{2}, \\
\sigma_{z}(r)=\sigma_{0}, \\
\varepsilon_{r}(r)=\frac{a^{2} \cdot\left(\sigma_{a}-\sigma_{0}\right)}{2 \cdot r^{2} \cdot \mu^{*}}, \\
\varepsilon_{t}(r)=\frac{a^{2} \cdot\left(\sigma_{0}-\sigma_{a}\right)}{2 \cdot r^{2} \cdot \mu^{*}} .
\end{gathered}
$$

The stresses in the hard and the weak layers are obtained by integrating their incremental stress-strain relationships (four last rows of Eq. 5.21 with the coefficients for case i), considering the initial stresses and the strains $\varepsilon_{r}(r)$ and $\varepsilon_{t}(r)$ :

$$
\sigma_{r, w}(r)=\sigma_{0}-\frac{a^{2}}{r^{2}} \cdot \frac{\mu_{w}}{\mu^{*}} \cdot\left(\sigma_{0}-\sigma_{a}\right)
$$




$$
\begin{aligned}
& \sigma_{t, w}(r)=\sigma_{0}+\frac{a^{2}}{r^{2}} \cdot \frac{\mu_{w}}{\mu^{*}} \cdot\left(\sigma_{0}-\sigma_{a}\right), \\
& \sigma_{r, h}(r)=\sigma_{0}-\frac{a^{2}}{r^{2}} \cdot \frac{\mu_{h}}{\mu^{*}} \cdot\left(\sigma_{0}-\sigma_{a}\right), \\
& \sigma_{t, h}(r)=\sigma_{0}+\frac{a^{2}}{r^{2}} \cdot \frac{\mu_{h}}{\mu^{*}} \cdot\left(\sigma_{0}-\sigma_{a}\right) .
\end{aligned}
$$

The elastic part of the ground response curve is given by Equation (5.24) with $r=a$.

The assumption of elastic behaviour is valid as long as the stress state at the tunnel boundary $(r=a)$ is within the elastic domain both in the weak and in the hard layers, i.e., as long as $\sigma_{t, w}(r=a) \leq m_{w} \cdot \sigma_{r, w}(r=a)+\sigma_{d, w}$ and $\sigma_{t, h}(r=a) \leq m_{h} \cdot \sigma_{r, h}(r=a)+\sigma_{d, h}$. Introducing $\sigma_{r, w}$, $\sigma_{t, w}, \sigma_{r, h}$ and $\sigma_{t, h}$ from Equations (5.30) to (5.33) into these equations leads to the condition $\sigma_{a} \geq \sigma_{r, \rho_{1}}=\max \left(\sigma_{r, \rho_{1}, w}, \sigma_{r, \rho_{1}, h}\right)$, where

$$
\begin{gathered}
\sigma_{r, \rho_{1}, w}=\frac{\left(1+m_{w}\right) \cdot \mu_{w} \cdot \sigma_{0}-\mu^{*} \cdot\left(\left(-1+m_{w}\right) \cdot \sigma_{0}+\sigma_{d, w}\right)}{\left(1+m_{w}\right) \cdot \mu_{w}}, \\
\sigma_{r, \rho_{1}, h}=\frac{\left(1+m_{h}\right) \cdot \mu_{h} \cdot \sigma_{0}-\mu^{*} \cdot\left(\left(-1+m_{h}\right) \cdot \sigma_{0}+\sigma_{d, h}\right)}{\left(1+m_{h}\right) \cdot \mu_{h}} .
\end{gathered}
$$

If $\sigma_{r, \rho 1, w}$ is greater than $\sigma_{r, \rho 1, h}$ (hereafter referred to as "case A"), then failure occurs first in the weak layers. Otherwise, if $\sigma_{r, \rho 1, w}$ is smaller than $\sigma_{r, \rho 1, h}$ ("case B"), then failure occurs first in the hard layers.

\subsubsection{GRC part for elasto-plastic behaviour either of the weak or the hard layers}

If the support pressure $\sigma_{a}$ is less than $\sigma_{r, \rho 1}$, then a plastic zone develops around the tunnel, in which either the weak layers or the hard layers yield (Fig. 5.4).

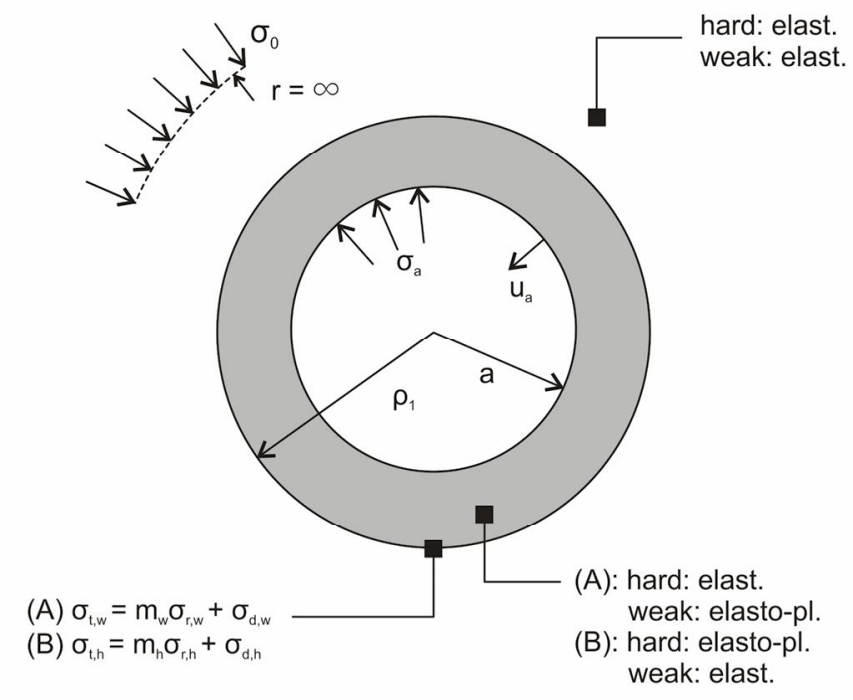

Figure 5.4. Plastic zone with yielding only of the hard or only of the weak layers in the inner ring. 
Due to the rotational symmetry, the plastic zone represents a thick-walled cylinder with an internal radius a and an external radius $\rho_{1}$, which is loaded by the external pressure $\sigma_{r, \rho 1}$. Outside this zone, both the weak and the hard layers behave linear-elastically. The plastic radius $\rho_{1}$ is initially unknown.

The stresses, strains and displacements in the elastic zone $\left(r \geq \rho_{1}\right)$ can be obtained by applying the equations derived in Section 5.2.3 to a tunnel with radius $\rho_{1}$ and support pressure $\sigma_{r, \rho 1}$ :

$$
\begin{gathered}
u=\frac{1}{2 \cdot \mu^{*}} \cdot\left(\sigma_{0}-\sigma_{r, \rho_{1}}\right) \cdot \frac{\rho_{1}^{2}}{r}, \\
\sigma_{r}=\sigma_{0}-\left(\sigma_{0}-\sigma_{r, \rho_{1}}\right) \cdot\left(\frac{\rho_{1}}{r}\right)^{2}, \\
\sigma_{t}=\sigma_{0}+\left(\sigma_{0}-\sigma_{r, \rho_{1}}\right) \cdot\left(\frac{\rho_{1}}{r}\right)^{2}, \\
\varepsilon_{r}=\frac{\rho_{1}^{2} \cdot\left(\sigma_{r, \rho_{1}}-\sigma_{0}\right)}{2 \cdot r^{2} \cdot \mu^{*}}, \\
\varepsilon_{t}=\frac{\rho_{1}^{2} \cdot\left(\sigma_{0}-\sigma_{r, \rho_{1}}\right)}{2 \cdot r^{2} \cdot \mu^{*}}, \\
\sigma_{r, w}(r)=\sigma_{0}-\frac{\rho_{1}^{2}}{r^{2}} \cdot \frac{\mu_{w}}{\mu^{*}} \cdot\left(\sigma_{0}-\sigma_{r, \rho_{1}}\right), \\
\sigma_{t, h}(r)=\sigma_{0}+\frac{\rho_{1}^{2}}{r^{2}} \cdot \frac{\mu_{h}}{\mu^{*}} \cdot\left(\sigma_{0}-\sigma_{r, \rho_{1}}\right) . \\
\sigma_{r, h}(r)=\sigma_{0}+\frac{\rho_{1}^{2}}{r^{2}} \cdot \frac{\mu_{w}}{\mu^{*}} \cdot\left(\sigma_{0}-\sigma_{r, \rho_{1}}\right), \\
\sigma_{1}^{2} \cdot \frac{\mu_{h}}{\mu^{*}} \cdot\left(\sigma_{0}-\sigma_{r, \rho_{1}}\right),
\end{gathered}
$$

Given the contact pressure $\sigma_{r, \rho 1}$ (Eq. 5.34 or 5.35, depending on whether the weak or the hard layers yield first), the stresses and strains $\left(\sigma_{t, \rho 1}, \sigma_{r, h, \rho 1}, \sigma_{t, h, \rho 1}, \sigma_{r, w, \rho 1}, \sigma_{t, w, \rho 1}, \varepsilon_{r, \rho 1}, \varepsilon_{t, \rho 1}\right)$ at the interface of the elastic and the plastic zone $\left(r=\rho_{1}\right)$ can be determined, using the Equations (5.37) to (5.45) for $r=\rho_{1}$ :

$$
\begin{gathered}
\sigma_{t, \rho_{1}}=2 \cdot \sigma_{0}-\sigma_{r, \rho_{1}}, \\
\sigma_{z, \rho_{1}}=\sigma_{0}, \\
\sigma_{r, w, \rho_{1}}=\sigma_{0}-\frac{\mu_{w}}{\mu^{*}}\left(\sigma_{0}-\sigma_{r, \rho_{1}}\right),
\end{gathered}
$$




$$
\begin{gathered}
\sigma_{t, w, \rho_{1}}=\sigma_{0}+\frac{\mu_{w}}{\mu^{*}}\left(\sigma_{0}-\sigma_{r, \rho_{1}}\right), \\
\sigma_{r, h, \rho_{1}}=\sigma_{0}-\frac{\mu_{h}}{\mu^{*}}\left(\sigma_{0}-\sigma_{r, \rho_{1}}\right), \\
\sigma_{t, h, \rho_{1}}=\sigma_{0}+\frac{\mu_{h}}{\mu^{*}}\left(\sigma_{0}-\sigma_{r, \rho_{1}}\right), \\
\varepsilon_{r, \rho_{1}}=-\frac{1}{2 \cdot \mu^{*}}\left(\sigma_{0}-\sigma_{r, \rho_{1}}\right), \\
\varepsilon_{t, \rho_{1}}=\frac{1}{2 \cdot \mu^{*}}\left(\sigma_{0}-\sigma_{r, \rho_{1}}\right) .
\end{gathered}
$$

The integration of the incremental stress-strain relationships (i.e., first two rows of Eq. 5.21), considering the boundary conditions at $r=\rho=\rho_{1}$, leads to the following relation between the stresses and strains inside the plastic zone $\left(a \leq r \leq \rho_{1}\right)$ :

$$
\left(\begin{array}{c}
\sigma_{r}-\sigma_{r, \rho} \\
\sigma_{t}-\sigma_{t, \rho}
\end{array}\right)=\left(\begin{array}{cc}
A_{1} & -A_{2} \\
A_{3} & -A_{4}
\end{array}\right) \cdot\left(\begin{array}{c}
\varepsilon_{r}-\varepsilon_{r, \rho} \\
\varepsilon_{t}-\varepsilon_{t, \rho}
\end{array}\right)
$$

where $A_{1}=A_{1, i i}, A_{2}=A_{2, i i}, A_{3}=A_{3, i i}$ and $A_{4}=A_{4, i i}$ in case A, while $A_{1}=A_{1, i i i}, A_{2}=A_{2, i i i}, A_{3}=A_{3, i i i}$ and $A_{4}=A_{4, i i i}$ in case B. (These coefficients are given in Appendices A.2 and A.3.) Equation (5.54) combined with the kinematic relationships (Eq. 5.1) and the equilibrium conditions (Eq. 5.2) leads to a second order differential equation for $u(r)$ :

$$
\frac{r\left(A_{1} \varepsilon_{r, \rho}-A_{3} \varepsilon_{r, \rho}-A_{2} \varepsilon_{t, \rho}+A_{4} \varepsilon_{t, \rho}-\sigma_{r, \rho}+\sigma_{t, \rho}+\left(-A_{1}+A_{2}+A_{3}\right) u^{\prime}(r)-A_{1} r u^{\prime \prime}(r)\right)-A_{4} u(r)}{r^{2}}=0
$$

the solution of which yields the stress, strain as well as the displacement field in the plastic zone:

$$
\begin{aligned}
u(r)= & \frac{r\left(A_{1} \varepsilon_{r, \rho}-A_{3} \varepsilon_{r, \rho}-A_{2} \varepsilon_{t, \rho}+A_{4} \varepsilon_{t, \rho}-\sigma_{r, \rho}+\sigma_{t, \rho}\right)}{A_{1}-A_{2}-A_{3}+A_{4}}+r^{\frac{-A+A_{2}+A_{3}}{2 A_{1}}} C[1]+r^{\frac{A+A_{2}+A_{3}}{2 A_{1}}} C[2], \\
\varepsilon_{r}(r)= & \frac{A_{1} \varepsilon_{r, \rho}-A_{3} \varepsilon_{r, \rho}-A_{2} \varepsilon_{t, \rho}+A_{4} \varepsilon_{t, \rho}-\sigma_{r, \rho}+\sigma_{t, \rho}}{A_{1}-A_{2}-A_{3}+A_{4}}+\frac{\left(-A+A_{2}+A_{3}\right) r^{-1+\frac{-A+A_{2}+A_{3}}{2 A_{1}}} C[1]}{2 A_{1}}+ \\
& \frac{\left(A+A_{2}+A_{3}\right) r^{-1+\frac{A+A_{2}+A_{3}}{2 A_{1}}} C[2]}{2 A_{1}} \\
\varepsilon_{t}(r)= & \frac{\frac{r\left(A_{1} \varepsilon_{r, \rho}-A_{3} \varepsilon_{r, \rho}-A_{2} \varepsilon_{t, \rho}+A_{4} \varepsilon_{t, \rho}-\sigma_{r, \rho}+\sigma_{t, \rho}\right)}{A_{1}-A_{2}-A_{3}+A_{4}}+r^{\frac{-A+A_{2}+A_{3}}{2 A_{1}}} C[1]+r^{\frac{A+A_{2}+A_{3}}{2 A_{1}} C[2]}}{r},
\end{aligned}
$$




$$
\begin{gathered}
\sigma_{r}(r)=\frac{\left(-A_{3}+A_{4}\right) \sigma_{r, \rho}+A_{2}\left(A_{3}\left(\varepsilon_{r, \rho}-\varepsilon_{t, \rho}\right)-\sigma_{t, \rho}\right)+A_{1}\left(-A_{4} \varepsilon_{r, \rho}+A_{4} \varepsilon_{t, \rho}+\sigma_{t, \rho}\right)}{A_{1}-A_{2}-A_{3}+A_{4}}+ \\
\frac{1}{2} r^{\frac{-A-2 A_{1}+A_{2}+A_{3}}{2 A_{1}}}\left(A\left(-C[1]+r^{\frac{A}{A_{1}}} C[2]\right)-\left(A_{2}-A_{3}\right)\left(C[1]+r^{\frac{A}{A_{1}}} C[2]\right)\right), \\
\sigma_{t}(r)=\frac{\left(-A_{3}+A_{4}\right) \sigma_{r, \rho}+A_{2}\left(A_{3}\left(\varepsilon_{r, \rho}-\varepsilon_{t, \rho}\right)-\sigma_{t, \rho}\right)+A_{1}\left(-A_{4} \varepsilon_{r, \rho}+A_{4} \varepsilon_{t, \rho}+\sigma_{t, \rho}\right)}{A_{1}-A_{2}-A_{3}+A_{4}}+ \\
\frac{1}{2 A_{1}} r^{\frac{-A-2 A_{4}+A_{2}+A_{3}}{2 A_{1}}}\left(-A \cdot A_{3}\left(C[1]-r^{\frac{A}{A_{1}}} C[2]\right)+\left(A_{2} A_{3}+A_{3}^{2}-2 A_{1} A_{4}\right)\left(C[1]+r^{\frac{A}{A_{1}}} C[2]\right),\right.
\end{gathered}
$$

where $\mathrm{C}[1]$ and $\mathrm{C}[2]$ are integration constants. Considering the continuity conditions at $r=\rho=\rho_{1}$, i.e.,

$$
\begin{gathered}
\sigma_{r}(\rho)=\sigma_{r, \rho}, \\
u(\rho)=\varepsilon_{t, \rho} \cdot \rho,
\end{gathered}
$$

the constants $C[1]$ and $C[2]$ can be obtained as a function of the plastic radius $\rho=\rho_{1}$ :

$$
\begin{aligned}
& C[1]=f(\rho)=-\frac{\rho{ }^{-\frac{-A-2 A_{1}+A_{2}+A_{3}}{2 A_{1}}}}{2\left(-A_{1}+A_{2}+A_{3}-A_{4}\right) A} \cdot\left(\left(A+A_{2}+A_{3}\right)\left(A_{3}\left(\varepsilon_{r, \rho}-\varepsilon_{t, \rho}\right)+\sigma_{r, \rho}-\sigma_{t, \rho}\right)+\right. \\
& \left.A_{1}\left(-A_{3} \varepsilon_{r, \rho}-2 A_{4} \varepsilon_{r, \rho}+A_{2}\left(\varepsilon_{r, \rho}-\varepsilon_{t, \rho}\right)+A_{3} \varepsilon_{t, \rho}+2 A_{4} \varepsilon_{t, \rho}+A\left(-\varepsilon_{r, \rho}+\varepsilon_{t, \rho}\right)-2 \sigma_{r, \rho}+2 \sigma_{t, \rho}\right)\right) \text {, } \\
& C[2]=f(\rho)=-\frac{\rho{ }^{-\frac{A-2 A_{1}+A_{2}+A_{3}}{2 A_{1}}}}{2\left(-A_{1}+A_{2}+A_{3}-A_{4}\right) A}\left(A _ { 1 } \left(A_{3} \varepsilon_{r, \rho}+2 A_{4} \varepsilon_{r, \rho}-A_{3} \varepsilon_{t, \rho}-2 A_{4} \varepsilon_{t, \rho}+A\left(-\varepsilon_{r, \rho}+\varepsilon_{t, \rho}\right)+\right.\right. \\
& \left.\left.A_{2}\left(-\varepsilon_{r, \rho}+\varepsilon_{t, \rho}\right)+2 \sigma_{r, \rho}-2 \sigma_{t, \rho}\right)+\left(A-A_{2}-A_{3}\right)\left(A_{3}\left(\varepsilon_{r, \rho}-\varepsilon_{t, \rho}\right)+\sigma_{r, \rho}-\sigma_{t, \rho}\right)\right)
\end{aligned}
$$

where

$$
A=\sqrt{\left(A_{2}+A_{3}\right)^{2}-4 A_{1} A_{4}} .
$$

Therefore, the stress-, strain- and displacement distributions $\left(\sigma_{r}(r), \sigma_{t}(r), \varepsilon_{r}(r), \varepsilon_{t}(r), u(r)\right)$ inside the plastic zone are only a function of the plastic radius $\rho=\rho_{1}$. The latter can be obtained (numerically) from the boundary condition,

$$
\sigma_{r}(a)=\sigma_{a}
$$

The stresses in the hard and the weak layers are obtained by integrating their incremental stress-strain relationships (four last rows of Eq. 5.21) considering their boundary conditions at $r=\rho=\rho_{1}$ :

$$
\begin{aligned}
& \sigma_{r, h}=\sigma_{r, h, \rho}+K_{1} \cdot\left(\varepsilon_{r}-\varepsilon_{r, \rho}\right)+K_{2} \cdot\left(\varepsilon_{t}-\varepsilon_{t, \rho}\right), \\
& \sigma_{t, h}=\sigma_{t, h, \rho}+K_{3} \cdot\left(\varepsilon_{r}-\varepsilon_{r, \rho}\right)+K_{4} \cdot\left(\varepsilon_{t}-\varepsilon_{t, \rho}\right), \\
& \sigma_{r, w}=\sigma_{r, w, \rho}+K_{5} \cdot\left(\varepsilon_{r}-\varepsilon_{r, \rho}\right)+K_{6} \cdot\left(\varepsilon_{t}-\varepsilon_{t, \rho}\right),
\end{aligned}
$$




$$
\sigma_{t, w}=\sigma_{t, w, \rho}+K_{7} \cdot\left(\varepsilon_{r}-\varepsilon_{r, \rho}\right)+K_{8} \cdot\left(\varepsilon_{t}-\varepsilon_{t, \rho}\right)
$$

where $K_{1}=K_{1, i i}, K_{2}=K_{2, i i}, K_{3}=K_{3, i i}, K_{4}=K_{4, i i}, K_{5}=K_{5, i i}, K_{6}=K_{6, i i}, K_{7}=K_{7, i i}, K_{8}=K_{8, i i}$ in case A, while $K_{1}=K_{1, i i i}, K_{2}=K_{2, i i i}, K_{3}=K_{3, i i i}, K_{4}=K_{4, i i i}, K_{5}=K_{5, i i i}, K_{6}=K_{6, i i i}, K_{7}=K_{7, i i i}, K_{8}=K_{8, i i i}$ in case B. (The coefficients are given in Appendices A.2 and A.3.)

Analogously, the stress in z-direction can be determined by integrating the incremental stress-strain relationship (third row of Eq. 5.21), considering the conditions at $r=\rho=\rho_{1}$ :

$$
\sigma_{z}=\sigma_{z, \rho}+B_{1} \cdot\left(\varepsilon_{r}-\varepsilon_{r, \rho}\right)+B_{2} \cdot\left(\varepsilon_{t}-\varepsilon_{t, \rho}\right)
$$

where $B_{1}=B_{1, i i}$ and $B_{2}=B_{2, i i}$ in case $A$, while $B_{1}=B_{1, i i i}$ and $B_{2}=B_{2, i i i}$ in case $B$. (The coefficients are given in Appendices A.2 and A.3.)

Equation (5.56) with $r=a$ represents the ground response curve of the homogenised rock mass.

The relations determined in this section are valid as long as the stresses in the elastic layers (i.e., the hard layers in case A or the weak layers in case B) at the tunnel boundary $(r=a)$ remain within the elastic domain:

$$
\begin{gathered}
\sigma_{t, h}(a) \leq m_{h} \cdot \sigma_{r, h}(a)+\sigma_{d, h} \text { for case A, and } \\
\sigma_{t, w}(a) \leq m_{w} \cdot \sigma_{r, w}(a)+\sigma_{d, w} \text { for case B. }
\end{gathered}
$$

As the radial and tangential stresses in the weak and in the hard layers (Eqs. 5.67 to 5.70 ) are functions of $\varepsilon_{r}$ and $\varepsilon_{t}$ (Eqs. 5.57 and 5.58) and thus depend only on the unknown radius $\rho_{1}$ of the plastic zone, $\rho_{1}$ can be determined such that Equations (5.72) or (5.73) hold with the equality sign. With this, the minimum support pressure $\sigma_{r, \rho 2}$ (at the tunnel boundary) can be determined (cf. Eq. 5.66). This has been done numerically.

\subsubsection{GRC part for elasto-plastic behaviour both of the weak and of the hard layers}

Once, the support pressure $\sigma_{a}$ is smaller than $\sigma_{r, \rho 2}$, an inner plastic zone having an external radius $\rho_{2}$ develops, in which both the hard and the weak layers yield (Fig. 5.5). In the outer plastic zone, either the weak layers (case B) or the hard layers (case A) are still elastic, depending on which layers yield first (see Section 5.2.4).

The static system can be now conceived as consisting of three components:

- Elastic zone $\left(r \geq \rho_{1}\right)$ with an internal radius $\rho_{1}$ and an internal pressure $\sigma_{r, \rho 1}$. The stress and displacement field is already known from Section 5.2.4. The stress-, strain- and displacement fields are given by Equations (5.36) to (5.45).

- Outer plastic zone $\left(\rho_{2} \leq r \leq \rho_{1}\right)$ : thick-walled cylinder with internal radius $\rho_{2}$, external radius $\rho_{1}$, internal pressure $\sigma_{r, \rho 2}$ and external pressure $\sigma_{r, \rho 1}$. In this zone, either the weak layers (case A) or the hard layers (case B) yield. The stress-, strain- and displacement fields can be obtained by applying the equations of Section 5.2.4 (Eqs. 5.56 to 5.65) to a tunnel having a radius $\rho_{2}$ and a support pressure $\sigma_{r, \rho 2}$. The plastic radius $\rho_{1}$ is obtained (analogously to Eq. 5.66) from the condition:

$$
\sigma_{r}\left(\rho_{2}\right)=\sigma_{r, \rho 2}
$$


Given, the support pressure $\sigma_{r, \rho 2}$, the interface stresses and strains at $r=\rho_{2}$ (i.e., $\sigma_{t, \rho 2}$, $\sigma_{r, w, \rho 2}, \sigma_{t, w, \rho 2}, \sigma_{r, h, \rho 2}, \sigma_{t, h, \rho 2}, \varepsilon_{r, \rho 2}$ and $\left.\varepsilon_{t, \rho 2}\right)$ can be determined using the Equations (5.57) to (5.70).

- Inner plastic zone $\left(a \leq r \leq \rho_{2}\right)$ : thick-walled cylinder with internal radius $a$, external radius $\rho_{2}$, internal pressure $\sigma_{a}$ and external pressure $\sigma_{r, \rho 2}$. In this zone, the stresses fulfil the failure criteria both of the weak and hard layers.

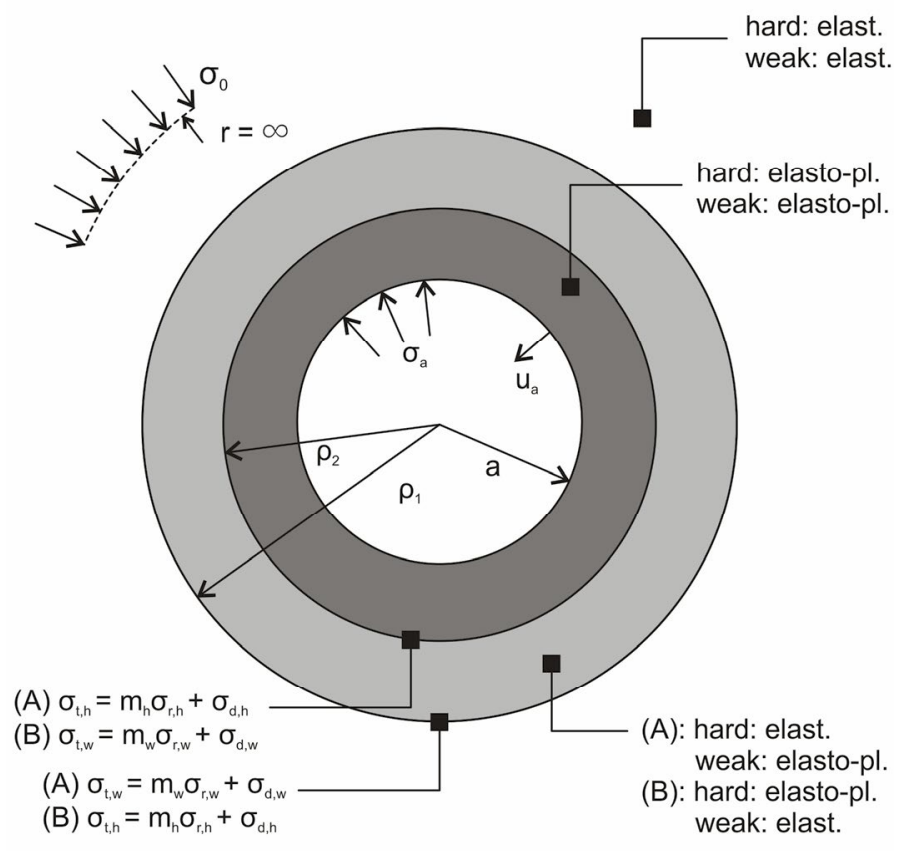

Figure 5.5. Plastic zones with yielding both of the hard and the weak layers in the inner ring.

For the inner plastic zone, the procedure for determining the stress-, strain- and displacement distribution is the same as in Section 5.2.4: By integrating the incremental stress-strain relationships (i.e., first two rows of Eq. 5.21), considering their boundary conditions at $r=\rho=\rho_{2}$, the following stress-strain relation can be obtained for the inner plastic zone:

$$
\left(\begin{array}{c}
\sigma_{r}-\sigma_{r, \rho} \\
\sigma_{t}-\sigma_{t, \rho}
\end{array}\right)=\left(\begin{array}{cc}
A_{1} & -A_{2} \\
A_{3} & -A_{4}
\end{array}\right) \cdot\left(\begin{array}{c}
\varepsilon_{r}-\varepsilon_{r, \rho} \\
\varepsilon_{t}-\varepsilon_{t, \rho}
\end{array}\right)
$$

where $A_{1}=A_{1, i v}, A_{2}=A_{2, i v}, A_{3}=A_{3, i v}$ and $A_{4}=A_{4, i v}$. The coefficients are given in Appendix A.4.

Equation (5.75) combined with the kinematic relationships (Eq. 5.1) and the equilibrium conditions (Eq. 5.2) leads to the second order differential equation (see Eq. 5.55) for $u(r)$, which delivers the stress, strain as well as the displacement field inside the plastic zone (Eqs. 5.56 to 5.65) as a function of the integration constants.

Taking account of the continuity conditions at $r=\rho=\rho_{2}$ (cf. Eqs. 5.61 and 5.62), i.e.,

$$
\begin{gathered}
\sigma_{r}\left(\rho_{2}\right)=\sigma_{r, \rho 2} \text { and } \\
u\left(\rho_{2}\right)=\varepsilon_{t, \rho 2} \cdot \rho_{2},
\end{gathered}
$$


the constants $C[1]$ and $C[2]$ (Eqs. 5.63 and 5.64) can be obtained as a function of the plastic radius $\rho=\rho_{2}$. The latter can be obtained (numerically) from the condition:

$$
\sigma_{r}(a)=\sigma_{a}
$$

Applying Equation (5.56) for $r=a$ yields the ground response curve of the homogenised rock mass.

Also the stresses in the weak and in the hard layers can be obtained by integrating their incremental stress-strain relationships (four last rows of Eq. 5.21) considering their boundary conditions at $r=\rho=\rho_{2}$ (i.e., $\sigma_{r, \rho}=\sigma_{r, \rho 2}, \sigma_{t, \rho}=\sigma_{t, \rho 2}, \sigma_{r, w, \rho}=\sigma_{r, w, \rho 2}, \sigma_{t, w, \rho}=\sigma_{t, w, \rho 2}, \sigma_{r, h, \rho}=\sigma_{r, h, \rho 2}$, $\sigma_{t, h, \rho}=\sigma_{t, h, \rho 2}, \varepsilon_{r, \rho}=\varepsilon_{r, \rho 2}, \varepsilon_{t, \rho}=\varepsilon_{t, \rho 2}$ ). The stresses are given in Equations (5.67) to (5.70), where $K_{1}=K_{1, i v}, K_{2}=K_{2, i v}, K_{3}=K_{3, i v}, K_{4}=K_{4, i v}, K_{5}=K_{5, i v}, K_{6}=K_{6, i v}, K_{7}=K_{7, i v}, K_{8}=K_{8, i v}$ are given in Appendix A.4, while the axial stress $\sigma_{z}$ can be obtained by inserting $B_{1}=B_{1, i v}$ and $B_{2}=B_{2, i v}$ into Equation (5.71).

\subsubsection{Verification of the analytical solution}

The analytical solution and its implementation in Matlab (MathWorks 2017) was checked by comparative axisymmetric numerical calculations which were carried out with the FEprogram Abaqus (Dassault Systèmes 2011). Specifically, an unsupported, cylindrical tunnel that crosses alternating hard and weak layers of a finite thickness of $h$ and $w$, respectively, will be studied (cf. Cantieni and Anagnostou 2007). The rock layers were modelled discretely as isotropic, linearly elastic and perfectly plastic material with the Mohr-Coulomb yield criterion.

For consistency with the closed-form solution, the subroutine of Clausen (2007) is used rather than the build-in subroutine of Abaqus, because the latter uses a smoothened plastic potential surface. Furthermore, a high Poisson's ratio is chosen both for the weak and the hard layers in order to avoid out-of-plane plastic flow (see, e.g., Reed 1988, Vrakas and Anagnostou 2014), which is not considered by the analytical solution of the present section.

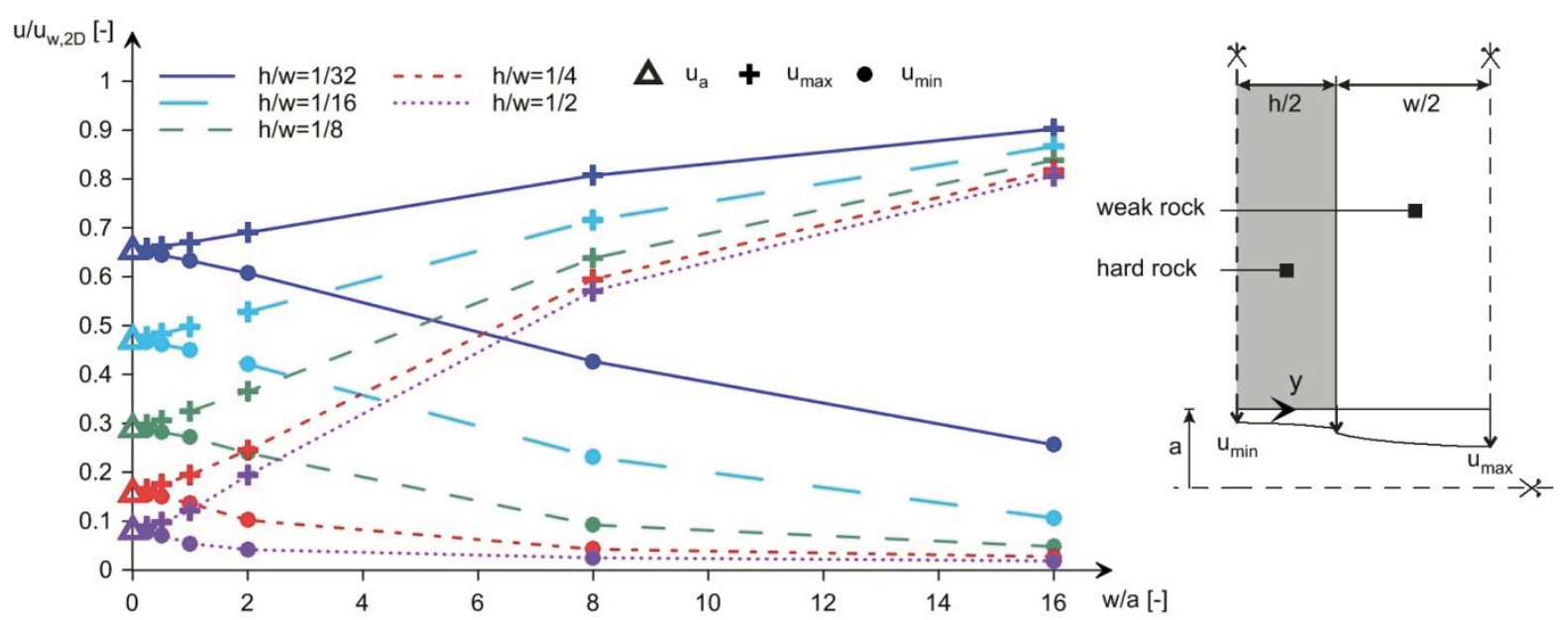

Figure 5.6. Maximum $u_{\max }$ and minimum displacements $u_{\min }$ (see r.h.s. sketch) normalised by the displacements of a very long weak zone $u_{w, 2 D}$ and compared with the displacements of the closed-form solution $u_{a}$ (unsupported tunnel, $a=4 \mathrm{~m}, \sigma_{0}=10 \mathrm{MPa}, E_{h}=10 \mathrm{GPa}, c_{h}=5 \mathrm{MPa}$, $\left.E_{w}=1 \mathrm{GPa}, c_{w}=0.5 \mathrm{MPa}, v_{h}=v_{w}=0.45, \varphi_{h}=\varphi_{w}=25^{\circ}, \psi_{h}=\boldsymbol{\psi}_{w}=5^{\circ}\right)$. 
Figure 5.6 shows the maximum $u_{\max }$ and the minimum displacements $u_{\min }$ (which occur in the middle of the weak and of the hard layer, respectively) in function of the layer thicknesses $w / a$, considering various ratios of the layer thicknesses $h / w$. The closed-form solution $u_{a}$ applies to thinly alternating layers (i.e., to w/a $\rightarrow 0$ ) and is marked by triangles on the ordinate axis of Figure 5.6. With decreasing layer thickness $w / a$, the difference between $u_{\text {min }}$ and $u_{\max }$ decreases (i.e., the longitudinal displacement profile becomes more and more uniform) and for w/a $\rightarrow 0$ the numerically determined displacements converge to those obtained by the closed-form solution.

Figures 5.7 and 5.8 compare the analytical with the numerical GRCs and stress fields, respectively, considering a small value of $w / a=0.25$, for which $u_{\max }$ is nearly equal to $u_{\min }$. (As will be seen later in Section 5.7, generally, the homogenised model is adequate if the thickness of the hard layers is less than $5 \%$ of the tunnel radius.) The GRCs agree very well (for the considered high Poisson's ratios), but the stress fields are different close to the tunnel, because out-of-plane plastic flow occurs $\left(\sigma_{t, h}=\sigma_{z, h}\right.$ according to the numerical results, in spite of the high Poisson's ratio), which is not considered by the analytical solution; the latter predicts tensile stresses close to the tunnel.
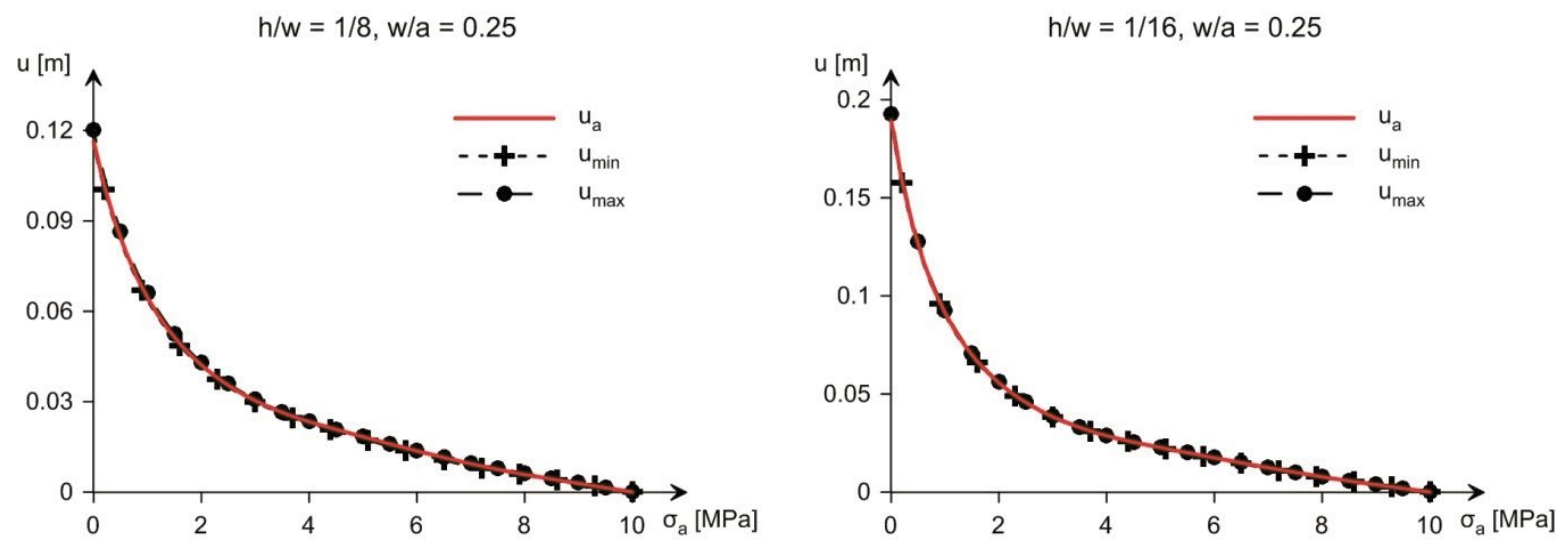

Figure 5.7. Analytically and numerically determined GRC $\left(a=4 \mathrm{~m}, \sigma_{0}=10 \mathrm{MPa}, E_{h}=10 \mathrm{GPa}, \mathrm{C}_{h}=5 \mathrm{MPa}\right.$, $\left.E_{w}=1 \mathrm{GPa}, c_{w}=0.5 \mathrm{MPa}, v_{h}=v_{w}=0.45, \varphi_{h}=\varphi_{w}=25^{\circ}, \psi_{h}=\psi_{w}=5^{\circ}\right)$.
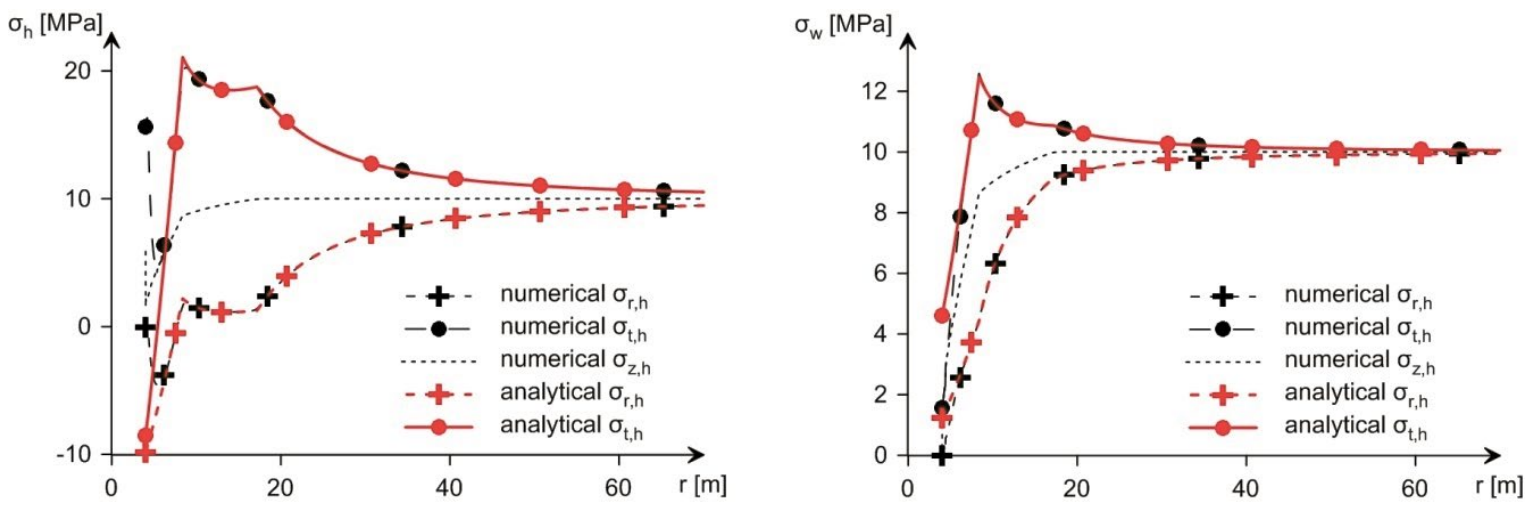

Figure 5.8. Analytically and numerically determined stress field in the hard layers (I.h.s diagram) and in the weak layers (r.h.s diagram) for $w / a=0.25$ and $h / w=1 / 8$ (unsupported tunnel, $a=4 \mathrm{~m}$, $\sigma_{0}=10 \mathrm{MPa}, E_{h}=10 \mathrm{GPa}, c_{h}=5 \mathrm{MPa}, E_{w}=1 \mathrm{GPa}, c_{w}=0.5 \mathrm{MPa}, v_{h}=v_{w}=0.45, \varphi_{h}=\varphi_{w}=25^{\circ}$, $\left.\boldsymbol{\Psi}_{h}=\boldsymbol{\Psi}_{w}=5^{\circ}\right)$. 
As the out-of-flow plastic flow is more pronounced for smaller Poisson's ratios, additional results are presented for Poisson's ratios of 0.3 (Fig. 5.9). According to Figure 5.9, the smaller the thickness fraction $x_{h}$ of the hard layers is, the bigger the error of disregarding outof-plane plastic flow will be.

If the thickness fraction $x_{h}$ of the hard layers is small and their Young's modulus $E_{h}$ is high, then the hard layers are loaded more and radial tensile stresses develop in tunnel proximity (r.h.s. diagrams of Fig. 5.9). (The observation that tensile stress will result in some layers, given sufficient contrast in the layer stiffnesses, was already made by Bourne (2003).) As in the state of failure the radial and the tangential stresses of the hard layers are interconnected by the Mohr-Coulomb criterion, the tangential stress may become smaller than the radial stress (particularly in the case of a low compressive strength). The closed-form solution of the present section does not consider this possibility (Section 5.2.2 assumes that the maximum and minimum principal stress act in $t$ - and $r$-direction, respectively; see Eqs. 5.17 and 5.18) and leads therefore to inaccurate results.
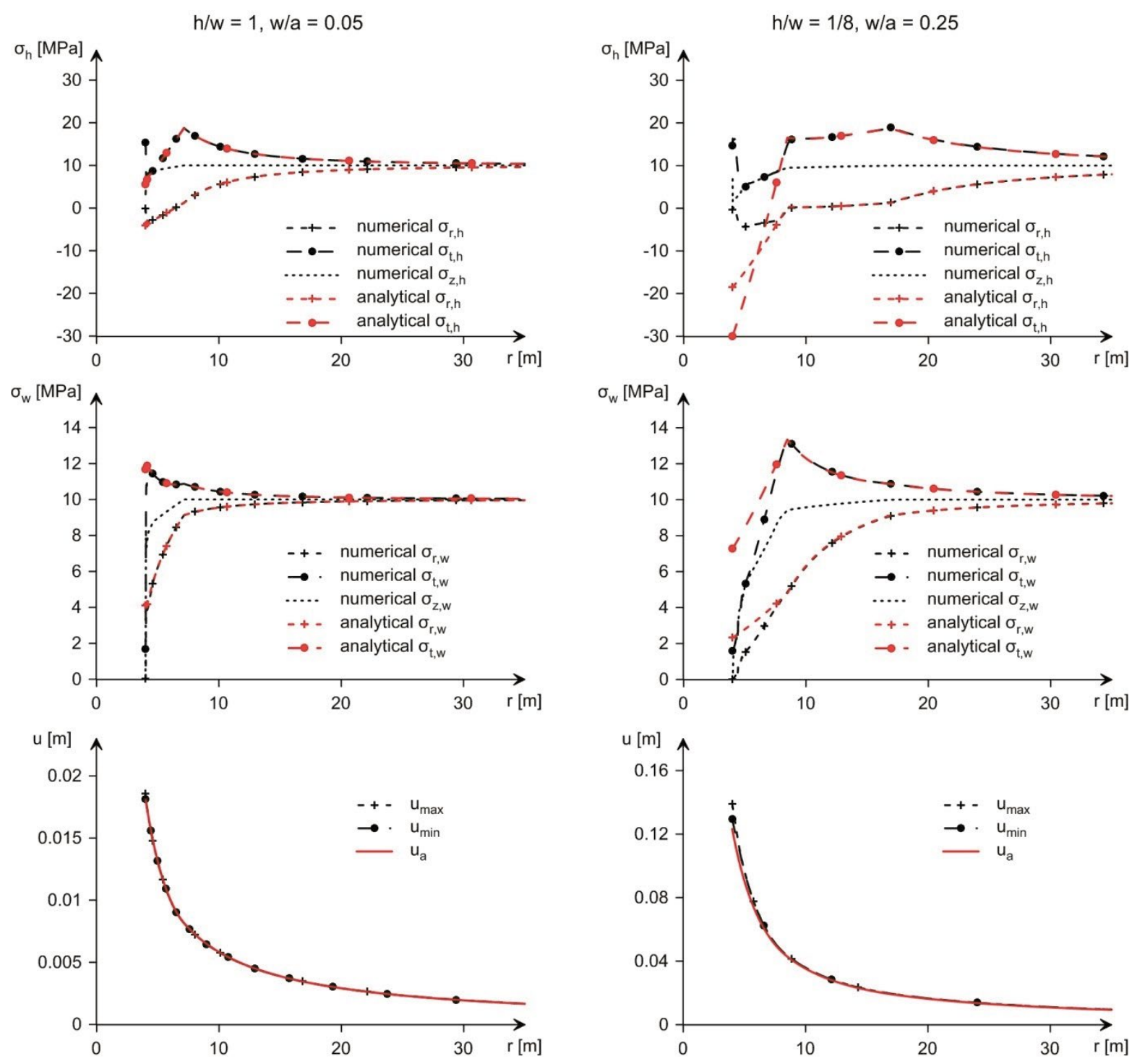

Figure 5.9. Stress and displacement fields in the hard and in the weak layers (unsupported tunnel, $a=4 \mathrm{~m}, \sigma_{0}=10 \mathrm{MPa}, E_{h}=10 \mathrm{GPa}, c_{h}=5 \mathrm{MPa}, E_{w}=1 \mathrm{GPa}, c_{w}=0.5 \mathrm{MPa}, v_{h}=v_{w}=0.3$, $\left.\varphi_{h}=\varphi_{w}=25^{\circ}, \psi_{h}=\varphi_{w}=5^{\circ}\right)$. 
This behaviour is pronounced for small Poisson's ratios and particularly if the friction angle $\varphi_{w}$ is much lower than $\varphi_{h}$. This can be seen from the analytical results of Figure 5.10 which assume a very small friction angle of the weak layers: for $\varphi_{h}=\varphi_{w}$, the radial stress $\sigma_{r}$ is smaller than the tangential stress $\sigma_{t}$ in most cases (except for very small $x_{h} / x_{w}$, cf. Fig. 5.9), while for $\varphi_{h}>\varphi_{w}$, the radial stress $\sigma_{r}$ may become higher than the tangential stress $\sigma_{t}$ in tunnel proximity and this even for rather large ratios $x_{h} / x_{w}$ of $1 / 4$ (compare the r.h.s diagrams with the I.h.s. diagrams of Fig. 5.10).

In conclusion, the closed-form solution gives accurate results for $\varphi_{h}=\varphi_{w}$ (error $<25 \%$ ). However, for $\varphi_{w}<\varphi_{h}$ and small $\sigma_{d, w} / \sigma_{0}$ values, calculations with different parameters as above show that the displacements obtained with the closed-form solution may be by $100 \%$ inaccurate.
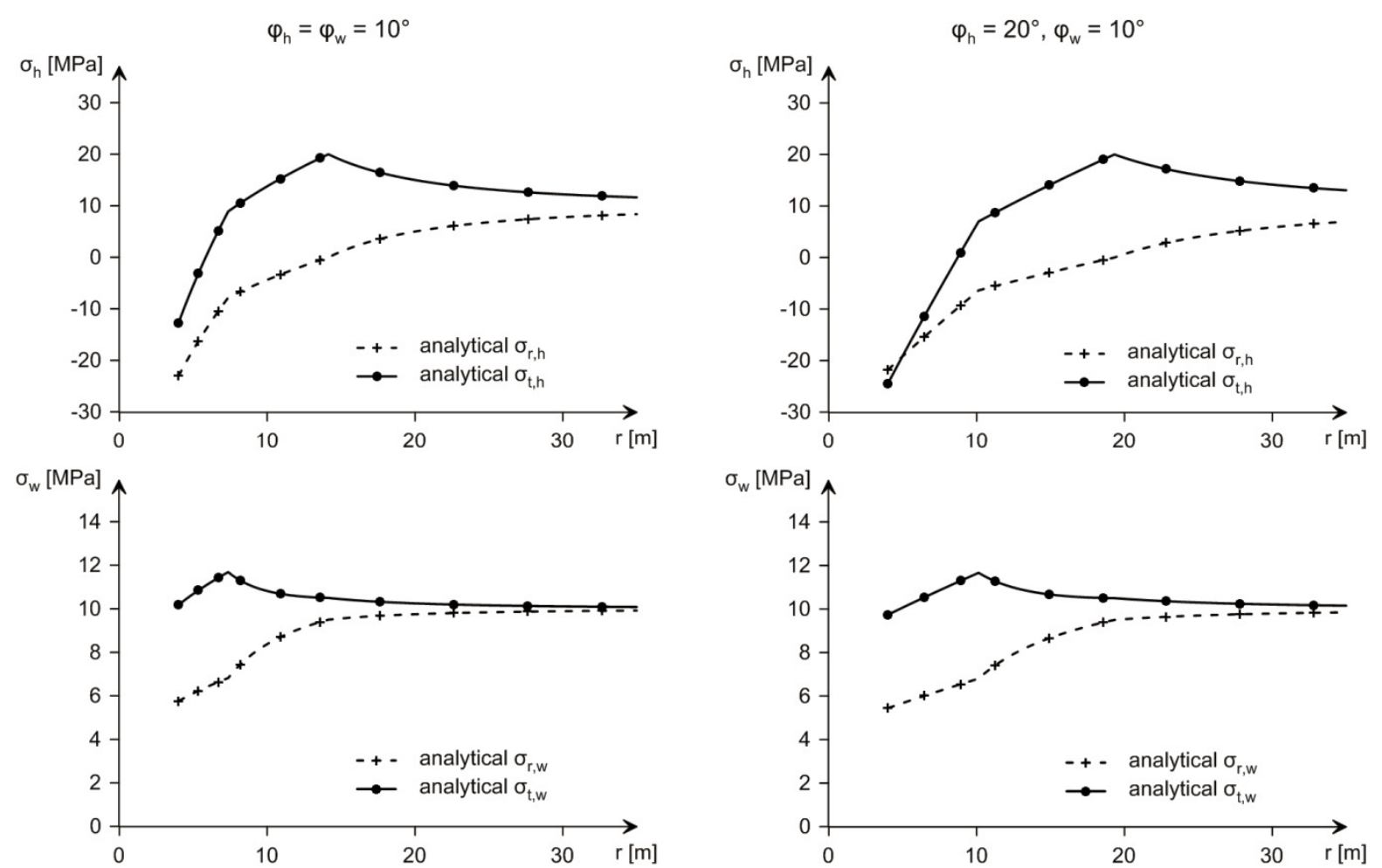

Figure 5.10. Stress field in the hard (upper diagrams) and in the weak layers (lower diagrams) (unsupported tunnel, $a=4 \mathrm{~m}, \sigma_{0}=10 \mathrm{MPa}, x_{h} / x_{w}=1 / 4, E_{h}=10 \mathrm{GPa} ; c_{h}=7 \mathrm{MPa}, E_{w}=0.5 \mathrm{GPa}$, $c_{w}=0.839 \mathrm{MPa}, v_{h}=v_{w}=0.3, \Psi_{h}=\psi_{w}=1^{\circ}$ ).

\subsubsection{Dimensionless diagrams}

One can readily verify (by inspecting the equations of the presented analytical solution) that the radial displacement $u_{a}$ of the excavation boundary can be expressed as follows:

$$
\frac{u_{a}}{u_{w, 2 D}}=f\left(\frac{\sigma_{a}}{\sigma_{0}}, \frac{x_{h}}{x_{w}}, \frac{\sigma_{d, h}}{\sigma_{d, w}}, \frac{E_{h}}{E_{w}}, \frac{\sigma_{d, w}}{\sigma_{0}}, v_{h}, \varphi_{h}, \psi_{h}, v_{w}, \varphi_{w}, \psi_{w}\right)
$$

where $u_{w, 2 D}$ is the displacement that would occur in the absence of the hard layers (i.e., for $x_{w}=1$ ) and can be computed using known equations (e.g., those of Anagnostou and Kovári 1993). The ratio $u_{a} / u_{w, 2 D}$ can be conceived as a "reduction factor" that expresses the stabilising effect of the hard layers. 
Appendix B presents dimensionless diagrams showing the normalised displacement $u_{a} / u_{w, 2 D}$ as a function of the normalised weak layer strength $\sigma_{d, w} / \sigma_{0}$ for the parameter values after Table 5.2. The considered parameter range is sufficiently wide for practical purposes. The diagrams allow easily constructing the practically relevant part of the GRC $\left(0 \leq \sigma_{a} / \sigma_{o} \leq 0.2\right)$.

As the underlying closed-form solution disregards out-of-plane plastic flow and the latter may be significant, particularly for very low $\sigma_{d, w} / \sigma_{0}$-values (see Section 5.2.6), the diagrams consider only the $\sigma_{d, w} / \sigma_{0}$ range, where the out-of-plane plastic flow is negligible.

Table 5.2. Considered parameter values in the dimensionless diagrams of Appendix B.

\begin{tabular}{|c|c|c|}
\hline$\sigma_{d, w} / \sigma_{o}$ & $\leq 2$ & $\begin{array}{l}\text { At higher strengths the weak layers } \\
\text { behave elastically even in the absence of } \\
\text { hard interlayers }\end{array}$ \\
\hline$\sigma_{a} / \sigma_{o}$ & $0.0,0.1,0.2$ & \\
\hline$x_{h} / x_{w}$ & $1 / 8,1 / 4,1 / 2,1,2$ & $\begin{array}{l}\text { The presence of weak layers is irrelevant } \\
\text { from the practical viewpoint if } x_{h} / x_{w}>2 \text {. }\end{array}$ \\
\hline$\sigma_{d, h} / \sigma_{d, w}$ & $2,6,10$ & \\
\hline$E_{h} / E_{w}$ & $0.5,1.0,1.5,2.0 \sigma_{d, h} / \sigma_{d, w}$ & $\begin{array}{l}\text { Considering that the Young's modulus is } \\
\text { in the order of } 500-1000 \text { times the } \\
\text { uniaxial strength. }\end{array}$ \\
\hline$\varphi_{h}$ & $20^{\circ}, 25^{\circ}, 30^{\circ}$ & \\
\hline$\varphi_{w}$ & $\varphi_{h}-\left(0^{\circ}, 5^{\circ}, 10^{\circ}\right)$ & \\
\hline$\psi_{h}$ & $\max \left(\varphi_{h}-20^{\circ}, 1^{\circ}\right)$ & cf. Vermeer and de Borst (1984) \\
\hline$\psi_{w}$ & $\max \left(\varphi_{w}-20^{\circ}, 1^{\circ}\right)$ & cf. Vermeer and de Borst (1984) \\
\hline$V h$ & 0.3 & \\
\hline$v_{w}$ & 0.3 & \\
\hline
\end{tabular}

\subsection{Extension of the closed-form solution for brittle behaviour of the hard layers}

\subsubsection{Derivation}

The closed-form solution of Section 5.2 can be easily extended, in order to consider the effect of brittle behaviour of the hard layers, with a sudden post-failure decrease in strength according to Figure 5.11.

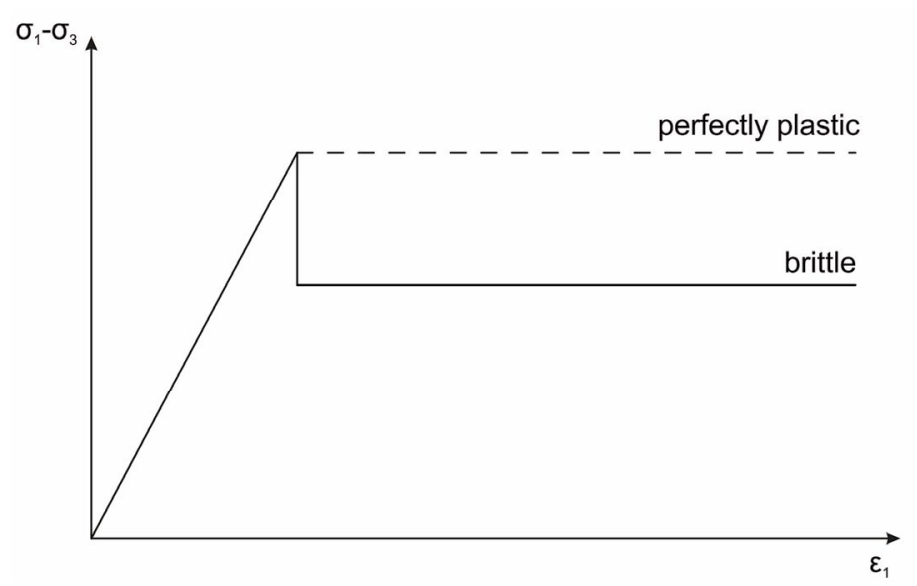

Figure 5.11. Constitutive law for the (brittle) hard layers. 
The Mohr-Coulomb failure criterion for the hard layers can be expressed as follows:

$$
\begin{gathered}
\sigma_{t, h}=m_{h, H} \cdot \sigma_{r, h}+\sigma_{d, h, H} \text { for peak strength and } \\
\sigma_{t, h}=m_{h, R} \cdot \sigma_{r, h}+\sigma_{d, h, R} \text { for post-peak strength, }
\end{gathered}
$$

where $m_{h, H}, \sigma_{\mathrm{d}, h, H}, m_{h, R}$ and $\sigma_{\mathrm{d}, h, R}$ are defined analogously to Equation (5.16), respectively, but in terms of the peak and residual cohesion and friction angle.

For the incremental stress-strain relations of the REV (which apply within the plastic zone; cf. Section 5.2.2), the Mohr-Coulomb failure criterion with the post-peak strength has to be considered, that is, instead of Equation (5.18),

$$
d \sigma_{t, h}=m_{h, R} \cdot d \sigma_{r, h} .
$$

The radial stress $\sigma_{r, h}$ is continuous at the elasto-plastic interface, while the tangential stress $\sigma_{t, h}$ experiences a sudden drop, caused by the decrease in strength (see, e.g., Park and Kim 2006). This leads to a sudden drop in $\sigma_{t}$ of the composite medium as well (cf. Eq. 5.10). Therefore, the boundary conditions used for integrating the incremental stress-strain relationships have to be modified as follows (while the "-" will denote the tangential stress after the drop):

- The minimum support pressure $\sigma_{r, p 1}$ for the case B (i.e., Eq. 5.35) can be calculated, analogously to Section 5.2.3, using the peak strength. This is also true for $\sigma_{r, w, \rho 1}$ (Eq. 5.48), $\sigma_{t, w, \rho 1}$ (Eq. 5.49), $\sigma_{r, h, \rho 1}$ (Eq. 5.50), $\varepsilon_{r, \rho 1}$ (Eq. 5.52) and $\varepsilon_{t, \rho 1}$ (Eq. 5.53). However, in order to consider the drop in the tangential stress mentioned above, the tangential stresses $\sigma_{t, h, \rho 1^{-}}$and $\sigma_{t, \rho 1^{-}}$have to be calculated as follows (instead of Eq. 5.51 and 5.46, respectively):

$$
\begin{gathered}
\sigma_{t, h, \rho_{1}}{ }^{-}=m_{h, R} \cdot \sigma_{r, \rho_{1}}+\sigma_{d, h, R}, \\
\sigma_{t, \rho_{1}}{ }^{-}=x_{h} \cdot \sigma_{t, h, \rho_{1}}{ }^{-}+x_{w} \cdot \sigma_{t, w, \rho_{1}} .
\end{gathered}
$$

The minimum support pressure $\sigma_{r, \rho 1}$ (i.e., Eq. 5.34) as well as all conditions at $r=\rho_{1}$ for case $A$ remain the same.

- For the case A, analogously, the minimum support pressure $\sigma_{r, \rho 2}$ (i.e., Eq. 5.72) as well as the boundary conditions $\sigma_{r, \rho 2}, \sigma_{r, w, \rho 2}, \sigma_{t, w, \rho 2}, \sigma_{r, h, \rho 2}, \varepsilon_{r, \rho 2}$ and $\varepsilon_{t, \rho 2}$ are calculated with the peak-strength, while the boundary conditions $\sigma_{t, h, \rho 2}{ }^{-}$and $\sigma_{t, \rho 2}{ }^{-}$have to be calculated with the following two equations:

$$
\begin{gathered}
\sigma_{t, h, \rho 2}{ }^{-}=m_{h, R} \cdot \sigma_{r, \rho_{2}}+\sigma_{d, h, R}, \\
\sigma_{t, \rho_{2}}{ }^{-}=x_{h} \cdot \sigma_{t, h, \rho_{2}}{ }^{-}+x_{w} \cdot \sigma_{t, w, \rho_{2}} .
\end{gathered}
$$

The closed-form solution considering a brittle behaviour of the hard layers differs from the one of Section 5.2 only in the interface conditions introduced above. All other equations for the $\mathrm{GRC}$ remain the same. 


\subsubsection{Verification of the analytical solution}

The analytical solution and its implementation in Matlab (MathWorks 2017) is verified by means of comparative numerical calculations (analogously to Section 5.2.6), which were performed with the program FLAC (Itasca Consulting Group 2001). The hard layers were modelled with the so-called "softening model", which considers the cohesion as a function of the deviatoric plastic strain. In order to reduce the effect of the out-of-plane plastic flow, the Poisson's ratio was taken equal to 0.45 .

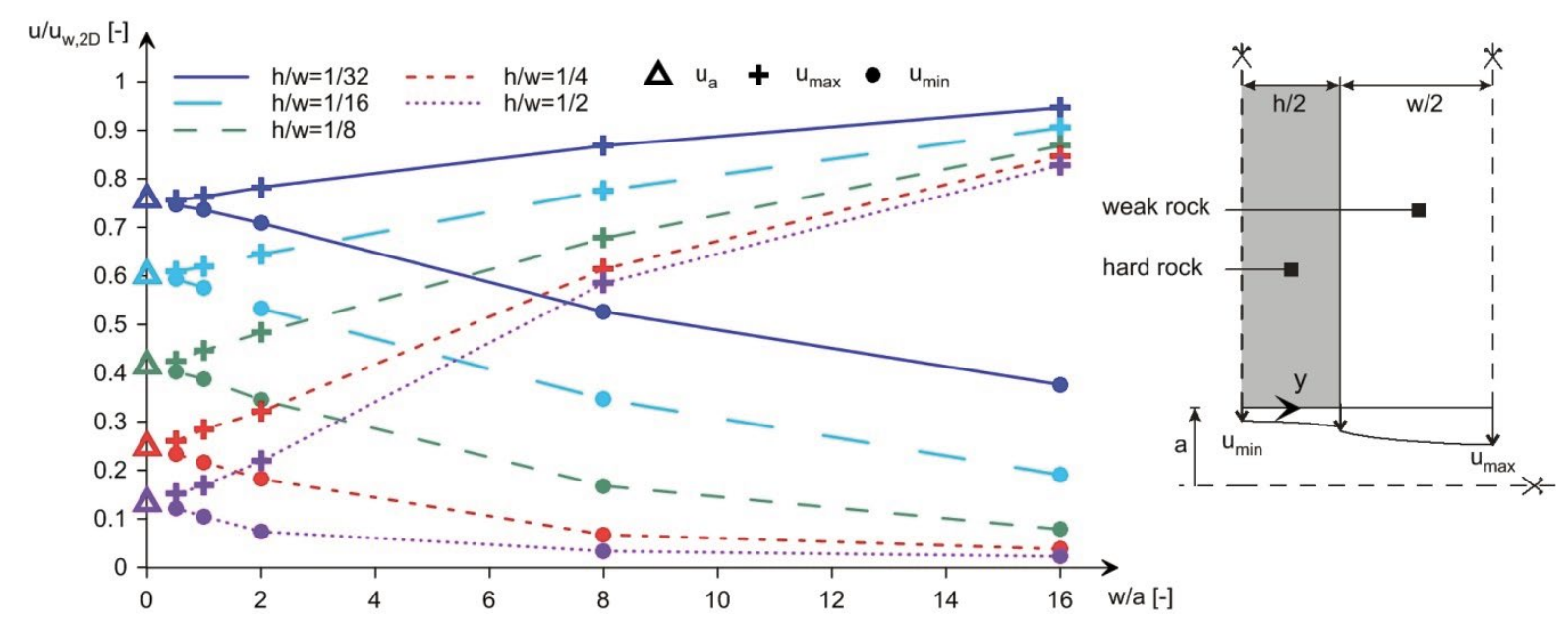

Figure 5.12. Maximum $u_{\max }$ and minimum displacements $u_{\min }$ (see r.h.s. sketch) normalised by the displacements of a very long weak zone $U_{w, 2 D}$ and compared with the displacements of the closed-form solution $U_{a}$ (unsupported tunnel, $a=4 \mathrm{~m}, \sigma_{0}=10 \mathrm{MPa}, E_{h}=10 \mathrm{GPa}, c_{h, H}=5 \mathrm{MPa}$, $\left.C_{h, R}=3 \mathrm{MPa}, E_{w}=1 \mathrm{GPa}, c_{w}=0.5 \mathrm{MPa}, v_{h}=v_{w}=0.45, \varphi_{h}=\varphi_{w}=25^{\circ}, \psi_{h}=\psi_{w}=5^{\circ}\right)$.
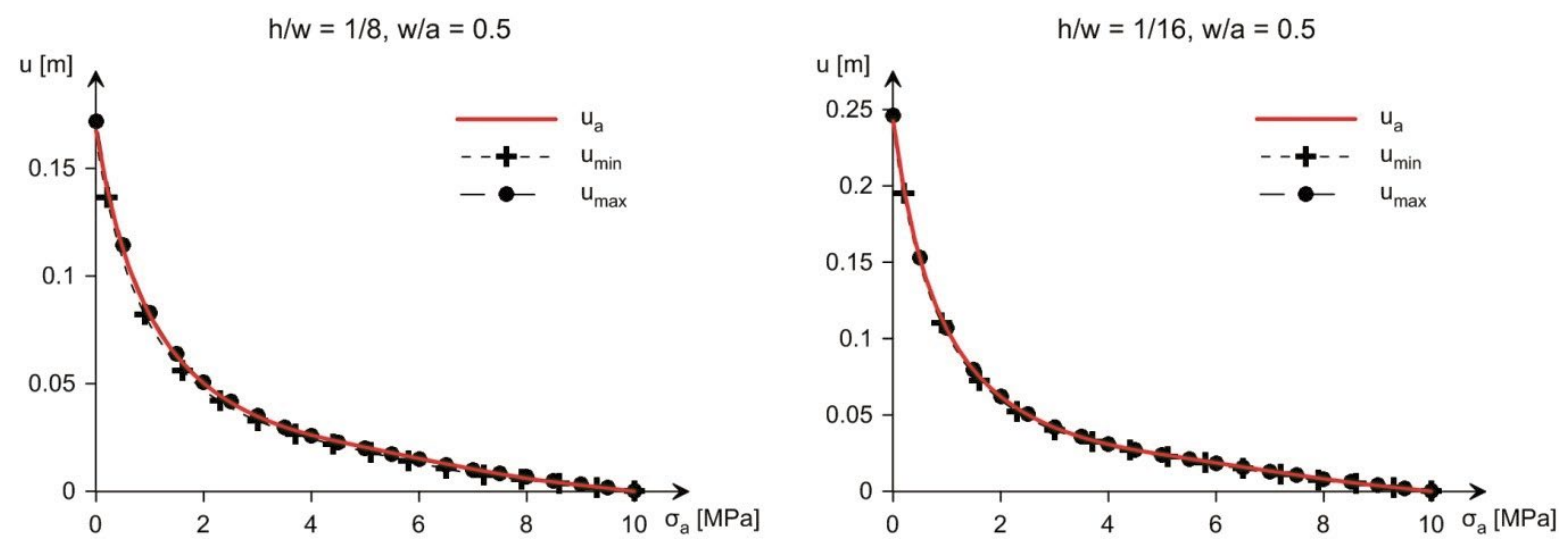

Figure 5.13. Analytically and numerically determined GRC $\left(a=4 \mathrm{~m}, \sigma_{0}=10 \mathrm{MPa}, E_{h}=10 \mathrm{GPa}\right.$, $c_{h, H}=5 \mathrm{MPa}, c_{h, R}=3 \mathrm{MPa}, E_{w}=1 \mathrm{GPa}, c_{w}=0.5 \mathrm{MPa}, v_{h}=v_{w}=0.45, \varphi_{h}=\varphi_{w}=25^{\circ}$, $\left.\boldsymbol{\psi}_{h}=\boldsymbol{\psi}_{w}=5^{\circ}\right)$.

Figure 5.12 shows the maximum $u_{\max }$ as well as the minimum displacement $u_{\min }$ (see inset) as a function of the layer thicknesses $w / a$, considering various ratios of the layer thicknesses $h / w$. With decreasing layer thickness $w / a$, the displacements $u_{\max }$ and $u_{\min }$ approach exactly the displacements obtained with the analytical solution. The analytical GRCs are marked by 
triangles (on the ordinate axis) and agree very well with the numerical results (Fig. 5.13). As mentioned in Section 5.2.6, disregarding the out-of-plane plastic flow may also in the present case lead to inaccurate displacements.

\subsection{Extension for out-of-plane plastic flow}

\subsubsection{Derivation}

The closed-form solution of Section 5.2 can be extended to include out-of-plane plastic flow analogously to Reed (1988). For a homogeneous material it is well known that at low support pressures, out-of-plane plastic flow develops in the inner part of the plastic zone, while yielding in the outer part occurs only in the plane of the tunnel cross-section. Analogously, for the thinly stratified rock considered here, two additional plastic zones may develop depending on the support pressure. The consideration of out-of-plane plastic flow is cumbersome because several special cases have to be considered in addition to cases $A$ and $B$ of Section 5.2, where a distinction was made depending on whether failure occurs first in the weak or in the hard layers. Specifically, one has further to determine, whether the outof-plane plastic flow occurs after (hereafter referred to as case I) or before (case II) both the hard and the weak layers reach in-plane failure.

Figure 5.14 shows the cases, which must be distinguished with respect to the sequence and type of plastic flow occurring as the support pressure decreases from its initial value to zero:

- Case $A(I)$ : the weak layers yield first. Out-of-plane failure occurs after the hard layers also reach in-plane failure. A distinction has to be made, depending on whether out-of-plane failure occurs earlier in the hard (subcase 1 in Fig. 5.14) or in the weak layers (subcase 2 in Fig. 5.14). After the weak (or the hard) layers experience out-of-plane yielding, the hard (or the weak) layers may also yield out-of-plane and this either in triaxial extension or in triaxial compression.

- Case $A(I I):$ the weak layers yield first and experience out-of-plane plastic flow before the hard layers yield; subsequently, the hard layers reach in-plane failure, followed by out-ofplane failure, either in triaxial extension or in triaxial compression.

- Cases $B(I)$ and $B(I I)$ are analogous to cases $A(I)$ and $A(I I)$, respectively, but with the hard layers yielding first. 
Case A (I): weak layers yield before hard layers, out-of-plane failure occurs after both layers reach in-plane failure

(1) hard layers reach out-of-plane failure before weak layers

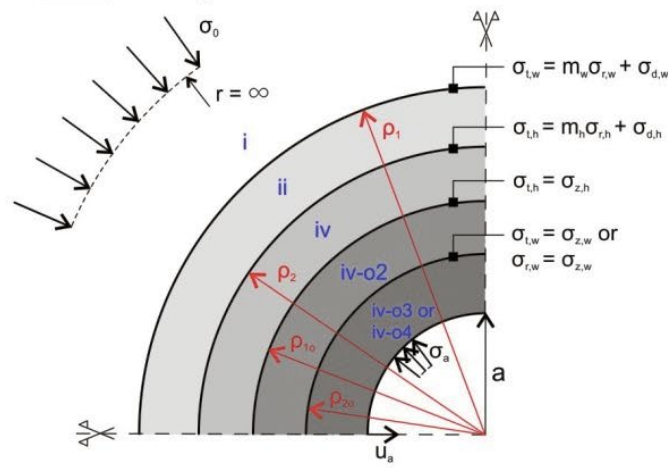

(2) hard layers reach out-of-plane failure after weak layers

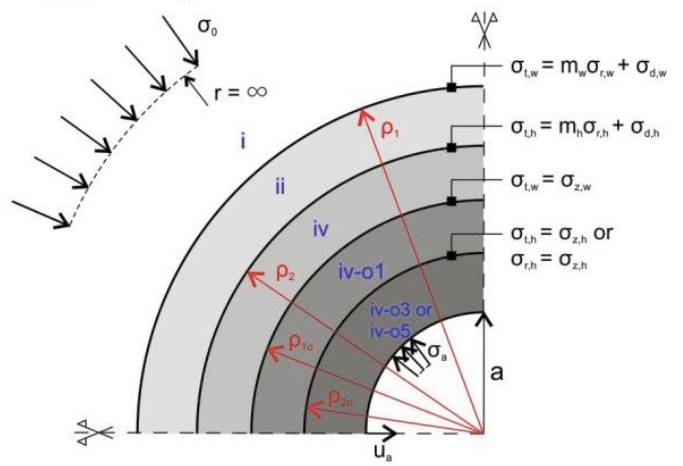

Case A (II): weak layers yield before hard layers, out-of-plane failure occurs before both layers reach in-plane failure

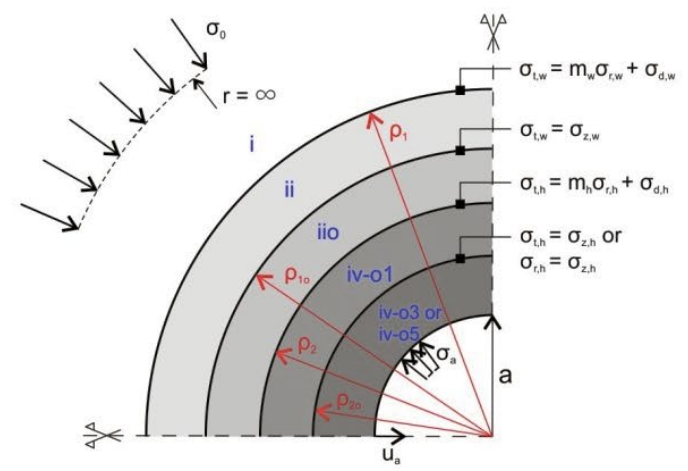

Case B (I): weak layers yield after hard layers, out-of-plane failure occurs after both layers reach in-plane failure

(1) hard layers reach out-of-plane failure before weak layers

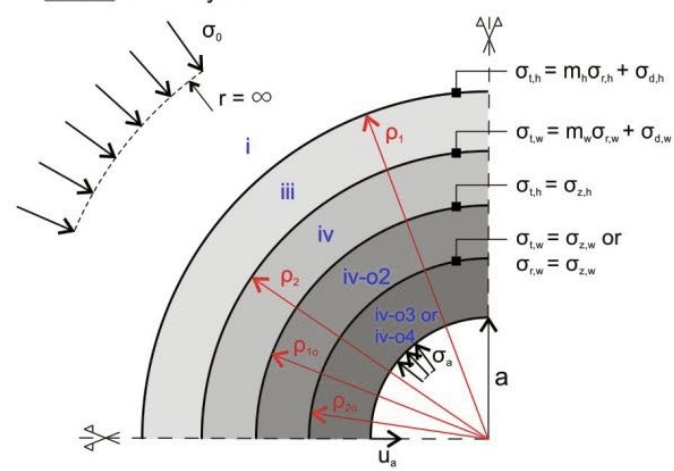

(2) hard layers reach out-of-plane failure

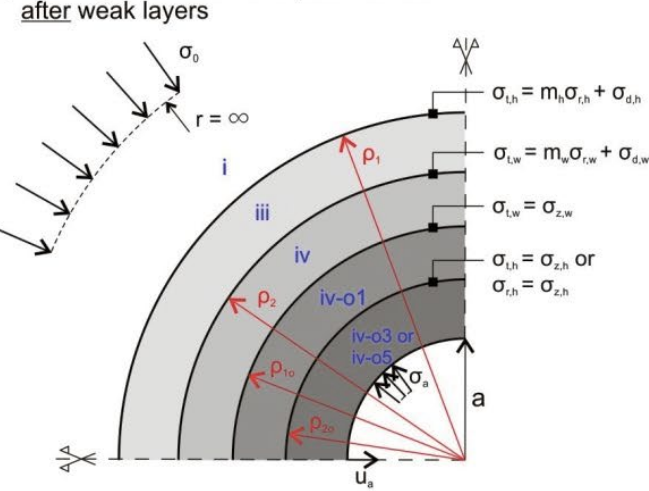

Case B (II): weak layers yield after hard layers, out-of-plane failure occurs before both layers reach in-plane failure

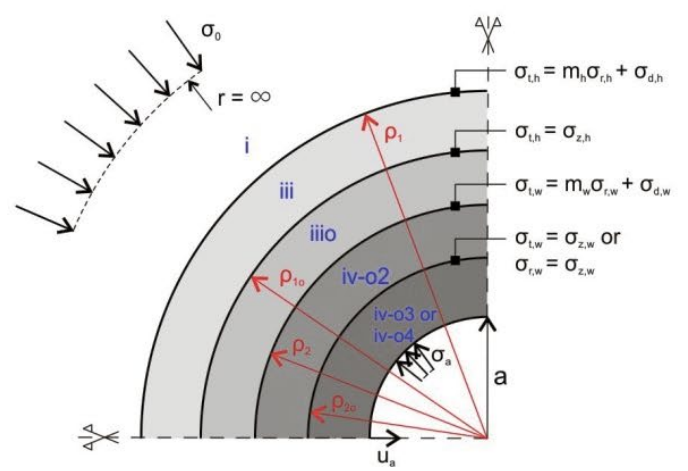

Figure 5.14. Plastic zones considering out-of-plane plastic flow. 
Table 5.3. Cases to be distinguished with respect to the incremental stress-strain relationships.

\begin{tabular}{|c|c|c|c|c|}
\hline \multirow[b]{2}{*}{ weak layers } & \multicolumn{4}{|c|}{ hard layers } \\
\hline & elastic & in-plane yielding & $\begin{array}{l}\text { out-of-plane } \\
\text { yielding (triaxial } \\
\text { extension) }\end{array}$ & $\begin{array}{l}\text { out-of-plane } \\
\text { yielding (triaxial } \\
\text { compression) }\end{array}$ \\
\hline elastic & $i^{(1)}$ & $\mathrm{iii}^{(1)}$ & iii-o & - (2) \\
\hline in-plane yielding & $\mathrm{ii}^{(1)}$ & iv (1) & iv-o2 & - (2) \\
\hline $\begin{array}{l}\text { out-of-plane yielding } \\
\text { (triaxial extension) }\end{array}$ & ii-o & iv-o1 & iv-o3 & iv-o5 \\
\hline $\begin{array}{l}\text { out-of-plane yielding } \\
\text { (triaxial compression) }\end{array}$ & - (2) & - (2) & iv-o4 & - (2) \\
\hline
\end{tabular}

(1) Cases i to iv were introduced in Section 5.2.2.

(2) These cases are not considered by the closed-form solution; a parametric study showed that, for realistic values of the Poisson's ratio (0.30), out-of-plane yielding in triaxial compression occurs either in the weak or in the hard layers and this only if the hard or weak layers, respectively, experience out-of-plane yielding in triaxial compression (cases iv-04 and iv-05, respectively).

In order to determine the stress-, strain- and displacement fields, additional incremental stress-strain relationships to those of Section 5.2.2 have to be derived (see Table 5.3), which account for out-of-plane plastic flow - either in one or in both layers. For this purpose one needs the plastic strain increments at the stress state lying on the intersection of two yield surfaces. They can be determined following the rule of Koiter (1953), according to which the plastic strain increment is equal to a linear combination of the normal vectors of the two potential surfaces. Specifically, for the weak layers, if $\sigma_{r, w}<\sigma_{t, w}=\sigma_{z, w}=m_{w} \sigma_{r, w}+\sigma_{d, w}$ (triaxial extension), then

$$
\left(d \varepsilon_{r, w}-d \varepsilon_{r, w}^{E I}\right)+\kappa_{w} \cdot\left(d \varepsilon_{t, w}-d \varepsilon_{t, w}^{E I}\right)+\kappa_{w} \cdot\left(d \varepsilon_{z, w}-d \varepsilon_{z, w}^{E I}\right)=0
$$

else if $\sigma_{t, w}=m_{w} \cdot \sigma_{r, w}+\sigma_{d, w}$ and $\sigma_{t, w}=m_{w} \cdot \sigma_{z, w}+\sigma_{d, w}\left(i . e\right.$. , if $\sigma_{r, w}=\sigma_{z, w}$; triaxial compression), then

$$
\left(d \varepsilon_{r, w}-d \varepsilon_{r, w}^{E I}\right)+\kappa_{w} \cdot\left(d \varepsilon_{t, w}-d \varepsilon_{t, w}^{E I}\right)+\left(d \varepsilon_{z, w}-d \varepsilon_{z, w}^{E I}\right)=0
$$

Analogously, for the hard layers, if $\sigma_{r, h}<\sigma_{t, h}=\sigma_{z, h}=m_{h} \sigma_{r, h}+\sigma_{d, h}$, then

$$
\left(d \varepsilon_{r, h}-d \varepsilon_{r, h}^{\boxminus I}\right)+\kappa_{h} \cdot\left(d \varepsilon_{t, h}-d \varepsilon_{t, h}^{E !}\right)+\kappa_{h} \cdot\left(d \varepsilon_{z, h}-d \varepsilon_{z, h}^{E I}\right)=0
$$

else if $\sigma_{t, h}=m_{h} \cdot \sigma_{r, h}+\sigma_{d, h}$ and $\sigma_{t, h}=m_{h} \sigma_{z, h}+\sigma_{d, h}\left(i . e .\right.$, if $\left.\sigma_{r, h}=\sigma_{z, h}\right)$, then

$$
\left(d \varepsilon_{r, h}-d \varepsilon_{r, h}^{E I}\right)+\kappa_{h} \cdot\left(d \varepsilon_{t, h}-d \varepsilon_{t, h}^{E I}\right)+\left(d \varepsilon_{z, h}-d \varepsilon_{z, h}^{E I}\right)=0
$$

The incremental stress-strain relationships (Eq. 5.21) of the REV, of the weak layers and of the hard layers result from the basic Equations (5.6) to (5.12), Hooke's law (Eqs. 5.13 and 5.14 ) and the case-specific equations of Table 5.4. The (case-specific) coefficients on the r.h.s. of Equation (5.21) are given in Appendix A (Sections A.5 to A.11).

The procedure for determining the stress $\sigma_{r}(r)$ and $\sigma_{t}(r)$, strain $\varepsilon_{r}(r)$ and $\varepsilon_{t}(r)$ as well as the displacement field $u(r)$ in the plastic zones of Figure 5.14 is the same as in Section 5.2. By integrating the incremental stress-strain relationships considering the conditions at the external radius $\rho$ of each plastic zone, the following generalised stress-strain relationship can be obtained: 


$$
\left(\begin{array}{c}
\sigma_{r}-\sigma_{r, \rho} \\
\sigma_{t}-\sigma_{t, \rho}
\end{array}\right)=\left(\begin{array}{ll}
A_{1} & -A_{2} \\
A_{3,} & -A_{4}
\end{array}\right) \cdot\left(\begin{array}{c}
\varepsilon_{r}-\varepsilon_{r, \rho} \\
\varepsilon_{t}-\varepsilon_{t, \rho}
\end{array}\right) .
$$

Considering additionally the kinematic relations (Eq. 5.1) and the equilibrium condition (Eq. 5.2), a second order differential equation is obtained for $u(r)$ (see Eq. 5.55), which is solved considering the radial stress and displacement continuity conditions,

$$
\begin{gathered}
\sigma_{r}(\rho)=\sigma_{r, \rho} \text { and } \\
u(\rho)=\varepsilon_{t, \rho} \cdot \rho,
\end{gathered}
$$

as boundary conditions. Consequently, as in Section 5.2, the stress-, strain- and displacement fields $\sigma_{r}(r), \sigma_{t}(r), \varepsilon_{r}(r), \varepsilon_{t}(r)$ and $u(r)$ around the tunnel are a function of the external radius of the plastic zone $\rho$. The latter can be obtained by considering either the support pressure at the tunnel boundary (for the innermost plastic zone) or the minimum support pressure at the inner radius of the plastic zone (for the outer zones). The latter can be obtained by determining the support pressure at the inner boundary, which is required in order that the failure criterion of the next inner plastic zone is not reached. (The failure criterion reached in every plastic zone is illustrated in Fig. 5.14.) All equations required for determining the stress $\sigma_{r}(r)$ and $\sigma_{t}(r)$, strain $\varepsilon_{r}(r)$ and $\varepsilon_{t}(r)$ as well as the displacement field $u(r)$ were already formulated in Section 5.2 .4 (cf. Eqs. 5.56 to 5.65). Appendix C provides the flow chart of a Matlab-code for calculating the GRC considering out-of-plane plastic flow,

\begin{tabular}{|c|c|c|}
\hline Case & Yield conditions & Flow rule \\
\hline ii-o & $\sigma_{t, w}=\sigma_{z, w}=m_{w} \cdot \sigma_{r, w}+\sigma_{d, w}$ & Eq. $5.87^{(1)}$ \\
\hline iii-o & $\sigma_{t, h}=\sigma_{z, h}=m_{h} \cdot \sigma_{r, h}+\sigma_{d, h}$ & Eq. $5.89^{(2)}$ \\
\hline \multirow{2}{*}{ iv-o1 } & $\sigma_{t, w}=\sigma_{z, w}=m_{w} \cdot \sigma_{r, w}+\sigma_{d, w}$ & Eq. 5.87 \\
\hline & $\sigma_{t, h}=m_{h} \cdot \sigma_{r, h}+\sigma_{d, h}\left(>\sigma_{z, h}>\sigma_{r, h}\right)$ & Eq. 5.20 \\
\hline \multirow{2}{*}{ iv-o2 } & $\sigma_{t, w}=m_{w} \cdot \sigma_{r, w}+\sigma_{d, w}\left(>\sigma_{z, w}>\sigma_{r, w}\right)$ & Eq. 5.19 \\
\hline & $\sigma_{t, h}=\sigma_{z, h}=m_{h} \cdot \sigma_{r, h}+\sigma_{d, h}$ & Eq. 5.89 \\
\hline \multirow{2}{*}{ iv-o3 } & $\sigma_{t, w}=\sigma_{z, w}=m_{w} \cdot \sigma_{r, w}+\sigma_{d, w}$ & Eq. 5.87 \\
\hline & $\sigma_{t, h}=\sigma_{z, h}=m_{h} \cdot \sigma_{r, h}+\sigma_{d, h}$ & Eq. 5.89 \\
\hline \multirow{2}{*}{ iv-o4 } & $\sigma_{t, w}=m_{w} \cdot \sigma_{r, w}+\sigma_{d, w}, \sigma_{t, w}=m_{w} \cdot \sigma_{z, w}+\sigma_{d, w}$ & Eq. 5.88 \\
\hline & $\sigma_{t, h}=\sigma_{z, h}=m_{h} \cdot \sigma_{r, h}+\sigma_{d, h}$ & Eq. 5.89 \\
\hline \multirow{2}{*}{ iv-o5 } & $\sigma_{t, w}=\sigma_{z, w}=m_{w} \cdot \sigma_{r, w}+\sigma_{d, w}$ & Eq. 5.87 \\
\hline & $\sigma_{t, h}=m_{h} \cdot \sigma_{r, h}+\sigma_{d, h}, \sigma_{t, h}=m_{h} \cdot \sigma_{z, h}+\sigma_{d, h}$ & Eq. 5.90 \\
\hline
\end{tabular}
while the Matlab-code is available as electronic supplementary material. ${ }^{4}$

Table 5.4. Case-specific equations for the incremental stress-strain relationships.

(1) for the hard layers: $d \varepsilon_{r, h}=d \varepsilon_{r, h}^{\boxminus l}$ and $d \varepsilon_{t, h}=d \varepsilon_{t, h}^{\boxminus}$

(2) for the weak layers: $d \varepsilon_{r, w}=d \varepsilon_{r, w}^{\boxminus}$ and $d \varepsilon_{t, w}=d \varepsilon_{t, w}^{\boxminus}$

Out-of-plane plastic flow can be considered in the same way also for brittle behaviour of the hard layers, provided that the hard layers experience first in-plane yielding, which is not always the case: If the softening-induced sudden decrease in strength is bigger than the prefailure difference between tangential and axial stress, then the hard layers experience right

\footnotetext{
${ }^{4}$ The Matlab-code can be downloaded from www.tunnel.ethz.ch.
} 
from the start yielding in the axial direction. In this case, $\sigma_{t, h}=\sigma_{z, h}$ and as $\sigma_{z, h}=\sigma_{z, w}$ (cf. Eq. 5.11), $\sigma_{z, w}$ may become smaller than $\sigma_{r, w}$; this case is not taken into account in Table 5.3.

\subsubsection{Verification of the analytical solution}

The analytical solution was verified analogously to Section 5.2.6, i.e. by comparative numerical calculations with the FE-program Abaqus (Dassault Systèmes 2011). The stress fields in Figure 5.15 show that out-of-plane plastic flow (in triaxial extension, as $\sigma_{t, h}=\sigma_{z, h}$ ) occurs both in the hard and in the weak layers. The closed-form solution is in perfect agreement with the numerical results. Solely, in the tunnel proximity, the results of the analytical solution deviate slightly from those of the numerical calculations. This is because, due to the discretisation of the layers in the numerical calculations, the radial stress (i.e., the support pressure) is zero at the tunnel boundary in the hard and the weak layers, while the analytical solution takes the radial stress of the REV equal to zero. However, the difference is negligible.
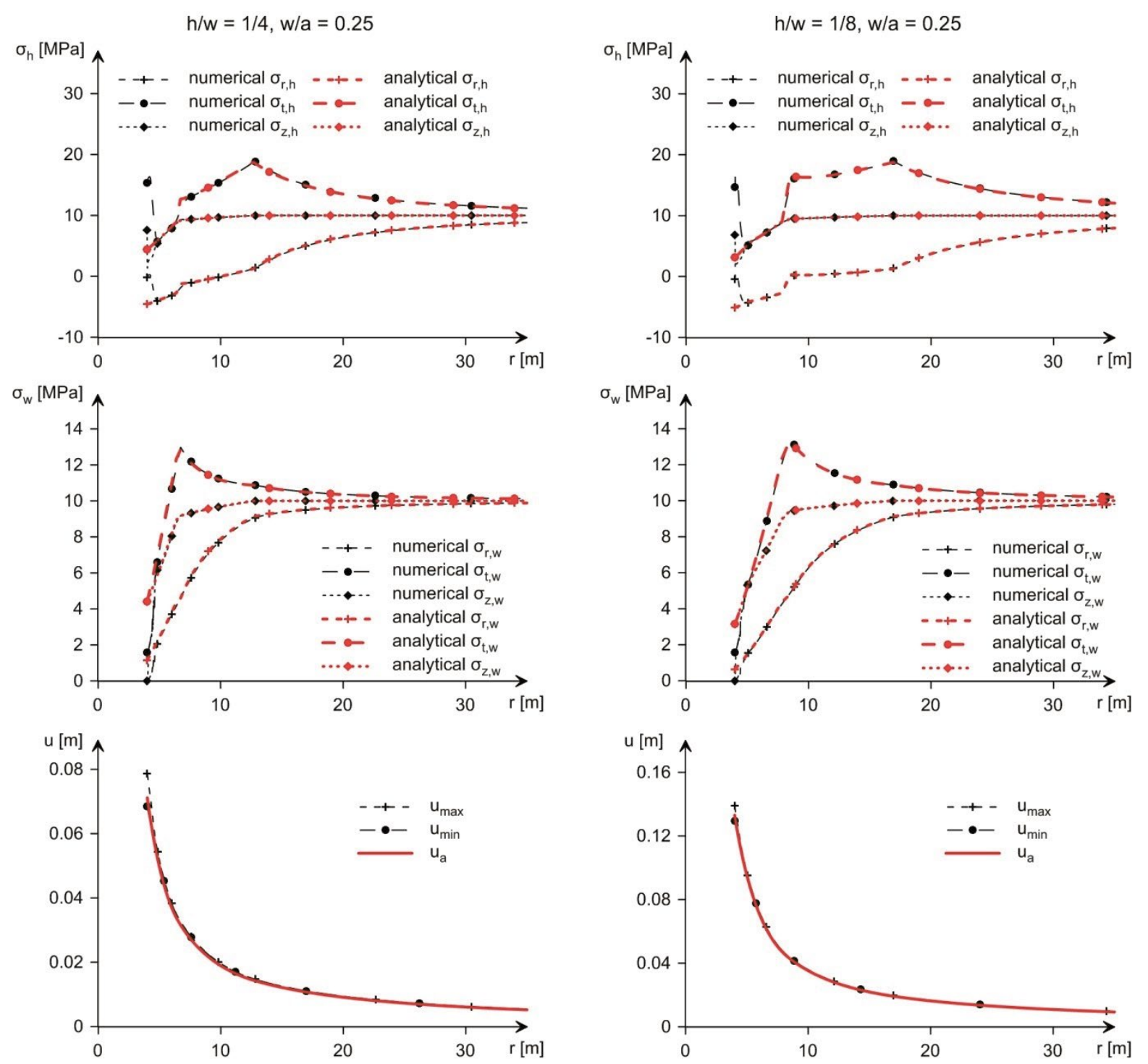

Figure 5.15. Analytically and numerically determined stress- and displacement fields in the hard and in the weak layers (unsupported tunnel, $a=4 \mathrm{~m}, \sigma_{0}=10 \mathrm{MPa}, E_{h}=10 \mathrm{GPa}, c_{h}=5 \mathrm{MPa}$, $\left.E_{w}=1 \mathrm{GPa}, c_{w}=0.5 \mathrm{MPa}, v_{h}=v_{w}=0.3, \varphi_{h}=\varphi_{w}=25^{\circ}, \psi_{h}=\psi_{w}=5^{\circ}\right)$. 


\subsection{Model behaviour}

\subsubsection{Perfectly plastic behaviour of the hard layers}

Some basic aspects of the model behaviour will be discussed by means of the results of Figure 5.16, which concerns an unsupported opening. The upper diagrams show the stress field (corresponding to the volumetric average of the stresses in the hard and in the weak layers; Eqs. 5.9 to 5.11) and the strain field of the equivalent continuum. The lower diagrams show the stress field in the hard and in the weak layers.
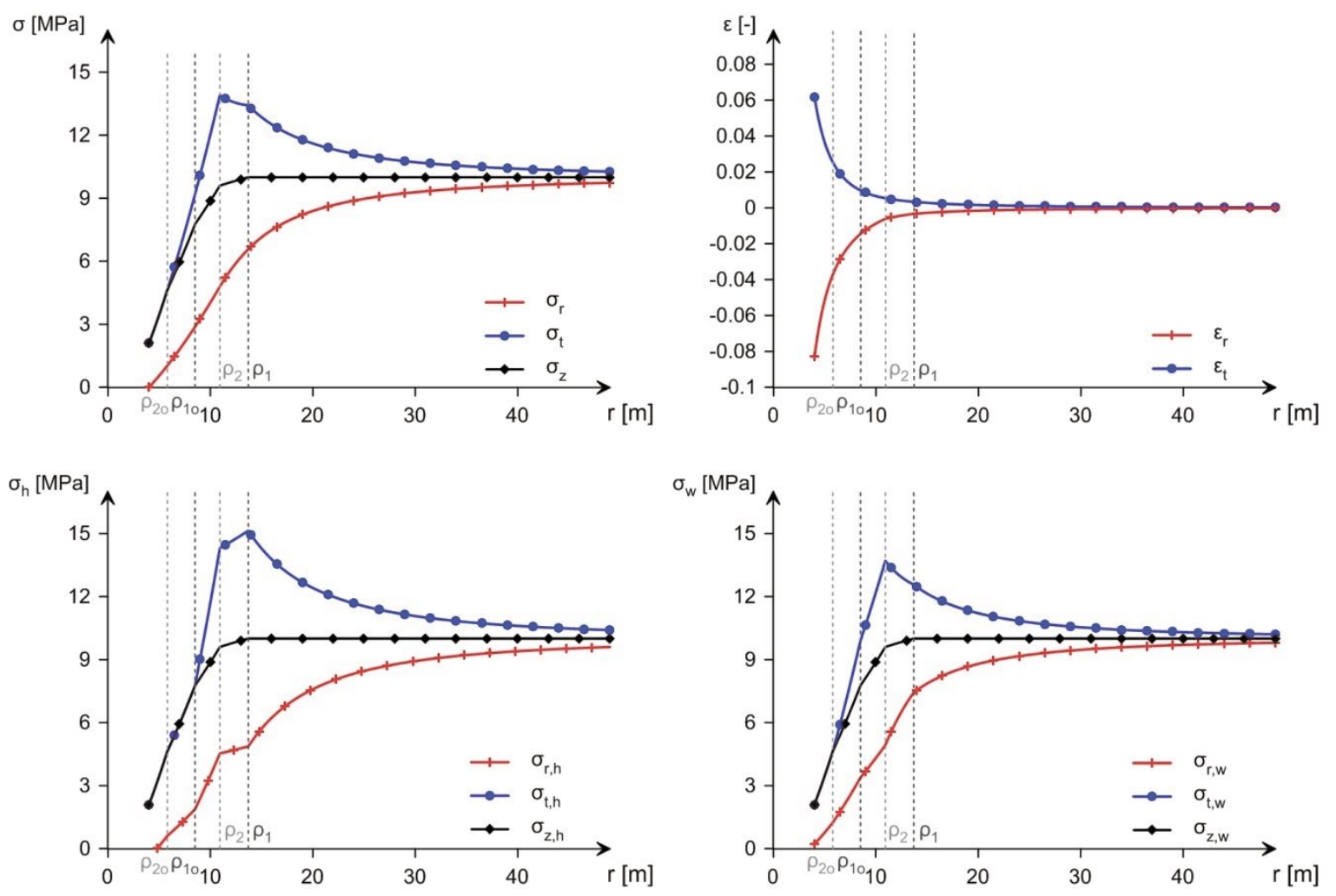

Figure 5.16. Upper diagrams: stress and strain field in the REV; lower diagrams: stress field in the hard and in the weak layers (unsupported tunnel, $a=4 \mathrm{~m}, \sigma_{0}=10 \mathrm{MPa}, x_{h} / x_{w}=0.5, E_{h}=2 \mathrm{GPa}$, $\left.c_{h}=1 \mathrm{MPa}, E_{w}=1 \mathrm{GPa}, c_{w}=0.5 \mathrm{MPa}, v_{h}=v_{w}=0.3, \varphi_{h}=\varphi_{w}=25^{\circ}, \psi_{h}=\psi_{w}=5^{\circ}\right)$.

The peaks in the tangential stress fields $\sigma_{t, h}$ and $\sigma_{t, w}$ in Figure 5.16 mark the boundary of the elastic zone in the hard and in the weak layers, respectively. Five zones can be distinguished (cf. Case B(I) in Fig. 5.14):

- $a=4 \mathrm{~m} \leq r \leq \rho_{20}=5.8 \mathrm{~m}$ : both the weak and the hard layers yield out-of-plane;

- $\rho_{20}=5.8 \mathrm{~m} \leq r \leq \rho_{10}=8.5 \mathrm{~m}$ : the hard layers yield out-of-plane and the weak layers yield in-plane;

- $\rho_{10}=8.5 \mathrm{~m} \leq r \leq \rho_{2}=10.9 \mathrm{~m}$ : both the hard and the weak layers yield in-plane;

- $\rho_{2}=10.9 \mathrm{~m} \leq r \leq \rho_{1}=13.7 \mathrm{~m}$ : hard layers yield in-plane; weak layers are elastic; and

- outer zone: all layers are in elastic state.

Generally, the extent of the plastic zone is greater in the hard layers due to their higher stiffness. The hard layers reach failure state earlier than the weak layers during the gradual decrease in the radial stress at the excavation boundary, because their tangential stress 
increases and their radial stress decreases on account of their higher stiffness by a factor $\mu_{h} / \mu_{w}$ (Eqs. 5.30 to 5.33) more rapidly than in the weak layers. However, the hard layers have a stabilising effect (in spite of their plastification in a more extended zone), because their deformations are (due to their higher stiffness and strength) small. (Note that the displacements of a linearly elastic, perfectly plastic material are inversely proportional to its Young's modulus; Anagnostou and Kovári 1993.)

$$
E_{h}=2 \mathrm{GPa}
$$

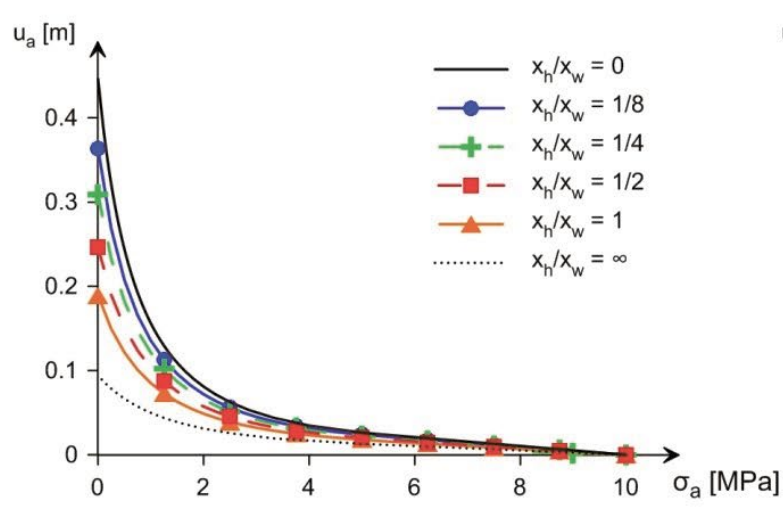

$$
x_{h} / x_{w}=1 / 2
$$

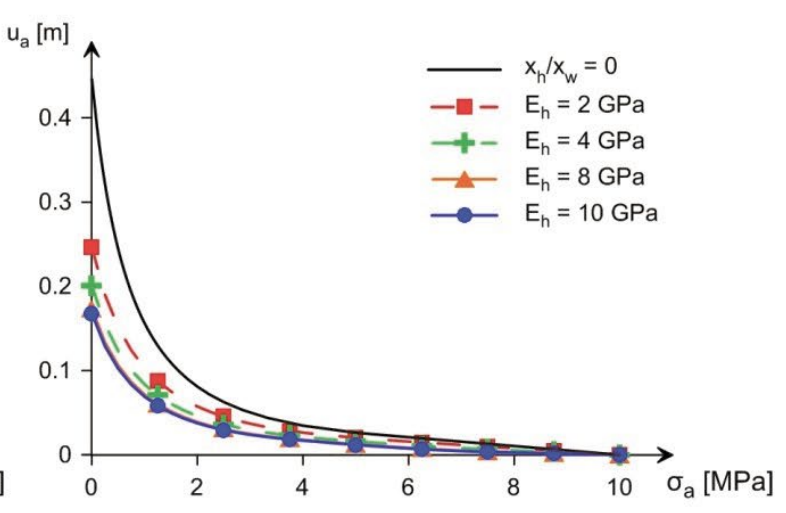

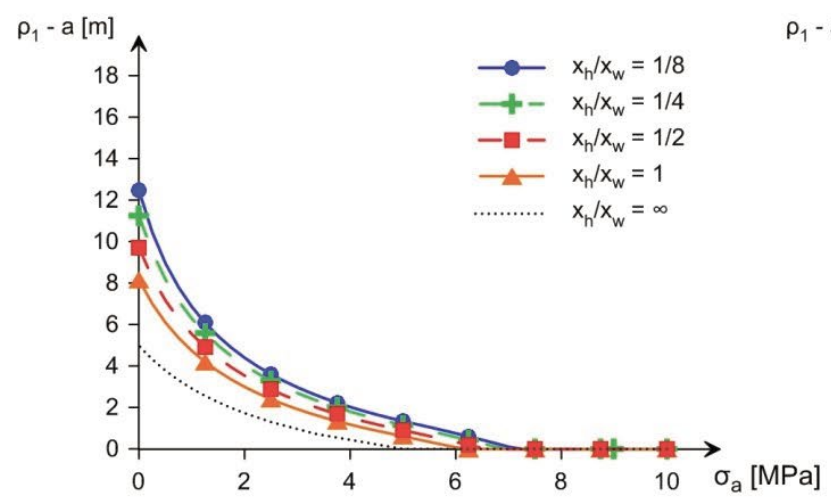
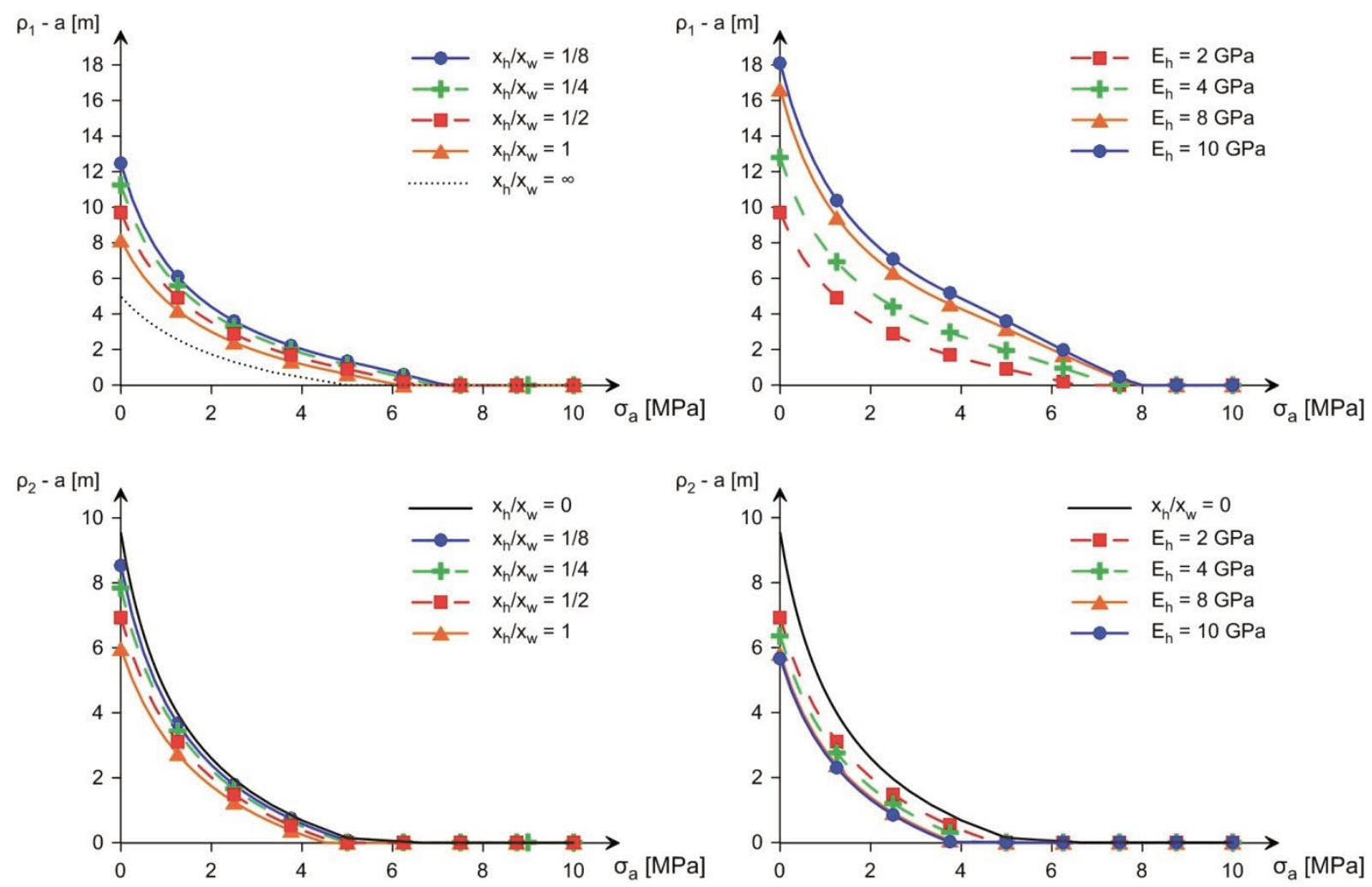

Figure 5.17. Influence of the thickness ratio $x_{h} / x_{w}$ (I.h.s. diagrams) and of the hard layer modulus $E_{h}$ (r.h.s. diagrams) on the GRC, on the extent $\rho_{1}$ of the outer plastic zone, in which the hard layers are at failure state, and on the extent $\rho_{2}$ of the inner plastic zone, in which both the hard and the weak layers are at failure state $\left(a=4 \mathrm{~m}, \sigma_{0}=10 \mathrm{MPa}, c_{h}=1 \mathrm{MPa}, E_{w}=1 \mathrm{GPa}\right.$, $\left.c_{w}=0.5 \mathrm{MPa}, v_{h}=v_{w}=0.3, \varphi_{h}=\varphi_{w}=25^{\circ}, \psi_{h}=\psi_{w}=5^{\circ}\right)$.

The stabilising effect of the hard layers is discussed further by means of the computational results of Figure 5.17; the latter shows the GRCs and the radiuses of plastic zones for various material constants and thickness fractions of the hard layers. According to the I.h.s. diagrams, a reduction of the thickness fraction of the hard layers leads generally to larger 
displacements, to a larger extent of the outer plastic radius $\rho_{1}$ (where the hard layers reach failure) and to a larger extent of the inner plastic radius $\rho_{2}$ (where both, the weak and the hard layers reach failure). For comparison also the analytical GRC for a tunnel crossing only weak $\left(x_{w}=1, x_{h}=0\right)$ or only competent rock $\left(x_{w}=0, x_{h}=1\right)$ is presented, illustrating that the hard rock layers have a stabilising effect even if their thickness fraction is small. With increasing stiffness of the hard layers $E_{h}$ (r.h.s. diagrams of Fig. 5.17) the stiffness of the REV increases, leading to smaller displacements. The stabilising effect increases with the stiffness of the hard layers; the inner plastic radius $\rho_{2}$ (where both layers reach failure) decreases although the outer plastic radius $\rho_{1}$ (where the hard layers reach failure) increases.

$$
E_{\mathrm{h}}=10 \mathrm{GPa}\left(\varphi_{\mathrm{w}}=25^{\circ}, \Psi_{\mathrm{w}}=5^{\circ}\right)
$$
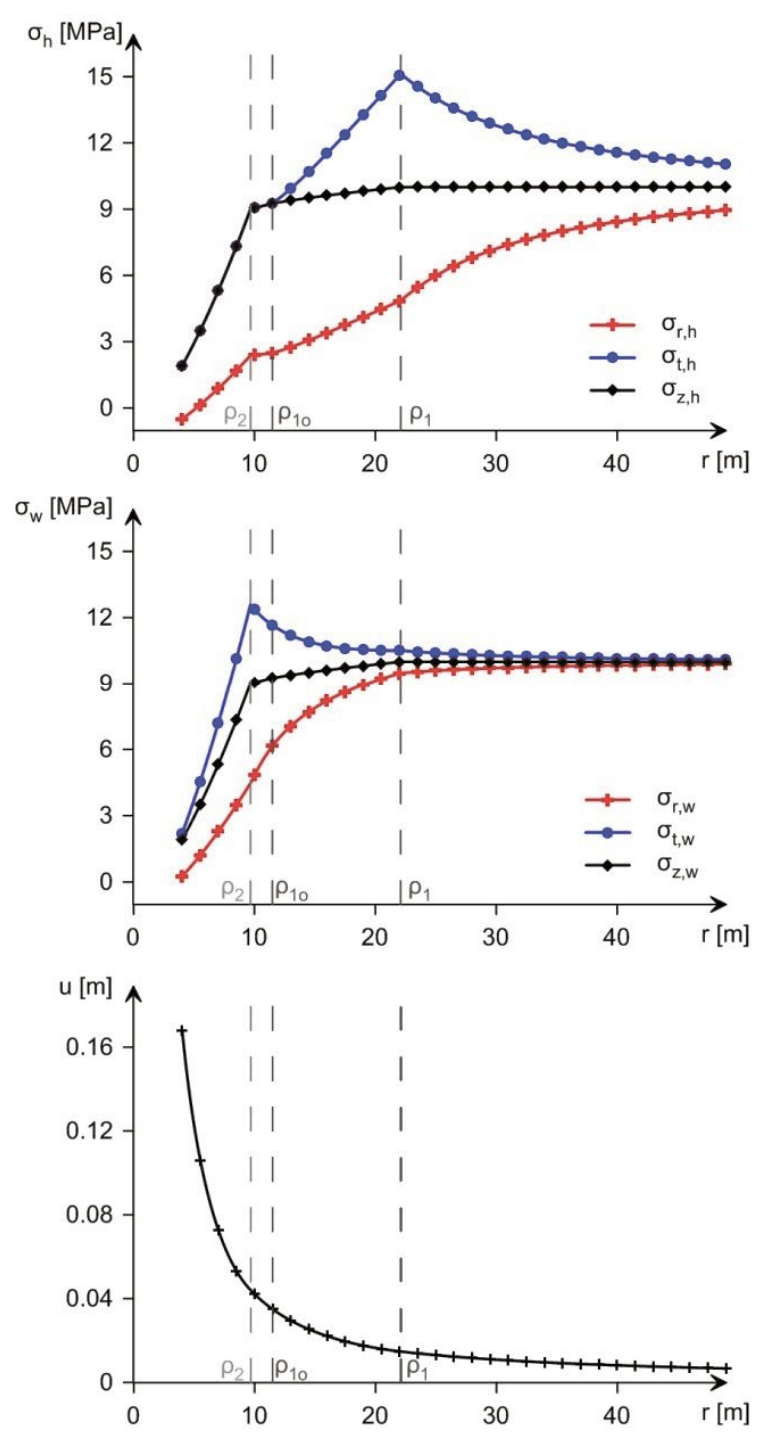

$$
\varphi_{\mathrm{w}}=15^{\circ}, \Psi_{\mathrm{w}}=1^{\circ}\left(\mathrm{E}_{\mathrm{h}}=2 \mathrm{GPa}\right)
$$
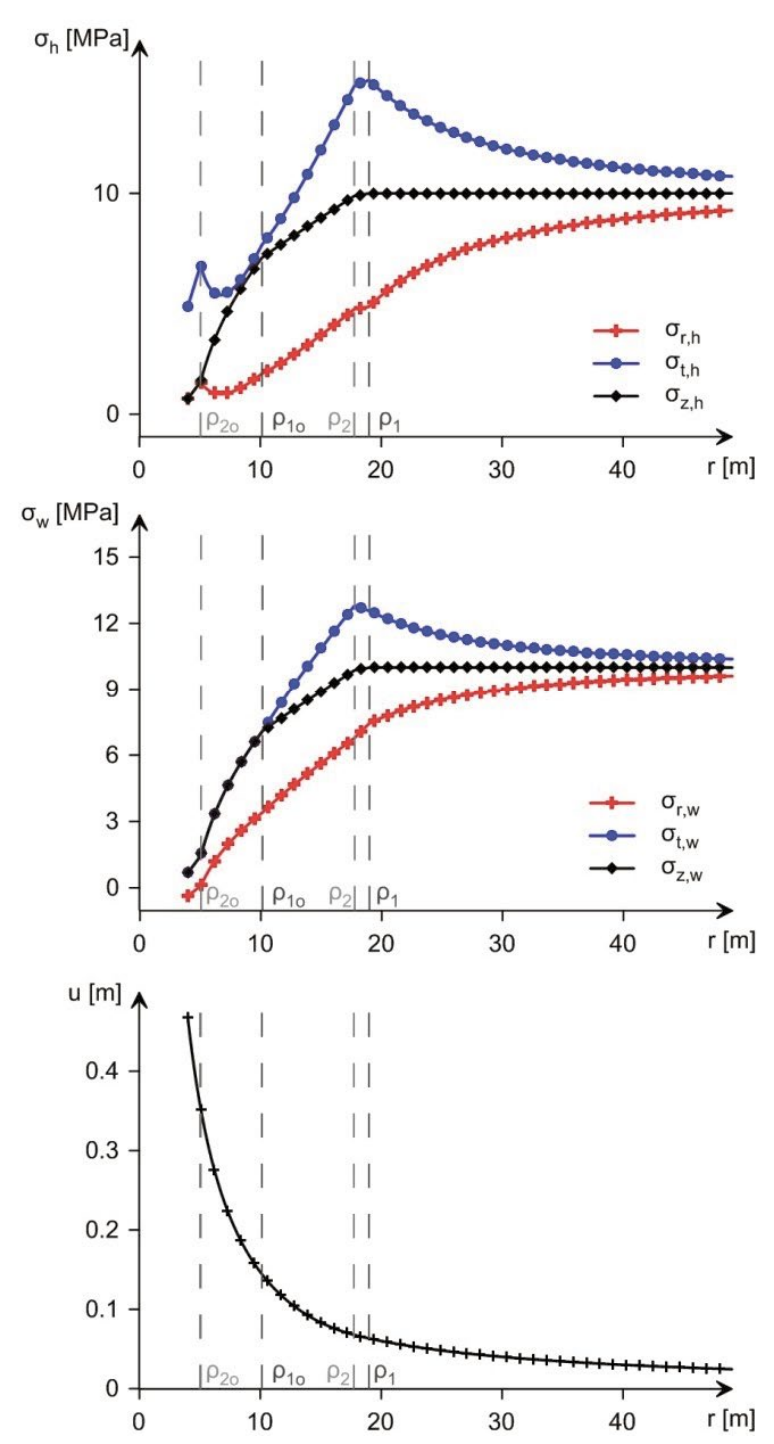

Figure 5.18. Upper diagrams: Stress fields in the hard layers and in the weak layers; lowermost diagrams: displacement field (unsupported tunnel, $a=4 \mathrm{~m}, \sigma_{0}=10 \mathrm{MPa}, x_{h} / x_{w}=0.5$, $\left.c_{h}=1 \mathrm{MPa}, E_{w}=1 \mathrm{GPa}, c_{w}=0.5 \mathrm{MPa}, v_{h}=v_{w}=0.3\right)$.

According to the I.h.s. diagrams of Figure 5.18, which concerns an unsupported tunnel, relatively stiff (e.g., $E_{h}=10 \mathrm{GPa}$ ) hard layers are loaded considerably, reach failure before the weak layers and experience an out-of-plane yielding $\left(\rho_{10}=11.5 \mathrm{~m}>\rho_{2}=9.7 \mathrm{~m}\right.$; cf. Case $B(I I)$ in Fig. 5.14), while the weak layers yield only in-plane. 
If the weak layers exhibit a small friction angle (usually combined with a small dilatancy angle; cf. Vermeer and de Borst 1984), out-of-plane failure in triaxial compression rather than in triaxial extension may occur (r.h.s. diagrams of Fig. 5.18). This happens, however, only under certain conditions (cases iv-04 and iv-05 of Table 5.3).

The effect of the hard layers strength is evident in Figure 5.19. The higher their cohesion (I.h.s. diagrams) or friction angle (r.h.s. diagrams) is, the less extended the plastic zone in the hard layers and the smaller the displacements will be.

$\varphi_{\mathrm{h}}=25^{\circ}$

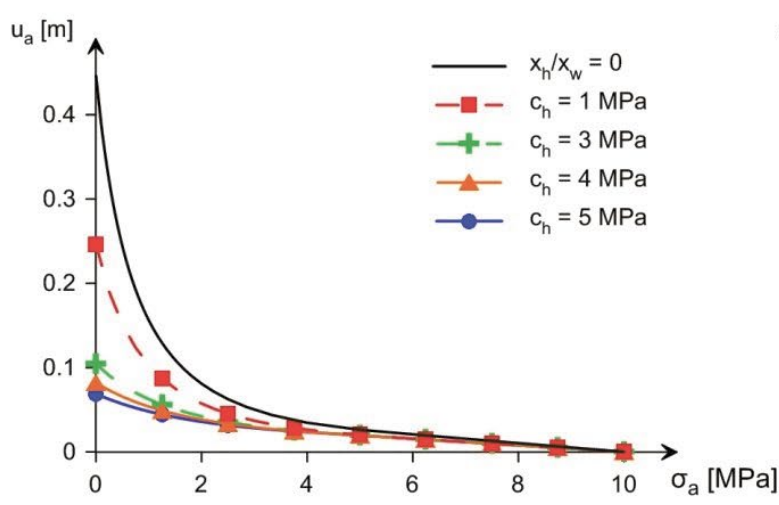

$c_{h}=1 \mathrm{MPa}$

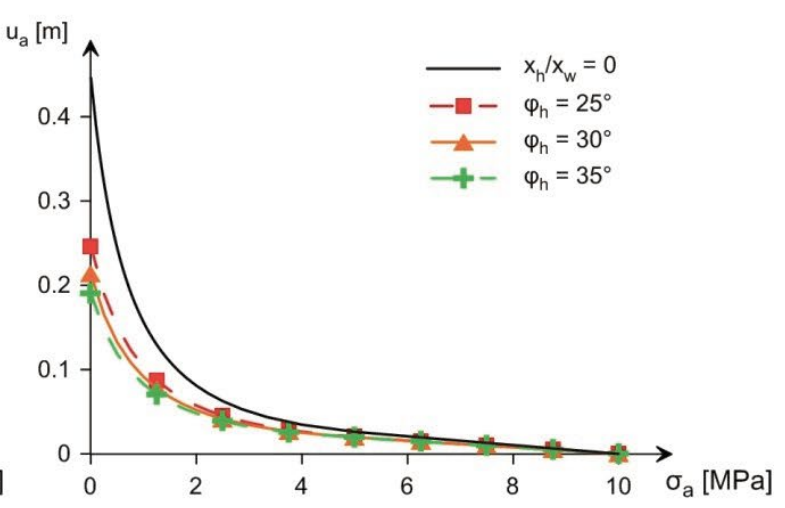

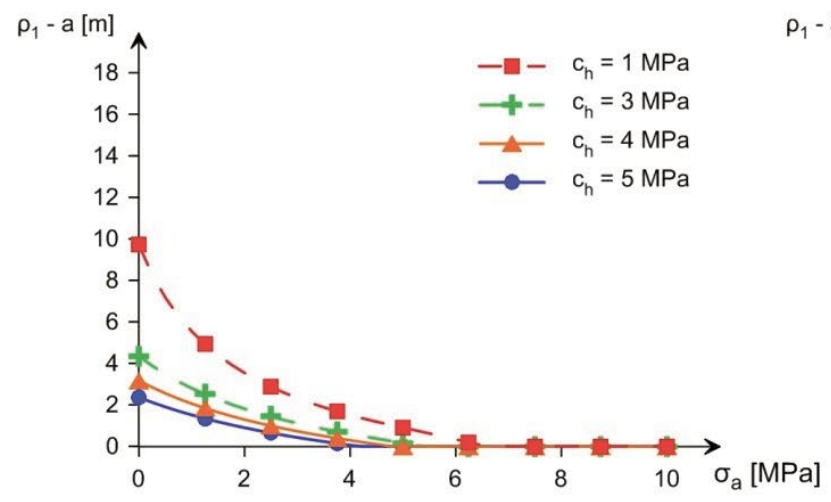
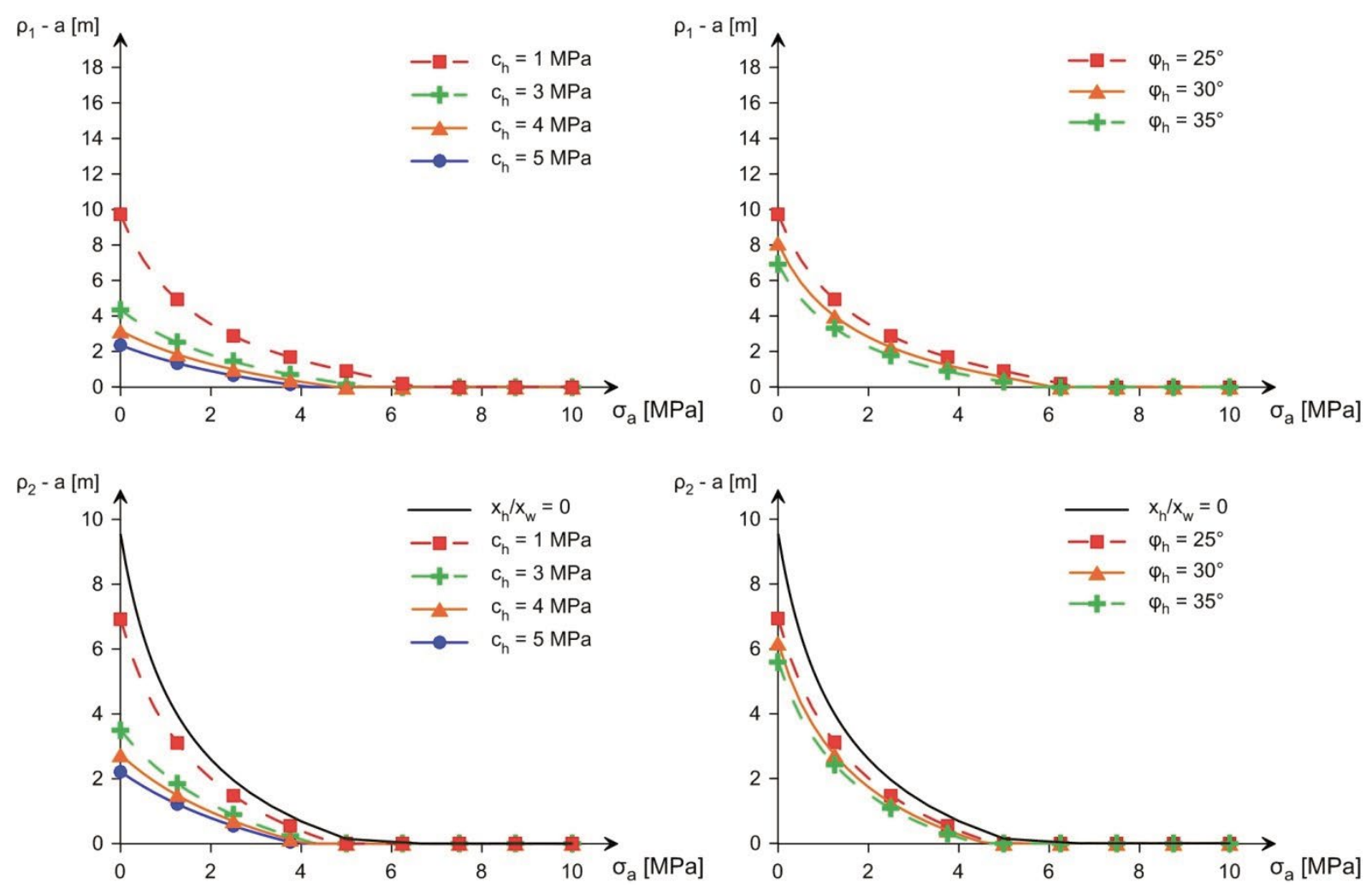

Figure 5.19. Influence of the strength parameters of the hard layers on the GRC, on the extent $\rho_{1}$ of the outer plastic zone, in which only the hard layers are at failure state, and on the extent $\rho_{2}$ of the inner plastic zone, in which both the weak and the hard layers are at failure state $\left(a=4 \mathrm{~m}, \sigma_{0}=10 \mathrm{MPa}, x_{h} / x_{w}=0.5, E_{h}=2 \mathrm{GPa}, E_{w}=1 \mathrm{GPa}, c_{w}=0.5 \mathrm{MPa}, v_{h}=v_{w}=0.3, \varphi_{w}=25^{\circ}\right.$, $\left.\boldsymbol{\Psi}_{h}=\boldsymbol{\psi}_{w}=5^{\circ}\right)$. 


\subsubsection{Effect of brittle behaviour of the hard layers}

Figure 5.20 compares the results obtained assuming brittle behaviour of the hard layers (red lines) with those obtained assuming perfectly plastic behaviour (black lines). It is evident that the stabilising effect of the hard layers is less pronounced in the case of brittle behaviour.

The hard layers reach failure state earlier than the weak layers (due to their higher stiffness; cf. Section 5.5.1) and experience a sudden drop in the tangential stress as soon as they fail. The decrease in strength results in a more extended plastic zone (as the plastic radius $\rho_{1}$ depends on the residual cohesion) and in larger displacements.
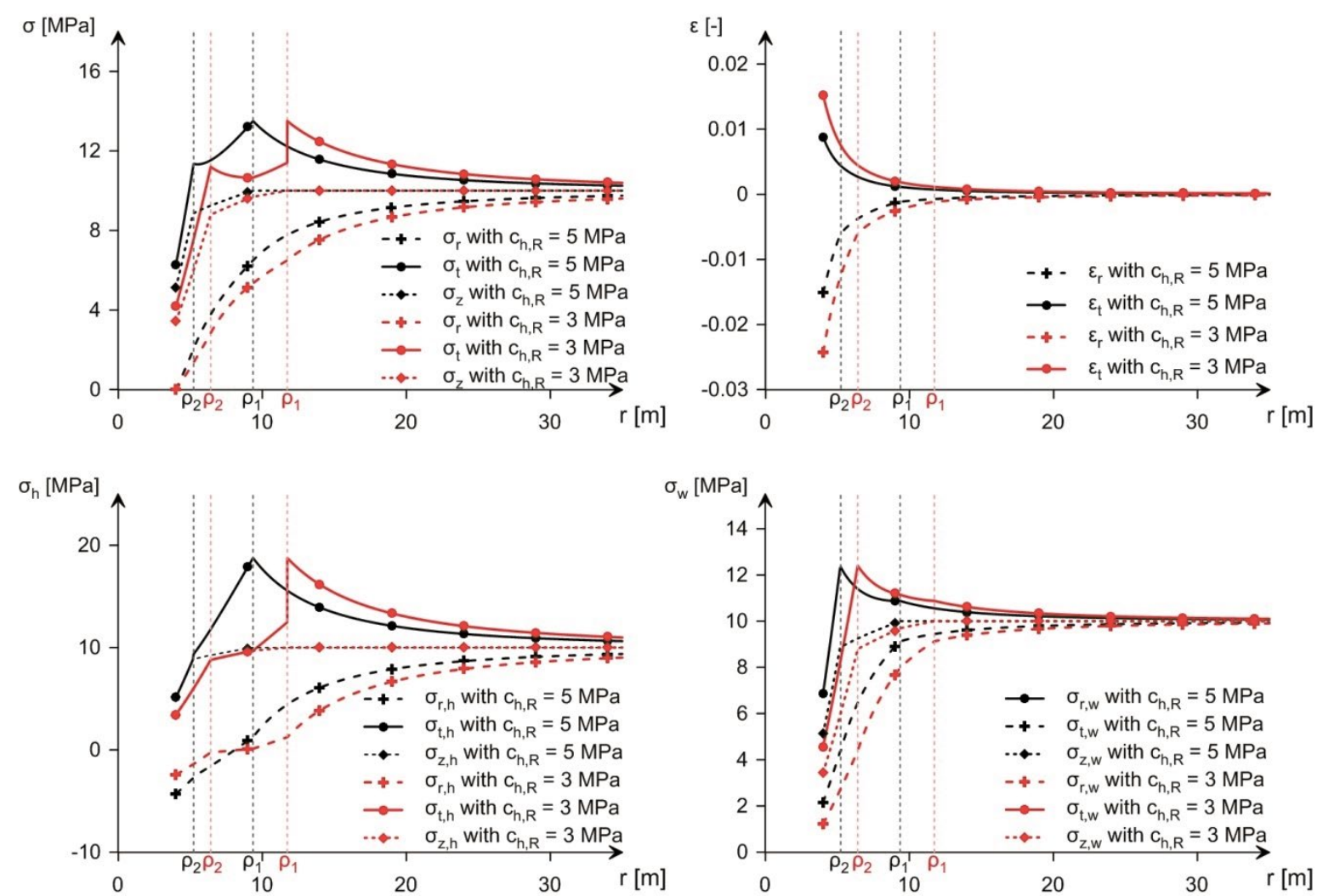

Figure 5.20. Upper diagrams: stress and strain field in the REV; lower diagrams: stress field in the hard and in the weak layers (unsupported tunnel, $a=4 \mathrm{~m}, \sigma_{0}=10 \mathrm{MPa}, x_{h} / x_{w}=0.5, E_{h}=10 \mathrm{GPa}$, $\left.c_{h, H}=5 \mathrm{MPa}, E_{w}=1 \mathrm{GPa}, c_{w}=0.5 \mathrm{MPa}, v_{h}=v_{w}=0.3, \varphi_{h}=\varphi_{w}=25^{\circ}, \psi_{h}=\psi_{w}=5^{\circ}\right)$.

\subsection{Relevance of hard interlayers}

This section investigates under which conditions the stabilising effect of the hard layers can be disregarded. As a measure for the stabilising effect, the ratio $u_{a} / u_{w, 2 D}$ will be considered (Eq. 5.79), where $u_{a}$ is the displacement of the composite material and $u_{w, 2 D}$ denotes the displacement that would occur in the absence of the hard layers (i.e., the displacement for $\left.x_{w} \rightarrow 1\right)$.

Figure 5.21 shows this ratio as a function of the thickness fraction of the hard layers for an unsupported opening, for a bigger and a smaller contrast in the mechanical parameters of the layers as well as for a high and a low in situ stress. 
The latter is considered as a borderline case where all the layers behave elastically and the reduction factor is given by the following simple equation:

$$
\frac{u_{a}}{u_{w, 2 D}}=\frac{\mu_{w}}{\mu^{*}}=\frac{1}{x_{h}\left(\frac{\mu_{h}}{\mu_{w}}-1\right)+1},
$$

where $\mu^{*}$ is the shear modulus of the composite material (Eq. A-6 given in Appendix A). One can recognise from Figure 5.21 that the hard layers have a significant stabilising effect even if their thickness fraction is as low as a few percent. For example, in the presence of $10 \%$ hard layers, the displacements decrease by a factor of 1.4-4 depending on the contrast in the mechanical parameters and on the in situ stress. In general, the higher the contrast in the mechanical parameters (see also Eq. 5.94) and the higher the in situ stress (and thus the squeezing potential), the more pronounced the stabilising effect will be for a given thickness fraction of the hard layers.

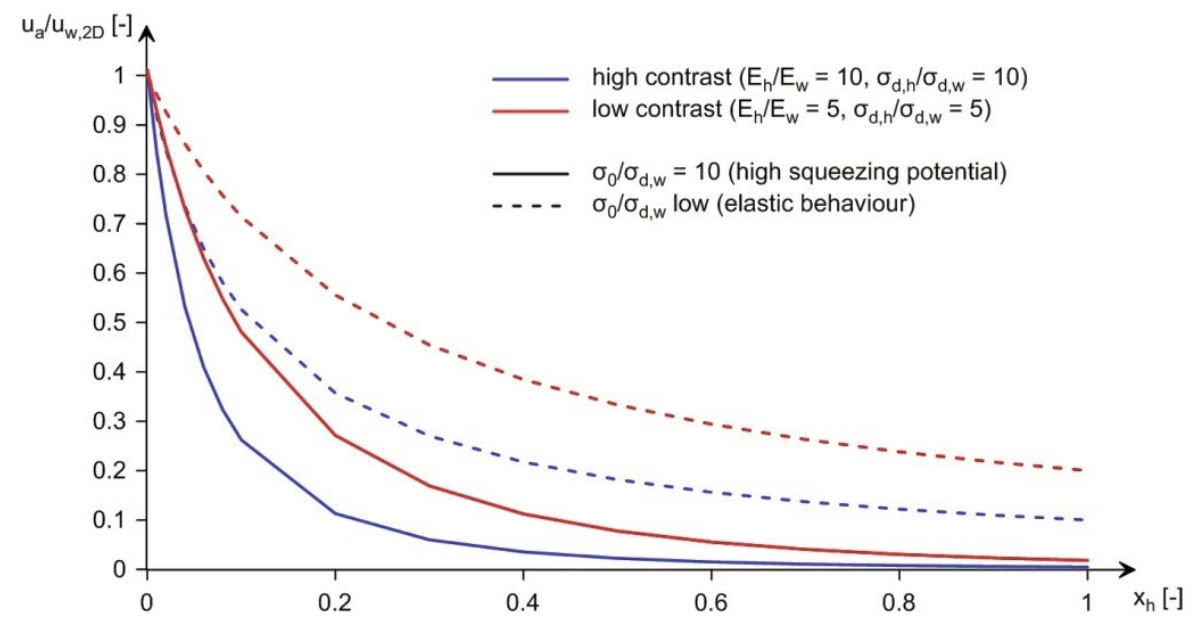

Figure 5.21. Ratio $u_{a} / u_{w, 2 D}$ as a function of the thickness fraction of the hard layers (unsupported tunnel, $\left.v_{h}=v_{w}=0.3, \varphi_{h}=\varphi_{w}=20^{\circ}, \psi_{h}=\psi_{w}=1^{\circ}\right)$.

\subsection{Remarks concerning the adequacy of the homogenised model}

As shown in Sections 5.2.6 and 5.3.2, the displacements that occur in the case of a stratified rock mass with finite layer thicknesses $w / a$ and $h / a$ converge to the closed-form solution for $w / a \rightarrow 0$. The numerical results (Figs. 5.6 and 5.12), besides validating the accuracy and the implementation of the closed-form solutions, provide an indication about the adequacy of the homogenised model: for the assumed mechanical parameters, the rock mass can be conceived practically as homogeneous, if the weak layers are less than about one tunnel radius thick. The purpose of the present section is to analyse whether the homogenisation is adequate or not for a stratified rock mass with given normalised layer thickness w/a. This depends mainly on the contrast in the mechanical parameters of the hard and weak layers:

If the mechanical parameters of the weak and of the hard layers are not very different, then the rock mass can obviously be considered as homogeneous even for very large values of the normalised thicknesses w/a. Conversely, in the case of a big contrast in the mechanical parameters, homogenisation would be adequate only for a very thinly stratified rock mass. This will be illustrated by means of additional computational results (see Fig. 5.22), which were obtained assuming a higher parameter contrast than the previous examples. 
The diagrams of Figure 5.22 show the error of the homogenised model as a function of the normalised layer thickness w/a (upper diagrams) and h/a (lower diagrams) for a series of thickness ratios $h / w$ and for two values of the initial stress (at the lower stress of $0.75 \mathrm{MPa}$ both the hard and the weak layers behave elastically for the assumed strength parameters). The error is defined as $\left(u_{a}-u_{\max }\right) / u_{\max }$, where $u_{\max }$ is the displacement at the vertical symmetry plane of the weak layer (see inset in Fig. 5.6) and $u_{a}$ denotes the displacement of the homogeneous, transversely isotropic material, which is strictly correct only for w/a $\rightarrow 0$ or $h / a \rightarrow 0$, respectively. Considering an underestimation of the displacements by maximally $20 \%$ as acceptable from the practical viewpoint, the homogenised model would be adequate solely if the hard layers would be up to $0.05-0.125$ a thick (see lower diagrams of Fig. 5.22), depending on the thickness ratio $h / w$ and on the initial stress. The error is bigger in the case of the lower initial stress (r.h.s. diagrams), where the behaviour is elastic, but this is irrelevant because the displacements in the elastic range are anyway small.

$$
\sigma_{0}=10 \mathrm{MPa}
$$
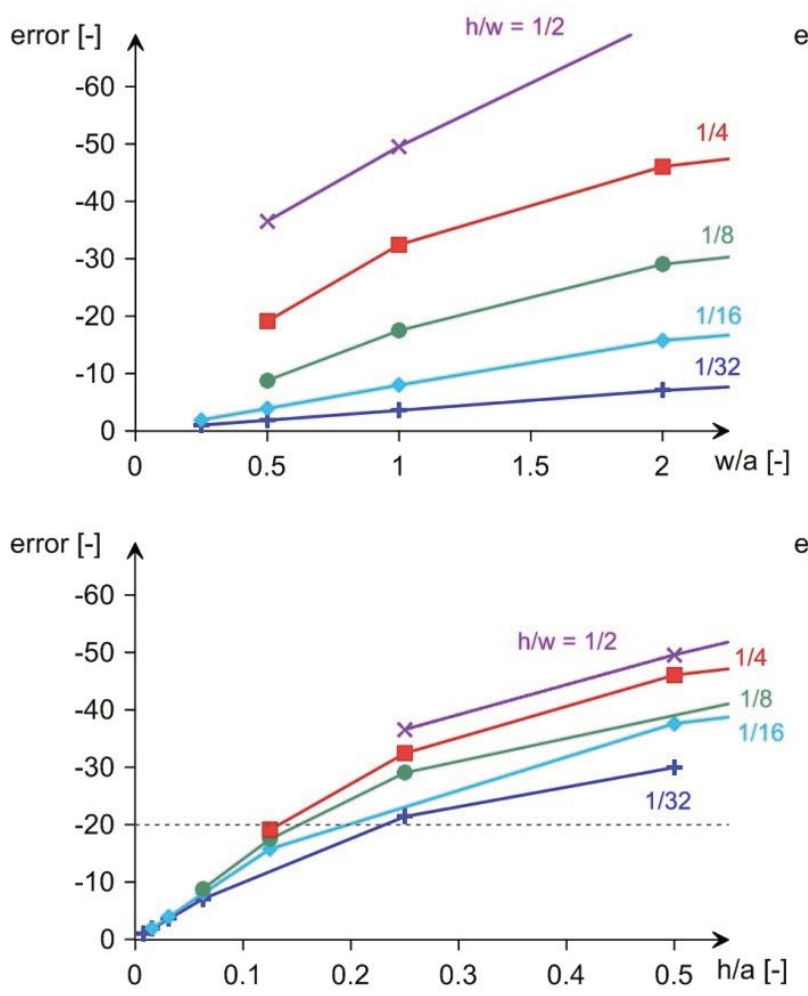

$$
\sigma_{0}=0.75 \mathrm{MPa}
$$
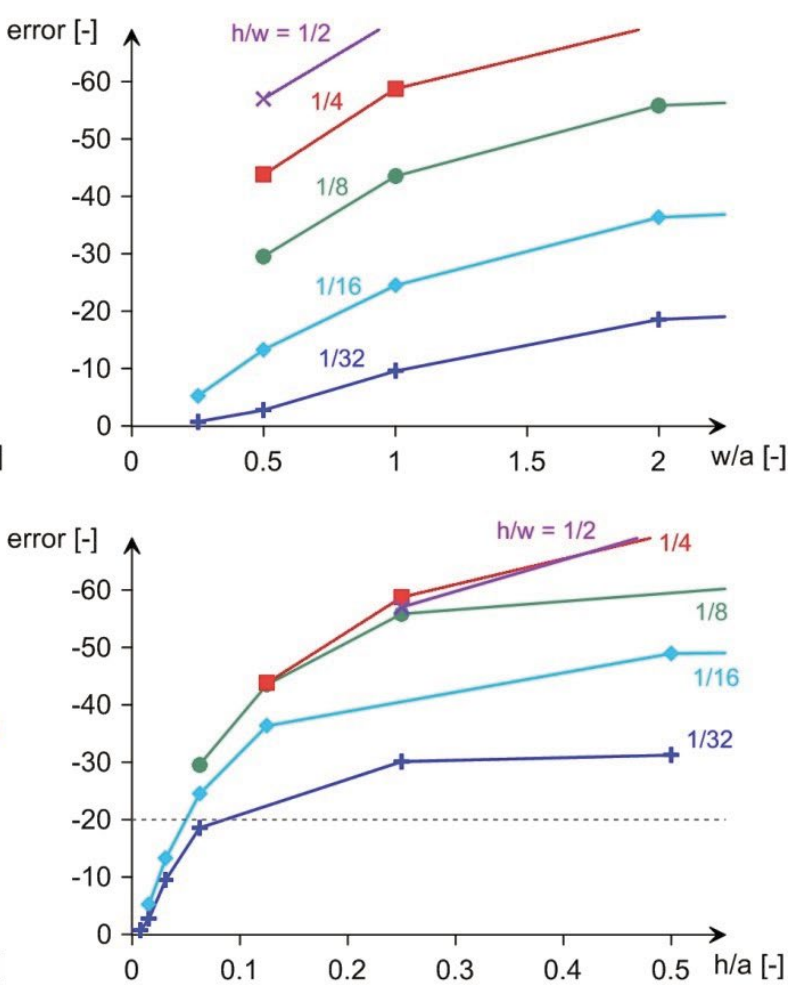

Figure 5.22. Error of the homogenised model as a function of the normalised thickness of the weak layers w/a (upper diagrams) and of the hard layers $h / a$ (lower diagrams; unsupported tunnel, $a=4 \mathrm{~m}, E_{h}=10 \mathrm{GPa}, c_{h}=5 \mathrm{MPa}, E_{w}=0.5 \mathrm{GPa}, c_{w}=0.5 \mathrm{MPa}, v_{h}=v_{w}=0.3, \varphi_{h}=\varphi_{w}=25^{\circ}$, $\left.\boldsymbol{\Psi}_{h}=\boldsymbol{\psi}_{w}=5^{\circ}\right)$.

\subsection{Conclusions}

In this chapter, an analytical solution for determining the displacements and the stresses of tunnels excavated in a sequence of thinly alternating weak and competent layers, assuming an equivalent homogeneous, transversely isotropic rock mass, was derived (under the assumptions of rotational symmetry and plane strain conditions). The derivation of this analytical solution was mathematically demanding, as a variety of cases regarding the failure state of the rock had to be considered (purely elastic or plastic behaviour of the layers - with 
and without brittle behaviour - considering a plastic flow either only in the tunnel crosssection plane or also perpendicular to it). This solution is particularly important for practical reasons, as the numerical modelling of thinly alternating hard and weak rocks is very timeconsuming.

Even if the hard rock layers make a small fraction of the composite (a few percent), in most tunnel problems they cannot be neglected during design. They have a considerable stabilising effect, which can be easily considered by using the developed analytical solution.

\section{Notation}

$A$
$a$
$A_{1}, A_{2}, A_{3}, A_{4}$
$C[1], C[2]$
$C_{h}, C_{w}$
$C_{h, H}, C_{h, R}$
$d \varepsilon_{r,} d \varepsilon_{t}, d \varepsilon_{z}$
$d \varepsilon_{r, h}, d \varepsilon_{t, h}, d \varepsilon_{z, h}$
$d \varepsilon_{r, h}^{E l}, d \varepsilon_{t, h}^{\boxminus}, d \varepsilon_{z h}^{\boxminus}$
$d \varepsilon_{r, w}, d \varepsilon_{t, w}, d \varepsilon_{z, w}$
$d \varepsilon_{r, w}^{\boxminus}, d \varepsilon_{t, w}^{E l}, d \varepsilon_{z, w}^{E l}$
$d \sigma_{r}, d \sigma_{t}, d \sigma_{z}$
$d \sigma_{r, h}, d \sigma_{t, h}, d \sigma_{z, h}$
$d \sigma_{r, w}, d \sigma_{t, w}, d \sigma_{z, w}$
$E_{h, E} E_{w}$
$f(\ldots)$
$h$
$K_{1}, K_{2}, K_{3}, K_{4}$
$K_{5}, K_{6}, K_{7}, K_{8}$
$m_{h}, m_{w}$
$m_{h, H}, m_{h, R}$
$u$
$u_{a}$

constant

tunnel radius

constants for defining the general incremental stress-strain relation of the equivalent continuum

integration constants

cohesion of the hard and of the weak layers

peak and residual (i.e., post-peak) cohesion of the hard layers

radial, tangential and axial (i.e., out-of-plane) strain increment of the equivalent continuum

radial, tangential and axial (i.e., out-of-plane) strain increment of the hard layers

radial, tangential and axial (i.e., out-of-plane) elastic strain increment of the hard layers

radial, tangential and axial (i.e., out-of-plane) strain increment of the weak layers

radial, tangential and axial (i.e., out-of-plane) elastic strain increment of the weak layers

radial, tangential and axial (i.e., out-of-plane) stress increment of the equivalent continuum

radial, tangential and axial (i.e., out-of-plane) stress increment of the hard layers

radial, tangential and axial (i.e., out-of-plane) stress increment of the weak layers

Young's modulus of the hard and of the weak layers

function of ...

thickness of the hard layers (see Fig. 5.6)

constants for defining the general incremental stress-strain relation of the hard layers

constants for defining the general incremental stress-strain relation of the weak layers

inclination of the failure surface of the hard and of the weak layers (function of the friction angle)

peak and residual (i.e., post-peak) inclination of the failure surface of the hard layers (function of the peak and residual friction angle)

radius, i.e., distance to the tunnel axis

radial displacements of the equivalent continuum

radial displacements of the equivalent continuum (consisting of weak and hard layers) at the tunnel boundary 


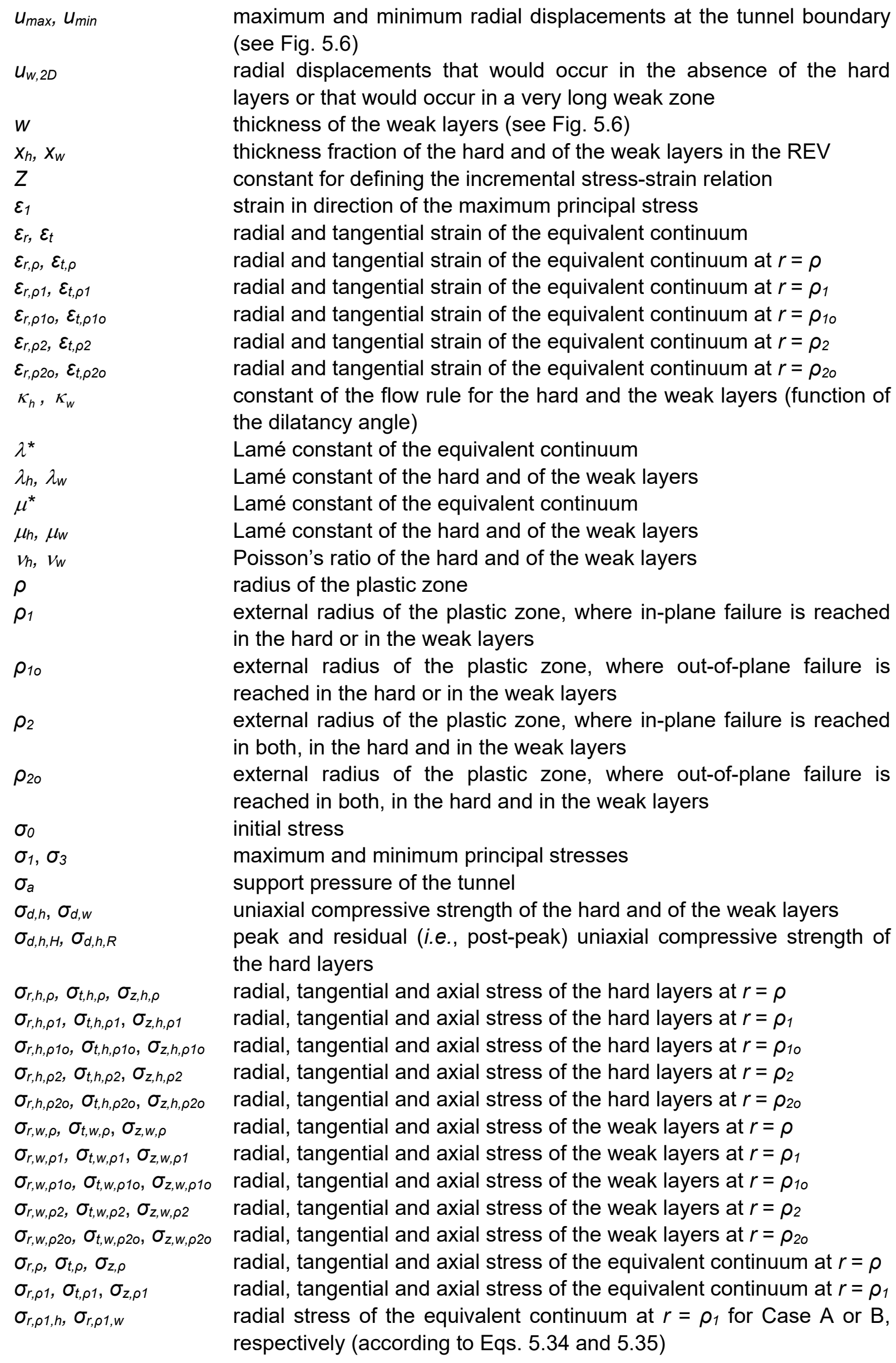

maximum and minimum radial displacements at the tunnel boundary (see Fig. 5.6)

radial displacements that would occur in the absence of the hard layers or that would occur in a very long weak zone thickness of the weak layers (see Fig. 5.6)

thickness fraction of the hard and of the weak layers in the REV constant for defining the incremental stress-strain relation strain in direction of the maximum principal stress radial and tangential strain of the equivalent continuum radial and tangential strain of the equivalent continuum at $r=\rho$ radial and tangential strain of the equivalent continuum at $r=\rho_{1}$ radial and tangential strain of the equivalent continuum at $r=\rho_{10}$ radial and tangential strain of the equivalent continuum at $r=\rho_{2}$ radial and tangential strain of the equivalent continuum at $r=\rho_{20}$ constant of the flow rule for the hard and the weak layers (function of the dilatancy angle)

Lamé constant of the equivalent continuum

Lamé constant of the hard and of the weak layers

Lamé constant of the equivalent continuum

Lamé constant of the hard and of the weak layers

Poisson's ratio of the hard and of the weak layers radius of the plastic zone

external radius of the plastic zone, where in-plane failure is reached in the hard or in the weak layers

external radius of the plastic zone, where out-of-plane failure is reached in the hard or in the weak layers

external radius of the plastic zone, where in-plane failure is reached in both, in the hard and in the weak layers

external radius of the plastic zone, where out-of-plane failure is reached in both, in the hard and in the weak layers

initial stress

maximum and minimum principal stresses

support pressure of the tunnel

uniaxial compressive strength of the hard and of the weak layers peak and residual (i.e., post-peak) uniaxial compressive strength of the hard layers

radial, tangential and axial stress of the hard layers at $r=\rho$ radial, tangential and axial stress of the hard layers at $r=\rho_{1}$ radial, tangential and axial stress of the hard layers at $r=\rho_{10}$ radial, tangential and axial stress of the hard layers at $r=\rho_{2}$ radial, tangential and axial stress of the hard layers at $r=\rho_{20}$ radial, tangential and axial stress of the weak layers at $r=\rho$ radial, tangential and axial stress of the weak layers at $r=\rho_{1}$ radial, tangential and axial stress of the weak layers at $r=\rho_{10}$ radial, tangential and axial stress of the weak layers at $r=\rho_{2}$ radial, tangential and axial stress of the weak layers at $r=\rho_{20}$ radial, tangential and axial stress of the equivalent continuum at $r=\rho$ radial, tangential and axial stress of the equivalent continuum at $r=\rho_{1}$ radial stress of the equivalent continuum at $r=\rho_{1}$ for Case $A$ or $B$, respectively (according to Eqs. 5.34 and 5.35) 
$\sigma_{r, \rho 10}, \sigma_{t, \rho 10}, \sigma_{z, \rho 10} \quad$ radial, tangential and axial stress of the equivalent continuum at $r=\rho_{10}$

$\sigma_{r, \rho 2}, \sigma_{t, \rho 2}, \sigma_{z, \rho 2}$

$\sigma_{r, \rho 2 o}, \sigma_{t, \rho 2 o}, \sigma_{z, \rho 2 o}$

$\varphi_{h}, \varphi_{w}$

$\varphi_{h, H}, \varphi_{h, R}$

$\psi_{h}, \psi_{w}$

radial, tangential and axial stress of the equivalent continuum at $r=\rho_{2}$ radial and tangential stress of the equivalent continuum at $r=\rho_{20}$ friction angle of the hard and of the weak layers peak and residual (i.e., post-peak) friction angle of the hard layers dilatancy angle of the hard and of the weak layers 


\section{Determination of equivalent parameters for a rock mass consisting of thinly alternating weak and competent rocks}

\subsection{Introduction}

The closed-form solution developed in Section 5 considers the thinly stratified rock mass as a homogeneous, transversely isotropic material, the parameters of which consist of those of the hard and weak layers and their thickness fractions. As the response of the homogenised rock mass to tunnel excavation is isotropic in the plane of the tunnel cross-section, it was investigated whether (and was found out that) it is possible to model the stratified rock mass as usually in design practice, i.e., by considering a homogeneous and isotropic (rather than transversely isotropic) linearly elastic - perfectly plastic material obeying Mohr-Coulomb failure criterion with a non-associated flow rule.

This approach makes it possible to analyse problems that do not meet the conditions of rotational symmetry or plane strain, applying known and commonly used methods (e.g., those of Cantieni and Anagnostou 2009b and of Ramoni and Anagnostou 2011b for yielding supports and TBM tunnelling, respectively, under squeezing conditions) also for the case of tunnelling perpendicularly to thinly stratified rocks.

Section 6.2 presents a method for the determination of the parameters of the equivalent isotropic material, which makes use of the closed-form solution for the GRC (of Chapter 5) and considers the parameters of the transversely isotropic material, i.e., the thickness fractions and mechanical parameters of the weak and the hard layers. By analysing the results of a parametric study, which was performed with the method of Section 6.2 , it is possible to express mathematically the parameters of the equivalent isotropic material as functions of the parameters of the transversely isotropic material; this is done in Section 6.3 using the closed-form solution of Section 5.2, which disregards out-of-plane plastic flow. Sections 6.4 and 6.5 discuss the accuracy and illustrate by means of examples the usefulness of the proposed method.

\subsection{Determination of the parameters of the equivalent isotropic medium}

The Young's modulus $E_{e q}$ and the Poisson's ratio $v_{e q}$ of the equivalent isotropic material will be taken such that the latter reproduces exactly the elastic response of the transversely isotropic material: 


$$
\begin{gathered}
E_{e q}=\frac{3 \mu_{e q} \cdot\left(\lambda_{e q}+2 \mu_{e q} / 3\right)}{\left(\lambda_{e q}+\mu_{e q}\right)}, \\
v_{e q}=\frac{\lambda_{e q}}{2 \cdot\left(\lambda_{e q}+\mu_{e q}\right)},
\end{gathered}
$$

where $\lambda_{e q}$ and $\mu_{e q}$ are taken equal to the Lamé constants of the REV:

$$
\begin{gathered}
\mu_{e q}=x_{h} \mu_{h}+x_{w} \mu_{w} \\
\lambda_{e q}=\frac{x_{w}{ }^{2} \lambda_{w}\left(\lambda_{h}+2 \mu_{h}\right)+x_{h}{ }^{2} \lambda_{h}\left(\lambda_{w}+2 \mu_{w}\right)+2 x_{h} x_{w}\left(\lambda_{h}\left(\lambda_{w}+\mu_{h}\right)+\lambda_{w} \mu_{w}\right)}{x_{w}\left(\lambda_{h}+2 \mu_{h}\right)+x_{h}\left(\lambda_{w}+2 \mu_{w}\right)}
\end{gathered}
$$

where $\mu_{h}$ and $\lambda_{h}$ as well as $\mu_{w}$ and $\lambda_{w}$ denote the Lamé constants of the hard and the weak layers, respectively.

The three plasticity parameters $\left(\sigma_{d, \mathrm{eq}}, \varphi_{\mathrm{eq}}\right.$ and $\left.\psi_{\mathrm{eq}}\right)$ of the equivalent isotropic medium will be determined such that the GRC of the latter, which in general can be expressed as:

$$
u_{a}=\frac{\sigma_{0} \cdot a}{E_{e q}} \cdot f_{l}\left(\frac{\sigma_{a}}{\sigma_{0}}, \frac{\sigma_{d, e q}}{\sigma_{0}}, v_{e q}, \varphi_{e q}, \psi_{e q}\right) \text {, }
$$

(cf. Anagnostou and Kovári 1993) coincides at three selected values of the support pressure with the GRC of the transversely isotropic material:

$$
u_{a}=\frac{\sigma_{0} \cdot a}{E_{w}} \cdot f_{T I}\left(\frac{\sigma_{a}}{\sigma_{0}}, \frac{x_{h}}{x_{w}}, \frac{E_{h}}{E_{w}}, \frac{\sigma_{d, h}}{\sigma_{d, w}}, \frac{\sigma_{d, w}}{\sigma_{0}}, v_{h}, \varphi_{h}, \psi_{h}, v_{w}, \varphi_{w}, \psi_{w}\right),
$$

i.e.,

$$
\left.\frac{E_{e q}}{E_{w}} f_{T I}\left(\frac{\sigma_{a k}}{\sigma_{0}}, \frac{x_{h}}{x_{w}}, \frac{E_{h}}{E_{w}}, \frac{\sigma_{d, h}}{\sigma_{d, w}}, \frac{\sigma_{d, w}}{\sigma_{0}}, v_{h}, \varphi_{h}, \psi_{h}, v_{w}, \varphi_{w}, \psi_{w}\right)=f_{l}\left(\frac{\sigma_{a k}}{\sigma_{0}}, \frac{\sigma_{d, e q}}{\sigma_{0}}, v_{e q}, \varphi_{e q}, \psi_{e q}\right) \text { (for } k=1,2,3\right) \text {, }
$$

or, considering (on account of Eqs. 6.1 to 6.4 ) that:

$$
\begin{gathered}
\frac{E_{e q}}{E_{w}}, v_{e q}=f_{0}\left(\frac{x_{h}}{x_{w}}, \frac{E_{h}}{E_{w}}, v_{h}, v_{w}\right), \\
\left.f\left(\frac{\sigma_{d, e q}}{\sigma_{0}}, \varphi_{e q}, \psi_{e q}, \frac{\sigma_{a k}}{\sigma_{0}}, \frac{x_{h}}{x_{w}}, \frac{E_{h}}{E_{w}}, \frac{\sigma_{d, h}}{\sigma_{d, w}}, \frac{\sigma_{d, w}}{\sigma_{0}}, v_{h}, \varphi_{h}, \psi_{h}, v_{w}, \varphi_{w}, \psi_{w}\right)=0 \text { (for } k=1,2,3\right) .
\end{gathered}
$$

Equation (6.9) represents a system of three nonlinear equations, the solution of which provides the normalised strength $\sigma_{d, e q} / \sigma_{0}$, the friction angle $\varphi_{\text {eq }}$ and the dilatancy angle $\psi_{\text {eq }}$ of the equivalent isotropic material for given thickness fraction $x_{h}$ and material constants of the hard and weak layers:

$$
\frac{\sigma_{d, \mathrm{eq}}}{\sigma_{0}}, \varphi_{e q}, \psi_{e q}=f\left(\frac{x_{h}}{x_{w}}, \frac{E_{h}}{E_{w}}, \frac{\sigma_{d, h}}{\sigma_{d, w}}, \frac{\sigma_{d, w}}{\sigma_{0}}, v_{h}, \varphi_{h}, \psi_{h}, v_{w}, \varphi_{w}, \psi_{w}\right)
$$


In this way the parameters of an isotropic material can be determined, the response curve of which is close to the one of the rigorously defined transversely isotropic material. Specifically, the GRC of the equivalent material has the same elastic part as the GRC of the transversely isotropic material and intersects the latter at the three selected values of the support pressure. The latter can be taken equal to $0,0.1 \sigma_{0}$ and $0.2 \sigma_{0}$ in order to capture the practically important portion of the GRC.

\subsection{Relationships between the material constants of the equivalent isotropic medium and those of the transversely isotropic medium}

The material constants of the equivalent isotropic medium will be expressed as functions of the material constants of the transversely isotropic medium. This is possible by postprocessing the results of a parametric study, which was carried out by applying the method of Section 6.2 to a large number of parameter sets $\left(x_{h} / x_{w}, E_{h} / E_{w}, \sigma_{d, h} / \sigma_{d, w}, \sigma_{d, w} / \sigma_{0}, v_{h}, \varphi_{h}, \psi_{h}\right.$, $\left.v_{w}, \varphi_{w}, \psi_{w}\right)$. The parametric study was carried out with the closed-form solution of Section 5.2, which disregards an out-of-plane plastic flow. The considered parameter sets are given in Table 5.2 and cover a range that is sufficiently wide for practical purposes. For the sake of economy, the Poisson's ratios were kept fixed to 0.3 and the dilatancy angles were taken equal to $\max \left(\varphi-20^{\circ} ; 1^{\circ}\right)$. In addition, use will be made of the fact that weaker materials are generally also softer, exhibiting a Young's modulus in the order of 500-1000 times the uniaxial strength, i.e., $E_{h}=\alpha_{h} \sigma_{d, h}$ and $E_{w}=\alpha_{w} \sigma_{d, w}$ (where $500 \leq \alpha_{h, w} \leq 1000$ ), which means that the moduli ratio amounts to $E_{h} / E_{w}=\alpha \sigma_{d, h} / \sigma_{d, w}$, where $0.5 \leq \alpha \leq 2$. In this way, the number of parameters to be considered decreases from ten (Eq. 6.10) to six:

$$
\frac{\sigma_{d, e q}}{\sigma_{0}}, \varphi_{e q}, \psi_{e q}=f\left(\frac{\sigma_{d, w}}{\sigma_{0}}, \frac{x_{h}}{x_{w}}, \alpha, \frac{\sigma_{d, h}}{\sigma_{d, w}}, \varphi_{h}, \varphi_{w}\right) .
$$

The computational results (obtained with the parameters of Table 6.1) were post-processed by representing graphically the parameters $\sigma_{d, \text { ed }} / \sigma_{0}, \varphi_{\text {eq }}$ and $\psi_{\text {eq }}$ of the isotropic medium as functions of the normalised strength $\sigma_{d w} / \sigma_{0}$ of the weak layers for each set of the last five parameters of Equation (6.11). Figure 6.1 shows an example of these diagrams for a specific parameter set; the computational results are represented by the black circular markers. By analysing these graphs, mathematical relationships were found between the parameters of the isotropic material and those of the transversely isotropic model (red curves in Fig. 6.1).

Table 6.1. Overview of the considered parameter ranges for determining the equivalent parameters.

\begin{tabular}{lc}
\hline$x_{h} / x_{w}$ & $1 / 8,1 / 4,1 / 2,1,2$ \\
$\sigma_{d, h} / \sigma_{d, w}$ & $2,6,10$ \\
$\alpha$ & $0.5,1,1.5,2$ \\
$\varphi_{h}$ & $20^{\circ}, 25^{\circ}, 30^{\circ}$ \\
$\varphi_{w}$ & $\varphi_{h}-\left(0^{\circ}, 5^{\circ}, 10^{\circ}\right)$ \\
\hline
\end{tabular}

As can be seen in the example of Figure 6.1, the relationships between the parameters of the isotropic model and the normalised strength $\sigma_{d, w} / \sigma_{0}$ consist of three parts, delimited by the characteristic values $\left(\sigma_{d, w} / \sigma_{0}\right)_{\text {crit }}$ and $\left(\sigma_{d, w} / \sigma_{0}\right)_{c o r}$. 


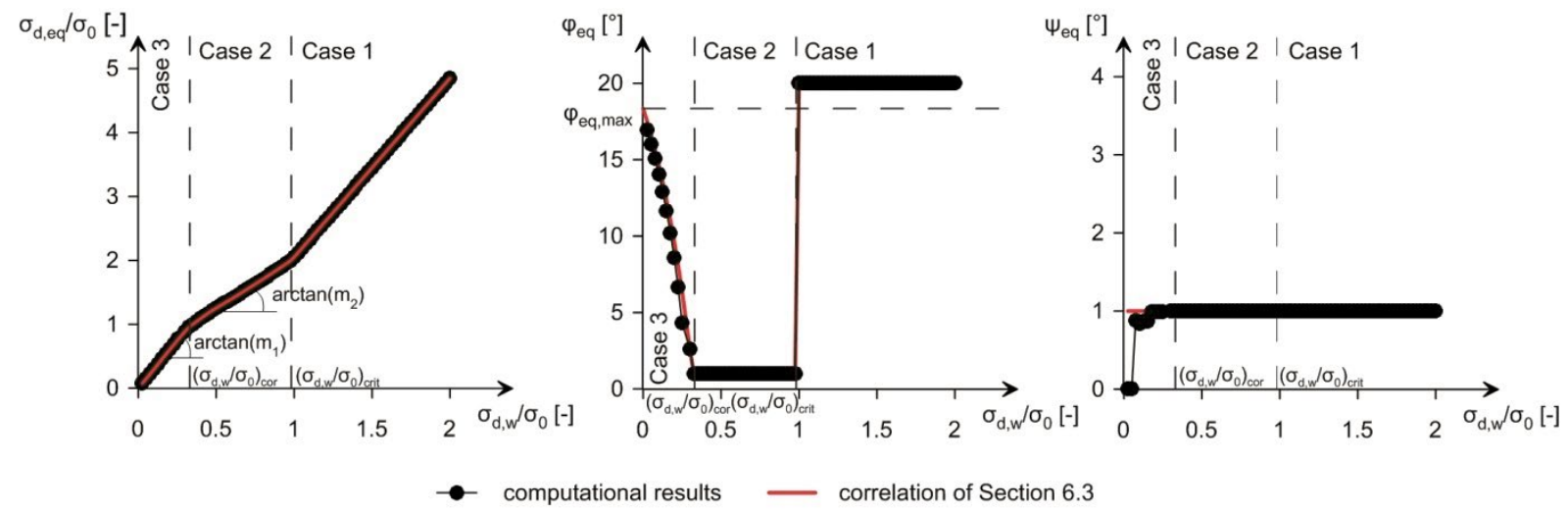

Figure 6.1. Parameters of the simplified isotropic model as functions of the normalised strength of the weak layers $\left(x_{h} / x_{w}=1 / 4, \alpha=1, \sigma_{d, h} / \sigma_{d, w}=10, \varphi_{h}=\varphi_{w}=20^{\circ}\right)$.

The first characteristic value is the strength, for which neither the weak nor the hard layers reach failure (even if $\sigma_{a} / \sigma_{0}=0$ ), and can be determined analytically based upon the equations that were derived for the transversely isotropic medium (cf. Section 5.2.3):

$$
\left(\frac{\sigma_{d, w}}{\sigma_{0}}\right)_{\text {crit }}=\max \left(\frac{-\mu_{\mathrm{eq}} \cdot\left(-1+m_{w}\right)+\left(1+m_{w}\right) \cdot \mu_{w}}{\mu_{\mathrm{eq}}}, \frac{-\mu_{\mathrm{eq}} \cdot\left(-1+m_{h}\right)+\left(1+m_{h}\right) \cdot \mu_{h}}{\mu_{\mathrm{eq}} \cdot \frac{\sigma_{d, h}}{\sigma_{d, w}} \cdot \frac{\sigma_{d, w}}{\sigma_{0}}}\right) .
$$

If the second r.h.s. term of this equation is greater than the first r.h.s. term, then the hard layers reach failure earlier than the weak layers. This is usually the case due to the higher stiffness of the hard layers (Section 5.5.1).

The second characteristic value, $\left(\sigma_{d, w} / \sigma_{0}\right)_{c o r}$, has no physical meaning. By trial-and-error, the following relationship could be found:

$$
\left(\frac{\sigma_{d, w}}{\sigma_{0}}\right)_{\text {cor }}=\left(-0.055 \cdot \frac{\sigma_{d, h}}{\sigma_{d, w}} \cdot \frac{m_{h}}{m_{w}}+1.45\right) \cdot \frac{E_{w}}{E_{e q}}
$$

The relationships between plasticity parameters of the isotropic model and the ground parameters can be approximated as follows.

If $\sigma_{d, w} / \sigma_{0} \geq\left(\sigma_{d, w} / \sigma_{0}\right)_{\text {crit }}$ (Case 1 of Fig. 6.1 ), the plasticity parameters are taken such, that the minimum support pressure $\sigma_{\rho}$ for which the rock remains elastic is the same for the isotropic and for the transversely isotropic material:

$$
\frac{\sigma_{d, e q}}{\sigma_{0}}=2-\frac{\sigma_{\rho}}{\sigma_{0}} \frac{2}{1-\sin \varphi_{\mathrm{eq}}}
$$

where

$$
\frac{\sigma_{\rho}}{\sigma_{0}}=\max \left(1-\frac{\mu_{\mathrm{eq}}}{\mu_{w}} \frac{m_{w}-1+\frac{\sigma_{d, w}}{\sigma_{0}}}{1+m_{w}}, 1-\frac{\mu_{\mathrm{eq}}}{\mu_{h}} \frac{m_{h}-1+\frac{\sigma_{d, h}}{\sigma_{d, w}} \cdot \frac{\sigma_{d, w}}{\sigma_{0}}}{1+m_{h}}\right),
$$




$$
\begin{gathered}
\varphi_{e q}=x_{h} \cdot \varphi_{h}+x_{w} \cdot \varphi_{w}, \\
\psi_{\text {eq }}=x_{h} \cdot \psi_{h}+x_{w} \cdot \psi_{w} .
\end{gathered}
$$

If $\left(\sigma_{d, w} / \sigma_{0}\right)_{c o r} \leq \sigma_{d, w} / \sigma_{0} \leq\left(\sigma_{d, w} / \sigma_{0}\right)_{c r i t}$ (Case 2 of Fig. 6.1), then

$$
\begin{gathered}
\frac{\sigma_{d, e q}}{\sigma_{0}}=\left(m_{1}-m_{2}\right)\left(\frac{\sigma_{d, w}}{\sigma_{0}}\right)_{c o r}+m_{2} \cdot \frac{\sigma_{d, w}}{\sigma_{0}}, \\
\varphi_{e q}=1^{\circ},
\end{gathered}
$$

and

$$
\psi_{e q}=x_{h} \cdot \psi_{h}+x_{w} \cdot \psi_{w}
$$

where

$$
\begin{gathered}
m_{1}=\frac{0.94}{\cos \left(\varphi_{h}\right)}+x_{h}\left(\frac{\sigma_{d, h}}{\sigma_{d, w}}-1\right) \cdot\left(0.96 \cos \left(\varphi_{h}\right)+0.088 m_{h} \frac{\sigma_{d, w}}{\sigma_{d, h}} \frac{E_{h}}{E_{w}}\right) \\
m_{2}=\max \left(\frac{2-m_{1} \cdot\left(\frac{\sigma_{d, w}}{\sigma_{0}}\right)_{c o r}}{\left(\frac{\sigma_{d, w}}{\sigma_{0}}\right)_{c r i t}-\left(\frac{\sigma_{d, w}}{\sigma_{0}}\right)_{c o r}} ; 0\right) .
\end{gathered}
$$

If $\sigma_{d, w} / \sigma_{0} \leq\left(\sigma_{d, w} / \sigma_{0}\right)_{c o r}$ (Case 3 of Fig. 6.1) then

$$
\begin{gathered}
\frac{\sigma_{d, e q}}{\sigma_{0}}=m_{1} \cdot \frac{\sigma_{d, w}}{\sigma_{0}}, \\
\varphi_{e q}=\frac{\left(1-e^{-3.5 \cdot \sigma_{s} \cdot\left(\frac{\sigma_{d, w}}{\sigma_{0}}\right)_{\text {cor }} \cdot\left(\left(\frac{\sigma_{d, w}}{\sigma_{0}}\right)_{\text {cor }}-\frac{\sigma_{d, w}}{\sigma_{0}}\right)}\right)}{\left(1-e^{-3.5 \cdot \sigma_{s} \cdot\left(\frac{\sigma_{d, w}}{\sigma_{0}}\right)_{\operatorname{cor}}^{2}} \cdot\left(1-0.6 \cdot \frac{m_{h}}{m_{w}} \cdot e^{-\left(12 \cdot x_{h}+3\right) \cdot E_{w} / E_{e q}}\right) \cdot\left(x_{h} \cdot \varphi_{h}+x_{w} \cdot \varphi_{w}\right),\right.} \\
\psi_{e q}=x_{h} \cdot \psi_{h}+x_{w} \cdot \psi_{w},
\end{gathered}
$$

where

$$
\sigma_{s}=\left(x_{h} \cdot \frac{\sigma_{d, h}}{\sigma_{d, w}}+x_{w}\right)
$$

\subsection{Accuracy of the proposed simplified model}

Note, that in certain cases, particularly if $E_{h} / E_{w}$ and $\sigma_{d, w} / \sigma_{d, h}$ are high and $\sigma_{d, w} / \sigma_{0}$ small, the middle section of the GRC cannot be captured perfectly (error up to $20 \%$ ) by using the 
isotropic model, no matter how well its parameters are chosen (compare green line with black markers in the I.h.s. diagram of Fig. 6.2).

However, the proposed equations for $\sigma_{d, e d} / \sigma_{0}, \varphi_{\text {eq }}$ and $\psi_{\text {eq }}$ reproduce well the computational results (compare red lines with black markers in Fig. 6.1). Generally, for $\varphi_{h}=\varphi_{w}$, the error is less than $10 \%$.

The equations of Section 6.3 provide in general satisfactory results also for $\varphi_{w}<\varphi_{h}$, but may result (particularly the equations for $\varphi_{e q}$ and $\psi_{e q}$; Eqs. 6.18 to 6.26) in an overestimation of the displacements (by maximum $50 \%$ ) in the range of $\sigma_{a} / \sigma_{0}=0.1-0.2$, especially if $E_{h} / E_{w}$ and $\sigma_{d, h} / \sigma_{d, w}$ are high and $\sigma_{d, w} / \sigma_{0}$ small (compare red line with black markers in the r.h.s. diagram of Fig. 6.2).

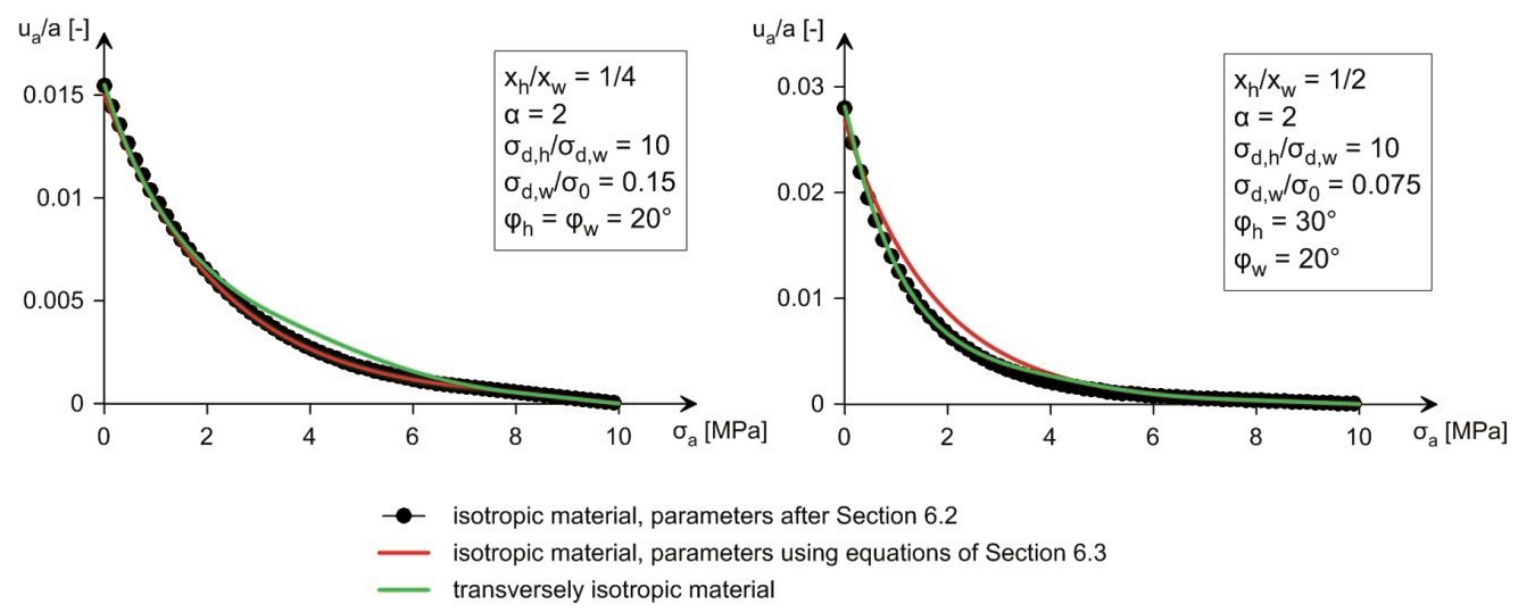

Figure 6.2. Ground response curves for $\sigma_{0}=10 \mathrm{MPa}$ and $E_{\mathrm{w}}=1 \mathrm{MPa}$ (all other rock parameters: see labels inside the diagrams).

$$
\varphi_{\mathrm{h}}=30^{\circ}, \varphi_{\mathrm{w}}=20^{\circ}
$$

(a)

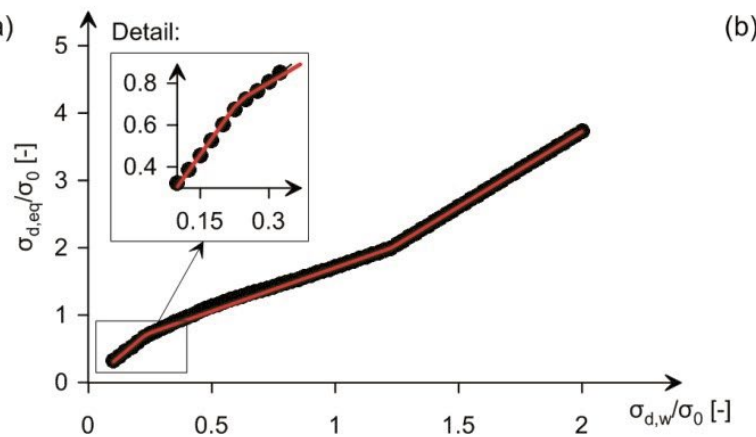

(b)

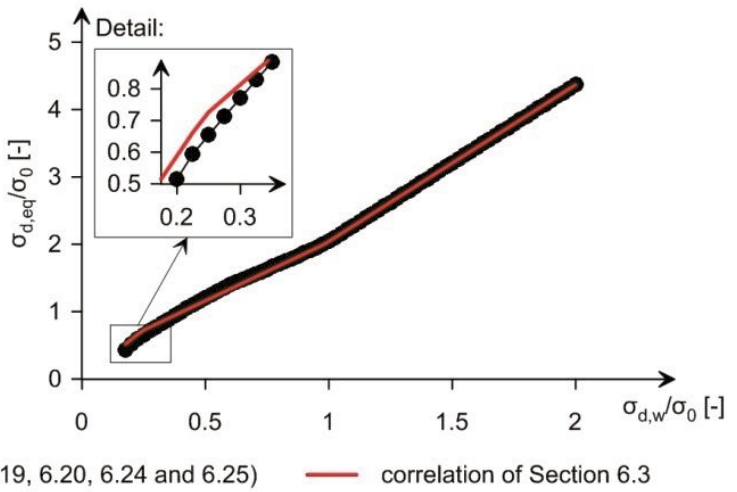

Figure 6.3. Equivalent parameters $\sigma_{d, \mathrm{eq}} / \sigma_{0}\left(x_{h} / x_{w}=1 / 4, \alpha=1, \sigma_{d, h} / \sigma_{d, w}=10\right)$.

Given $\varphi_{\text {eq }}$ and $\psi_{\text {eq }}$ according to Equations (6.19), (6.20), (6.24) and (6.25), the value of $\sigma_{d, e q} / \sigma_{0}$ that results from Equations (6.18) and (6.23) deviates by maximum $20 \%$ from the value that would reproduce the correct displacement at $\sigma_{a} / \sigma_{0}=0$ (compare red line with black markers in Fig. 6.3). Curve fitting by the proposed equations is significantly better at higher $\varphi_{\mathrm{h}}$ values (compare detail in Fig. 6.3a with detail in Fig. 6.3b). The maximum error occurs again for high $E_{h} / E_{w}$ and $\sigma_{d, w} / \sigma_{d, h}$ values in combination with low $\sigma_{d, w} / \sigma_{0}$ values. This is particularly problematic, as especially under a high initial stress $\sigma_{0}$, a small error in $\sigma_{d \text {, eq }}$ can 
produce a considerable error in the displacements. However, considering the usual uncertainties with respect to the rock parameters, a threshold error of $20 \%$ (Fig. $6.3 \mathrm{~b}$ ) can be considered as acceptable.

\subsection{Application examples}

The usefulness and accuracy of the proposed simplified equivalent isotropic model will be illustrated by means of four tunnelling problems (considering an initial hydrostatic stress field of $10 \mathrm{MPa}$ ): (1) The ground response curve of a cylindrical tunnel (shown for evaluating the accuracy of the equivalent parameters; Fig. 6.4); (2) The longitudinal displacement profile of an unsupported tunnel (Fig. 6.5); (3) The boundary displacements of an unsupported tunnel with a horseshoe profile assuming plane strain conditions (Fig. 6.6); (4) The ground pressure $p$ developing upon the shield and the lining in shielded TBM tunnelling (Fig. 6.7).

The exact solutions were obtained either analytically (for problem 1) or numerically (for the problems 2 to 4 ) using the Abaqus finite element code, where the transversely isotropic material model was implemented (see Chapter 9). All the numerical details for problem 2 can be found in Chapter 12; Fig. 6.5). Problem 4 was solved by modelling tunnel excavation and support installation step-by-step; further numerical details can be found in Ramoni and Anagnostou 2010b, as well as in Chapter 14; Fig. 6.7). Table 6.2 shows the assumed parameters of the weak and of the hard layers as well as the parameters of the equivalent isotropic material (calculated after Section 6.3).

Table 6.2. Rock parameters and equivalent parameters.

\begin{tabular}{cccccccccccccccc}
\hline$x_{h} / x_{w}$ & $\begin{array}{c}E_{h} \\
{[-]}\end{array}$ & $v_{h}$ & $\varphi_{h}$ & $c_{h}$ & $\psi_{h}$ & $E_{w}$ & $v_{w}$ & $\varphi_{w}$ & $c_{w}$ & $\psi_{w}$ & $E_{\text {eq }}$ & $v_{\text {eq }}$ & $\varphi_{\text {eq }}$ & $c_{\text {eq }}$ & $\psi_{\text {eq }}$ \\
$1 / 8$ & 6 & 0.3 & 20 & 2.626 & 1 & 1 & 0.3 & 20 & 0.438 & 1 & 1.536 & 0.28 & 18.6 & 0.719 & 1 \\
\hline
\end{tabular}

The comparative computations show that the simplified isotropic model with the parameters after Section 6.3 is sufficiently accurate even for problems that do not meet rotational symmetry and plane strain conditions. The error amounts to less than $10 \%$ and is due to the out-of-plane plastic flow, which was not considered in the determination of the parameters of the equivalent isotropic material. (The out-of-plane is negligible in this case, as $\varphi_{h}=\varphi_{w}$; see Section 5.2.6).

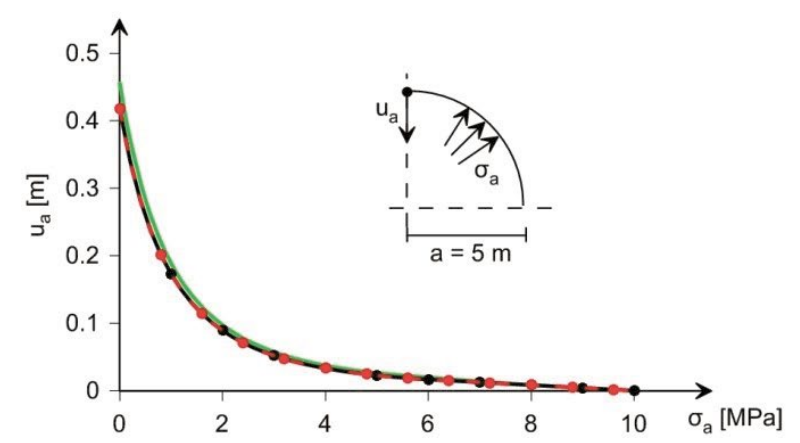

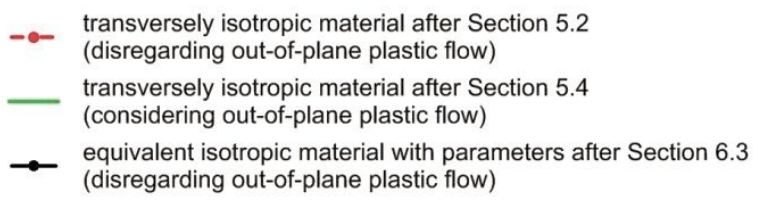

Figure 6.4. GRC of a cylindrical tunnel. 


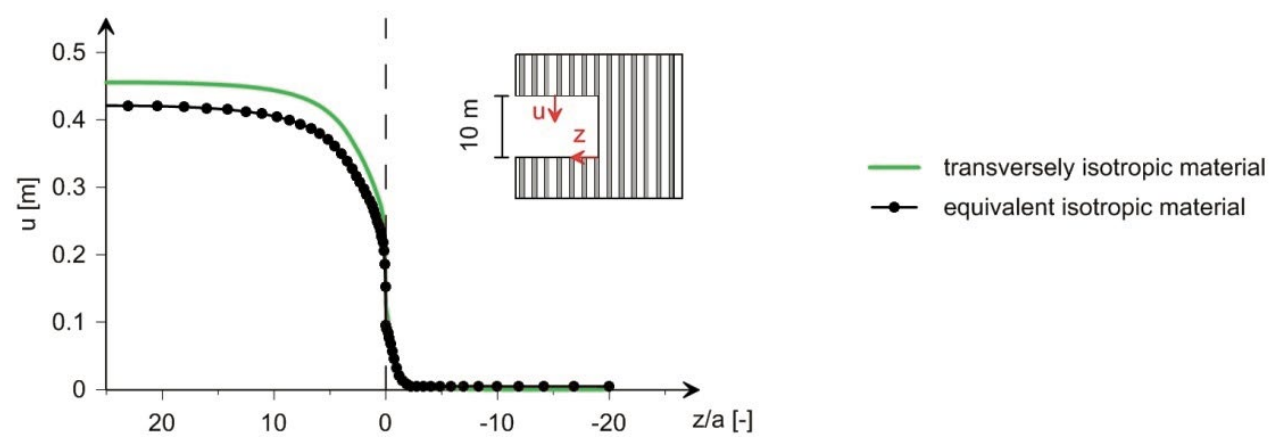

Figure 6.5. Longitudinal displacement profile of an unsupported tunnel.

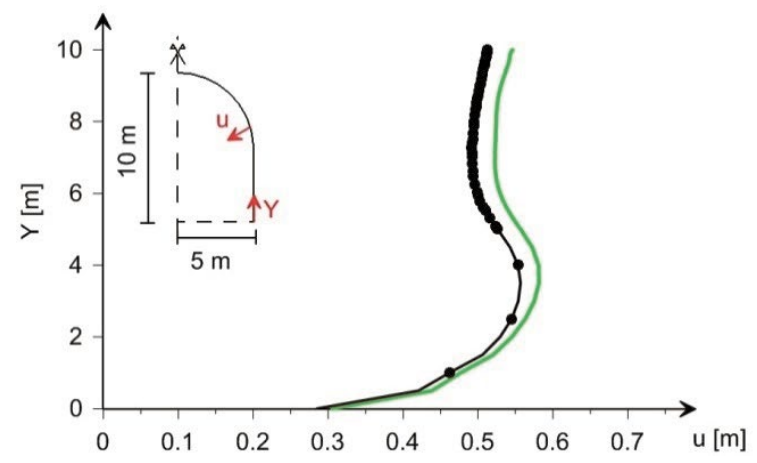

transversely isotropic material

$\rightarrow$ equivalent isotropic material

Figure 6.6. Magnitude of the displacement vector along the unsupported tunnel boundary of a horseshoe profile.

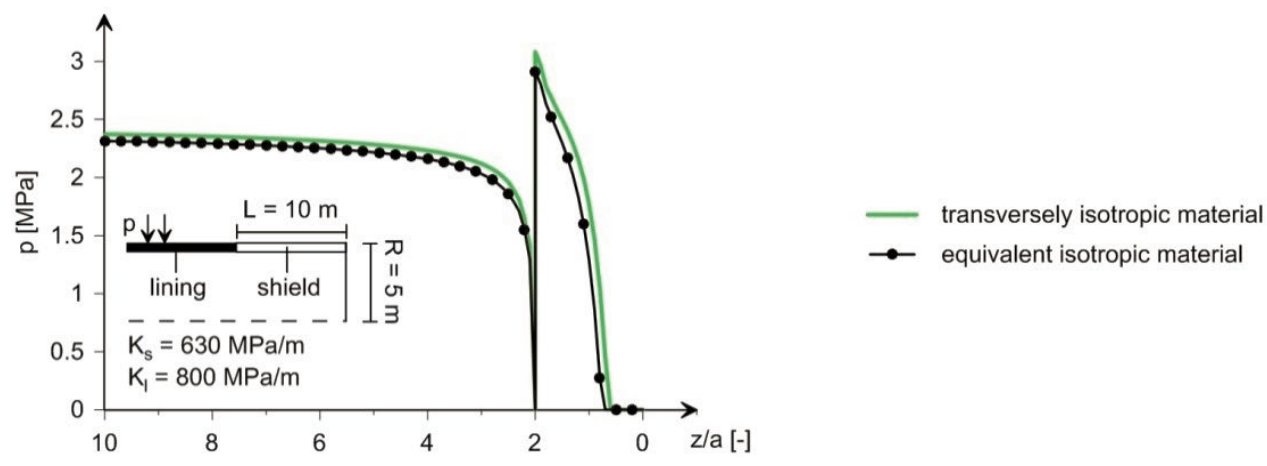

Figure 6.7. Ground pressure $p$ acting upon the shield and the lining in TBM-tunnelling.

\subsection{Closing remarks}

Section 6.2 explained how the analytical solutions of Chapter 5 for the ground response curve can be used in order to determine the parameters of an equivalent homogeneous, isotropic and elasto-plastic material for given parameters of the weak and the hard layers of a thinly stratified rock mass. This is very useful for design purposes since it allows the use of common calculation methods and programs to solve problems that do not meet the conditions of rotational symmetry or plane strain.

Using the method of Section 6.2, relationships between the parameters of the equivalent isotropic model and those of the rigorous transversely isotropic model were developed in Section 6.3. These equations were found by applying the closed-form solution of Section 5.2, which disregards out-of-plane plastic flow. Therefore, they may lead to an underestimation of the rock displacements and pressures. In most cases the effect of out-of-plane plastic flow is 
negligible. However, if $\varphi_{h}$ is considerably higher than $\varphi_{w}$ and the strength $\sigma_{d, w} / \sigma_{0}$ of the weak layers very low, neglecting the out-of-plane plastic flow may lead to inaccurate results (Section 5.2.6). The effect of the out-of-plane plastic flow can be assessed for given parameters of the hard and of the weak layers using the closed-form solution of Chapter 5 . If this effect is significant, then the parameters of the simplified equivalent isotropic material can be determined with the procedure shown in Section 6.2 .

\section{Notation}

a

$C_{e q}$

$E_{e q}$

$E_{h}, E_{w}$

$f(\ldots)$

$f_{0}(\ldots)$

$f_{l}(\ldots)$

$f_{T 1}(\ldots)$

$F_{1}$

$F_{2}$

$K_{s}$

$K_{l}$

$L$

$m_{1}$

$m_{2}$

$m_{e q}$

$m_{h}, m_{w}$

$m_{\varphi=20^{\circ}}$

$p$

$R$

$u$

$u_{a}$

$u_{w, 2 D}$
$x_{h}, x_{w}$
$z$
$\alpha$
$\alpha_{h}$
$\alpha_{w}$
$\lambda_{e q}$
$\mu_{e q}$
$v_{e q}$
$\lambda_{h}, \lambda_{w}$
$\mu_{h}, \mu_{w}$
$v_{h}, v_{w}$
$\sigma_{0}$

$X_{h}, X_{W}$

Z

$\alpha_{w}$

$\lambda_{\text {eq }}$

$\lambda_{h}, \lambda_{w}$

$\mu_{h}, \mu_{w}$

$\sigma_{0}$ tunnel radius

equivalent cohesion of the simplified isotropic rock mass

equivalent Young's modulus of the simplified isotropic rock mass

Young's modulus of the hard and of the weak layers

function of ...

function of ...

function of ...

function of ....

value for the determination of the equivalent parameters

value for the determination of the equivalent parameters

stiffness of the shield (cf. Ramoni and Anagnostou 2010b)

stiffness of the lining (cf. Ramoni and Anagnostou 2010b)

length of the shield

value for the determination of the equivalent parameters

value for the determination of the equivalent parameters

equivalent inclination of the failure surface of the simplified isotropic rock mass (function of the friction angle)

inclination of the failure surface of the hard and of the weak layers (function of the friction angle)

inclination of the failure surface for $\varphi=20^{\circ}$

ground pressure acting upon the shield and the lining

boring radius for TBM tunnelling

displacements of the ground

radial displacements of the equivalent continuum (consisting of weak and hard layers) at the tunnel boundary

radial displacements of a very long weak zone at the tunnel boundary thickness fraction of the hard and of the weak layers

axial co-ordinate (distance behind the tunnel face)

ratio between $E_{h} / E_{w}$ and $\sigma_{d, h} / \sigma_{d, w}$

ratio between the Young's modulus $E_{h}$ and the uniaxial compressive strength of the hard layers $\sigma_{d, h}$

ratio between the Young's modulus $E_{w}$ and the uniaxial compressive strength of the hard layers $\sigma_{d, w}$

Lamé constant of the equivalent continuum

Lamé constant of the equivalent continuum

equivalent Poisson's ratio of the simplified isotropic rock mass

Lamé constant of the hard and of the weak layers

Lamé constant of the hard and of the weak layers

Poisson's ratio of the hard and of the weak layers

initial stress 
$\sigma_{a}$

$\sigma_{d, e q}$

$\sigma_{d, h}, \sigma_{d, w}$

$\left(\sigma_{d, w} / \sigma_{0}\right)_{c o r}$

$\left(\sigma_{d, w} / \sigma_{0}\right)_{c r i t}$

$\sigma_{s}$

$\sigma_{\rho}$

$\varphi_{\text {eq }}$

$\varphi_{\text {eq, max }}$

$\varphi_{h}, \varphi_{w}$

$\psi_{\text {eq }}$

$\psi_{h}, \psi_{w}$ support pressure of the tunnel

equivalent uniaxial compressive strength of the simplified isotropic rock mass

uniaxial compressive strength of the hard and of the weak layers

characteristic value for the determination of the equivalent parameters

characteristic value for the determination of the equivalent parameters

value for the determination of the equivalent parameters

minimum support pressure for which the rock mass remains elastic

equivalent friction angle of the simplified isotropic rock mass

value for the determination of the equivalent parameters

friction angle of the hard and of the weak layers

equivalent dilatancy angle of the simplified isotropic rock mass

dilatancy angle of the hard and of the weak layers 


\section{Influence of the heterogeneity scale on the squeezing variability along the tunnel}

\subsection{Introduction}

Rock mass heterogeneity may lead - depending on the heterogeneity scale (Anagnostou et al. 2014) - to significant variations in the squeezing intensity during tunnelling (see Chapter 4). Section 7.2 investigates how the heterogeneity scale (represented here by the normalised thickness w/a of the weak layers) affects the longitudinal distribution of the displacements in tunnelling perpendicularly to a sequence of weak and hard layers of finite thickness. With the exception of extremely wide weak zones (for which known closed-form solutions can be used) or thinly stratified rocks consisting of weak and hard interlayers (for which the analytical solution of Chapter 5 applies), the displacements can be determined only by means of numerical computations. However, as shown in Section 7.3, one can obtain a reasonably accurate estimation of the displacements in weak zones also analytically, by means of a simple equation.

\subsection{Longitudinal distribution of the displacements}

Figure 7.1 shows the maximum $u_{\max }$ and the minimum displacements $u_{\min }$ (occurring in the middle of the weak and of the hard layer, respectively) as a function of the layer thicknesses $w / a$ for various thickness ratios $h / w$ (computational model as in Section 5.2.6). Figure 7.2 shows the longitudinal displacement distribution for a fixed thickness ratio $h / w=0.5$ and various w/a-values.

The computational results allow drawing the following conclusions:

- There is clearly an interaction between the hard and the weak layers, since the displacement in the cross-section in the middle of a weak layer $\left(u_{\max }\right)$ or of a hard layer $\left(u_{\min }\right)$ may be bigger or smaller depending on the distance between these cross-sections and the layer interface (i.e. depending on w/a and $h / a$, respectively). The hard layers have a stabilising effect on the weak layers and, vice versa, the latter have a destabilising effect on the hard layers.

- The interaction between the layers is maximum for $w / a \rightarrow 0$ (and arbitrary $h / w$ ), i.e., when the layers are very thin in relation to the tunnel radius. In this case, the displacements are practically uniformly distributed and can be obtained by means of the closed-form solution of Chapter 5 .

- With increasing layer thicknesses (i.e., for increasing w/a- and arbitrary $h / w$-values), the interaction between the layers (the stabilising effect of the hard layers and the 
destabilising effect of the weak layers) becomes less and less pronounced. The displacement $u_{\max }$ in the middle of the weak layer increases, while the displacement $u_{\min }$ in the middle of the hard layer decreases (see also Fig. 7.2). The squeezing intensity varies along the tunnel within a scale of a few metres (cf. Cantieni and Anagnostou 2007).

- For w/a $\rightarrow \infty$ (which practically means for w/a $>16$ in the example of Fig. 7.1), the displacements in the middle of the weak and of the hard layer approach the displacements $u_{w, 2 D}$ and $u_{h, 2 D}$, respectively, obtained assuming plane strain conditions with the parameters of the weak and of the hard layers, respectively; the interaction between the layers is limited to the vicinity of their interface (Fig. 7.2).

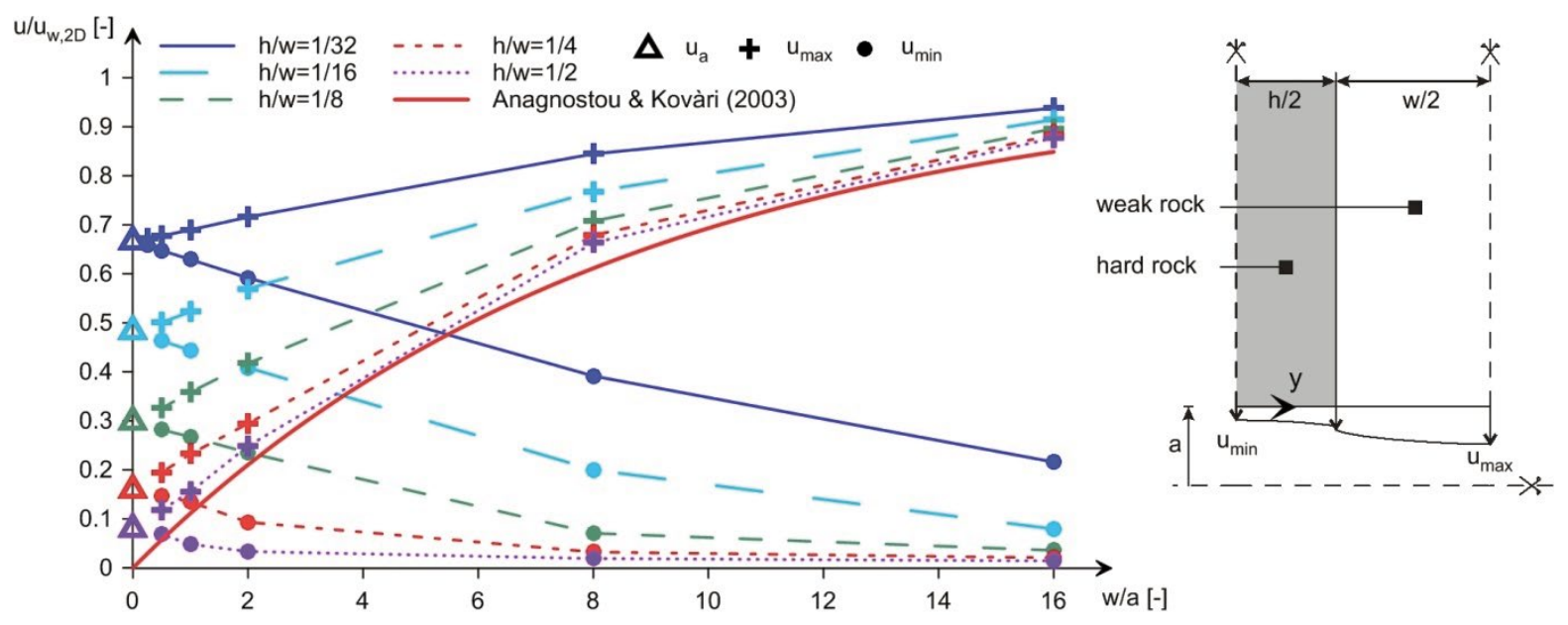

Figure 7.1. Normalised displacement in the middle of the weak layer $\left(u_{\max }\right)$ and of the hard layer ( $u_{\min }$; unsupported tunnel, $a=4 \mathrm{~m}, \sigma_{0}=10 \mathrm{MPa}, E_{h}=10 \mathrm{GPa}, c_{h}=5 \mathrm{MPa}, E_{w}=1 \mathrm{GPa}, c_{w}=0.5 \mathrm{MPa}$, $\left.v_{h}=v_{w}=0.3, \varphi_{h}=\varphi_{w}=25^{\circ}, \psi_{h}=\psi_{w}=5^{\circ}\right)$.

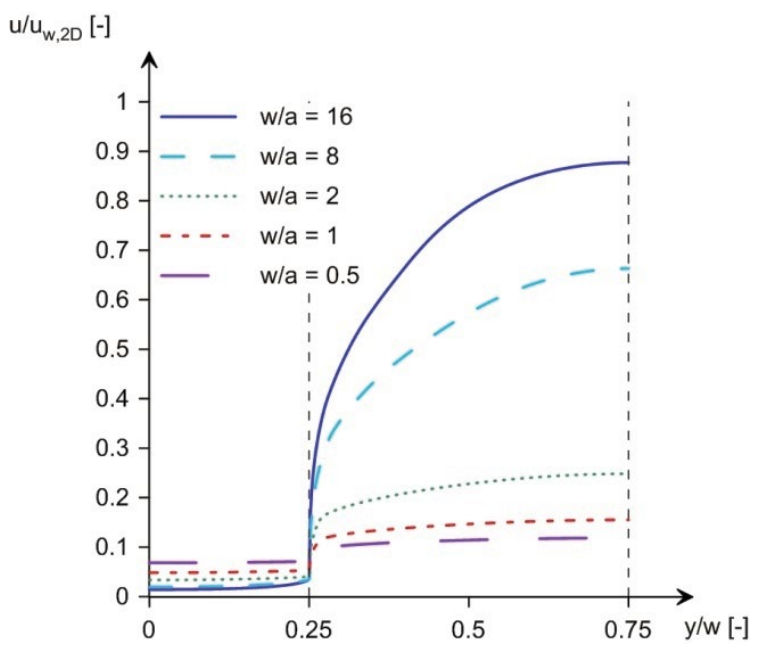

Figure 7.2. Longitudinal displacement distribution for $h / w=1 / 2$ (unsupported tunnel, $a=4 \mathrm{~m}$, $\sigma_{0}=10 \mathrm{MPa}, E_{h}=10 \mathrm{GPa}, c_{h}=5 \mathrm{MPa}, E_{w}=1 \mathrm{GPa}, c_{w}=0.5 \mathrm{MPa}, v_{h}=v_{w}=0.3, \varphi_{h}=\varphi_{w}=25^{\circ}$, $\left.\boldsymbol{\psi}_{h}=\boldsymbol{\psi}_{w}=5^{\circ}\right)$.

Similar remarks apply to the case of hard layers that exhibit brittle behaviour, the only difference being that the stabilising effect of the hard layers is smaller if they become overstressed and experience brittle failure. The latter was already shown in Section 5.5.2 for a thinly stratified rock mass and is also true for layers of finite thickness. Figure 7.3 compares the results obtained by numerical computations (FLAC analyses as in Section 5.3.2) under 
the assumption of brittle behaviour of the hard layers (red lines) with those obtained assuming perfectly plastic behaviour (black lines). The effect of brittle behaviour is evident. It results in larger displacements (both in the hard and in the weak layers) particularly for small ratios $w / a$ and $h / w$, where the hard layers are heavily loaded (cf. Cantieni and Anagnostou 2007).

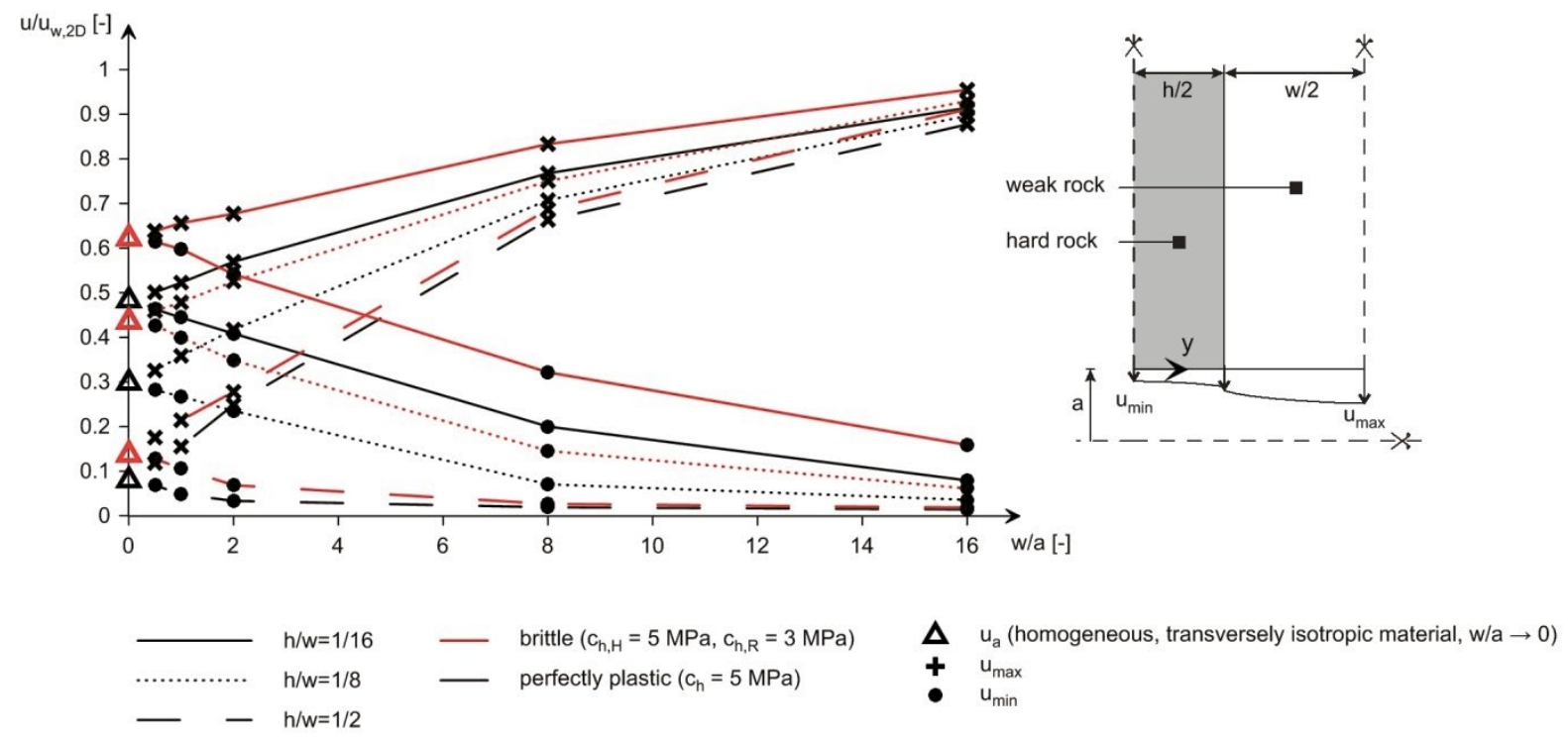

Figure 7.3. Normalised displacements in the weak $\left(u_{\max }\right)$ and in the hard $\left(u_{\min }\right)$ layers as a function of the normalised thickness w/a for perfectly plastic or brittle behaviour of the hard layers (unsupported tunnel, $a=4 \mathrm{~m}, \sigma_{0}=10 \mathrm{MPa}, E_{h}=10 \mathrm{GPa}, E_{w}=1 \mathrm{GPa}, c_{w}=0.5 \mathrm{MPa}$, $\left.v_{h}=v_{w}=0.3, \varphi_{h}=\varphi_{w}=25^{\circ}, \psi_{h}=\psi_{w}=5^{\circ}\right)$.

\subsection{A simple equation for the displacements in a weak zone considering a wall-effect}

Anagnostou and Kovári (2003) proposed the following equation for estimating the displacements $u_{\max }$ in a weak zone of limited length $w$ bounded by competent rock:

$$
u_{\max }=\delta u_{w, 2 D}
$$

where $u_{w, 2 D}$ denotes the displacement that would occur in an infinitely long weak zone while $\delta$ represents a reduction factor $(0<\delta<1)$ that accounts for the stabilising effect of the adjacent competent rock:

$$
\delta=1-e^{-0.4 w / \rho_{w, 2 D}}
$$

where $\rho_{w, 2 D}$ denotes the radius of the plastic zone in the case of an infinitely long weak zone. As both $u_{w, 2 D}$ and $\rho_{w, 2 D}$ can be computed applying the commonly used closed-form GRC solution, the equations above allow a quick estimation of the wall-effect without performing numerical analyses.

The equation for the reduction factor $\delta$ was found by analysing the results of a parametric study, which was carried out assuming that the competent rock is rigid. Due to this assumption, the equation of Anagnostou and Kovári (2003), in general, overestimates the wall-effect and underestimates the displacements in the weak zone, particularly if the latter is bounded by relatively thin layers of competent rock. In the numerical example of Figure 7.1, 
the equation of Anagnostou and Kovári (2003) (red solid line) underestimates the displacements considerably if $h / w<0.5$.

In the present thesis, it was investigated whether the equation of Anagnostou and Kovári (2003) can be modified, so that it includes the effects of deformation and failure of the competent rock. Intuitively, by studying numerical results as those of Figure 7.1 and by considering some important conditions (given below), the following simple equation was constructed:

$$
\frac{u_{\max }}{u_{w, 2 D}}=\delta \cdot\left(1-\frac{u_{a}}{u_{w, 2 D}}\right)+\frac{u_{a}}{u_{w, 2 D}}
$$

where $u_{a}$ is the displacement that would occur in the case of a thinly stratified rock mass (i.e., for $w / a \rightarrow 0$ and arbitrary $h / w$ ) and can be computed analytically after Chapter 5 . The modified equation fulfils the following three conditions:

- $u_{\max } \rightarrow u_{a}$ for $w / a \rightarrow 0$ (note that $\delta \rightarrow 0$ in this case; Eq. 7.2);

- $u_{\max } \rightarrow u_{w, 2 D}$ for $w / a \rightarrow \infty$ (note that $\delta \rightarrow 1$ in this case; Eq. 7.2);

- $u_{\max }=\delta \cdot u_{w, 2 D}$ (i.e., equation of Anagnostou and Kovári 2003) if the competent rock is rigid (note that $u_{a}=0$ in this case).

Figure 7.4 plots the displacements after Equation (7.3) and the exact ones obtained from the numerical calculations as a function of $w / a$. For this specific parameter set, the equation is reasonably accurate.

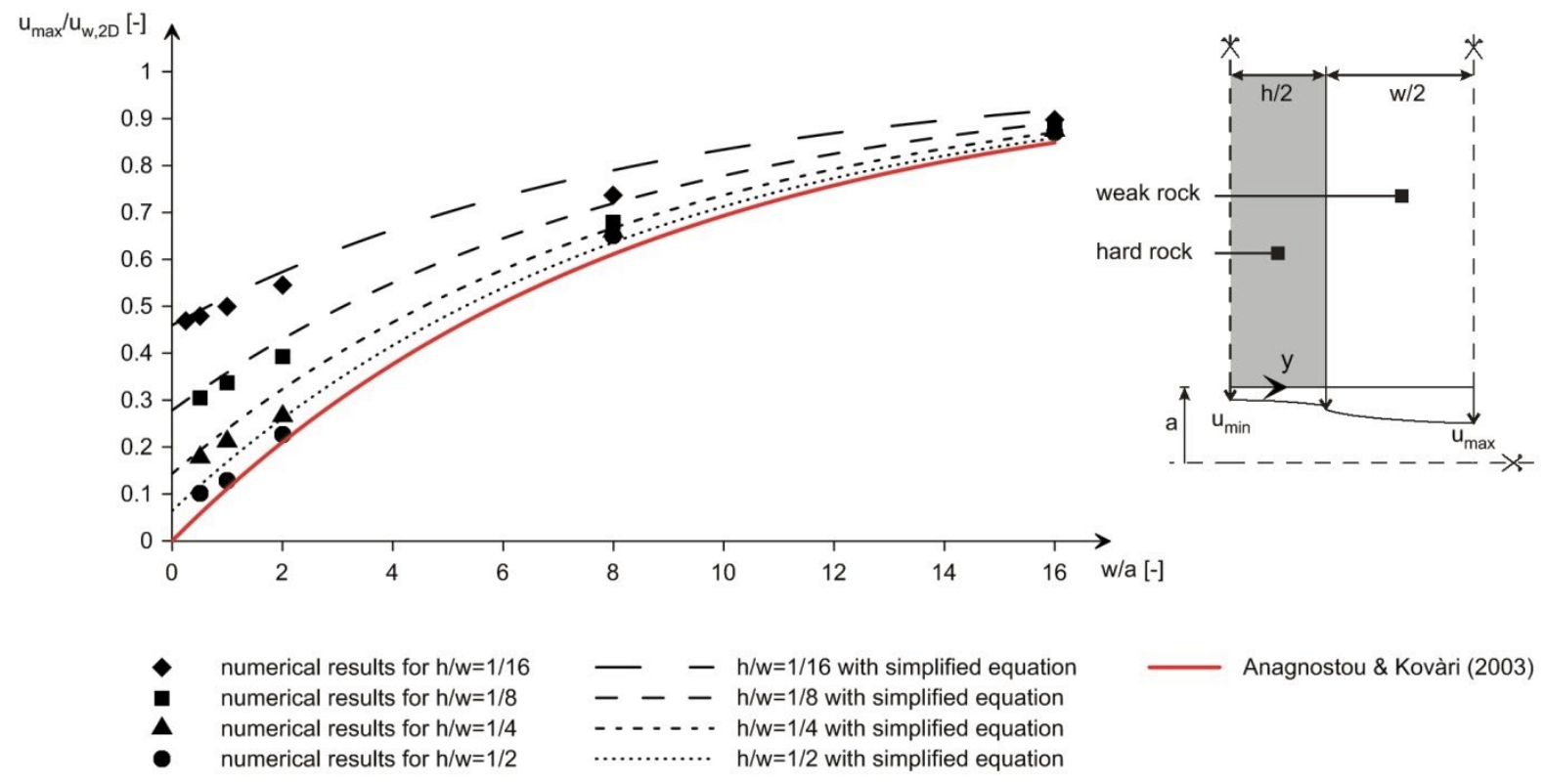

Figure 7.4. Accuracy of the simplified Equation (7.3) for perfectly plastic behaviour of the hard layers: Normalised displacement $u_{\max }$ as a function of the normalised weak layer thickness (unsupported tunnel, $a=4 \mathrm{~m}, \sigma_{0}=10 \mathrm{MPa}, E_{h}=10 \mathrm{GPa}, c_{h}=5 \mathrm{MPa}, E_{w}=1 \mathrm{GPa}, c_{w}=0.5 \mathrm{MPa}$, $\left.v_{h}=v_{w}=0.3, \varphi_{h}=\varphi_{w}=25^{\circ}, \psi_{h}=\psi_{w}=5^{\circ}\right)$.

The accuracy of the proposed equation was further checked by means of a comprehensive parametric study considering the significant parameters of the problem (disregarding brittle behaviour of the hard layers): 


$$
\frac{u_{\max }}{a}=f\left(\frac{h}{w}, \frac{w}{a}, \frac{\sigma_{a}}{\sigma_{0}}, \frac{\sigma_{d, w}}{\sigma_{0}}, \frac{\sigma_{d, h}}{\sigma_{d, w}}, \frac{E_{h}}{E_{w}}, v_{h}, \varphi_{h}, \psi_{h}, v_{w}, \varphi_{w}, \psi_{w}\right) \text {, }
$$

with the values after Table 7.1. Figure 7.5 plots the displacements after Equation (7.3) as a function of the exact ones and shows that the equation is reasonably accurate.

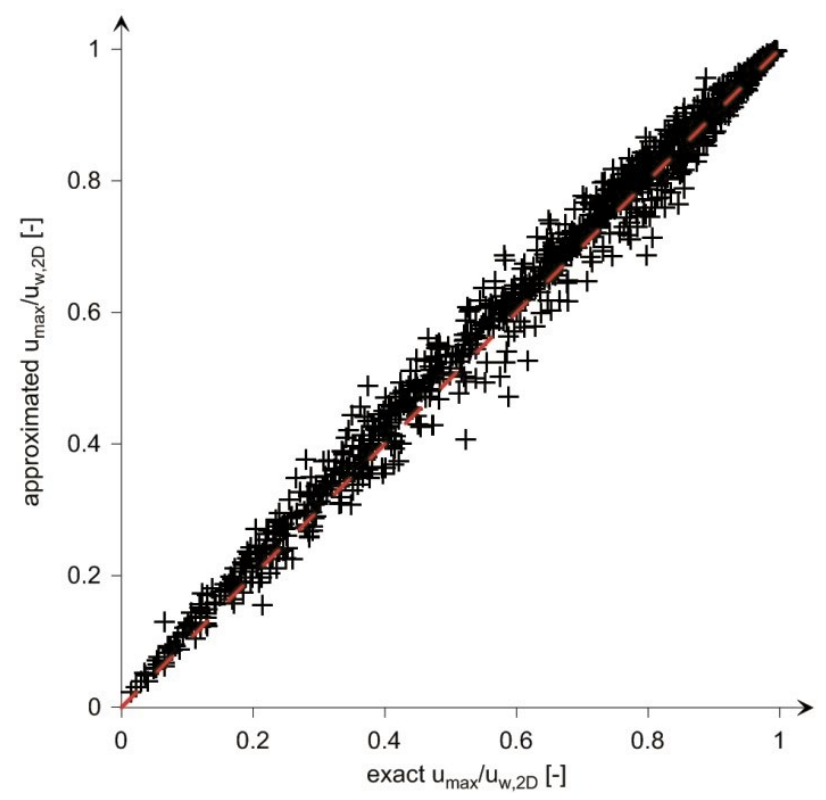

Figure 7.5. Accuracy of the simplified Equation (7.3): Normalised (approximated) displacement after Equation (7.3) versus exact normalised displacement.

Equation (7.3) was not checked comprehensively for the case of brittle behaviour of the hard layers, but holds probably also for this case, as indicated by the computational example of Figure 7.6.

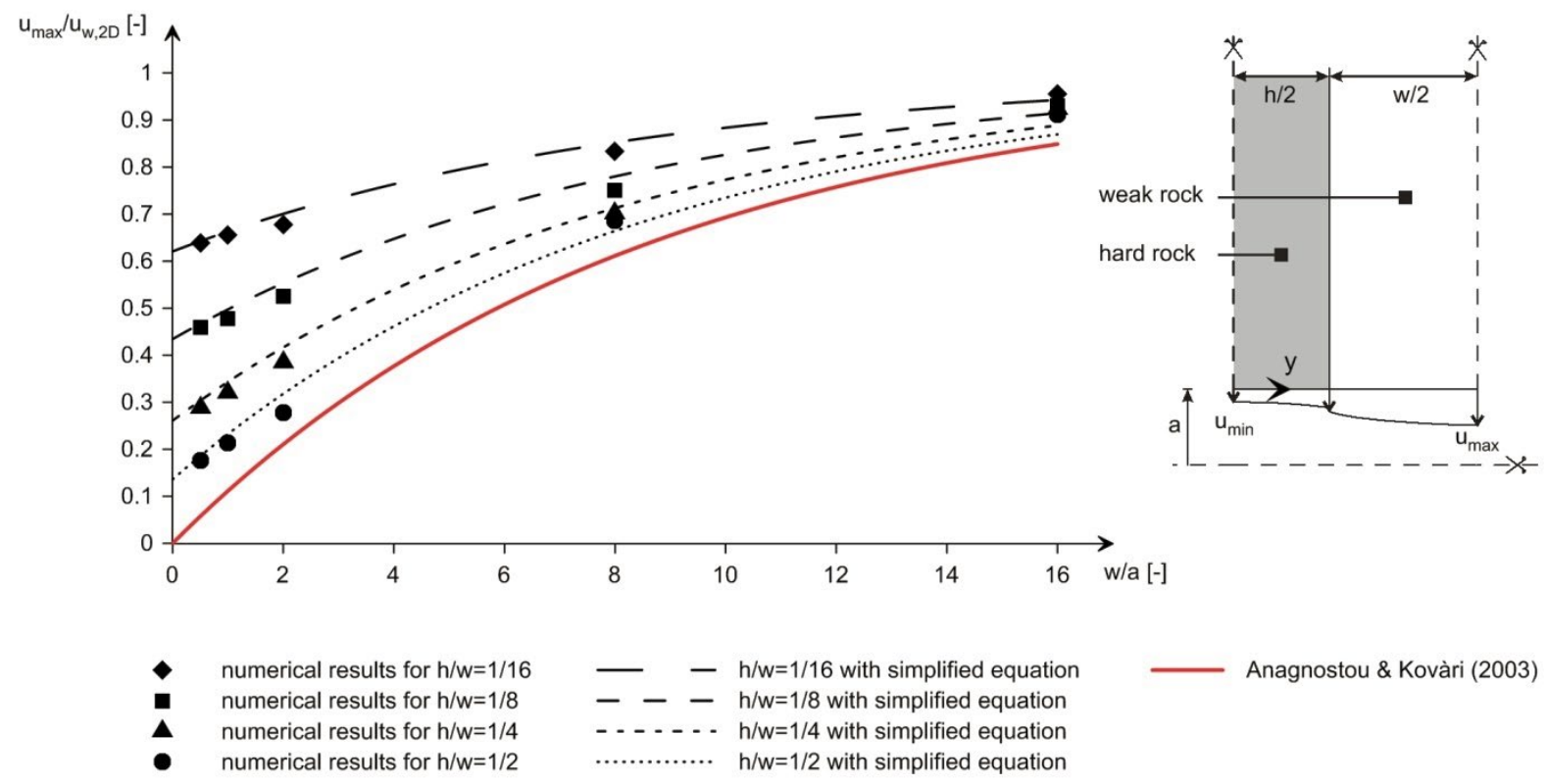

Figure 7.6. Accuracy of the simplified Equation (7.3) for brittle behaviour of the hard layers: Normalised displacement $u_{\max }$ as a function of the normalised weak layer thickness (unsupported tunnel, a $=4 \mathrm{~m}, \sigma_{0}=10 \mathrm{MPa}, E_{h}=10 \mathrm{GPa}, c_{h, H}=5 \mathrm{MPa}, c_{h, R}=3 \mathrm{MPa}, E_{w}=1 \mathrm{GPa}, c_{w}=0.5 \mathrm{MPa}$, $v_{h}=v_{w}=0.3, \varphi_{h}=\varphi_{w}=25^{\circ}, \psi_{h}=\psi_{w}=5^{\circ}$ ). 
Table 7.1. Parameter range considered for checking accuracy of Equation (7.3).

\begin{tabular}{|c|c|c|c|}
\hline$h / w[-]$ & \multicolumn{3}{|c|}{$1 / 2 ; 1 / 8 ; 1 / 32$} \\
\hline w/a [-] & \multicolumn{3}{|c|}{$0.5 ; 1 ; 2 ; 8 ; 16$} \\
\hline$\sigma_{a} / \sigma_{0}[-]$ & \multicolumn{3}{|c|}{0} \\
\hline$\sigma_{d, w} / \sigma_{0}[-]$ & \multicolumn{3}{|c|}{$0.15 ; 0.3 ; 0.45$} \\
\hline$\sigma_{d, h} / \sigma_{d, w}[-]$ & 2 & 6 & 10 \\
\hline$E_{h} / E_{w}[-]$ & $1 ; 4$ & $3 ; 12$ & $5 ; 20$ \\
\hline$v_{h}=v_{w}[-]$ & \multicolumn{3}{|c|}{0.3} \\
\hline$\varphi_{h}\left[{ }^{\circ}\right]$ & \multicolumn{3}{|c|}{$20 ; 30$} \\
\hline$\varphi_{w}\left[^{\circ}\right]$ & \multicolumn{3}{|c|}{$\varphi_{h}-\left(5^{\circ}, 10^{\circ}\right)$} \\
\hline$\psi_{h, w}\left[{ }^{\circ}\right]$ & \multicolumn{3}{|c|}{$\begin{array}{c}\varphi_{h, w}-20^{\circ} \text { for } \varphi_{h, w}>20^{\circ} \\
\text { (cf. Vermeer and de Borst 1984) }\end{array}$} \\
\hline
\end{tabular}

Disregarding the wall-effect introduces an error on the safe side (it overestimates the deformations in the weak zone), which however is small if the weak zone is sufficiently long in relation to tunnel radius. Expressing the error as $\left(u_{w, 2 D}-u_{\max }\right) / u_{\max }$, taking $u_{\max }$ after Equation (7.3) and denoting the acceptable error by $\varepsilon_{t o l}$, the wall-effect can be neglected if the weak zone thickness is:

$$
w>2.5 \rho_{w, 2 D} \ln \left(\frac{\varepsilon_{t o l}+1}{\varepsilon_{t o l}}\left(1-\frac{u_{a}}{u_{w, 2 D}}\right)\right),
$$

where the plastic radius $\rho_{w, 2 D}$ and the radial displacement $u_{w, 2 D}$ depend on the in situ stress $\sigma_{0}$ and on the mechanical parameters of the weak zone and can be computed using the common GRC equations, while the radial displacement $u_{a}$ depends on the in situ stress $\sigma_{0}$, on the thickness ratio $h / w$ and on the mechanical parameters of both layers and can be determined using either the closed-form solution of Chapter 5 or the diagrams of Appendix $B$. Figure 7.7 represents graphically Equation (7.5).

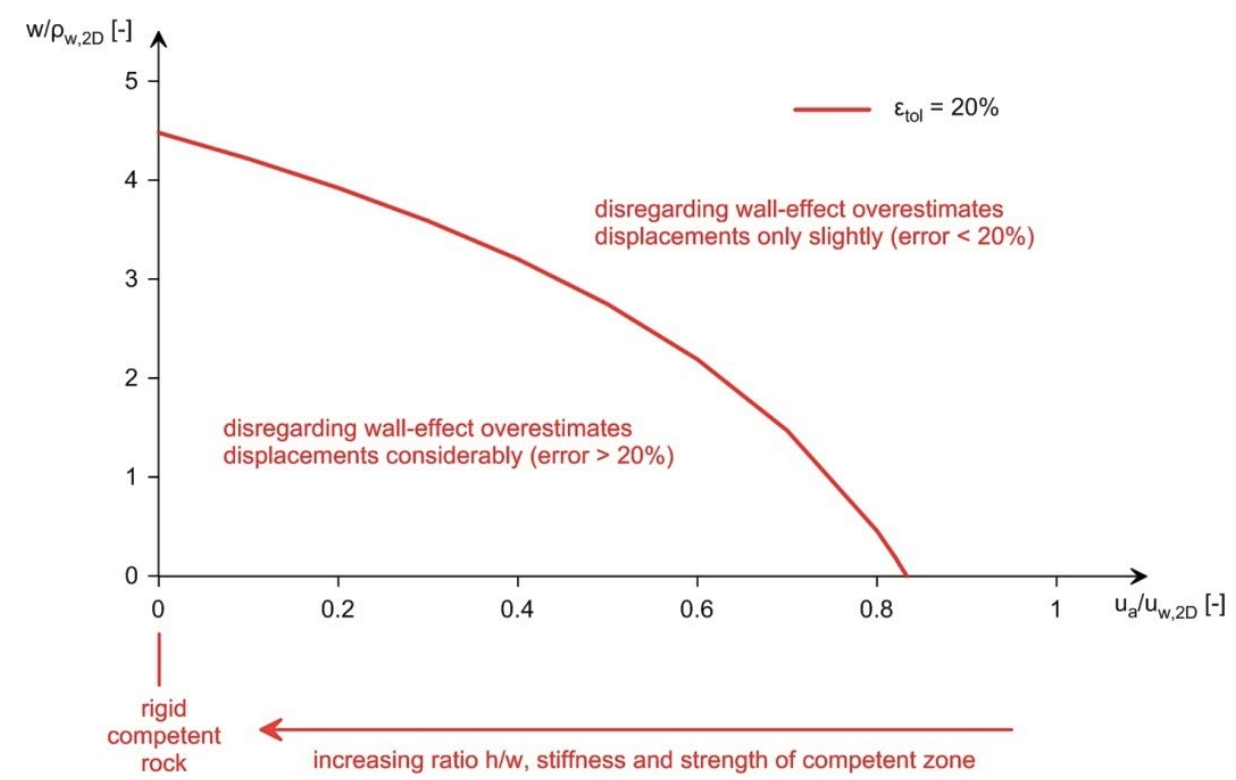

Figure 7.7. Normalised weak zone length for which disregarding wall-effect overestimates displacements by $20 \%$. 


\subsection{Conclusions}

At the current state of research, the displacements in tunnelling perpendicularly to a sequence of weak and hard layers of finite thickness can be determined only by means of numerical computations. For this, in this chapter, a simple analytical equation was developed based on the solution of Anagnostou and Kovári (2003), which makes it possible to estimate the displacements in a weak zone considering the wall-effect of the hard layers and which is accurate enough for practical purposes. This equation seems also to hold for the case of brittle behaviour of the hard layers, where the stabilising effect is less pronounced.

\section{Notation}

$\begin{array}{ll}a & \text { tunnel radius } \\ C_{h}, c_{w} & \text { cohesion of the hard and of the weak layers } \\ c_{h, H}, C_{h, R} & \text { peak and residual (i.e., post-peak) cohesion of the hard layers } \\ E_{h}, E_{w} & \text { Young's modulus of the hard and of the weak layers } \\ h & \text { thickness of the hard layers (see inset of Fig. 7.1) } \\ u_{a} & \text { radial displacements of the homogenised model at the tunnel bound- } \\ & \text { ary } \\ u_{h, 2 D} & \text { radial displacements of a very long competent zone at the tunnel } \\ & \text { boundary } \\ u_{\max }, u_{\min } & \text { maximum and minimum radial displacements at the tunnel boundary } \\ u_{w, 2 D} & \text { (see inset of Fig. 7.1) } \\ w & \text { radial displacements of a very long weak zone at the tunnel boundary } \\ y & \text { thickness of the weak layers (see inset of Fig. 7.1) } \\ \delta & \text { axial co-ordinate (see inset of Fig. 7.1) } \\ \varepsilon_{t o l} & \text { reduction factor } \\ \rho_{w, 2 D} & \text { the acceptable error for disregarding the wall-effect } \\ \sigma_{0} & \text { radius of the plastic zone for a very long weak zone } \\ v_{h,}, v_{w} & \text { initial stress } \\ \varphi_{h,}, \varphi_{w} & \text { Poisson's ratio of the hard and of the weak layers } \\ \psi_{h}, \psi_{w} & \text { friction angle of the hard and of the weak layers } \\ & \text { dilatancy angle of the hard and of the weak layers }\end{array}$





\section{PART III: TUNNELLING PARALLEL TO ALTERNATING WEAK AND COMPETENT ROCK LAYERS}

This part of the thesis investigates the excavation-induced tunnel displacements in a stratified rock mass, consisting of frequently alternating weak and hard layers that strike parallel to the tunnel axis. If the layers are very thick and their interface lies at a great distance to the tunnel, then the displacements of the tunnel profile will be practically uniform and thus the heterogeneity of the ground can be neglected. Otherwise, the convergence of the tunnel profile will be non-uniform and this even if the layers are very thin in relation to the tunnel radius. Hence, attention must be paid to the heterogeneity scale. Rock response is analysed here computationally first by considering an equivalent homogeneous model and subsequently by modelling the individual layers. Furthermore, this part of the thesis investigates the excavation-induced tunnel displacements in schistous rock masses, which can be conceived from the mechanical viewpoint as a special case of a stratified rock mass. 



\section{Introduction}

When tunnelling parallel to alternating hard and weak layers, the tunnel profile may experience non-uniform deformations, but the displacements do not vary along the tunnel axis. Although there is no squeezing variability in tunnelling parallel to the bedding, this case is analysed in this thesis because it represents a borderline case for the general case of an arbitrary orientation of the anisotropy plane, which is studied in Part IV.

(a)

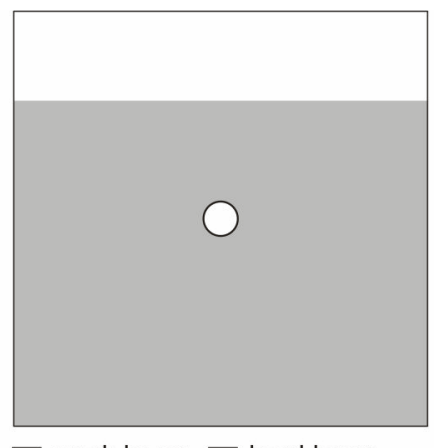

$\square$ weak layer $\square$ hard layer (b)

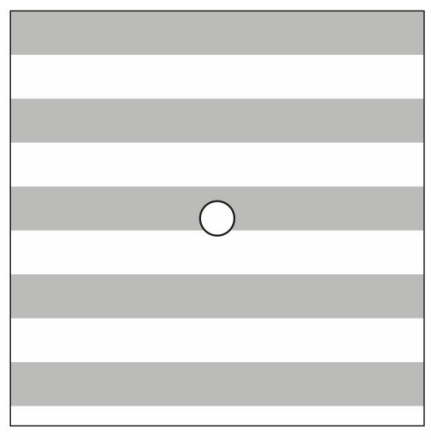

Figure 8.1. Tunnelling through a sequence of, (a), very thick weak and competent rock formations, (b), alternating weak and competent layers of medium thickness, (c), thinly alternating weak and competent layers.

Depending on the heterogeneity scale, the following three cases can be distinguished (cf. Fig. 8.1):

- If the weak (or the hard) layers are very thick and their interface lies far away from the tunnel (Fig. 8.1a), their unfavourable (or stabilising) effect will not be noticeable in the convergence distribution.

- If the alternating weak and hard rock layers are very thin relatively to the tunnel radius (Fig. 8.1c), then the rock mass can be conceived as a homogeneous, but, in view of the rock structure, transversely isotropic material.

- Otherwise (Fig. 8.1b), the heterogeneity of the rock mass has to be considered explicitly in the numerical modelling.

The present part of the thesis is structured as follows:

Chapter 9 deals with the special case of a very thinly stratified rock mass (Fig. 8.1c). The constitutive model of Chapter 5.2 is formulated in 3D and implemented in Abaqus. This model serves to investigate the tunnelling-induced displacements under plane strain conditions and to develop design diagrams that allow to estimate quickly the ground response for a wide range of conditions in terms of the mechanical parameters and the thickness fractions of the weak and hard layers. 
Chapter 10 analyses the effect of the rock structure on squeezing deformations paying attention to the various heterogeneity scales of Figure 8.1. Specifically, numerical (plane strain) calculations are performed, where the individual layers are modelled discretely. The ratio of the thickness of the layers to the tunnel radius is considered as a measure of the heterogeneity scale. The numerical results indicate, (i), under which conditions it is adequate to consider the homogenised model rather than the individual layers (Section 10.2), (ii), in which cases the heterogeneity of the ground can be neglected in the design (Section 10.3), (iii), or must be considered explicitly in numerical modelling (Section 10.4).

Chapter 11 deals with schistous rocks, as they represent, from the mechanical point of view, a special case (and their behaviour exhibits similarities to that) of a stratified rock mass. The excavation-induced displacements are investigated using a constitutive model, which was formulated (using the homogenisation technique) and implemented in Abaqus. For this purpose, design diagrams were also worked out that allow assessing quickly the effect of schistosity on the squeezing deformations. 


\section{Response of a thinly stratified rock mass, striking parallel to tunnel axis}

\subsection{Introduction}

The squeezing behaviour in tunnelling parallel to a sequence of thinly alternating weak and hard rocks has not been analysed so far. This will be done in the present section based upon a homogeneous, transversely isotropic material model. An overview about stratified rock mass and homogenisation techniques can be found in Chapter 5. Contrarily to Chapter 5, however, a closed-form solution cannot be formulated for the GRC, because the problem is not rotationally symmetric. Therefore, the problem will be solved numerically. To this end, a constitutive model was formulated and implemented into the FE-program Abaqus (Dassault Systèmes 2011; Section 9.2) and validated by several examples (Appendix D and Section 9.3). Section 9.3 discusses the tunnelling-induced displacements and stresses by means of computational examples considering a circular tunnel cross-section under plane strain conditions, which corresponds to the situation that prevails in deep tunnels far behind the face. Subsequently, based upon a comprehensive parametric study, dimensionless diagrams are worked out in Section 9.4 that allow depicting the tunnelling-induced displacements for practically arbitrary conditions. These dimensionless diagrams can be used to determine the mechanical parameters of an isotropic homogeneous elasto-plastic material that can be characterised as "equivalent" to the transversely isotropic material in the sense that it experiences the same maximum or minimum displacements (Section 9.5). By using this equivalent isotropic material in combination with existing computational methods or design nomograms (e.g., those of Ramoni and Anagnostou 2011b) one can estimate the range of rock pressures or deformations developing when tunnelling parallel to thinly stratified rocks.

\subsection{Constitutive model}

\subsubsection{Formulation of the constitutive model}

For the formulation of appropriate constitutive equations (accounting for the thickness fractions and mechanical properties of the individual rock layers), homogenisation techniques for a sequence of alternating weak and hard layers have been proposed in the literature, initially considering linearly elastic behaviour (cf., e.g., Salamon 1968, Gerrard 1982) and later on extended for elasto-plastic behaviour of the layers (Elomri and Sidoroff 1991; Pruchnicki and Shahrour 1994; Lourenço 1995) and shear failure along their interfaces (Nejad Ensan and Shahrour 2003). 
The representative elementary volume (REV), which consists of hard and weak layers lying perpendicular to the $y$-axis, considered for the formulation of the constitutive model is presented in Figure 9.1. The constitutive model of the REV is equivalent to the one of Chapter 5, but is formulated for the general 3D case (and not solely for plane strain conditions). An arbitrary bedding orientation can be considered by appropriate coordinate transformations: the stresses and the strains are transformed from the global into the local coordinate system (in which the $y$-axis is perpendicular to the layers) and, after having performed all the computations in the local coordinate system, the resulting stresses are back-calculated into the global coordinate system. The only input needed for the coordinate transformation is the normal vector of the layers.

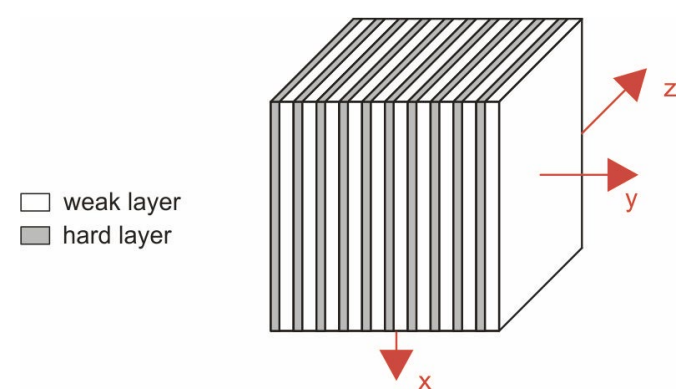

Figure 9.1. REV consisting of a sequence of hard and weak layers.

The model - whose elasto-plastic parameters depend on thickness fraction, strength and stiffness properties of the alternating layers - was formulated and implemented in the finite element code Abaqus (Dassault Systèmes 2011) based upon the general homogenisation procedure of Lourenço (1995), the only difference being that Lourenço (1995) used the von Mises rather than the Mohr-Coulomb yield criterion. Therefore, as usual in design practice, the individual layers are modelled as linearly elastic - perfectly plastic materials obeying Mohr-Coulomb failure criterion with a non-associated flow rule (no tension cut-off, no brittle behaviour), using the stress return algorithm after Clausen (2007), which, of course, considers an out-of-plane plastic flow. Details concerning the formulation of the constitutive model can be found in Appendix D. Furthermore, this appendix contains validation examples.

\subsubsection{Rock element behaviour}

In order to illustrate some basic aspects of the material behaviour and to validate the numerical implementation of the elasto-plastic equations, simple displacement-controlled element tests (considering a single quadratic element, $1 \mathrm{~m} \times 1 \mathrm{~m}$ big, under plane strain conditions) were performed computationally. An uniform displacement $u$ is applied either perpendicularly or parallel to the layers (see insets of Fig. 9.2). For simplicity, the thickness fractions of the hard and weak layers were taken equal to $50 \%$.

The I.h.s. diagrams of Figure 9.2 show the results for the case of a displacement applied in the $y$-direction, i.e. perpendicularly to the layers. The stresses in $y$-direction are the same in the hard and in the weak layers and thus also in the REV (i.e., $\sigma_{y y, h}=\sigma_{y y, w}=\sigma_{y y}$ ). The weak layers experience, due to their lower stiffness, a bigger compressive strain in $y$-direction than the hard layers and, in the absence of the hard layers, would expand also more laterally (in $x$-direction). As they are retained by the hard layers in $x$-direction, compressive stresses develop in the weak layers (i.e., $\sigma_{x x, w}=\sigma_{\max , w}>0$ ) and tensile stresses in the hard layers (i.e., $\sigma_{x x, h}=\sigma_{\max , h}<0$, as $\left.\sigma_{x x}=x_{h} \sigma_{x x, h}+x_{w} \sigma_{x x, w}\right)$. The development of tensile stress in the harder layers was already described by Bourne (2003). It results in a tensile failure (at $\varepsilon_{y y} \approx 0.0037$ 
in the present example) and so in a slight decrease in the stiffness. Subsequently, also the weak layers fail (at $\varepsilon_{y y} \approx 0.004$ ) and the stress remains subsequently constant.
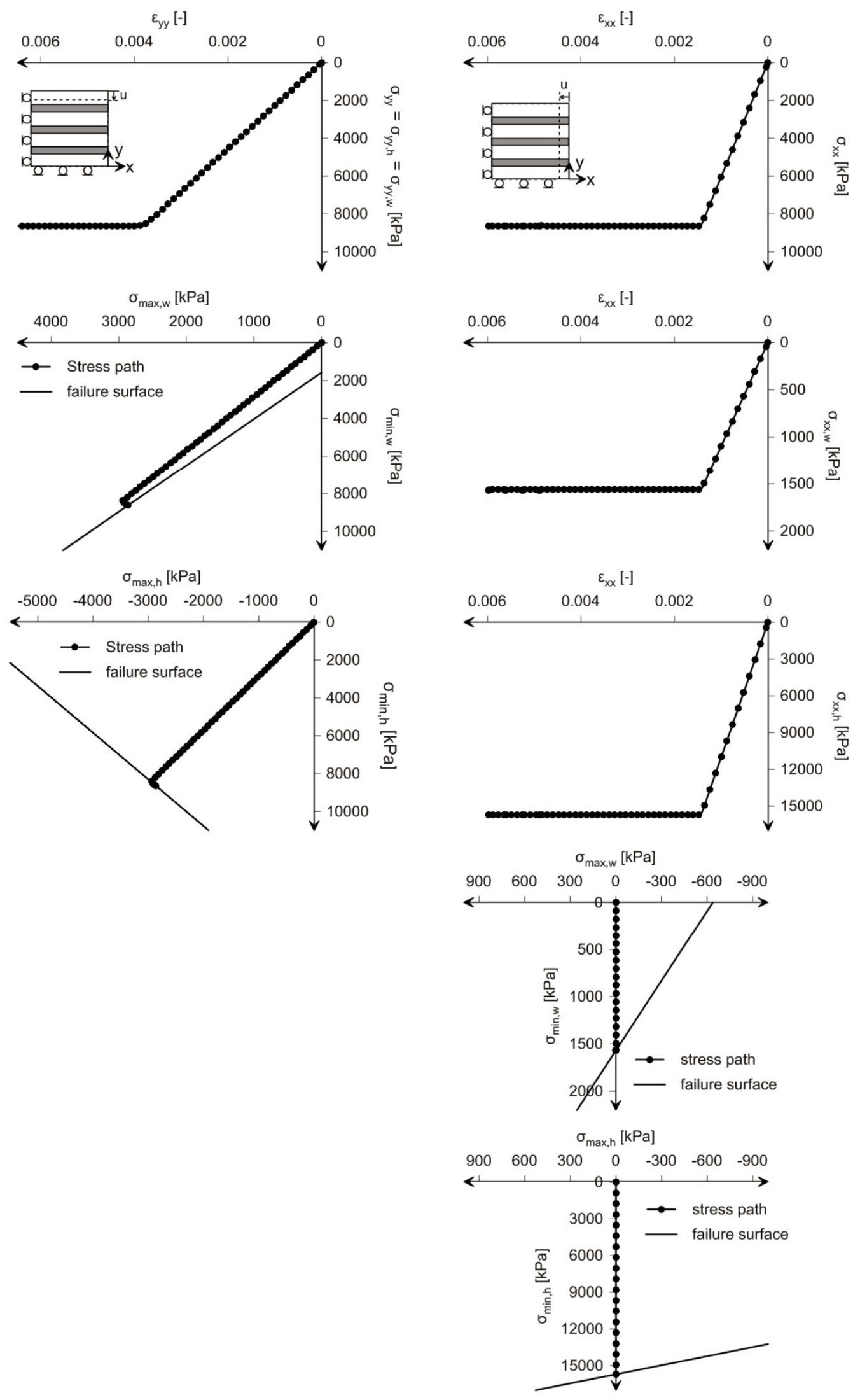

Figure 9.2. Results of the single element tests for displacement application perpendicularly (I.h.s.) or parallel (r.h.s) to the layers $\left(x_{h}=x_{w}=0.5, E_{h}=10 \mathrm{GPa}, c_{h}=5 \mathrm{MPa}, E_{w}=1 \mathrm{GPa}, c_{w}=0.5 \mathrm{MPa}\right.$, $\left.v_{h}=v_{w}=0.3, \varphi_{h}=\varphi_{w}=25^{\circ}, \psi_{h}=\psi_{w}=5^{\circ}\right)$. 
The r.h.s. diagrams of Figure 9.2 show the results for a displacement applied in the $x$ direction, i.e. parallel to the layers. The hard and the weak layers experience the same stresses in $y$-direction but different stresses in the $x$-direction. Expectedly, the stress in the REV corresponds to the volumetric averages of the stresses in the different layers (i.e., $\left.\sigma_{x x}=x_{h} \sigma_{x x, h}+x_{w} \sigma_{x x, w}\right)$. The hard layers are by a factor 10 stiffer than the weak layers and experience, therefore, in the elastic state, a 10 times higher stress in the $x$-direction. As the uniaxial compressive strength of the hard layers was taken 10 times higher than the one of the weak layers, the weak and the hard layers fail at the same time.

Furthermore, the composite behaves expectedly considerably softer when it is loaded perpendicularly to the layers (compare r.h.s with I.h.s curves) and the stiffnesses agree to the analytical predictions. Specifically, Hooke's law for the composite reads as follows (Salamon 1968):

$$
\left(\begin{array}{l}
\varepsilon_{x x} \\
\varepsilon_{y y} \\
\varepsilon_{z z} \\
\gamma_{x y}
\end{array}\right)=\left(\begin{array}{cccc}
\frac{1}{E_{1}} & -\frac{v_{2}}{E_{1}} & -\frac{v_{1}}{E_{1}} & 0 \\
-\frac{v_{2}}{E_{1}} & \frac{1}{E_{2}} & -\frac{v_{2}}{E_{1}} & 0 \\
-\frac{v_{1}}{E_{1}} & -\frac{v_{2}}{E_{1}} & \frac{1}{E_{1}} & 0 \\
0 & 0 & 0 & \frac{1}{G_{2}}
\end{array}\right) \cdot\left(\begin{array}{c}
\sigma_{x x} \\
\sigma_{y y} \\
\sigma_{z z} \\
\tau_{x y}
\end{array}\right)=\left(\begin{array}{cccc}
s_{11} & s_{12} & s_{13} & 0 \\
s_{12} & s_{22} & s_{12} & 0 \\
s_{12} & s_{12} & s_{33} & 0 \\
0 & 0 & 0 & s_{44}
\end{array}\right) \cdot\left(\begin{array}{l}
\sigma_{x x} \\
\sigma_{y y} \\
\sigma_{z z} \\
\tau_{x y}
\end{array}\right),
$$

where

$$
\begin{gathered}
v_{1}=\frac{E_{h} v_{h}\left(-1+v_{w}{ }^{2}\right)\left(-1+x_{w}\right)-E_{w}\left(-1+v_{h}{ }^{2}\right) v_{w} x_{w}}{E_{h}\left(-1+v_{w}{ }^{2}\right)\left(-1+x_{w}\right)-E_{w}\left(-1+v_{h}{ }^{2}\right) x_{w}}, \\
v_{2}=\frac{\left(-E_{h}\left(1+v_{w}\right)\left(-1+x_{w}\right)+E_{w}\left(1+v_{h}\right) x_{w}\right)\left(-v_{w} x_{w}+v_{h}\left(-1+v_{w}+x_{w}\right)\right)}{-E_{h}\left(-1+v_{w}{ }^{2}\right)\left(-1+x_{w}\right)+E_{w}\left(-1+v_{h}{ }^{2}\right) x_{w}}, \\
E_{1}=-\frac{E_{h}{ }^{2}\left(-1+v_{w}{ }^{2}\right)\left(-1+x_{w}\right)^{2}-2 E_{h} E_{w}\left(-1+v_{h} v_{w}\right)\left(-1+x_{w}\right) x_{w}+E_{w}{ }^{2}\left(-1+v_{h}{ }^{2}\right) x_{w}{ }^{2}}{E_{h}\left(-1+v_{w}{ }^{2}\right)\left(-1+x_{w}\right)-E_{w}\left(-1+v_{h}{ }^{2}\right) x_{w}}, \\
Z=E_{w}{ }^{2}\left(-1+v_{h}+2 v_{h}{ }^{2}\right)\left(-1+x_{w}\right) x_{w}+E_{h}{ }^{2}\left(-1+v_{w}+2 v_{w}{ }^{2}\right)\left(-1+x_{w}\right) x_{w}- \\
E_{h} E_{w}\left(-1+2 x_{w}+\left(-2+v_{h}\right) x_{w}{ }^{2}+v_{w}\left(-1+x_{w}\right)\left(-1+x_{w}+4 v_{h} x_{w}\right)\right) \\
E_{h}=\frac{E_{h} E_{w}\left(E_{h}\left(-1+v_{w}\right)\left(-1+x_{w}\right)-E_{w}\left(-1+v_{h}\right) x_{w}\right)}{E_{w}}, \\
G_{2}=\frac{E_{w}\left(-E_{w}\left(1+v_{h}\right)\left(-1+x_{w}\right)+E_{h}\left(1+v_{w}\right) x_{w}\right)}{2}
\end{gathered}
$$


assuming plane strain conditions (i.e., $\varepsilon_{z z}=0$ ),

$$
\left(\begin{array}{l}
\varepsilon_{x x} \\
\varepsilon_{y y} \\
\gamma_{x y}
\end{array}\right)=\left(\begin{array}{ccc}
s_{11}-\frac{s_{13}^{2}}{s_{11}} & s_{12}-\frac{s_{13} s_{12}}{s_{11}} & 0 \\
s_{12}-\frac{s_{13} s_{12}}{s_{11}} & s_{22}-\frac{s_{12}^{2}}{s_{11}} & 0 \\
0 & 0 & s_{44}
\end{array}\right) \cdot\left(\begin{array}{l}
\sigma_{x x} \\
\sigma_{y y} \\
\tau_{x y}
\end{array}\right)=\left(\begin{array}{ccc}
S_{11} & S_{12} & 0 \\
S_{21} & S_{22} & 0 \\
0 & 0 & S_{33}
\end{array}\right) \cdot\left(\begin{array}{l}
\sigma_{x x} \\
\sigma_{y y} \\
\tau_{x y}
\end{array}\right) \text {. }
$$

The two upper diagonal terms represent the stiffness of the composite perpendicular and parallel to the layers and are equal to $2278 \mathrm{MPa}$ and $6044 \mathrm{MPa}$, respectively, which agrees with those obtained from the numerical calculations.

Furthermore, the uniaxial compressive strength of the stratified is equal to $8633 \mathrm{kPa}$ (i.e., $\sigma_{d}=x_{h} \sigma_{d, h}+x_{w} \sigma_{d, w}$ ) and is the same, when loaded perpendicular or parallel to the layers (cf. Taliercio and Sacchi Landriani 1988).

The failure of the stratified rock mass, accounting for the thickness fractions and mechanical properties of the rock layers, was investigated by various authors (e.g., Taliercio and Sacchi Landriani 1988, Lydzba et al. 2003, Triantafyllidis and Gerolymatou 2014). The uniaxial compressive strength of the stratified rock mass depends on the direction of loading (cf. Fig. 9.3). The lowest uniaxial compressive strength occurs at an angle $\delta=45^{\circ}-\varphi_{w} / 2$ between the layers and the loading direction. Hence, at this orientation, the composite behaves as though it was provided with the strength properties $\left(c_{w}, \varphi_{w}\right)$ of the weakest layers (cf. Taliercio and Sacchi Landriani 1988). The largest uniaxial compressive strengths occur at an angle of $\delta=0^{\circ}$ and $90^{\circ}$ (cf. Fig. 9.3).

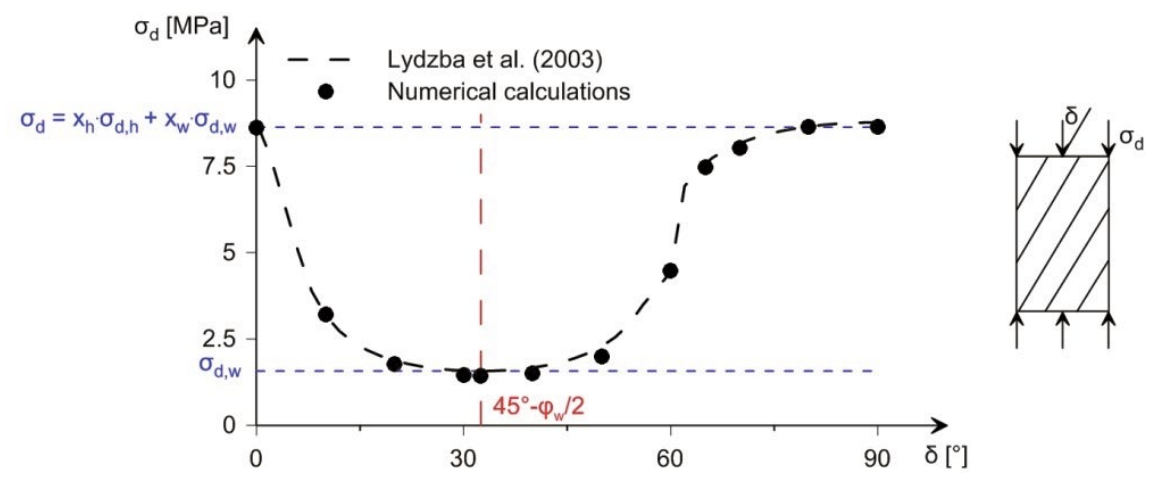

Figure 9.3. Uniaxial compressive strength $\sigma_{d}$ of the stratified rock mass as a function the angle between loading direction and bedding $\left(x_{h}=x_{w}=0.5, E_{h}=10 \mathrm{GPa}, c_{h}=5 \mathrm{MPa}, E_{w}=1 \mathrm{GPa}\right.$, $\left.c_{w}=0.5 \mathrm{MPa}, v_{h}=v_{w}=0.3, \varphi_{h}=\varphi_{w}=25^{\circ}, \psi_{h}=\psi_{w}=5^{\circ}\right)$.

\subsection{Basic aspects of the bedded rock response to excavation}

The ground response to tunnel excavation parallel to the layers is analysed by plane strain numerical computations (as in Chapter 10) for a series of layer thicknesses considering, due to symmetry, only a quarter of the system (Fig. 9.4).

According to Figure 9.5, which presents the displacement as a function of the support pressure for two points (Fig. 9.5a) as well as the displacement distribution along an unsupported excavation boundary (Fig. 9.5b), respectively, the results of the discrete models 
converge to those of the homogenised model with decreasing layer thickness, thus validating the formulation and numerical implementation of the homogenisation procedure.
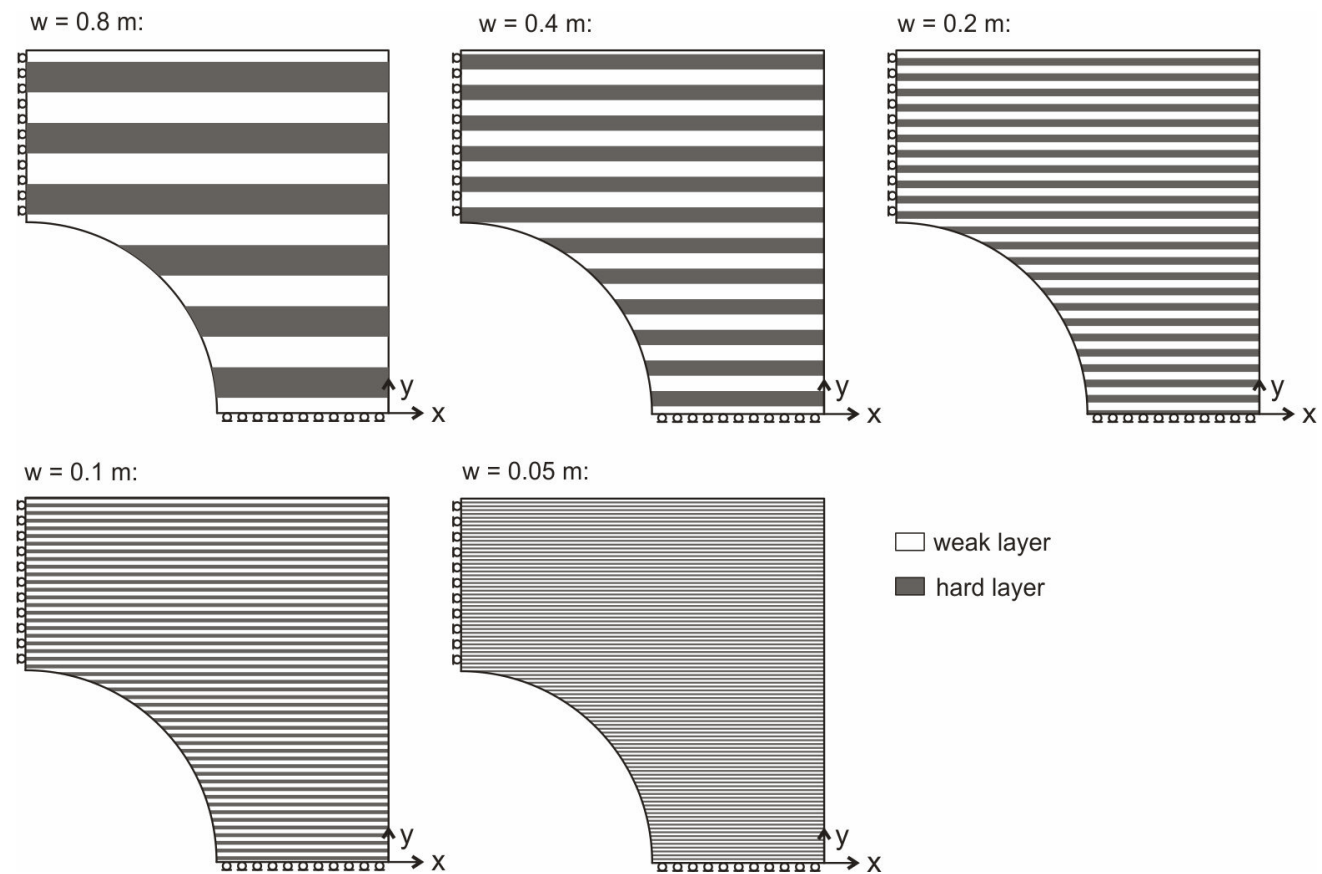

Figure 9.4. Discrete models for a rock mass consisting of alternating, $\mathbf{0 . 0 5}$ to $0.8 \mathrm{~m}$ thick competent and weak layers.

(a)

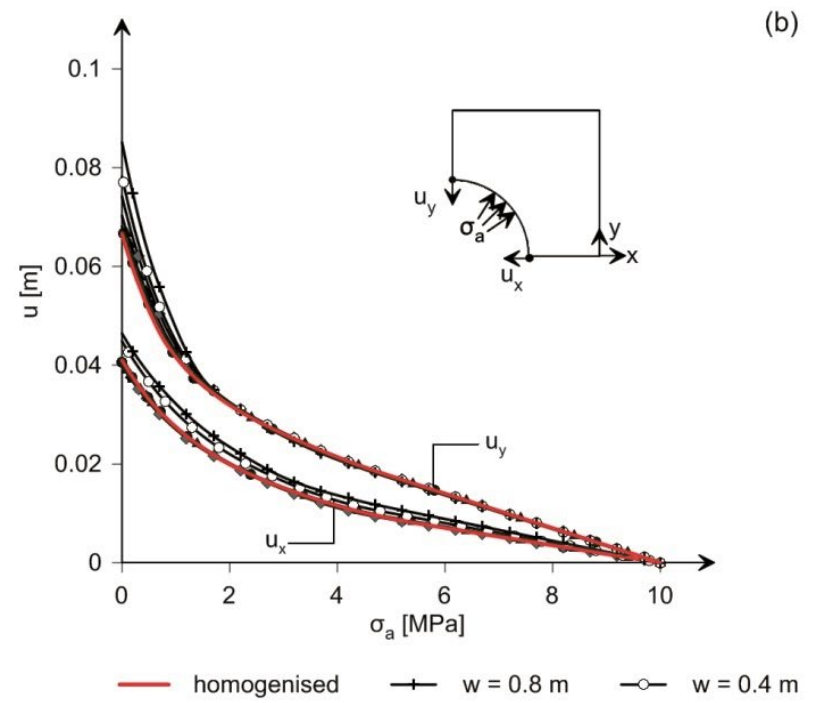

)

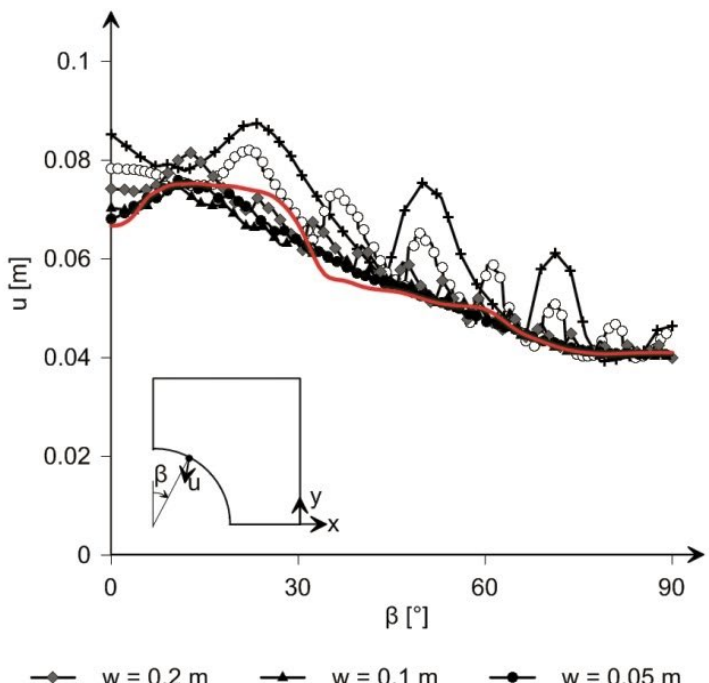

Figure 9.5. (a) Horizontal wall displacement and vertical crown displacement as a function of the support pressure, (b), magnitude of the displacement vector along an unsupported tunnel boundary according to the homogenised model (red line) as well as according to the discrete models of Figure 9.4 (black lines; $a=5 \mathrm{~m}, \sigma_{0}=10 \mathrm{MPa}, x_{h}=x_{w}=0.5, E_{h}=10 \mathrm{GPa}$, $\left.c_{h}=5 \mathrm{MPa}, E_{w}=1 \mathrm{GPa}, c_{w}=0.5 \mathrm{MPa}, v_{h}=v_{w}=0.3, \varphi_{h}=\varphi_{w}=25^{\circ}, \psi_{h}=\psi_{w}=5^{\circ}\right)$. 


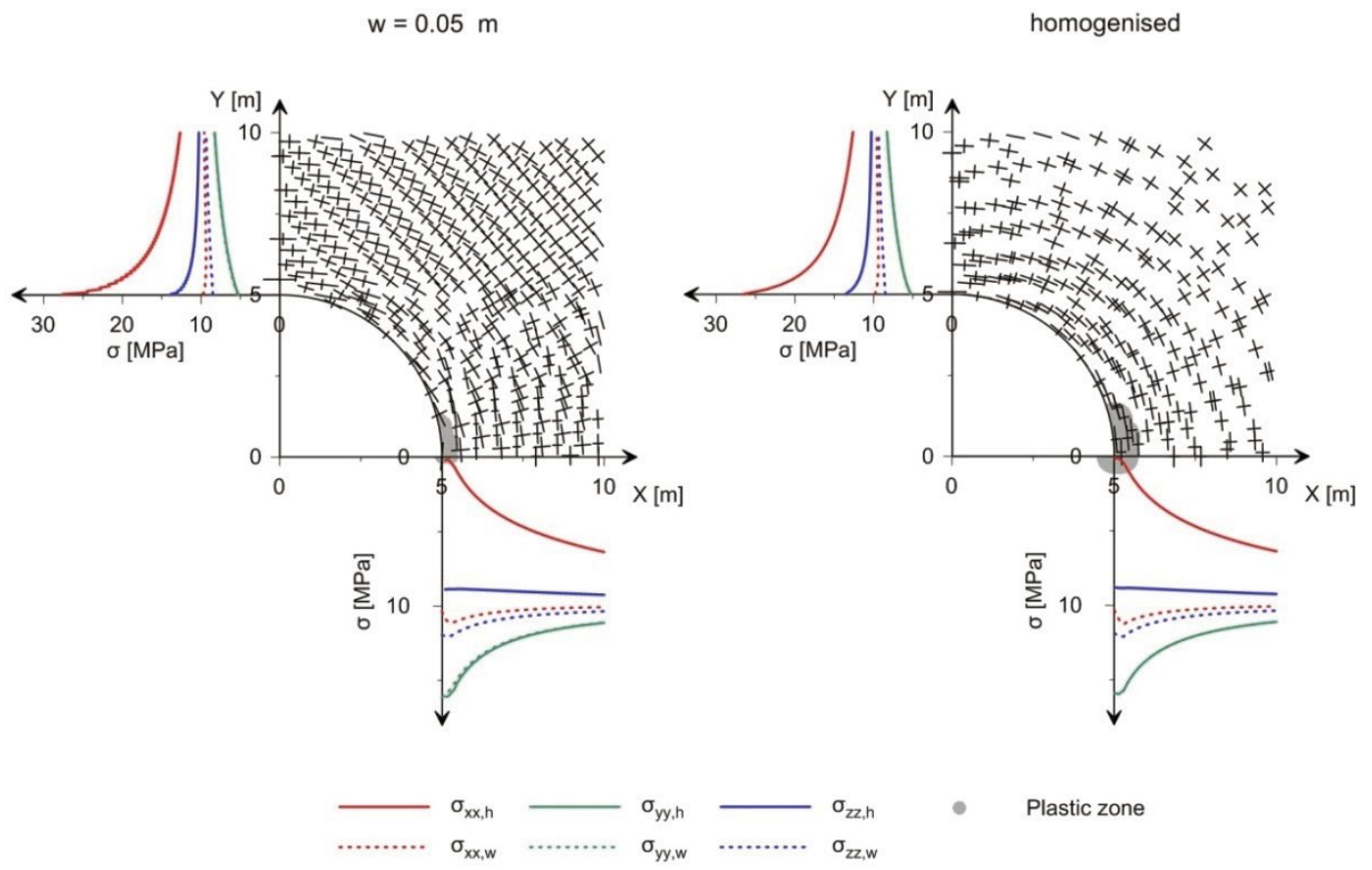

Figure 9.6. Principal stress orientation as well as stress field beside the tunnel and above the crown according to the homogenised model (r.h.s.) and to the discrete model of $0.05 \mathrm{~m}$ thick alternating layers (I.h.s.) for $\sigma_{a}=5.2 \mathrm{MPa}\left(a=5 \mathrm{~m}, \sigma_{0}=10 \mathrm{MPa}, x_{h}=x_{w}=0.5, E_{h}=10 \mathrm{GPa}\right.$, $\left.c_{h}=5 \mathrm{MPa}, E_{w}=1 \mathrm{GPa}, c_{w}=0.5 \mathrm{MPa}, v_{h}=v_{w}=0.3, \varphi_{h}=\varphi_{w}=25^{\circ}, \psi_{h}=\psi_{w}=5^{\circ}\right)$.
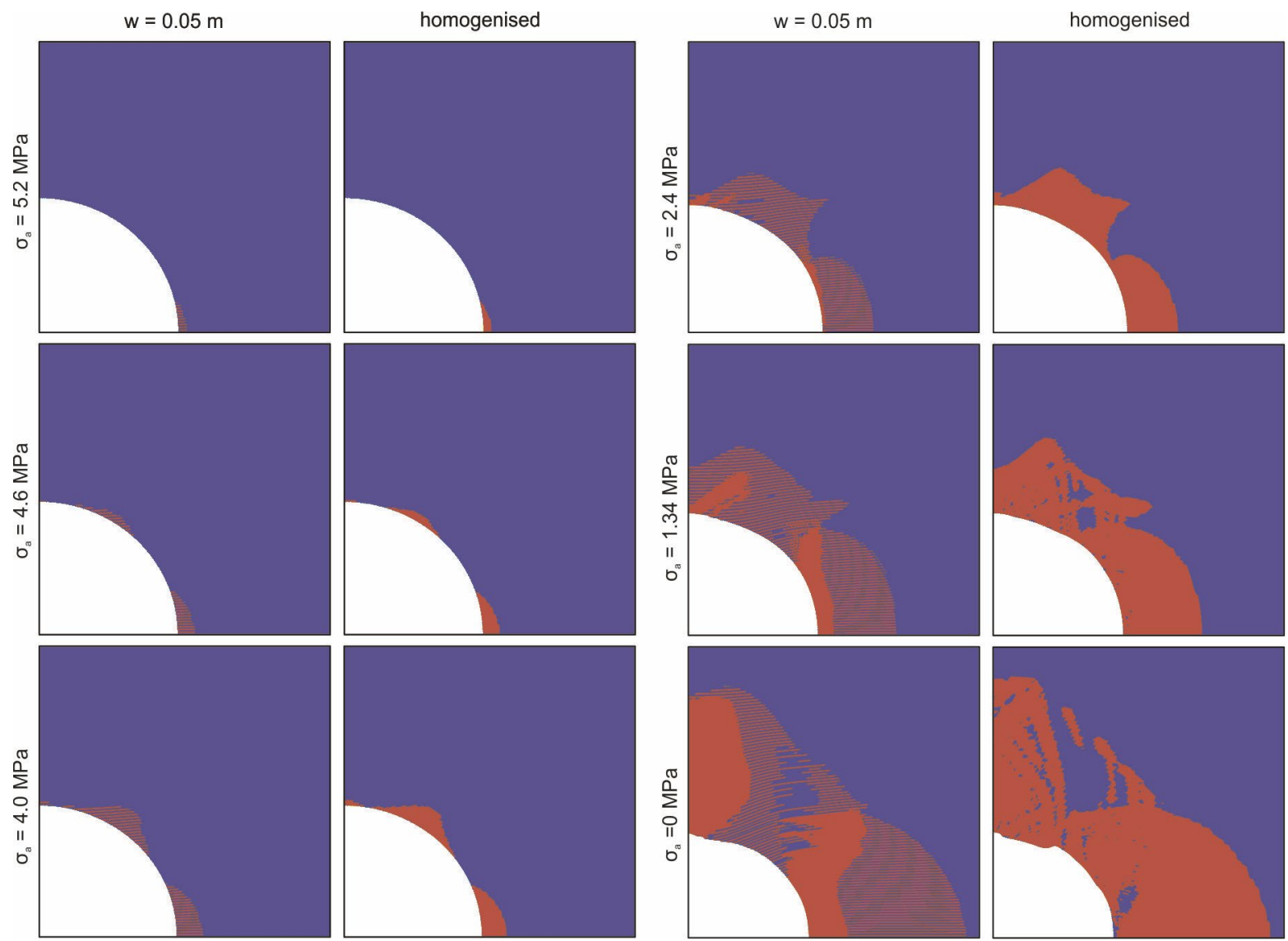

Figure 9.7. Plastic zone and deformed mesh (magnified by factor 20) at different support pressures $\sigma_{a}$ according to the homogenised model as well as to a discrete model of $0.05 \mathrm{~m}$ thick layers (red: plastic points; blue: elastic points; $a=5 \mathrm{~m}, \sigma_{0}=10 \mathrm{MPa}, x_{h}=x_{w}=0.5, E_{h}=10 \mathrm{GPa}$, $\left.c_{h}=5 \mathrm{MPa}, E_{w}=1 \mathrm{GPa}, c_{w}=0.5 \mathrm{MPa}, v_{h}=v_{w}=0.3, \varphi_{h}=\varphi_{w}=25^{\circ}, \psi_{h}=\psi_{w}=5^{\circ}\right)$. 
Additional evidence is provided by Figure 9.6, which presents the principal stress orientation as well as the stress field beside and above the crown, and by Figure 9.7, which presents the evolution of the plastic zone and of the deformation field during the reduction of the support pressure according to the homogenised model and to a discrete model of very thin alternating layers.

The computational results of Figures 9.5 to 9.7 , besides validating the implementation of the homogenised model, provide valuable insight in the response of a thinly stratified rock to tunnel excavation.

According to Figure 9.5, the crown displacement $u_{y}$ is larger than the wall displacement $u_{x}$. The reason for this ovalisation of the tunnel cross-section is that the layers beside the tunnel experience a larger tangential strain than the layers above the crown. This is such because the composite stiffness perpendicular (i.e., $E_{y y}=E_{2}$ ) to the layers is higher than parallel thereto (i.e., $E_{x x}=E_{1}$, cf. Section 9.2.2).

The ovalisation is recognisable already in the elastic range (i.e., for $\sigma_{a}>$ ca. $5.8 \mathrm{MPa}$ in the present example), where the radial $u_{a, r}$ and the tangential displacement $u_{a, t}$ as well as the tangential stress $\sigma_{t}$ along the tunnel boundary (i.e., as a function of $\theta=90^{\circ}-\beta$, with $\beta$ after Fig. 9.5b) can be calculated after Hefny and Lo (1999):

$$
\begin{gathered}
\sigma_{t}=\frac{2+2\left(\gamma_{1}+\gamma_{2}\right)^{2}-2 \gamma_{1}^{2} \gamma_{2}^{2}-4\left(\gamma_{1}+\gamma_{2}\right) \cos (2 \theta)}{\left(1+\gamma_{1}^{2}-2 \gamma_{1} \cos (2 \theta)\right)\left(1+\gamma_{2}^{2}-2 \gamma_{2} \cos (2 \theta)\right)}\left(\sigma_{0}-\sigma_{a}\right)+\sigma_{a}, \\
u_{a, r}=\frac{a\left(\sigma_{0}-\sigma_{a}\right)}{2\left(\gamma_{1}-\gamma_{2}\right)}\left(\left(\gamma_{1} \rho_{2}-\gamma_{2} \rho_{2}\right)+\left(\gamma_{1} \delta_{2}-\gamma_{2} \delta_{1}\right) \cos (2 \theta)\right),
\end{gathered}
$$

and

$$
u_{a, t}=\frac{a\left(\sigma_{0}-\sigma_{a}\right)}{2\left(\gamma_{1}-\gamma_{2}\right)}\left(\gamma_{2} \delta_{1}-\gamma_{1} \delta_{2}\right) \cos (2 \theta)
$$

with

$$
\begin{gathered}
\gamma_{1}=\frac{\alpha_{1}-1}{\alpha_{1}+1},\left|\gamma_{1}\right|<1, \gamma_{2}=\frac{\alpha_{2}-1}{\alpha_{2}+1},\left|\gamma_{2}\right|<1, \\
\alpha_{1}^{2} \alpha_{2}^{2}=\frac{S_{11}}{S_{22}}, \alpha_{1}^{2}+\alpha_{2}^{2}=\frac{2 S_{12}+S_{33}}{S_{22}}, \\
\delta_{1}=\left(1+\gamma_{1}\right) \beta_{2}-\left(1-\gamma_{1}\right) \beta_{1}, \delta_{2}=\left(1+\gamma_{2}\right) \beta_{1}-\left(1-\gamma_{2}\right) \beta_{2}, \\
\rho_{1}=\left(1+\gamma_{1}\right) \beta_{2}+\left(1-\gamma_{1}\right) \beta_{1}, \rho_{2}=\left(1+\gamma_{2}\right) \beta_{1}+\left(1-\gamma_{2}\right) \beta_{2}, \\
\beta_{1}=S_{12}-S_{22} \alpha_{1}^{2}, \beta_{2}=S_{12}-S_{22} \alpha_{2}^{2},
\end{gathered}
$$

and $S_{12}, S_{22}, S_{21}$ and $S_{33}$ after Section 9.2.2. (Note that the displacement and the tangential stress along the tunnel boundary for a given support pressure, which originates from Hefny and Lo (1999), was corrected in this chapter. The tangential stress obtained with the original formula of Hefny and Lo (1999) amounts to zero and the displacements are unequal to zero 
for $\sigma_{a}=\sigma_{0}$, which is obviously wrong.) According to Equation (9.9), the tangential stress is slightly higher in the crown than in the side wall (cf. Fig. 9.6), while the lowest tangential stress occurs at about $\beta \approx 40^{\circ}$.

With decreasing support pressure, yielding occurs first at the side wall and this in the hard rather than in the weak layers (see results for $\sigma_{a}=5.2 \mathrm{MPa}$ in Fig. 9.7). This result is somehow surprising at the first glance because the hard layers are subjected to the same tangential stress as the weak layers $\left(\sigma_{y y, w}=\sigma_{y y, h} \approx 16.0 \mathrm{MPa}\right.$; Fig. 9.6) and the later exhibit a lower strength. The hard layers fail first because of the constraint they impose to the lateral (horizontal) extension of the weak layers, which results to a very low radial stress $\left(\sigma_{x x, h} \approx 0\right)$ in the hard layers (cf. Section 9.2.2). It should be noted that, at $\sigma_{a}=5.2 \mathrm{MPa}$, the hard layers are subjected to even higher tangential stresses than at the side wall $\left(\sigma_{x x, h} \approx 26.6 \mathrm{MPa}\right.$, cf. Fig. 9.6), but remain elastic; this is such because of the high minimum principal stress (the radial stress at the crown corresponds to the support pressure, i.e. $\sigma_{y y, h}=5.2 \mathrm{MPa}$ both in the hard and in the weak layers; Fig. 9.6).

A further decrease in the support pressure $\sigma_{a}$ results in yielding of the weak layers within a second plastic zone that starts developing at $\beta \approx 45^{\circ}-\varphi_{w} / 2=32.5^{\circ}$ (see results for $\sigma_{a}=4.6 \mathrm{MPa}$ in Fig. 9.7). At this angle (which corresponds to the angle between the maximum principal stress and the planes of anisotropy), the composite exhibits the lowest uniaxial compressive strength (Section 9.2.2). Therefore, one might expect that failure, due to the decrease in support pressure, would first occur at this angle $\beta$ and not at the side wall of the tunnel. However, due to the different stiffness in horizontal and vertical direction $\left(E_{1} \gg E_{2}\right)$, the tangential stresses in this area $\left(\right.$ at $\left.\beta \approx 40^{\circ}\right)$ are reduced, leading thus to a later failure.

As the support pressure $\sigma_{a}$ decreases further, the hard layers reach failure also at the tunnel crown (see results for $\sigma_{a}=4.0 \mathrm{MPa}$ in Fig. 9.7) and afterwards the three plastic zones increase in size. Due to the direction-dependent strength of the composite (cf. Fig. 9.3), the second plastic zone does not increase uniformly and symmetrically. As soon as in certain areas both the weak and the hard layers reach their failure criterion, the deformations increase significantly in these areas. This is for example the case, when the second plastic zone meets the third plastic zone (at $\sigma_{a}=2.4 \mathrm{MPa}$ in Fig. 9.7), thus leading to high deformations in the area of the crown (see $u$ at $\beta \approx 10-25^{\circ}$ in Fig. 9.5b).

Further insight into the model behaviour and into the effect of the parameters of the hard layers provide the computational results of Figure 9.8. Specifically, the figure shows for the same parameter set as Figures 9.5 to 9.7 as well as for four additional parameter sets $\left(x_{h}, E_{h}\right.$, $C_{h}, \varphi_{h}$; given in the leftmost column):

- the GRC at the crown and the side wall, including for comparison the GRC in the absence of the hard layers (dashed line; I.h.s diagrams);

- the magnitude of the displacement vector along the tunnel boundary for $\sigma_{a}=0$ (middle diagrams); and

- the plastic zones at support pressures of 0 to $5.2 \mathrm{MPa}$ (r.h.s. diagrams). 
(a)

$\mathrm{E}_{\mathrm{h}}=10 \mathrm{GPa}$

$\mathrm{Xh}_{\mathrm{h}} / \mathrm{X}_{\mathrm{W}}=1$

$\mathrm{Ch}=5 \mathrm{MPa}$

$\varphi \mathrm{h}=25^{\circ}$

(b)

$\mathrm{E}_{\mathrm{h}}=10 \mathrm{GPa}$

$\mathrm{xh}_{\mathbf{h}} / \mathrm{x}_{\mathrm{w}}=\mathbf{0 . 2 5}$

$\mathrm{Ch}=5 \mathrm{MPa}$

$\varphi \mathrm{h}=25^{\circ}$

(c)

$\mathrm{E}_{\mathrm{h}}=\mathbf{2} \mathrm{GPa}$ $\mathrm{xh}_{\mathrm{h}} / \mathrm{x}_{\mathrm{w}}=1$ $\mathrm{Ch}=5 \mathrm{MPa}$ $\varphi \mathrm{h}=25^{\circ}$

(d)

$\mathrm{E}_{\mathrm{h}}=10 \mathrm{GPa}$ $\mathrm{xh}_{\mathrm{h}} / \mathrm{x}_{\mathrm{w}}=1$

$\mathrm{Ch}_{\mathrm{h}}=\mathbf{3} \mathrm{MPa}$

$\varphi \mathrm{h}=25^{\circ}$
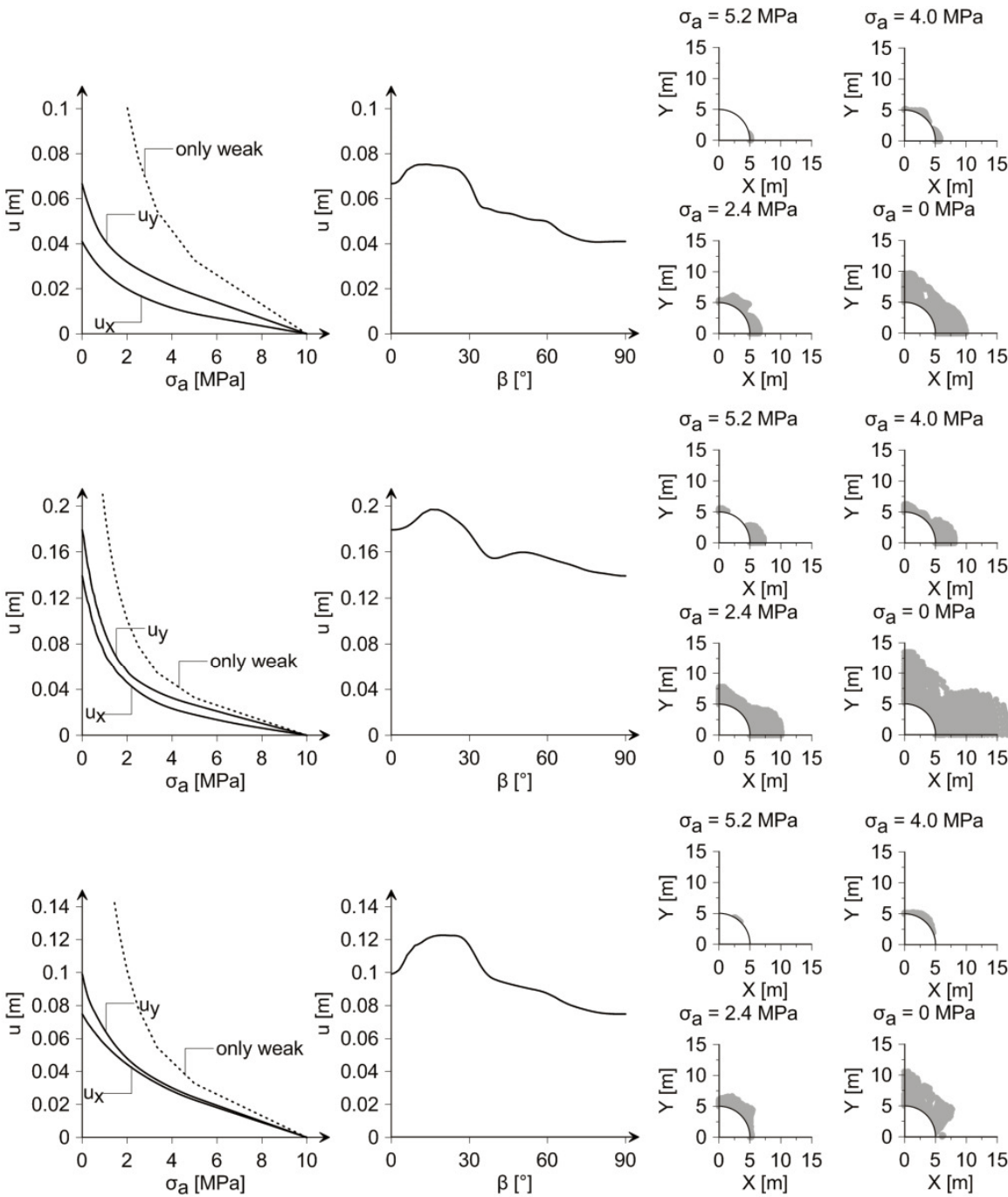

$\sigma_{\mathrm{a}}=5.2 \mathrm{MPa}$

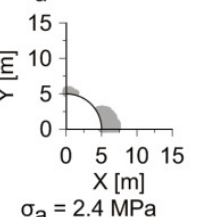

$\sigma_{\mathrm{a}}=4.0 \mathrm{MPa}$
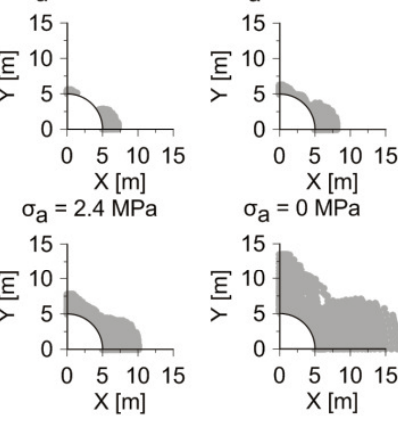

$\sigma_{\mathrm{a}}=5.2 \mathrm{MPa}$

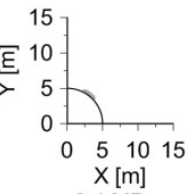

$\sigma_{\mathrm{a}}=2.4 \mathrm{MPa}$

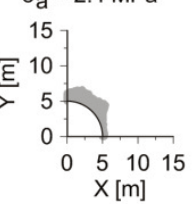

$\sigma_{\mathrm{a}}=4.0 \mathrm{MPa}$

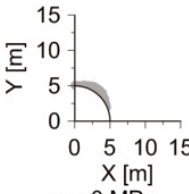

$\sigma_{\mathrm{a}}=0 \mathrm{MPa}$
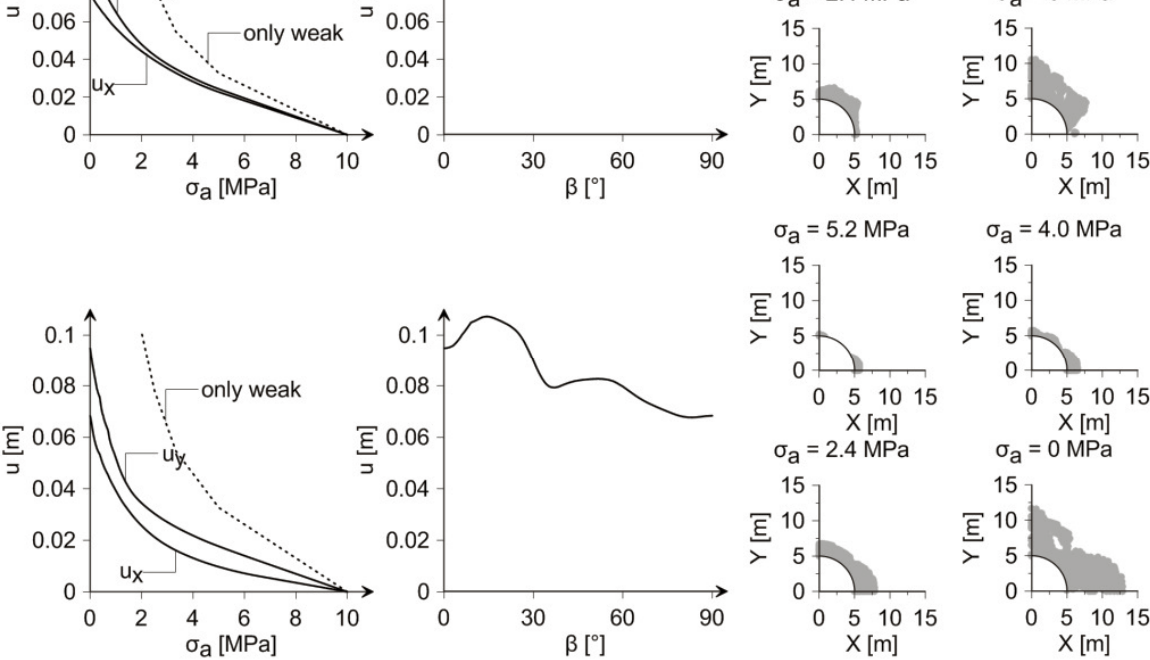

(e)

$\mathrm{E}_{\mathrm{h}}=10 \mathrm{GPa}$

$\mathrm{xh}_{\mathrm{h}} / \mathrm{x}_{\mathrm{w}}=1$

$\mathrm{Ch}=5 \mathrm{MPa}$

$\varphi \mathrm{h}=35^{\circ}$
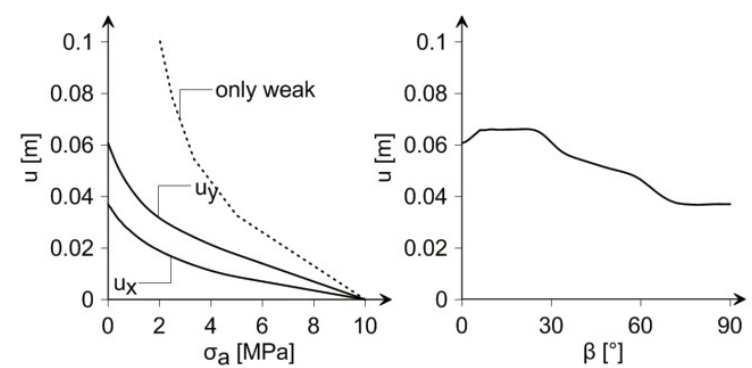

$\sigma_{\mathrm{a}}=5.2 \mathrm{MPa}$
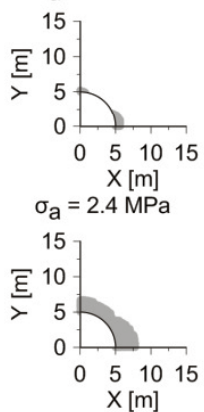

$\sigma_{\mathrm{a}}=4.0 \mathrm{MPa}$
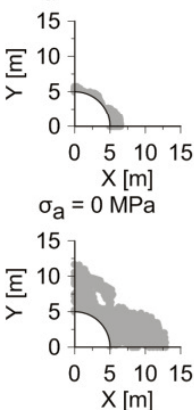

$$
\sigma_{\mathrm{a}}=5.2 \mathrm{MPa}
$$
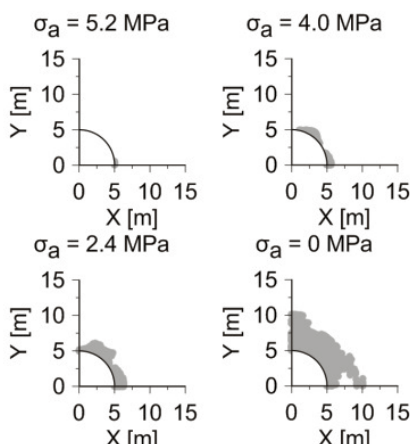

Figure 9.8. GRC (I.h.s. diagrams), magnitude of the displacement vector along the tunnel boundary for $\sigma_{a}=0$ (middle digrams) and plastic zone (r.h.s. diagrams; $a=5 \mathrm{~m}, \sigma_{0}=10 \mathrm{MPa}, c_{h}=5 \mathrm{MPa}$, $E_{w}=1 \mathrm{GPa}, c_{w}=0.5 \mathrm{MPa}, v_{h}=v_{w}=0.3, \varphi_{h}=\varphi_{w}=25^{\circ}, \psi_{h}=\psi_{w}=5^{\circ}$ ). 
The following conclusions can be drawn:

- The hard layers have a considerable stabilising effect (compare dashed with solid lines in the I.h.s. diagrams).

- A decrease in the thickness fraction of the hard layers (from $x_{n} / x_{w}=1$ to 0.25 ) results in a less pronounced but still remarkable stabilising effect. Particularly, the first and the third plastic zones (developing at the side wall and the crown of the tunnel, respectively), in which the hard layers reach failure, increase considerably, as the latter are more loaded (compare, e.g., results for $\sigma_{a}=5.2 \mathrm{MPa}$ in Figs. 9.8a and 9.8b).

- A decrease in the stiffness of the hard layers from $E_{h}=10$ to $2 \mathrm{GPa}$ results to an overall lower stiffness and larger displacements. As the contrast in $E$ of the weak and the hard layers is smaller than before, the stiffness in horizontal direction (parallel to bedding) is only slightly higher than the one in vertical direction (perpendicular to bedding) and, therefore, the displacement distribution in the elastic range is nearly uniform along the tunnel boundary. Therefore, the weak layers reach failure first (at $\beta \approx 45^{\circ}-\varphi_{w} / 2=32.5^{\circ}$; see results for $\sigma_{a}=5.2 \mathrm{MPa}$ in Fig. 9.8c). With a further decrease in the support pressure, the non-uniformity of the displacements in the tunnel profile increases (compare $u_{y}$ and $u_{x}$ in I.h.s. diagram of Fig. 9.8c), due to the considerable direction-dependent strength of the composite (cf. Section 9.2.2).

- A decrease in the cohesion of the hard layers to $c_{h}=3 \mathrm{MPa}$ leads to a more extended plastification of the composite and thus to considerably larger displacements (Fig. 9.8d). Particularly, the plastic zones, in which the hard layers reach failure, increase considerably.

- An increase in the friction angle of the hard layers (from $\varphi_{h}=25^{\circ}$ to $35^{\circ}$ ) results expectedly in smaller displacements (Fig. 9.8e). However, the effect of the friction angle of the hard layers is rather small compared to the other factors discussed above.

In conclusion, due to the strength and stiffness anisotropy of a stratified rock, a tunnel drive parallel to the bedding is characterised by a very non-uniform deformation of the profile. Principally, the largest deformations occur at locations where the layers are almost (but not exactly) parallel to the excavation boundary (i.e., in the crown for a horizontal stratification).

The displacement distribution may be even more non-uniform if the in situ stress field is nonhydrostatic; for example, according to Hefny and Lo (1999), the crown displacement increases, while the side wall displacement decreases with decreasing initial stress ratio $K_{0}$.

\subsection{Development of nomograms}

This section develops dimensionless diagrams for the minimum, average and maximum magnitude of the displacement vector of the tunnel boundary.

The magnitude of the displacement vector in a specific location $\beta$ of the tunnel boundary depends on all parameters of the problem under consideration:

$$
u=f\left(x_{h}, E_{h}, v_{h}, \varphi_{h}, \sigma_{d, h}, \psi_{h}, x_{w}, E_{w}, v_{w}, \varphi_{w}, \sigma_{d, w}, \psi_{w}, \sigma_{0}, \sigma_{a}, a\right) .
$$

A dimensional analysis in combination with the findings of Chapter 5 and with the general property of elasto-plastic continua, according to which the displacements are inversely 
proportional to Young's modulus (Anagnostou and Kovári 1993), suggests that the displacement $u$ can be expressed as follows:

$$
\frac{u}{u_{w, 2 D}}=f\left(\frac{x_{h}}{x_{w}}, \frac{E_{h}}{E_{w}}, \frac{\sigma_{d, h}}{\sigma_{d, w}}, \frac{\sigma_{d, w}}{\sigma_{0}}, \frac{\sigma_{a}}{\sigma_{0}}, v_{h}, \varphi_{h}, \psi_{h}, v_{w}, \varphi_{w}, \psi_{w}\right),
$$

where $u_{w, 2 D}$ is the radial displacement of a tunnel crossing only weak rock and can be computed using known closed-form equations (e.g., those of Anagnostou and Kovári 1993). As the aforementioned property of elasto-plastic continua was proven only for an isotropic material, the correctness of the normalisation of Equation (9.18) was checked by performing a series of numerical calculations: As can be seen from Figure 9.9, the displacements $u / u_{w, 2 D}$ obtained for different values of the initial stress $\sigma_{0}$ and the Young's modulus of the weak layers $E_{w}$ fall on one single curve.

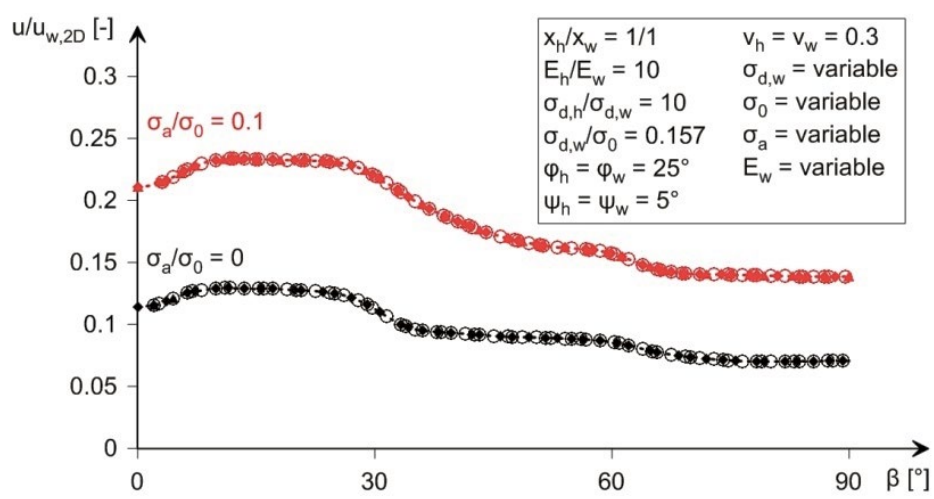

Figure 9.9. Normalised magnitude of the displacement vector along the tunnel boundary.

In order to reduce the computational effort, the numerical analyses were only carried out for specific ground parameters (according to Table 9.1). For the sake of simplicity, only Poisson's ratios of the hard and the weak layers amounting to 0.3 will be considered. Moreover, it can be assumed that the dilatancy angles $\psi_{h}$ and $\psi_{w}$ are interconnected with the friction angles. Furthermore, use will be made of the fact that weaker materials are generally also softer, exhibiting a Young's modulus in the order of 500 to 1000 times the uniaxial strength, which means that solely specific moduli ratio $E_{h} / E_{w}$ have to be considered for given ratio $\sigma_{d, h} / \sigma_{d, w}(c f$. Chapter 6$)$.

Table 9.1. Overview of the considered parameter ranges for the nomograms.

\begin{tabular}{|c|c|c|c|}
\hline$x_{h} / x_{w}[-]$ & \multicolumn{3}{|c|}{$1 / 8-2$} \\
\hline$\sigma_{d, h} / \sigma_{d, w}[-]$ & 2 & 6 & 10 \\
\hline$E_{h} / E_{w}[-]$ & $1-4$ & 3-12 & $5-20$ \\
\hline$v_{h}=v_{w}[-]$ & \multicolumn{3}{|c|}{0.3} \\
\hline$\varphi=\varphi_{h}=\varphi_{w}\left[^{\circ}\right]$ & \multicolumn{3}{|c|}{$20 ; 30$} \\
\hline$\psi_{h}=\psi_{w}\left[{ }^{\circ}\right]$ & \multicolumn{3}{|c|}{$\begin{array}{l}10 \text { for } \varphi_{h}=\varphi_{w}=30^{\circ} \\
g \text { to Vermeer and de Borst 1984) }\end{array}$} \\
\hline$\sigma_{a} / \sigma_{0}[-]$ & \multicolumn{3}{|c|}{$0 ; 0.1 ; 0.2$} \\
\hline
\end{tabular}

As the friction angle $\varphi_{h}$ of the hard layers has a rather small influence on the tunnel displacements (cf. Section 9.3) and, for given cohesion of the hard layers, the assumption of a lower friction angle is on the safe side, the dimensionless diagrams were worked out only for $\varphi_{h}=\varphi_{w}=\varphi=20^{\circ}$ or $30^{\circ}$ although $\varphi_{h}$ is usually higher than $\varphi_{w}$. 
In order to cover a wide range of parameters, but to reduce the computational effort anyway, only specific values of the thickness fraction $x_{h} / x_{w}$, of the strength ratio $\sigma_{d, h} / \sigma_{d, w}$ and of the normalised support pressure $\sigma_{a} / \sigma_{0}$ will be considered. The latter was chosen such as to cover the practically important part of the GRC (at $\sigma_{a 1}=0, \sigma_{a 2}=0.1 \sigma_{0}$ and $\sigma_{a 3}=0.2 \sigma_{0}$ ).

Bearing in mind that some of the parameters have been fixed, the maximum, minimum and averaged displacement along the tunnel boundary depend on the following parameters:

$$
\frac{u_{\max }}{u_{w, 2 D}}, \frac{u_{\min }}{u_{w, 2 D}}, \frac{u_{\text {average }}}{u_{w, 2 D}}=f\left(\frac{\sigma_{d, w}}{\sigma_{0}}, \frac{x_{h}}{x_{w}}, \frac{E_{h}}{E_{w}}, \frac{\sigma_{d, h}}{\sigma_{d, w}}, \frac{\sigma_{a}}{\sigma_{0}}, \varphi\right) \text {. }
$$

The nomograms of Appendix $E$ show these three normalised displacement values as a function of the normalised strength $\sigma_{d, w} / \sigma_{0}$ of the weak layers in a series of figures. Each figure applies to a certain parameter set $\left(\sigma_{a} / \sigma_{0}, \sigma_{d, h} / \sigma_{d, w}, x_{h} / x_{w}\right)$ and contains a number of diagrams (each applying to another set $\left(\varphi, E_{h} / E_{w}\right)$ ).

As the normalised displacements on the I.h.s. of Equation (9.19) are equal to 1 in the absence of hard layers, the nomograms of Appendix $E$ show directly the stabilising effect of the hard layers.

For an unsupported tunnel, the ratio $u / u_{w, 2 D}$ decreases (i.e., the stabilising effect becomes more pronounced) with decreasing normalised strength $\sigma_{d, w} / \sigma_{0}$ of the weak layers. Therefore, the nomograms are useful particularly for squeezing rocks. For large values of $\sigma_{d, w} / \sigma_{0}$, the weak and the hard layers behave elastically and the normalised displacements remain constant. In this case, the displacements can be calculated also after Hefny and Lo (1999), but the nomograms can be used for a fast estimation of the tunnel displacements.

\subsection{Parameters of an equivalent isotropic material}

\subsubsection{Procedure}

The response of a transversely isotropic material to tunnel excavation is very different from that of an isotropic material. It is thus obvious that an "equivalent" or "practically equivalent" isotropic material cannot exist. The notion of "equivalent material" is used here for simplicity and only in the following sense: It is equivalent in the sense that its (uniform) excavationinduced displacement is equal to the maximum, to the average or to the minimum displacement of the transversely isotropic rock. In spite of its obvious limitations, such an equivalent material model is valuable because it allows to determine an upper and a lower limit of the deformations of the profile.

The parameters of the equivalent isotropic elasto-plastic material can be determined analogously to Chapter 6, but using the nomograms of Appendix E. However, contrarily to Chapter 6, the displacements in a tunnel drive parallel to the bedding are very non-uniform along the tunnel boundary, which makes the determination of equivalent parameters more difficult. In the following, the procedure will be described based upon an example.

Section 9.3 showed that the bigger the contrast in the mechanical parameters of the weak and of the hard layers is, the less uniform the displacements of the tunnel profile will be. Hence, an extreme case with $E_{h} / E_{w}=20$ and $c_{h} / c_{w}=10$ will be considered here, for which the 
non-uniformity of the displacement-distribution is considerable (Fig. 9.10a). For design purposes, usually the range of the displacements is assessed, considering therefore the maximum and the minimum displacement of the tunnel profile. (The consideration of the average values might make sense for dimensioning a stiff lining. Preliminary computational investigations show that the rock pressure distribution is approximately uniform even in a transversely isotropic rock; Fig. 9.10b.)

(a)

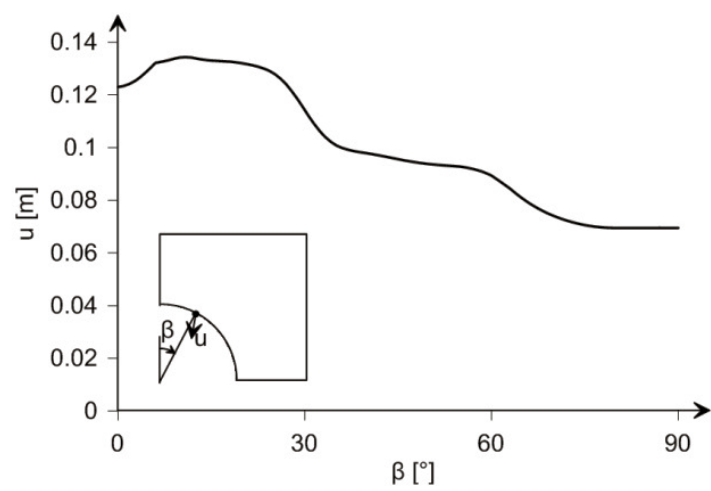

(b)

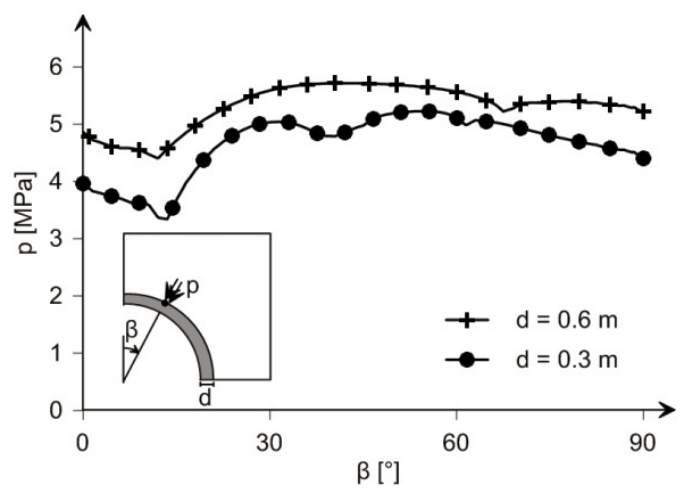

Figure 9.10. (a) Magnitude of the displacement vector along the tunnel boundary for $\sigma_{a}=0$; (b) Radial pressure along a stiff lining $\left(a=5 \mathrm{~m}, \sigma_{0}=10 \mathrm{MPa}, x_{h} / x_{w}=0.25, E_{h}=20 \mathrm{GPa}, c_{h}=10 \mathrm{MPa}\right.$, $E_{w}=1 \mathrm{GPa}, c_{w}=1 \mathrm{MPa}, v_{h}=v_{w}=0.3, \varphi_{h}=\varphi_{w}=20^{\circ}, \psi_{h}=\psi_{w}=1^{\circ}$, lining thickness 0.3-0.6 m, Young's modulus of concrete $=30 \mathrm{GPa}$, frictionless rock-lining interface, $20 \%$ predeformation).

The GRC of the equivalent isotropic model can be written as follows:

$$
u_{a}=\frac{\sigma_{0} \cdot a}{E_{e q}} \cdot f_{2}\left(\frac{\sigma_{a}}{\sigma_{0}}, \frac{\sigma_{d, e q}}{\sigma_{0}}, v_{e q}, \varphi_{e q}, \psi_{e q}\right) \text {, }
$$

where $E_{e q}, v_{\mathrm{eq}}, \sigma_{d, \mathrm{eq}}, \varphi_{\mathrm{eq}}$ and $\psi_{\mathrm{eq}}$ denote the equivalent Young's modulus, Poisson's ratio, uniaxial compressive strength, friction angle and dilatancy angle. Parameters are sought that reproduce either the minimum or the maximum displacement of the transversely isotropic rock mass.

In the following, for the sake of simplicity, only the case of equal Poisson's ratios $\left(v_{e q}=v_{h}=v_{w}\right)$ will be considered. The equivalent Young's modulus $E_{e q}$ can be calculated by setting the displacement of an elastic unsupported isotropic rock mass equal either to the maximum $\left(u_{\max }\right.$; in order to estimate an upper limit of the displacements of the transversely isotropic material) or to the minimum $\left(u_{\min }\right.$; in order to estimate a lower limit of the displacements) magnitude of the displacement vector in an elastic, transversely isotropic rock mass:

$$
\begin{aligned}
& \left(\sqrt{u_{a, r}^{2}+u_{a, t}^{2}}\right)_{\text {max }}=\frac{1+v_{e q}}{E_{e q, \max }} \cdot \sigma_{0} \cdot a, \\
& \left(\sqrt{u_{a, r}^{2}+u_{a, t}^{2}}\right)_{\text {min }}=\frac{1+v_{e q}}{E_{e q, \min }} \cdot \sigma_{0} \cdot a,
\end{aligned}
$$

where the radial and tangential displacements of the transversely isotropic rock mass $\left(u_{a, r}\right.$, $\left.u_{a, t}\right)$ can be calculated after Hefny and Lo (1999); Eqs. 9.10 and 9.11). 
Setting the maximum displacement $u_{\max }$ (or the minimum displacement $u_{\min }$ ) of the transversely isotropic material $\left(u_{\max }\right.$ and $u_{\min }$ can be obtained from the nomograms of Appendix E) equal to those of the equivalent isotropic model (Eq. 9.20) for three selected values of the support pressure $\left(\sigma_{a 1}, \sigma_{a 2}, \sigma_{a 3}\right.$; preferably in the practical relevant range), provides a system of three nonlinear equations for the unknown plasticity parameters $\sigma_{d, \text { eq }} / \sigma_{0}$, $\varphi_{e q}$ and $\psi_{e q}$ :

$$
\begin{aligned}
u_{\text {max }} & =\frac{\sigma_{0} \cdot a}{E_{w}} \cdot f\left(\frac{x_{h}}{x_{w}}, \frac{E_{h}}{E_{w}}, \frac{\sigma_{d, h}}{\sigma_{d, w}}, \frac{\sigma_{d, w}}{\sigma_{0}}, \frac{\sigma_{a k}}{\sigma_{0}}, v_{h}, \varphi_{h}, \psi_{h}, v_{w}, \varphi_{w}, \psi_{w}\right) \\
& =\frac{\sigma_{0} \cdot a}{E_{e q, \max }} \cdot f_{2}\left(\frac{\sigma_{a k}}{\sigma_{0}}, \frac{\sigma_{d, e q, \max }}{\sigma_{0}}, v_{e q}, \varphi_{e q, \max }, \psi_{e q, \max }\right) \quad(\text { for } k=1,2,3), \\
u_{\text {min }} & =\frac{\sigma_{0} \cdot a}{E_{w}} \cdot f\left(\frac{x_{h}}{x_{w}}, \frac{E_{h}}{E_{w}}, \frac{\sigma_{d, h}}{\sigma_{d, w}}, \frac{\sigma_{d, w}}{\sigma_{0}}, \frac{\sigma_{a k}}{\sigma_{0}}, v_{h}, \varphi_{h}, \psi_{h}, v_{w}, \varphi_{w}, \psi_{w}\right) \\
& \left.=\frac{\sigma_{0} \cdot a}{E_{e q, \min }} \cdot f_{2}\left(\frac{\sigma_{a k}}{\sigma_{0}}, \frac{\sigma_{d, e q, \min }}{\sigma_{0}}, v_{e q}, \varphi_{e q, \min }, \psi_{e q, \min }\right) \quad \text { (for } k=1,2,3\right) .
\end{aligned}
$$

Applying this procedure to the parameters of the example of Figure 9.10 provides the equivalent parameters given in Table 9.2. Figure 9.11 compares the two GRCs of the isotropic rock mass (obtained with the two sets of equivalent parameters - one set based upon $u_{\min }$ and one set based upon $u_{\max }$ ) with the GRCs of the transversely isotropic material and shows that the equivalent isotropic model reproduces well the maximum or minimum displacement of the composite material and this even in the case of a big contrast in the mechanical parameters of the layers.

Table 9.2. Parameters of the hard and weak layers and corresponding parameters of the equivalent isotropic material.

\begin{tabular}{lcccccc}
\hline & $x$ & $E$ & $v$ & $c$ & $\varphi$ & $\psi$ \\
& {$[-]$} & {$[\mathrm{GPa}]$} & $\begin{array}{c}v \\
{[-]}\end{array}$ & $\begin{array}{c}\mathrm{MPa}] \\
{\left[{ }^{\circ}\right]}\end{array}$ & {$\left[{ }^{\circ}\right]$} \\
\hline hard layers & 0.20 & 20 & 0.3 & 10 & 20 & 1 \\
weak layers & 0.80 & 1 & 0.3 & 1 & 20 & 1 \\
& & & & & & \\
Equivalent isotropic material & & & & & & \\
fitted based upon: & - & 1.26 & 0.3 & 2.12 & 20 & 1 \\
$u_{\max }$ & - & 2.95 & 0.3 & 3.45 & 1 & 1 \\
$u_{\min }$ & - & & & & & \\
\hline
\end{tabular}

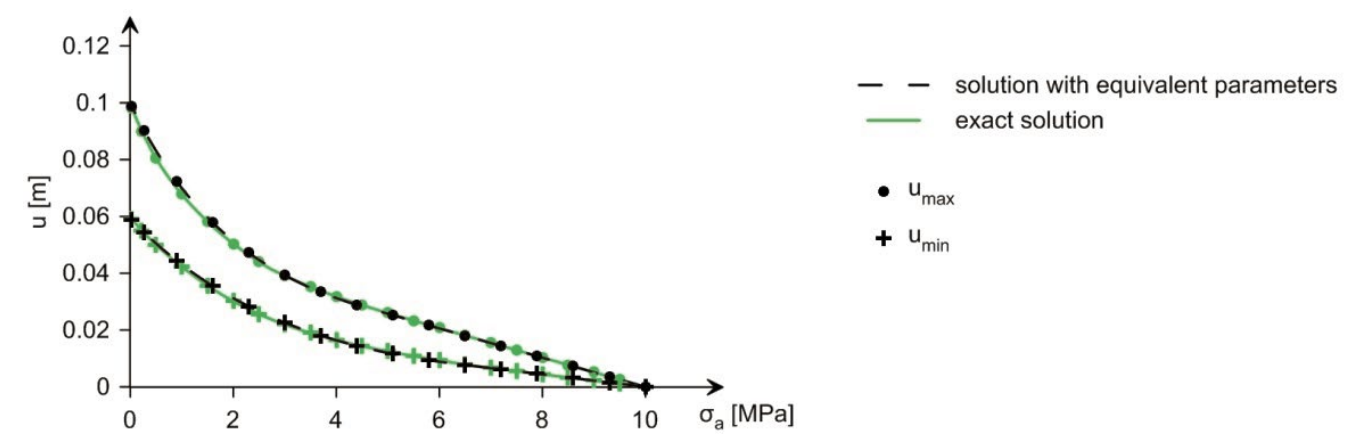

Figure 9.11. Maximum and minimum displacement at the tunnel boundary of a cylindrical tunnel as a function of the support pressure, obtained with the exact model and with the isotropic model considering equivalent parameters $\left(a=5 \mathrm{~m}, \sigma_{0}=10 \mathrm{MPa}\right)$. 


\subsubsection{Application examples}

The value of using the proposed equivalent isotropic model will be illustrated by means of two tunnelling problems (considering an initial hydrostatic stress field of $10 \mathrm{MPa}$ ): (1) The longitudinal displacement profile of an unsupported tunnel (Fig. 9.12a); (2) The boundary displacements of an unsupported tunnel with a horseshoe profile assuming plane strain conditions (Fig. 9.12b).

These problems were solved numerically, either using the exact transversely isotropic material model of Section 9.2.1 (with the parameters given in the two first rows of Table 9.2) or considering an equivalent isotropic rock mass (with the parameters given in the last two rows of Table 9.2). (Numerical details for problem 1 can be found in Chapter 12.)

Figure 9.12 shows that the exact solution lies in-between the two solutions obtained considering an isotropic material. Using latter allows thus to bound satisfactorily the response of transversely anisotropic rock mass.

(a)

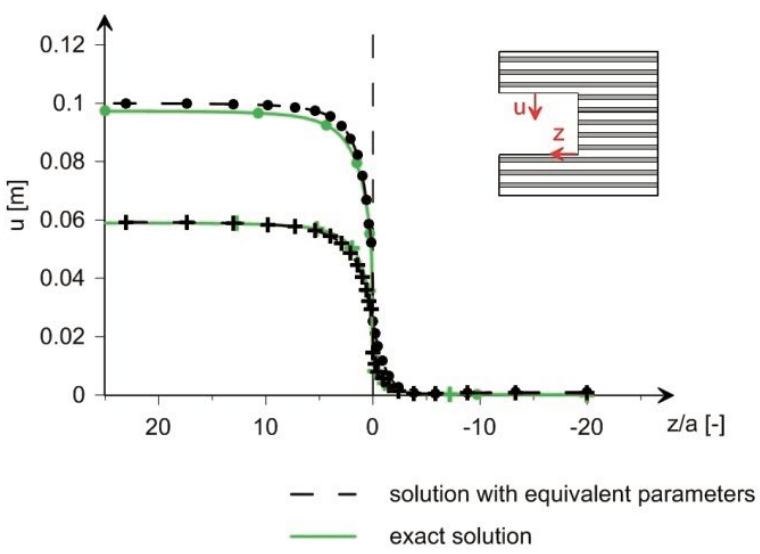

(b)

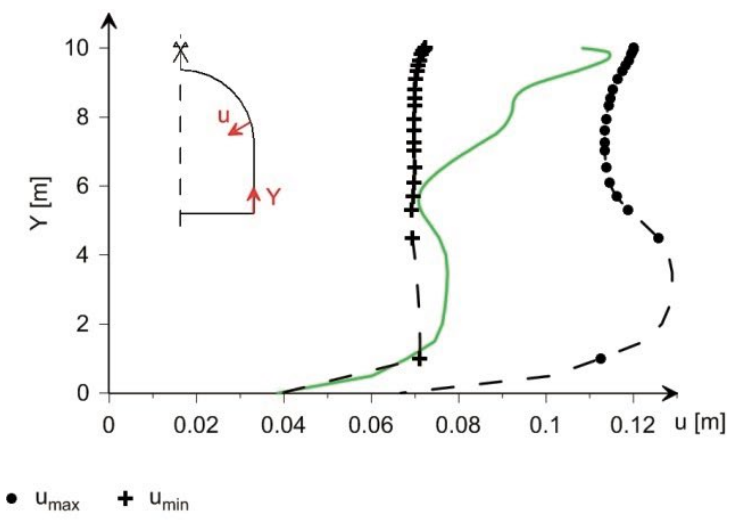

Figure 9.12. (a) Maximum and minimum longitudinal displacement profile of an unsupported tunnel. (b) Maximum and minimum magnitude of the displacement vector along the unsupported tunnel boundary of a horseshoe profile, obtained with the exact model and with the equivalent isotropic model (parameters after Table 9.2).

\subsection{Conclusions}

This chapter investigated the squeezing behaviour during tunnelling parallel to a sequence of thinly alternating weak and hard rocks, taking the rock mass as a homogeneous, transversely isotropic material. By means of application examples, the rock response to tunnelling was discussed, by specially focussing on the non-uniformity of the displacements in the tunnel profile, which may be considerable in certain cases. In order to facilitate the estimation of the displacements along the tunnel profile for given geotechnical conditions, design diagrams were developed that represent a valuable tool for engineering practice, as they enable to determine easily the maximum and minimum displacements in the tunnel profile (far behind the tunnel face) for a wide range of geotechnical conditions.

Furthermore, these diagrams allow determining the parameters of an equivalent isotropic rock mass based upon the maximum or the minimum displacement of the transversely isotropic material. Considering an isotropic material with these parameters makes it possible 
to estimate the range of deformations or pressures in problems that do not meet plane strain or rotationally symmetric conditions.

\section{Notation}

$a$
$c_{e q}$
$c_{h}, c_{w}$
$d$
$D_{h}$
$D_{h, e p}$
$E_{1}, E_{2}$
$E_{e q}$
$E_{e q, \max }, E_{\text {eq,min }}$

$E_{h}, E_{w}$

$E_{x x}, E_{y y}$

$f(\ldots)$

$f_{1}(\ldots)$

$f_{i}$

$g_{i}$

$\mathrm{G}_{2}$

$h$

$K_{0}$

I

$m_{i}$

\section{$n$}

$p$

$P_{e}, P_{t}$

$r$

$s_{11}, s_{12}, s_{13}$

$s_{22}, s_{33}, s_{44}$

$S_{11}, S_{12}, S_{21}$

$S_{22}, S_{33}$

$T_{t i}$

$u$

$u_{a, r}, u_{a, t}$

$u_{\text {average, }} u_{\max }, u_{\min }$

$u_{P, x}, u_{P, y}, u_{P, z}$

$U_{w, 2 D}$

$u_{x}, u_{y}$

W tunnel radius

equivalent cohesion of the simplified isotropic rock mass

cohesion of the hard and of the weak layers

thickness of the lining

elastic homogenised stiffness matrix

consistent constitutive matrix for the homogenised rock mass

Young's modulus of the composite parallel and perpendicular to the layers

equivalent Young's modulus of the simplified isotropic rock mass

equivalent Young's modulus of the simplified isotropic rock mass

fitted with the maximum or minimum GRC of the equivalent continuum

Young's modulus of the hard and of the weak layers

Young's modulus of the composite in $x$ - and $y$-direction

function of ...

function of ...

Mohr-Coulomb criterion of the $i^{\text {th }}$ layer

plastic potential of the $i^{\text {th }}$ layer

shear modulus of the composite

thickness of the hard layers

initial stress ratio

identity matrix

inclination of the failure surface of the $i^{\text {th }}$ layer (function of the friction angle)

stage

radial pressure along the lining

projection matrix into the strain space and into the stress space

radius, i.e., distance to the tunnel axis

constants for defining Hooke's law of the composite

constants for defining Hooke's law of the composite

constants for defining Hooke's law of the composite for plane strain conditions

constants for defining Hooke's law of the composite for plane strain conditions

transformation matrix

magnitude of the displacement vector at the tunnel boundary

displacement at the tunnel boundary in radial and tangential direction

averaged, maximum and minimum magnitude of the displacement vector along the tunnel boundary

displacement of the point $P$ in $x-, y$ - and z-direction

radial displacement of the homogeneous weak ground

displacement at the side wall (in $x$-direction) and at the crown (in $y$ direction)

thickness of the weak layers 


\begin{tabular}{|c|c|}
\hline$X$ & co-ordinate \\
\hline$x$ & co-ordinate \\
\hline$X_{h}, X_{w}$ & thickness fraction of the hard and of the weak layers \\
\hline Y & co-ordinate \\
\hline$y$ & co-ordinate \\
\hline$z$ & axial co-ordinate (distance behind the tunnel face) \\
\hline Z & constant \\
\hline$\alpha_{1}, \alpha_{2}$ & constants according to Hefny and Lo (1999) \\
\hline$\beta$ & angle between the tangential direction of the tunnel and the layers \\
\hline$\beta_{1}, \beta_{2}$ & constants according to Hefny and Lo (1999) \\
\hline$\gamma_{1}, \gamma_{2}$ & constants according to Hefny and Lo (1999) \\
\hline$\gamma_{x y}$ & shear strain of the composite \\
\hline$\delta$ & angle between the layers and the loading direction \\
\hline$\delta_{1}, \delta_{2}$ & constants according to Hefny and Lo (1999) \\
\hline$\Delta \varepsilon_{n+1}$ & homogenised strain increment \\
\hline$\Delta \varepsilon_{n+1, i}$ & strain increment in the $i^{\text {th }}$ layer \\
\hline$\left.\Delta \lambda_{n+1, i} D_{i} \frac{\partial g_{i}}{\partial \sigma_{i}}\right|_{n+1}$ & plastic corrector stress of the $i^{\text {th }}$ layer (cf. Clausen 2007) \\
\hline$\theta$ & angle according to Hefny and Lo (1999) \\
\hline$\varepsilon_{x x}, \varepsilon_{y y}, \varepsilon_{z z}$ & strain of the equivalent continuum in $x-, y$ - and $z$-direction \\
\hline$\varepsilon_{x y}, \varepsilon_{x z}, \varepsilon_{y z}$ & shear strain of the equivalent continuum in the $x y-, x z$ - and $y z$-plane \\
\hline$\kappa_{i}$ & $\begin{array}{l}\text { constant of the flow rule for the } i^{\text {th }} \text { layer (function of the dilatancy } \\
\text { angle) }\end{array}$ \\
\hline$V_{e q}$ & equivalent Poisson's ratio of the simplified isotropic rock mass \\
\hline$V_{h}, V_{w}$ & Poisson's ratio of the hard and of the weak layers \\
\hline$v_{1}, v_{2}$ & $\begin{array}{l}\text { Poisson's ratio of the composite parallel and perpendicular to the } \\
\text { layers }\end{array}$ \\
\hline$\sigma_{0}$ & initial stress \\
\hline$\sigma_{1}, \sigma_{3}$ & maximum and minimum principal stresses \\
\hline$\sigma_{a}$ & support pressure of the tunnel \\
\hline$\sigma_{d}$ & uniaxial compressive strength of the equivalent continuum \\
\hline$\sigma_{d, e q}$ & $\begin{array}{l}\text { equivalent uniaxial compressive strength of the simplified isotropic } \\
\text { rock mass }\end{array}$ \\
\hline$\sigma_{d, e q, \max }, \sigma_{d, e q, \min }$ & $\begin{array}{l}\text { equivalent uniaxial compressive strength of the simplified isotropic } \\
\text { rock mass fitted with the maximum or minimum GRC of the equiv- } \\
\text { alent continuum }\end{array}$ \\
\hline$\sigma_{d, h,} \sigma_{d, w}$ & uniaxial compressive strength of the hard and the weak layers \\
\hline $\begin{array}{l}\sigma_{d, i} \\
\sigma_{i}^{\text {trial }}\end{array}$ & $\begin{array}{l}\text { uniaxial compressive strength of the } i^{i t h} \text { layer } \\
\text { elastic predictor stress state in the } i^{\text {th }} \text { layer (i.e., so-called trial stress) }\end{array}$ \\
\hline$\sigma_{\max , h}, \sigma_{\min , h}$ & maximum and minimum stress of the hard layers \\
\hline$\sigma_{\max , w}, \sigma_{\min , w}$ & maximum and minimum stress of the weak layers \\
\hline$\sigma_{n}$ & homogenised stress at previous stage (at state $n$ ) \\
\hline$\sigma_{n, i}$ & stress in the $i^{\text {th }}$ layer at previous stage (at state $n$ ) \\
\hline$\sigma_{n+1}$ & update of the homogenised stress (at state $n+1$ ) \\
\hline$\sigma_{n+1, i}$ & $\begin{array}{l}\text { update of the stress in the } i^{\text {th }} \text { layer (i.e., in the weak or in the hard } \\
\text { layers) }\end{array}$ \\
\hline$\sigma_{r}, \sigma_{t}, \sigma_{z}$ & radial, tangential and axial stress of the equivalent continuum \\
\hline$\sigma_{r, h}, \sigma_{t, h}, \sigma_{z, h}$ & radial, tangential and axial stress of the hard layers \\
\hline$\sigma_{r, w}, \sigma_{t, w}, \sigma_{z, w}$ & radial, tangential and axial stress of the weak layers \\
\hline$\sigma_{x x}, \sigma_{y y}, \sigma_{z z}$ & f the equivalent continuum in $x$-, $y$ - and $z$-direction \\
\hline
\end{tabular}


$\sigma_{x x, h}, \sigma_{y y, h}, \sigma_{z z, h}$

$\sigma_{x x, w}, \sigma_{y y, w}, \sigma_{z z, w}$

$T_{x y}, T_{x z}, T_{y z}$

$\varphi_{\text {eq }}$

$\varphi_{\text {eq,max }}, \varphi_{\text {eq,min }}$

$\varphi_{h}, \varphi_{w}$

$\psi_{\text {eq }}$

$\psi_{\text {eq,max }}, \psi_{\text {eq,min }}$

$\psi_{h}, \psi_{w}$ stress of the hard layers in $x-, y$ - and $z$-direction

stress of the weak layers in $x-, y$ - and $z$-direction

shear stress of the equivalent continuum in $x y-, x z$ - and $y z$-direction equivalent friction angle of the simplified isotropic rock mass equivalent friction angle of the simplified isotropic rock mass fitted with the maximum or minimum GRC of the equivalent continuum friction angle of the hard and of the weak layers equivalent dilatancy angle of the simplified isotropic rock mass equivalent dilatancy angle of the simplified isotropic rock mass fitted with the maximum or minimum GRC of the equivalent continuum dilatancy angle of the hard and of the weak layers 



\section{Influence of the heterogeneity scale on the distribution of the ground displacements in the profile}

\subsection{Introduction}

Alternating weak and hard layers lying parallel to the tunnel axis may lead - depending on the heterogeneity scale (see Chapter 8) - to a considerable non-uniformity of the displacement distribution in the tunnel profile. The present chapter investigates: (i), under which conditions the homogenised model of Chapter 9 is adequate; (ii), in which cases the heterogeneity of the ground can be neglected in the design; and, (iii), whether the simple equation of Section 7.3 can be applied to the case of alternating layers that strike parallel to the tunnel axis.

\subsection{Adequacy of the homogenised model}

Obviously, the homogenised model of a transversely isotropic rock mass is adequate if the layers are sufficiently thin relative to the tunnel radius. It is also obvious that if the contrast in the mechanical parameters of the layers is small, then homogenisation is adequate even for relatively thick layers. The bigger the contrast in the mechanical parameters, the thinner the layers must be in order to consider the rock mass as a homogeneous material in the scale of the tunnel cross-section.

This will be illustrated by means of computational examples. The ground response to tunnel excavation is analysed by plane strain numerical calculations for a series of thicknesses of the hard $h$ and the weak $w$ layers as in Section 9.3. Besides an initial stress $\sigma_{0}$ of $10 \mathrm{MPa}$ (which in combination with the weak layer parameters results in severe squeezing conditions) also a low initial stress was considered $(0.75 \mathrm{MPa})$ in order to check, whether the statements of this section also apply to slightly (or non-)squeezing conditions. (For the lower initial stress and the assumed strength parameters, both the hard and the weak layers behave elastically.) 

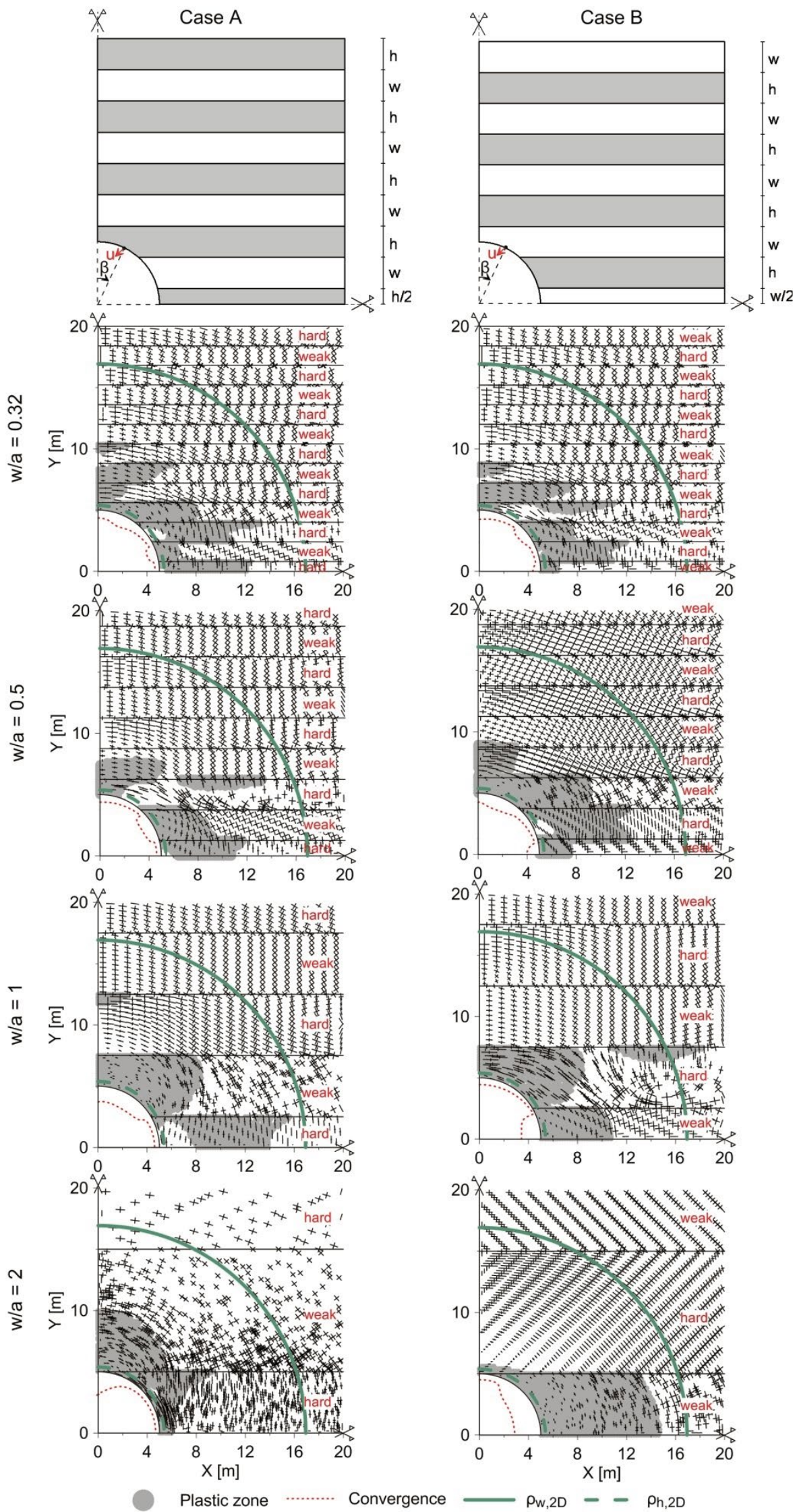

Figure 10.1. Convergence $u$ (magnified by factor 5), plastic zone (hatched area) and principal stress orientation for different thickness and location of the weak and of the hard layers $\left(\sigma_{0}=10 \mathrm{MPa}, a=5 \mathrm{~m}, h / w=1, E_{h}=10 \mathrm{GPa}, c_{h}=5 \mathrm{MPa}, E_{w}=0.5 \mathrm{GPa}, c_{w}=0.5 \mathrm{MPa}\right.$, $\left.v_{h}=v_{w}=0.3, \varphi_{h}=\varphi_{w}=25^{\circ}, \psi_{h}=\psi_{w}=5^{\circ}\right)$. 

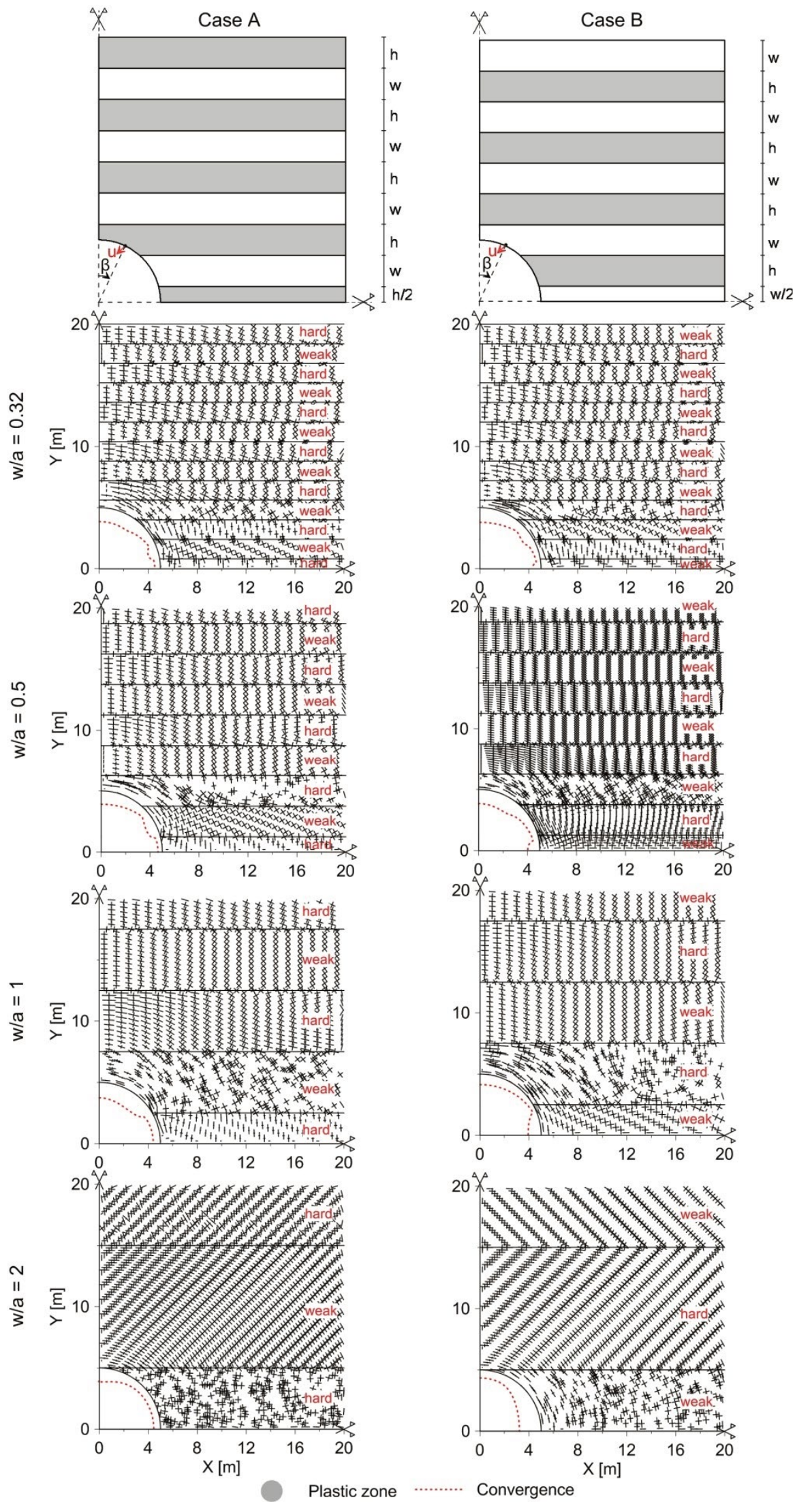

Figure 10.2. Convergence $u$ (magnified by factor 250), plastic zone (hatched area) and principal stress orientation for different thickness and location of the weak and of the hard layers $\left(\sigma_{0}=0.75 \mathrm{MPa}, a=5 \mathrm{~m}, h / w=1, E_{h}=10 \mathrm{GPa}, c_{h}=5 \mathrm{MPa}, E_{w}=0.5 \mathrm{GPa}, c_{w}=0.5 \mathrm{MPa}\right.$, $\left.v_{h}=v_{w}=0.3, \varphi_{h}=\varphi_{w}=25^{\circ}, \psi_{h}=\psi_{w}=5^{\circ}\right)$. 
For simplicity, an unsupported tunnel crossing equally thick weak and hard layers will be considered first; the effect of the thickness ratio $h / w$ will be discussed later.

Figure 10.1 shows the deformed tunnel boundary (red dotted line), the stress trajectories (crosses) and the plastic zone (grey) for a series of w/a-ratios, two only slightly different layer sequences ("case A/B"; see sketches at the top) and an initial stress of $10 \mathrm{MPa}$. For comparison, also the plastic radius of a homogeneous isotropic material (with the parameters either of the weak or of the hard layers) is presented (green lines). Figure 10.3 shows the distribution of the magnitude of the displacement vector along the tunnel boundary for the same w/a-ratios as Figure 10.1 and the mentioned cases $A$ and B. Figures 10.2 and 10.4 present the same results for the lower initial stress of $0.75 \mathrm{MPa}$. The following conclusions can be drawn from these figures:

- The exact location of the layers in the tunnel profile can affect considerably the displacement distribution, particularly if the layers are not very thin; compare Case $A$ (hard layer at the springline) with Case B (weak layer at the springline) in Figures 10.1 to 10.4 .

- The principal stress axes deviate from the radial and tangential direction, indicating an arching in the weak layers against the hard layers, leading thus to an additional loading of the latter (Figs. 10.1 and 10.3).

- The stabilising effect (but also the overstressing) of the hard layers is particularly evident for thin layers under a high initial stress; the plastic zone would be considerably more extended in the absence of the hard layers and much more narrow in the absence of the weak layers.

- Due to arching, the maximum displacements for $\sigma_{0}=10 \mathrm{MPa}$ amount to about $25 \%$ of those that would develop in the absence of the hard layers (Fig. 10.3). The stabilising effect of the latter is smaller for $\sigma_{0}=0.75 \mathrm{MPa}\left(u_{\max } / u_{\mathrm{w}, 2 D} \approx 0.55\right.$; Fig. 10.4).

- With decreasing layer thickness, the results of the discrete models converge to those of the homogenised models (Figs. 10.3 and 10.4). In these example, homogenisation is adequate if $w / a=h / a<0.16$.

- Expectedly, the displacements along the tunnel profile are very non-uniform even for the homogenised model. This is such because the composite stiffness perpendicular to the layers is lower than parallel thereto (cf., e.g., Bobet 2011, Vu et al. 2013).

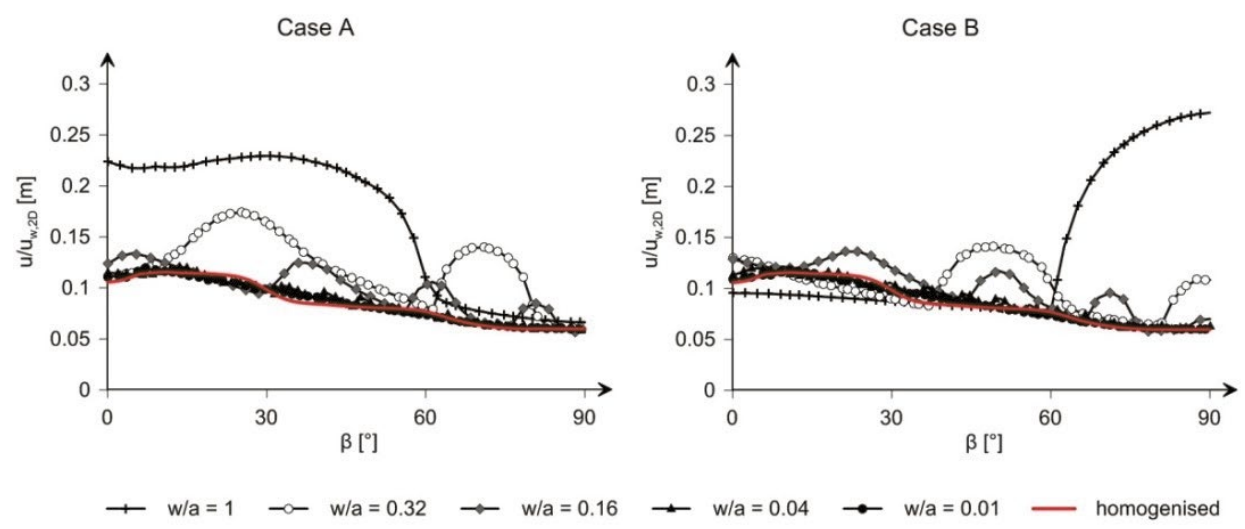

Figure 10.3. Magnitude of the displacement vector along the tunnel boundary according to the homogenised model (red line) as well as according to the discrete models (black lines; cf. inset of Fig. 10.1; $\sigma_{0}=10 \mathrm{MPa}, a=5 \mathrm{~m}, h / w=1, E_{h}=10 \mathrm{GPa}, c_{h}=5 \mathrm{MPa}, E_{w}=0.5 \mathrm{GPa}$, $\left.c_{w}=0.5 \mathrm{MPa}, v_{h}=v_{w}=0.3, \varphi_{h}=\varphi_{w}=25^{\circ}, \psi_{h}=\psi_{w}=5^{\circ}\right)$. 


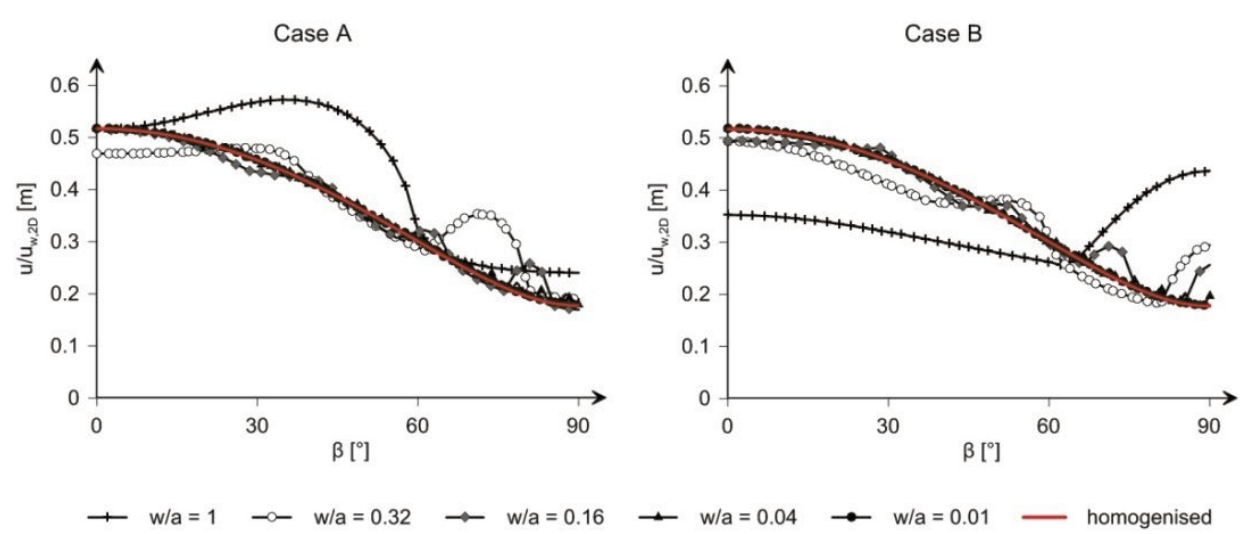

Figure 10.4. Magnitude of the displacement vector along the tunnel boundary according to the homogenised model (red line) as well as according to the discrete models (black lines; cf. inset of Fig. 10.2; $\sigma_{0}=0.75 \mathrm{MPa}, a=5 \mathrm{~m}, h / w=1, E_{h}=10 \mathrm{GPa}, c_{h}=5 \mathrm{MPa}, E_{w}=0.5 \mathrm{GPa}$, $\left.c_{w}=0.5 \mathrm{MPa}, v_{h}=v_{w}=0.3, \varphi_{h}=\varphi_{w}=25^{\circ}, \psi_{h}=\psi_{w}=5^{\circ}\right)$.

In order to estimate the applicability limit of the homogenisation, it is sufficient to consider the maximum and the minimum displacement at the tunnel boundary. The I.h.s. diagrams of Figures 10.5 and 10.6 show (for $\sigma_{0}=10$ and $0.75 \mathrm{MPa}$, respectively) these displacements as a function of the weak layer thickness $w / a$ and of the thickness-ratio $h / w$. The homogenised solution (which is strictly correct only for w/a $\rightarrow 0$ or $h / a \rightarrow 0$ ) is marked by the red symbols on the ordinate axis.

The middle and r.h.s. diagrams of the Figures 10.5 and 10.6 show the ratio $u_{\max } / u_{\max }$ hom as a function of the normalised layer thickness $w / a$ and $h / a$, respectively, for a series of thickness ratios $h / w$. The error of the homogenised solution is zero if the ratio $u_{\max } / u_{\max , h o m}=1$ and increases with the ratio $u_{\max } / u_{\max , h o m}$.

From these diagrams one may conclude that the error increases with $h / w$, particularly in the case of the high initial stress (Fig. 10.5); for the low initial stress of $0.75 \mathrm{MPa}$, the displacements $u_{\max , h o m}$ are very close to $u_{w, 2 D}$. The homogenised solution $u_{\max , h o m}$ becomes considerably smaller than $u_{w, 2 D}$ only when the weak layers begin to yield. Consequently, the case of $\sigma_{0}=10 \mathrm{MPa}$ is decisive for estimating the applicability limit of the homogenisation.

Analogous to Section 5.7, a general statement can be made based on the ratio $h / a$ (r.h.s. diagrams of Fig. 10.5): For $h / a<0.05$, the ratio $u_{\max } / u_{\max }$,hom is smaller than 1.25 (and consequently the error is less than $20 \%$ ) for all $h / w$-values. Would the rock parameters, however, be less different than in the examined extreme case, the error when using the homogenised model would be smaller.

If the layers were oriented perpendicular to the tunnel axis, homogenisation would be adequate for $h / a<$ ca. 0.10 (see results of Section 5.7 for $\sigma_{0}=10 \mathrm{MPa}$ ). In the present case (strike parallel to the tunnel axis) the layers would have to be thinner. Homogenisation is thus easier in the case of tunnelling perpendicular to the layers, because the stabilising effect of the hard layers is more pronounced ( $u_{\min }$ becomes considerably larger and $u_{\max }$ smaller, so that the values are closer to the homogenised solution).

Due to this smaller stabilising effect of the hard layers in the case of tunnelling parallel to the layers, the influence of the brittle behaviour of the hard layers is less pronounced than in Chapter 5 and was therefore not considered here. 

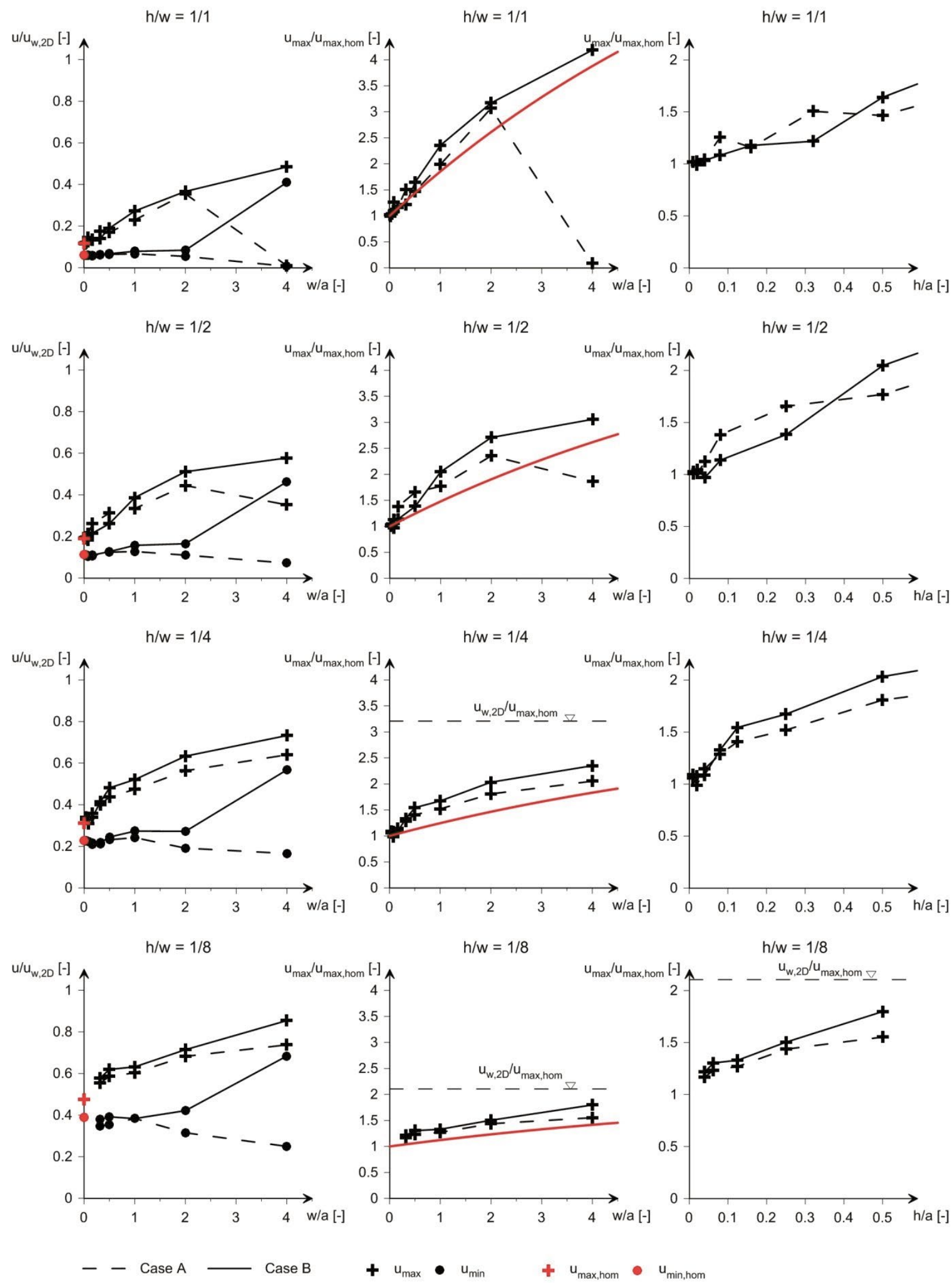

Eq. (10.1)

Figure 10.5. Maximum $u_{\max }$ and minimum $u_{\min }$ displacements (normalised by the displacements of a homogeneous weak ground $\left.u_{w, 2 D}\right)$, compared with the displacements of the maximum $u_{\text {max,hom }}$ and minimum $u_{\text {min,hom }}$ displacements of the homogenised solution $\left(\sigma_{0}=10 \mathrm{MPa}\right.$, $a=5 \mathrm{~m}, E_{h}=10 \mathrm{GPa}, c_{h}=5 \mathrm{MPa}, E_{w}=0.5 \mathrm{GPa}, c_{w}=0.5 \mathrm{MPa}, v_{h}=v_{w}=0.3, \varphi_{h}=\varphi_{w}=25^{\circ}$, $\psi_{h}=\psi_{w}=5^{\circ}$; cases A and B: see inset at the top of Fig. 10.1). 

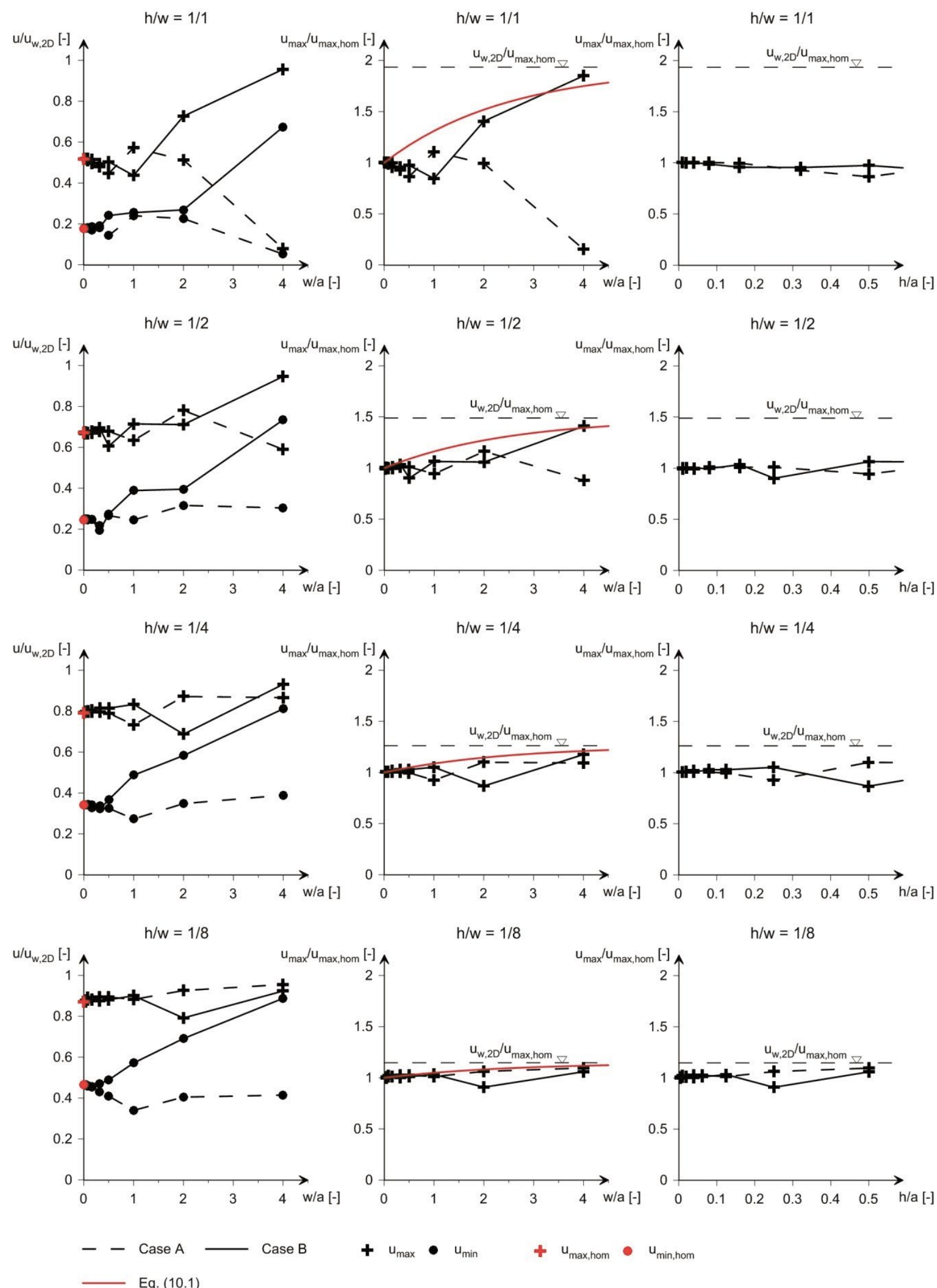

Figure 10.6. Maximum $u_{\max }$ and minimum $u_{\min }$ displacements (normalised by the displacements of a homogeneous weak ground $\left.u_{w, 2 D}\right)$, compared with the displacements of the maximum $u_{\text {max,hom }}$ and minimum $u_{\text {min,hom }}$ displacements of the homogenised solution $\left(\sigma_{0}=0.75 \mathrm{MPa}\right.$, $a=5 \mathrm{~m}, E_{h}=10 \mathrm{GPa}, c_{h}=5 \mathrm{MPa}, E_{w}=0.5 \mathrm{GPa}, c_{w}=0.5 \mathrm{MPa}, v_{h}=v_{w}=0.3, \varphi_{h}=\varphi_{w}=25^{\circ}$, $\psi_{h}=\psi_{w}=5^{\circ}$; cases A and B: see inset at the top of Fig. 10.2). 


\subsection{Tunnelling parallel to the interface between a weak and a competent formation}

In this section, the theoretical case of infinite layer thickness (w/a $\rightarrow \infty$ and $h / a \rightarrow \infty$ ) will be investigated. The distribution of the deformations in the tunnel profile depends on the distance of the formation interface from the tunnel: If the interface intersects (or is close to) the tunnel, then the displacements of the tunnel profile will be non-uniform (cf. Fig. 10.8).

This section investigates numerically, how far the formation interface has to be in order that the heterogeneity of the ground can be neglected (i.e., in order to assume $u_{w, 2 D}$ for the weak formation or $u_{h, 2 D}$ for the hard formation, respectively). A weak formation overlain by a competent formation will be considered. The formation interface is located at a distance $H$ above the tunnel axis (with $H<0$ meaning that the interface lies below the tunnel axis). Figure 10.8 shows the deformed profile (red dotted line), the stress trajectories and the plastic zone for a series of $H$-values. For the considered rock parameters and initial stress (10 MPa), the competent formation has a remarkable effect only if it crosses partially the tunnel.

The Figure 10.7 shows the results of a parametric study into the effect of the distance $H$ of the hard formation to the tunnel axis. Specifically, the diagram shows the maximum $\left(u_{\max }\right)$ and the minimum $\left(u_{\min }\right)$ displacements of the tunnel cross-section, normalised by the convergence $u_{w, 2 D}$ (upper diagrams) and $u_{h, 2 D}$ (lower diagrams), which would develop in a homogeneous weak or competent ground, respectively. If $H>5 a$, the stabilising effect of the hard formation is negligible $\left(u_{\min } / u_{w, 2 D} \approx 0.8\right.$; upper diagram of Fig. 10.7) and, vice versa, if $H<-5 a$, then the unfavourable effect of the nearby weak formation can be neglected $\left(u_{\max } / u_{h, 2 D} \approx 1.2\right.$; lower diagram of Fig. 10.7). These results can be regarded as generally valid, because they assume a big contrast in the mechanical parameters of the two zones and a high initial stress.
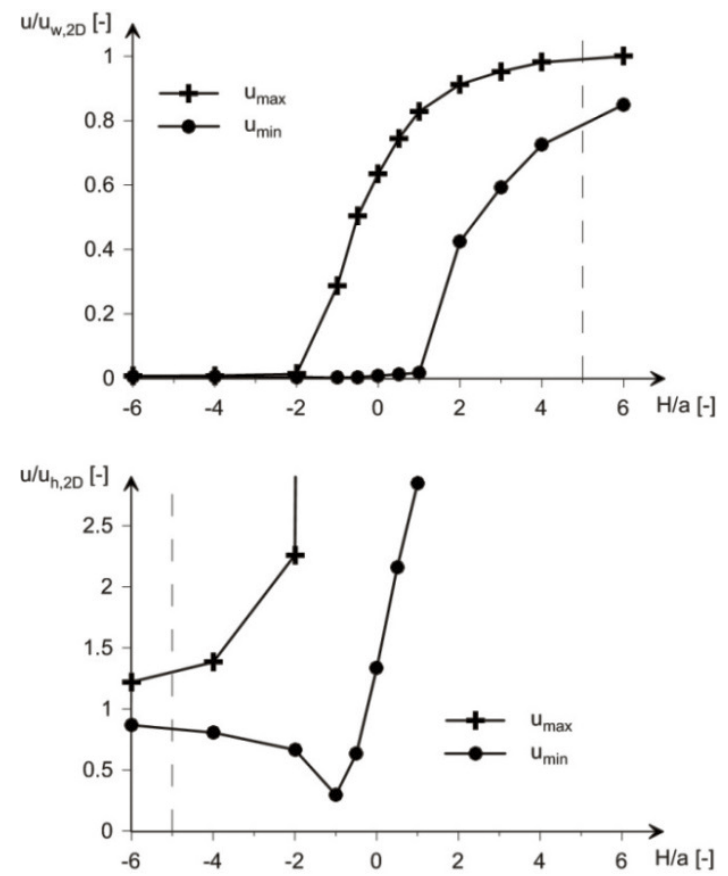

Figure 10.7. Maximum $u_{\max }$, minimum $u_{\min }$ displacements of the tunnel profile (normalised by the convergence $U_{w, 2 D}$ or $U_{h, 2 D}$ which would develop in a homogeneous weak or hard ground, respectively) for different distances $H$ of the hard formation to the tunnel axis (cf. Fig. 10.8; $\sigma_{0}=10 \mathrm{MPa}, a=5 \mathrm{~m}, E_{h}=10 \mathrm{GPa}, c_{h}=5 \mathrm{MPa}, E_{w}=0.5 \mathrm{GPa}, c_{w}=0.5 \mathrm{MPa}, v_{h}=v_{w}=0.3$, $\varphi_{h}=\varphi_{w}=25^{\circ}, \boldsymbol{\psi}_{h}=\boldsymbol{\psi}_{w}=5^{\circ}$ ). 

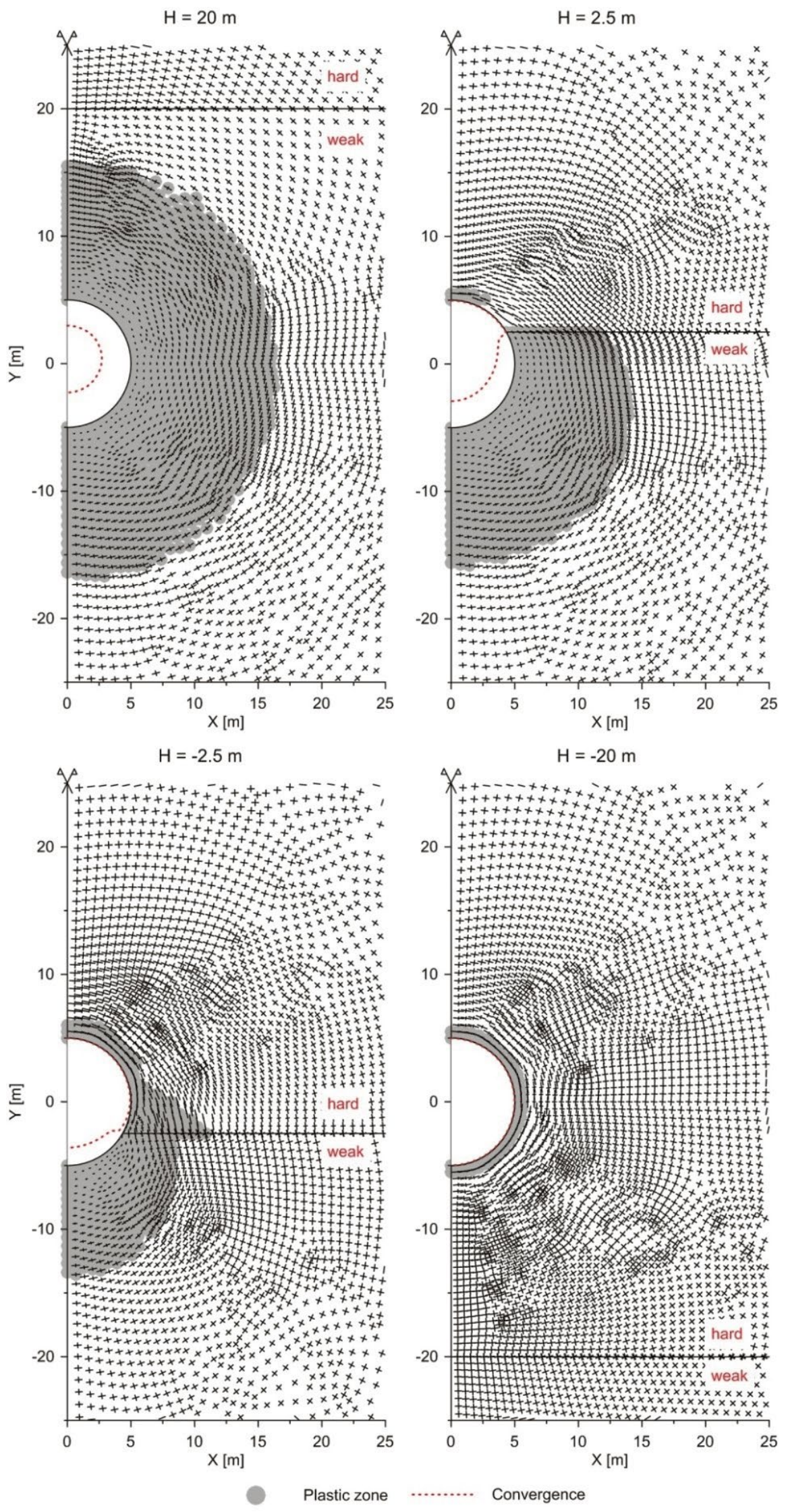

Figure 10.8. Convergence $u$ (magnified by factor 2.5), plastic zone (hatched area) and principal stress orientation for different distances $H$ of the hard formation to the tunnel axis $\left(\sigma_{0}=10 \mathrm{MPa}\right.$, $a=5 \mathrm{~m}, E_{h}=10 \mathrm{GPa}, c_{h}=5 \mathrm{MPa}, E_{w}=0.5 \mathrm{GPa}, c_{w}=0.5 \mathrm{MPa}, V_{h}=v_{w}=0.3, \varphi_{h}=\varphi_{w}=25^{\circ}$, $\left.\boldsymbol{\Psi}_{h}=\boldsymbol{\Psi}_{w}=5^{\circ}\right)$. 


\subsection{Alternating weak and competent layers of medium thickness}

In this section, it is investigated whether the equation

$$
\frac{u_{\max }}{u_{w, 2 D}}=\delta \cdot\left(1-\frac{u_{\text {max }, h o m}}{u_{w, 2 D}}\right)+\frac{u_{\text {max }, \text { hom }}}{u_{w, 2 D}},
$$

where

$$
\delta=1-e^{-0.4 w / \rho_{w, 2 D}}
$$

which is practically identical with the one proposed in Chapter 7 for tunnelling perpendicularly to alternating weak and hard layers, can also be applied for estimating the maximum displacements in the case of tunnelling parallel to the layers. The red solid lines in the middle diagrams of Figures 10.5 and 10.6 show the displacements obtained with this equation. It is remarkable that this simple equation underestimates only slightly the maximum displacements for $w / a$ smaller than a value of ca. 2 (by less than $25 \%$ for $\sigma_{0}=10 \mathrm{MPa}$, and even lesser for $\sigma_{0}=0.75 \mathrm{MPa}$ ). A better agreement could be achieved by selecting another constant (larger than 0.4) in the exponent of the last r.h.s. term of Equation (10.2). A definitive statement cannot be made, however, without checking Equation (10.1) for a wide parameter range, analogously to Chapter 7 .

\subsection{Conclusions}

The homogenised model is adequate only if the hard layers are thinner than $5 \%$ of the tunnel radius. As this criterion is valid both for a tunnel parallel and a tunnel perpendicular to the layers, it seems that this criterion is applicable for any orientation of the layers to the tunnel axis.

For very thick formations, the influence of the adjacent hard or weak formation can be neglected, if the weak or the hard rock formation lies at a distance to the tunnel axis of at least 5 times the tunnel radius. Otherwise, numerical calculations have to be performed in order to estimate the displacements of the tunnel profile.

For medium layer thicknesses, depending on the location of the layers in the tunnel profile, the tunnel displacements may vary considerably. Therefore, numerical calculations have to be performed that consider the exact location of the weak and the hard layers in the profile.

\section{Notation}

$\begin{array}{ll}a & \text { tunnel radius } \\ C_{h}, C_{w} & \text { cohesion of the hard and of the weak layers } \\ E_{h}, E_{w} & \text { Young's modulus of the hard and of the weak layers } \\ h & \text { thickness of the hard layers } \\ H & \text { distance of the hard formation to the tunnel axis } \\ r & \text { radius, i.e., distance to the tunnel axis } \\ u & \text { magnitude of the displacement vector at the tunnel boundary }\end{array}$




\begin{tabular}{|c|c|}
\hline$u_{\text {average }}, u_{\max }, u_{\min }$ & $\begin{array}{l}\text { averaged, maximum and minimum magnitude of the displacement } \\
\text { vector along the tunnel boundary }\end{array}$ \\
\hline$U_{\text {average,hom, }} U_{\max , h o m}$ & $\begin{array}{l}\text { averaged and maximum magnitude of the displacement vector of the } \\
\text { homogenised rock mass along the tunnel boundary }\end{array}$ \\
\hline$u_{\min , h o m}$ & $\begin{array}{l}\text { minimum magnitude of the displacement vector of the homogenised } \\
\text { rock mass along the tunnel boundary }\end{array}$ \\
\hline$U_{h, 2 D}$ & radial displacement of the homogeneous competent ground \\
\hline$U_{w, 2 D}$ & radial displacement of the homogeneous weak ground \\
\hline$w$ & thickness of the weak layers \\
\hline$X$ & co-ordinate \\
\hline Y & co-ordinate \\
\hline$\beta$ & $\begin{array}{l}\text { angle between the tangential direction of the tunnel and the schis- } \\
\text { tosity plane }\end{array}$ \\
\hline$\delta$ & reduction factor \\
\hline$\rho_{h, 2 D}$ & radius of the plastic zone of the homogeneous competent ground \\
\hline$\rho_{w, 2 D}$ & radius of the plastic zone of the homogeneous weak ground \\
\hline$\sigma_{0}$ & initial stress \\
\hline$v_{h}, v_{w}$ & Poisson's ratio of the hard and of the weak layers \\
\hline$\varphi_{h}, \varphi_{w}$ & iction angle of the hard and of the weak layers \\
\hline$\psi_{h}, \psi_{w}$ & angle of the hard and of the weak layers \\
\hline
\end{tabular}





\section{Response of a schistous rock mass striking parallel to the tunnel axis}

\subsection{Introduction}

From the literature it is well known that planes of anisotropy may affect rock behaviour adversely, particularly if their strike direction forms a small angle with (or is parallel to) the tunnel axis (Barla 2002, Goricki et al. 2005). This chapter will thus focus on the most adverse situation, which is tunnelling parallel to schistosity; the influence of the angle between schistosity plane and tunnel axis will be investigated in Chapter 12.

The influence of the planes of anisotropy, especially the schistosity planes, on the tunnel displacements was already described in a number of publications. On the one hand, the measured displacements occurring in some particular tunnel sections were examined empirically as well as numerically (Panet 1996, Thuro et al. 2004, Huber et al. 2005, Merlini and Falanesca 2013, Malaguti et al. 2013, etc.; see also Chapter 1). On the other hand, the influence of the schistosity for the tunnel problem was investigated analytically (Daemen 1983) and numerically (Shen and Barton 1997, Hsu et al. 2004, Button et al. 2006, Leitner et al. 2006, Wang and Huang 2014, etc.). All investigations could show that the geometrical and mechanical properties of schistosity play an important role for the development of the displacements.

The influence of the discontinuities on the tunnel displacements is investigated in the literature either by continuum or by discontinuum models. In the discontinuum models, planes of anisotropy are explicitly represented in the numerical model, while in the continuum models, a homogenised rock mass is considered. As this chapter will focus on schistous rocks (e.g., phyllites and schists) that are characterised by a large number of very closely spaced discontinuities, a continuum model is applied (cf. Sulem and Mühlhaus 1997). With the continuum models, an opening of discontinuities or a complete detachment of the elements cannot be simulated (cf. Jing 1998, Barla and Barla 2000, Lisjak et al. 2014). However, these mechanisms are relevant mostly for stability problems (e.g., loosening or rock fall) and less for the analysis of squeezing (where large tangential stresses around the tunnel occur). For thinly layered rocks, a continuum model can adequately represent ground behaviour (Clark 2006, Leitner et al. 2006, Sainsbury et al. 2008). Of course, with this kind of model, the influence of spacing cannot be investigated (cf. Leitner et al. 2006). However, from Leitner et al. (2006), it is known that the spacing may have an important influence on the tunnel displacements. This chapter will focus on schistous rocks characterised by very small spacing, which is the most critical case (cf. Leitner et al. 2006). 
In the current state of research a systematic, quantitative investigation of the influence of the properties of the schistosity planes on squeezing behaviour is missing. Furthermore, one misses practical guidelines on how this influence can be considered in the design.

From the mechanical point of view, schistosity can be conceived as the borderline case of a stratified rock mass with extremely thin weak layers. Therefore, the constitutive model of Section 9.2 could be used also in order to analyse the response of a schistous rock to tunnel excavation. This can be seen also in Figure 11.1, which presents the uniaxial compressive strength as a function of the angle $\delta$ (defined as the angle between the direction of the maximum principal stress and the schistosity planes). For a tunnel drive parallel to the layers, the angle $\delta$ varies along the tunnel boundary from $0^{\circ}$ (at the crown for a horizontal stratification) to $90^{\circ}$ (at the side wall). Therefore, for a tunnel drive parallel to the planes of anisotropy, the influence of the weak layers does not vanish with decreasing thickness fraction of the weak layers. If the latter is very small $\left(x_{w}=0.01\right)$, the rock behaviour converges to the known behaviour of schistous rocks (which can be obtained analytically according to Jaeger and Cook 1976). With decreasing thickness fraction of the weak layers, the horizontal stiffness approaches the vertical stiffness of the composite, so that $E_{1}=E_{2}=E_{h}$ (cf. Eqs. 9.4 and 9.5).

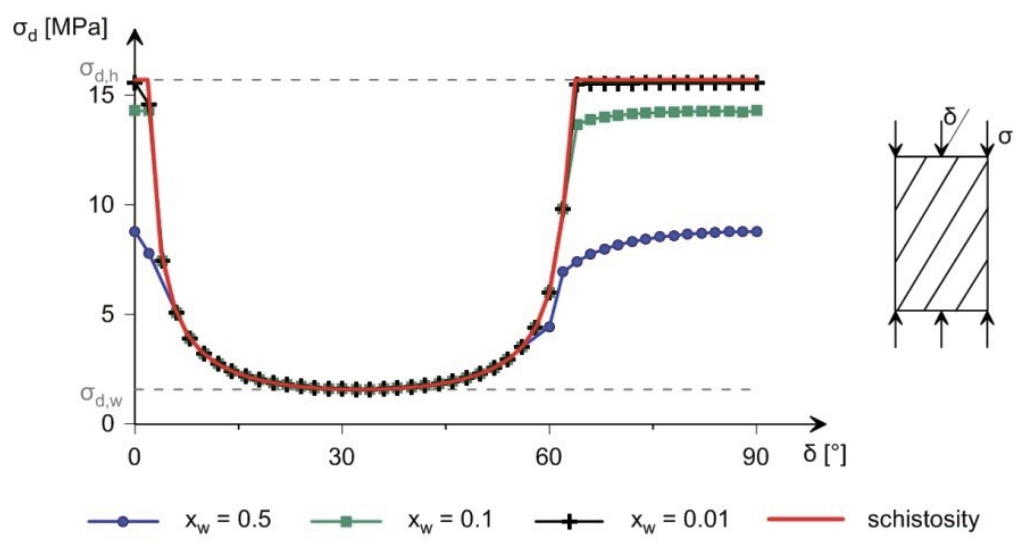

Figure 11.1. Uniaxial compressive strength $\sigma_{d}$ of the stratified rock mass depending on the direction of loading for different thickness fractions $x_{w}$ of the weak layers, obtained with Lydzba et al. (2003) and compared to the analytical uniaxial compressive strength for the schistosity according to Jaeger and Cook (1976) $\left(E_{h}=10 \mathrm{GPa}, c_{h}=5 \mathrm{MPa}, E_{w}=1 \mathrm{GPa}, c_{w}=0.5 \mathrm{MPa}\right.$, $\left.v_{h}=v_{w}=0.3, \varphi_{h}=\varphi_{w}=25^{\circ}, \psi_{h}=\psi_{w}=5^{\circ}\right)$.

The displacements in a tunnel drive parallel to the layers can be obtained by numerical calculations, as in Chapter 9, with the constitutive model of the stratified rock mass. The results (Fig. 11.2) were compared to those obtained with constitutive models for the schistosity implemented in the commercially available software programs Plaxis (Brinkgreve et al. 2016; considering a Mohr-Coulomb failure criterion for the rock mass; more details concerning the implementation and verification: see Azami et al. 2012 and Azami et al. 2013) and Abaqus (Dassault Systèmes 2011; considering a Drucker-Prager failure criterion for the rock mass and determining the Drucker-Prager material parameters with a plane strain matching to the Mohr-Coulomb parameters). The results of all the constitutive models are in good agreement. However, if $x_{w}$ approaches the value of zero, the constitutive model of Section 9.2 is extremely costly in terms of computer time.

Therefore, in Section 11.2, a constitutive model for the schistous rock mass is formulated and then validated for a series of boundary problems. By means of this constitutive model, in 
Section 11.3, numerical calculations (assuming plane strain conditions) were carried out, in order to analyse the response of schistous rocks striking parallel to the tunnel axis. Based on these investigations, dimensionless diagrams are presented in Section 11.4 that allow a quick estimation of the displacements (occurring far behind the tunnel face) to be made for a wide range of geotechnical conditions. Finally, Section 11.5 shows that considering an isotropic homogeneous model with appropriate parameters can be very valuable in spite of its inherent limitations and differences from a transversely isotropic rock mass.

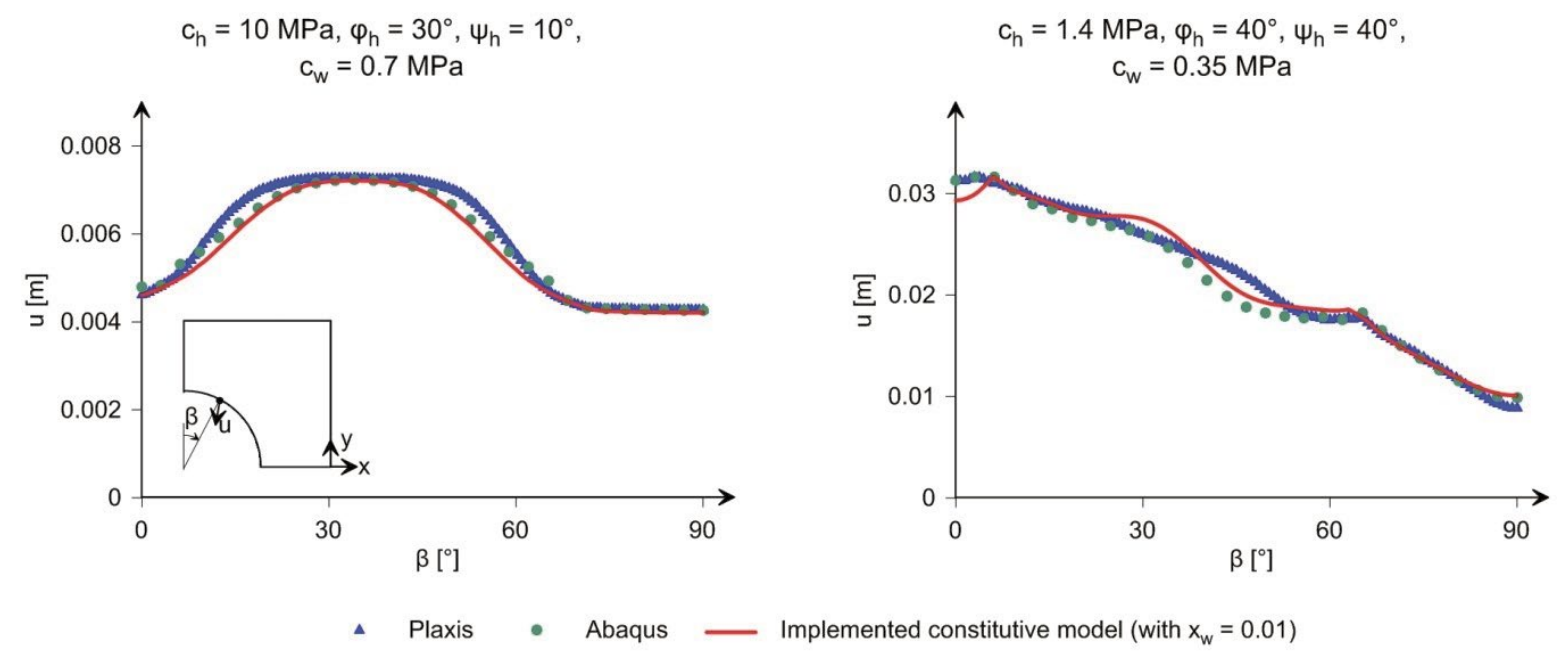

Figure 11.2. Magnitude of the displacement vector along the tunnel boundary obtained with the constitutive model of Section 9.2 and those implemented in Plaxis and Abaqus (unsupported tunnel, $a=5 \mathrm{~m}, \sigma_{0}=10 \mathrm{MPa}, E_{h}=E_{w}=20 \mathrm{GPa}, \varphi_{w}=20^{\circ}, \boldsymbol{\psi}_{w}=20^{\circ}, v_{h}=v_{w}=0.3$ ).

\subsection{Constitutive model}

\subsubsection{Formulation of the constitutive model}

The mechanical behaviour of anisotropic rock mass was investigated over the last decades by many authors, particularly by means of laboratory tests. The main focus of these studies was the directional dependence of rock strength. An extensive review on this topic can be found in Pietruszczak et al. (2002) or Duveau et al. (1998).

Besides analysing the mechanical behaviour of anisotropic rock mass, various failure criteria have been proposed (cf. Duveau et al. 1998, Ambrose 2014). Amongst these, the most representative model is the so-called "single plane of weakness theory" proposed by Jaeger (1960). Jaeger (1960) considers a (Mohr-Coulomb) failure criterion for the rock matrix and one for the planes of weakness:

$$
\begin{gathered}
\tau=c_{m}+\sigma \tan \left(\varphi_{m}\right), \\
\tau=c_{s}+\sigma \tan \left(\varphi_{s}\right),
\end{gathered}
$$

respectively. In order to model the schistous rocks realistically, elasto-plastic behaviour of the matrix should be considered (cf. Lisjak et al. 2014). Duveau et al. (1998) could show that there is a good agreement between the model of Jaeger (1960) and experiments. The model of Jaeger (1960) was further developed by many authors (e.g., Walsh and Brace 1964, Hoek 
and Brown 1980), leading however to more complex failure criteria, which require more parameters than usually available, while the material model of Jaeger (1960) can be described by means of a small number of easily interpretable material parameters, even in the case of anisotropy (Kovári 1977).

A schistous rock mass may be stiffer for loading parallel to the schistosity than perpendicular thereto (Amadei 1996, Park and Min 2015, Ismael and Konietzky 2017). For the sake of simplicity, stiffness anisotropy is not considered here.

Constitutive models, which consider the failure of the rock mass and the planes of weakness according to the material model of Jaeger (1960) have already been formulated (e.g., Kovári 1977, Chen 1986, Cai and Horii 1993, Itasca Consulting Group 2007, Xu et al. 2010, Zhao and $\mathrm{He} 2011$ ) and implemented in the commercially available software programs Plaxis (Brinkgreve et al. 2016) and Abaqus (Dassault Systèmes 2011). However, in Chapter 13, the squeezing variability due to orientation changes of the anisotropy planes along the alignment will be investigated, which necessitates to formulate and implement the constitutive model of Jaeger (1960) in Abaqus (Dassault Systèmes 2011), based on the homogenisation technique (see Chapters 5 and 9 ). This section will only show the most essential aspects of the formulation of the constitutive model. More details concerning the formulation of the constitutive model can be found in Appendix F.
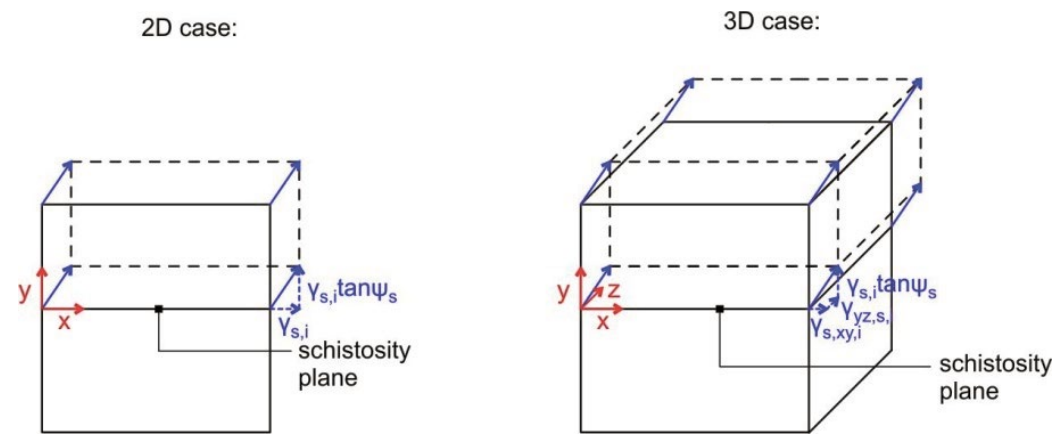

Figure 11.3. REV consisting of a single schistosity plane.

For the derivation of the constitutive law, a representative elementary volume (REV) is considered, which consists of $N$ schistosity planes and the surrounding matrix. The schistosity planes are perpendicular to the $y$-axis (cf. Fig. 11.3). At failure state, an irreversible shear strain $\gamma_{s, i}$ occurs due to slip along the schistosity plane. This, in turn, leads to a normal strain perpendicular to schistosity plane, which amounts to $\gamma_{s, t a n} \psi_{s}$, where $\psi_{s}$ denotes the dilatancy angle of the schistosity (cf. Fig. 11.3). The shear strain of the REV due to failure in the schistosity planes is equal to

$$
\gamma_{s}=N \cdot \gamma_{s, i}
$$

where $N$ denotes the number of schistosity planes in the REV. Of course, slipping may only occur along the schistosity planes. This means that in the $2 \mathrm{D}$ case, the shearing strain increment occurs in $x y$-direction, while in the 3D case, it occurs in $x y$ - and $y z$-direction (cf. Fig. 11.3). In general,

$$
\gamma_{s}=\sqrt{\Delta \gamma_{x y, s}^{2}+\Delta \gamma_{y, s}^{2}},
$$

whereby the last r.h.s. term is equal to zero in the $2 \mathrm{D}$ case. 
By definition, the variation of stresses and strains across the REV can be neglected, meaning that the resulting strains and stresses of the homogenised medium are the volumetric averages of the strain and stress components in the matrix and the schistosity planes. As the thickness fraction of the schistosity planes is small in comparison to the one of the surrounding matrix, the relations for the homogenised stress and strain increments (introduced in the Chapters 5 and 9 ) can be simplified as follows:

$$
\begin{aligned}
& \Delta \sigma_{x x}=\Delta \sigma_{x x, m} \quad \Delta \varepsilon_{x x}=\Delta \varepsilon_{x x, m} \\
& \Delta \sigma_{y y}=\Delta \sigma_{y y, m}=\Delta \sigma_{y y, s} \quad \Delta \varepsilon_{y y}=\Delta \varepsilon_{y y, m}+\gamma_{s} \cdot \tan \psi_{s} \\
& \begin{array}{l}
\Delta \sigma_{z z}=\Delta \sigma_{z z, m} \\
\Delta \tau_{x y}=\Delta \tau_{x y, m}=\Delta \tau_{x y, s}
\end{array} \quad \text { and } \begin{array}{l}
\Delta \varepsilon_{z z}=\Delta \varepsilon_{z z, m} \\
\Delta \gamma_{x y}=\Delta \gamma_{x y, m}+\Delta \gamma_{x y, s}
\end{array} \\
& \Delta \tau_{x z}=\Delta \tau_{x z, m} \quad \Delta \gamma_{x z}=\Delta \gamma_{x z, m} \\
& \Delta \tau_{y z}=\Delta \tau_{y z, m}=\Delta \tau_{y z, s} \quad \Delta \gamma_{y z}=\Delta \gamma_{y z, m}+\Delta \gamma_{y z, s}
\end{aligned}
$$

where the subscript $m$ denotes the strain and stress increments in the matrix and $s$ those in the schistosity planes. As usual in design practice, the rock will be considered as linearly elastic and perfectly plastic, considering a Mohr-Coulomb failure criterion with a nonassociated flow rule for the schistosity and the matrix. For the matrix the stress return algorithm after Clausen (2007) will be used (see also Appendix F).

As mentioned before, in the constitutive model, a REV is considered whose schistosity planes lie perpendicular to the $y$-axis. By performing appropriate coordinate transformations, arbitrary orientations of the schistosity planes can be considered: the stresses and the strains are transformed from the global into the local coordinate system (where the $y$-axis is perpendicular to the planes of schistosity) and, after having performed all the computations in the local coordinate system, the resulting stresses are back-calculated into the global coordinate system. The only input needed for the coordinate transformation is the normal vector of the schistosity planes.

\subsubsection{Rock element behaviour}

In order to illustrate the material behaviour, single element tests were performed considering plane strain conditions. These computations serve also to validate the numerical implementation of the elasto-plastic equations. (Further validation examples are presented in Appendix F.)

According to the analytical solution of Jaeger and Cook (1976), the uniaxial compressive strength of the schistous rock mass under a specific loading direction is the minimum between the one governed by the matrix and the schistosity and reads as follows:

$$
\sigma_{d}=\min \left(\frac{2 \cdot c_{s}}{\sin 2 \delta\left(1-\tan \varphi_{s} \tan \delta\right)} ; \frac{2 \cdot c_{m} \cdot \cos \varphi_{m}}{\left(1-\sin \varphi_{m}\right)}\right) .
$$

The compressive strength reaches its minimum at an angle of $\delta=45-\varphi_{s} / 2=35^{\circ}$ between the schistosity planes and the loading direction and is given by the following equation:

$$
\sigma_{d, s}=\frac{2 \cdot c_{s} \cdot \cos \varphi_{s}}{\left(1-\sin \varphi_{s}\right)}
$$


If the angle $\delta$ is greater than $90^{\circ}-\varphi_{s}=70^{\circ}$ or equals $0^{\circ}$, then failure in the schistosity planes cannot occur. The analytical uniaxial compressive strength is presented in Figure 11.4 and compared with the results obtained with numerical calculations; the schistous rock mass model (and thus the implemented elasto-plastic algorithm) behaves as expected.

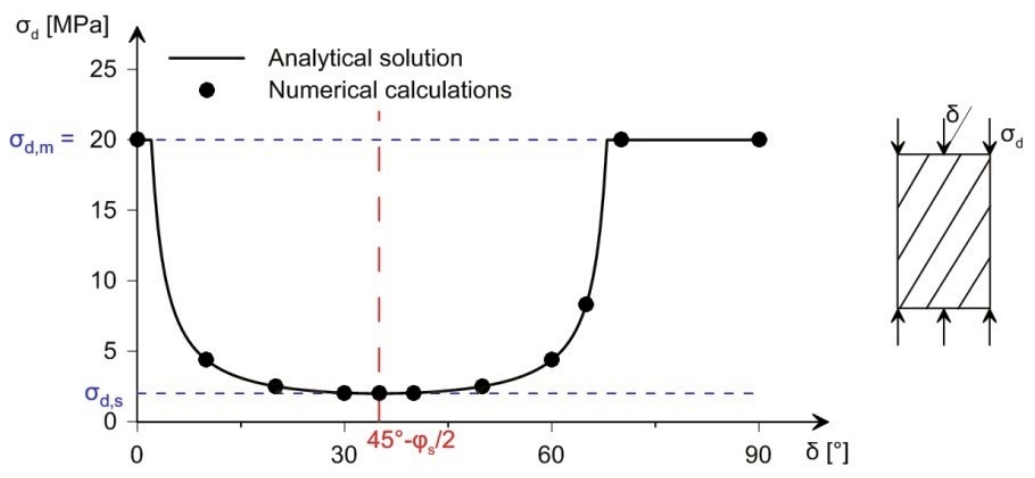

Figure 11.4. Uniaxial compressive strength $\sigma_{d}$ of the REV depending on the direction of loading, obtained with numerical calculations and compared to the analytical solution of Jaeger and Cook (1976) $\left(E=20 \mathrm{GPa}, c_{m}=5.77 \mathrm{MPa}, \varphi_{m}=30^{\circ}, \psi_{m}=10^{\circ}, c_{s}=0.7 \mathrm{MPa}, \varphi_{s}=20^{\circ}, \psi_{s}=20^{\circ}, v=0.3\right)$.

If a lateral pressure $\sigma_{3}$ is applied, the maximum axial strength $\sigma_{1}$ that can be sustained amounts to:

$$
\sigma_{1}=\min \left(\frac{\tan \left(\delta+\varphi_{s}\right)}{\tan (\delta)} \sigma_{3}+\frac{2 \cdot c_{s}}{\sin 2 \delta\left(1-\tan \varphi_{s} \tan \delta\right)} ; \frac{1+\sin \varphi_{m}}{1-\sin \varphi_{m}} \sigma_{3}+\frac{2 \cdot c_{m} \cdot \cos \varphi_{m}}{\left(1-\sin \varphi_{m}\right)}\right)
$$

Therefore, the axial strength increases with the lateral pressures (see, e.g., line for $\sigma_{3}=10 \mathrm{MPa}$ in Fig. 11.5). Furthermore, the range of $\delta$, in which failure in the schistosity planes occurs, decreases. However, the strength minimum occurs still at $\delta=45^{\circ}-\varphi_{s} / 2=35^{\circ}$.

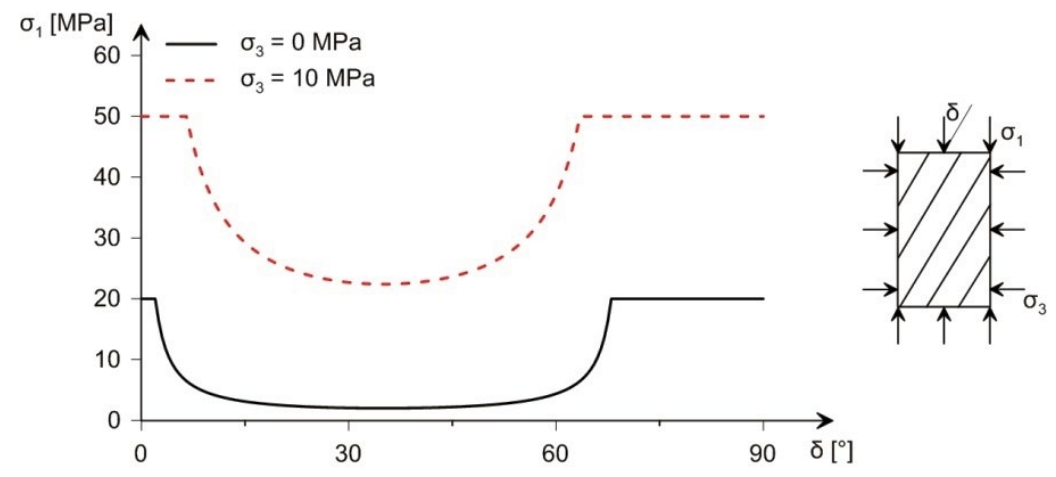

Figure 11.5. Influence of the lateral pressure $\sigma_{3}$ on the maximum axial strength that can be sustained, depending on the direction of loading $\left(E=20 \mathrm{GPa}, c_{m}=5.77 \mathrm{MPa}, \varphi_{m}=30^{\circ}, \Psi_{m}=10^{\circ}\right.$, $\left.c_{s}=0.7 \mathrm{MPa}, \varphi_{s}=20^{\circ}, \psi_{s}=20^{\circ}, v=0.3\right)$.

\subsection{Basic aspects of the schistous rock response to excavation}

In this section, some basic aspects of tunnelling through schistous rock mass will be analysed. 
Considering the failure criterion of the schistosity (Eq. 11.2) and that, in the elastic state, the maximum principal stress (in tangential direction) amounts to $2 \sigma_{0}-\sigma_{a}$ and the minimum principal stress (in radial direction) to $\sigma_{a}$, the support pressure at which failure in the planes of weakness occurs first amounts to

$$
\sigma_{a}=\frac{2 \sigma_{0}-\sigma_{d, s}}{m_{s}-1}=5.9 \mathrm{MPa}, \text { where } m_{s}=\frac{1+\sin \varphi_{s}}{1-\sin \varphi_{s}}
$$

and is located at $\beta=45^{\circ}-\varphi_{s} / 2=35^{\circ}$ (Fig. 11.6; cf. Daemen 1983). The support pressure at which failure in the matrix would occur first amounts to (assuming elastic state and thus no failure in the schistosity):

$$
\sigma_{a}=\frac{2 \sigma_{0}-\sigma_{d, m}}{m_{m}-1}=-7.32 \mathrm{MPa}, \text { where } m_{m}=\frac{1+\sin \varphi_{m}}{1-\sin \varphi_{m}} .
$$

As this value is negative, it can be assumed that no failure in the matrix will occur, even for $\sigma_{a}=0 \mathrm{MPa}$, as the largest deviatoric stresses occur in the elastic state. In reality, due to the failure in the planes of schistosity, the deviatoric stresses would be smaller than in the elastic state and thus the support pressure, at which failure in the matrix occurs, would be even smaller.

With a further decrease in support pressure, the plastic zone increases at the segment $0^{\circ}<\beta<90^{\circ}-\varphi_{s}$ (as failure at $\beta=0^{\circ}$ and $\beta \geq 90^{\circ}-\varphi_{s}$ is not possible, cf. Section 11.2.2). Due to failure in these areas, stress redistribution occurs around the plastic zone, so that the principal stress axes are not tangential and radial anymore (see results for $\sigma_{a}=3.5 \mathrm{MPa}$ in Fig. 11.6).
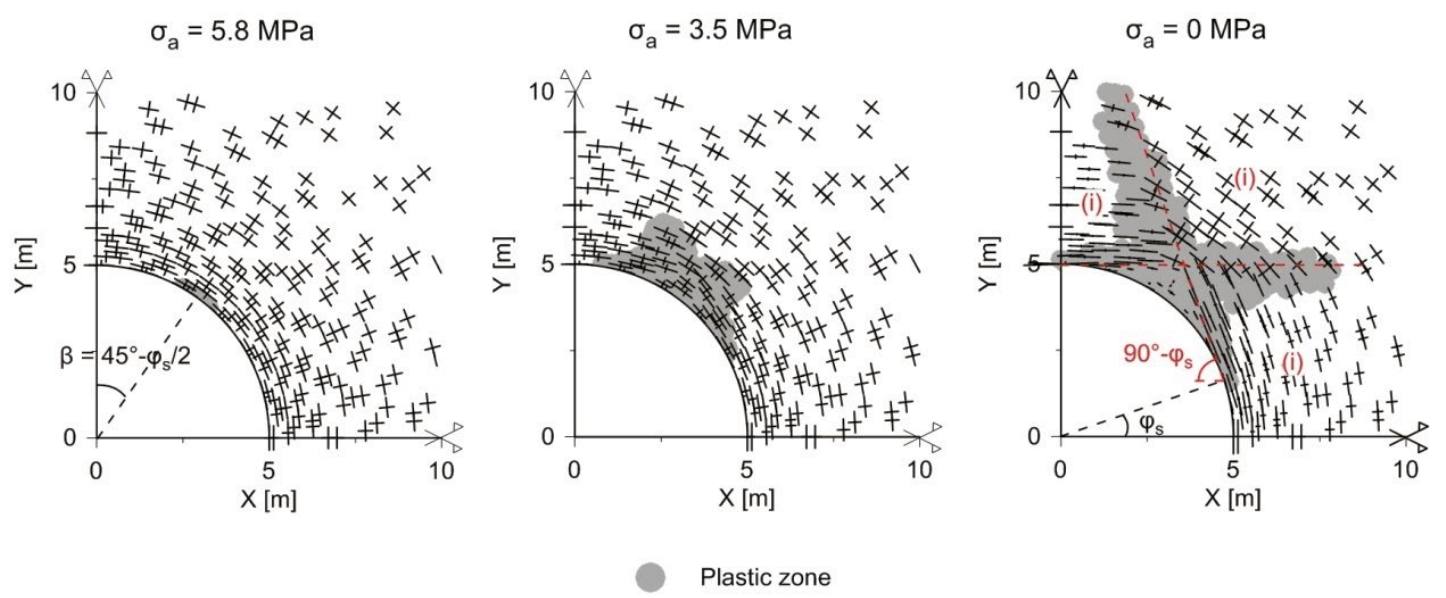

Figure 11.6. Principal stress orientation as well as plastic zone for different support pressures $(a=5 \mathrm{~m}$, $\sigma_{0}=10 \mathrm{MPa}, E=20 \mathrm{GPa}, c_{m}=10 \mathrm{MPa}, \varphi_{m}=30^{\circ}, \psi_{m}=10^{\circ}, c_{s}=0.7 \mathrm{MPa}, \varphi_{s}=20^{\circ}, \psi_{s}=20^{\circ}$, $v=0.3)$.

Due to this stress redistribution, three areas can be distinguished with respect to the direction of the principal axes (see results for $\sigma_{a}=0 \mathrm{MPa}$ in Fig. 11.6): (i) the area of the crown of the tunnel, where the maximum principal stress is horizontal; (ii) the area around $\beta=45^{\circ}-\varphi_{s} / 2$, where the maximum principal stress forms an angle of about $45^{\circ}-\varphi_{s} / 2$ with the horizontal; and, (iii), the area besides the tunnel, where the angle of the maximum principal stress to the horizontal is greater than $90^{\circ}-\varphi_{s}$. As in the area (ii), the minimum principal stress is nearly as large as the maximum principal stress, no failure can occur there. Furthermore, due to the 
fact that failure in the schistosity may only occur at an angle $\beta$ between $0^{\circ}$ and $90^{\circ}-\varphi_{s}$, the failure in the schistosity can solely propagate in the proximity of the two red lines drawn in Figure 11.6, which indicate the respective borders of the areas introduced before. The extent of the plastic zone along these two lines is approximately the same, so that the largest displacements occur exactly at $\beta=45^{\circ}-\varphi_{s} / 2=35^{\circ}$ (cf. Fig. 11.7).

The influence of the rock parameters on the tunnel displacements will be discussed by the results of a parametric study (Figs. 11.7 and 11.8).

Influence of $c_{s}\left(\right.$ where $\left.\varphi_{\mathrm{s}}=\psi_{\mathrm{s}}=20^{\circ}\right)$

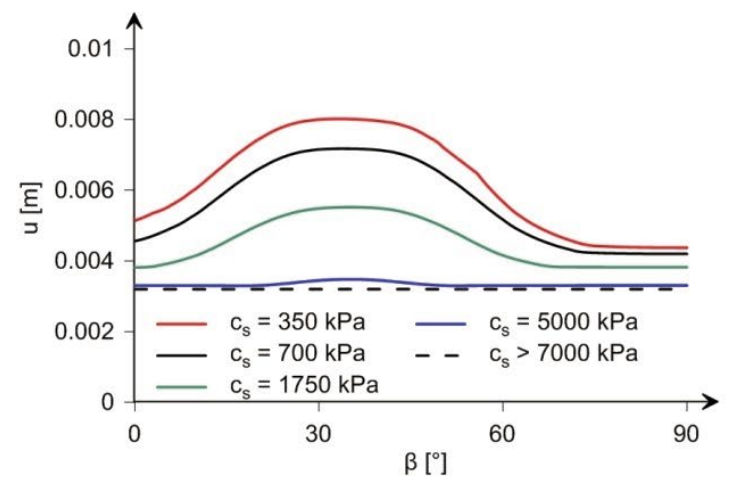

Influence of $\varphi_{\mathrm{s}}$ (where $\mathrm{c}_{\mathrm{s}}=0.7 \mathrm{MPa}, \psi_{\mathrm{s}}=\varphi_{\mathrm{s}}$ )

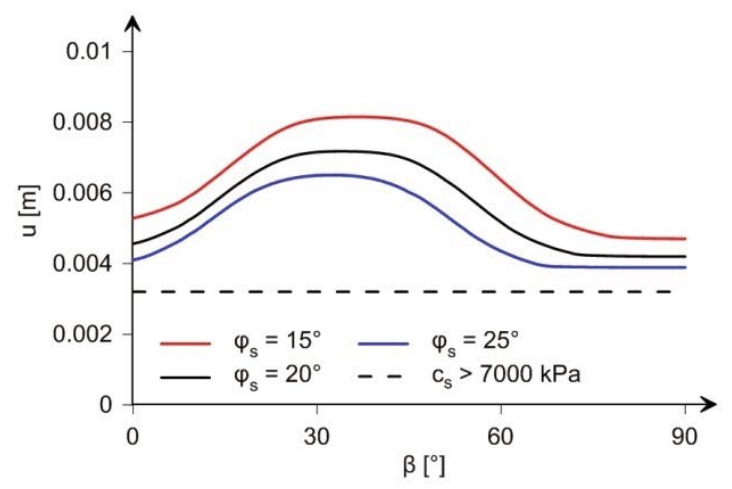

Influence of $\varphi_{\mathrm{s}}\left(\right.$ where $\left.\mathrm{c}_{\mathrm{s}}=0.7 \mathrm{MPa}, \psi_{\mathrm{s}}=20^{\circ}\right)$

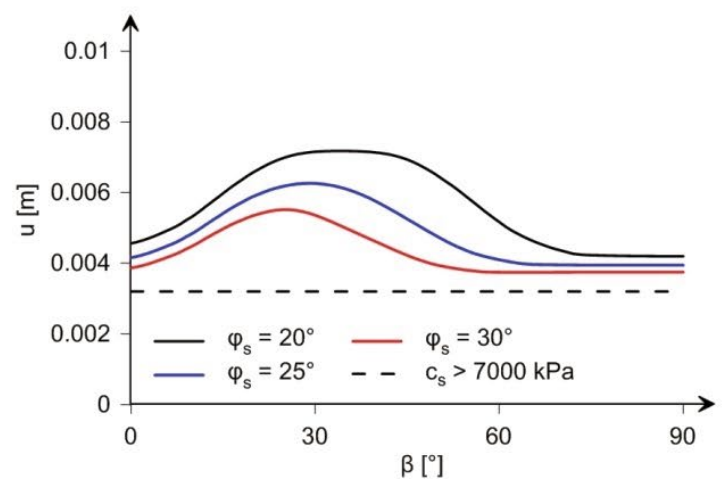

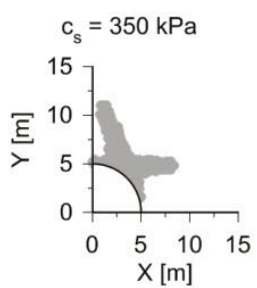
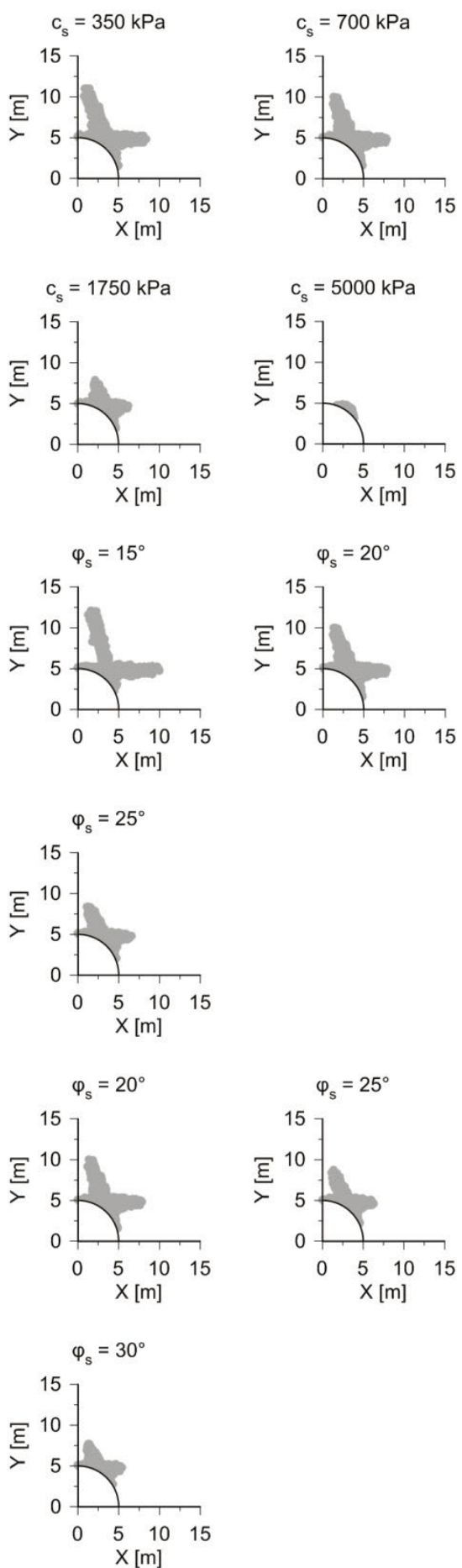

Figure 11.7. Magnitude of the displacement vector along the tunnel boundary (I.h.s.) as well as extent of the plastic zone (r.h.s.) for varying parameters (unsupported tunnel, $a=5 \mathrm{~m}, \sigma_{0}=10 \mathrm{MPa}$, $\left.E=20 \mathrm{GPa}, c_{m}=10 \mathrm{MPa}, \varphi_{m}=30^{\circ}, \Psi_{m}=10^{\circ}, v=0.3\right)$. 
The upper diagrams of Figure 11.7 show the influence of the schistosity cohesion on the magnitude of the displacements along an unsupported tunnel boundary (I.h.s. diagram) and on the plastic zone (r.h.s. diagrams). A decrease in the cohesion of the schistosity leads to a larger extent of the plastic zone and consequently to larger deformations. According to Equation (11.6), failure in the schistosity planes only occurs, for an unsupported tunnel, if

$$
c_{s} \leq \sigma_{0} \sin 2 \beta\left(1-\tan \varphi_{s} \tan \beta\right) \text {, where } \beta=45^{\circ}-\varphi_{s} / 2 \text {. }
$$

Therefore, $c_{s}$ should be smaller than $7 \mathrm{MPa}$, in order that failure occurs in the schistosity plane. For $c_{s}>7 \mathrm{MPa}$, the displacements are equal to those occurring in the absence of the schistosity. A decrease in $c_{s}$ may lead to a considerable increase of the displacements. The maximum displacement along the unsupported tunnel, for $c_{s}=350 \mathrm{kPa}$, may be about 2.5 times larger than those that would occur in the absence of the schistosity $\left(u_{m, 2 D}=0.0032 \mathrm{~m}\right)$.

The middle diagrams of Figure 11.7 show that larger friction angles in the schistosity plane lead generally to smaller plastic zones and consequently to smaller displacements. For $\psi_{s}=\varphi_{s}$, the largest displacements occur at $\beta=45^{\circ}-\varphi_{s} / 2$. An increase of $\varphi_{s}$ leads therefore to moving of the location of maximum displacement towards the crown (i.e., towards smaller $\beta)$. Furthermore, for a high $\varphi_{s}$, the development of the almost vertical plastic zone is less steep, as the latter develops at an angle $90^{\circ}-\varphi_{\text {s }}$ to the horizontal direction (cf. Fig. 11.6).

If the dilatancy angle remains constant and solely the friction angle of the schistosity plane increases (lowermost diagrams of Fig. 11.7), the displacements decrease and the maximum displacements move towards the crown. This occurs also for decreasing dilatancy angles (see upper diagrams of Fig. 11.8) and can be explained as follows: As the extent of the plastic zones remains constant for decreasing dilatancy angles, the displacement occurring in vertical direction decreases (as it amounts to $\gamma_{s} \tan \psi_{s}$ according to Eq. 11.5) given the same portion of displacements in horizontal direction (i.e., $\gamma_{s}$ ). The displacement vectors at the tunnel boundary, therefore, experience larger horizontal than vertical displacements, which leads to moving of the deformation shape to the left (i.e., towards smaller $\beta$ ).

If the cohesion in the matrix decreases to less than $5.77 \mathrm{MPa}$ (for an unsupported tunnel), failure occurs also in the matrix (see lower diagrams of Fig. 11.8). This happens in the areas, where the schistosity cannot fail (i.e., in the areas (i) to (iii) of Fig. 11.6). With decreasing cohesion of the matrix, the displacements of the tunnel profile increase considerably (see Fig. 11.8). Furthermore, the largest displacements no longer occur at about $\beta=45^{\circ}-\varphi_{s} / 2$, but near the crown.

Figure 11.9 shows the displacements along the tunnel boundary as well as the extent of the plastic zone for three cases: (i), when solely the matrix reaches failure, (ii), when solely the schistosity reaches failure and, (iii), when both the matrix and the schistosity reach failure. (Of course, in reality, the cohesion of the schistosity is smaller than the one of the rock mass.) If solely the matrix or the schistosity reaches failure, the maximum displacements are smaller than $0.01 \mathrm{~m}$, while for a failure of both, the displacements can be by a factor 4 larger.

In conclusion, it is the combined effect of failure in the schistosity planes and failure in the matrix which leads to very large deformations of the tunnel profile. 
Influence of $\Psi_{\mathrm{s}}\left(\right.$ where $\left.\mathrm{c}_{\mathrm{m}}=10 \mathrm{MPa}\right)$

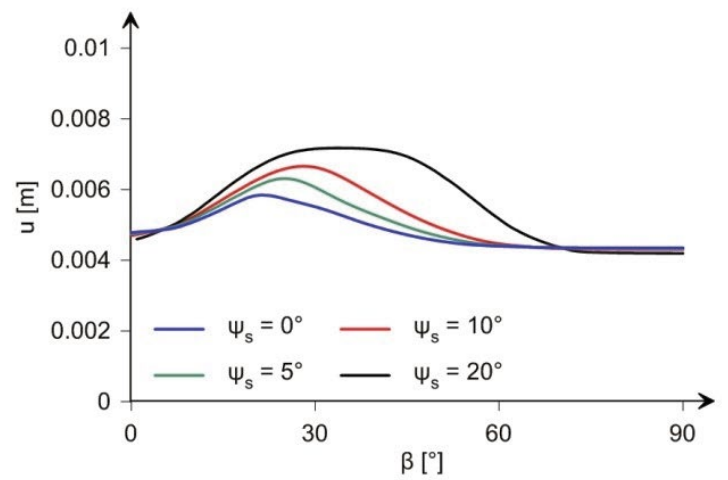

Influence of $\mathrm{c}_{\mathrm{m}}\left(\right.$ where $\left.\Psi_{\mathrm{s}}=20^{\circ}\right)$

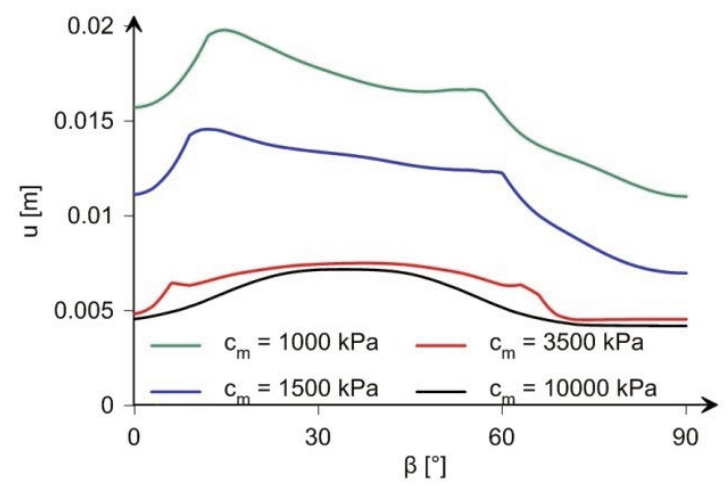

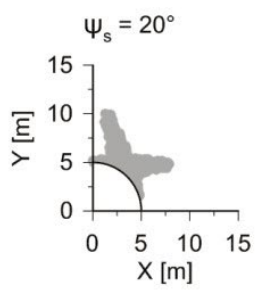
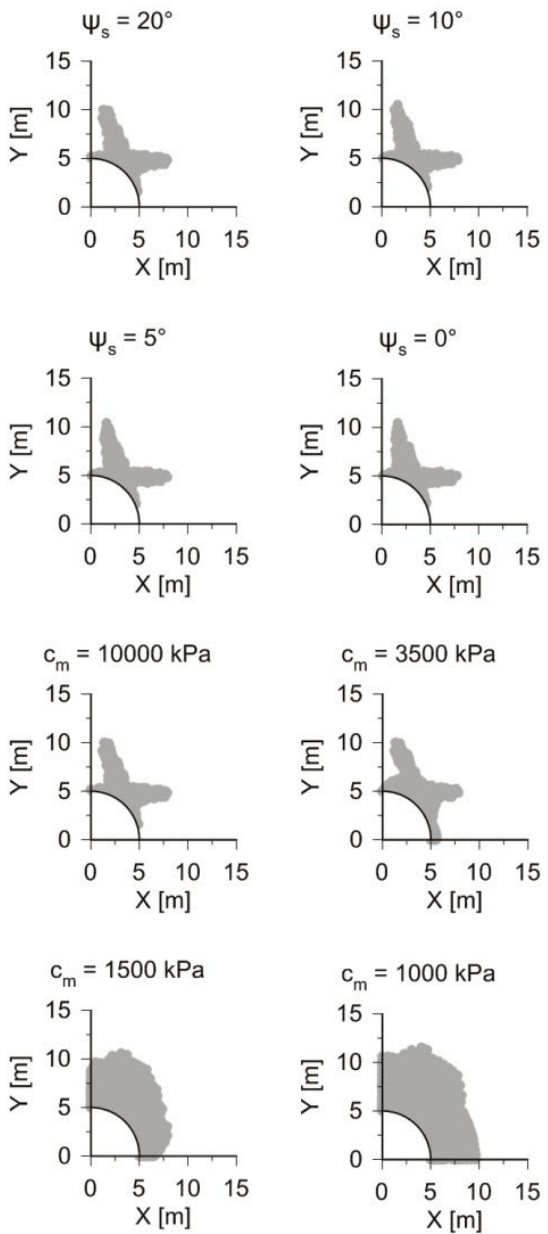

Figure 11.8. Magnitude of the displacement vector along the tunnel boundary (I.h.s.) as well as extent of the plastic zone (r.h.s.) for varying parameters (unsupported tunnel, $a=5 \mathrm{~m}, \sigma_{0}=10 \mathrm{MPa}$, $\left.E=20 \mathrm{GPa}, \varphi_{m}=30^{\circ}, \psi_{m}=10^{\circ}, c_{s}=0.7 \mathrm{MPa}, \varphi_{s}=20^{\circ}, v=0.3\right)$.
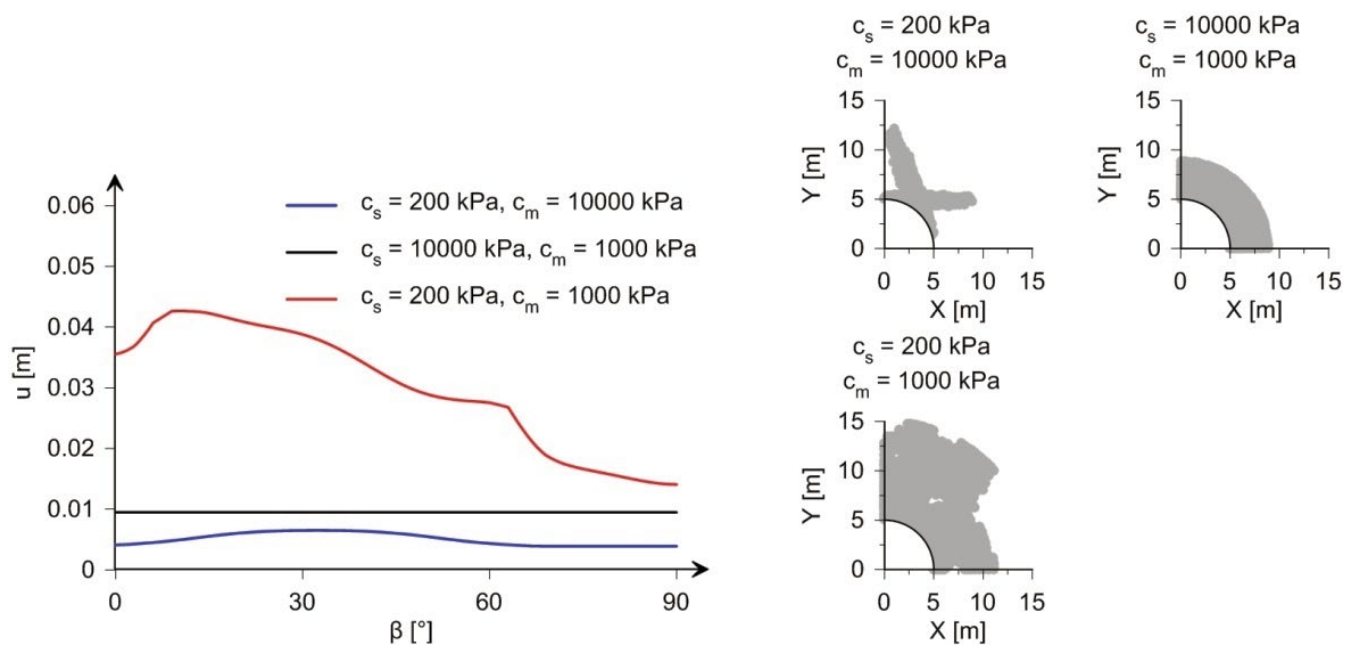

Figure 11.9. Magnitude of the displacement vector along the tunnel boundary (I.h.s.) as well as extent of the plastic zone (r.h.s.) for varying parameters (unsupported tunnel, $a=5 \mathrm{~m}, \sigma_{0}=10 \mathrm{MPa}$, $\left.E=20 \mathrm{GPa}, \varphi_{m}=30^{\circ}, \psi_{m}=10^{\circ}, \varphi_{s}=20^{\circ}, \psi_{s}=20^{\circ}, v=0.3\right)$. 


\subsection{Development of nomograms}

The estimation of the displacements when tunnelling parallel to schistosity planes (see Section 11.3) shall be facilitated by dimensionless nomograms. The displacements at a specific location (i.e., at a specific angle $\beta$ ) of the tunnel boundary generally depend on all the parameters of the problem under consideration:

$$
u=f\left(E, v, \varphi_{m}, \sigma_{d, m}, \psi_{m}, \varphi_{s}, \sigma_{d, s}, \psi_{s}, \sigma_{0}, \sigma_{a}, a\right)
$$

where

$$
\sigma_{d, s}=\frac{2 \cdot c_{s} \cdot \cos \varphi_{s}}{\left(1-\sin \varphi_{s}\right)} \text { and } \sigma_{d, m}=\frac{2 \cdot c_{m} \cdot \cos \varphi_{m}}{\left(1-\sin \varphi_{m}\right)}
$$

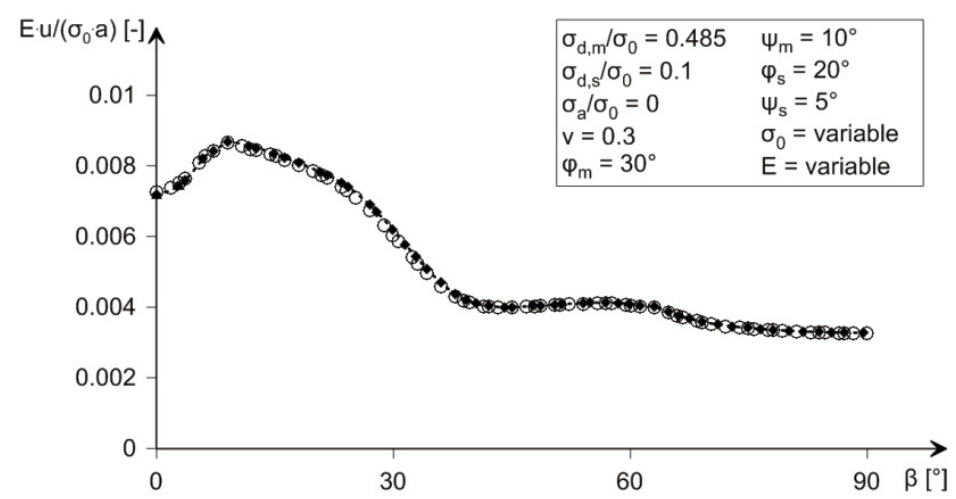

Figure 11.10. Magnitude of the displacement vector $E \cdot u /\left(\sigma_{0 .} a\right)$ along the unsupported tunnel boundary for various values of $\sigma_{0}$ and $E$.

The number of parameters can be reduced by performing a dimensional analysis and by taking into account the findings of Chapter 9, considering that the schistosity represents from the mechanical point of view a special case of a stratified rock mass:

$$
u=\frac{\sigma_{0} \cdot a}{E} \cdot f\left(\frac{\sigma_{d, m}}{\sigma_{0}}, \frac{\sigma_{d, s}}{\sigma_{0}}, \frac{\sigma_{a}}{\sigma_{0}}, v, \varphi_{m}, \psi_{m}, \varphi_{s}, \psi_{s}\right)
$$

This theoretical hypothesis was investigated for the problem under consideration by performing a series of numerical calculations. Figure 11.10 shows the considered parameter sets and the normalised magnitude of the displacement vector $E \cdot u /\left(\sigma_{0}\right.$.a) along the tunnel boundary. The results were obtained for different parameter sets but fall on one single curve.

As the displacements of the tunnel profile are very non-uniform, only the maximum $\left(u_{\max }\right)$, the minimum $\left(u_{\min }\right)$ as well as the averaged $\left(u_{\text {average }}\right)$ displacement at the tunnel boundary will be shown in the nomograms; these values are essential for design purposes.

In order to cover a wide range of parameters, but also to limit the computational effort, the numerical calculations (cf. Section 11.3) were only carried out for specific parameter sets (according to Table 11.1). The friction angles of the matrix $\varphi_{m}$ were chosen to be larger than those of the schistosity $\varphi_{s}$. The support pressure was chosen such as to cover the practically important portion of the ground response curve (at $\sigma_{a 1}=0, \sigma_{a 2}=0.1 \sigma_{0}$ and $\sigma_{a 3}=0.2 \sigma_{0}$ ). Moreover, it can be assumed that the dilatancy angle of the matrix $\psi_{m}$ is interconnected with its friction angle $\varphi_{m}$ (Vermeer and de Borst 1984). The dilatancy angle of the schistosity is usually not constant during the shearing process, but decreases gradually until it approaches 
zero for large shear displacements (Nguyen and Konietzky 2016). In order to be on the safe side (see upper diagrams of Fig. 11.8), a rather high threshold value of $5^{\circ}$ will be chosen.

For the sake of simplicity and considering that the effect of Poisson's ratio is subordinate, the Poisson's ratio was kept fixed to 0.3 .

Table 11.1. Overview of the considered parameter ranges for the nomograms.

\begin{tabular}{lc}
\hline$\sigma_{d, s} / \sigma_{0}[-]$ & $0.05 ; 0.1 ; 0.2 ; 0.3, \infty$ \\
$v[-]$ & 0.3 \\
$\varphi_{m}\left[^{\circ}\right]$ & $20 ; 25 ; 30$ \\
$\varphi_{s}\left[^{\circ}\right]$ & $10 ; 15 ; 20$ \\
& 1 for $\varphi_{m}=20^{\circ}$ \\
$\psi_{m}\left[^{\circ}\right]$ & 5 for $\varphi_{m}=25^{\circ}$ \\
& 10 for $\varphi_{m}=30^{\circ}$ \\
$\psi_{s}\left[^{\circ}\right]$ & (according to Vermeer and de Borst 1984) \\
$\sigma_{a} / \sigma_{0}[-]$ & $5^{\circ}$ \\
\hline
\end{tabular}

Bearing in mind that some of the parameters have been fixed, the maximum, minimum and averaged displacement along the tunnel boundary depend on the following parameters:

$$
\frac{u_{\max } \cdot E}{\sigma_{0} \cdot a}, \frac{u_{\min } \cdot E}{\sigma_{0} \cdot a}, \frac{u_{\text {average }} \cdot E}{\sigma_{0} \cdot a}=f\left(\frac{\sigma_{d, m}}{\sigma_{0}}, \frac{\sigma_{d, s}}{\sigma_{0}}, \frac{\sigma_{a}}{\sigma_{0}}, \varphi_{m}, \varphi_{s}\right) .
$$

This equation is represented in form of nomograms in Appendix G. Each figure of Appendix $\mathrm{G}$ applies to a certain value of $\varphi_{s}, \varphi_{m}$ and $\sigma_{a} / \sigma_{0}$, while each diagram applies to a different value of $\sigma_{d, s} / \sigma_{0}$ (where $\sigma_{d, s}$ can be obtained with Eq. 11.13). Each curve shows the normalised maximum $E_{\max } u /\left(\sigma_{0 .} a\right)$, minimum $E_{\min } u /\left(\sigma_{0 .} a\right)$ or averaged displacement $E_{\text {average }} u /\left(\sigma_{0} a\right)$ in function of the normalised strength $\sigma_{d, w} / \sigma_{0}$. Consequently, one can determine easily the maximum, minimum and averaged displacements for an unsupported as well as for a supported, cylindrical tunnel for given initial stress and mechanical parameters.

Furthermore, the nomograms allow assessing the influence of schistosity on the squeezing deformations easily, by comparing the displacements for the schistous rock mass (with the given rock parameters) with those in the absence of the schistosity planes (given by the lines for $\sigma_{d, d} d \sigma_{0}=\infty$ in the nomograms).

For the considered combinations of minimum uniaxial compressive strength and friction angle of the schistosity, it may happen in some cases that the schistosity cohesion is higher than the matrix cohesion. Nevertheless, for all the parameters of the nomograms, failure occurs first in the planes of weakness and not in the matrix (cf. Eqs. 11.9 and 11.10).

The displacements for other values than those considered in the nomograms can be estimated by interpolating between the respective curves of the nomograms. Generally, the error due to the interpolation is less than $20 \%$. For example, the displacements for a friction angle $\varphi_{m}$ of the matrix of $22.5^{\circ}$ (and thus of a dilatancy angle $\psi_{m}$ of $3^{\circ}$ ) can be estimated by interpolating between the nomograms of $\varphi_{m}=20^{\circ}$ and $25^{\circ}$. (Of course, the estimated displacements for dilatancy angles smaller than $3^{\circ}$ would be on the safe side.) 


\subsection{Parameters of an equivalent isotropic material}

Analogously to Section 9.5, the range of the (non-uniformly distributed) displacements of a schistous rock mass can be estimated by considering an isotropic elasto-plastic material with appropriately selected mechanical parameters. As the schistosity represents, from the mechanical point of view, a special case of the stratified rock mass, the parameters of the isotropic elasto-plastic model can be determined using the same procedure as in Section 9.5, the only difference being that the elasticity parameters of the equivalent material are equal to the actual ones and that the plasticity parameters are obtained (based upon the maximum or the minimum displacements of the anisotropic model) using the diagrams of Appendix $G$.

The goal of this section is to show, how equivalent parameters for an isotropic homogeneous rock mass can be determined for the case of a tunnel drive parallel to the layers, using the nomograms of Appendix $G$ (introduced in Section 11.4). With these equivalent parameters, the assessment of the rock mass response to tunnelling can be facilitated, as the calculation methods commonly used in tunnelling can be applied for tunnels through schistous rocks, whose schistosity planes lie parallel to the tunnel axis. As the schistosity represents, from the mechanical point of view, a special case of the stratified rock mass, the same procedure as was already outlined in Section 9.5 can be used for determining the equivalent parameters for an isotropic homogeneous rock mass, by requesting that the maximum $u_{\max }$ or minimum $u_{\min }$ displacements of the exact model (obtained with the nomograms) are equal to those of the equivalent isotropic model for three selected values of the support pressure $\left(\sigma_{a 1}, \sigma_{a 2}, \sigma_{a 3}\right)$.

Figure 11.11a compares the GRCs of the anisotropic model (obtained for the parameters in the first two rows of Table 11.2) with the GRCs obtained considering the two parameter sets of the equivalent isotropic material after Table 11.2 (last two rows). The equivalent isotropic material reproduces well the displacement range of the schistous material. Solely, the middle sections of the GRC cannot be fitted properly, no matter how well chosen the equivalent parameters are.

Table 11.2. Rock parameters.

\begin{tabular}{|c|c|c|c|c|c|}
\hline & $\begin{array}{c}E \\
{[\mathrm{GPa}]}\end{array}$ & $\begin{array}{c}v \\
{[-]}\end{array}$ & $\begin{array}{c}c \\
{[\mathrm{MPa}]}\end{array}$ & $\begin{array}{c}\varphi \\
{\left[{ }^{\circ}\right]}\end{array}$ & $\begin{array}{c}\psi \\
{\left[{ }^{\circ}\right]}\end{array}$ \\
\hline Matrix & 20 & 0.3 & 1.155 & 30 & 10 \\
\hline Schistosity & - & - & 0.419 & 10 & 5 \\
\hline \multicolumn{6}{|c|}{ Equivalent isotropic material } \\
\hline based upon $u_{\max }$ & 20 & 0.3 & 0.518 & 20.0 & 1.0 \\
\hline based upon $u_{\min }$ & 20 & 0.3 & 0.449 & 30.0 & 1.0 \\
\hline
\end{tabular}

The usefulness of the proposed equivalent isotropic model will be illustrated by means of two further tunnelling problems assuming the parameters of Table 11.2 (considering an initial hydrostatic stress field of $10 \mathrm{MPa}$ ): (1) The longitudinal displacement profile of an unsupported tunnel (Fig. 11.11b); (2) The boundary displacements of an unsupported tunnel with a horseshoe profile assuming plane strain conditions (Fig. 11.11c). These problems were solved numerically, using the exact schistous material model and the equivalent isotropic model. (Numerical details for problem 1 can be found in Chapter 12.)

According to Figure 11.11, the results obtained with the equivalent isotropic model (fitted with the minimum and the maximum GRC) bound the exact solution. However, the predeformations obtained with equivalent isotropic model are larger than the exact ones. This is 
such because the middle section of the GRCs of the exact model does not fit perfectly with those obtained with equivalent parameters. However, the rock response to tunnelling obtained with equivalent parameters is accurate enough for practical purposes at least at the preliminary design stage.

(a)

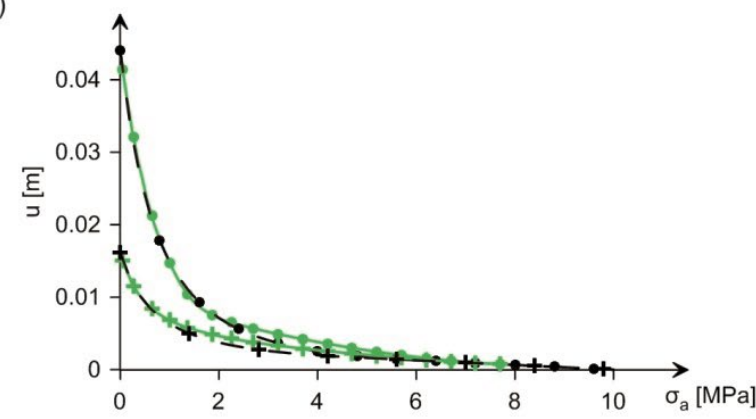

(b)

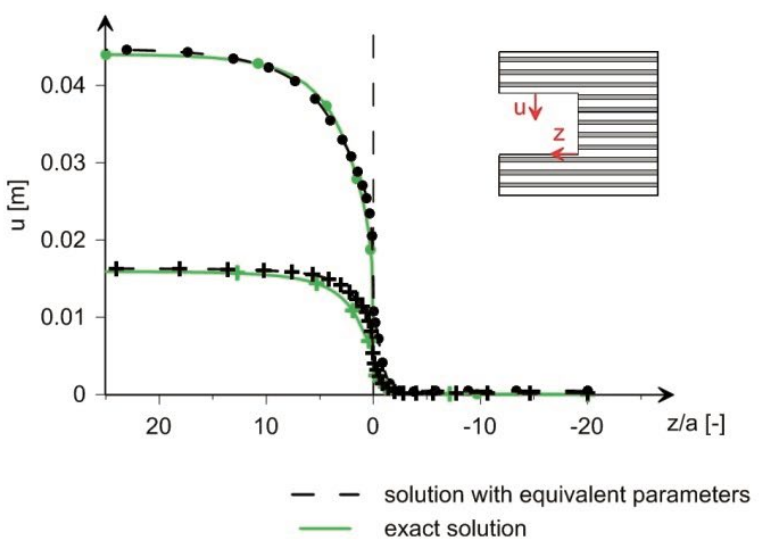

(c)

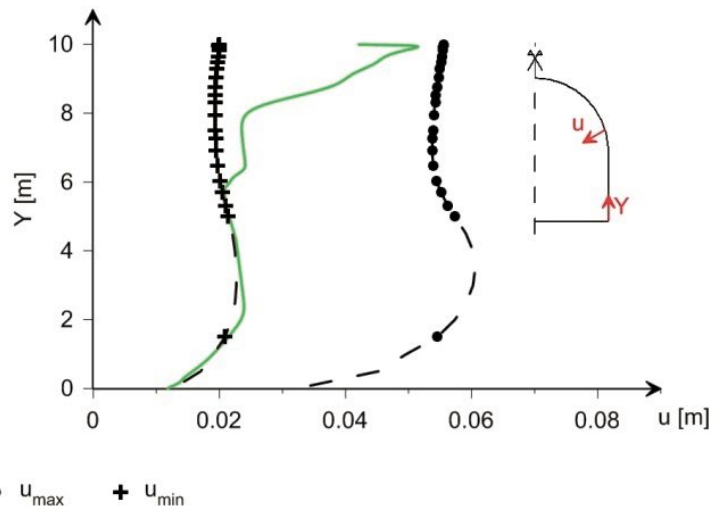

Figure 11.11. (a) Maximum and minimum displacement at the tunnel boundary of a cylindrical tunnel as a function of the support pressure (GRC). (b) Maximum and minimum longitudinal displacement profile of an unsupported tunnel. (c) Maximum and minimum magnitude of the displacement vector along the unsupported tunnel boundary of a horseshoe profile, obtained with the exact model and with the equivalent isotropic model (parameters after Table 11.2).

\subsection{Conclusions}

This chapter investigated the response of schistous rock to tunnel excavation parallel to schistosity, considering a homogeneous rock mass with strength anisotropy.

In order to facilitate the assessment of squeezing in schistous rocks, dimensionless diagrams were worked out based upon the results of a parametric study. These diagrams serve to determine the parameters of an isotropic elasto-plastic model, which can be used for estimating an upper and a lower bound of the non-uniformly distributed deformations of the schistous rock. 


\section{Notation}

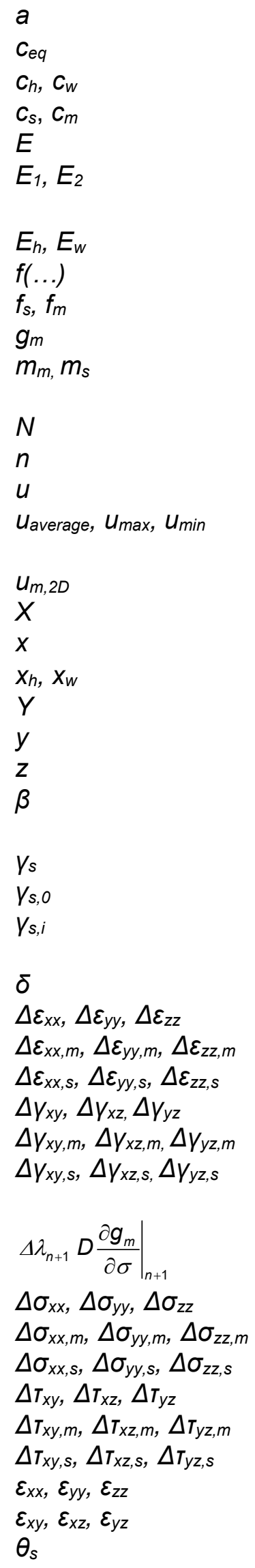

tunnel radius

equivalent cohesion of the simplified isotropic rock mass

cohesion of the hard and of the weak layers

cohesion of the schistosity and the surrounding matrix

Young's modulus of the rock mass

Young's modulus of the composite parallel and perpendicular to the layers

Young's modulus of the hard and of the weak layers

function of ...

Mohr-Coulomb criterion of the schistosity and the surrounding matrix plastic potential of the matrix

inclination of the failure surface of the schistosity and the surrounding matrix (function of the friction angle)

number of schistosity planes in the REV

stage

magnitude of the displacement vector at the tunnel boundary

averaged, maximum and minimum magnitude of the displacement vector along the tunnel boundary

radial displacement of the non-schistous rock mass

co-ordinate

co-ordinate

thickness fraction of the hard and of the weak layers

co-ordinate

co-ordinate

axial co-ordinate (distance behind the tunnel face)

angle between the tangential direction of the tunnel and the schistosity planes

shearing strain increment

initial guess of the shearing strain increment $\gamma_{s}$

shearing strain increment along a single schistosity plane due to schistosity slip

angle between the planes of weakness and the loading direction

homogenised normal strain increment in $x-, y$ - and $z$-direction

normal strain increment in the matrix in $x-, y$ - and $z$-direction

normal strain increment in the schistosity in $x-, y$ - and $z$-direction

homogenised shearing strain increment in the $x y-, x z-$ and $y z$-plane

shearing strain increment in the matrix in the $x y-, x z$ - and $y z$-plane

shearing strain increment along the schistosity in the $x y-, x z-$ and $y z-$ plane

plastic corrector stress of the matrix (cf. Clausen 2007)

homogenised normal stress increment in $x-, y$ - and $z$-direction

normal stress increment in the matrix in $x-, y$ - and $z$-direction

normal stress increment in the schistosity in $x-, y$ - and $z$-direction

homogenised shear stress increment in the $x y-, x z$ - and $y z$-plane

shear stress increment in the matrix in the $x y-, x z$ - and $y z$-plane

shear stress increment in the schistosity in $x y-, x z$ - and $y z$-plane

homogenised strain in $x$-, $y$ - and $z$-direction

homogenised shear strain in the $x y-, x z$ - and $y z$-plane

angle between the tunnel axis and the strike direction of the schistosity planes 


$K_{m}$
$V$
$\sigma$
$\sigma_{0}$
$\sigma_{1}, \sigma_{3}$
$\sigma_{a}$
$\sigma_{d}$
$\sigma_{d, h}, \sigma_{d, w}$
$\sigma_{d, s}, \sigma_{d, m}$
$\sigma_{n}$
$\sigma_{n+1}$
$T$
$T_{x y}, T_{x z}, T_{y z}$
$\varphi_{h}, \varphi_{w}$
$\varphi_{s}, \varphi_{m}$
$\psi_{h}, \Psi_{w}$
$\psi_{s}, \psi_{m}$
$\omega_{s}$

$K_{m}$

$\sigma$

$\sigma_{1}, \sigma_{3}$

$\sigma_{a}$

$\sigma_{d, h,} \sigma_{d, w}$

$\sigma_{n}$

$\sigma_{n+1}$

$T_{x y}, T_{x z}, T_{y z}$

$\varphi_{h}, \varphi_{w}$

$\varphi_{s}, \varphi_{m}$

$\psi_{s}, \psi_{m}$

$\omega_{s}$

constant of the flow rule for the matrix (function of the dilatancy angle) Poisson's ratio of the rock mass

normal stress

initial stress

maximum and minimum principal stress

support pressure of the tunnel

uniaxial compressive strength of the schistous rock mass

uniaxial compressive strength of the hard and the weak layers

uniaxial compressive strength of the schistosity (according to Eq.

11.7) and the surrounding matrix

homogenised stress at previous stage (at state $n$ )

update of the homogenised stress (at state $n+1$ )

shear stress

shear stress of the equivalent continuum in $x y-, x z$ - and $y z$-direction

friction angle of the hard and of the weak layers

friction angle of the schistosity and of the surrounding matrix

dilatancy angle of the hard and of the weak layers

dilatancy angle of the schistosity and of the surrounding matrix

dip angle of the schistosity planes 


\section{PART IV: TUNNELLING WITH AN ARBITRARY ANGLE TO THE SCHISTOSITY OR BEDDING PLANES}

This part of the thesis investigates the influence of the orientation of schistosity or bedding on the convergences (considering also the pre-deformation occurring ahead of the tunnel face) and shows how this influence can be considered in the design. Specifically, simple convergence estimation methods are presented that render costly spatial numerical analyses unnecessary in the preliminary design stage. Furthermore, the variability of squeezing in tunnelling through folded rock masses is studied. 



\section{Influence of the orientation of the anisotropy planes on the squeezing deformations}

\subsection{Introduction}

The orientation of planes of anisotropy, particularly of the bedding and schistosity planes, influences considerably the magnitude and distribution of the squeezing deformations (Barla 2002, Goricki et al. 2005, Button et al. 2006). Particularly adverse are planes of anisotropy that strike parallel or with a small angle (less than $25^{\circ}$ ) to the tunnel axis (cf. Cording and Mahar 1974). This was observed also in the case histories of Part $I$.

In this chapter also the convergences are investigated, which are the displacements far behind the face minus the displacements that occur ahead of the face (hereafter referred to as "pre-deformation"). A review of pre-deformation estimation methods for homogeneous and isotropic materials can be found in Cantieni and Anagnostou (2009a). There are only a few investigations concerning the pre-deformations in layered or schistous rocks. CarranzaTorres and Fairhurst (2000) investigated the application of the convergence-confinement method for rock masses that satisfy the Hoek-Brown failure criterion. However, the failure of the planes of weakness was not considered explicitly. Tonon and Amadei (2002) investigated numerically the effect of elastic anisotropy on the pre-deformations. They found out that the existing expressions (valid for isotropic rock masses under a uniform state of stress) are applicable only if the plane of transverse isotropy strikes parallel to the tunnel axis; otherwise three-dimensional analyses are necessary. Klopčič and Logar (2014) and Madkour (2012) showed that a large portion of displacements occurs ahead of the tunnel face especially when tunnelling with the dip, but did not consider failure of the rock matrix. Schubert and Mendez (2017) also investigated the influence of the orientation of the schistosity on the tunnel behaviour using the example of the Galgenberg Tunnel.

In the current state of research a systematic, quantitative investigation of the influence of the orientation of the planes of anisotropy on the convergences and easy-to-use, design-oriented methods of analysis are missing. This chapter shows that the tunnel convergences can be determined approximately based upon the solutions developed for the borderline cases of tunnelling parallel or perpendicularly to the anisotropy planes (Parts II and III of the thesis) and the "schistosity influence factor" introduced in Chapter 1, which expresses the combined effect of the dip angle $\omega_{s}$ and of the angle $\theta_{s}$ between the strike direction of the planes of anisotropy and the tunnel axis.

The plane strain assumption is only valid, if the planes of anisotropy are parallel or perpendicular to the tunnel axis (cf. Amadei and Goodman 1981): Plane strain calculations presuppose that (cf. Amadei and Goodman 1981, Zienkiewicz et al. 2015) the normal strain $\varepsilon_{z}$, as well as the shear strains $\gamma_{y z}$ and $\gamma_{x z}$ are equal zero, i.e., that one principal stress direction coincides with the tunnel axis. For a stratified or schistous rock mass with 
anisotropy planes, which are neither parallel nor perpendicular to the tunnel axis, the axial strain $\varepsilon_{z}$ far behind the tunnel face equals zero, which is however not true for the shear strains $\gamma_{y z}$ and $\gamma_{x z}$. According to Zienkiewicz et al. (2015), the displacements in a crosssection far behind the tunnel face could be obtained by a so-called "complete plane strain analysis" (see, e.g., Amadei and Goodman 1981, Rencis and Huang 1992, Zienkiewicz et al. 2015). In the present case, however, full 3D analyses are indispensable because the predeformations also have to be determined.

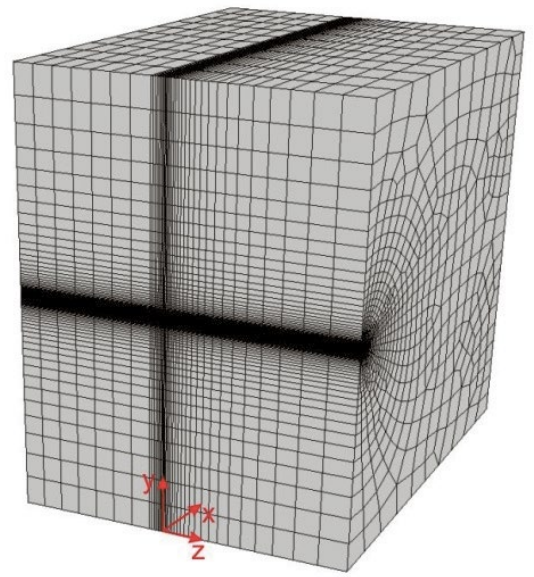

Figure 12.1. Numerical model for the strike direction of the planes of anisotropy perpendicular to the tunnel axis.

In order to determine the convergences, the ground response to tunnel excavation is analysed by 3D numerical calculations (using Abaqus; Dassault Systèmes 2011), which consider the advancing tunnel face. Figure 12.1 shows the numerical model. An unsupported tunnel is considered and the excavation is simulated through a stepwise reduction of the tractions along the entire boundary and at the face from $\sigma_{0}$ to zero (Fig. 12.2); a step-by-step simulation of tunnel advance is unnecessary for an unsupported tunnel.
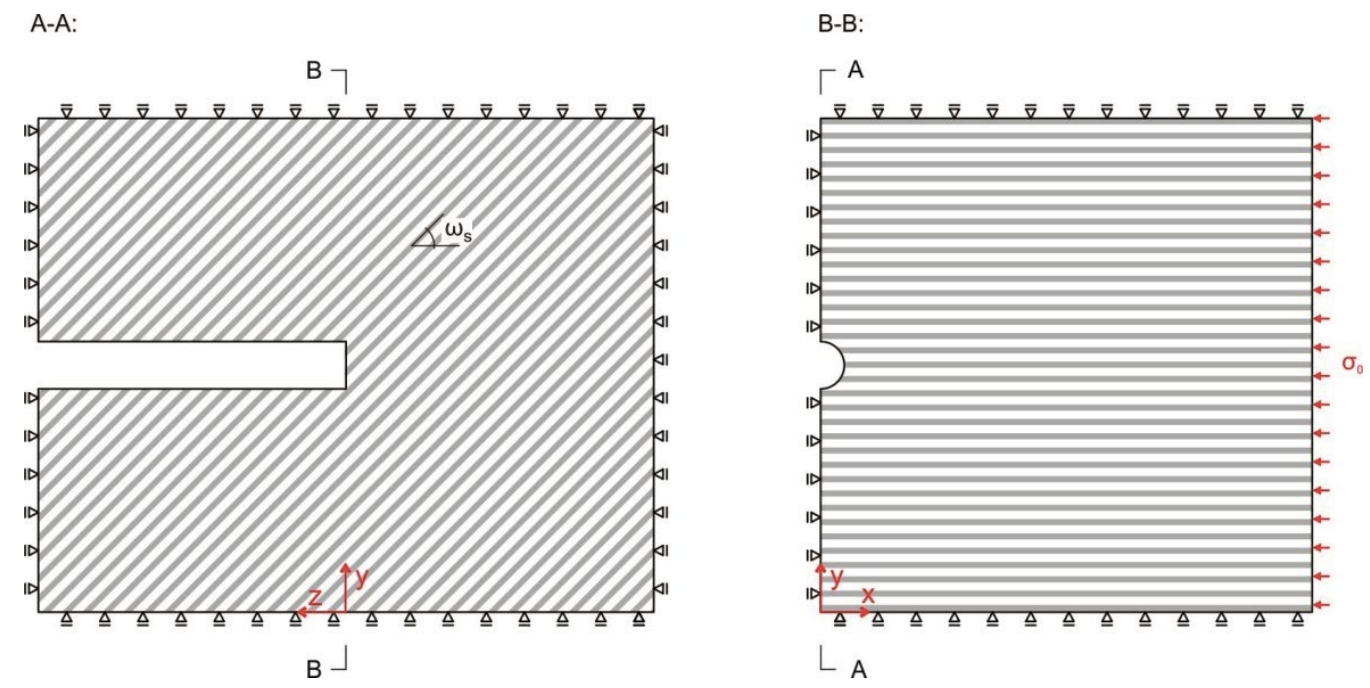

Figure 12.2. Problem layout with boundary conditions for the strike direction of the planes of anisotropy perpendicular to the tunnel axis.

Figure 12.2 shows the assumed boundary conditions. As the displacements and the shear stresses $T_{y z}$ and $T_{x z}$ at the left vertical boundary are fixed to zero (and this contradicts the actual behaviour, if the anisotropy planes are not parallel or perpendicular to the tunnel axis), 
boundary effects appear (see Fig. 12.3; cf. Zienkiewicz et al. 2015). In order to eliminate the effect of the boundary condition, a sufficient long model is considered (50 times the tunnel radius).

The rock mass is taken as a homogeneous transversely isotropic material with the constitutive model after Chapter 9 (thinly stratified rock) or 11 (schistous rock). If the strike direction of the planes of anisotropy is perpendicular to the tunnel axis (as in Sections 12.2 and 12.3), only half of the system needs to be considered (Fig. 12.2). Otherwise (as in Section 12.4), symmetry is lost and the whole system has to be considered.

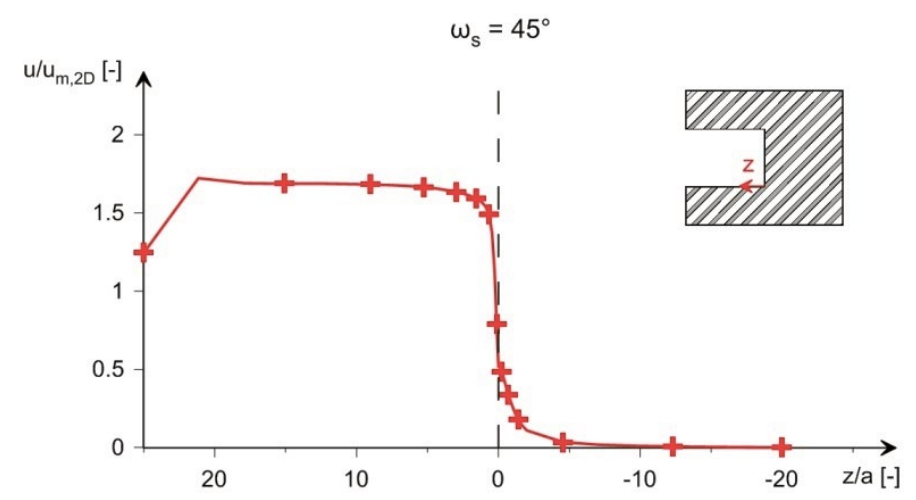

Figure 12.3. Normalised magnitude of the displacement vector $u / u_{m, 2 D}$ at the crown along a tunnel through schistous rock $\left(a=5 \mathrm{~m}, \sigma_{0}=10 \mathrm{MPa}, E=1 \mathrm{GPa}, c_{m}=6 \mathrm{MPa}, \varphi_{m}=30^{\circ}, \psi_{m}=10^{\circ}\right.$, $\left.c_{s}=0.8 \mathrm{MPa}, \varphi_{s}=10^{\circ}, \psi_{s}=5^{\circ}, v=0.3\right)$.

\subsection{Tunnelling in schistous rock, perpendicular to the strike direction}

In this section, the influence of the dip angle $\omega_{s}$ (see inset of Fig. 12.5) on the displacements (Section 12.2.1), on the pre-deformations (Section 12.2.2) and on the convergences (Section 12.2.3) will be shown.

The analyses were performed by considering a friction angle and cohesion of the schistosity, which are considerably smaller than those of the matrix. Two different values of the cohesion of the surrounding matrix $c_{m}$ will be analysed: A rather small cohesion of $c_{m}=1 \mathrm{MPa}$ (where the matrix yields) and a larger value of $c_{m}=6 \mathrm{MPa}$, where the matrix remains elastic. The latter was chosen, in order to investigate if the statements of this section are valid also for less severe squeezing conditions, where yielding occurs only along the schistosity plane.

\subsubsection{Influence of the dip angle on the displacements}

The Figure 12.4 shows, for a dip angle $\omega_{s}$ of $0^{\circ}, 45^{\circ}$ or $90^{\circ}$, (a), the longitudinal distribution of the magnitude of the displacement vector (hereafter referred as "the displacement") at the crown, at the invert and at the side wall. The displacements are normalised by the displacement $u_{m, 2 D}$, which would occur in the absence of schistosity $\left(u_{m, 2 D}\right.$ can be determined analytically); (b) the plastic zone in the vertical symmetry plane of the tunnel; and, (c), the longitudinal distribution of the magnitude of the longitudinal component of the displacement vector as well as the magnitude of the projection of the displacement vector in the plane of the tunnel cross-section (at the tunnel crown and invert; normalised by the displacement $\left.u_{m, 2 D}\right)$. 
For $\omega_{s}=90^{\circ}$, schistosity is irrelevant for the displacements far behind the face, because failure in the schistosity plane cannot occur there. With decreasing dip angle the displacements increase and the profile deforms non-uniformly.

(a)
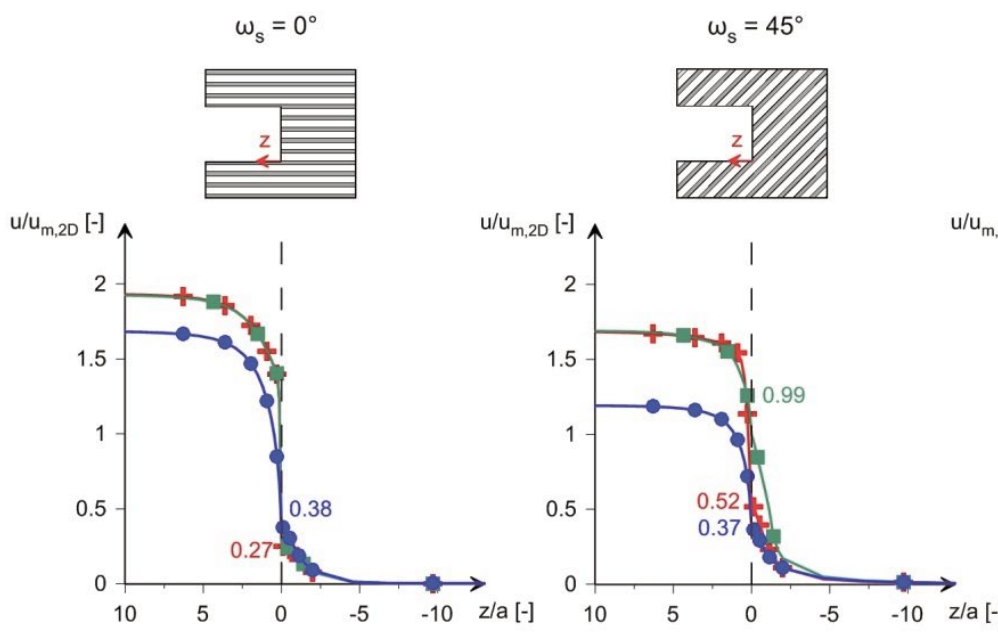

$\omega_{\mathrm{s}}=45^{\circ}$
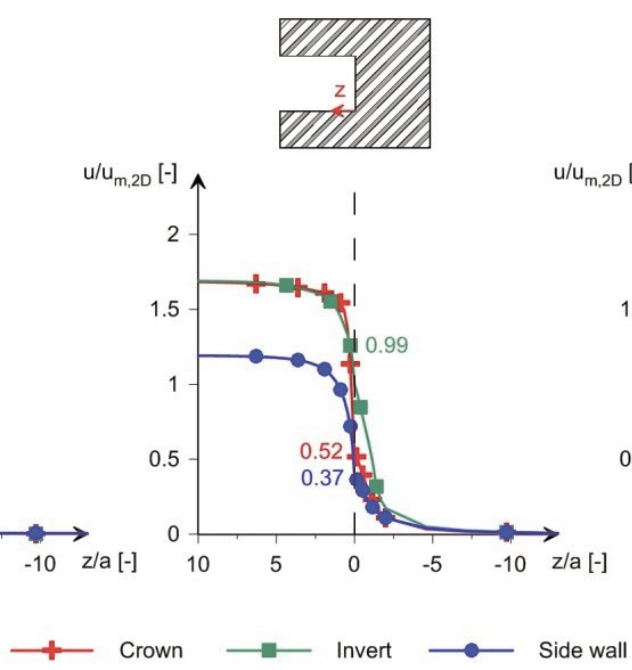

(b)
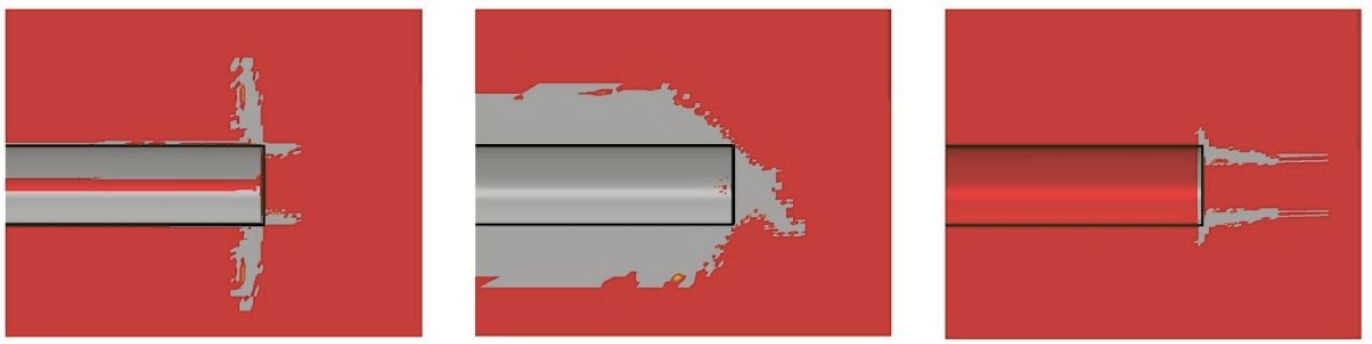

(c)
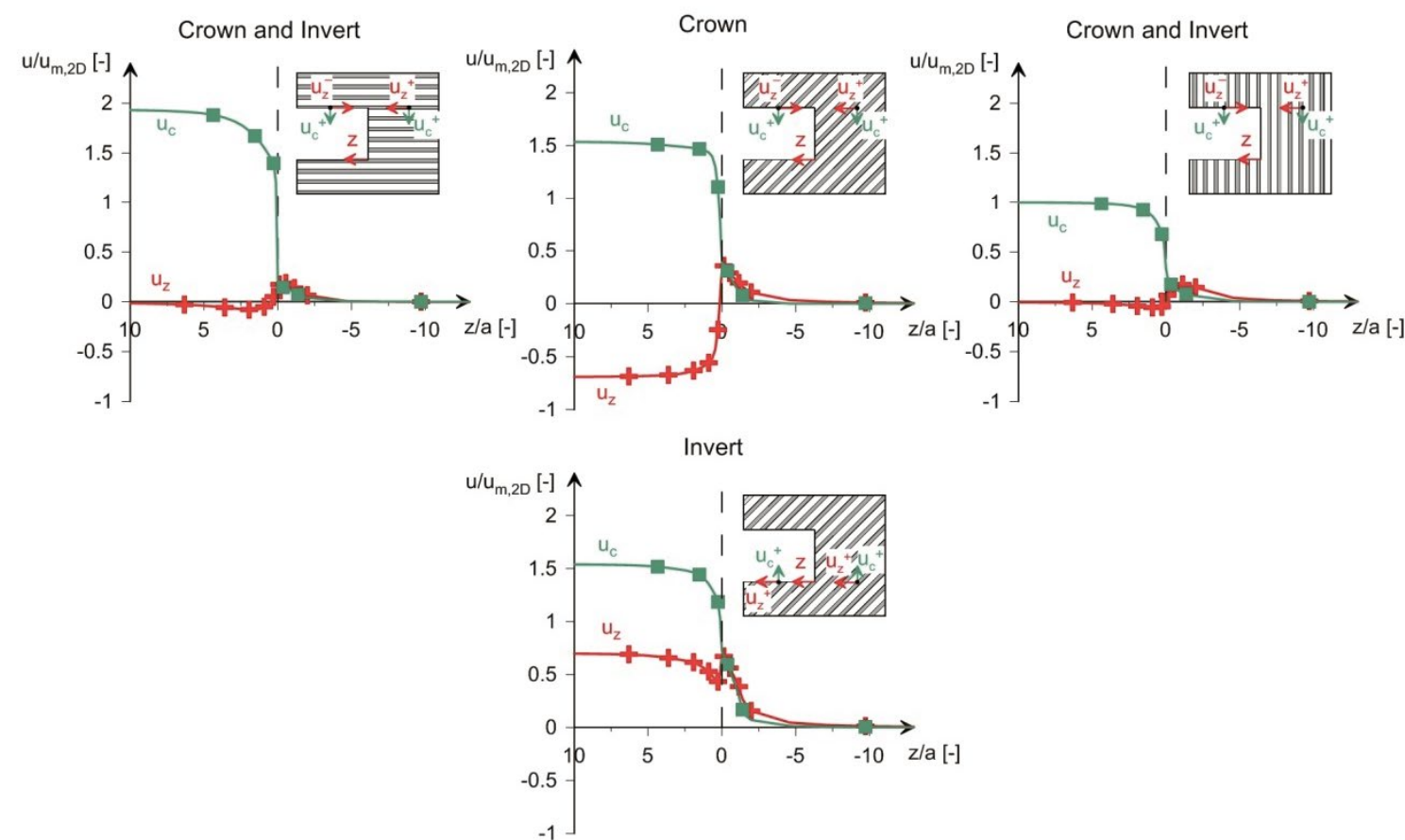

Figure 12.4. (a) Normalised displacement $u / u_{m, 2 D}$ along the tunnel; (b) extent of the plastic zone; and, (c), normalised longitudinal displacement $\left(u_{z} / u_{m, 2 D}\right)$ and normalised cross-sectional displacement $\left(u_{c} / u_{m, 2 D}\right)$ along the tunnel $\left(a=5 \mathrm{~m}, \sigma_{0}=10 \mathrm{MPa}, E=1 \mathrm{GPa}, c_{m}=6 \mathrm{MPa}\right.$, $\varphi_{m}=30^{\circ}, \psi_{m}=10^{\circ}, c_{s}=0.8 \mathrm{MPa}, \varphi_{s}=10^{\circ}, \psi_{s}=5^{\circ}, v=0.3$ ). 
In the following, the maximum and minimum displacements of the profile will be considered rather than the displacements at specific points of the profile. (Note that the maximum displacements do not occur at the profile locations considered in Figure 12.4a and therefore the results of Figure 12.4a cannot be compared with those of the next figures.)
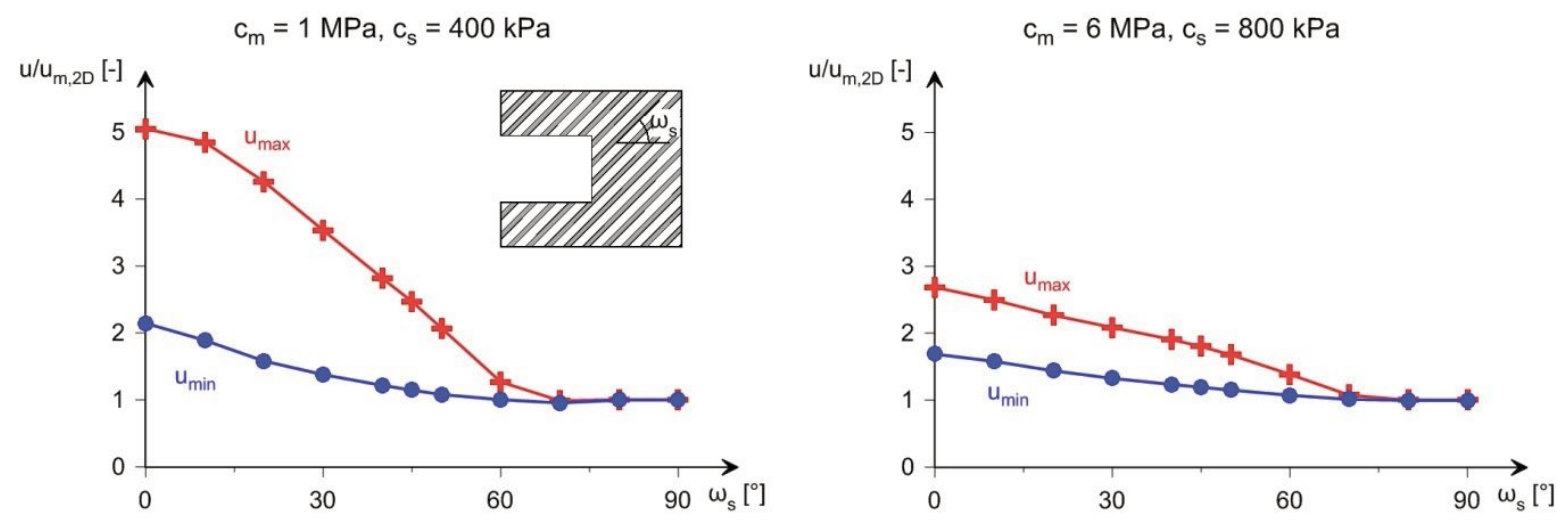

Figure 12.5. Normalised maximum and minimum displacements as a function of the dip angle $\omega_{s}$ $\left(a=5 \mathrm{~m}, \sigma_{0}=10 \mathrm{MPa}, E=1 \mathrm{GPa}, \varphi_{m}=30^{\circ}, \psi_{m}=10^{\circ}, \varphi_{s}=10^{\circ}, \psi_{s}=5^{\circ}, v=0.3\right.$ ).

$\mathrm{c}_{\mathrm{m}}=1 \mathrm{MPa}, \mathrm{c}_{\mathrm{s}}=400,800 \mathrm{kPa}$

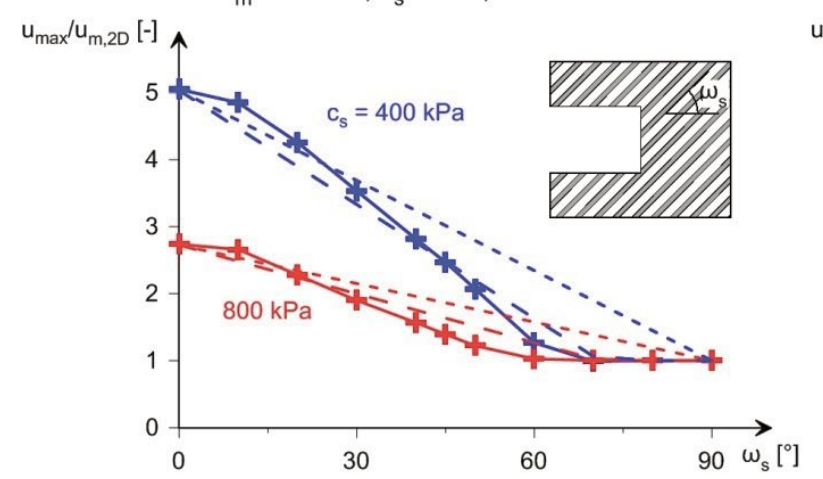

$\mathrm{c}_{\mathrm{m}}=6 \mathrm{MPa}, \mathrm{c}_{\mathrm{s}}=400,800 \mathrm{kPa}$

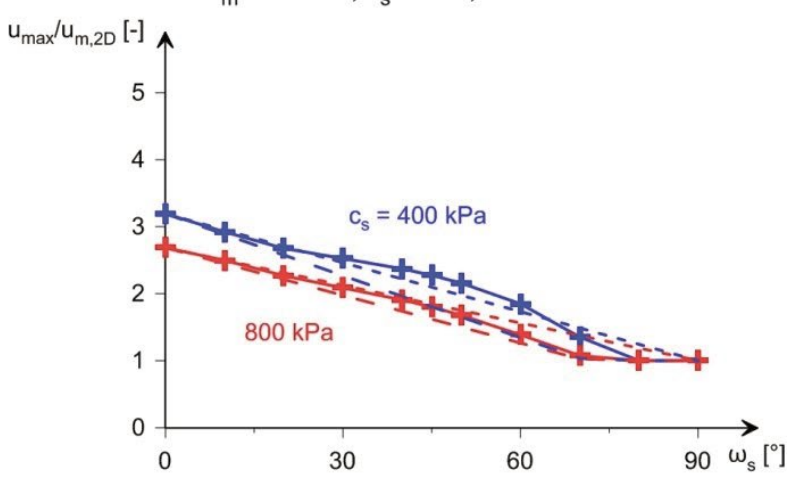

+ numerical calculations $\quad--$ approximation with $\mathrm{S} \quad-$ approximation with improved S

Figure 12.6. Effect of matrix and schistosity cohesion: Normalised maximum displacements as a function of the dip angle $\omega_{s}\left(a=5 \mathrm{~m}, \sigma_{0}=10 \mathrm{MPa}, E=1 \mathrm{GPa}, \varphi_{m}=30^{\circ}, \psi_{m}=10^{\circ}, \varphi_{s}=10^{\circ}\right.$, $\left.\boldsymbol{\psi}_{s}=5^{\circ}, v=0.3\right)$.

Figures 12.5 and 12.6 show the normalised maximum and minimum displacements as a function of the dip angle for a matrix cohesion of 1 or $6 \mathrm{MPa}$ and a schistosity cohesion of 400 or $800 \mathrm{kPa}$. The following conclusions can be drawn:

- The dip angle has a remarkable influence both on the maximum and on the minimum displacement (Fig. 12.5);

- The non-uniformity of the displacements in the tunnel profile is maximum at $\omega_{s}=0^{\circ}$ and decreases monotonously with increasing dip angle (Fig. 12.5);

- Schistosity does not play a role for the maximum and minimum displacements $\left(u=u_{m, 2 D}\right)$ if the dip angle is greater than ca. $70^{\circ}$;

- The displacements in tunnelling parallel to schistosity plane can be considerably larger than in tunnelling perpendicular to the schistosity plane;

- The lower the schistosity cohesion, the bigger the effect of the dip angle will be (Fig. 12.6; I.h.s. diagram). 
- It is the combination of a low schistosity cohesion with a low matrix cohesion which produces very large displacements: A by $50 \%$ lower cohesion in the schistosity planes results only in slightly larger displacements if the matrix is strong $\left(c_{m}=6 \mathrm{MPa}\right.$; r.h.s. diagram of Fig. 12.6), but to twice as big displacements if the matrix is weak $\left(c_{m}=1 \mathrm{MPa}\right.$; I.h.s. diagram of Fig. 12.6).

According to Figure 12.6, the maximum displacement can be approximated as a linear (or better bilinear) function of the dip angle. The linear function can be defined in terms of the schistosity influence factor $S$ (Chapter 1), which combines the dip angle $\omega_{s}$ and the angle $\theta_{s}$ between the strike direction and the tunnel axis:

$$
u_{\max }=\left(u_{\max , I I}-u_{\perp}\right) \cdot S+u_{\perp},
$$

where

$$
S=1-\frac{\omega_{s}}{90} \frac{\theta_{s}}{90} \in[0,1]
$$

$\left(\theta_{s}=90^{\circ}\right.$ in the present case) and $u_{\max , \| l}$ and $u_{\perp}$ are the displacements in tunnelling parallel and perpendicular to the schistosity planes, respectively. $u_{\max , \|}$ can be determined after Chapter 11 , while $u \perp$ is equal to $u_{m, 2 D}$, which can be determined analytically.

The linear approximation (Eq. 12.1 with $S$ after Eq. 12.2) is satisfactory for small dip angles, but overestimates the maximum displacement for steep schistosity planes, which - as mentioned before - do not play a role. The latter suggests a bilinear relationship, using an improved schistosity influence factor,

$$
S=\max \left(1-\frac{\omega_{s}}{90-\varphi_{s}} \frac{\theta_{s}}{90-\varphi_{s}} ; 0\right) \in[0,1]
$$

which takes into account that failure is not possible for dip angles larger than $90^{\circ}-\varphi_{s}$. The $S$ versus $\left(\omega_{s}, \theta_{s}\right)$ plot is given in Figure 12.7.

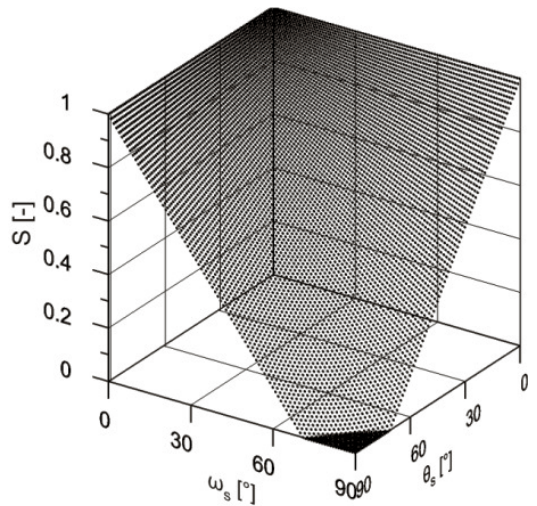

Figure 12.7. Improved schistosity influence factor $S$ as a function of the schistosity dip angle $\omega_{s}$ and the angle $\theta_{s}$ between the strike direction of the schistosity and the tunnel axis $\left(\varphi_{s}=10^{\circ}\right)$.

The accuracy of the improved schistosity influence factor for arbitrary strike directions will be demonstrated in Section 12.4. 
The minimum displacements can be expressed analogously (see Fig. 12.5):

$$
u_{\min }=\left(u_{\min , l l}-u_{\perp}\right) \cdot S+u_{\perp} \text {. }
$$

The relationships of this section provide a simple estimation of the displacements for different dip angles, which are satisfactory enough for practical purposes.

In Chapter 3 , the so-called "schistosity angle" $\beta$ (defined as the angle between the normal vector of the schistosity plane and the radial direction of the tunnel) was considered as a possible measure of the influence of the schistosity orientation on the displacement at a specific location of the tunnel profile. The underlying idea was that the largest displacements along the tunnel boundary occur at locations where the schistosity is almost parallel to the excavation boundary, while the smallest displacement occur at locations where the schistosity is almost perpendicular to the excavation boundary (provided that both, the schistosity and the matrix reach failure; see, e.g., Goricki et al. 2005, Button et al. 2006). However, no clear correlation could be found in Chapter 3 between the displacements and the schistosity angle. This can be explained by means of numerical calculations. Figure 12.8 shows the displacements at the tunnel boundary as a function of the schistosity angle $\beta$ and of the dip angle $\omega_{s}$. The displacements decrease with increasing schistosity angle, but only if the dip angle is fixed. The schistosity angle allows to estimate only the location of the tunnel boundary with the maximum displacement.

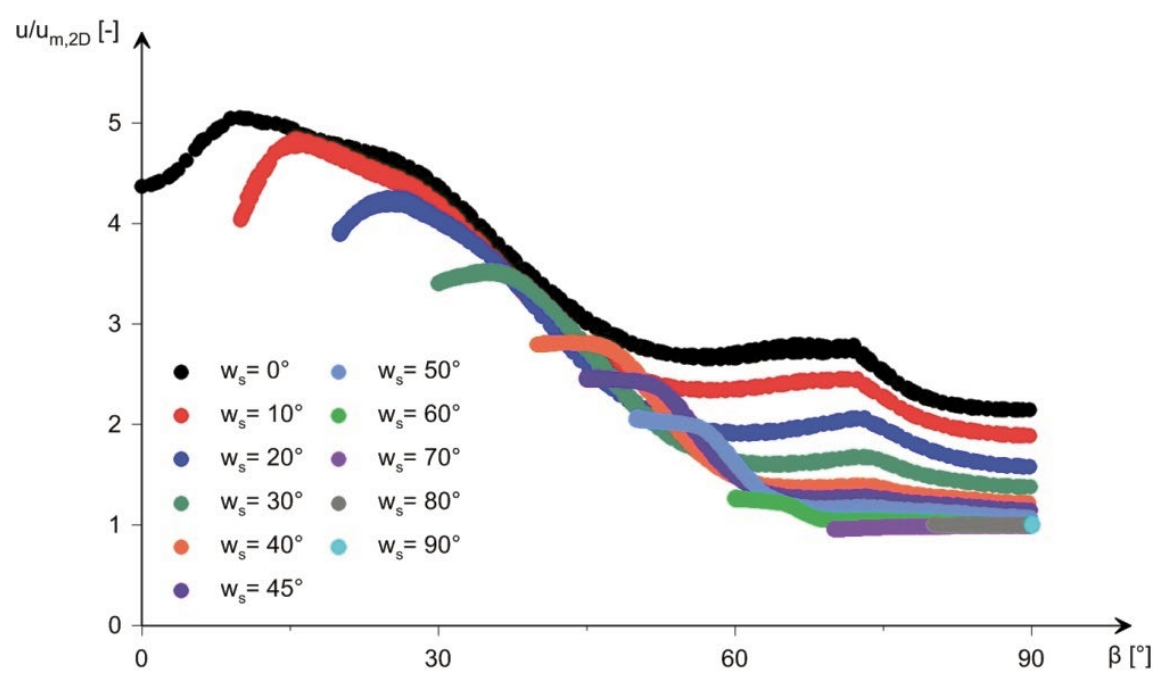

Figure 12.8. Normalised magnitude of the displacement vector $u / u_{m, 2 D}$ as a function of the schistosity angle $\beta$ for various dip angles $\omega_{s}\left(a=5 \mathrm{~m}, \sigma_{0}=10 \mathrm{MPa}, E=1 \mathrm{GPa}, c_{m}=1 \mathrm{MPa}, \varphi_{m}=30^{\circ}\right.$, $\Psi_{m}=10^{\circ}, c_{s}=0.4 \mathrm{MPa}, \varphi_{s}=10^{\circ}, \Psi_{s}=5^{\circ}, v=0.3$ ).

\subsubsection{Influence of the dip angle on the pre-deformations}

The numbers besides the $z=0$ line in Figure 12.4a give the normalised displacement at the face, i.e. the pre-deformation. The latter is considerably bigger for a dip angle $\omega_{s}$ of $45^{\circ}$ than for $\omega_{s}=0^{\circ}$ or $90^{\circ}$ or for an isotropic material $\left(0.27 \cdot u_{m, 2 D}\right.$ in the example of Fig. 12.4). The existing pre-deformation estimation methods, which were developed for isotropic materials, underestimate the pre-deformation (and, consequently, overestimate the convergence) for schistous rocks with medium dip angles, but seem to be adequate for very steep or subhorizontal schistosity planes. 
The large pre-deformations occurring in the case of $\omega_{s}=45^{\circ}$ are caused by failure in the schistosity plane in an extended zone in the ground ahead of the face (over the entire face; Fig. 12.4b). The failure in the schistosity planes results also in bigger longitudinal displacements (Fig. 12.4c). For a tunnel drive perpendicular or parallel to the tunnel axis, the pre-deformations at the crown and the invert are the same (cf. Fig. 12.4) and lie almost in the cross-sectional plane of the tunnel. For $\omega_{s}=45^{\circ}$, however, the longitudinal deformations are considerable at the face and along the entire tunnel; furthermore, the pre-deformations are considerably larger in the invert than in the crown (Fig. 12.4c), because the plastic zone ahead of the face is not symmetric with respect to tunnel axis (Fig. 12.4b). Klopčič and Logar (2014) obtained similar results and therefore concluded that tunnel advance with the dip is advantageous because in this case the major part of the displacements occurs ahead of the face and, consequently, the convergences are smaller. This conclusion is correct concerning the crown displacement, but disregards that the convergence at the invert, which under squeezing conditions is equally important, will be considerably bigger.

Figure 12.9 presents additional computational results, for other values of the schistosity- and matrix-cohesion. The diagrams show the maximum of the "pre-deformation fraction" over the profile circumference as a function of the dip angle. (The "pre-deformation fraction" is defined as $u / u(0)$, where $u$ is the displacement magnitude at a profile point far behind the face and $u(0)$ denotes the displacement magnitude of the same profile point at the face.) The highest pre-deformation fractions occur at dip angles between $20^{\circ}$ and $70^{\circ}$, irrespective of the cohesion of the schistosity and of the matrix.

$$
\mathrm{c}_{\mathrm{m}}=1 \mathrm{MPa}, \mathrm{c}_{\mathrm{s}}=400,800 \mathrm{kPa}
$$

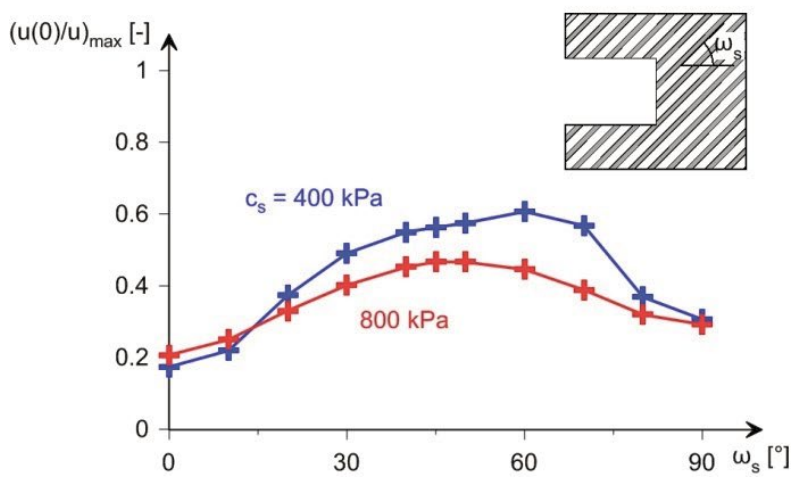

$\mathrm{C}_{\mathrm{m}}=6 \mathrm{MPa}, \mathrm{c}_{\mathrm{s}}=400,800 \mathrm{kPa}$

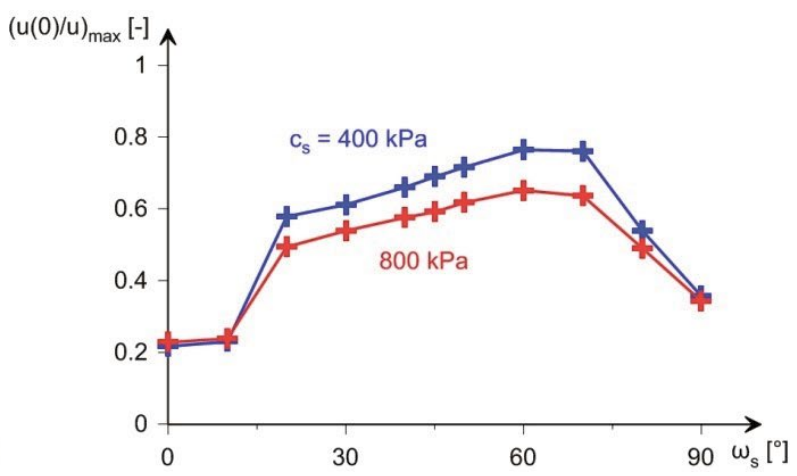

Figure 12.9. Maximum pre-deformation fraction as a function of the dip angle $\omega_{s}\left(a=5 \mathrm{~m}, \sigma_{0}=10 \mathrm{MPa}\right.$, $\left.E=1 \mathrm{GPa}, \varphi_{m}=30^{\circ}, \psi_{m}=10^{\circ}, \varphi_{s}=10^{\circ}, \psi_{s}=5^{\circ}, v=0.3\right)$.

\subsubsection{Influence of the dip angle on the convergences}

Figure 12.10 shows the maximum and minimum convergence as a function of the dip angle. One can recognise that:

- The dip angle influences considerably both the maximum and the minimum convergence;

- Schistosity does not play a role if the schistosity planes are steeper than about $70^{\circ}$;

- The dip angle influences the convergences more than the displacements (for $c_{m}=1 \mathrm{MPa}$ and $c_{s}=0.4 \mathrm{MPa}$, the maximum convergences for $\omega_{s}=0^{\circ}$ are by a factor of 6.5 higher than those for $\omega_{s}=90^{\circ}$, while the maximum displacements for $\omega_{s}=0^{\circ}$ are by a factor of 5 higher than those for $\omega_{s}=90^{\circ}$, see Fig. 12.5). 
$\mathrm{c}_{\mathrm{m}}=1 \mathrm{MPa}, \mathrm{c}_{\mathrm{s}}=400 \mathrm{kPa}$

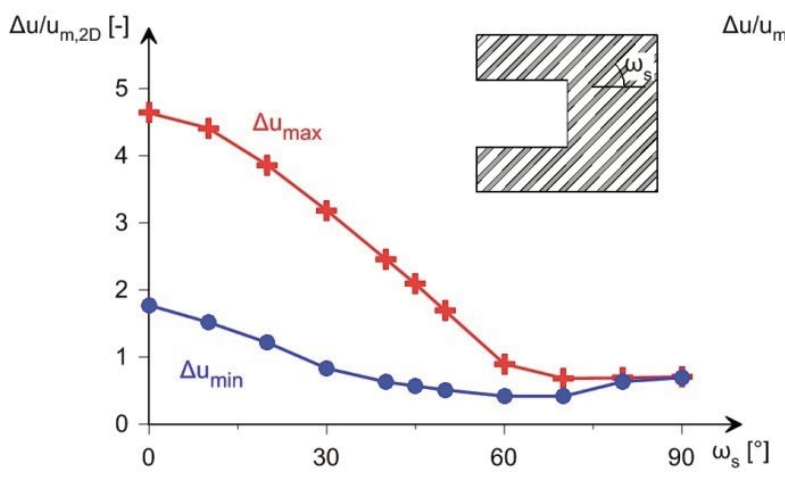

$\mathrm{c}_{\mathrm{m}}=6 \mathrm{MPa}, \mathrm{c}_{\mathrm{s}}=800 \mathrm{kPa}$

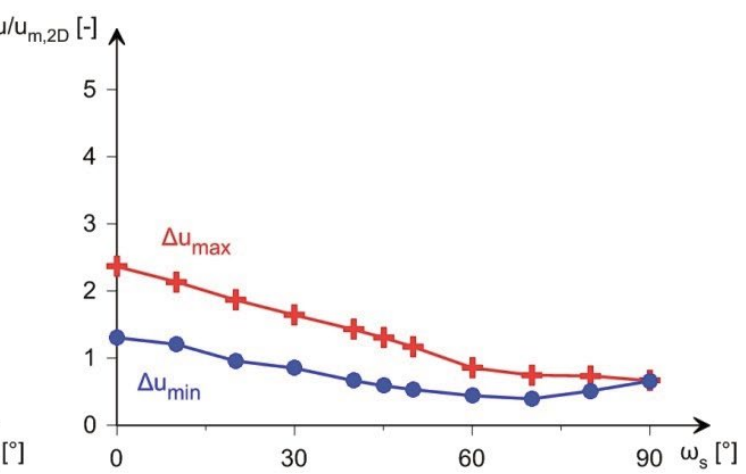

Figure 12.10. Normalised maximum and minimum convergences as a function of the dip angle $\omega_{s}(a=5 \mathrm{~m}$, $\left.\sigma_{0}=10 \mathrm{MPa}, E=1 \mathrm{GPa}, \varphi_{m}=30^{\circ}, \Psi_{m}=10^{\circ}, \varphi_{s}=10^{\circ}, \psi_{s}=5^{\circ}, v=0.3\right)$.

The relationship between the minimum or maximum convergence and dip angle can be approximated analogously to Section 12.2 .1 , i.e. by a linear or bilinear function (see Fig. 12.11):

$$
\begin{aligned}
& \Delta u_{\max }=\left(\Delta u_{\max , \| l}-\Delta u_{\perp}\right) \cdot S+\Delta u_{\perp}, \\
& \Delta u_{\text {min }}=\left(\Delta u_{\text {min, }, I}-\Delta u_{\perp}\right) \cdot S+\Delta u_{\perp} .
\end{aligned}
$$

where $S$ denotes the schistosity influence factor after Equation (12.2) or (12.3); $\Delta u_{\max , I l}$ and $\Delta u_{\text {min,ll }}$ denote the maximum and minimum convergence, respectively, when tunnelling parallel to the schistosity planes and can be determined as usual for isotropic elasto-plastic materials (see Cantieni and Anagnostou 2009a) with the parameters of the equivalent isotropic material after Chapter 11; and $\Delta u \perp$ is the convergence when tunnelling perpendicular to the schistosity planes and can be determined as usual for isotropic elastoplastic materials with the parameters of the matrix. ( $\Delta u_{\perp}$ will be slightly overestimated, because the pre-deformations occurring in the absence of the schistosity are slightly smaller than those occurring in schistous rocks.)

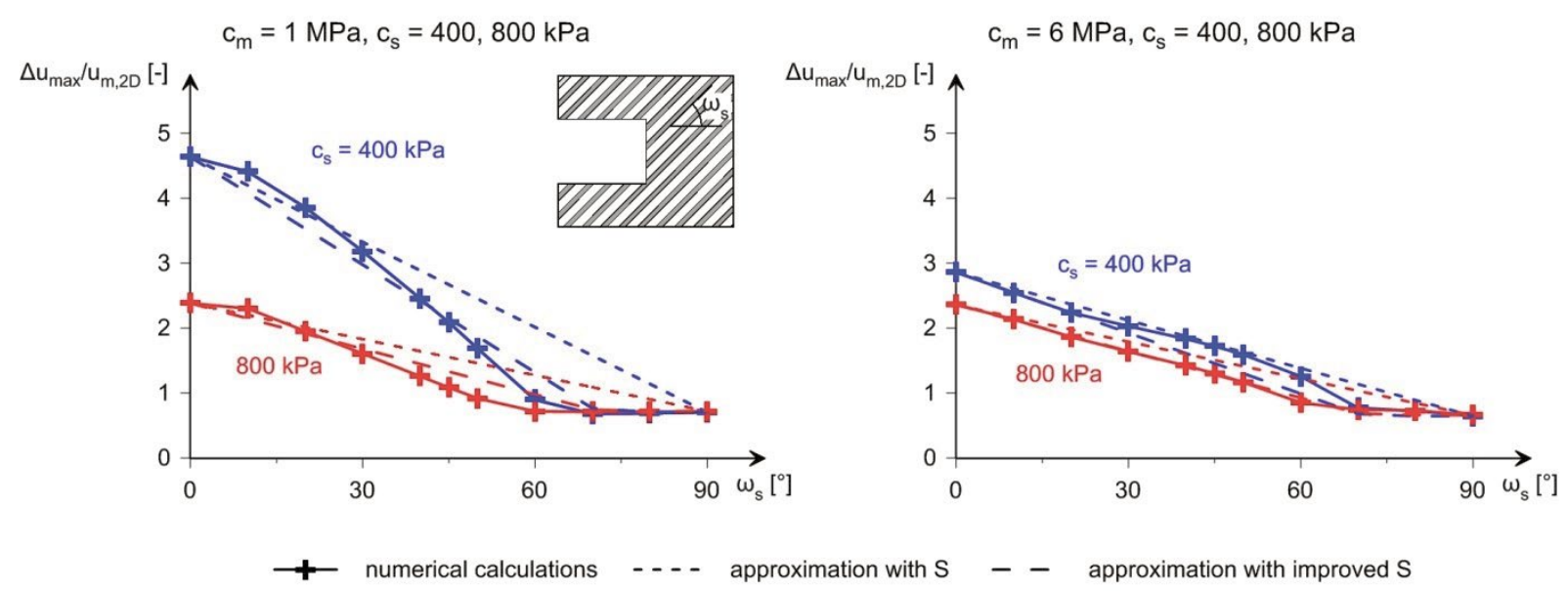

Figure 12.11. Effect of matrix and schistosity cohesion: Normalised maximum convergences as a function of the dip angle $\omega_{s}\left(a=5 \mathrm{~m}, \sigma_{0}=10 \mathrm{MPa}, E=1 \mathrm{GPa}, \varphi_{m}=30^{\circ}, \psi_{m}=10^{\circ}, \varphi_{s}=10^{\circ}, \psi_{s}=5^{\circ}\right.$, $v=0.3)$. 


\subsection{Tunnelling in thinly stratified rock, perpendicular to the strike direction}

This section discusses differences and similarities between schistous and stratified rocks with respect to the influence of the dip angle.

\subsubsection{Influence of the dip angle on the displacements}

Figure 12.12 shows the displacement (the maximum one along the tunnel boundary in a cross-section far behind the tunnel face; normalised by the displacement that would occur in the absence of hard interlayers) as a function of the dip angle. As in the case of a schistous rock (Section 12.2), the dip angle has a remarkable influence on the displacements.

The dashed lines in Figure 12.12 represent the linear interpolation (Eqs. 12.1 and 12.2) between the displacements $u_{\perp}$ and $u_{m a x, I l}$ occurring in the borderline cases of horizontal and vertical bedding, whereby $u \perp$ can be determined analytically after Chapter 5 , while $u_{\text {max, }, I}$ can be estimated using the dimensionless diagrams introduced in Chapter 9.

Contrarily to a schistous rock mass, the bilinear approximation ( $S$ after Eq. 12.3) is not meaningful; due to the direction-dependent stiffness of the stratified rock mass, the displacements for dip angles larger than about $90^{\circ}-\varphi_{w}=65^{\circ}$ are not constant.

The linear approximation underestimates the displacements considerably for the low initial stress $\sigma_{0}$ of $0.75 \mathrm{MPa}$ (for which both the weak and the hard layers remain elastic) but provides satisfactory results for the practically relevant case of a high initial stress $\left(\sigma_{0}=10 \mathrm{MPa}\right.$ in the example of Fig. 12.12). Therefore, in the next sections, only the case of $\sigma_{0}=10 \mathrm{MPa}$ will be analysed.

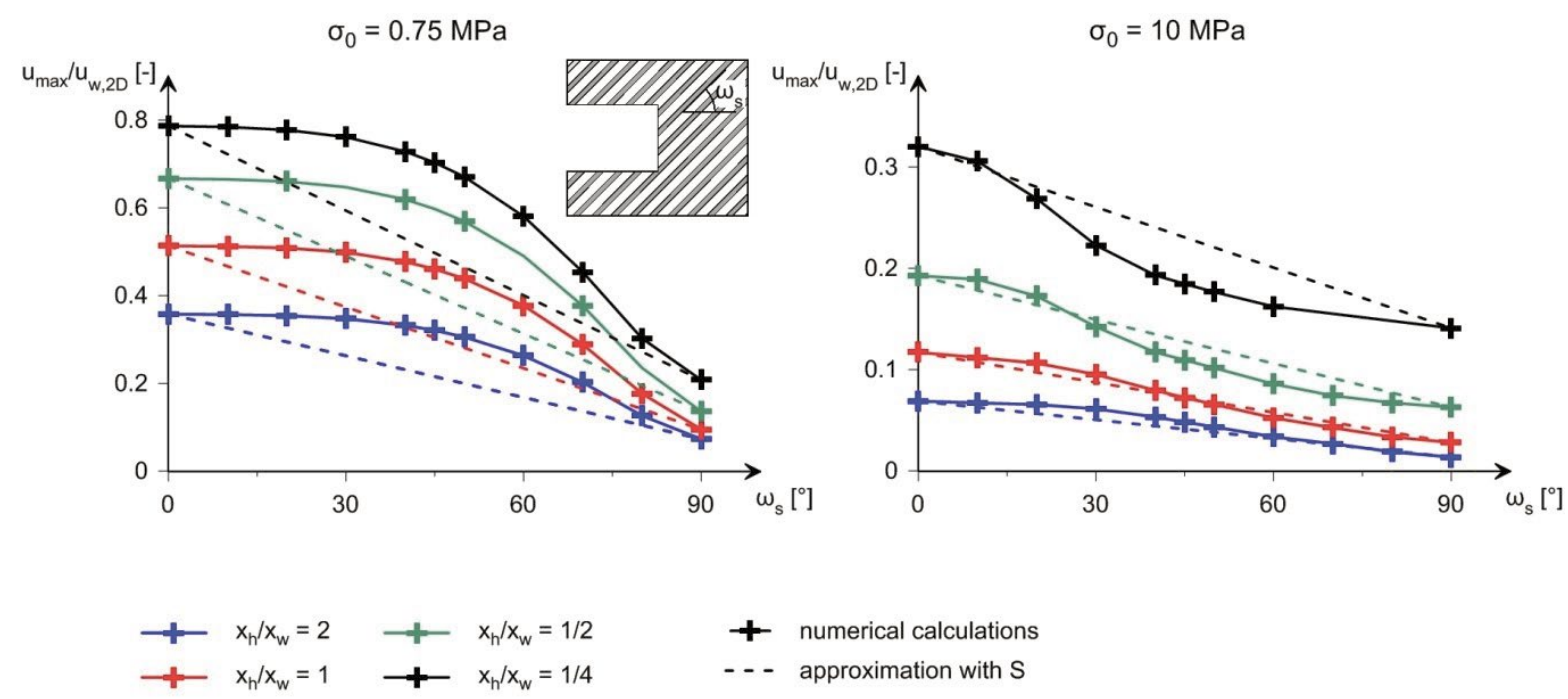

Figure 12.12. Normalised maximum displacement as a function of the dip angle $\omega_{s}\left(a=5 \mathrm{~m}, E_{h}=10 \mathrm{GPa}\right.$, $\left.c_{h}=5 \mathrm{MPa}, E_{w}=0.5 \mathrm{GPa}, c_{w}=0.5 \mathrm{MPa}, v_{h}=v_{w}=0.3, \varphi_{h}=\varphi_{w}=25^{\circ}, \psi_{h}=\psi_{w}=5^{\circ}\right)$.

\subsubsection{Influence of the dip angle on the pre-deformations}

As for a schistous rock, the pre-deformation in the invert is large for $\omega_{s}=45^{\circ}$ (Fig. 12.13a). (For comparison, the pre-deformation for a tunnel drive in only hard rocks would amount to 
$0.016 \cdot u_{w, 2 D}$ and those in only weak rocks to $0.25 \cdot u_{w, 2 D}$.) However, contrary to schistous rocks, considerable pre-deformation fractions occur also for a dip angle of $90^{\circ}$.

The large pre-deformations in tunnelling perpendicular to the layers are associated with the large longitudinal deformations (Fig. 12.13b), which occur due to the considerably lower stiffness perpendicular to the layers: As the ground deforms more ahead of the tunnel face, the rock behind the face experiences a longitudinal displacement towards the excavated part of the tunnel. The opposite happens in a tunnel drive parallel to the layers: As the ground deforms more behind the tunnel face, it experiences a longitudinal displacement towards the tunnel face.

(a)
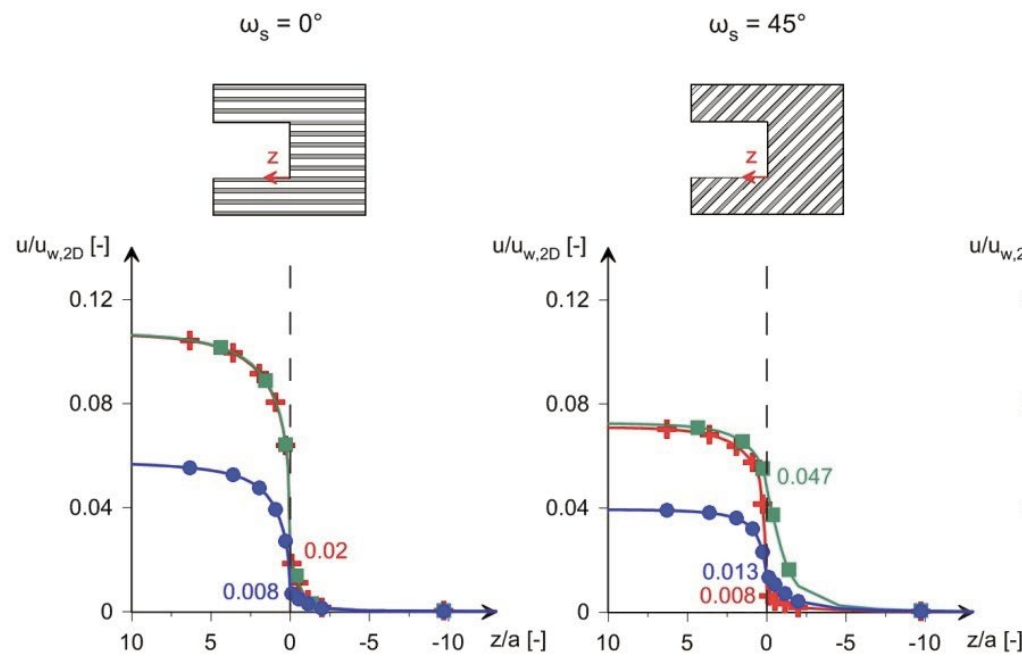

$\omega_{\mathrm{s}}=45^{\circ}$
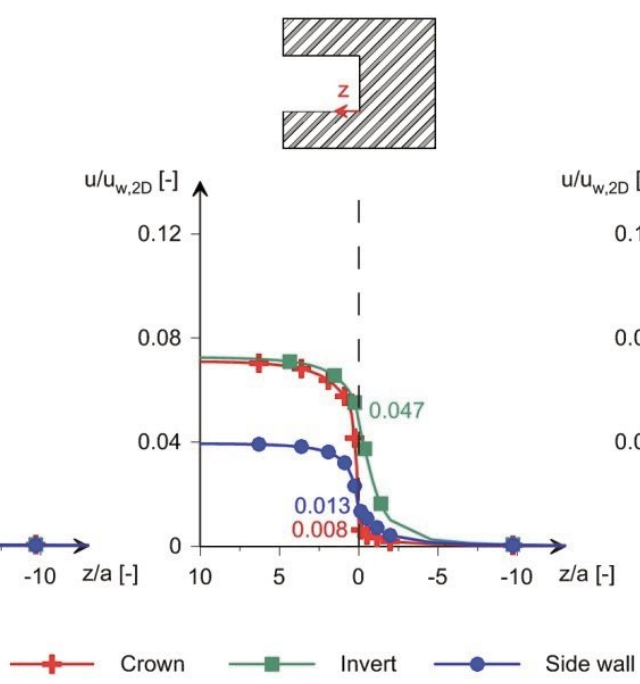

(b)
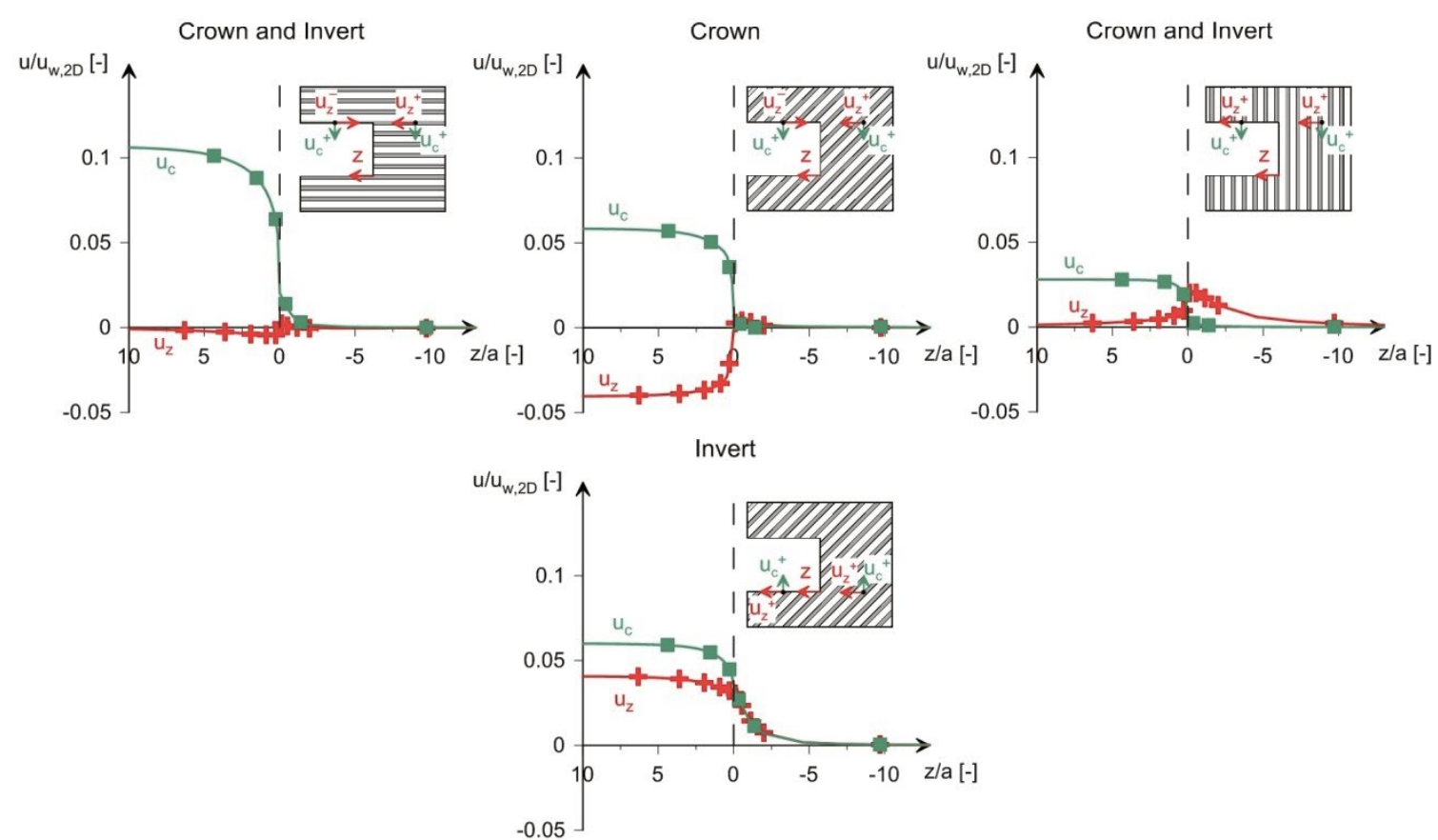

Figure 12.13. (a) Normalised displacement $u / u_{w, 2 D}$ along the tunnel; and, (b), normalised longitudinal displacement $\left(u_{z} / u_{w, 2 D}\right)$ and normalised cross-sectional displacement $\left(u_{c} / u_{w, 2 D}\right)$ along the tunnel $\left(a=5 \mathrm{~m}, \sigma_{0}=10 \mathrm{MPa}, X_{h} / X_{w}=1, E_{h}=10 \mathrm{GPa} ; c_{h}=5 \mathrm{MPa}, E_{w}=0.5 \mathrm{GPa}, c_{w}=0.5 \mathrm{MPa}\right.$, $\left.v_{h}=v_{w}=0.3, \varphi_{h}=\varphi_{w}=25^{\circ}, \psi_{h}=\psi_{w}=5^{\circ}\right)$. 
As for schistous rocks, large longitudinal deformations ahead of the face take also place for a dip angle of $\omega_{s}$ of $45^{\circ}$ (Fig. 12.13b); they are considerably larger in the invert than in the crown. This aspect is more pronounced than for the schistous rock mass and was already observed by Tonon and Amadei (2002): A transversely isotropic rock mass is stiffer in direction parallel to the layers and more deformable in direction normal to them. When excavating against dip, the rock mass at the invert is more deformable towards the excavated part of the tunnel. On the contrary, the rock mass at the crown is more deformable towards the non-excavated part of the tunnel, leading thus to smaller pre-deformations at the crown (cf. Tonon and Amadei 2002).

Tunnelling against dip
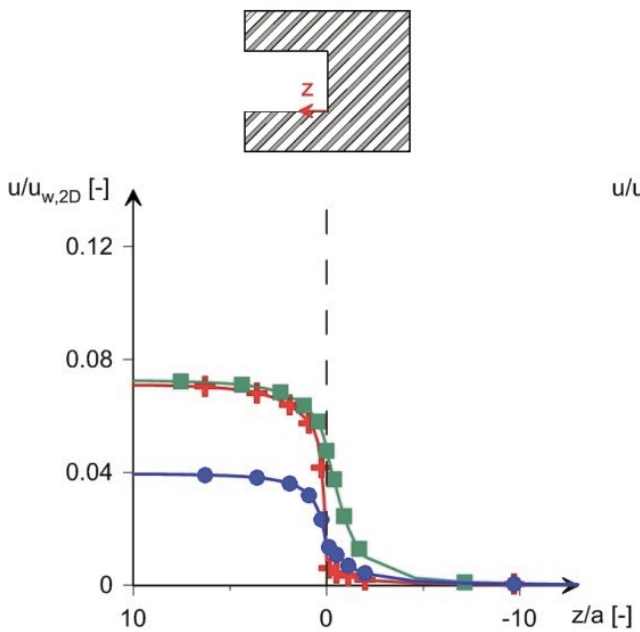

+- Crown
Tunnelling with dip
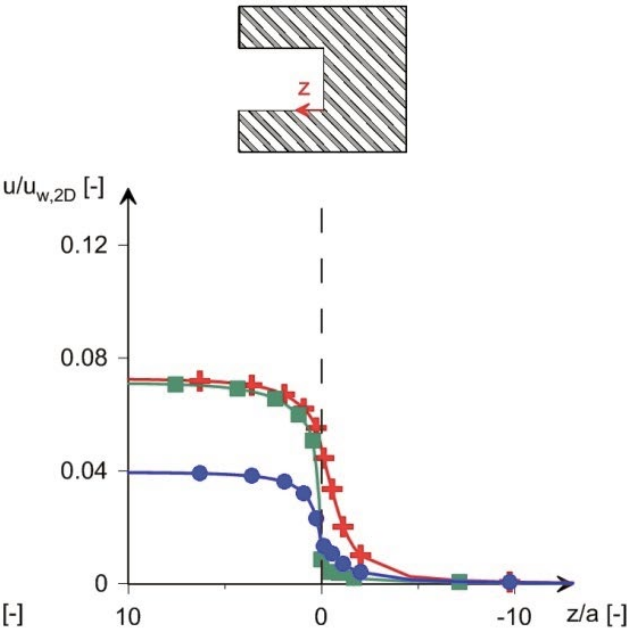

Side wall

Figure 12.14. Normalised magnitude of the displacement vector $u / u_{w, 2 D}$ along the tunnel for a dip angle $\omega_{s}$ of $45^{\circ}\left(a=5 \mathrm{~m}, \sigma_{0}=10 \mathrm{MPa}, x_{h} / x_{w}=1, E_{h}=10 \mathrm{GPa} ; c_{h}=5 \mathrm{MPa}, E_{w}=0.5 \mathrm{GPa}, c_{w}=0.5 \mathrm{MPa}\right.$, $\left.v_{h}=v_{w}=0.3, \varphi_{h}=\varphi_{w}=25^{\circ}, \psi_{h}=\psi_{w}=5^{\circ}\right)$.

Therefore, according to Tonon and Amadei (2002), larger tunnel convergences develop when tunnelling with dip (as when tunnelling against dip), as the pre-deformations occurring ahead of the tunnel are larger (while the total displacements are the same). Figure 12.14, however, shows that the tunnel convergences when tunnelling with or against dip are exactly the same, but do not develop at the same location in the tunnel (i.e., mirroring the tunnel problem). Tonon and Amadei (2002) only considered the pre-deformations in the crown and did not take into account that the pre-deformations in the invert (when advancing in dip direction) are considerably smaller. Of course, the excavation direction can be important in tunnelling - as for example tunnelling against dip is more critical in respect to the stability of the tunnel face (due to loosening) - but not in respect to squeezing.

According to Figure 12.15, which shows the pre-deformation fraction as a function of the dip angle, the pre-deformation fraction reaches considerable values for dip angles between $20^{\circ}$ and $70^{\circ}$. However in contrast to schistous rocks, for dip angles of about $90^{\circ}$, the predeformation fraction can also be large, because large longitudinal deformations occur ahead of the face due to the stiffness difference parallel and perpendicular to the layers. 


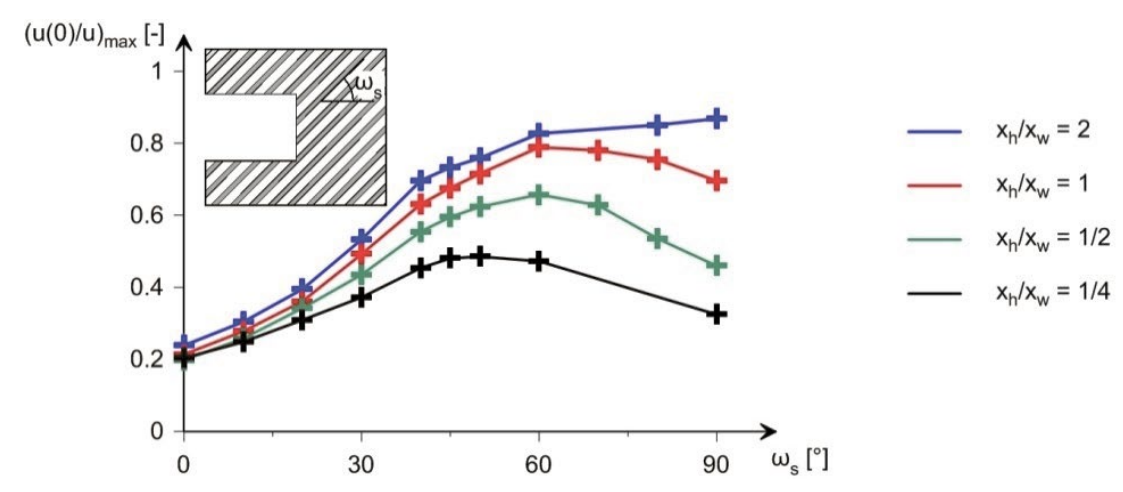

Figure 12.15. Maximum pre-deformation fraction as a function of the dip angle $\omega_{s}\left(a=5 \mathrm{~m}, \sigma_{0}=10 \mathrm{MPa}\right.$, $E_{h}=10 \mathrm{GPa}, c_{h}=5 \mathrm{MPa}, E_{w}=0.5 \mathrm{GPa}, c_{w}=0.5 \mathrm{MPa}, v_{h}=v_{w}=0.3, \varphi_{h}=\varphi_{w}=25^{\circ}, \psi_{h}=\psi_{w}=5^{\circ}$ ).

\subsubsection{Influence of the dip angle on the convergences}

The linear approximation (Eq. 12.5) can also be applied for stratified rocks, whereby $\Delta u \perp$ and $\Delta u_{m a x, l l}$ can be determined as usual for isotropic elasto-plastic materials (see Cantieni and Anagnostou 2009a) with the parameters of an equivalent isotropic material after Chapter 6 and 9, respectively. The linear approximation is satisfactory for stratified rocks (Fig. 12.16), although not so good as for schistous rocks.

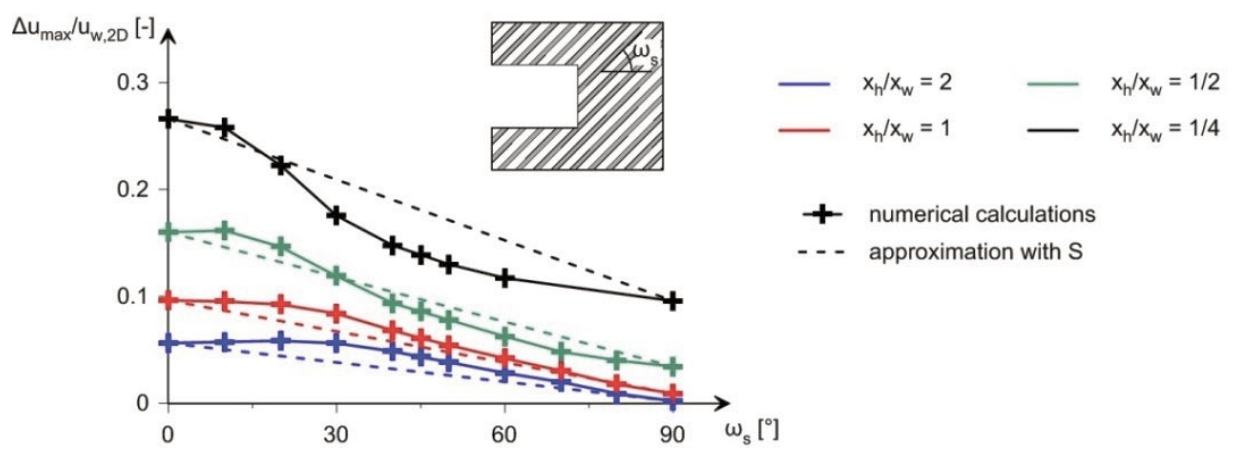

Figure 12.16. Normalised maximum tunnel convergences as a function of the dip angle $\omega_{s}(a=5 \mathrm{~m}$, $\sigma_{0}=10 \mathrm{MPa}, E_{h}=10 \mathrm{GPa} ; c_{h}=5 \mathrm{MPa}, E_{w}=0.5 \mathrm{GPa}, c_{w}=0.5 \mathrm{MPa}, v_{h}=v_{w}=0.3, \varphi_{h}=\varphi_{w}=25^{\circ}$, $\boldsymbol{\Psi}_{h}=\boldsymbol{\psi}_{w}=5^{\circ}$ ).

\subsection{Arbitrary strike}

The linear or bilinear approximations of Section 12.2 (Eqs. 12.1 to 12.6) are sufficiently accurate also for arbitrary dip angles $\omega_{s}$ and strike directions $\theta_{s}$. This will be shown by the computational results of a parametric study considering schistous rock. (The behaviour of thinly stratified rocks is mostly the same in this respect; see Section 12.3.)

Figures 12.17 and 12.18 show the numerically obtained displacement versus $\left(\omega_{s}, \theta_{s}\right)$ and the convergence versus $\left(\omega_{s}, \theta_{s}\right)$ diagrams for less and more severe squeezing conditions, respectively. (In the first case, yielding occurs only in the schistosity plane.) 
The diagrams of Figures 12.17 and 12.18 are very similar to the $S$ versus $\left(\omega_{s}, \theta_{s}\right)$ diagram of Chapter 1 and, particularly, to the one of Figure 12.7 (improved schistosity influence factor $S$ ), supporting thus the conclusion that the linear and, particularly, the bilinear approximation introduced in Section 12.2 are sufficiently accurate for practical purposes for any strike direction to the tunnel axis.

$$
\mathrm{c}_{\mathrm{s}}=800 \mathrm{kPa}, \mathrm{c}_{\mathrm{m}}=6000 \mathrm{kPa}
$$
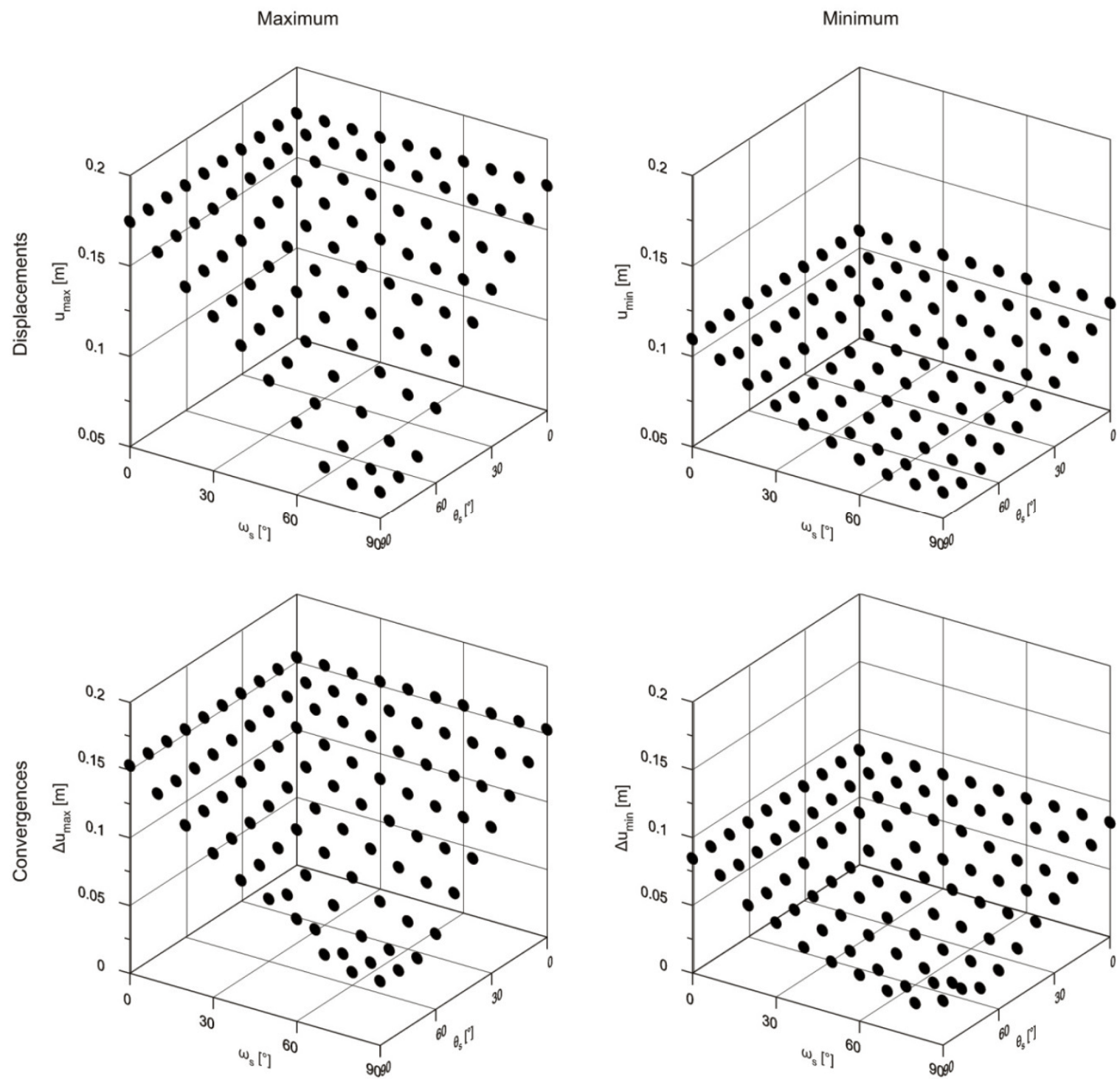

Figure 12.17. Maximum and minimum displacements and convergences as a function of the dip angle $\omega_{s}$ and the angle $\theta_{s}$ between the schistosity strike direction and tunnel axis $(a=5 \mathrm{~m}$, $\sigma_{0}=10 \mathrm{MPa}, E=1 \mathrm{GPa}, c_{m}=6 \mathrm{MPa}, \varphi_{m}=30^{\circ}, \psi_{m}=10^{\circ}, c_{s}=0.8 \mathrm{MPa}, \varphi_{s}=10^{\circ}, \psi_{s}=5^{\circ}$, $v=0.3)$. 

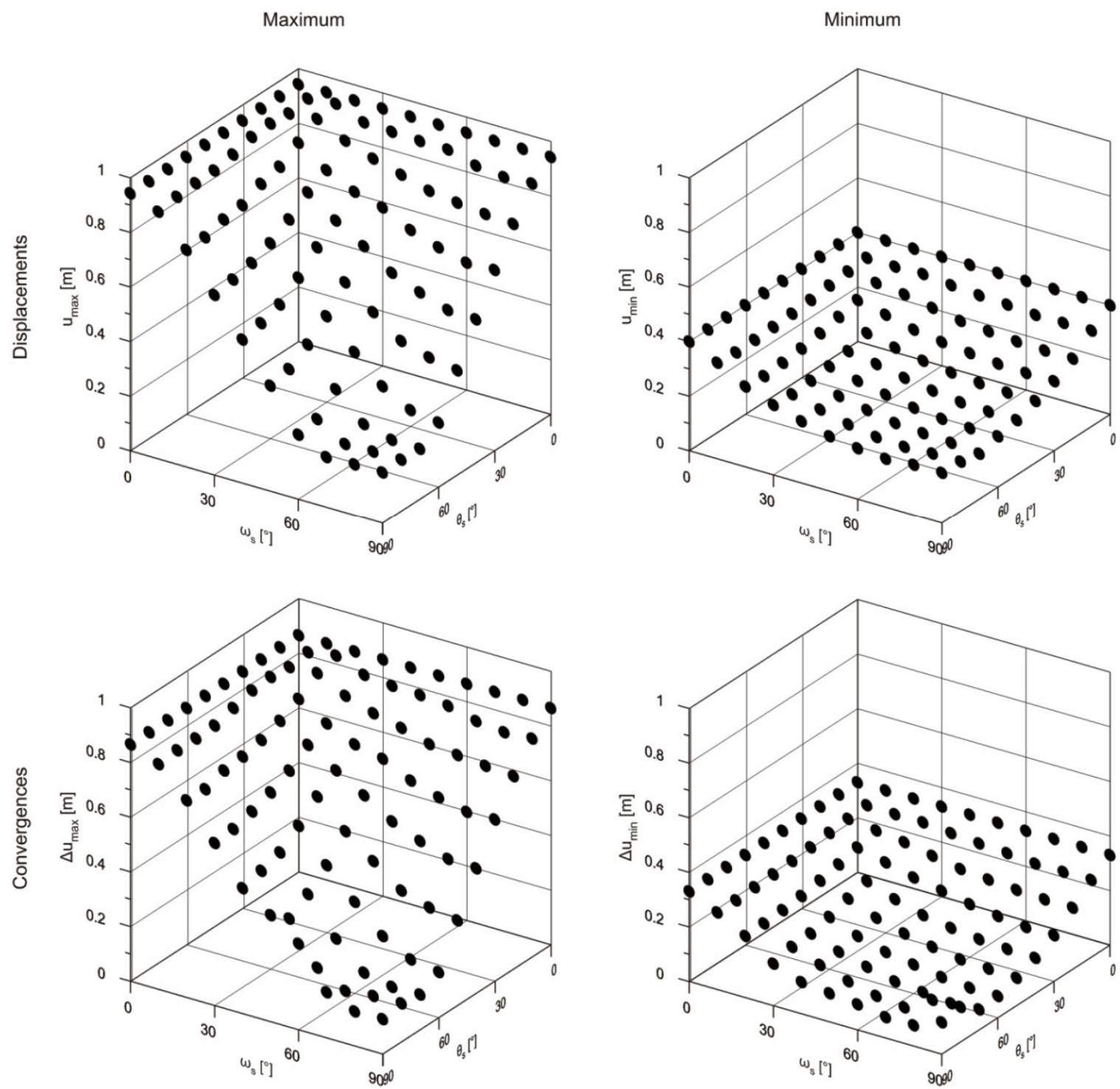

Figure 12.18. Maximum and minimum displacements and convergences as a function of the dip angle $\omega_{s}$ and the angle $\theta_{s}$ between the schistosity strike direction and tunnel axis $(a=5 \mathrm{~m}$, $\sigma_{0}=10 \mathrm{MPa}, E=1 \mathrm{GPa}, c_{m}=1 \mathrm{MPa}, \varphi_{m}=30^{\circ}, \psi_{m}=10^{\circ}, c_{s}=0.4 \mathrm{MPa}, \varphi_{s}=10^{\circ}, \psi_{s}=5^{\circ}$, $v=0.3)$.

\subsection{Conclusions}

This chapter investigated the influence of bedding and schistosity planes using spatial calculation models with arbitrary orientation of the planes of anisotropy relative to the tunnel axis and showed that the orientation of the planes of anisotropy to the tunnel has a considerable influence on the convergences (considering the pre-deformation occurring ahead of the tunnel face), when tunnelling through thinly stratified and schistous rock masses.

Furthermore, this chapter could show that there are relevant differences to the well-known case of an isotropic rock mass and that the numerical calculations reproduce well the empirically determined relationship between convergence and spatial orientation of the schistosity considering the so-called "schistosity influence factor", which combines the dip $\omega_{s}$ 
and the strike direction $\theta_{s}$ of the planes of anisotropy to the tunnel axis and was developed based on the data from the Gotthard Base Tunnel (Chapter 1). This relationship allows to estimate the displacement for an arbitrary orientation of the anisotropy planes to the tunnel axis based upon the displacements for the borderline cases of vertical or horizontal anisotropy planes, rendering thus spatial numerical analyses unnecessary at least in the preliminary design stage.

\section{Notation}

\begin{tabular}{|c|c|}
\hline a & tunnel radius \\
\hline$c_{h}, c_{w}$ & cohesion of the hard and of the weak layers \\
\hline$C_{s}, C_{m}$ & cohesion of the schistosity and the surrounding matrix \\
\hline E & Young's modulus of the schistous rock mass \\
\hline$E_{h}, E_{w}$ & Young's modulus of the hard and of the weak layers \\
\hline$S$ & influence factor of the schistosity \\
\hline$u$ & magnitude of the displacement vector at the tunnel boundary \\
\hline$u(0)$ & displacements occurring ahead of the tunnel face \\
\hline$u_{c}$ & $\begin{array}{l}\text { magnitude of the projections of the displacement vectors in the cross- } \\
\text { sectional plane of the tunnel }\end{array}$ \\
\hline$u_{m, 2 D}$ & $\begin{array}{l}\text { radial displacement of the non-schistous rock mass (far behind the } \\
\text { face) }\end{array}$ \\
\hline$u_{\max }, u_{\min }$ & $\begin{array}{l}\text { maximum and minimum magnitude of the displacement vector along } \\
\text { the tunnel boundary }\end{array}$ \\
\hline$u_{\max , I I}, u_{\min , \|}$ & $\begin{array}{l}\text { maximum and minimum magnitude of the displacement vector along } \\
\text { the tunnel boundary for a tunnel drive parallel to the planes of aniso- } \\
\text { tropy }\end{array}$ \\
\hline$u \perp$ & $\begin{array}{l}\text { magnitude of the displacement vector along the tunnel boundary for a } \\
\text { tunnel drive perpendicular to the planes of anisotropy }\end{array}$ \\
\hline$u_{w, 2 D}$ & $\begin{array}{l}\text { radial displacement of the homogeneous weak ground (occurring far } \\
\text { behind the face) }\end{array}$ \\
\hline$u_{z}$ & longitudinal displacement \\
\hline$x_{h}, x_{w}$ & thickness fraction of the hard and of the weak layers \\
\hline$x$ & co-ordinate (see Fig. 12.2) \\
\hline$y$ & co-ordinate (see Fig. 12.2) \\
\hline$z$ & axial co-ordinate (distance behind the tunnel face; see Fig. 12.2) \\
\hline$\beta$ & $\begin{array}{l}\text { angle between the tangential direction of the tunnel boundary and the } \\
\text { planes of weakness }\end{array}$ \\
\hline$\Delta u$ & $\begin{array}{l}\text { convergences, i.e., displacements far behind the face minus pre- } \\
\text { deformations (defined as } u-u(0) \text { ) }\end{array}$ \\
\hline$\Delta u_{\max , I I}, \Delta u_{\min , I l}$ & $\begin{array}{l}\text { maximum and minimum convergence for a tunnel drive parallel to the } \\
\text { planes of anisotropy }\end{array}$ \\
\hline$\Delta u \perp$ & $\begin{array}{l}\text { convergence for a tunnel drive perpendicular to the planes of aniso- } \\
\text { tropy }\end{array}$ \\
\hline$\theta_{s}$ & $\begin{array}{l}\text { angle between the tunnel axis and the strike direction of the planes of } \\
\text { anisotropy }\end{array}$ \\
\hline$v$ & Poisson's ratio of the schistous rock mass \\
\hline$V_{h}, V_{w}$ & Poisson's ratio of the hard and of the weak layers \\
\hline$\sigma_{0}$ & initial stress \\
\hline$\sigma_{a}$ & support pressure of the tunnel \\
\hline
\end{tabular}



$T_{x y}, T_{x z}, T_{y z}$
$\varphi_{h}, \varphi_{w}$
$\varphi_{s}, \varphi_{m}$
$\psi_{h}, \psi_{w}$
$\psi_{s}, \psi_{m}$
$\omega_{s}$

shear stress in $x y-, x z$ - and $y z$-direction

friction angle of the hard and of the weak layers

friction angle of the schistosity and of the surrounding matrix

dilatancy angle of the hard and of the weak layers

dilatancy angle of the schistosity and of the surrounding matrix dip angle of the planes of anisotropy 



\section{Variability of squeezing deformations in folded rocks}

\subsection{Introduction}

Chapter 12 showed that the orientation of bedding or schistosity plays a very important role for the magnitude and distribution of the deformations in the cross-section of the tunnel. Specifically, planes of anisotropy may affect rock behaviour adversely, particularly if their strike direction forms a small angle with (or is parallel to the tunnel axis). The orientation of bedding or schistosity may change frequently along a tunnel through folded rocks and result in variable squeezing intensity.

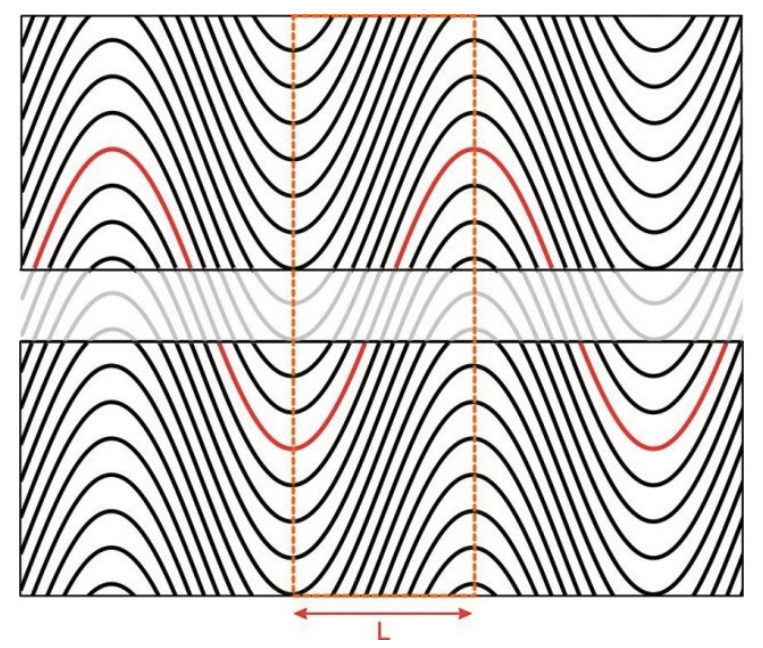

Figure 13.1. Tunnelling through a folded structure with strike direction of the planes of anisotropy perpendicular to the tunnel axis.

In this chapter, a folded rock mass is considered, whose strike direction is perpendicular to the tunnel, while the dip angle changes along the alignment. A folded structure is characterised by tunnel sections, where the schistosity planes lie parallel to the tunnel axis and tunnel sections with larger dip angles (see Fig. 13.1). From Chapter 12, it is known that the most adverse conditions occur, when the schistosity lies parallel to the tunnel axis and the most favourable conditions occur, when the dip angle of the schistosity becomes maximum. Due to the interaction between tunnel sections with more favourable anisotropy plane orientations and tunnel sections with adverse anisotropy plane orientations, the deformations may become more uniform along the tunnel, so that the squeezing variability may even disappear completely. This can be the case in an extremely folded rock mass, where the orientation of the anisotropy planes changes within few meters. The influence of frequent orientation changes of the planes of anisotropy in folded rocks on the squeezing behaviour has not been investigated so far. 
Starting with the problem definition and the geometric modelling of a folded rock mass in Section 13.2, it will be shown that even small variations of the orientation of the planes of anisotropy may cause a significant variability of the intensity of the convergences, and this both for schistous (Section 13.3) and for thinly stratified rock masses (Section 13.4).

Finally, in Section 13.5, the influence of the variation of the orientation of the planes of anisotropy on the tunnel convergences will be shown by means of an application example concerning the Sedrun section of the Gotthard Base Tunnel, where heavily squeezing conditions were encountered during construction (Chapter 1), and the predictive capacity of the so-called "schistosity influence factor" $S$, which was already introduced in Chapter 1 , will be discussed.

\subsection{Problem definition}

Due to buckling of the formation, a folding may develop that has a symmetric, periodic, sinusoidal shape (cf., e.g., Burg 2018). The geometry of the folding can be simplified as a sinusoidal function in the z-direction (Fig. 13.2):

$$
y=A \cdot \sin \left(\frac{(z-L / 2) \cdot \pi}{L}\right)
$$

where $L$ denotes half the period and $A$ the amplitude of the folds.

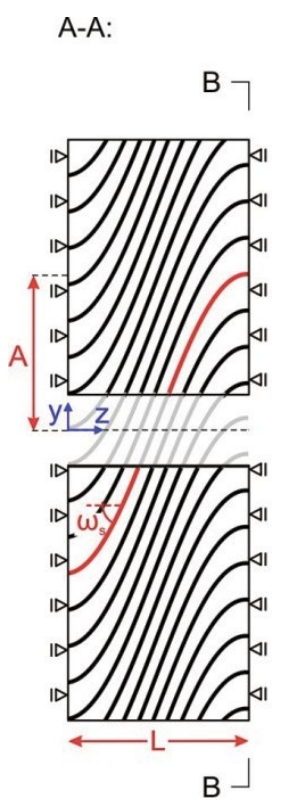

B-B:<smiles>C1CCCCC1</smiles>

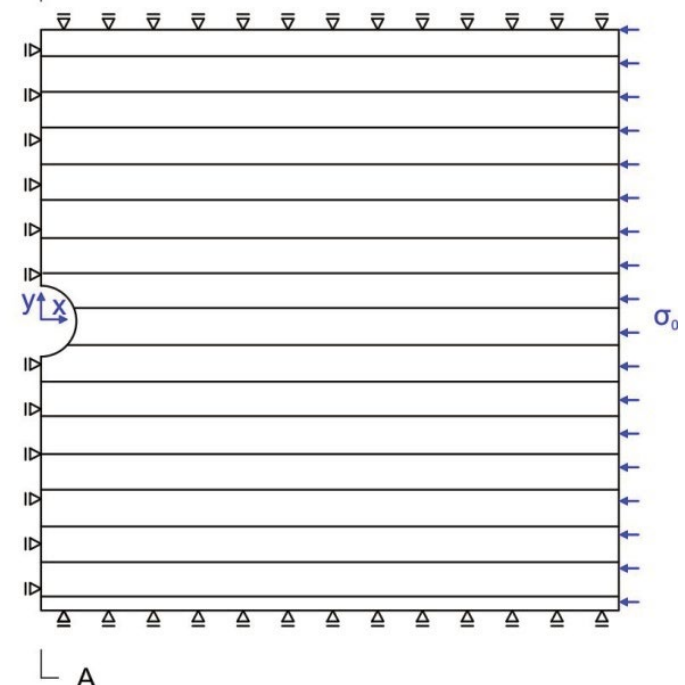

Figure 13.2. Problem layout with boundary conditions assuming that the strike direction of the planes of anisotropy is perpendicular to the tunnel axis.

The dip angle $\omega_{s}$ varies then along the tunnel according to the equation:

$$
\omega_{s}=\left|\arctan \left(A \cdot \frac{\pi}{L} \cos \left(\frac{(z-L / 2) \cdot \pi}{L}\right)\right)\right| \in[0 ; 90],
$$


while the maximum dip angle of the folds reads as follows:

$$
\omega_{s, \max }=\arctan \left(A \cdot \frac{\pi}{L}\right)
$$

Folding is taken into account numerically on the level of the constitutive model, by considering a position-dependent normal vector to the anisotropy surface:

$$
n=\left(\begin{array}{c}
\mathbf{n} \\
0 \\
1 \\
-\frac{A \cdot \pi}{L} \cdot \cos \left(\frac{(z-L / 2) \cdot \pi}{L}\right)
\end{array}\right) .
$$

According to Equation (13.4), the geometry of the folded rock mass is defined solely by the maximum dip angle $\omega_{s, \max }$ (defined by $A \pi / L$; Eq. 13.3) and the period $2 L$ of the sinuisodal folds.

(a)

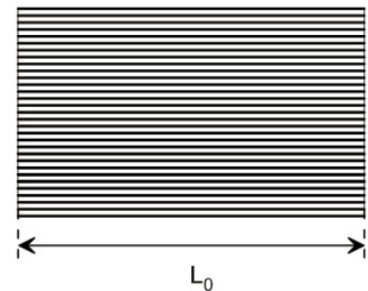

(b)

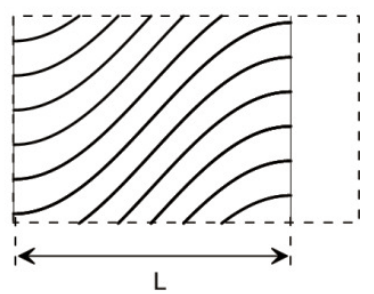

Figure 13.3. (a) Undeformed formation, (b), folding due to shortening of the formation by $20 \%$.

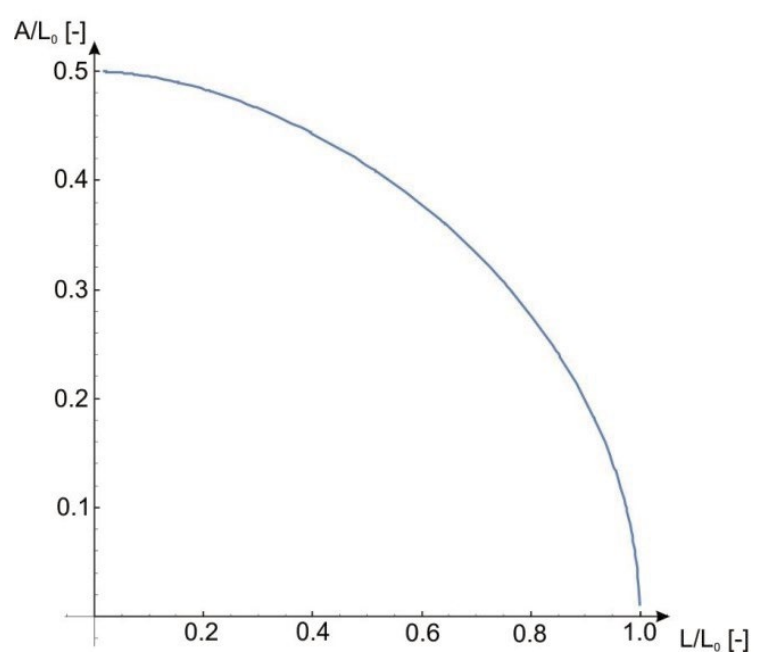

Figure 13.4. Amplitude of the folding $A / L_{0}$ according to Equation (13.5) as a function of $L / L_{0}$.

Physically meaningful pairs of these two parameters could be chosen by considering an initially unfolded formation of length $L_{0}$ (Fig. 13.3a), which buckles under the action of horizontal tectonic stresses and becomes compressed to a length $L$ (Fig. 13.3b). The corresponding amplitude of the folding (cf. Eq. 13.1) can be calculated with the requirement that the arc length of the folding corresponds to the original length of the formation: 


$$
L_{0}=\int_{0}^{L} \sqrt{1+\left(y^{\prime}\right)^{2}} \cdot d z=\int_{0}^{L} \sqrt{1+\left(\frac{A \cdot \pi}{L} \cos \left(\frac{\pi \cdot z}{L}\right)\right)^{2}} \cdot d z
$$

which can be solved with respect to $A$ by using the elliptic integral of the function. The results are represented in Figure 13.4. The more the formation is compressed, the larger the amplitude and thus the maximum dip angle $\omega_{s, \max }$ of the folding will be (Eq. 13.3).

(a)

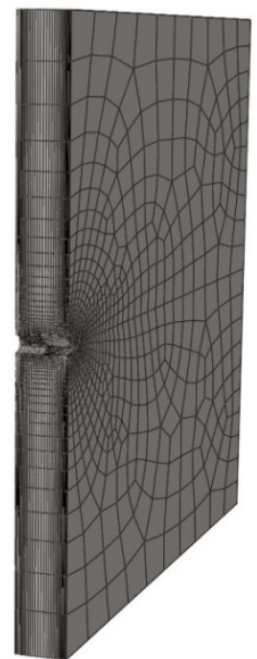

(b)

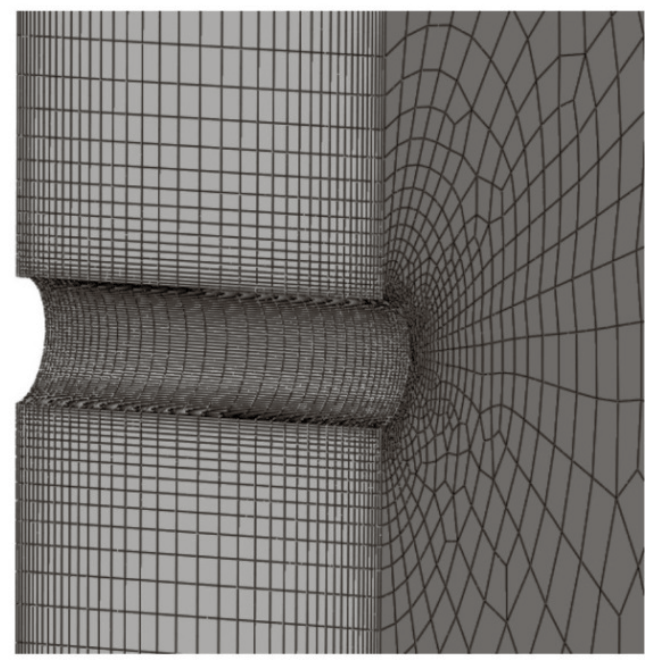

Figure 13.5. (a) Numerical model for $L=25 \mathrm{~m}$ for the strike direction of the planes of anisotropy perpendicular to the tunnel axis, (b), Detail of the numerical model.

The numerical model is presented in Figure 13.2 and is delimited by the symmetry planes of the folding, having thus a length equal to half a period $L$ (cf. Fig. 13.1). If the strike direction of the planes of anisotropy is perpendicular to the tunnel axis (due to symmetry), only half of the system has to be considered; otherwise the whole system has to be considered (as in Section 13.5).

An unsupported tunnel will be considered and the excavation will be simulated through a stepwise reduction of the tractions at the excavation boundary from $\sigma_{0}$ to zero along the entire tunnel (Fig. 13.2). The displacements, that are calculated in this way, include the predeformations. Only the magnitude of the displacement vector will be considered (hereafter referred to as "displacement"). (The longitudinal displacement is small, which means that the main component of the displacement vector is the cross-sectional one.) In order to reduce the sensitivity of the results to the mesh discretisation, sufficiently fine meshes were chosen (e.g., Fig. 13.5).

The rock structure is taken as a homogeneous, transversely isotropic material, which accounts either for the presence of schistosity planes, or for the mechanical parameters and thickness fractions of thinly alternating weak and hard layers. The constitutive models were already presented and validated in the Chapters 9 and 11, respectively. The computations are carried out with Abaqus (Dassault Systèmes 2011). 


\subsection{Schistous rocks}

According to Chapter 12, the largest displacements develop when yielding occurs both in the schistosity planes and in the matrix. Therefore, low cohesion values will be chosen both for the matrix and the schistosity planes (see legend of Fig. 13.6).

The I.h.s. diagrams of Figure 13.6 show the longitudinal displacement distribution (maximum, minimum and averaged values, normalised by the tunnel radius) for an unfolded formation as well as for the folding resulting from a formation compression $\varepsilon_{f}$ by $3.5 \%, 20 \%$ and $50 \%$. The r.h.s. diagrams show the displacement distribution along the circumference of the tunnel cross-section at the fold peak ( $z=0$; the most unfavourable cross-section) as well as at the location of the maximum dip angle ( $z=L / 2$; the most favourable cross-section). In the following, the magnitude and distribution of the displacements will be discussed, considering the ratio of the maximum displacements at the two aforementioned cross-sections (at $z=0$ and $L / 2$ ) as a measure of the squeezing variability. (The minimum and averaged displacements remain nearly constant along the tunnel.) Studying the computational results of Figure 13.6 from top to down, one can readily recognise the effect of an increasing tectonic compression $\varepsilon_{f}$ and folding of the formation:

- The maximum dip angle $\omega_{s, \text { max }}$ increases and consequently (Chapter 12) the displacements at $z=L / 2$ decrease. For $\varepsilon_{f} \geq 50 \%$, the anisotropy plane becomes so steep at the cross-section $z=L / 2\left(\omega_{s, \max }>68^{\circ}\right)$, that the schistosity does not play a role anymore (cf. Chapter 12). The displacements at the cross-section $z=L / 2$ are approximately equal to the displacements that would occur if the dip angle were constant $\left(=\omega_{s, \max }\right)$ along the tunnel. (This is, for example, recognisable for $\varepsilon_{f}=20 \%$, as $u_{\text {max }} / a$ for the constant $\omega_{s, \max }$ amounts to ca. 0.1 and is the same as $u_{\max }(z=L / 2) / a \approx 0.1$.) This means that the tunnel section with steep anisotropy planes is not influenced by the nearby zones where the anisotropy planes are subhorizontal.

- The displacements at the fold peak $(z=0)$ decrease (by a factor of two for a compression $\varepsilon_{f}$ of $50 \%$ ); the adjacent tunnel sections with steep anisotropy planes have a stabilising effect. Note that in the case of intensive folding $\left(\varepsilon_{f}=50 \%\right)$, particularly large displacements occur only very locally, in the very short tunnel sections with almost horizontal schistosity planes. The tunnel sections with steep schistosity planes are much more extended, which explains the observation made above that the displacements in these tunnel sections are not affected by the fold peaks.

- The displacement distribution becomes more uniform along the circumference of the tunnel cross-section (compare the r.h.s. diagrams for $\varepsilon_{f}=0 \%$ with those for $\varepsilon_{f}=50 \%$ ). In the case of intensive folding $\left(\varepsilon_{f}=50 \%\right)$, the displacements are non-uniformly distributed along the circumference of the tunnel cross-section only in the close vicinity of the fold peaks.

- The squeezing variability increases in the considered range of $0 \%<\varepsilon_{f}<50 \%$. For $\varepsilon_{f}=50 \%$, the maximum displacement at the fold peak is by a factor 2.5 higher than the maximum displacement in the tunnel section with steep schistosity planes. It should be noted that the squeezing variability is maximum at $\varepsilon_{f}=50 \%$. If the formation were more intensively folded, then the displacements at $z=L / 2$ would remain constant (schistosity does not play a role for such big dip angles; cf. Chapter 12), but the displacements at $z=0$ (and consequently the variability, too) would decrease (due to the stabilising influence of the adjacent areas with steep schistosity planes). This will be shown later. 


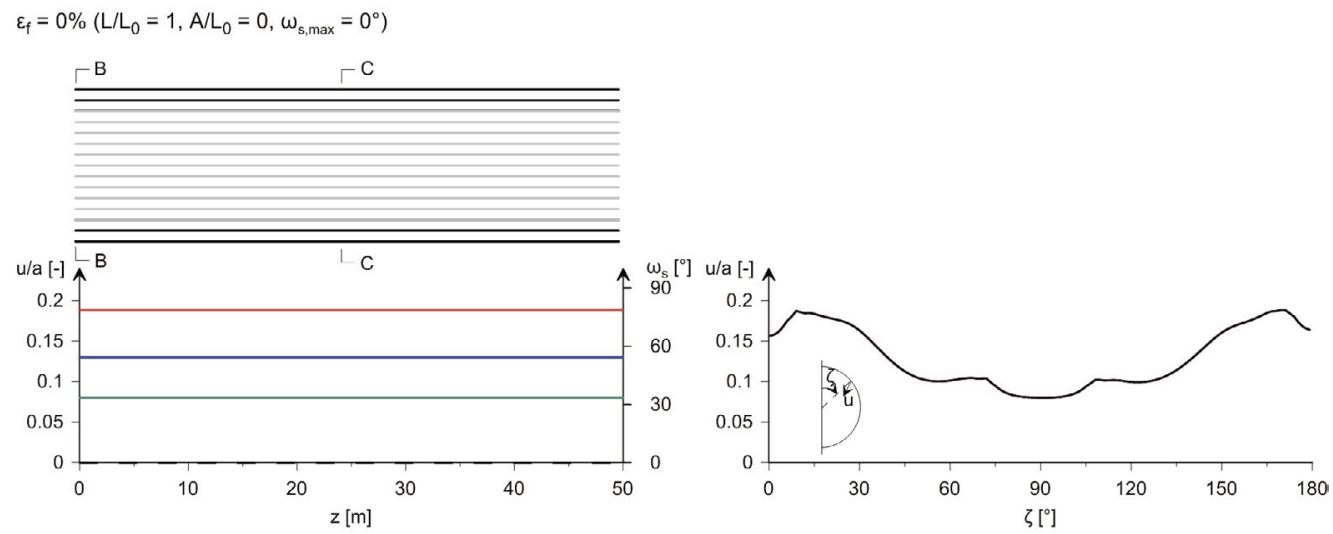

$\varepsilon_{f}=3.5 \%\left(L / L_{0}=0.965, A / L_{0}=0.119, \omega_{s, \max }=21^{\circ}\right)$

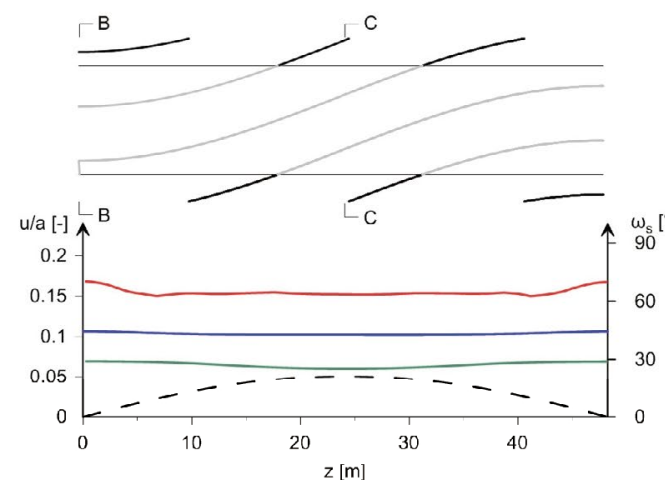

$\varepsilon_{\mathrm{f}}=20 \%\left(L / L_{0}=0.8, \mathrm{~A} / \mathrm{L}_{0}=0.276, \omega_{\mathrm{s}, \max }=45^{\circ}\right)$
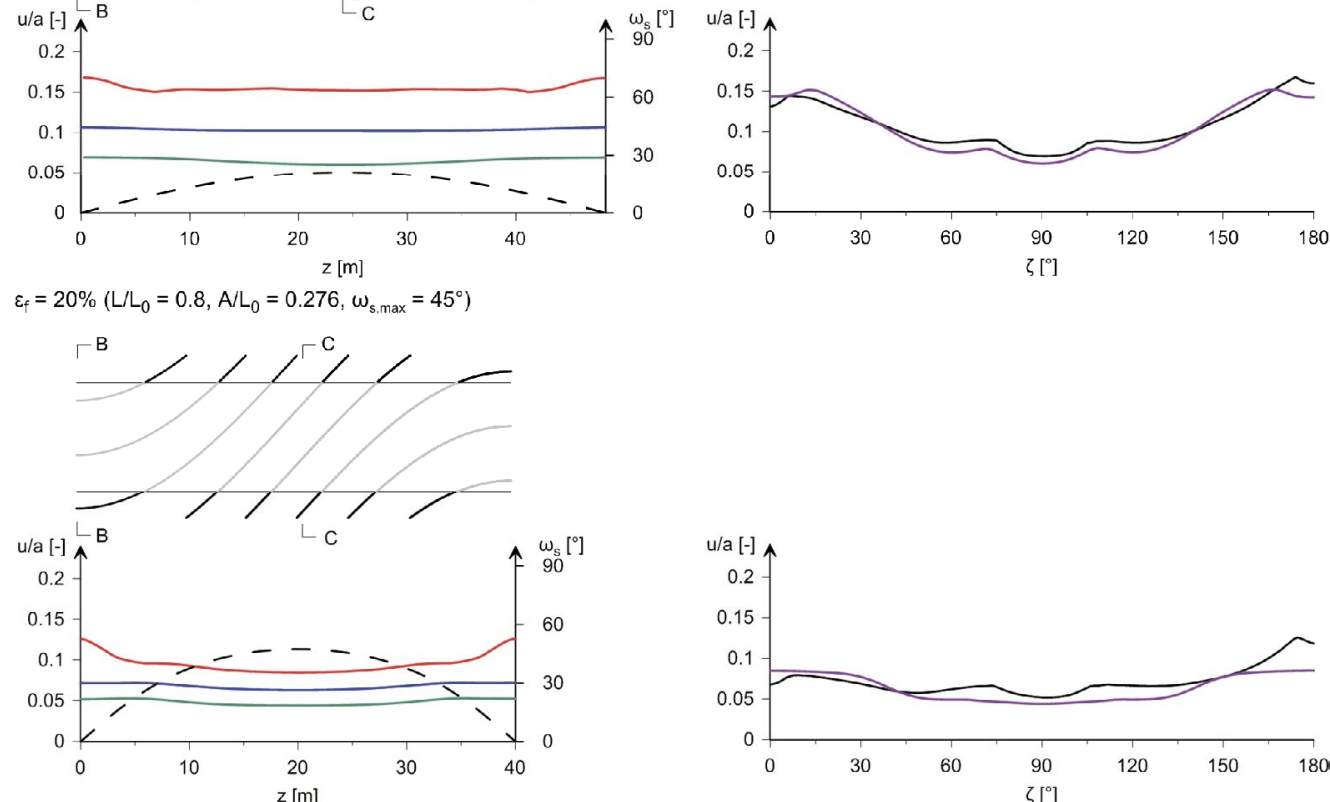

$\varepsilon_{f}=50 \%\left(L / L_{0}=0.5, A / L_{0}=0.414, \omega_{s, \max }=68^{\circ}\right)$
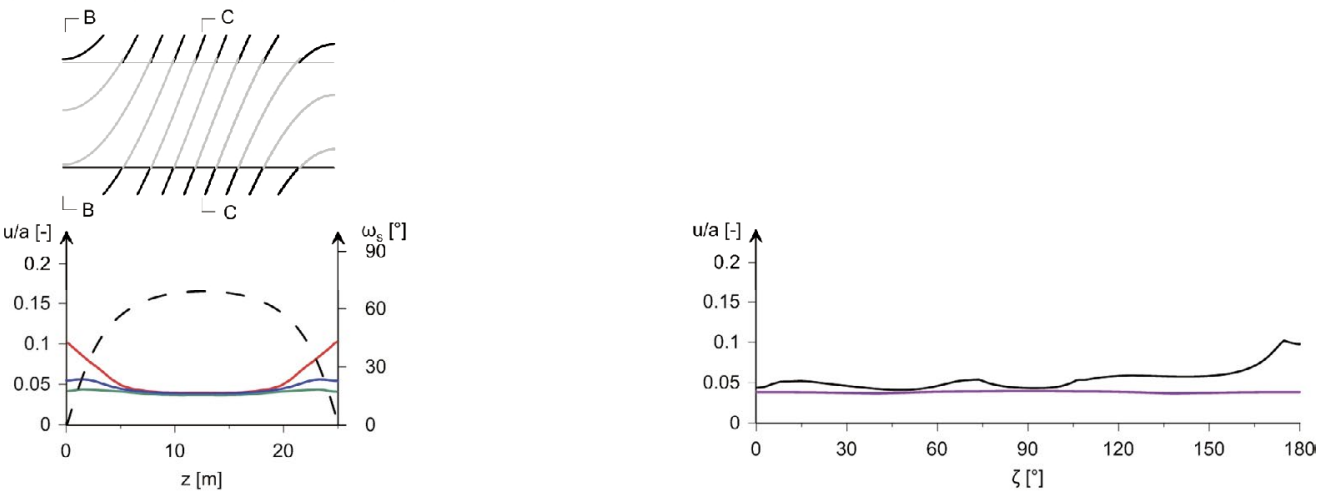

$-\mathrm{u}_{\max }$ in the cross-section
$-\mathrm{u}_{\text {average in the cross-section }}--\mathrm{u}_{\mathrm{sin}}$ in the cross-section

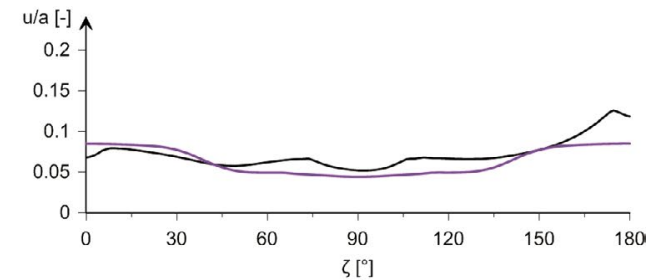
ments along the tunnel (I.h.s.) and displacements along the tunnel boundary at $z=0$ and at $z=L / 2$ (r.h.s.; $a=5 \mathrm{~m}, \sigma_{0}=10 \mathrm{MPa}, E=1 \mathrm{GPa}, c_{m}=1 \mathrm{MPa}, \varphi_{m}=30^{\circ}, \psi_{m}=10^{\circ}, c_{s}=0.4 \mathrm{MPa}$, $\left.\varphi_{s}=10^{\circ}, \psi_{s}=5^{\circ}, v=0.3\right)$. 


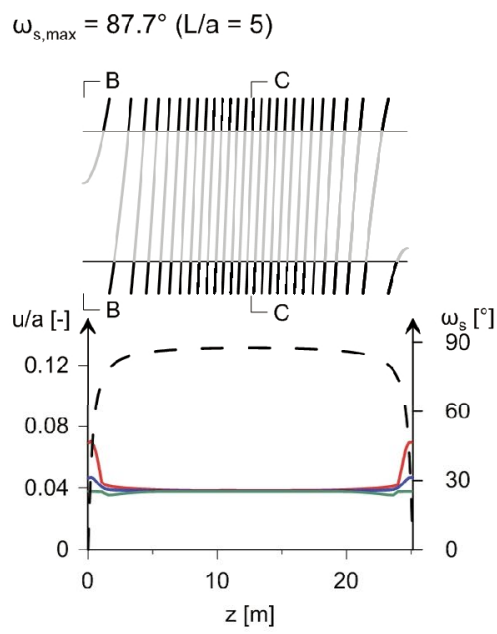

$\omega_{s, \max }=68^{\circ}(\mathrm{L} / \mathrm{a}=5)$

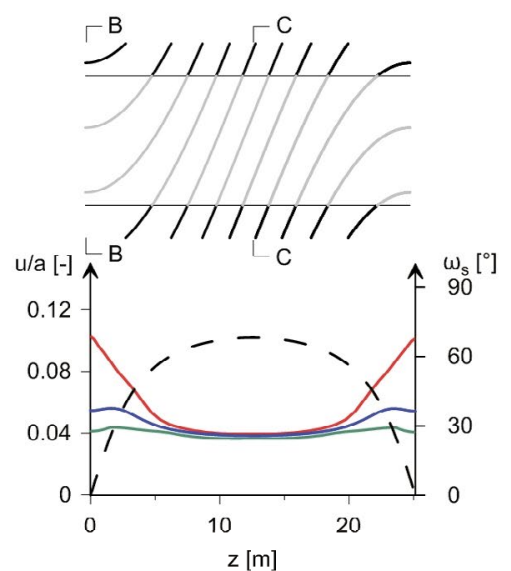

$\omega_{\mathrm{s}, \max }=51^{\circ}(\mathrm{L} / \mathrm{a}=5)$
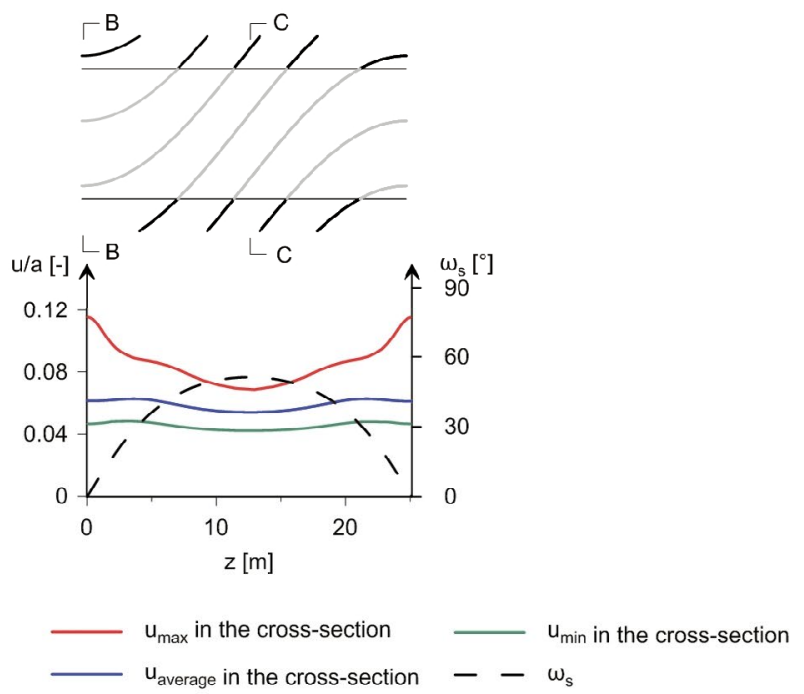
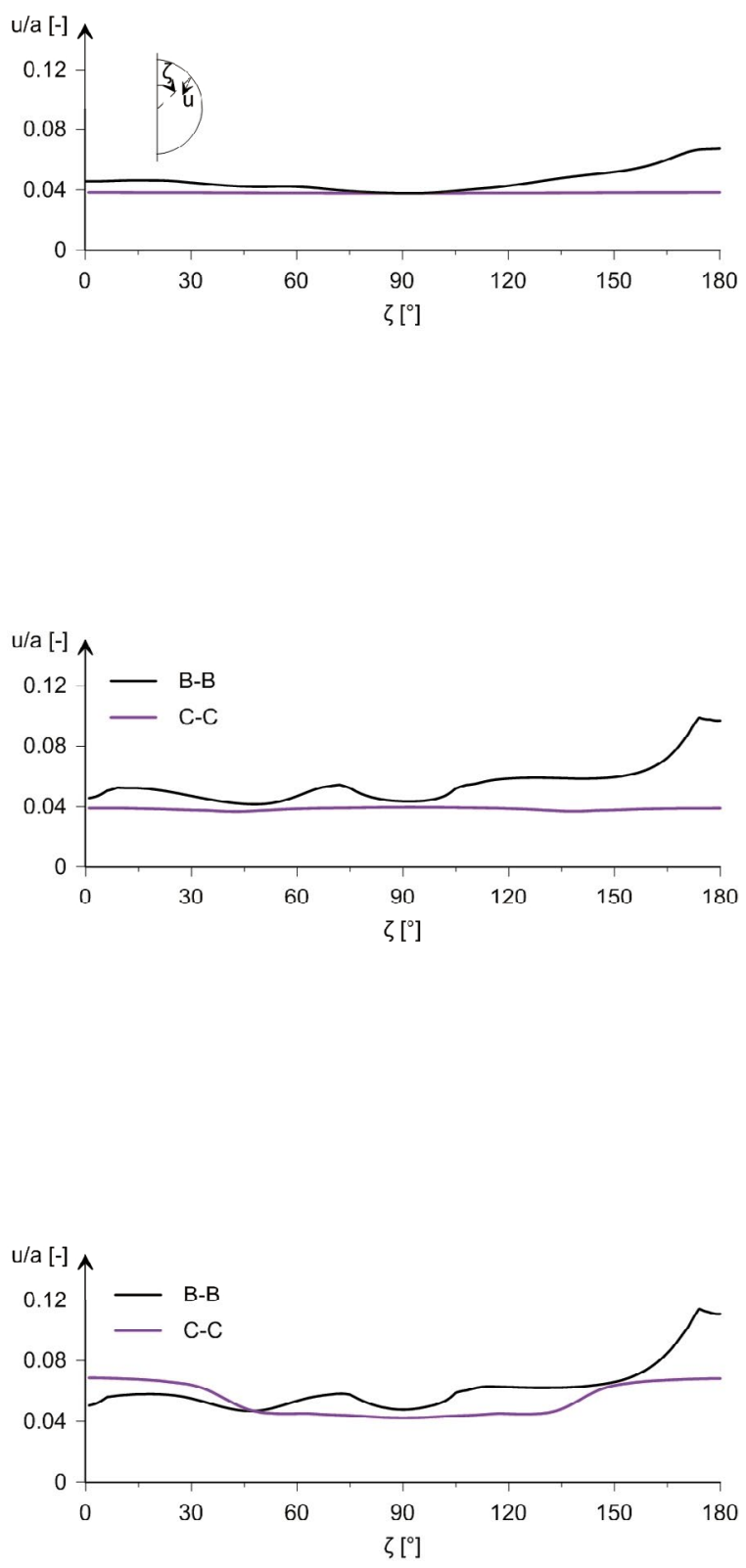

- $u$ at $z=0$ (B-B)

- $u$ at $z=\mathrm{L} / 2(\mathrm{C}-\mathrm{C})$

Figure 13.7. Influence of the maximum dip angle. Maximum, minimum and averaged tunnel displacements along the tunnel (I.h.s.) and displacements along the tunnel boundary at $z=0$ and at $z=L / 2$ (r.h.s.; $a=5 \mathrm{~m}, \sigma_{0}=10 \mathrm{MPa}, E=1 \mathrm{GPa}, c_{m}=1 \mathrm{MPa}, \varphi_{m}=30^{\circ}, \psi_{m}=10^{\circ}, c_{s}=0.4 \mathrm{MPa}$, $\left.\varphi_{s}=10^{\circ}, \psi_{s}=5^{\circ}, v=0.3\right)$. 
In the parametric study of Figure 13.6, the maximum dip angle $\omega_{s, \max }$ and the fold period are varied simultaneously. Next, the effect of these two parameters will be investigated separately. In Figure 13.7, the fold period is kept constant and the influence of the maximum dip angle $\omega_{s, \max }$ (or, equivalently, the fold amplitude; see Eq. 13.3) is investigated. The following conclusions can be drawn:

- The maximum displacements along the tunnel increase with decreasing $\omega_{s, \max }$ (The influence of $\omega_{s, \max }$ on the averaged and minimum displacements is small.)

- A decrease in $\omega_{s, \max }$ from $87.7^{\circ}$ to $68^{\circ}$ results in a larger squeezing variability along the tunnel (the ratio of the maximum displacements at the cross-sections $z=0$ and $z=L / 2$ increases from 1.8 to 2.6). This is because the displacements at $z=L / 2$ remain constant (schistosity does not play a role, if the schistosity planes are so steep; cf. Chapter 12), but the maximum displacements at the fold peak $(z=0)$ decrease due to the stabilising influence of the adjacent areas with steeply inclined schistosity planes.

- A further decrease in $\omega_{s, \max }$ from $68^{\circ}$ to $51^{\circ}$ leads to a smaller squeezing variability along the tunnel (the aforementioned ratio decreases from ca. 2.6 to 1.7), but to a bigger nonuniformity of the displacements in the tunnel profile, and this over the entire tunnel. (Note that, therefore, squeezing variability is maximum at $\omega_{s, \max }=68^{\circ}$.)

- The displacements in the long tunnel section with steep schistosity planes are not influenced by the adjacent tunnel sections with subhorizontal schistosity planes; they are almost equal to those that would occur if the dip angle were constant $\left(=\omega_{s, \max }\right)$. As will be shown later, this is such due to the assumed, rather large, fold period (of $L / a=5$ ).

Next, the influence of the fold period is investigated, keeping the maximum dip angle $\omega_{s, \max }$ equal to almost $90^{\circ}$ (Fig. 13.8). The following conclusions can be drawn:

- The displacements at the fold peaks increase with increasing fold period. The reason is that the tunnel sections with subhorizontal schistosity planes become longer. However, large displacements occur only locally (around the fold peaks), as the tunnel sections with steep schistosity planes become also very long.

- Squeezing variability along the tunnel also increases with increasing fold period, but is remarkable also for a relatively small fold period. (For $L / a=2.5$, the maximum deformations $u_{\max } / a$ vary between 0.04 to 0.07 ). 


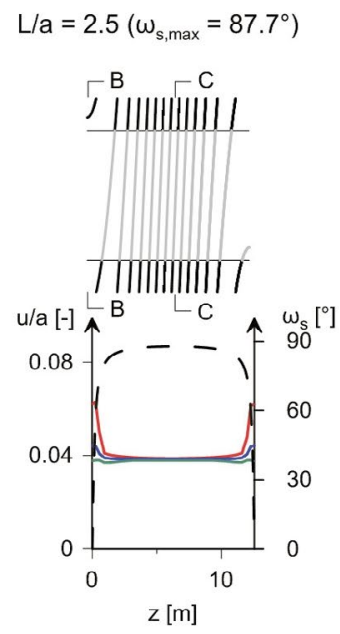

$L / a=5\left(\omega_{\mathrm{s}, \max }=87.7^{\circ}\right)$

$\Gamma^{\mathrm{B}}+{ }^{\mathrm{C}}$

| ||||||||||||||||||||||||||

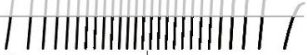

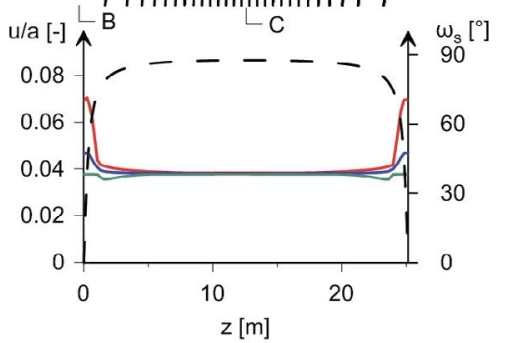

$\mathrm{L} / \mathrm{a}=10\left(\omega_{\mathrm{s}, \max }=87.7^{\circ}\right)$

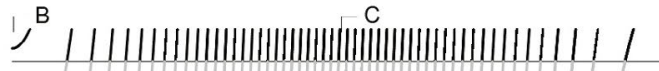
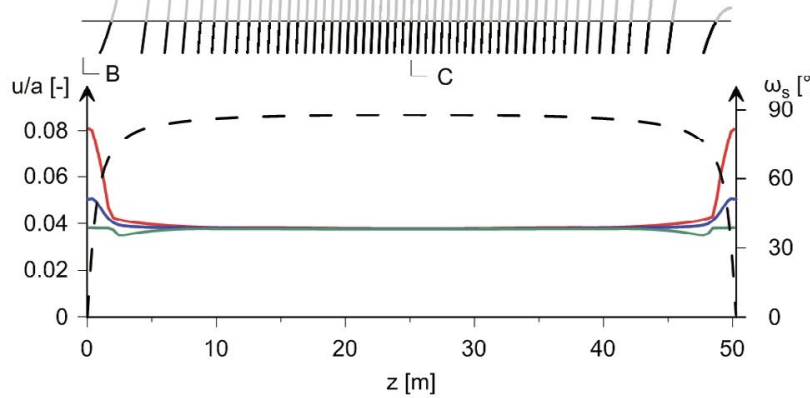

$\begin{array}{lll}-\mathrm{u}_{\max } \text { in the cross-section } & - & \mathrm{u}_{\min } \text { in the cross-section } \\ - & \mathrm{u}_{\text {average in the cross-section }}-\mathrm{w}_{\mathrm{s}}\end{array}$
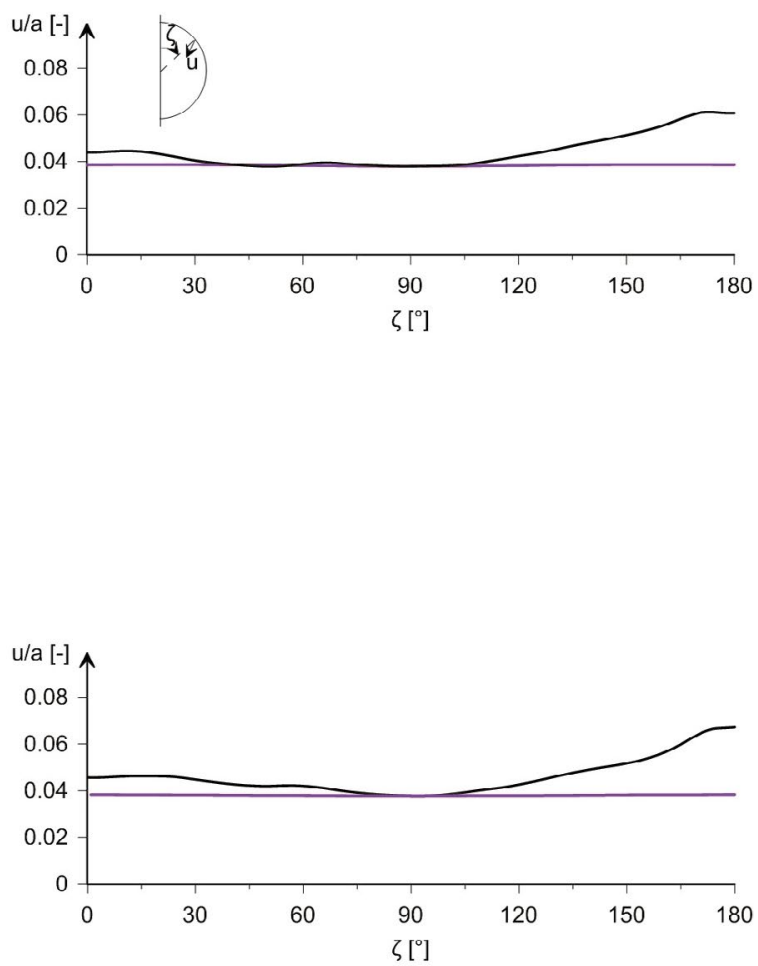

$\zeta\left[^{\circ}\right]$

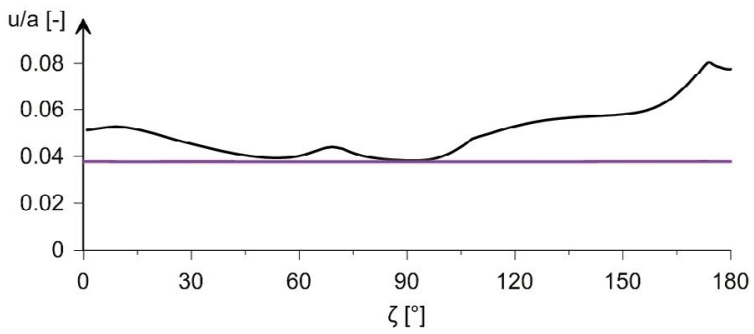

Figure 13.8. Influence of the fold period. Maximum, minimum and averaged tunnel displacements along the tunnel (I.h.s.) and the displacement distribution along the tunnel boundary at $z=0$ and at $z=L / 2$ (r.h.s.; $a=5 \mathrm{~m}, \sigma_{0}=10 \mathrm{MPa}, E=1 \mathrm{GPa}, c_{m}=1 \mathrm{MPa}, \varphi_{m}=30^{\circ}, \psi_{m}=10^{\circ}, c_{s}=0.4 \mathrm{MPa}$, $\left.\varphi_{s}=10^{\circ}, \Psi_{s}=5^{\circ}, v=0.3\right)$. 
In order to identify the conditions under which folding can be neglected in design, a parametric study was performed. The maximum displacements $u_{\max } / a$ of the cross-sections at the fold peaks $(z=0)$ and at the largest dip angle $(z=L / 2)$ generally depend on the geometric parameters of the folding,

$$
\left.\frac{u_{\max }}{a}\right|_{z=0},\left.\frac{u_{\max }}{a}\right|_{z=\frac{L}{2}}=f\left(\frac{A}{L}, \frac{L}{a}\right) \text { or } f\left(\omega_{s, \max }, \frac{L}{a}\right),
$$

and on the mechanical parameters of the schistous rock mass (see Chapter 11).

Figure 13.9 presents the maximum displacements at $z=0$ and $z=L / 2$ as a function of the fold period L/a for maximum dip angles of $20^{\circ}$ to $87.7^{\circ}$. With increasing fold period, the displacements at the fold peaks $(z=0)$ and at the locations with the steepest schistosity planes $(z=L / 2)$ approach asymptotically the displacements that would occur if the schistosity planes had a constant dip angle (of $0^{\circ}$ or $\omega_{s, \max }$, respectively) along the entire tunnel. This happens relatively quickly in the locations with steep schistosity planes (cross-section $z=L / 2$ ), but very slowly (at much bigger fold periods) in the fold peaks (cross-section $z=0$ ). The reason is that the tunnel sections with steeply inclined planes are considerably longer than the peak zones. According to Figure 13.9, folding can be practically disregarded (the displacements at the fold peaks are by maximum $20 \%$ lower than in the case of uniformly horizontal schistosity planes), if the tunnel sections with subhorizontal schistosity planes (dip angle $<10^{\circ}$ ) have a length of minimum 4 times the tunnel radius. This leads to the following condition:

$$
\frac{L}{a}=\frac{4}{1-\frac{2}{\pi} \arccos \left(\frac{\tan \left(10^{\circ}\right)}{\tan \left(\omega_{s, \text { max }}\right)}\right)} \quad\left(=12.4-30 \text { for } \omega_{s, \text { max }}=20^{\circ}-40^{\circ}\right) .
$$

The maximum displacements at the fold peaks $(z=0)$ decrease monotonously with decreasing fold period, because the tunnel sections with subhorizontal schistosity planes become shorter and the stabilising effect of the adjacent sections with steeply inclined schistosity planes becomes more pronounced. The effect of the fold period on the maximum displacements at $z=L / 2$ is more complex (note the minima in the blue lines in Fig. 13.9), because of two opposite effects: With decreasing fold period,

- on the one hand, the adjacent tunnel sections with subhorizontal schistosity planes have an increasingly unfavourable effect, which tentatively increases the deformations at $z=L / 2$;

- on the other hand, the vertical symmetry planes at $z=0$ and $z=L$ (where the axial displacements are equal zero) have an increasingly favourable effect (particularly for small $\omega_{s, \max }$ angles; cf. Chapter 12 ), which tentatively reduces the deformations at $z=L / 2$.

For $L / a$ of approximately 1 , the maximum tunnel deformations are practically uniform along the tunnel, but considerably smaller than the displacements that would occur in a tunnel drive parallel to the schistosity. Therefore, it can be concluded, that even small scale folding may have a considerable influence on the tunnel displacements. 

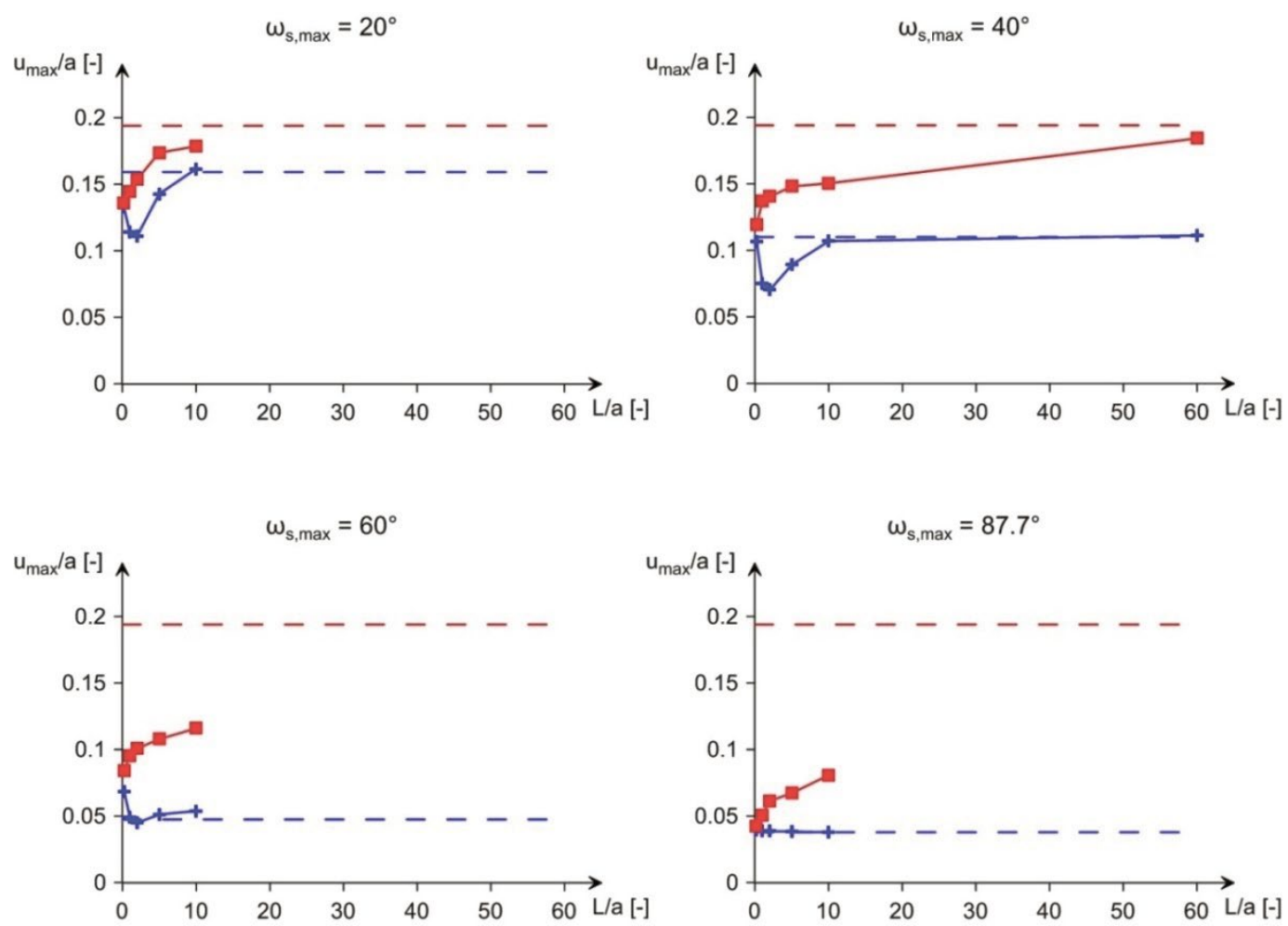

$$
\begin{aligned}
& \longrightarrow \text { at } z=0 \text { (folded rocks) } \quad-\quad-\text { for } \omega_{\mathrm{s}}=0^{\circ} \text { (unfolded rocks) } \\
& \square \text { at } z=\mathrm{L} / 2 \text { (folded rocks) } \quad-\quad \text { for } \omega_{\mathrm{s}}=\omega_{\mathrm{s}, \max } \text { (unfolded rocks) }
\end{aligned}
$$

Figure 13.9. Influence of the period of the folding on the maximum tunnel displacements (normalised by the tunnel radius $a)$ at $z=0$ and at $z=L / 2\left(a=5 \mathrm{~m}, \sigma_{0}=10 \mathrm{MPa}, E=1 \mathrm{GPa}, c_{m}=1 \mathrm{MPa}\right.$, $\left.\varphi_{m}=30^{\circ}, \Psi_{m}=10^{\circ}, c_{s}=0.4 \mathrm{MPa}, \varphi_{s}=10^{\circ}, \psi_{s}=5^{\circ}, v=0.3\right)$.

\subsection{Stratified rocks}

This section investigates similarities and differences of schistous and thinly stratified rocks with respect to the influence of folding. An extreme case is considered, for which the hard layers are considerably stronger and harder than the weak layers (see parameter values in the legend of Fig. 13.10). The thickness fractions of the layers are taken equal to 0.5. The initial stress $\sigma_{0}$ amounts to $10 \mathrm{MPa}$ or $0.75 \mathrm{MPa}$ in order to check whether squeezing plays a role. (Under an initial stress of $0.75 \mathrm{MPa}$, the rock mass behaves elastically.)

Figures 13.10 and 13.11 show the longitudinal distribution of the displacements (normalised by the displacement that would occur in the absence of hard interlayers) for $\sigma_{0}=0.75 \mathrm{MPa}$ and $10 \mathrm{MPa}$, respectively, and various fold periods. One can recognise that:

- Folding results in considerable squeezing variability also in the case of thinly stratified rocks.

- The maximum deformations increase with the length of the tunnel sections with subhorizontal bedding.

- These tunnel sections are, nevertheless, much more extended than in the case of schistous rocks (compare Figs. 13.10 and 13.11 with Fig. 13.8). This was observed already in Chapter 12: In tunnelling through schistous rocks the displacements remain constant for dip angles of $80^{\circ}$ to $90^{\circ}$ (since no failure can occur in the schistosity), but in stratified rocks even small deviations from $90^{\circ}$ lead to bigger displacements. This is 
particularly evident for $\sigma_{0}=0.75 \mathrm{MPa}$ (due to the considerably larger deformability perpendicular to the layers than parallel thereto).

$L / a=5\left(\omega_{s, \max }=87.7^{\circ}\right)$

|||||||||||||||||||||||||| $\mid$

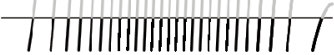

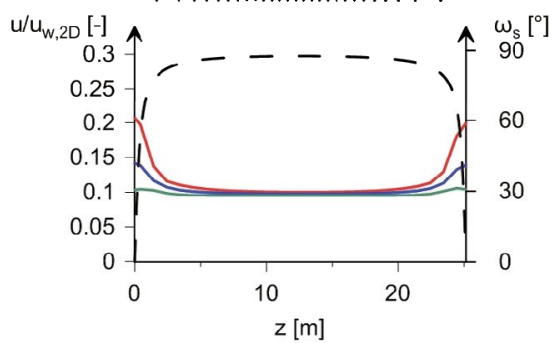

$\mathrm{L} / \mathrm{a}=10\left(\omega_{\mathrm{s}, \max }=87.7^{\circ}\right)$
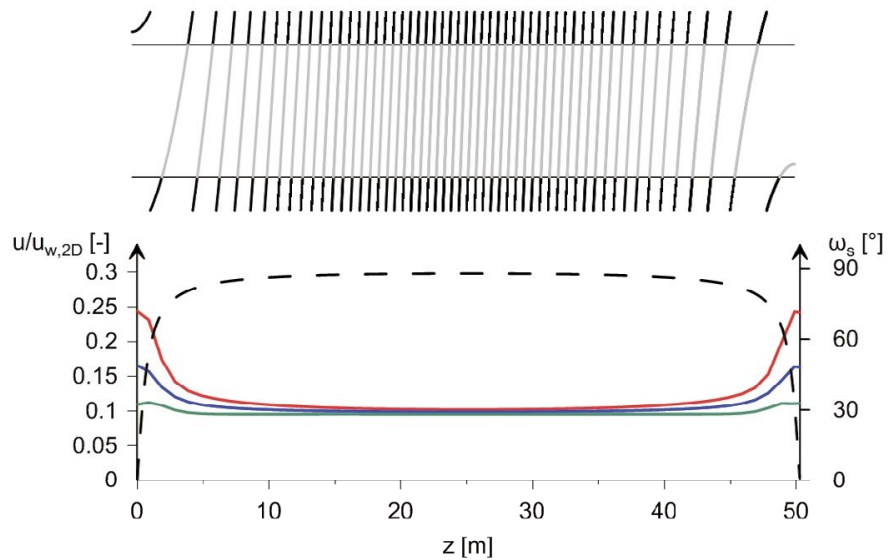

$L / a=20\left(\omega_{s, \max }=87.7^{\circ}\right)$
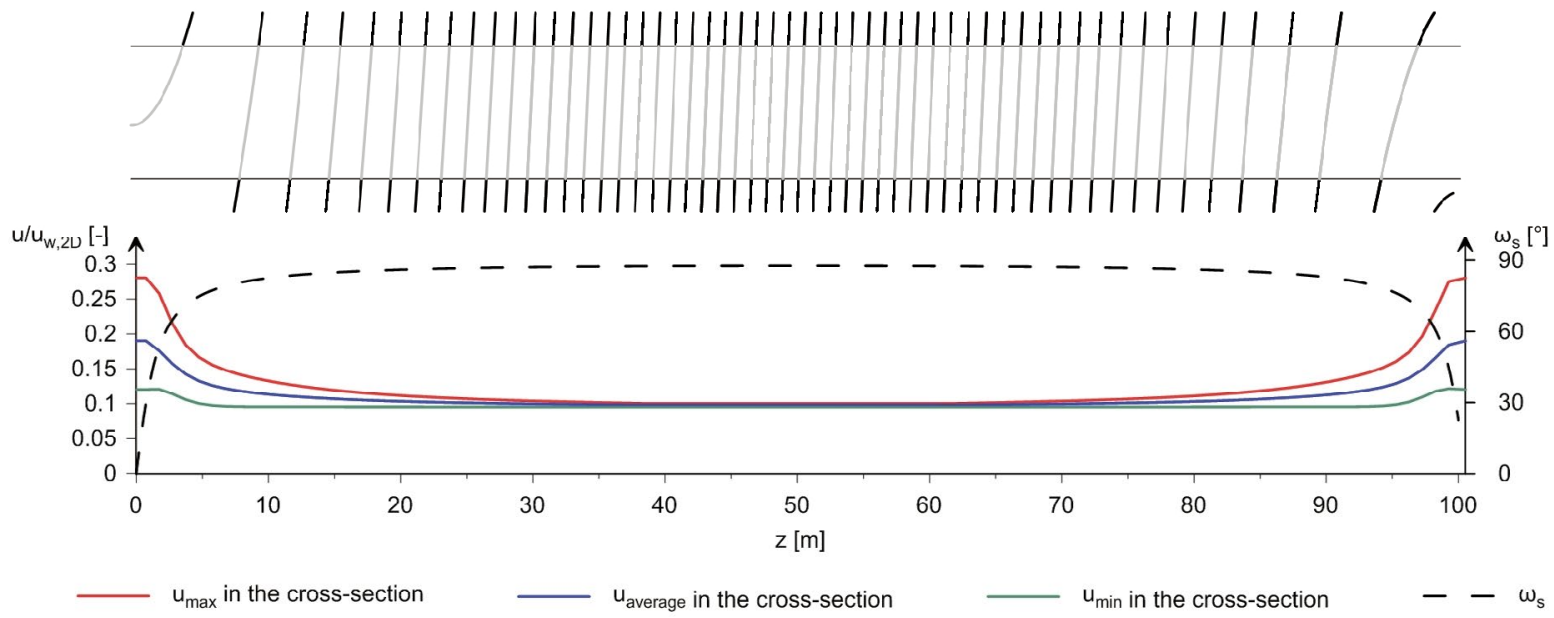

Figure 13.10. Influence of the fold period of stratified rocks $\left(\sigma_{0}=0.75 \mathrm{MPa}\right)$. Maximum, minimum and averaged tunnel displacements along the tunnel $\left(a=5 \mathrm{~m}, \sigma_{0}=0.75 \mathrm{MPa}, x_{h}=x_{w}=0.5\right.$, $\left.E_{h}=10 \mathrm{GPa}, c_{h}=5 \mathrm{MPa}, E_{w}=0.5 \mathrm{GPa}, c_{w}=0.5 \mathrm{MPa}, v_{h}=v_{w}=0.3, \varphi_{h}=\varphi_{w}=25^{\circ}, \psi_{h}=\psi_{w}=5^{\circ}\right)$. 
$\mathrm{L} / \mathrm{a}=5\left(\omega_{\mathrm{s}, \max }=87.7^{\circ}\right)$

| |||||||||||||||||||||||||| |

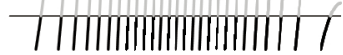

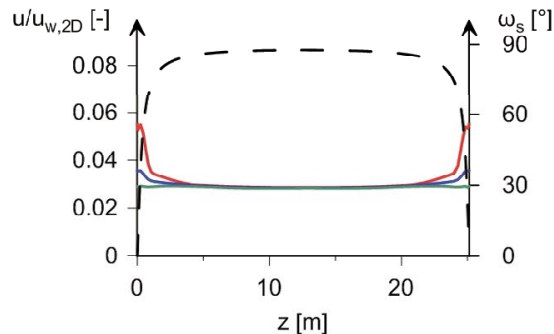

$\mathrm{L} / \mathrm{a}=10\left(\omega_{\mathrm{s}, \max }=87.7^{\circ}\right)$

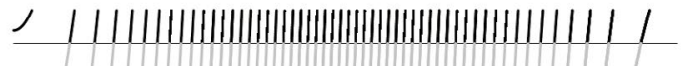
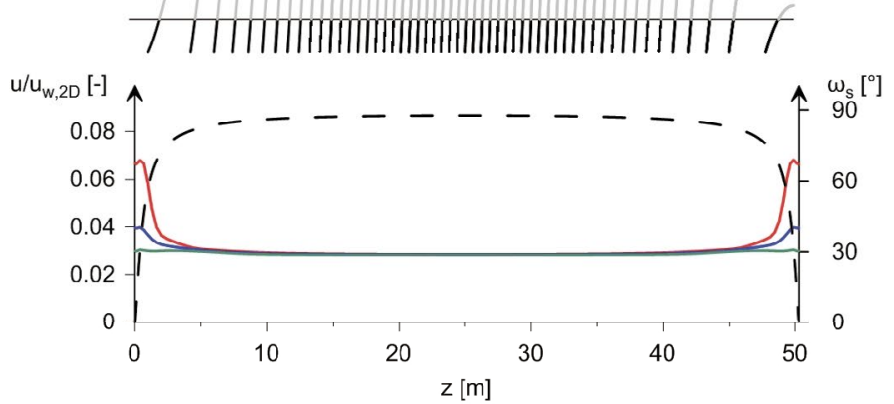

$\mathrm{L} / \mathrm{a}=20\left(\omega_{\mathrm{s}, \max }=87.7^{\circ}\right)$
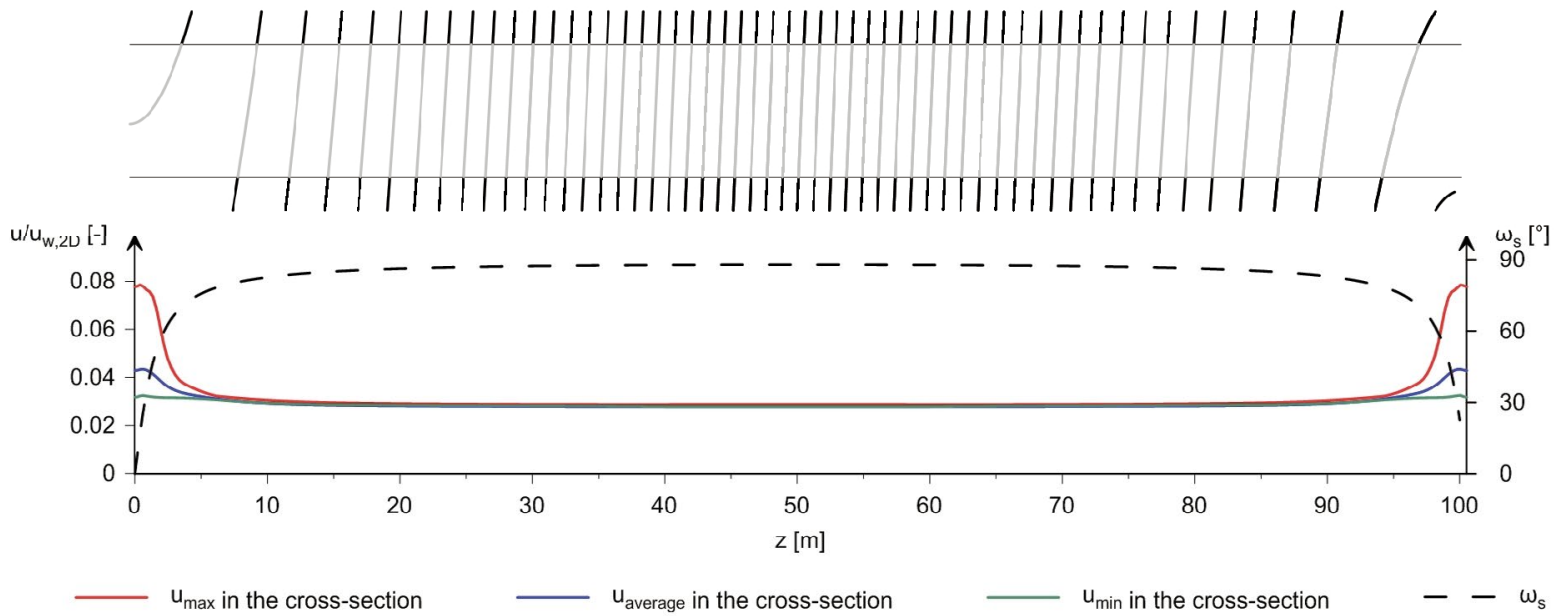

Figure 13.11. Influence of the fold period of stratified rocks $\left(\sigma_{0}=10 \mathrm{MPa}\right)$. Maximum, minimum and averaged tunnel displacements in the cross-section along the tunnel $\left(a=5 \mathrm{~m}, \sigma_{0}=10 \mathrm{MPa}\right.$, $x_{h}=x_{w}=0.5, E_{h}=10 \mathrm{GPa}, c_{h}=5 \mathrm{MPa}, E_{w}=0.5 \mathrm{GPa}, c_{w}=0.5 \mathrm{MPa}, v_{h}=v_{w}=0.3, \varphi_{h}=\varphi_{w}=25^{\circ}$, $\left.\boldsymbol{\Psi}_{h}=\boldsymbol{\Psi}_{w}=5^{\circ}\right)$. 


\subsection{Case history of the Sedrun section of the Gotthard Base Tunnel}

According to Chapter 1 , the "schistosity influence factor" $S$, which combines the dip angle and the strike direction of the planes of weakness to the tunnel axis, can be used as an indicator, in order to predict the tunnel convergences. This was particularly evident in Chapter 1 for a tunnel reach with constant degree of shearing of 4 (for the definition of the degree of shearing: see Chapter 1): The large variability of the averaged convergences (over all the measuring points) in this case was solely due to the change of the schistosity orientation (cf. Fig. 13.12a). Therefore, this section will analyse this tunnel reach (NE tube, chainage 1760-1900) and show, this time by means of numerical calculations, whether the schistosity influence factor $S$ can adequately express the squeezing variability.

A comprehensive description of the Sedrun Section and the related experiences can be found in Chapter 1 . The depth of cover amounts to about $800 \mathrm{~m}\left(\sigma_{0}=20 \mathrm{MPa}\right)$. The rock parameters of Table 13.1 correspond to a rock with medium degree of kakiritization (as the shearing degree in this tunnel section amounts to 4 according to Chapter 1) and were chosen on the basis of the report of Ingenieurgemeinschaft Gotthard-Basistunnel Süd (2009). The computational model was already introduced in Section 13.2 (however, here the whole 3D model must be considered, as there are no planes of symmetry). The dip angle and the strike direction of the schistosity were implemented in the numerical model, considering the geological records made during tunnel advance (cf. Guntli and Weber 2009). Since the tectonic units are very disturbed, contrarily to Section 13.3, no equation for the folding of the schistosity can be defined and thus dip angle and strike direction of the schistosity were taken section-wise constant (see values in Fig. 13.12b). The resulting schistosity influence factors (obtained with Eq. 1.1) are also given in Figure 13.12b.

Table 13.1. Rock parameters (according to Ingenieurgemeinschaft Gotthard-Basistunnel Süd 2009).

\begin{tabular}{cccccccc}
\hline$E$ & $C_{m}$ & $\varphi_{m}$ & $\psi_{m}$ & $c_{s}$ & $\varphi_{s}$ & $\psi_{s}$ & $v$ \\
{$[\mathrm{GPa}]$} & {$[\mathrm{MPa}]$} & {$\left[{ }^{\circ}\right]$} & {$\left[^{\circ}\right]$} & {$[\mathrm{kPa}]$} & {$\left[^{\circ}\right]$} & {$\left[^{\circ}\right]$} & {$[-]$} \\
4.4 & 1.3 & 30 & 10 & 600 & 20 & 5 & 0.3 \\
\hline
\end{tabular}

Figure 13.12c shows the numerically determined maximum, minimum and averaged displacements along the tunnel, while Figure 13.12d compares them with the measured values. One can recognise that the deformations obtained with the numerical calculations are greater than the measured deformations. There are two main reasons for this: the computed displacements include the pre-deformations; the computations consider an unsupported tunnel. The pre-deformations and the installed support could be taken into account computationally by a step-by-step simulation of tunnel excavation, but this simulation would be very time-consuming without adding much value since the selection of appropriate parameters itself is a difficult task (due to the natural variability of the rock mass along the tunnel stretch). Hence, the calculated displacements can be compared only qualitatively with the measured tunnel convergences.

Figure $13.12 \mathrm{~d}$ presents the measured as well as calculated displacements $\bar{u}_{c}$ (according to Chapter 1). They correspond to the average values of the five to seven measuring points of each monitoring station. Depending on the position of the measuring points (which is not exactly known), these calculated average values may be subject of some uncertainty. 
(a)

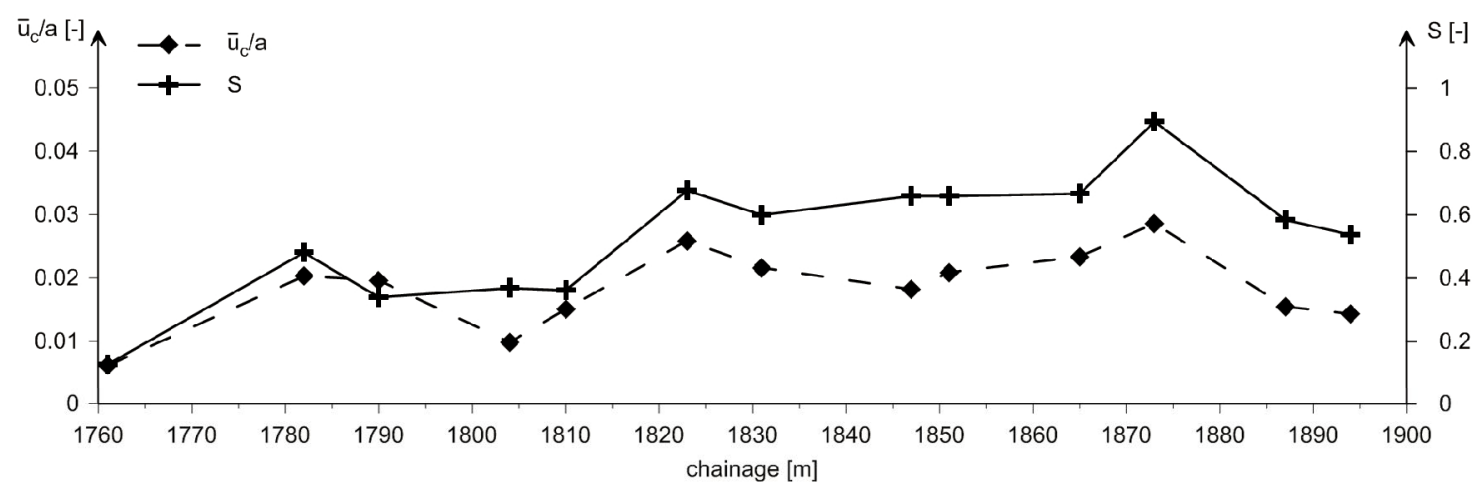

(b)

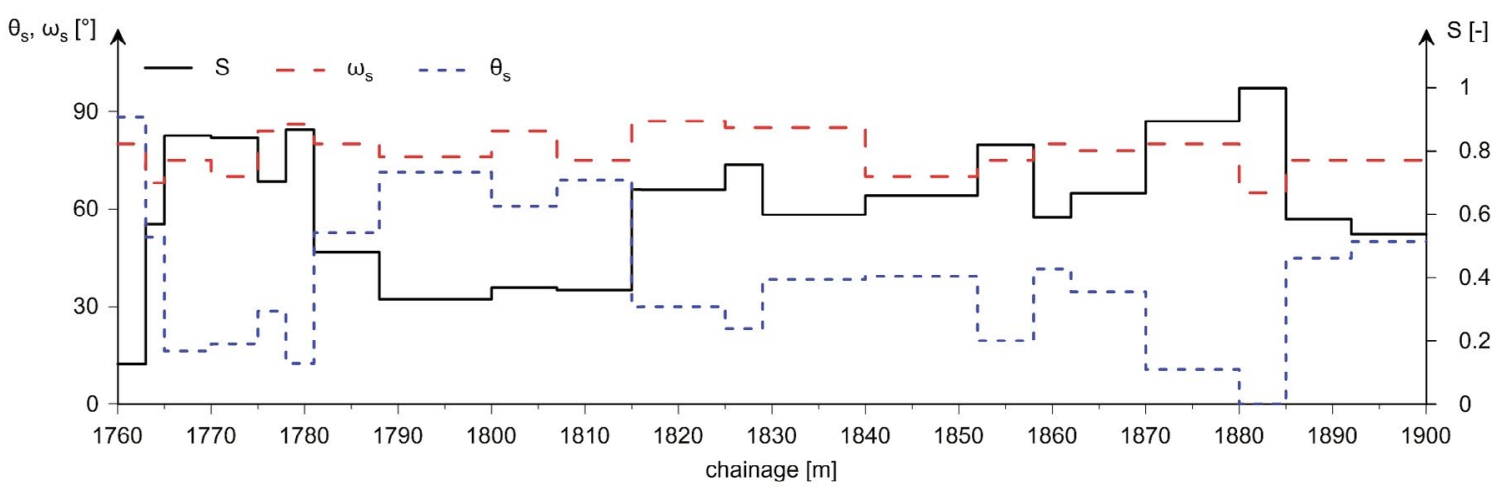

(c)

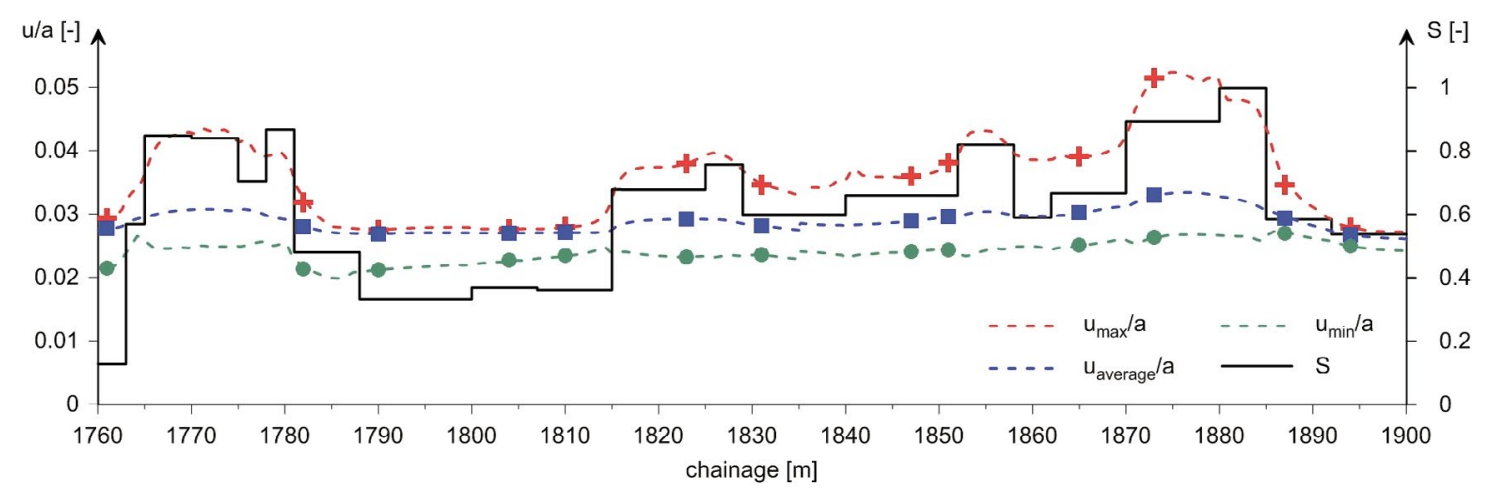

(d)

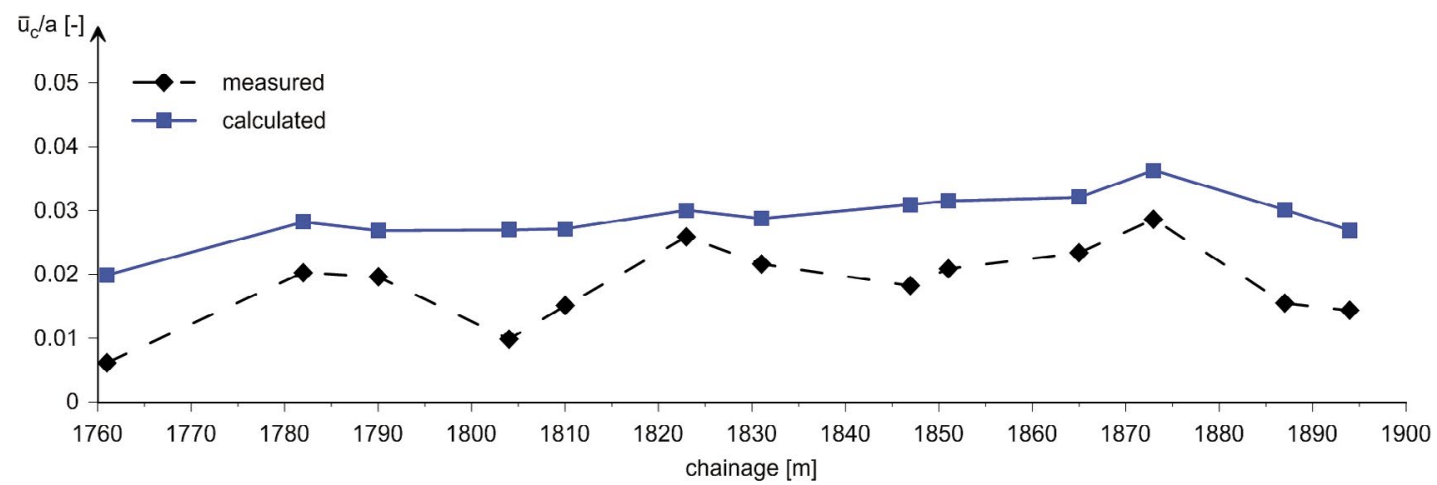

Figure 13.12. (a) Average magnitude of the projections of the displacement vectors in the cross-sectional plane of the tunnel normalised by the tunnel radius $\left(\bar{u}_{c} / a\right)$ along the tunnel as well as schistosity influence factor for chainage 1760-1900 of the NE tube, according to Chapter 1; (b) Implemented dip angle $\omega_{s}$ and angle $\theta_{s}$ between the tunnel axis and the strike direction of the planes of weakness, as well as resulting schistosity influence factor $S$; (c) Maximum, minimum and averaged displacements of the tunnel boundary obtained with numerical calculations along the tunnel as well as schistosity influence factor $S$; (d) Comparison between the measured convergences and the total displacements obtained from the numerical calculations. 
Nevertheless, one can readily recognise that the distribution of the calculated displacements along the tunnel is very similar to that of the measured ones, but the squeezing variability is less pronounced than actually observed. According to Chapter 12 , the squeezing variability would be larger, had the tunnel convergences (rather than the total displacements) been considered in the numerical modelling. The deformations would then be on average $30 \%$ smaller (see Chapter 12) and thus the results of the numerical modelling would be in better agreement with the measured convergences. The squeezing variability would also be larger and thus more visible in the development of the displacements, if an even smaller value for $c_{s}$ was chosen.

Despite all the uncertainties mentioned before, the analysed case history shows that the schistosity influence factor is a reliable indicator, which enables to determine in which tunnel sections larger deformations have to be expected during tunnelling. Of course, this presupposes that the schistosity orientation can be identified in advance. This information can be obtained by means of advance probing (cf. Chapter 1 ).

\subsection{Conclusions}

The variation of the orientation of the planes of anisotropy is an important factor for the squeezing variability and this, when tunnelling through schistous or stratified rock mass. The results of numerical investigations indicate that, there is a mutual influence of the alternating areas of favourable and unfavourable inclination of the anisotropy planes along the tunnel. However, despite of this mutual influence, the folding leads to a considerable variability of the rock deformations along the tunnel.

Furthermore, this chapter could show that the squeezing variability observed in the Sedrun Section of the Gotthard Base Tunnel can be well understood with the help of numerical calculations taking into account the orientation of the schistosity to the tunnel axis. In order to predict the tunnel convergences, the "schistosity influence factor" $S$, which combines the dip angle and the strike direction of the planes of weakness to the tunnel axis, can be used as an indicator thus providing, in combination with advance core drilling, reliable indications of the squeezing intensity.

As was already illustrated in Section 13.3, depending on the length of the tunnel sections, which are parallel to the schistosity, and on the stabilising vicinity, the maximum displacement may vary considerably. Of course, this influence is not taken into account when predicting the convergences solely by means of the schistosity influence factor.

\section{Notation}

a

A

$C_{h}, C_{w}$

$C_{s}, C_{m}$

E

$E_{h}, E_{w}$

$f(\ldots)$

$L$

$L_{0}$ tunnel radius

amplitude of the folding

cohesion of the hard and of the weak layers

cohesion of the schistosity and the surrounding matrix

Young's modulus of the schistous rock mass

Young's modulus of the hard and of the weak layers

function of ...

half of the period of the folding

initial length of the planes of anisotropy before folding 


$\vec{n}$
$S$
$u$
$u_{\text {average }}, u_{\text {max }}, u_{\min }$
$u_{c}$
$\bar{u}_{c}$
$u_{w, 2 D}$
$x_{h}, x_{w}$
$y$
$z$
$\varepsilon_{f}$
$\zeta$
$\theta_{s}$
$v$
$v_{h}, v_{w}$
$\sigma_{0}$
$\varphi_{h}, \varphi_{w}$
$\varphi_{s}, \varphi_{m}$
$\psi_{h}, \psi_{w}$
$\psi_{s}, \psi_{m}$
$\omega_{s}$
$\omega_{s, \max }$

normal vector of the planes of anisotropy influence factor of the schistosity magnitude of the displacement vector at the tunnel boundary averaged, maximum and minimum magnitude of the displacement vector along the tunnel boundary in the cross-section

magnitude of the projection of the displacement vector in the crosssectional plane of the tunnel

average magnitude of the projections of the displacement vectors in the cross-sectional plane of the tunnel (according to Chapter 1)

radial displacement of the homogeneous weak ground

thickness fraction of the hard and of the weak layers

co-ordinate

axial co-ordinate

compression of the folding

angle between the crown and the displacement in the cross-sectional plane of the tunnel (see inset of Fig. 13.6)

angle between the tunnel axis and the strike direction of the planes of anisotropy

Poisson's ratio of the schistous rock mass

Poisson's ratio of the hard and of the weak layers

initial stress

friction angle of the hard and of the weak layers

friction angle of the schistosity and of the surrounding matrix

dilatancy angle of the hard and of the weak layers

dilatancy angle of the schistosity and of the surrounding matrix

dip angle of the planes of anisotropy

maximum dip angle of the planes of anisotropy 



\section{PART V: DEALING WITH THE SQUEEZING VARIABILITY IN SHIELDED TBM TUNNELLING}

Shielded TBMs are, in contrast to conventional tunnelling, particularly vulnerable to squeezing due to the very limited space available: Relatively small convergences may lead to considerable difficulties in the machine area or in the back-up area. Besides the sticking of the cutter head or the jamming of the shield, one main hazard of shielded TBM tunnelling in squeezing rock is the overstressing of the segmental lining. In order to deal with the squeezing variability in TBM tunnelling, variable lining types (switch from normal to higher strength segments or from a stiff to a deformable lining of the same thickness) or even lining thicknesses may be installed. Therefore, this part of the thesis investigates the extent to which the application range of shielded TBMs could be broadened in squeezing conditions by either using stiff linings of higher bearing capacity or deformable lining systems. 



\section{Higher capacity segmental lining systems ${ }^{5}$}

\subsection{Introduction}

Overstressing of the segmental lining is the main hazard of TBM tunnelling in squeezing rock (Ramoni and Anagnostou 2010a). Due to their stiffness, segmental linings allow only very limited convergence, thus resulting in higher rock pressures than light or deformable rock supports. Ramoni et al. (2011b) investigated the rock pressure developing upon segmental linings taking account of the backfilling materials and procedures, and presented design nomograms for the lining loading. Under certain geotechnical conditions, the rock pressure exceeds the resistance of the usual segmental linings, thus necessitating higher capacity linings in order to extend the operational possibilities for TBMs in squeezing rock.

The first and easiest solution is to increase the thickness of the segments. Usually, lining segments with a thickness of 20 to $50 \mathrm{~cm}$ are used, but thicknesses of up to $70 \mathrm{~cm}$ have been implemented in the past (e.g., Sparvo Highway Project, $4^{\text {th }}$ tube of the Elb Tunnel, Orlovski Tunnel). However, should it be necessary to further increase lining resistance (beyond the limits of manageable thicknesses and weights), either segmental linings made of high or ultra-high performance concretes (abbreviated to HPC and UHPC, respectively), or lining systems consisting of two segmental rings can be used (Ramoni and Anagnostou 2010a).

The present chapter starts with an overview of these basic options for increasing the bearing capacity of segmental linings (Section 14.2), and continues with a discussion of the different solutions from the perspectives of TBM technology, the construction process and materials technology (Sections 14.3 to 14.6). The range of rock pressures that can be sustained by these lining systems is then estimated (Section 14.7) and design aids are presented that allow a quick estimate of the actual lining loading to be made for a wide range of geotechnical conditions (Section 14.8). Finally, Section 14.9 compares the costs of the different systems under specific geological situations and using the structural design aids in Sections 14.7 and 14.8 .

\subsection{Lining systems}

The bearing capacity of a segmental lining can be increased, (i), by increasing its thickness, (ii), by using higher-strength concrete or, (iii), by installing an additional inner ring made

5 This chapter has been published in: Mezger, F., Ramoni, M., Anagnostou, G., Dimitrakopoulos, A., Meystre, N. 2017. Evaluation of higher capacity segmental lining systems when tunnelling in squeezing rock. Tunnelling and Underground Space Technology. Vol. 65: 200-214. 
either of prefabricated segments or of cast in-situ concrete (double-shell lining). Basically, four systems are possible (Fig. 14.1): a single shell segmental lining made of normal-strength concrete (Fig. 14.1a); a single shell segmental lining made of HPC or UHPC (Fig. 14.1b); a double shell lining consisting of two segmental rings (Fig. 14.1c); and a double shell lining with an inner ring of cast in-situ, normal-strength concrete (Fig. 14.1d). For the first three lining systems, it is also possible to install an inner ring made by cast in-situ concrete for reasons of serviceability as well. In this case, the serviceability requirements for the segmental lining (cracking limits, fire safety, waterproofing) are less stringent.

(a)

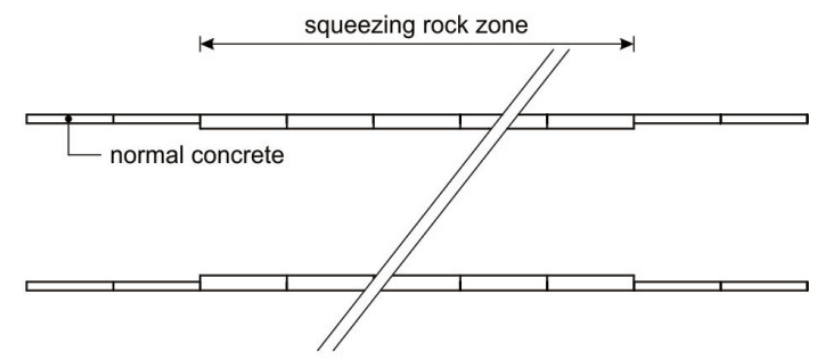

(b)

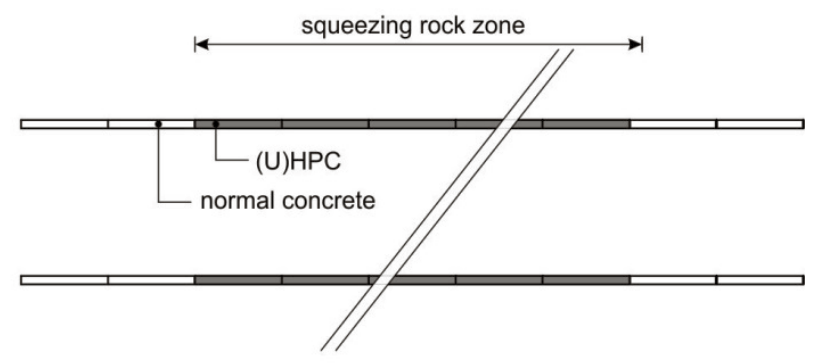

(c)

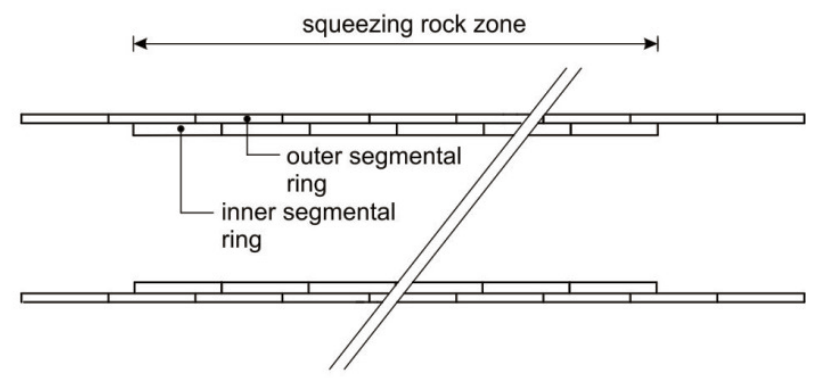

(d)

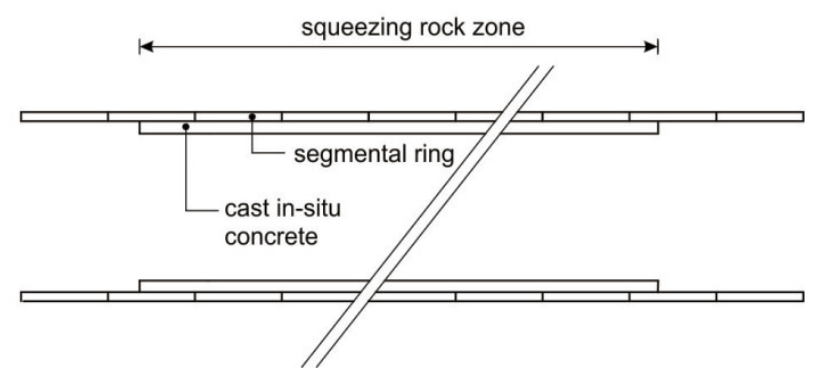

Figure 14.1. (a) Single shell segmental lining made of locally thicker normal-strength concrete segments; (b) single shell segmental lining of uniform thickness, locally made from high performance or ultra-high performance concrete (U)HPC; (c) double shell lining consisting of two segmental rings; d) double shell lining with segments and cast in-situ concrete. 
Table 14.1. Qualitative comparison between the lining systems.

\begin{tabular}{|c|c|c|c|c|}
\hline \multirow[b]{2}{*}{ Criterion } & \multicolumn{4}{|c|}{ Lining system } \\
\hline & $\begin{array}{l}\text { Single shell } \\
\text { segmental } \\
\text { lining made of } \\
\text { normal-strength } \\
\text { concrete }\end{array}$ & $\begin{array}{l}\text { Single shell } \\
\text { segmental } \\
\text { lining made of } \\
\text { HPC or UHPC }\end{array}$ & $\begin{array}{l}\text { Double shell } \\
\text { lining consisting } \\
\text { of two } \\
\text { segmental rings }\end{array}$ & $\begin{array}{l}\text { Double shell } \\
\text { lining with inner } \\
\text { ring of cast in- } \\
\text { situ concrete }{ }^{(1)}\end{array}$ \\
\hline $\begin{array}{l}\text { Requirements for the } \\
\text { manufacturing and placement } \\
\text { precision of the segments }\end{array}$ & medium & medium & high & medium \\
\hline $\begin{array}{l}\text { Complexity of segment } \\
\text { manufacture (factory requirements) }\end{array}$ & medium & high & low & low \\
\hline $\begin{array}{l}\text { Requirements of the shielded TBM } \\
\text { (e.g. gripper systems, suction } \\
\text { plates) }\end{array}$ & medium & normal & high & high \\
\hline $\begin{array}{l}\text { Complexity and logistical problems } \\
\text { in the installation of the lining } \\
\text { system }\end{array}$ & normal & normal & medium & difficult \\
\hline $\begin{array}{l}\text { Problems with large deformations } \\
\text { (e.g. waterproofing, force } \\
\text { application) }\end{array}$ & medium & medium-difficult & difficult & medium \\
\hline $\begin{array}{l}\text { Size of boring diameter (volume of } \\
\text { underground excavation) }\end{array}$ & medium & small & large & large \\
\hline $\begin{array}{l}\text { Size of segments (transport, } \\
\text { machine handling) }\end{array}$ & normal-big & normal & normal & normal \\
\hline Changes in the cross-section & yes & no & yes & yes \\
\hline $\begin{array}{l}\text { Time for installation (and thus } \\
\text { advance rate) in squeezing rock } \\
\text { zones }^{(2)}\end{array}$ & normal & normal & normal & normal \\
\hline $\begin{array}{l}\text { Changes in support concept in a } \\
\text { longitudinal direction }\end{array}$ & normal & normal & big & big \\
\hline Costs of the lining system & medium & medium-high & high & medium-high \\
\hline Technological risk & low-medium & medium & medium-high & high \\
\hline $\begin{array}{l}\text { Short-term stiffness of the lining } \\
\text { system }^{(3)}\end{array}$ & normal & medium & high & normal \\
\hline $\begin{array}{l}\text { Long-term stiffness of the lining } \\
\text { system }^{(4)}\end{array}$ & normal & medium & medium-high & high \\
\hline $\begin{array}{l}\text { Long-term resistance of the lining } \\
\text { system to rock pressure }\end{array}$ & normal & medium-high & high & medium \\
\hline $\begin{array}{l}\text { Short-term resistance of the lining } \\
\text { system to rock pressure }\end{array}$ & normal & medium-high & high & normal \\
\hline $\begin{array}{l}\text { Serviceability problems (cracking, } \\
\text { fire, waterproofing) }\end{array}$ & medium & high & medium & normal \\
\hline \multicolumn{5}{|c|}{$\begin{array}{l}\text { (1) Installed close to the shield (see Section 14.6). } \\
\text { (2) Prerequisite: all the technical measures for the shielded TBM (see Sections } 14.3 \text { to 14.6) are implemented. } \\
\text { (3) A higher early stiffness favours longitudinal arching action around the shield, thus resulting in lower thrust } \\
\text { demand. } \\
\text { (4) A higher final stiffness results in a higher final rock pressures. }\end{array}$} \\
\hline
\end{tabular}

The above-mentioned lining systems are discussed in the following Sections 14.3 to 14.6; Table 14.1 provides a comparative overview. The fundamental issues concerning TBM technology and the construction process of the different lining systems were clarified with a machine manufacturer (Burger 2012). Generally, novel lining systems are inherently characterised by higher technological risks. This is due, on the one hand, to the high requirements placed on the shielded TBM and, on the other hand, to the complexity of the logistics and of the installation and manufacturing procedure. 


\subsection{Single shell segmental lining made of normal-strength concrete}

Thicker segments can be applied either over the entire length of the tunnel or only in certain critical squeezing rock zones. To maintain the minimum clearance profile, the boring diameter is anyway chosen for the maximum segment thickness. A solution with segmental lining of variable thickness is envisaged, e.g., for the Bossler Tunnel, where thicker segments shall be installed over a $1.7 \mathrm{~km}$ long section (about $20 \%$ of the total length; Edelhoff et al. 2015).

Thicker segments are heavier and more difficult to manufacture, transport and handle during erection. Specifically, the manufacturing tolerances are more difficult to achieve for thick segments. In addition, transport (rather than the machine handling, i.e. erection) limits the weight and thus the dimensions of the segments. Considering a weight limit of 20 to $22 \mathrm{t}$, segment thicknesses of up to 1.0-1.2 $\mathrm{m}$ are feasible.

If the segments are too heavy, the vacuum system of the erector can no longer be used and the segments have to be gripped and installed mechanically by the erector. In this case, the segments are subject to concentrated loads, which have to be considered in their design.

If the thicker segments are installed only in the critical squeezing zones (Fig. 14.1a), the lining has a variable thickness, while the boring diameter is of course constant. Consequently, the following points have to be considered in the planning stage:

- As soon as the thicker segments have to be installed in a potentially critical squeezing zone, the suction plates and gripping systems have to be exchanged. This requires one to three weeks and has to take place during a planned standstill, in good time before advancing into the squeezing zone. Reliable advance ground probing is essential in this respect. If additional suction plates and gripping systems are installed on the TBM (e.g., a double erector), no standstill is required. This, however, might necessitate a longer shield, which is unfavourable under squeezing conditions (higher risk of shield jamming).

- The machine should advance with the same arrangement of hydraulic jacks over the entire length of the tunnel (i.e. also in the squeezing zones). This can be achieved by using the same geometrical subdivision for the segments. In the tunnel sections with thicker segments, however, the hydraulic jacks are arranged eccentrically on the segmental lining. This is not a problem for the machine. As usual, the segmental lining has to be designed so that it has the capacity to bear the thrust force safely. Due to the eccentric arrangement of the hydraulic jacks, the lining may experience deformations leading to a misalignment of the segments (Fig. 14.2a), which in turn may result in them spalling at the edges. In order to avoid an eccentric arrangement of the jacks in the squeezing rock zone, the jack pressure ring can be built so as to be moveable. However, this would lead to a much greater expenditure (of time and money) in the construction of the machine and possibly to reduced machine reliability.

- Waterproofing of the ring joints often necessitates double sealing profiles between the segmental rings. In order to ensure waterproofing also for the joints between segmental rings of different thicknesses, the sealing profiles of the first thicker ring must be placed asymmetrically. Alternatively, transitional segmental rings of varying thickness might be envisaged (Fig. 14.2b). Such rings could, in spite of their different geometry, also be gripped by the erector and placed in position. The development of such special segments anyway requires a consultation with machine and segment manufacturers in order to consider the aspects of transport, handling and installation. 
- A transitional segmental ring may also be necessary in high-speed railway tunnels for aerodynamic reasons (to reduce air turbulence and resistance during operation). By arranging several transitional segmental rings, the transition length can be adjusted to the aerodynamic requirements. However, this solution leads to considerably higher costs for the formwork in the production of the segments. Alternatively, one could also smoothen the transition by applying cast in-situ concrete.

- A locally thicker segmental lining results in a step in the invert, which poses logistics problems during construction. This problem can be solved by placing prefabricated concrete elements on the invert of the thinner rings. With the Swiss segmental lining system (fixed location of the invert segments and of the key stone, see Fig. 14.3), a thicker invert segment can be placed throughout the entire tunnel.

(a)

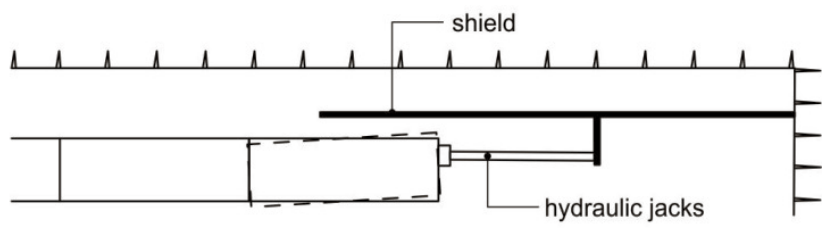

(b)

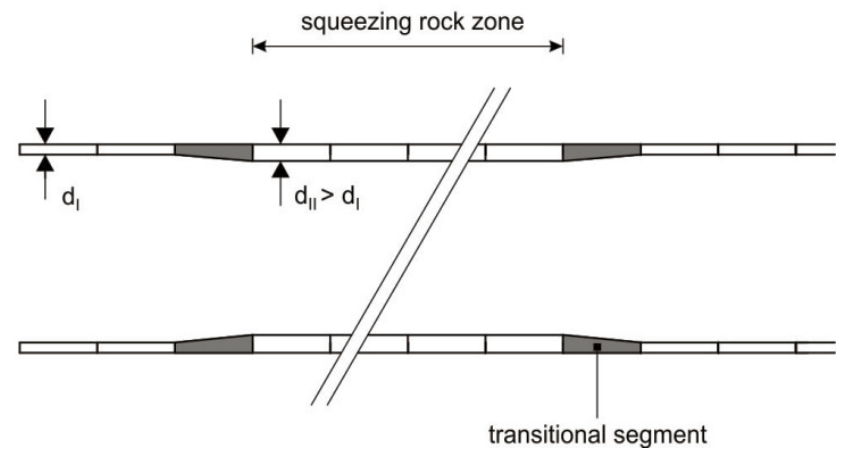

Figure 14.2. (a) Misalignment of the segments due to eccentric arrangement of the hydraulic jacks; (b) installation of a transitional segment.

From the economic point of view, the choice of a greater segment thickness only locally in a squeezing rock zone seems to be worthwhile, but imposes higher requirements on the shielded TBM with regard to the suction plates and the gripper system, and therefore implies a higher technological risk (see Table 14.1).

(a)

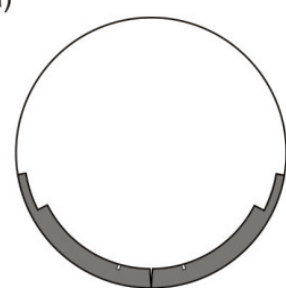

(b)

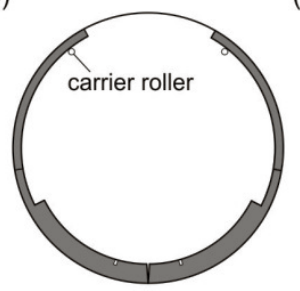

(c)

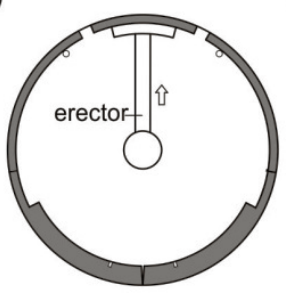

(d)

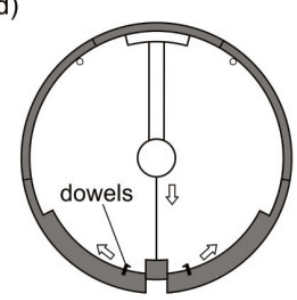

(e)

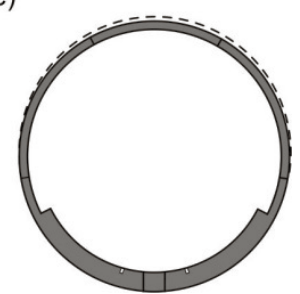

Figure 14.3. Installation process using the Swiss method (after Maidl et al. 2011): (a) withdrawal of the pressure ring and installation of the two invert segments; (b) installation of the side segments; (c) installation of the top segment; (d) spreading of the invert segments with dowels and insertion of the keystone; (e) final position of the segmental ring, advancing the thrust pressure ring. 


\subsection{Single shell segmental lining made of (U)HPC}

This solution consists in the installation of higher-strength segments in the critical squeezing zones (Fig. 14.1b). In contrast to the previous solution, the thickness of the lining can be kept constant over the entire TBM drive; the squeezing zones are thus not decisive in terms of the boring diameter. In the following sections, the most important aspects of (U)HPC are discussed.

\subsubsection{Principle and manufacture}

(U)HPC is characterised by high compressive strength ( $\geq 50 \mathrm{MPa}$ for $\mathrm{HPC}$ and $\geq 100 \mathrm{MPa}$ for UHPC), low porosity and high density (see, e.g., Richard and Cheyrezy 1995).

Due to the low water/cement ratios, high demands are placed on the mix technology in order to achieve a homogeneous mix, particularly if a high fibre content is required (Dehn 2003). Mixing order and time are decisive for the properties of the concrete, whereby the mix and batching technologies known from normal-strength concrete can generally be used. Additional information on the manufacture of (U)HPC can be found in, e.g., Brüggemann (1998), Dehn (2003), Schmidt (2003), Fehling et al. (2005), Fehling et al. (2012), Schmidt and Fehling (2006), DAfStb (2008) and Leutbecher (2008). Prefabrication of the segments under controlled conditions makes it possible to fulfil the high demands on the mixing technology (e.g. also using heat treatment) and thus to achieve higher compressive strengths.

It is indispensable to carry out preliminary tests on the applied mix and to conduct permanent accompanying tests for quality assurance. Suggestions regarding methods for the quality assurance can be found in DAfStb/DIN (1995) and DAfStb (2008).

\subsubsection{Mechanical properties}

With today's standard technologies, uniaxial compressive strengths of over $100 \mathrm{MPa}$ can be easily achieved. Under laboratory conditions and using special compression treatment as well as a vacuum mixer, the uniaxial compressive strengths can even reach $800 \mathrm{MPa}$ (König et al. 2001). Schmidt and Herget (2007) reported on prefabricated elements of a pedestrian bridge exhibiting compressive strengths as high as 175-200 MPa.

Unlike normal-strength concrete, the failure surface of (U)HPC runs through the aggregate particles rather than along the aggregate-matrix interface (Leutbecher 2008). (U)HPC is thus particularly brittle. As a sudden failure (without forewarning) is undesirable and a redistribution of the section forces to less intensively loaded parts of the structure is preferable in the case of statically-indeterminate structures, the ductility of (U)HPC should be increased. Ductility can be achieved to certain degree through the addition of a sufficient quantity of steel fibres (cf., e.g., Kützing and König 2001); Fehling et al. (2005) showed experimentally that UHPC (with 2.5 vol-\% fibres, $9 \mathrm{~mm}$ long) exhibits a strength of $80-140 \mathrm{MPa}(40-70 \%$ of the peak strength) even after compression by $7 \%$. Even with fibres, however, the post-peak behaviour of (U)HPC is not pronouncedly ductile (Schnellenbach-Held and Prager 2011). An aligned micro-reinforcement consisting of thin steel wires eliminated several disadvantages of the steel fibres (e.g. their random orientation and distribution as well as the corrosion spots resulting from near-surface fibres), but did not improve ductility greatly (Schnellenbach-Held and Prager 2011). Generally, experimental investigations into the deformation capacity are essential for any confident use of (U)HPC. 
UHPC exhibits a higher tensile strength than normal-strength concrete and HPC, but it should be noted that tensile strength increases sub-linearly with compressive strength. Further information on uniaxial tensile strength, splitting tensile strength, the shear force capacity of construction elements and the bond strength between the UHPC matrix and reinforcement bars or steel fibres can be found in AFGC / SETRA (2002), Fehling et al. (2005), Leutbecher (2008) and DAfStb (2008). Since tensile strength increases sub-linearly with compressive strength, failure due to partial area loading occurs earlier (in the areas where tensile splitting develops; Reinhardt and Koch 1998, Klotz and Holschemacher 2003), which results in spalling of the concrete cover. For this reason, fibre reinforcement is necessary, as it also acts near the surface of the concrete.

The Young's modulus of (U)HPC increases sub-linearly, too, with its compressive strength. It is equal to about 45-55 GPa for UHPC (König et al. 2001, DAfStb 2008), but can reach $70 \mathrm{GPa}$ using aggregates from dense igneous rocks (DAfStb 2008). The higher value of the Young's modulus for (U)HPC has to be taken into account when determining the section forces of statically-indeterminate structures (e.g. in the analysis of lining-rock interaction).

The creep coefficient of $(U) H P C$ is lower than that of normal-strength concrete (Brisard 2006). This leads, on the one hand, to a greater sensitivity of the structural elements with respect to non-uniform deformations and, on the other hand, to a slower and smaller relaxation of restraint forces (König et al. 2001).

Due to the high cement content and the low w/c ratio of UHPC, the greater part of the shrinkage deformation takes place within the first few hours after setting (Leutbecher 2008, Fehling et al. 2005). Shrinkage is irrelevant, however, for prefabricated segmental linings.

\subsubsection{Design}

The Swiss code SIA 262 (2003) as well as the Eurocode 2 (2011) - in combination with the national annex for Germany - provide rules for the use and design of normal-strength and high performance concretes. However, structural elements whose strength exceeds strength class C100/115 cannot be designed on the basis of these codes of practice. The design of steel fibre reinforced HPC (up to strength class C100/115) can be performed under SIA 162/6 (1999). For the designs using UHPC one has to turn either to the SIA 2052 (2016) or to the relevant international guidelines. The following international guidelines are essentially relevant: AFGC / SETRA (2002), JSCE (2004), DAfStb (2008) and Fehling et al. (2012).

Due to the lack of experience in the application of UHPC and some still insufficiently investigated aspects of this new construction material, Leutbecher (2008) recommends fullscale tests on the structural elements.

\subsubsection{Durability}

The durability of $(U) H P C$ is generally higher than that of normal-strength concrete. This is mainly due to its lower porosity. HPC still possesses a high portion of permeable capillary pores and thus does not achieve a durability higher than normal-strength concrete and thus allowing, e.g., a reduction in the concrete cover. UHPC, however, is practically free of permeable capillary pores. Thereby, according to Schmidt and Fehling (2006), it is very impervious to chloride ions, carbonises much more slowly and also (without air-entraining additives) exhibits a very high de-icing salt resistance. Thus, the concrete cover can be 
reduced in the case of UHPC. The addition of steel fibres to the concrete does not impair its durability (König et al. 2001). However, it is possible that corrosion of the fibres will occur close to the surface (AFGC / SETRA 2002, Leutbecher 2008).

\subsubsection{Fire behaviour}

Important causes of failure in the case of fire are: concrete spalling due to the development of high pore pressures; thermal stresses; explosion of the aggregates; or a change in the crystal phase of the quartz aggregates resulting in an increase in their volume. After spalling, the reinforcement is exposed to high temperatures and the structural element loses its resistance. Due to the high density of the (U)HPC matrix, the problem of spalling is more critical for (U)HPC than for a normal-strength concrete (König et al. 2001, Schmidt and Fehling 2005).

For ensuring fire safety with $(U) \mathrm{HPC}$, the use of fire-resistant plasters as thermal barriers and polypropylene fibres (PP fibres) has proved successful (Klingsch et al. 2013, Klingsch 2014). These two concepts are briefly discussed below:

- Fire-resistant plasters as thermal barriers provide a quick and simple solution for increasing the fire resistance of a structure and can be sprayed directly onto structural elements. In contrast to the use of PP fibres the renewal of plaster after a fire is much simpler, since the structure remains intact and only the plaster has to be replaced. According to Klingsch et al. (2013) plaster thicknesses of less than $10 \mathrm{~mm}$ are not recommended and strong curvature conditions $<0.021 / \mathrm{mm}$ should be avoided. Fireresistant plasters with a thickness of at least $15 \mathrm{~mm}$ will keep the temperature of the reinforcement below $300{ }^{\circ} \mathrm{C}$ after two hours of ISO-fire exposure (Klingsch et al. 2013). The fire-resistant plaster must also be weather-resistant, especially in the case of gypsum-mortar. The biggest disadvantages of fire-resistant plasters are the restrictions they impose on inspections of the tunnel structure (due to the spraying of the plaster onto the structure) and their relatively short lifespan (25 to 35 years).

- The addition of PP fibres has proved to be effective in fires (see, e.g., Diederichs 1999, Dehn and König 2003, Bornemann et al. 2001). The PP fibres melt and increase permeability, whereby the water vapour pressure is reduced and thus extensive spalling is avoided. The fibres increase the inhomogeneity of the concrete and may affect its workability. However, a high fibre content does not necessarily result in lower compressive strengths (Klingsch et al. 2013); tests carried out at the Institute of Structural Engineering of the ETH Zurich (Klingsch et al. 2013) showed that UHPC with a compressive strength of $\mathrm{ca}$. $150 \mathrm{MPa}$ (without fibres) exhibits a strength of about $80 \mathrm{MPa}$ up to a temperature of $700^{\circ}$ after adding $3 \mathrm{~kg} / \mathrm{m}^{3}$ PP fibres (together with 2 vol.- $\%$ steel fibres and quartzite aggregates). This strength is well above the recommendations of Eurocode 2 (2011). Moreover, no spalling was observed. The latter contradicts the findings of Schulte (2011) and other authors, however, who observed that even the addition of $6 \mathrm{~kg} / \mathrm{m}^{3}$ of PP fibres could not prevent distinct spalling in specimens made of UPHC (strength class C135/150). According to Klingsch (2014), this was because inappropriate PP fibres (of too large a diameter and length) were used in these fire tests. In any case, it is recommended that the effectiveness of fire resistance using PP fibres should be verified by carrying out fire tests. 


\subsubsection{The use of (U)HPC for segmental linings}

The highly brittle behaviour of (U)HPC is potentially problematic for the joints upon which concentrated loads act. Adequate joint design is very important. Tongue-and-groove joints provide a good guide during installation and avoid unwanted relative displacements (which might impair sealing) between the neighbouring segmental rings. However, concrete spalling can occur if the available joint play is only slightly exceeded (Maidl et al. 2011). This problem is especially pronounced for (U)HPC (due to its greater brittleness). To reduce this danger, the tongue-and-groove joints should have a sufficiently large amount of play (DAUB 2001). Flat circumferential joints are usually less prone to spalling and seem, therefore, to be more suitable. Flat joints or tongue-and-groove joints with a large amount of play permit larger relative displacements, but this problem is of subordinate importance in the presence of a sufficiently high axial force (the friction between the segmental rings prevents differential displacements; Grübl 2012).

In addition to joint design, ductility of the segments is desirable so that stress can be redistributed to less loaded areas. The ductility for (U)HPC can be increased to a certain degree by adding steel fibres (Section 14.4.2). They are effective particularly for the corner regions, which are more prone to spalling.

With regard to the problem of joint spalling, the erection of the segments has also to be taken into account. Specifically, the friction occurring during insertion of the wedge-shaped keystone may lead to spalling. Lubrication of the contact areas of the segments is important for the sealing (Grübl 2012), but does not reduce the friction sufficiently. The friction as well as the restraint forces are reduced, however, by the "Swiss Method", in which the segments are forced apart before the keystone is installed (see Fig. 14.3).

The transport route of $(\mathrm{U}) \mathrm{HPC}$ segments must be given greater attention than with normal segments, so that no spalling occurs at the edges. Although impact loading causes only local damage in the case of (U)HPC (Grübl 2012), steel-fibre reinforcement of the corner regions is recommended. In addition, (U)HPC segments have to be placed more carefully than normal-strength segments, but the usual ring installation times can be achieved (Grübl 2012). When using the "Swiss Method", spalling is more likely to occur (due to lower installation precision).

Leucker et al. (2009) showed (based upon large-scale tests on segments with compressive strengths of 80,120 and $136 \mathrm{MPa}$ ) that segments of HPC can resist very high loads and that the load-bearing capacity of the joints could be considerably increased by using higher strength concrete. Furthermore, they could show that the dimensioning methods applied for HPC agreed very well with the tests and thus are suitable for design purposes.

Joint spalling is of subordinate importance if an inner lining of cast-in-situ concrete is envisaged; the serviceability requirements are less stringent in this case.

Due to their density, (U)HPC segments are less fire-resistant (Section 14.4.5). In the tunnel design stage, preliminary project-specific tests must be carried out to investigate the suitability of $(U) H P C$. The problem can be overcome through the admixture of PP fibres or through the use of fire-resistant plasters. Moreover, the consequences of a lining failure in squeezing rock is small compared, for example, to urban tunnelling in soft ground (where a cave-in can propagate up to the ground surface) or below a body of water (complete loss of the tunnel in the case of lining collapse). 


\subsection{Double shell lining consisting of two segmental rings}

The two rings can be installed by the following procedure (Fig. 14.4): The outer segmental ring is constructed as usual. The position of the keystone is therefore variable. In order to install the inner segmental ring without the need for hydraulic jacks, it can be constructed in the same way as a non-waterproof segmental lining with the keystone at the bottom (as is usually the case in Switzerland, Fig. 14.3; Maidl et al. 2011). The segments of the two rings can be installed using a double erector. As the inner segmental ring is placed directly onto the outer ring, the requirements are high with respect to the manufacturing and erection precision of the segments.
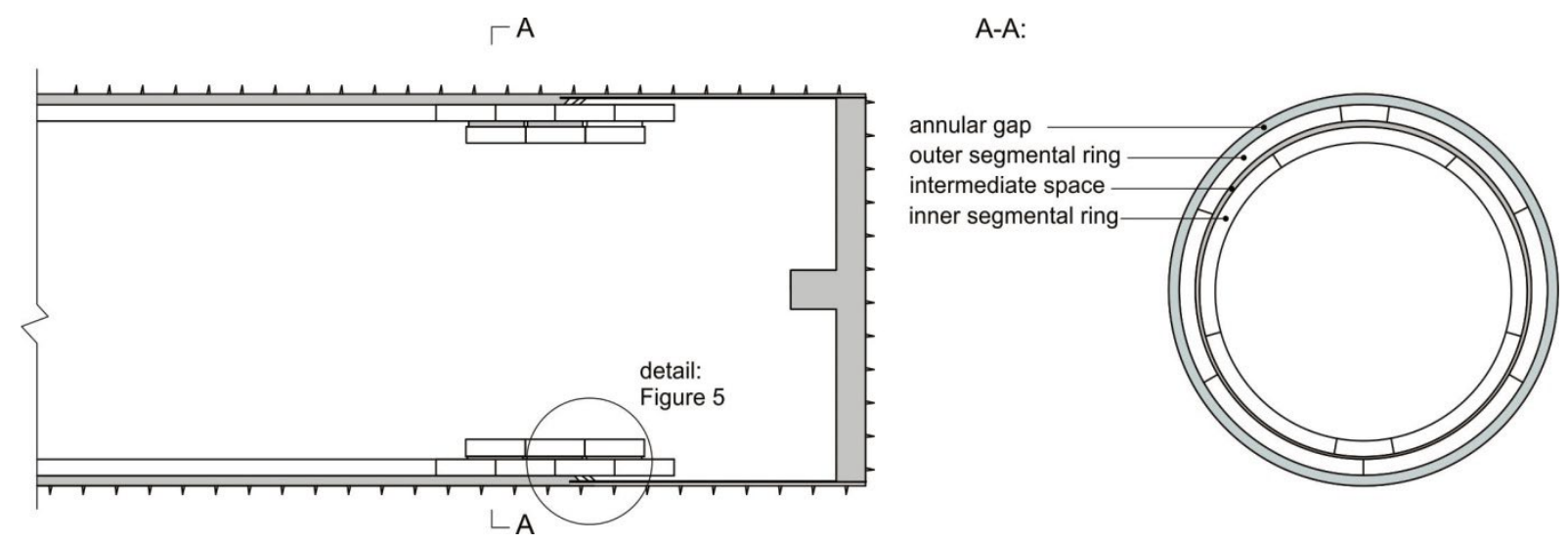

Figure 14.4. Longitudinal section and cross-section of the double shell lining with segmental rings.

In order to enable a certain shear bond between the inner and outer shell, the intermediate space between the two segmental rings has to be grouted. For this purpose, either a stopend formwork on the jack pressure ring or a sealing frame with flexible spacer disks (cellular rubber frame) is fixed to every segment (Fig. 14.5). The annular gap is grouted directly through the shield tail ("1" in Fig. 14.5a); the intermediate space is grouted through openings in the inner segmental ring ("2" in Fig. 14.5a). Thereby, both the space between the two rings and the annular gap can be grouted immediately behind the shield. To ensure a good-quality bedding of the outer segmental ring, the annular gap must be grouted first. Furthermore, in order to avoid direct seepage flow paths, the circumferential and the longitudinal joints of the second segmental ring have to be installed with an offset (Fig. 14.4).

From the logistics point of view, it would be simpler to install the second segmental ring during TBM standstills. However, as standstills are unfavourable under squeezing conditions, the inner segmental ring should be installed during TBM advance (standstills would then be necessary only to install the outer segmental rings). This can be achieved provided that, (i), erectors are installed that can work in parallel, (ii), the hydraulic jacks are not needed for installation of the inner ring and, (iii), the two segmental rings can be delivered at practically the same time. These requirements can probably be fulfilled only with large machine diameters and may necessitate a longer shield (due to the space needed for storage and installation of the segmental rings), which would be unfavourable under squeezing conditions.

Since the inner segmental ring is only installed in squeezing zones, particular attention must be paid to the transition (see Section 14.3). Such a lining system requires a non-standard TBM, which must be planned and designed in close cooperation with the machine manufacturer. 

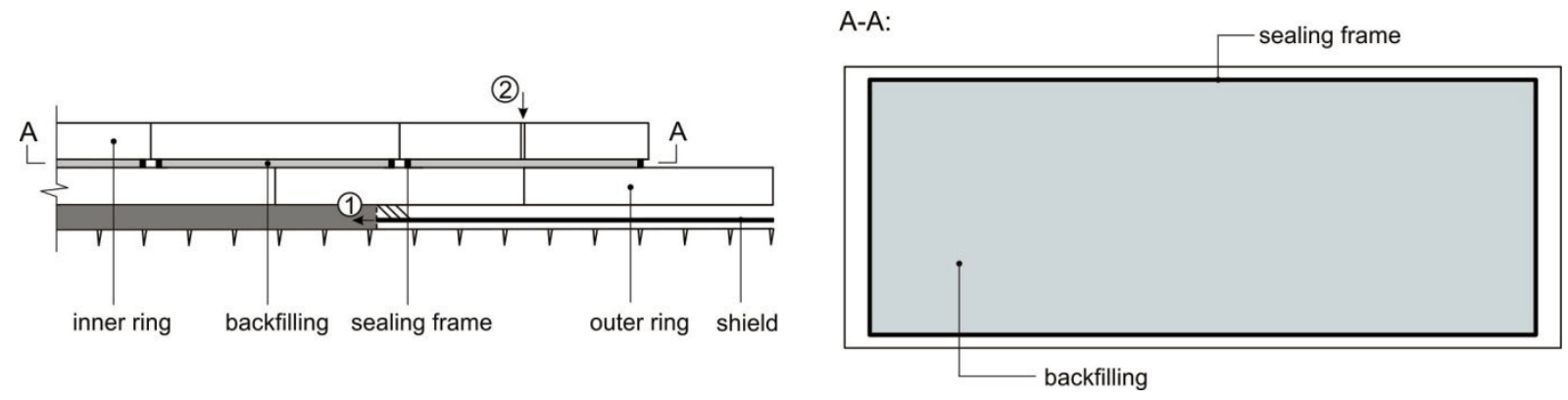

Figure 14.5. Double shell lining system consisting of two segmental rings: (a) Detail of Figure 14.4; (b) Sketch of the internal ring segment with sealing frame.

\subsection{Double shell lining with inner ring of cast in-situ concrete}

From the perspective of structural behaviour, the difference between this lining system and a double segmental lining is that it develops its full bearing capacity later, due to the later installation of the inner lining and the setting-time of the cast in-situ concrete. A further difference is that the inner ring must be made of normal-strength concrete, as (U)HPC cannot be cast in-situ; factory production would be necessary to achieve high strengths and ensure quality (Section 14.4.1).

In order to achieve a high support pressure early on, the inner lining should be installed as soon as possible behind the shield, which is very demanding; it can be constructed only in the back-up area, after the space used for temporary segment storage (the available space directly behind the shield is very limited). Such a system presupposes, therefore, that the rock pressure develops so slowly over time that it reaches its final value in the back-up area far behind the TBM. Reliable predictions about the time-development of rock pressure are, nevertheless, often impossible.

The various forms of lifting gear and the formwork (including the stop-end formwork) which is needed to concrete the inner ring should ideally be part of the back-up equipment. The formwork can, for example, be fixed to a carrier star, or a stop-end formwork consisting of steel ribs could be used. However, the stop-end formwork should be constructed so that standstills in squeezing zones can be avoided.

The casting in-situ of a $10 \mathrm{~m}$ long concrete lining block requires at least one working day. The TBM, however, can often advance further than that in the same time. To avoid standstills in squeezing zones, the TBM drive and the construction of the inner ring have to be uncoupled by providing a sufficient length of the back-up area (for placing the cast in-situ concrete) and a sufficient freedom of movement for the lifting gear (fixed at the TBM). Furthermore, it would be advantageous if a sealing membrane could additionally be installed between the segmental ring and the cast in-situ concrete. This, however, requires a sealing carrier in the back-up area.

For the third tube of the Belchen Tunnel, a double shell lining with an inner ring of cast in-situ concrete is planned. The latter is required to resist the high loads from swelling and is to be installed after the back-up area of the shielded TBM, at latest four months after excavation (Röthlisberger 2015). This lining system is very challenging with regard to logistics (the formwork always interferes with the path of the segments, so that crossing material flows arise) and requires a lot of manual work, so standstills may occur; the technological risk is therefore assessed as high (Table 14.1). Prefabricated elements (e.g. the lining system in 
Section 14.5) would therefore be a simpler option, particularly in difficult geotechnical zones. Such machines would pose major challenges to the machine manufacturer.

\subsection{Load bearing capacity of the lining systems}

\subsubsection{Single or double shell systems of uniform quality concrete}

For a preliminary assessment, the maximum rock pressure $p_{\infty}$ that can be resisted by a stiff lining may be determined by considering the lining as a thick-walled cylinder under external pressure and by setting the maximum hoop stress equal to the compressive strength of the concrete $\sigma_{d}$. Incorporating a safety factor of 4.0 (which accounts in a simplified manner for material imperfections, bending moments and the higher stresses at the longitudinal joints), maximum rock pressure is obtained:

$$
p_{\infty}=\frac{\sigma_{d}}{8}\left(1-\left(\frac{R_{\text {int }}}{R_{\text {int }}+d}\right)^{2}\right),
$$

where $d$ and $R_{\text {int }}$ denote the thickness and the inner radius of the lining, respectively.

(a)

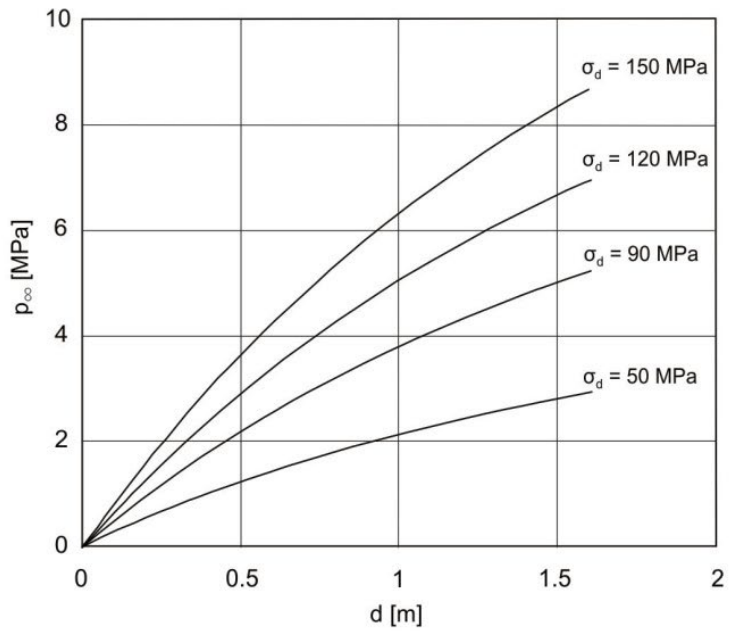

(b)

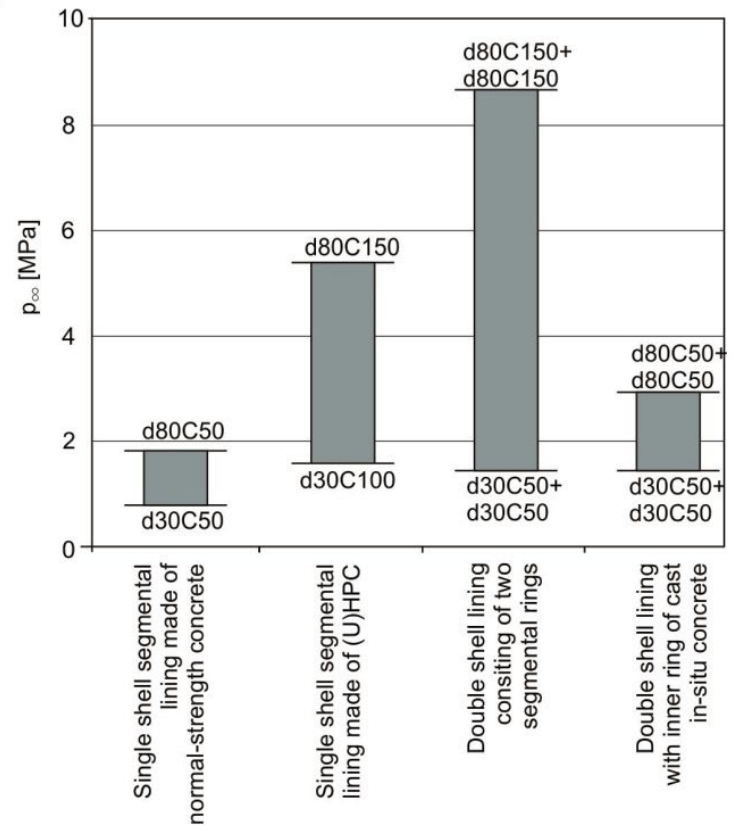

Figure 14.6. (a) Lining resistance $p_{\infty}$ as a function of the lining thickness $d$ and the concrete compressive strengths $\sigma_{d}$; (b) Lining resistance $p_{\infty}$ for the different lining systems (defined by the thickness $d$ and the concrete type $C$ ).

Figure 14.6a shows the lining resistance as a function of the thickness $d$ and of the compressive strength of the concrete $\sigma_{d}$ for an inner radius $R_{\text {int }}$ of $4.35 \mathrm{~m}$, which is typical for single-lane traffic tunnels. The diagram also applies to double shell lining systems, provided that the two rings exhibit the same strength. Figure 14.6b shows the range of resistance offered by the various lining systems in relation to their minimum and maximum thicknesses $d$ (in $\mathrm{cm}$ ) and concrete strength $C$ (in $\mathrm{MPa}$ ). The Figure shows that a single shell segmental lining made of normal strength concrete can resist only limited rock pressure (of ca. $2 \mathrm{MPa}$ ), even when using $1 \mathrm{~m}$ thick segments. A further increase in the thickness of the lining is 
generally of little benefit, as it results in only slightly higher resistance (ca. $3 \mathrm{MPa}$ in the case of a $160 \mathrm{~cm}$ thick lining), but also in greater stiffness and a larger boring radius (in order to maintain the minimum clearance profile) and thus also in higher rock pressures acting on the lining (see Section 14.8). Segments made of (U)HPC, however, can resist high rock pressures, even when a single shell lining is installed (Fig. 14.6b).

Under the simplifying assumption that the stiff lining system is subjected only to normal forces (the safety factor of 4 introduced above also accounts for bending moments), there is no difference between a single and a double shell lining of the same thickness and concrete strength with respect to the ultimate load. In the case of a highly non-uniform rock pressure, a double shell lining would, however, exhibit a lower bearing capacity than a single ring of the same thickness because its resistance to bending is lower in the absence of shear bond between the two shells. In this respect, quick and proper backfilling is of prime importance for the bedding of the segmental lining and thus for its structural resistance.

\subsubsection{Double shells of different quality concretes}

Where the rings of a double shell lining are made of concrete of different strengths, Figure 14.6 is only approximately correct. The bearing capacity of the double shell can be estimated by considering two interacting thick-walled cylinders of thicknesses $d_{1}$ (inner ring) and $d_{2}$ (outer ring). The outer cylinder is loaded with an external pressure $p_{\infty}$ and supported by the contact pressure $\sigma_{k}$ of the two rings; the inner cylinder is loaded with $\sigma_{k}$ (Fig. 14.7).

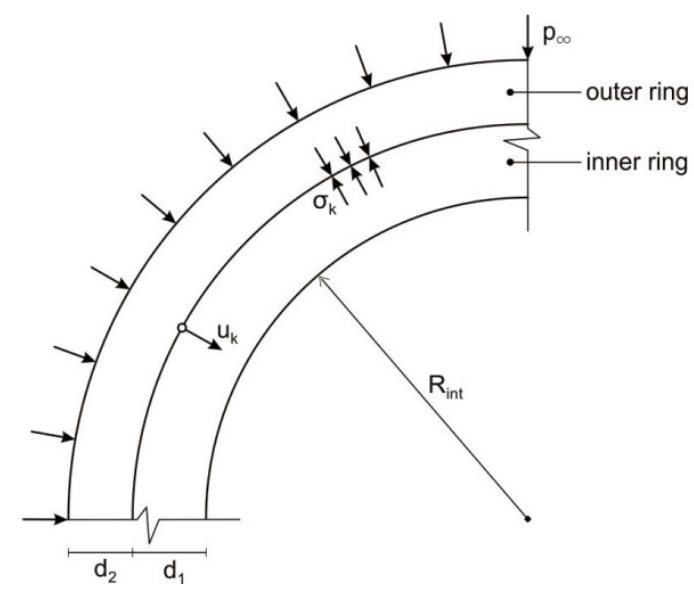

Figure 14.7. Problem layout of the double shell lining made of different strength concretes.

The continuity condition for the radial displacement at $r=R_{\text {int }}+d_{1}$ allows a determination to be made of the contact pressure $\sigma_{k}$, which in turn is used to calculate the maximum hoop stresses $\sigma_{t 1}\left(R_{\text {int }}\right)$ and $\sigma_{t 2}\left(R_{\text {int }}+d_{1}\right)$ in the inner and in the outer ring, respectively. Setting the hoop stress $\sigma_{t 1}\left(R_{\text {int }}\right)$ equal to the strength $\sigma_{d 1}$ of the inner ring concrete, the maximum rock pressure $p_{\infty 1}$, at which the inner ring would fail, is obtained:

$$
p_{\infty 11}=\sigma_{d 1} f_{1}\left(R_{\text {int }}, d_{1}, d_{2}, v_{1}, v_{2}, \frac{E_{2}}{E_{1}}\right),
$$

where

$$
f_{1}=d_{1} R_{1}\left(\frac{E_{2}}{E_{1}} \frac{d_{2} R_{2}}{b_{1}^{2} b_{2}^{2}}\left(\frac{R_{\text {int }}^{2}}{d_{1} R_{1}}+1-v_{1}\right)+\frac{1-v_{2}}{2 b_{2}^{2}}+\frac{1+v_{2}}{2 b_{1}^{2}}\right)
$$




$$
R_{1}=R_{\text {int }}+0.5 d_{1}, \quad R_{2}=R_{\text {int }}+d_{1}+0.5 d_{2}, \quad b_{1}=R_{\text {int }}+d_{1}, \quad b_{2}=R_{\text {int }}+d_{1}+d_{2} .
$$

The rock pressure, at which the outer ring would fail, is obtained analogously, i.e. by setting its maximum hoop stress $\sigma_{t 2}\left(R_{\text {int }}+d_{1}\right)$ equal to $\sigma_{d 2}$ :

$$
p_{\infty 2}=\sigma_{d 2} f_{2}\left(R_{\text {int }}, d_{1}, d_{2}, v_{1}, v_{2}, \frac{E_{2}}{E_{1}}\right),
$$

where

$$
f_{2}=\frac{d_{2} R_{2}}{b_{2}^{2}}+\frac{2 d_{1} R_{1}+d_{2} R_{2}+R_{\text {int }}^{2}}{b_{2}^{2}\left(v_{2}+\frac{E_{2}}{E_{1}}\left(1-v_{1}+\frac{R_{\text {int }}^{2}}{d_{1} R_{1}}\right)\right)} .
$$

With a safety factor of 4 (cf. Section 14.7.1), the bearing capacity of the double shell lining reads as follows:

$$
p_{\infty}=\frac{\min \left(p_{\infty, 1}, p_{\infty, 2}\right)}{4}
$$

In certain cases, particularly if the difference between the concrete strengths is large and the outer ring is thicker than the inner ring, Equation (14.7) may predict that the bearing capacity of the double shell lining will be lower than the bearing capacity of a lining consisting only of the outer ring (Eq. 14.1). This is a paradox of elastic analysis, which disregards stress redistribution (and unrealistically assumes that the entire structure fails) when the stress at a given point of the structure reaches its concrete strength. However, the paradox does not occur if the inner ring is thicker than the outer ring as in the examples of Section 14.9.

\subsection{Rock pressure}

For a structural evaluation of the lining systems, it is necessary to know (in addition to their load bearing capacity) the rock pressure $p_{\infty}$ developing far behind the TBM. The latter can be estimated with the aid of the upper diagrams of Figure 14.8. The diagrams were worked out from the results of numerical analyses (details follow) and cover a wide range of geotechnical conditions (in terms of overburden and rock parameters) and apply to a typical single-lane traffic tunnel (useable radius $R_{\text {int }}=4.35 \mathrm{~m}$ ) excavated by means of a single shielded TBM. 
$\square \mathrm{f}_{\mathrm{c}}=1 \mathrm{MPa} \quad \boldsymbol{\Delta} \mathrm{f}_{\mathrm{c}}=3 \mathrm{MPa} \quad \triangle \mathrm{f}_{\mathrm{c}}=5 \mathrm{MPa}$
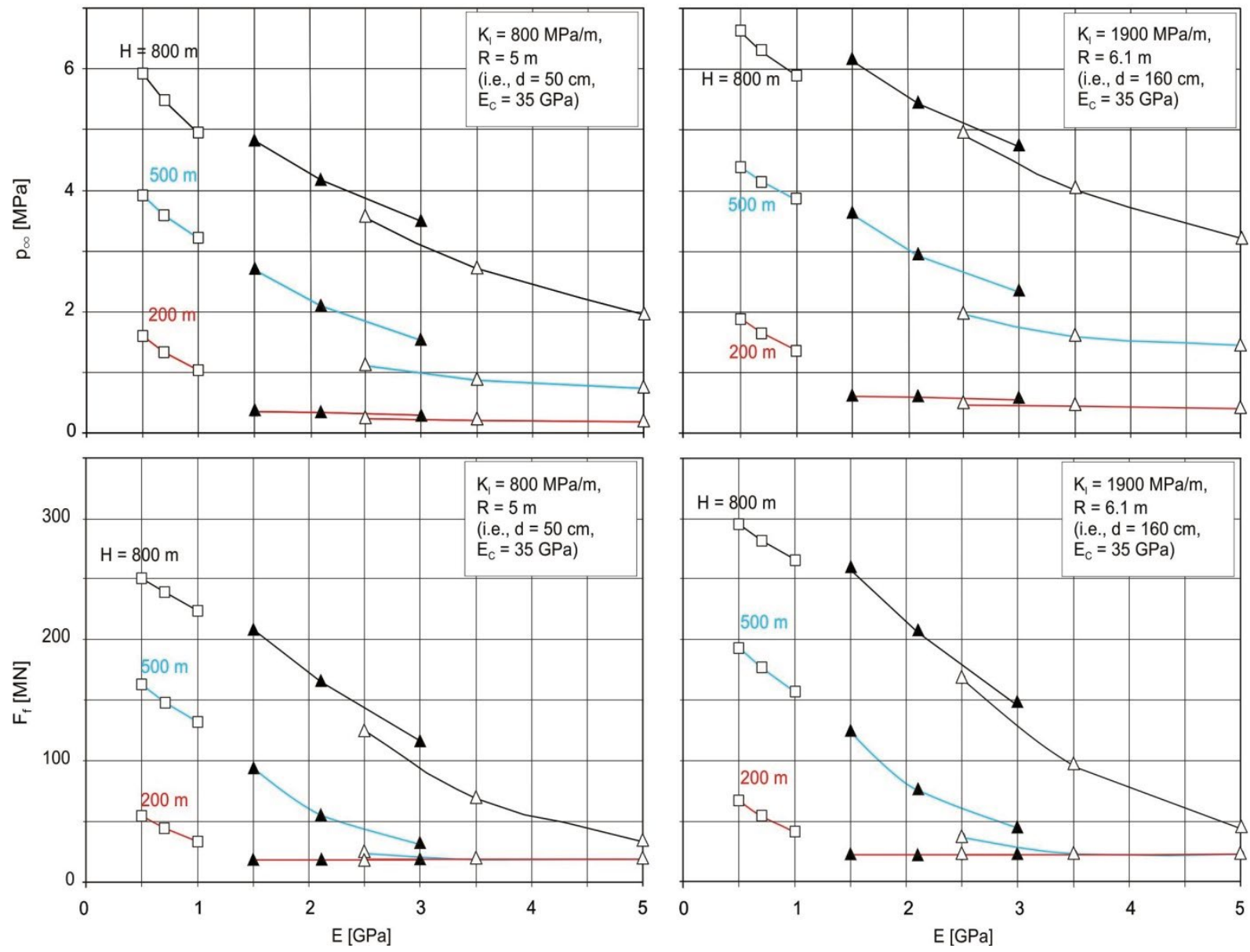

Figure 14.8. Diagrams for estimating the final lining pressure $p_{\infty}$ and the required thrust force $F_{f}$ (parameters according to Table 14.2).

The numerical analyses were carried out taking the boring radius and the lining stiffness for two cases (Fig. 14.9): a $50 \mathrm{~cm}$ thick lining, which can be regarded as the minimum reasonable thickness for squeezing conditions and which results in a boring radius of $5 \mathrm{~m}$ and a stiffness of $800 \mathrm{MPa} / \mathrm{m}$; and a $160 \mathrm{~cm}$ thick lining, which is the maximum feasible thickness of a double shell lining and which results in a boring radius of $6.1 \mathrm{~m}$ and a stiffness of $1900 \mathrm{MPa} / \mathrm{m}$.

(a)

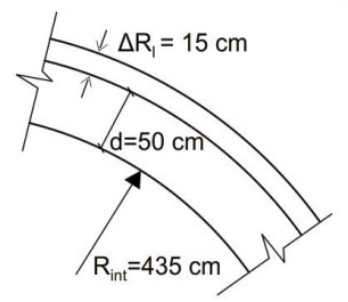

(b)

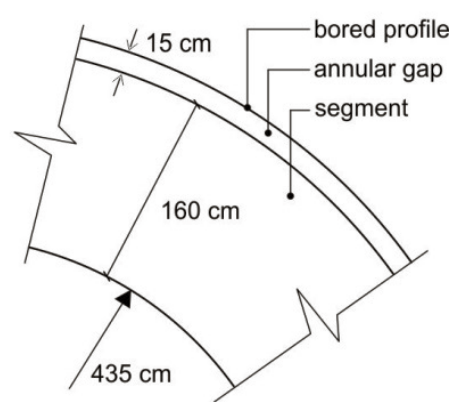

Figure 14.9. Investigated lining systems (minimum clearance radius $R_{\text {int }}=4.35 \mathrm{~m}$; annulus width $\left.\Delta R_{I}=0.15 \mathrm{~m}\right)$.

In contrast to Mezger et al. (2015a) and Ramoni et al. (2011b), where the lining stiffness was calculated using a thin-walled cylinder $\left(K_{l}=d E_{c} / R^{2}\right)$, in this chapter a thick-walled cylinder with an inner radius of $R_{\text {int }}$ and an external radius of $R_{\text {int }}+d$ is considered, which leads to 


$$
K_{I}=\frac{E_{c} d}{\left(R_{\text {int }}+d\right)^{2}} \frac{2-\bar{d}}{2-\bar{d}(2-\bar{d})\left(1+v_{c}\right)}\left(\text { where } \bar{d}=\frac{d}{R_{\text {int }}+d}\right)
$$

The stiffness of a double shell lining (Fig. 14.7) made of different strength concretes, and thus having different Young's moduli, is defined as the rock pressure that causes a unit radial displacement of the outer ring extrados and is calculated as outlined in Section 14.7.2. It reads as follows:

$$
K_{l}=\frac{-2 d_{2} E_{2} R_{2}}{b_{2}\left(2 b_{1}^{2} f_{3}-\left(1-v_{2}\right) b_{2}^{2}-\left(1+v_{2}\right) b_{1}^{2}\right)},
$$

where

$$
f_{3}=\frac{2 b_{2}^{2}}{\frac{d_{2} E_{2} R_{2}}{d_{1} E_{1} R_{1}}\left(\left(1+v_{1}\right) R_{\text {int }}{ }^{2}+\left(1-v_{1}\right) b_{1}^{2}\right)+\left(1-v_{2}\right) b_{1}^{2}+\left(1+v_{2}\right) b_{2}^{2}}
$$

\begin{tabular}{|c|c|c|}
\hline TBM and lining & & \\
\hline Young's modulus of the lining $E_{C}$ & \multicolumn{2}{|c|}{$35 \mathrm{GPa}$} \\
\hline Poisson's ratio of the lining $v_{c}$ & \multicolumn{2}{|c|}{0.2} \\
\hline Inner radius of the lining $R_{\text {int }}$ & \multicolumn{2}{|c|}{$435 \mathrm{~cm}$} \\
\hline Annular gap $\Delta R_{l}$ & \multicolumn{2}{|c|}{$15 \mathrm{~cm}$} \\
\hline Lining thickness $d$ & $50 \mathrm{~cm}$ & $160 \mathrm{~cm}$ \\
\hline Boring radius $R$ & $500 \mathrm{~cm}$ & $610 \mathrm{~cm}$ \\
\hline Number of cutters ${ }^{(1)}$ & 67 & 82 \\
\hline Stiffness of lining $K_{l}{ }^{(2)}$ & $800 \mathrm{MPa} / \mathrm{m}$ & $\begin{array}{c}1,900 \\
\mathrm{MPa} / \mathrm{m}\end{array}$ \\
\hline Shield length $L$ & \multicolumn{2}{|c|}{$10 \mathrm{~m}$} \\
\hline Radial overcut $\Delta R^{(3)}$ & \multicolumn{2}{|c|}{$5 \mathrm{~cm}$} \\
\hline Shield thickness $d_{s}$ & \multicolumn{2}{|c|}{$7.5 \mathrm{~cm}$} \\
\hline Young's modulus of the shield $E_{s}$ & \multicolumn{2}{|c|}{$210 \mathrm{GPa}$} \\
\hline Shield stiffness $K_{s}{ }^{(4)}$ & \multicolumn{2}{|c|}{$630 \mathrm{MPa} / \mathrm{m}$} \\
\hline Sliding friction coefficient $\mu^{(5)}$ & \multicolumn{2}{|c|}{0.10} \\
\hline Static friction coefficient $\mu^{(5)}$ & \multicolumn{2}{|c|}{0.15} \\
\hline Boring force per cutter (6) & \multicolumn{2}{|c|}{$267 \mathrm{kN}$} \\
\hline \multicolumn{3}{|l|}{ Geotechnical conditions } \\
\hline Young's modulus $E$ & $500 f_{c}, 700$ & or $1000 f_{c}$ \\
\hline Poisson's ratio $v$ & \multicolumn{2}{|c|}{0.25} \\
\hline Uniaxial compressive strength $f_{c}$ & \multicolumn{2}{|c|}{1,3 or $5 \mathrm{MPa}$} \\
\hline Angle of internal friction $\varphi$ & \multicolumn{2}{|c|}{$25^{\circ}$} \\
\hline Dilatancy angle $\psi$ & \multicolumn{2}{|c|}{$5^{\circ}$} \\
\hline Unit weight $y$ & \multicolumn{2}{|c|}{$25 \mathrm{kN} / \mathrm{m}^{3}$} \\
\hline Depth of cover $H$ & \multicolumn{2}{|c|}{200,500 or $800 \mathrm{~m}$} \\
\hline
\end{tabular}

Table 14.2. Assumed parameter values for working out the design charts.

\footnotetext{
(1) $6.7 \times$ (boring diameter)/m (Vigl et al. 1999)

(2) $K$ I according to Equation (14.8)

(3) Can be achieved by changing the position of the gauge cutters (Herrenknecht et al. 2010)

(4) $K_{s}=E_{s} d_{s} / R^{2}$; calculated for $R=500 \mathrm{~cm}$ (Ramoni et al. 2011b)

(5) With lubrication of shield skin (Ramoni and Anagnostou 2011b)

(6) Sänger (2006)
} 
The numerical analyses did consider the interaction between the advancing shield, the ground and the tunnel lining. Assuming homogeneous and isotropic ground and a hydrostatic and uniform initial stress field, an axially symmetric elasto-plastic model was applied (Fig. 14.10a). The time-dependency of ground behaviour was not taken into account. The shield and the lining were numerically modelled by prescribing appropriate boundary conditions: After the closure of the annular gap around the shield a rock pressure develops, which is taken as linearly elastic (stiffness $K_{s}$, Fig. 14.10b); the stiff lining, too, is taken as linearly elastic with a constant stiffness $K_{I}$ (Fig. 14.10c), which presupposes that the annular gap is grouted directly behind the shield. The calculations were carried out using the finite element program HYDMEC of the ETH Zurich and the so-called "steady state method" (Anagnostou 2007), which solves the advancing heading problem in just one computational step by taking into account the progress of the shield and the installation of the lining. Further details can be found in Ramoni and Anagnostou (2010b) and Ramoni and Anagnostou (2011b). Table 14.2 summarises the assumed model parameters.

(a)

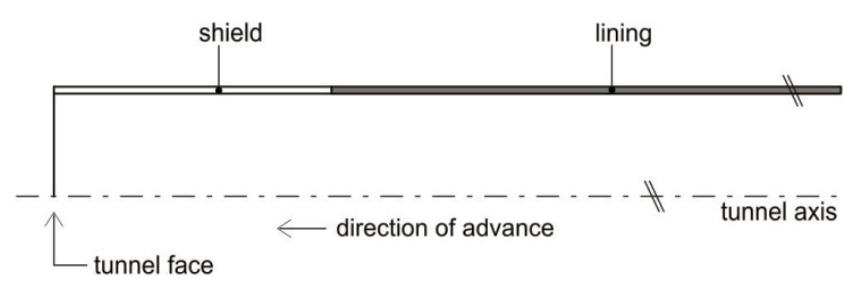

(b)

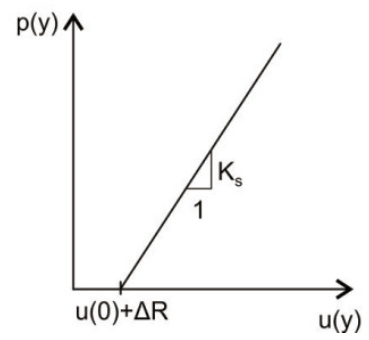

(c)

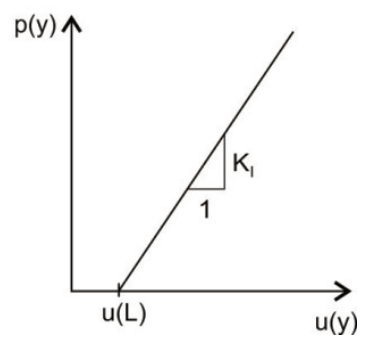

Figure 14.10. Problem layout (a) and boundary conditions at the tunnel wall in the shield area (b) and along the lining (c).

An evaluation of the various segmental lining options would not make sense if squeezing is of such a high intensity that it could immobilise the shield. Conditions under which the thrust force required to overcome shield friction is prohibitively high (more than 200-300 MN; Ramoni and Anagnostou 2010a), even when all possible measures are taken (short shield, adequate overcut, lubrication of the shield, immediate grouting of the annular gap, auxiliary hydraulic jacks), do not need to be considered in the lining evaluation. In order to avoid investigating cases necessitating an excessive thrust force, additional diagrams are provided in Figure 14.8 for estimating the required thrust force. The latter was determined from the numerically computed rock pressure distribution along the shield, assuming that the shield extrados is lubricated and considering the conditions both during boring (in this case thrust force must overcome sliding friction and the rock's resistance to boring) and during TBM restart after a standstill (when the thrust force must overcome static friction).

Generally, the installation of a stiff lining immediately behind the shield favours longitudinal arching in the surrounding rock, thus resulting in lower shield loading and helping to avoid shield jamming (Ramoni and Anagnostou 2011a). The numerical calculations assume that the lining offers resistance to rock deformation with its full stiffness immediately behind the shield. This assumption presupposes that the annular gap is grouted immediately via the 
shield tail and it is approximately valid for a double shell lining with an inner cast in-situ concrete ring far behind the shield (Section 14.6). Improper annulus grouting or backfilling with pea gravel allow deformations to occur (Ramoni et al. 2011b), which is favourable with respect to the lining, but increases the risk of shield jamming. In these cases, Figure 14.8 underestimates the required thrust force, but is anyway on the safe side with respect to the lining. Furthermore, the numerical calculations do not consider the increased deformability of the lining due to the joints. Therefore, for the segmental linings (without an inner shell of cast in-situ concrete), Figure 14.8 tentatively underestimates the required thrust force and overestimates the lining pressure. Due to the large normal forces, this effect is however small in squeezing rocks.

The rock pressure for linings with a different thickness, concrete modulus $E_{c}$ and boring radius can be estimated by calculating the lining stiffness $K_{l}$ using Equation (14.8) for single shell linings and Equation (14.9) for double shell linings (of different strength concretes) and interpolating or extrapolating between the diagrams given for $K_{l}=800$ and $1900 \mathrm{MPa} / \mathrm{m}$. The interpolated rock pressures are on the safe side, provided that the Young's modulus of the lining $E_{c}$ (which affects the lining stiffness) is equal to minimum $35 \mathrm{GPa}$. The reason for this is that the r.h.s. diagram of Figure 14.8 assumes not only a greater stiffness, but also a bigger boring radius. Assume, for example, that the actual lining thickness and boring radius are exactly the same as assumed in the I.h.s. diagram, but a higher quality concrete is used that exhibits a higher Young's modulus (e.g. HPC with $E_{c}=44 \mathrm{GPa}$ instead of $35 \mathrm{GPa}$ - the value assumed by the I.h.s. diagram). The lining stiffness in this case would be about $1000 \mathrm{MPa} / \mathrm{m}$. Interpolation would overestimate rock pressure because the I.h.s. diagram (which applies to $K_{l}=800 \mathrm{MPa} / \mathrm{m}$ ) assumes the same boring radius, but the r.h.s diagram (which applies to $K_{l}=1900 \mathrm{MPa} / \mathrm{m}$ and is used in the interpolation) assumes a higher than actual radius. In general, the higher the Young's modulus is, the less exact are the interpolated or extrapolated rock pressures. For example for a $40 \mathrm{~cm}$ thick lining made of $\mathrm{HPC}\left(E_{c}=44 \mathrm{GPa}\right)$ and a boring radius $\mathrm{R}=4.9 \mathrm{~m}$, the interpolation-induced overestimation of the rock pressure amounts to $2 \%$, and for a $120 \mathrm{~cm}$ thick lining made of HPC $\left(E_{c}=44 \mathrm{GPa}\right.$ ) and a boring radius $\mathrm{R}=5.7 \mathrm{~m}$ to $6 \%$ (for $E=1.5 \mathrm{GPa}, f_{c}=3 \mathrm{MPa}$ and $H=500 \mathrm{~m})$.

It should be noted that the diagrams of Figure 14.8 apply for a specific shield diameter, shield length and shield stiffness (Table 14.2). For other TBM parameters the nomograms of Ramoni and Anagnostou (2010b) and Ramoni et al. (2011b) should be used, keeping in mind that these nomograms incorporate simplifying assumptions on the safe side. More specifically, they assume a normalised shield stiffness $K_{s} R / E$ of 10 , which is sufficiently accurate for $K_{s} R / E>2$, but overestimates the lining load and thrust force required for lower values $\left(K_{s} R / E=0.7-7\right.$ in the calculations of the present chapter).

\subsection{Comparative cost analysis}

This section compares the segmental lining systems of Figure 14.1 from the cost viewpoint by means of an example which also illustrates the use of the design diagrams. The example is complex insofar as it involves zones of variable length and squeezing intensity, in which the rock pressure is in the order of 1 to $3 \mathrm{MPa}$, where all of the lining systems of Sections 14.3 to 14.6 are feasible from the bearing capacity viewpoint according to Figure $14.6 \mathrm{~b}$. Specifically, a fictitious tunnel with a minimum clearance radius of $4.35 \mathrm{~m}$ will be considered, crossing non-squeezing, moderately squeezing and heavily squeezing ground (hereafter referred to as ground types 1, 2 and 3, respectively) with the parameters in Table 14.3. 
Table 14.3. Ground types considered.

\begin{tabular}{llcccc}
\hline$j$ & Ground type & Length & $\begin{array}{c}H \\
{[\mathrm{~m}]}\end{array}$ & $\begin{array}{c}E \\
{[\mathrm{GPa}]}\end{array}$ & $\begin{array}{c}f_{c} \\
{[\mathrm{MPa}]}\end{array}$ \\
\hline 1 Non-squeezing & variable & 500 & - & - \\
2 & Moderately & variable & 500 & 3.0 & 3.0 \\
$\begin{array}{l}\text { squeezing } \\
3\end{array}$ & Heavily squeezing & variable & 500 & 2.6 & 2.0 \\
\hline
\end{tabular}

First, the minimum lining thickness has to be determined, considering the parameters of each ground type and the overburden, as well as the strength and the Young's modulus of the concrete types used $\left(E_{C 50}=37 \mathrm{GPa}, E_{C 90}=44 \mathrm{GPa}, E_{C 120}=50 \mathrm{GPa}\right)$. In general, this must be done iteratively, because the necessary lining thickness depends on the rock pressure, which in turn depends on the initially unknown lining stiffness. The iterative procedure for a single shell lining is as follows:

1. Estimate the rock pressures $p_{\infty, 800 \mathrm{MPa} / \mathrm{m}}$ and $p_{\infty, 1900 \mathrm{MPa} / \mathrm{m}}$ (for a lining stiffness $K_{/}$of $800 \mathrm{MPa} / \mathrm{m}$ and $1900 \mathrm{MPa} / \mathrm{m}$, respectively) using the nomograms of Figure 14.8.

2. Make a guess at the actual lining stiffness. (It is recommended to start by assuming $K_{l}=800 \mathrm{MPa} / \mathrm{m}$. )

3. Estimate rock pressure taking the last guess for the lining stiffness. As the latter will generally differ from the stiffness values assumed by the design charts, one has to

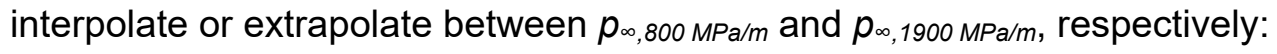

$$
p_{\infty}=p_{\infty, 800 \mathrm{MPa} / \mathrm{m}}+\left(p_{\infty, 1900 \mathrm{MPa} / \mathrm{m}}-p_{\infty, 800 \mathrm{MPa} / \mathrm{m}}\right) \frac{K_{l}[\mathrm{MPa} / \mathrm{m}]-800}{1100} .
$$

4. Calculate the required lining thickness for the rock pressure from step 3 and considering the concrete strength (Eq. 14.1):

$$
d=R_{\text {int }}\left(\sqrt{\frac{\sigma_{d}}{\sigma_{d}-8 p_{\infty}}}-1\right) .
$$

5. Calculate the actual lining stiffness $K_{l}$ (Eq. 14.8) for the lining thickness from step 4 and considering the elasticity constants of the concrete.

6. If the actual stiffness is equal to the stiffness assumed by step 3, then the rock pressure has been estimated correctly and the computation stops here. Otherwise one has to repeat steps 3 to 6 until the lining stiffness and thus the rock pressure remains constant.

The procedure for a double shell lining made of given (different) concrete strengths is basically the same, the main difference being that there are two design parameters in this case (the two lining thicknesses), one of which, e.g. the thickness of the outer ring, has to be chosen a priori. The thickness of the other ring can then be determined as above, using of course Eqs. (14.7) and (14.9) in steps 4 and 5, respectively.

The iterative procedure provides the theoretical minimum required thickness. However, a greater thickness may be chosen for practical reasons. In this case, it must be considered that a thicker lining is stiffer, which results in a higher load. Therefore, a final check has to be made by comparing its resistance (Eq. 14.1 or Fig. 14.6 for single shell linings, Eq. 14.7 for double shell linings of different strength concretes) with the rock pressure corresponding to its stiffness (Eq. 14.11). 
It should be noted that step 1 has to be carried out only once. For the moderately squeezing zone of the example considered ( $\left.E=3 \mathrm{GPa}, f_{c}=3 \mathrm{MPa}, H=500 \mathrm{~m}\right)$, the rock pressures can be depicted directly (1.53 and $2.31 \mathrm{MPa}$; marked red in Fig. 14.11, which reproduces Fig. 14.8). For the heavily squeezing zone ( $\left.E=2.6 \mathrm{GPa}, f_{c}=2 \mathrm{MPa}, H=500 \mathrm{~m}\right)$, however, the rock pressures have to be estimated by extrapolation. The curves for $f_{c}=3$ and $5 \mathrm{MPa}$ give values of $p_{\infty, 800 \mathrm{MPa} / \mathrm{m}}=2.11 \mathrm{MPa}$ and $p_{\infty, 1900 \mathrm{MPa} / \mathrm{m}}=2.93 \mathrm{MPa}$ (marked green in Fig. 14.11); comparative numerical calculations with the exact data showed that the extrapolation-induced error is less than $5 \%$.
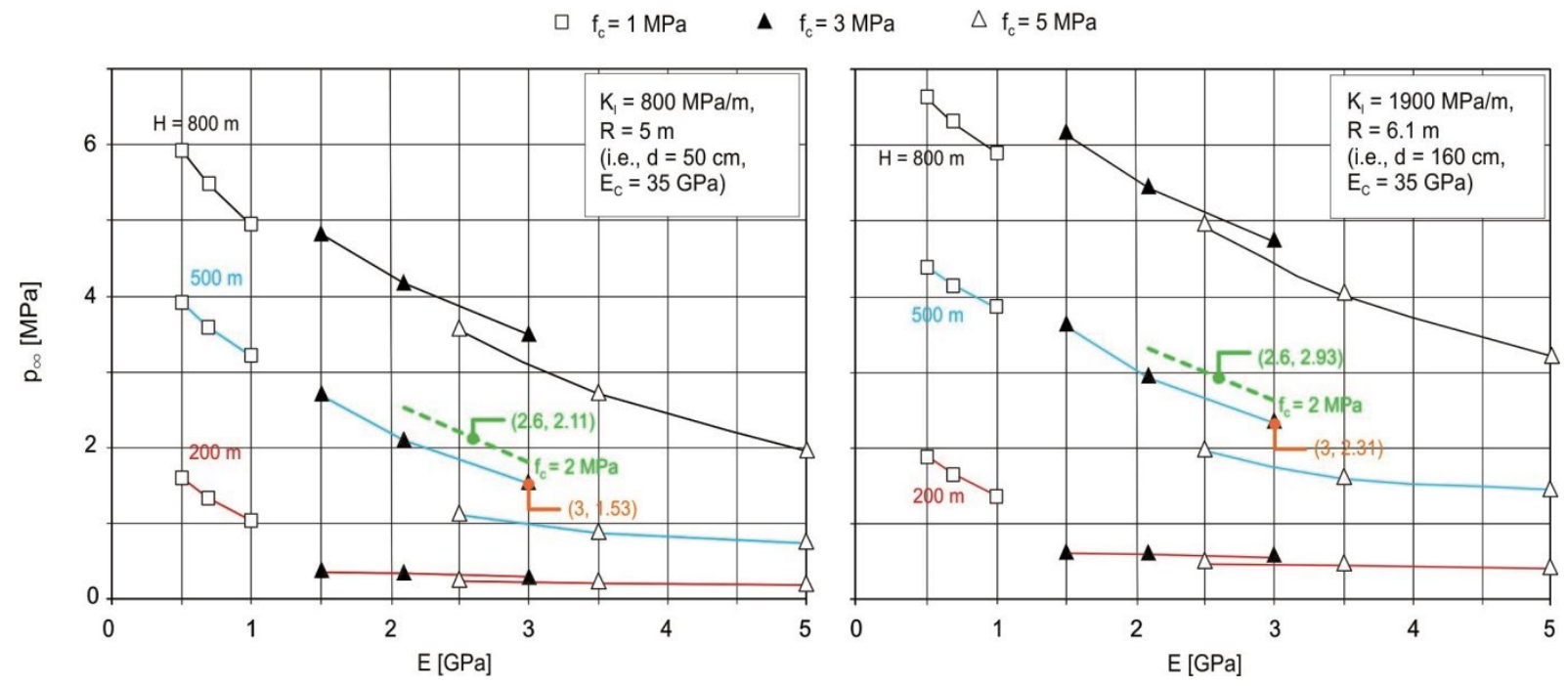

Figure 14.11. Detail from Figure 14.8 with final rock pressure for the ground parameters and overburden from Table 14.3 and a lining stiffness of $K_{I}=800 \mathrm{MPa} / \mathrm{m}$ or $K_{I}=1900 \mathrm{MPa} / \mathrm{m}$.

Table 14.4 shows the iteration progress for the example considered. The estimated rock pressures are given in brackets in the last columns. The corresponding lining thicknesses are given next to the rock pressures (values outside brackets). In the first iteration, the rock pressure is the same for all qualities of concrete, because the first iteration step assumes a fixed value of the lining stiffness $\left(K_{l}=800 \mathrm{MPa} / \mathrm{m}\right)$, i.e. it does not account for the differences in the concrete moduli $E_{c}$ and of course does not need an interpolation $\left(p_{\infty}=p_{\infty}, 800 \mathrm{MPa} / \mathrm{m}\right)$. The iterations converge rapidly. Depending on the concrete quality and the ground type, the lining has to be minimum $0.21-1.78 \mathrm{~m}$ thick (Table 14.4 , last iteration).

Table 14.4. Iterative determination of the final rock pressure $p_{\infty}([\mathrm{MPa}]$, in brackets) and the corresponding minimum lining thickness $d[\mathrm{~m}]$.

\begin{tabular}{|cccc|}
\hline \multicolumn{4}{|c|}{ Ground type $j=2-$ moderately squeezing } \\
$\left(H=500 \mathrm{~m}, E=3 \mathrm{GPa}, f_{c}=3 \mathrm{MPa}\right)$ \\
\hline Iter. & $\mathrm{C} 50$ & $\mathrm{C} 90$ & $\mathrm{C} 120$ \\
\hline 1 & $(1.53) 0.66$ & $(1.53) 0.33$ & $(1.53) 0.24$ \\
2 & $(1.71) 0.76$ & $(1.46) 0.31$ & $(1.38) 0.22$ \\
3 & $(1.81) 0.81$ & $(1.43) 0.31$ & $(1.34) 0.21$ \\
4 & $(1.85) 0.84$ & $(1.42) 0.30$ & $(1.33) 0.21$ \\
5 & $(1.89) 0.86$ & $(1.42) 0.30$ & $(1.33) 0.21$ \\
6 & $(1.89) 0.86$ & $(1.42) 0.30$ & $(1.33) 0.21$ \\
\hline
\end{tabular}

\begin{tabular}{|cccc|}
\hline \multicolumn{3}{|c|}{$\begin{array}{l}\text { Ground type } j=3-\text { heavily squeezing } \\
\left(H=500 \mathrm{~m}, E=2.6 \mathrm{GPa}, f_{c}=2 \mathrm{MPa}\right)\end{array}$} \\
\hline Iter. & $\mathrm{C} 50$ & $\mathrm{C} 90$ & $\mathrm{C} 120$ \\
\hline 1 & $(2.11) 0.99$ & $(2.11) 0.48$ & $(2.11) 0.34$ \\
2 & $(2.61) 1.35$ & $(2.23) 0.51$ & $(2.12) 0.35$ \\
3 & $(2.86) 1.56$ & $(2.27) 0.52$ & $(2.13) 0.35$ \\
4 & $(2.99) 1.67$ & $(2.29) 0.52$ & $(2.13) 0.35$ \\
5 & $(3.08) 1.76$ & $(2.29) 0.53$ & $(2.13) 0.35$ \\
6 & $(3.08) 1.76$ & $(2.29) 0.53$ & $(2.13) 0.35$ \\
7 & $(3.09) 1.77$ & $(2.30) 0.53$ & $(2.13) 0.35$ \\
8 & $(3.10) 1.78$ & $(2.30) 0.53$ & $(2.13) 0.35$ \\
\hline
\end{tabular}




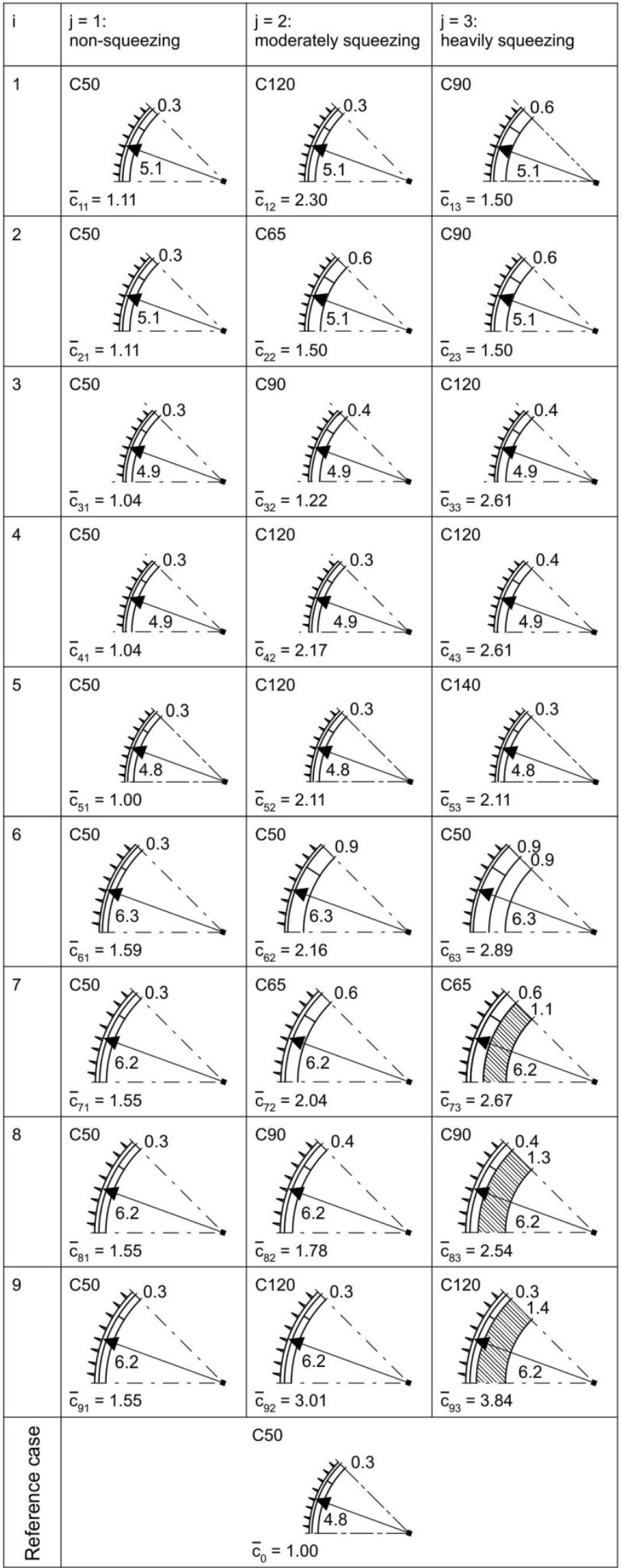

Figure 14.12. Considered lining alternatives $i$ for the ground types $j$ of Table 14.3 as well as for the reference case without squeezing (hatched: cast in-situ concrete of concrete strength C50). 
Taking account of the minimum lining thicknesses from Table 14.4 and a necessary minimum clearance radius of $4.35 \mathrm{~m}$, a series of technical solutions were considered, which differ with respect to the lining in the three ground types $j$ (Fig. 14.12). Only the technically meaningful solutions were considered in the cost analysis. For example, it is not meaningful to apply UHPC (C120) in the moderately squeezing zone (in order to reduce the lining thickness to $30 \mathrm{~cm}$ ) and then use a $150 \mathrm{~cm}$ thick double shell lining made of normal-strength concrete (in combination with a large boring radius) in the heavily squeezing zone. In fact, only normalstrength concrete (as (U)HPC is expensive) or (U)HPC (in order to reduce the boring radius and thus the excavation costs) would be used throughout the entire tunnel. Furthermore, in order to reduce the formwork costs and to avoid the disadvantages associated with a variable lining (Section 14.3), the segment thickness should remain the same in all three zones if possible.

The cost $c_{i j}$ per linear metre tunnel for each ground type $j$ of each solution $i$ of Figure 14.12 reads as follows:

$$
c_{i j}=\alpha \sum_{n} Q_{i j n} p_{n}
$$

where $Q_{i j n}$ and $p_{n}$ denote the quantities per linear metre and their unit prices, respectively, while the factor $\alpha$ considers site installations, unforeseen costs and the acquisition of the TBM. For simplicity, this factor is assumed to be the same for all technical solutions, which basically implies that a more costly lining generally requires a more costly installation and TBM (logistics, greater TBM diameter, a TBM design allowing for the installation of special lining systems).

The following quantities per linear metre were considered: excavation $\left(\mathrm{m}^{3} / \mathrm{m}^{\prime}\right)$, annulus grouting $\left(\mathrm{m}^{3} / \mathrm{m}^{\prime}\right)$, outer segmental ring $\left(\mathrm{m}^{3} / \mathrm{m}^{\prime}\right)$, inner segmental ring (if any; $\left.\mathrm{m}^{3} / \mathrm{m}^{\prime}\right)$ and inner cast in-situ concrete ring (if any; $\mathrm{m}^{3} / \mathrm{m}^{\prime}$ ). Table 14.5 shows the assumed unit prices $p_{n}$, normalised by the excavation unit price $p_{1}$. For simplicity, the latter was assumed to be independent of the geology. The normal-strength concrete and HPC unit prices for the segments include the costs for the minimum reinforcement $\left(90 \mathrm{~kg} / \mathrm{m}^{3}\right)$, while the UHPC unit price includes steel fibres $(3 \%$-vol.). Furthermore, the inner shell made of cast in-situ concrete (hatched in Fig. 14.12, solutions 7 to 9 ) is assumed to be unreinforced. Therefore, the price for the cast in-situ concrete is lower than the one for segments made of normalstrength concrete.

Table 14.5. Assumed unit prices.

\begin{tabular}{llc}
\hline $\mathrm{n}$ & Quantity & Unit price $\left[-/ \mathrm{m}^{3}\right]$ \\
\hline 1 & Excavation & 1.00 \\
2 & Annulus grouting & 2.35 \\
3 & Normal-strength concrete segments $\left(\sigma_{d}=50 \mathrm{MPa}\right.$, & 2.90 \\
& reinforced) & 3.90 \\
4 & HPC segments $\left(\sigma_{d}=65\right.$ or $90 \mathrm{MPa}$, reinforced $)$ & 17.00 \\
5 & UHPC segments $\left(\sigma_{d}=120\right.$ or $140 \mathrm{MPa}$, reinforced $)$ & 2.00 \\
6 & Cast in-situ concrete $\left(\sigma_{d}=50 \mathrm{MPa}\right.$, unreinforced $)$ & \\
\hline
\end{tabular}

In order to better illustrate the squeezing-induced costs, the costs $c_{i j}$ were normalised by the cost $c_{0}$ per linear metre in the reference case of a tunnel without squeezing (bottom of Fig. 14.12), 


$$
\bar{c}_{i j}=\frac{c_{i j}}{c_{0}}
$$

where

$$
c_{0}=\alpha \sum_{n} Q_{0 n} p_{n}
$$

The normalised costs $\bar{c}_{i j}$ are given in Figure 14.12. The normalised average tunnel cost per linear metre for each alternative solution $i$ then reads as follows:

$$
\bar{C}_{i}=\sum_{j} \bar{C}_{i j} \chi_{j}
$$

where $X_{j}$ denotes the fraction of ground type $j$. For simplicity, no account is taken of the potential additional costs associated with squeezing variability. Specifically, according to Equation (14.16), the cost of a tunnel with a single $500 \mathrm{~m}$ long squeezing zone would be the same as that of a tunnel crossing 5 distinct $100 \mathrm{~m}$ long squeezing zones. (In fact, frequently alternating geological zones generally cause additional costs because they necessitate either frequent advance probing and frequent changes in the support type or application of a suboptimum support type.)

Figure 14.13 shows the average normalised tunnel cost $\bar{C}_{i}$ for each solution $i$ of Figure 14.12 as a function of the total length $\left(X_{2}+X_{3}\right)$ of the squeezing zones assuming that the tunnel crosses, (a), only moderately squeezing ground (i.e., $\left.X_{3}=0\right)$, (b), moderately and heavily squeezing ground over equal lengths (i.e., $X_{2}=X_{3}$ ) and, (c), only heavily squeezing ground (i.e., $X_{2}=0$ ).

The following conclusions can be drawn from Figure 14.13:

- Solutions 2, 3 and 5 exhibit the lowest costs, independently of the lengths of the two squeezing ground types. All three solutions involve single shell linings made of (U)HPC in the squeezing zones. The use of normal-strength concrete in the squeezing zones would necessitate partial double shell linings (solutions 6 to 9) and is in general not competitive (even when using unreinforced cast in-situ concrete), because they result in a larger boring radius over the entire tunnel and thus a higher total excavation cost. Applying $(U) H P C$ segments in the squeezing zones reduces excavation costs, which compensates for the higher expenditure on (U)HPC.

- If the squeezing rock zones are rather extended $\left(X_{2}+X_{3}>\right.$ ca. 15\%), solutions with HPC segments and a medium boring diameter are more economical than solutions with UHPC segments and a smaller boring diameter. Specifically, lining systems with the smallest possible lining thickness (in the squeezing zones) that can be achieved with the use of HPC (i.e. C90) are the most cost-effective, as the cost of HPC is low relative to that of UHPC.

- UHPC is very expensive and therefore represents an attractive option only if the squeezing zones make up a small percentage $\left(X_{2}+X_{3}\right)$ of the tunnel length. The maximum length of the squeezing zones for which the use of UHPC is economically worthwhile depends on the boring radius that can be saved when using UHPC compared to HPC. If the tunnel crosses only moderately squeezing ground $\left(X_{3}=0\right)$, UHPC allows for a reduction in boring radius of just $10 \mathrm{~cm}(4.8 \mathrm{~m}$ vs. $4.9 \mathrm{~m})$ and is therefore economically attractive only if the squeezing zone is very short $\left(X_{2}<5 \%\right)$. Under heavily squeezing conditions $\left(X_{2}=0\right)$, however, the difference between the boring radius 
amounts to $30 \mathrm{~cm}$ ( $4.8 \mathrm{~m}$ vs. $5.1 \mathrm{~m}$ ) and thus UHPC is economically worthwhile even if the overall length of the squeezing zones reaches $15 \%$ of the total length.
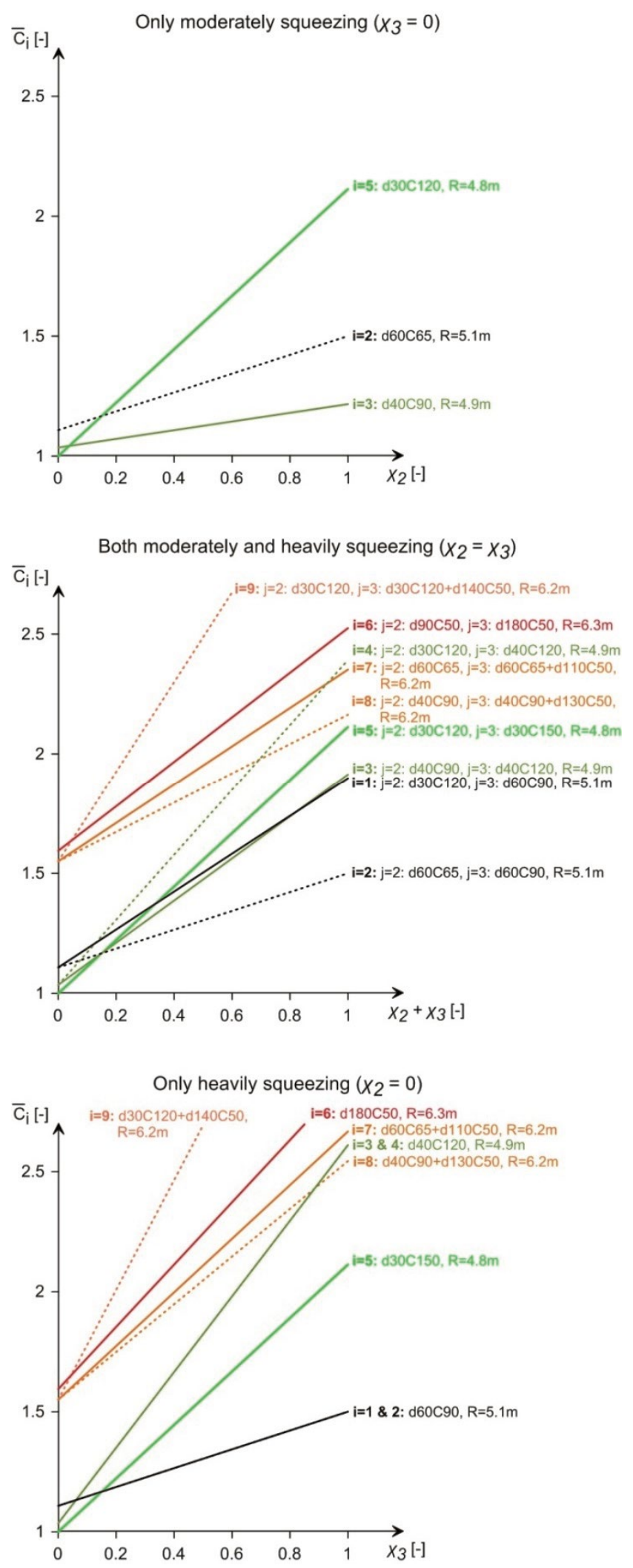

Figure 14.13. Normalised averaged tunnel cost per linear metre as a function of the percentage of the squeezing zones for the lining alternatives $i$ of Figure 14.12 (whereupon $d$ denotes the thickness of the lining, $C$ the concrete strength and $R$ the boring radius). 


\subsection{Conclusions}

This chapter investigated several conceivable high capacity lining systems for TBM tunnelling under squeezing conditions. Besides the obvious solution of thicker segments, resistance can be increased by using a double shell lining consisting of two segmental rings or by using segmental linings made of HPC or UHPC. These lining systems are principally feasible, but introduce a higher technological risk due to the high requirements placed on the shielded TBM, the complexity of the logistics and the non-standard installation and manufacturing procedures. The main problems of (U)HPC are its greater brittleness and, due to its greater density, lower fire-resistance. These problems can be overcome by adding steel- or PP-fibres or by using fire-resistant plasters. When installing an inner ring of cast insitu concrete, the serviceability requirements become less stringent for the external ring. However, double shell lining systems with an inner ring of cast in-situ concrete installed directly behind the shield do not appear promising. Such systems are only meaningful when the rock pressure develops so slowly over time that it reaches its final value in the back-up area far behind the TBM.

The structural analyses showed that a single shell segmental lining made of normal-strength concrete can resist only rather limited rock pressures, even when using $1 \mathrm{~m}$ thick segments. A further increase in the thickness of the lining is generally of little benefit, as it results only in slightly increased resistance, but also in greater stiffness and a larger boring radius and thus in higher rock pressure. Therefore, lining systems consisting of normal-strength concrete soon reach their application limits in squeezing rocks. The structurally most effective way to deal with high rock pressures in squeezing rocks is the use of segments made of (U)HPC. However, the higher Young's modulus of (U)HPC (i.e. the higher stiffness of the lining) has to be taken into account when determining the rock pressures.

Linings made of normal-strength concrete are generally not competitive because they result in a larger boring radius over the entire tunnel and thus higher total excavation costs; the most cost-effective solutions involve HPC or UHPC linings in the squeezing zones. UHPC allows a constant lining thickness and a smaller boring diameter over the whole TBM drive. UHPC is nevertheless very expensive and therefore economically viable only if the squeezing zone makes up a very small percentage of the total tunnel length. Otherwise, solutions with HPC segments and a medium boring diameter are more economical.

\section{Notation}

$b_{1}$

$b_{2}$

C

$c_{i}$

$c_{0}$

$\bar{C}_{i j}$

$\bar{C}_{i}$

$d$

$d_{1}$

$d_{2}$

$d_{s}$

E outer radius of the inner ring

outer radius of the outer ring

type of concrete (defined by its uniaxial compressive strength $\sigma_{d}$ )

cost per linear metre of tunnel for lining solution $i$ in ground type $j$

reference cost per linear metre (tunnel without squeezing)

cost per linear metre of tunnel for lining solution $i$ in ground type $j$ normalised by the reference cost $c_{0}$ normalised average tunnel cost per linear metre for solution $i$

thickness of the lining

thickness of the inner ring

thickness of the outer ring

thickness of the shield

Young's modulus of the rock 


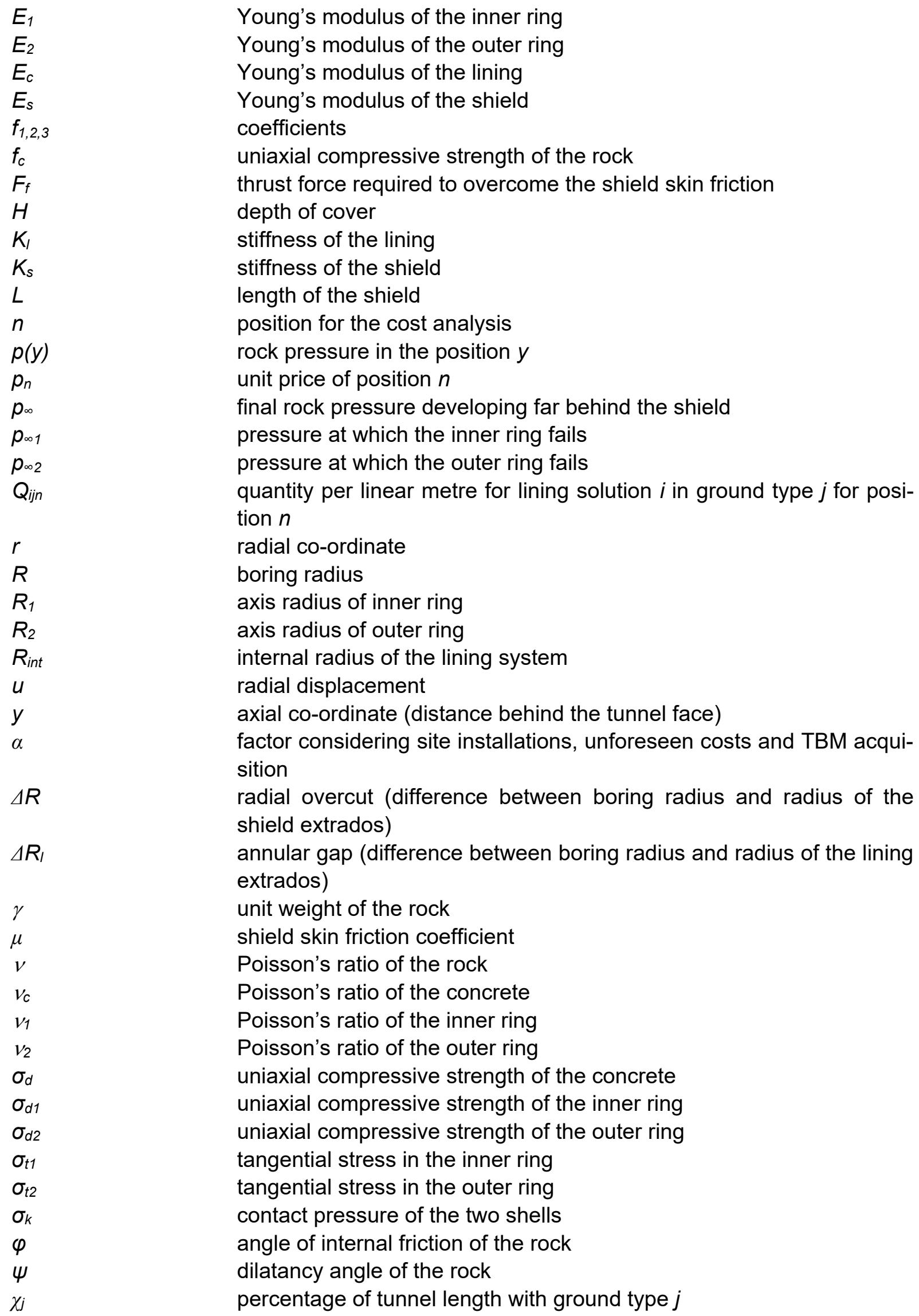




\section{Deformable segmental lining systems ${ }^{6}$}

\subsection{Introduction}

One major hazard of shield tunnelling in squeezing rock is the overstressing of the segmental lining (Ramoni and Anagnostou 2010a). Basically, there are two main support principles for squeezing rock: the resistance principle and the yielding principle (Kovári 1998). Stiff lining systems can be classified as tunnel supports which follow the resistance principle. Their effectiveness and limitations specifically for shield tunnelling through squeezing ground was investigated in the Chapter 14 . According to the yielding principle, the rock pressure and thus the thickness of the lining can be reduced by allowing deformations to occur. (In reality, a situation always arises somewhere between these two extreme cases. For the sake of simplicity, however, the resistance principle will denote practically rigid concrete linings and the yielding principle will denote systems incorporating deformable components.)

(a)

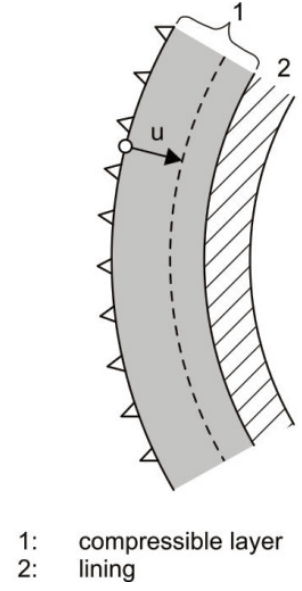

(b)

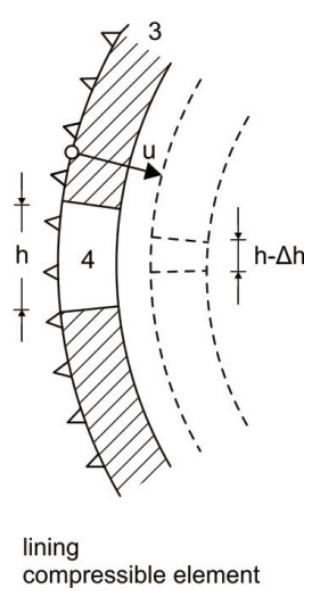

Figure 15.1. (a) Radially deformable, (b), tangentially yielding supports (after Cantieni and Anagnostou 2009).

Two basic options are available for yielding supports (Cantieni and Anagnostou 2009): radially deformable supports, which allow rock deformations to occur outside the lining extrados (Fig. 15.1a; see also John Mowlem and Company Limited 1979), and tangentially deformable linings, which can accommodate a reduction in their circumference (Fig.15.1b). Combinations thereof are also possible at least in principle (Fig. 15.2a).

6 This chapter has been published in: Mezger, F., Ramoni, M., Anagnostou, G. 2018. Options for deformable segmental lining systems for tunnelling in squeezing rock. Tunnelling and Underground Space Technology. Vol. 76: 64-75. 
(a)

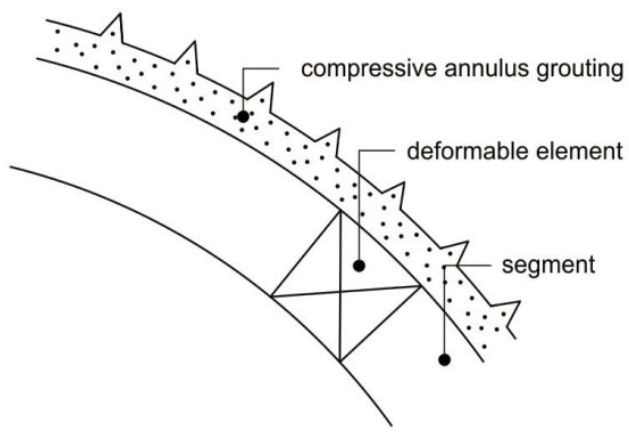

(b)

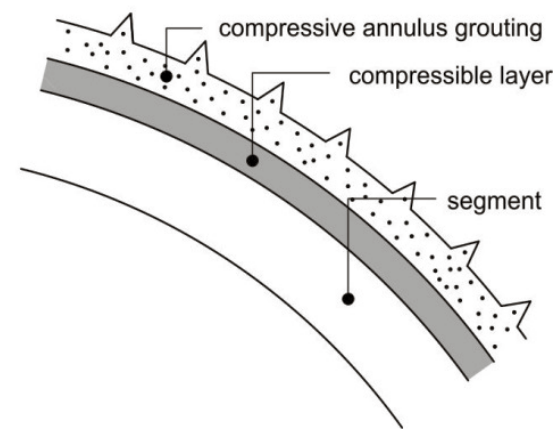

Figure 15.2. Tangentially deformable segmental ring, (a), with yielding elements in the longitudinal joints or, (b), radially deformable composite segments, both in combination with a compressible annulus grout.

Radially deformable segmental linings can be implemented by annulus grouting with a compressible backfill or by using pre-fabricated composite segments made of concrete with a deformable layer at their extrados. Another solution proposed in the past is the so-called "convergence-compatible" segmental lining ("CO-CO"), which incorporates ribs on its extrados providing support to the rock, so that the rock can deform in the spaces between the ribs (Vigl 2003). This system presents the disadvantage of potentially high concentrated loads on the segments (Schneider and Spiegl 2010). In this regard, composite segments made of concrete (without ribs) and a compressible outer layer (Fig. 15.2b) are more favourable. They can be applied in combination with a compressible annulus grout such as "Compex" (Schneider et al. 2005), "DeCo-Grout" (Billig et al. 2007a) or with a backfill made of compressible clay pebbles instead of pea gravel (Semeraro et al. 2014). No example of these systems has yet been implemented in practice: Compressive mortars were applied only once, in a tunnel through non-squeezing rocks excavated by a slurry shield (Jenbach Tunnel, Austria; Gamper et al. 2009). Compressible clay pebbles were planned (but not applied) as backfill in the squeezing section of the Fréjus tunnel (Semeraro et al. 2014).

(a)

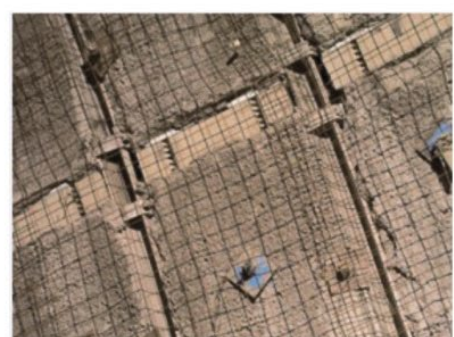

(d)

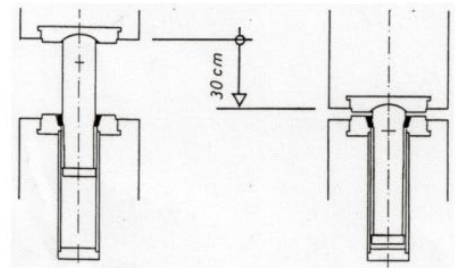

(b)

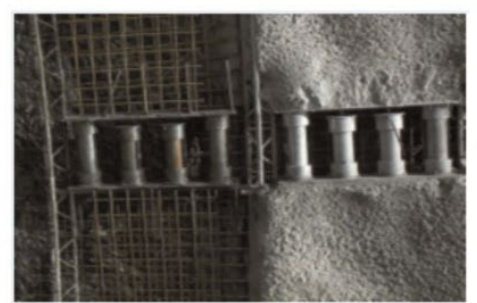

(e)

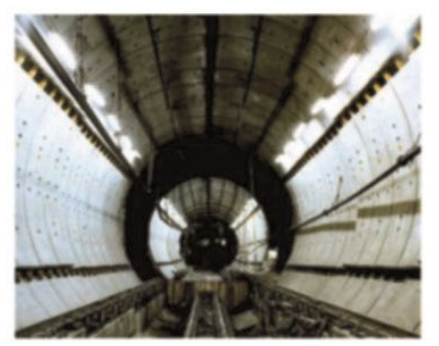

(c)

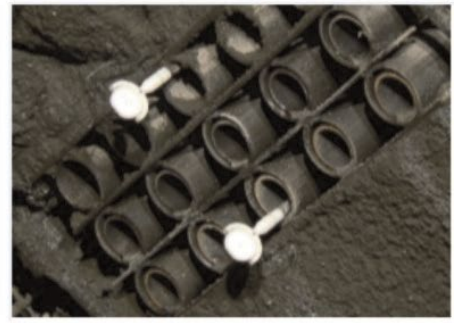

Figure 15.3. (a) Yielding shotcrete lining with hiDCon elements (Kovári 2005) and steel ribs in the Lötschberg Base Tunnel (Solexperts 2013); (b) Lining Stress Controller elements (“LSC", Schubert 1996) with four yielding steel tubes (Moritz 2011); (c) Wabe elements (Podjadtke and Weidig 2010, Moritz 2011); (d) Meypo element (Brunar and Powondra 1985); (e) Meypo elements in a coalmine in Ibbenbüren (Germany, Haecker 1987). 
Tangentially deformable supports incorporate special compressible elements made of concrete, steel or plastic (Fig. 15.3). They have been applied frequently in conventionally driven tunnels (Table 15.1) and occasionally in tunnelling with gripper TBMs, and they could also be implemented in shield tunnelling by arranging compressible elements in the longitudinal joints of the segments.

Table 15.1. Projects with deformable support elements.

\begin{tabular}{|c|c|c|}
\hline Project & Type & References \\
\hline Galgenberg Tunnel & LSC & Schubert (1996) \\
\hline \multirow[t]{3}{*}{ Gotthard Base Tunnel } & hiDCon $^{(1)}$ & Ramoni and Anagnostou (2010a) \\
\hline & Styrofoam ${ }^{(1)}$ & Ramoni and Anagnostou (2010a) \\
\hline & LSC & Billig et al. (2007) \\
\hline Ibbenbüren coalmine & Meypo & $\begin{array}{l}\text { Brunar and Powondra (1985) } \\
\text { Baumann and Zischinski (1994) }\end{array}$ \\
\hline \multirow[t]{2}{*}{ Lötschberg Base Tunnel } & hiDCon & Kovári (2005), Thut et al. (2006) \\
\hline & LSC & Keller (2005) \\
\hline Semmering pilot tunnel & LSC & Schubert et al. (2000) \\
\hline St. Martin La Porte Tunnel & hiDCon & Barla et al. (2008) \\
\hline Strenger Tunnel & LSC & Budil et al. (2004) \\
\hline Tauern Tunnel, 2nd tube & Wabe & Podjadtke and Weidig (2010) \\
\hline
\end{tabular}

(1) Tunnelling by gripper TBM in the Faido-Sedrun section

As that no yielding system has yet been implemented in squeezing rock in combination with a shielded TBM, this chapter evaluates the feasibility of deformable segmental lining systems while taking into account the aspects of structural safety, construction management, TBM technologies and construction costs. The chapter starts with a qualitative discussion of conceivable systems from the viewpoints of deformation capacity, installation procedure, serviceability etc. (Table 15.2). Subsequently the structural interaction between rock and deformable lining systems is investigated and design aids are presented that allow a quick estimation to be made of the lining loading for a wide range of geotechnical conditions. Finally, the last two sections of the chapter compare deformable with stiff linings and investigate the geological conditions for which the use of deformable lining systems is structurally adequate (Section 15.4) and economical (Section 15.5).

Table 15.2. Qualitative comparison of the yielding lining systems.

\begin{tabular}{|l|c|c|c|c|c|c|}
\hline Criterion & Meypo & Wabe & hiDCon & LSC & DeCo-Grout & Compex \\
\hline Initial stiffness & high & medium & high & medium & low & low \\
\hline Yield pressure & high & medium & high & high & low & low \\
\hline $\begin{array}{l}\text { Deformation } \\
\text { capacity }\end{array}$ & high & high & high & high & low & low \\
\hline $\begin{array}{l}\text { Installation } \\
\text { procedure }\end{array}$ & medium & difficult & difficult & difficult & simple & simple \\
\hline Serviceability & difficult & difficult & difficult & difficult & simple & simple \\
\hline Costs & high & high & medium & medium & low & low \\
\hline
\end{tabular}

\subsection{Basic considerations}

\subsubsection{Deformation capacity}

From the structural point of view, the main criteria for the choice of a yielding lining system are the amount of deformation that it can accommodate (hereafter referred to as "yield 
deformation" $u_{y}$ ) and, as will be explained in the next section, its initial stiffness and the support pressure it exerts during the deformation stage (hereafter referred to as "yield pressure" $\left.p_{y}\right)$.

Radially deformable systems with compressible annulus grouts can only accommodate relatively small rock deformations, particularly if squeezing develops rapidly and the rock comes into contact with the shield (i.e., if the convergence $\Delta u$ of the bored profile in the shield area is about the same as the radial overcut $\Delta R$; Fig. 15.4). In this case, the thickness of the compressible grout is equal to the difference between the external radii of the segmental ring and of the shield ( $s$ in Fig. 15.4). As the thickness of the shield tail seals cannot be increased arbitrarily, the space available for the annulus grout is limited. Considering that, for constructional reasons, the width of the sealing gap is 60-95 $\mathrm{mm}$ with a working range for the seals of $30-50 \mathrm{~mm}$ (the so-called "ring building clearance"; Maidl et al. 2011), the thickness of the compressible annulus is potentially small, which limits the effectiveness of this solution.

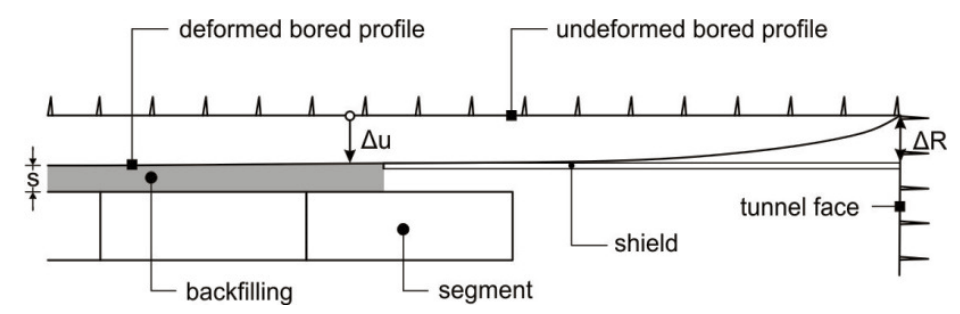

Figure 15.4. Actual thickness of the backfill s (after Ramoni et al. 2011a).

A larger and, very importantly, more predictable rock deformation might be accommodated by radially deformable systems using pre-fabricated composite segments with a compressible layer at their extrados. However, as these outer layers cannot be compressed completely, only a certain fraction of the outer layer thickness (in the order of $30 \%$ ) can be utilised for deformations ( $u$ in Fig. 15.1a).

Tangentially deformable linings are more favourable in this respect. Specifically, if an increase in the boring diameter is considered for allowing rock deformations, this extra space can be used fully for this purpose only with a tangentially deformable system. The compressible elements in the joints are compressed in the tangential direction ( $\Delta h$ in Fig. $15.1 \mathrm{~b}$ ) and the whole available space ( $u$ in Fig. 15.1b) can be utilised. For example, if six compressible elements are incorporated into the longitudinal joints, each having a deformation capacity of $20 \mathrm{~cm}$, the lining radius can decrease by about $20 \mathrm{~cm}$. A radially deformable composite segmental lining with a $20 \mathrm{~cm}$ thick compressible layer at the segment extrados would allow a convergence only of about $6 \mathrm{~cm}$.

\subsubsection{Load-displacement and stress-strain behaviour}

If the lining system can develop a high support pressure immediately behind the shield, then shield loading and thrust demand will be lower because of the longitudinal arching action in the rock around the shield (Ramoni and Anagnostou 2011a). Lining systems exhibiting a low stiffness or deforming at a low yield pressure are thus unfavourable with respect to shield loading. In addition, a low yield pressure of the lining results in a higher final rock pressure in most cases (Cantieni and Anagnostou 2009). Therefore, a high yield pressure should be the aim. 
This requirement cannot be satisfied with radially deformable lining systems utilising compressible annulus grouting mortars, as the latter exhibit low initial stiffnesses, due to their slow hardening process (cf. curves for young and $28 \mathrm{~d}$ old Compex in Fig. 15.5a). In addition, they may result in insufficient lateral embedment of the lining and development of significant bending moments in the case of loosening pressure. To ensure a certain level of rock support right from the start, compressible annular grouts (such as "Compex" or "DeCoGrout"; Schneider et al. 2005, Billig et al. 2007a) should be injected directly behind the shield, ideally simultaneously with TBM advance, through openings in the shield tail.

(a)

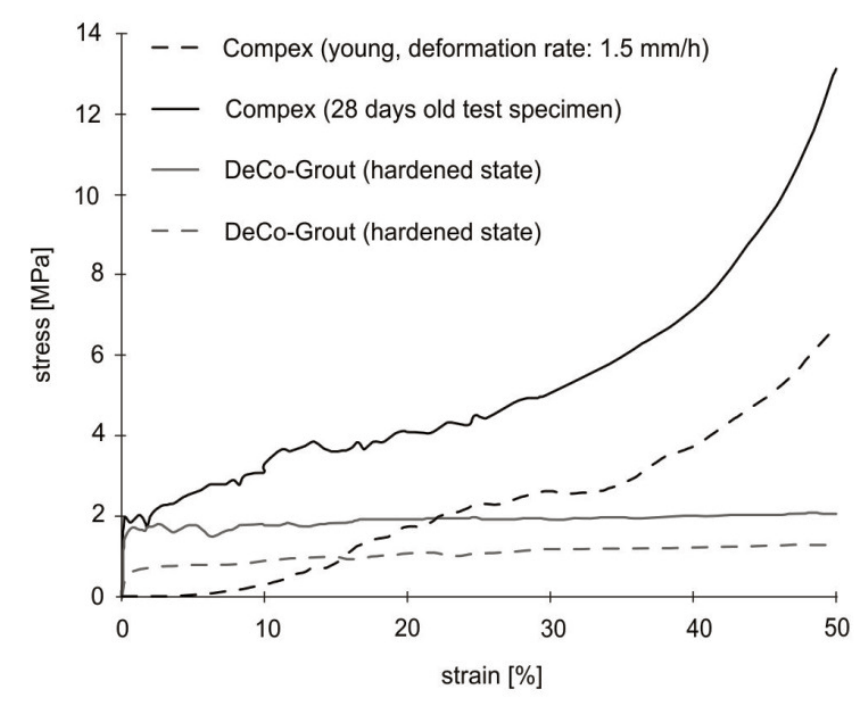

(b)

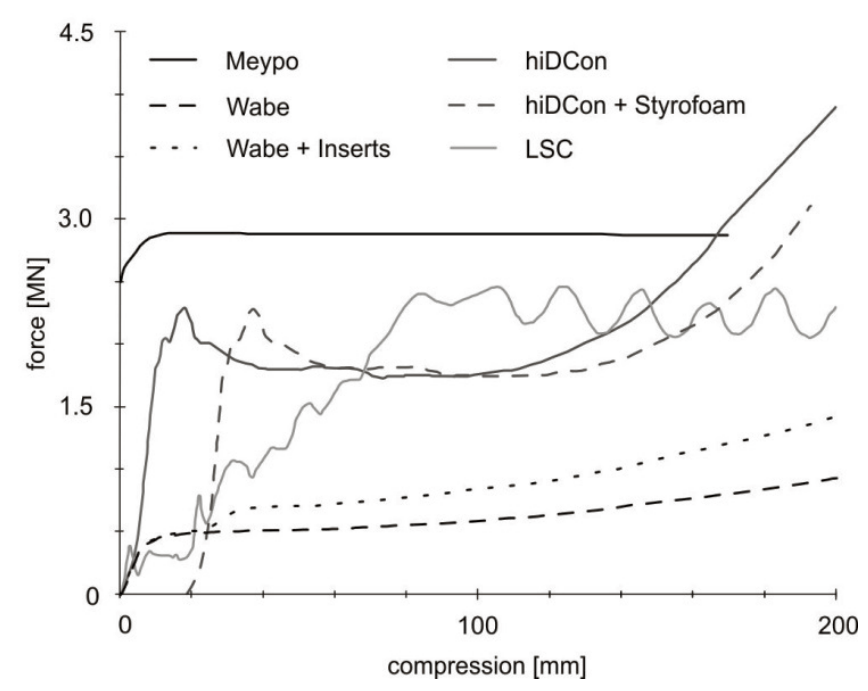

Figure 15.5. (a) Examples of stress-strain curves for compressible mortars (after Schneider et al. 2005, Billig et al. 2007a); (b) examples of load-displacement curves for tangentially deformable elements (hiDCon-elements with dimensions $25 \mathrm{~cm}$ x $100 \mathrm{~cm}$ and $40 \mathrm{~cm}$ high; Wabe-element with 3 rows and 5 elements; LSC with 4x Type A II; after Moritz 2011, Brunar and Powondra 1985).

The requirements for high initial stiffness and high yield pressure can be achieved either with radially deformable linings made of composite segments or with tangentially deformable linings (incorporating yielding elements built into the joints). Based on the results of experimental investigations (Fig. 15.5b; cf. Wiese 2011 and Opolony et al. 2011) the load- 
displacement behaviour of existing yielding elements (Table 15.1 and Figure 15.3) can be outlined as follows:

- The hiDCon elements exhibit practically linearly elastic, perfectly plastic behaviour, characterised by a high initial stiffness and a high yield pressure (4-15 MPa; Solexperts 2013) up to a strain of about $30 \%$ (Thut et al. 2006).

- LSC elements (consisting of a group of four yielding steel tubes), initially exhibit an almost linear increase with low initial stiffness. Subsequently, the load oscillates between about 2050 and $2500 \mathrm{kN}$, due to the buckling of the tubes (Moritz 2011).

- Wabe elements exhibit a relatively quick load-bearing capacity at small compression. The yield load, however, amounts to about $500 \mathrm{kN}$, but can be increased to about $700 \mathrm{kN}$ by inserting additional tubes (Moritz 2011).

- Meypo elements exhibit practically linearly elastic, perfectly plastic behaviour. They are stiff up to about $85 \%$ of the residual load capacity and maintain their load capacity during the deformation stage (Brunar and Powondra 1985). The effectiveness of Meypo elements could be verified for compression of the plunger of up to $17 \mathrm{~cm}$ (Baumann and Zischinski 1994).

\subsubsection{Installation procedure}

The compressible elements of the tangentially deformable segmental linings can be fixed in advance (during pre-fabrication) to the longitudinal joints of the segments. The segments should be installed carefully in order to avoid damage to the yielding elements or their early compression during the forcible insertion of the wedge-shaped key segment. Radially deformable linings made of pre-fabricated composite segments can be installed as usual, but also need care during handling to avoid damage to the outer compressible layer.

In general, the annular gap has to be backfilled in order to ensure a proper embedment of the lining and thus maintain the shape of the system.

\subsubsection{Serviceability}

A tangentially deformable lining can bear considerable normal forces, but only very limited shear forces and bending moments. Asymmetric rock loading can, therefore, lead to misalignment of the longitudinal joints, loss of water tightness or even damage to the lining (Schneider et al. 2005). This problem, however, can be mitigated by offset installation of the longitudinal joints or by including a final inner lining. The latter is not necessary for radially deformable systems (at least from the serviceability viewpoint), because rock deformations occur outside the lining, without affecting the sealing gaskets in the joints.

\subsubsection{Bearing capacity}

Provided that the structural detailing of the longitudinal joints is adequate, the bearing capacity of a deformable segmental lining increases with segment thickness and concrete quality. Basic considerations concerning these factors, including the potential benefits and problems of using high or ultra high performance concretes (HPC, UHPC) for segmental linings according to the resistance principle, can be found in Chapter 14 . They are also valid for radially deformable linings, where the rock deformations occur outside the lining without affecting its shape. However, the use of (U)HPC segments for tangentially deformable linings 
appears to be unreasonable considering that such segments are of high quality (and very expensive), but in combination with deformable longitudinal joints may experience large displacements.

\subsubsection{Monitoring}

Yielding linings adjust their shape to rock deformations. They are vulnerable to non-uniform pressures; the latter can displace the lining entirely (Ramoni and Anagnostou 2010a). An underestimation of the magnitude and distribution of the rock deformations may result in violations of the clearance profile or damage to the segmental lining.

Advance probing can identify potentially critical zones ahead of the tunnel face and enable the timely implementation of planned measures. Depending on the design, decision-making during the TBM drive may have to address: the boring diameter (to provide more space for deformations); the lining type (switch from normal to higher strength segments or from a stiff to a deformable lining of the same thickness); or, as discussed in Chapter 14, even the lining thickness.

Control measurements should be performed to observe the behaviour of the rock and check the effectiveness of a yielding lining. The deformations of tangentially deformable lining systems can be monitored using standard techniques. In the case of radially deformable linings, the surrounding rock and the outer yielding layer may experience relevant deformations while the lining entrados remains practically undeformed. In such cases, extensometers and strain gauges have to be installed (see Semeraro et al. 2014).

\subsubsection{Costs}

Generally, radially deformable systems consisting of a compressible annulus grouting are cheaper than tangentially deformable systems. Tangentially deformable steel elements are expensive (particularly Wabe elements, because of their high steel content; Schubert 2008).

\subsection{Structural assessment}

\subsubsection{Computational model}

The rock pressure $p_{\infty}$ developing upon the lining far behind the TBM depends on the geotechnical conditions (rock mass properties, overburden), on the shield stiffness, shield length and overcut, and on the characteristic line of the lining system (Ramoni et al. 2011a). The latter represents the resistance $p$ offered by the lining system to rock deformation $u$ and depends on the structural detailing of the lining and the type of backfill. The most important parameters of deformable linings are their yield pressure $p_{y}$ and yield deformation $u_{y}$.

The interaction between the advancing shield, the ground and the tunnel lining will be studied here numerically considering an axially symmetric elasto-plastic model (Fig. 15.6a, Ramoni and Anagnostou 2010b). The potentially time-dependent behaviour of the rock is not considered. The shield and the yielding lining are taken into account by introducing appropriate boundary conditions: After the closure of the overcut $\Delta R$, a rock pressure develops on the shield (taken as linearly elastic with stiffness $K_{s}$; Fig. 15.6b) and later on the lining. The deformability of the latter is accounted for by imposing a mixed boundary 
condition according to its characteristic line (Fig. 15.6c), which presupposes that the annular gap is grouted directly behind the shield. This assumption is on the safe side with respect to the lining, as leaving the annular gap open over a certain interval would allow deformations to occur and result in a lower lining loading.

(a)

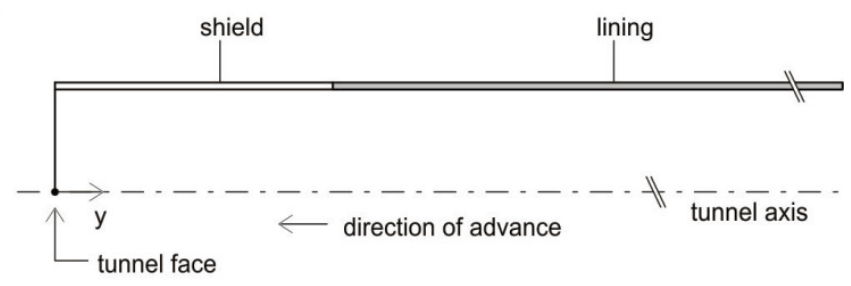

(b)

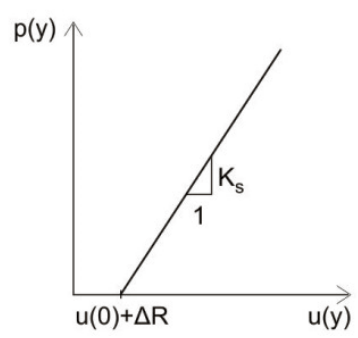

(c)

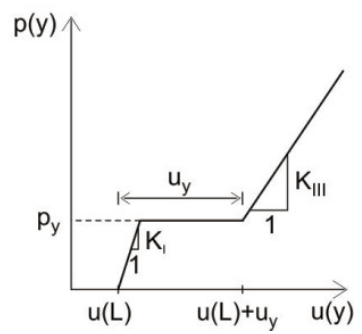

Figure 15.6. (a) Problem layout and boundary conditions at the tunnel wall for the simulation, (b), of the shield and, (c), of the yielding lining.

Modelling details can be found in Ramoni and Anagnostou (2010b, 2011a). The numerical calculations were carried out with the finite element program HYDMEC of ETH Zurich, using the so-called "steady state method" (Anagnostou 2007).

\subsubsection{Computational example}

The system behaviour and the importance of the yield pressure will be illustrated by means of a computational example (Fig. 15.7). Two linings are considered, which can accommodate a radial displacement of $5 \mathrm{~cm}$ either without resistance $\left(p_{y}=0\right)$ or at a yield pressure of $1 \mathrm{MPa}$. The parameters are given in Figure 15.7a. The three diagrams show - for the two yield pressures $p_{y}$ - the radial displacement $u$ of the ground at the tunnel boundary (Fig. 15.7a); the convergence $u-u(0)$ of the bored profile, i.e., the total radial displacement $u$ of the rock minus the pre-deformation $u(0)$ that occurs ahead of the tunnel face (Fig. 15.7b); and the distribution of the rock pressure $p$ acting on the shield and the lining (Fig. 15.7c).

After the deformations have used up the overcut (of $\Delta R=5 \mathrm{~cm}$, Fig. 15.7a), the rock starts to load the shield (Fig. 15.7c). The shield deforms elastically under the rock pressure (Fig. 15.6b). As the lining is installed and backfilled directly behind the shield, it interacts immediately with the rock, begins to yield after a small deformation (due to its high initial stiffness; $K_{l}=400 \mathrm{MPa} / \mathrm{m}$ ) and reaches its yield pressure $p_{y}$ almost instantly (at $y \approx L$; Fig. 15.7c). Only after the yield deformation $\left(u_{y}=5 \mathrm{~cm}\right)$ has been used up does the pressure increase again and reach its final value $p_{\infty}$.

A high yield pressure results in a lower final pressure (Fig. 15.7c; cf. Cantieni and Anagnostou 2009) and, due to longitudinal arching around the shield, in lower shield loads and thus a lower thrust force requirement (Fig. 15.7c; cf. Ramoni and Anagnostou 2011a). This effect is relatively small in the present example, but can be significant under other conditions. 
(a)

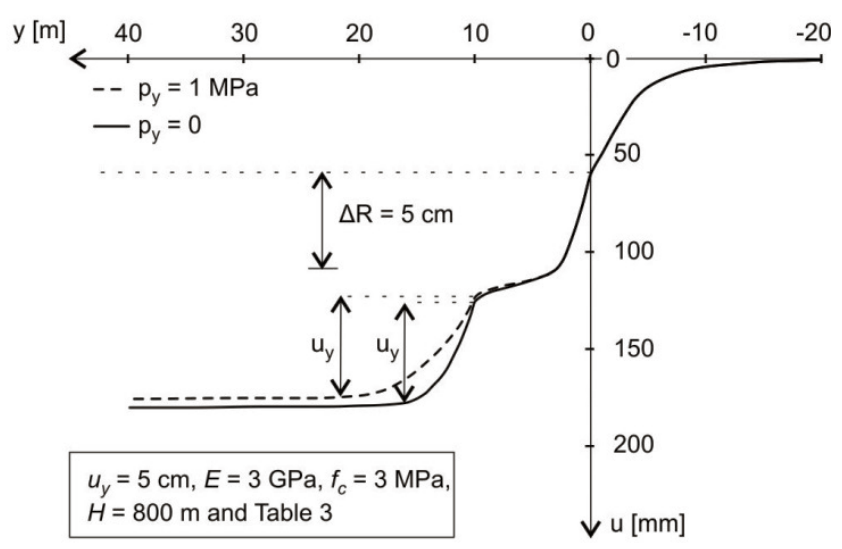

(b)

(c)

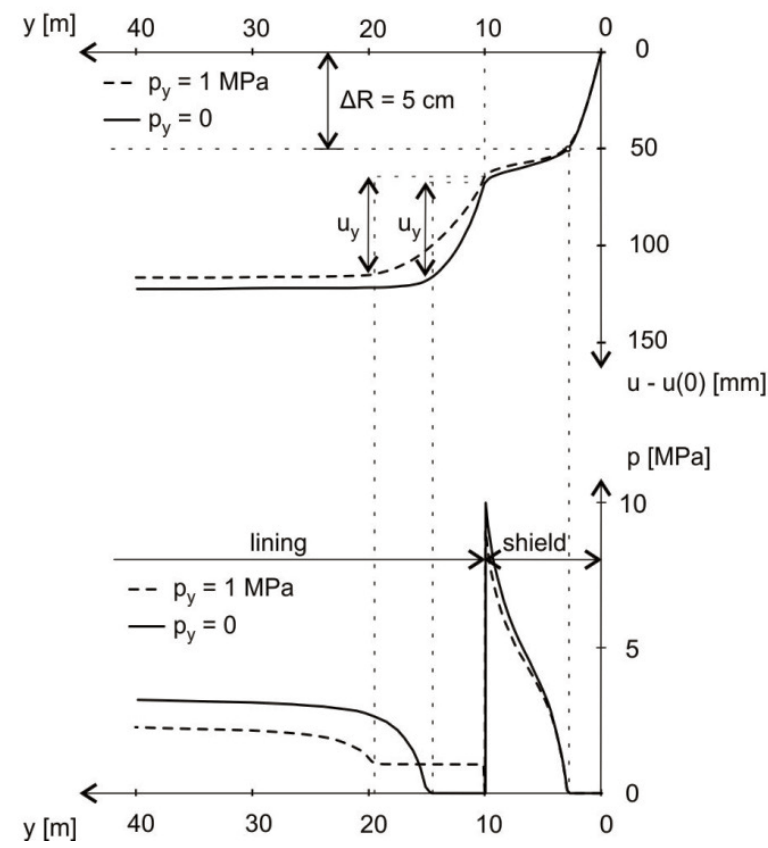

Figure 15.7. Interaction between the advancing shield, the ground and the deformable segmental lining $\left(u_{y}=5 \mathrm{~cm}\right)$ for a specific geotechnical situation $\left(f_{c}=3 \mathrm{MPa}, E=3 \mathrm{GPa}, H=800 \mathrm{~m}\right)$ : (a) radial displacement $u$ of the ground, (b), radial displacement $u-u(0)$ of the bored profile, (c), rock pressure acting on the shield and the lining.

\subsubsection{Design diagrams}

Based on a suite of such numerical simulations, design diagrams were worked out (Fig. 15.8, upper diagrams) which allow a quick estimation to be made of the rock pressure for the typical case of a single-lane traffic tunnel with a boring radius of $5 \mathrm{~m}$. Table 15.3 summarises the rock parameters, depths of cover, TBM data and yielding lining parameters considered. The initial stiffness assumed for the lining is typical for a segmental lining incorporating hiDCon elements in the longitudinal joints. The yielding pressure was taken equal to 0 or $1 \mathrm{MPa}$. In order to cover a wide range of deformable linings and to be on the safe side, a high value was chosen for the lining stiffness $K_{\text {III }}$ after the deformation phase (see Table 15.3; cf. Ramoni et al. 2011a). As a yielding segmental lining permits only limited radial displacements, the yielding deformation was taken equal to 5 or $20 \mathrm{~cm}$ (cf. Section 15.2.1). As a simplification, the boring radius was kept constant at $5 \mathrm{~m}$ for all computations, although a bigger deformation margin implies a bigger boring radius for a given tunnel internal radius. 
This simplification is sufficiently accurate for $R=5 m \pm 5 \%$, which corresponds to the usual range of the boring radius for the deformable lining systems under consideration.

The diagrams of Figure 15.8 can be used for radially deformable linings made of composite segments as well as for tangentially deformable linings, because the characteristic line of Figure $15.6 \mathrm{c}$ applies to both types. This is not the case for radially deformable lining systems with a compressible backfill, because the actual thickness ( $s$ in Fig. 15.4) and thus also the stiffness of the backfill also depend on the rock deformation, thus necessitating an iterative computational procedure (Ramoni et al. 2011a).

Table 15.3. Assumed parameter values.

\begin{tabular}{lc}
\hline TBM and lining & \\
\hline Boring radius $R$ & $500 \mathrm{~cm}$ \\
Annular gap $\Delta R_{I}$ & $15 \mathrm{~cm}$ \\
Number of cutters ${ }^{(1)}$ & 67 \\
Stiffness of lining $K_{l}{ }^{(2)}$ & $400 \mathrm{MPa} / \mathrm{m}$ \\
Stiffness of lining $K_{\text {III }}{ }^{(3)}$ & $800 \mathrm{MPa} / \mathrm{m}$ \\
Yield pressure $p_{y}$ & $0 ; 1 \mathrm{MPa}($ variable) \\
Yield deformation $u_{y}$ & $5 ; 20 \mathrm{~cm}($ variable) \\
Shield length $L$ & $10 \mathrm{~m}$ \\
Radial overcut $\Delta R^{(4)}$ & $5 \mathrm{~cm}$ \\
Shield thickness $d_{s}$ & $7.5 \mathrm{~cm}$ \\
Young's modulus of the shield $E_{s}$ & $210 \mathrm{GPa}$ \\
Shield stiffness $K_{s}{ }^{(5)}$ & $630 \mathrm{MPa} / \mathrm{m}$ \\
Sliding friction coefficient $\mu^{(6)}$ & 0.10 \\
Static friction coefficient $\mu^{(6)}$ & 0.15 \\
Boring force per cutter ${ }^{(7)}$ & $267 \mathrm{kN}$ \\
\hline Geotechnical conditions & \\
\hline Young's modulus $E$ & $500 \mathrm{f}_{\mathrm{c}}, 700 \mathrm{\textrm {f } _ { \mathrm { c } }}$ or $1000 \mathrm{f}_{\mathrm{c}}$ \\
Poisson's ratio $v$ & 0.25 \\
Uniaxial compressive strength $f_{c}$ & 1,3 or $5 \mathrm{MPa}$ \\
Angle of internal friction $\varphi$ & $25^{\circ}$ \\
Dilatancy angle $\psi$ & $5^{\circ}$ \\
Unit weight $Y$ & $25 \mathrm{kN} / \mathrm{m}^{3}$ \\
Depth of cover $H$ & $200,500 \mathrm{or} 800 \mathrm{~m}$ \\
\hline
\end{tabular}

(1) $6.7 \times$ (boring diameter)/m (Vigl et al. 1999)

(2) typical for a segmental lining incorporating hiDCon elements

(3) $K_{I I I}=E_{c} d / R^{2}$ with $E_{c}=40 \mathrm{GPa}$ and $d=50 \mathrm{~cm}$

(4) Can be achieved by changing the position of the gauge cutters (Herrenknecht et al. 2010)

(5) $K_{s}=E_{s} d_{s} / R^{2}$ (Ramoni et al. $2011 \mathrm{~b}$ )

(6) With lubrication of shield skin (Ramoni and Anagnostou 2011b)

(7) Sänger (2006)

The rock pressure $p_{\infty}$ in Figure 15.8 can be used to estimate the necessary lining thickness. Specifically, assuming hydrostatic loading and a safety factor of 4 (which accounts in a simplified manner for load eccentricities or concentrated loads at the longitudinal joints; cf. Chapter 14), the lining thickness $d$ can be taken equal to $4 R p_{\infty} / \sigma_{d}$, where $\sigma_{d}$ denotes the uniaxial compressive strength of the concrete. The upper diagrams in Figure 15.8 thus make it possible to study (for given geological conditions) structurally equivalent lining alternatives characterised by different deformation capacities $u_{y}$, yield pressures $p_{y}$, segment thicknesses $d$ and concrete qualities $\sigma_{d}$.

Investigating the structural performance of a lining makes sense only for geological conditions under which the required thrust force is not prohibitively high. Conditions under 
which shield jamming may occur (even after all possible counter-measures are implemented, such as selecting a short shield, adequate overcut, lubrication of the shield, immediate grouting of the annular gap, auxiliary hydraulic jacks) do not need to be considered in the evaluation of the lining systems. Therefore, diagrams for estimating the thrust force required to overcome the shield skin friction $F_{f}$ are provided additionally in Figure 15.8 (lower diagrams). The required thrust force was determined from the numerically computed rock pressure distribution along the shield, assuming that the shield extrados is lubricated and considering the conditions both during boring (in this case thrust force must overcome sliding friction and the rock's resistance to boring) and during TBM restart after a standstill (when the thrust force must overcome static friction).

$\square \mathrm{f}_{\mathrm{c}}=1 \mathrm{MPa}$
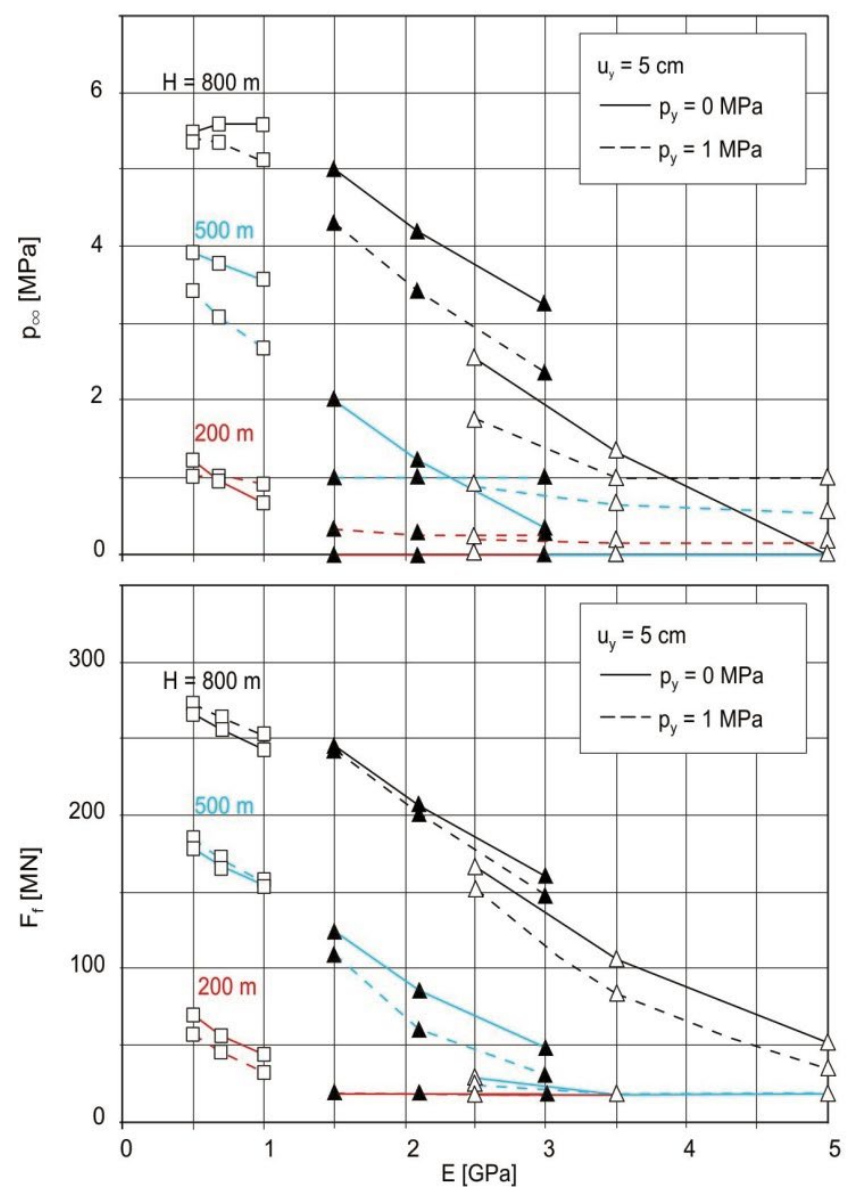

$\Delta \mathrm{f}_{\mathrm{c}}=3 \mathrm{MPa}$

$\triangle f_{c}=5 M P a$
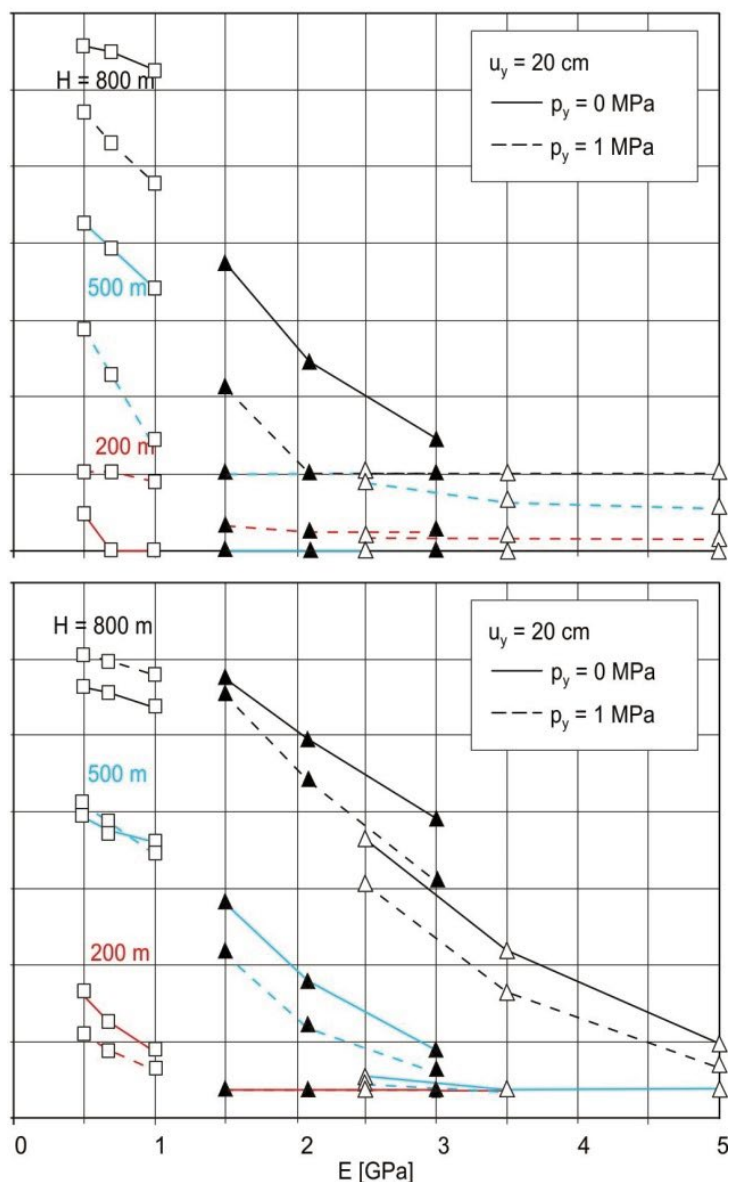

Figure 15.8. Diagrams for estimating the final lining pressure $p_{\infty}$ and the required thrust force $F_{f}$ (parameters according to Table 15.3).

As can be seen in the diagrams of Figure 15.8, a lower yield pressure $p_{y}$ generally results in a higher final rock pressure $p_{\infty}$. However, when the yield deformation $u_{y}$ is not used up, the final pressure is of course less than or equal to the chosen yield pressure (and does not decrease with it).

A larger yield deformation $u_{y}$ generally leads to a smaller final pressure $p_{\infty}$, but also to higher shield loading and higher thrust demand due to the reduced rock support behind the shield. In some cases, an increase in the yielding deformation $u_{y}$ from 5 to $20 \mathrm{~cm}$ does not lead to a further reduction in the final pressure, because the ground deformations that develop behind the shield are smaller than $5 \mathrm{~cm}$ and therefore the final rock pressure is the same (less than or equal to the yield pressure) both for a yielding deformation of $5 \mathrm{~cm}$ and for a yielding 
deformation of $20 \mathrm{~cm}$. As soon as the yield deformation has been used up, the pressure increases significantly (the stiffness of the lining $K_{I I I}$ is high). Therefore, a small increase in the overburden can lead to a considerably higher final pressure.

In a few (mostly non-critical) geological conditions, an increase in the uniaxial compressive strength or in the yield pressure results in higher final pressure $p_{\infty}$ and required thrust force $F_{f}$ (Fig. 15.8), while an increase in the yield deformation may lead to an increase in the final pressure. A discussion of this paradox can be found in Cantieni and Anagnostou (2011), Ramoni and Anagnostou (2010b) and Ramoni and Anagnostou (2011a).

\subsection{Structural comparison: resistance vs. yielding principle}

In the following, the conditions under which deformable linings offer structural advantages over stiff linings will be investigated.

Figure 15.9 compares a $50 \mathrm{~cm}$ thick, stiff segmental lining with a deformable lining (yielding at $p_{y}=1 \mathrm{MPa}$ and able to accommodate a radial displacement $u_{y}=20 \mathrm{~cm}$, which is the maximum feasible yield deformation) for various rock parameters and an overburden of $200 \mathrm{~m}$ or $800 \mathrm{~m}$. The lines for the stiff lining (resistance principle) were taken from Figure 14.8 (Chapter 14), while those for the yielding lining are based on Figure 15.8 of the present chapter. Both Figures consider the same TBM- and geotechnical parameters (cf. Table 15.3).

As expected, the yielding lining systems result in smaller lining loads, but are rather unfavourable with respect to shield loading. As the longitudinal arching action is reduced, the thrust force required to overcome the skin friction and keep the machine advancing is higher than in the case of a stiff lining. At the same time, the resistance of a yielding lining to the axial thrust force is probably lower, because its deformability may result in load eccentricity. The problem of shield jamming is therefore more critical (Ramoni and Anagnostou 2010a).

Consider first a deep tunnel $(H=800 \mathrm{~m})$. If the quality of the rock is poor (e.g., $f_{c}=1 \mathrm{MPa}$, $E=0.5 \mathrm{GPa}$ ), then a very high rock pressure of about $6 \mathrm{MPa}$ develops far behind the TBM, even if deformations are allowed to occur (points $A$ and $A^{\prime}$ in Fig. 15.9a). In addition, a very high thrust force would be required to overcome shield skin friction (points $A$ and $A^{\prime}$ in Fig. 15.9b). Such geotechnical conditions would be beyond the feasibility limits of both lining systems. In a better quality rock (e.g., $f_{c}=5 \mathrm{MPa}, E=2.5-5 \mathrm{GPa}$ ), the rock pressure developing upon a stiff lining would still be high (2-3.5 MPa, points B and C in Fig. 15.9a). However, allowing a small convergence to occur would result in a significantly lower rock pressure of $1 \mathrm{MPa}$ (points $\mathrm{B}^{\prime}$ and $\mathrm{C}^{\prime}$ in Fig. 15.9a), thus making mechanised tunnelling feasible (in combination with a yielding segmental lining), provided that the lining can sustain the thrust force that is needed to overcome shield skin friction $\left(F_{f} \approx 150 \mathrm{MN}\right.$ for $f_{c}=5 \mathrm{MPa}$, $E=2.5 \mathrm{GPa}$, point $\mathrm{B}^{\prime}$ in Fig. 15.9b).

Under a smaller depth of cover $(H=200 \mathrm{~m})$, the rock pressure is manageable with a stiff lining, even if the rock quality is poor (point D in Fig. 15.9a; cf. Chapter 14). Installing a deformable lining would lead only to a small reduction in the rock pressure (point D' in Fig. 15.9a). This means, in combination with the potential problems of a yielding lining system (clearance profile deformation, serviceability and higher thrust force), that the resistance principle is more advantageous in the case of small depths of cover. 
In conclusion, a lining based on the yielding principle appears to be appropriate specifically for deep tunnels through rock of relatively high stiffness and strength (cf. Ramoni et al. 2011a and Mezger et al. 2015a).

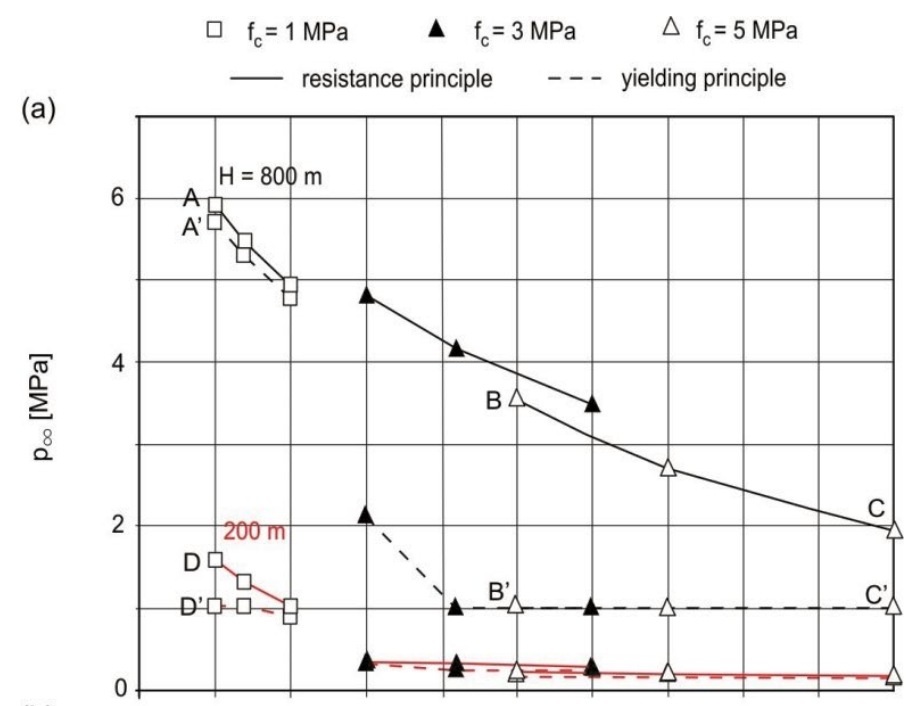

(b)

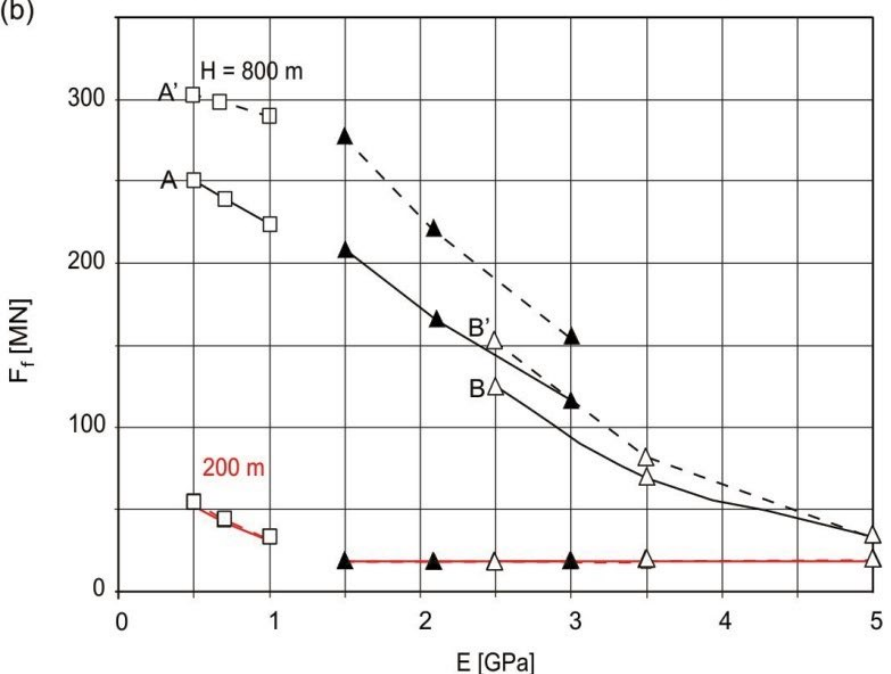

Figure 15.9. Comparison between the required thrust force $F_{f}$ and the final rock pressure $p_{\infty}$ acting on the lining for the yielding (yield deformation: $u_{y}=20 \mathrm{~cm}$, yield pressure: $p_{y}=1 \mathrm{MPa}$ ) and stiff lining systems (thickness of the lining $d=50 \mathrm{~cm}$, see Chapter 14).

A more detailed explanation will be provided below of the statement that, in slightly or moderately squeezing rocks under a great depth of cover, deformable linings allow for a more effective use of the available bored profile. More specifically, the following question will be addressed: Given the boring diameter, how can the space available for the lining be best used? Is it better to install a thicker lining (resistance principle) or to install a thinner deformable segmental lining and use the remaining space for accommodating deformations (yielding principle)?

Table 15.4 shows the safety factor SF of various segmental linings for a series of geotechnical conditions, characterised by different overburdens, rock stiffnesses and rock strengths (rows 2 to 4). For each geotechnical condition, the convergence $u_{a} / R$ of an unsupported opening is also given as a rough indicator of the squeezing severity (row 5 ).

Besides a boring radius of $5.05 \mathrm{~m}$, which (for the clearance profile radius of $4.35 \mathrm{~m}$ and a $0.15 \mathrm{~m}$ thick annular gap) leaves a space of $55 \mathrm{~cm}$ for lining and convergence (upper half of 
Table 15.4), the option of increasing the boring radius to $5.2 \mathrm{~m}$ is also considered (lower half of Table 15.4); such an increase in boring radius would offer more space for accommodating deformations or for installing a thicker lining. Both tangentially and radially deformable linings are considered. The latter, however, will only be considered for the bigger boring diameter, where a $20 \mathrm{~cm}$ outer layer, compressible by $25 \%$, would allow a convergence of $5 \mathrm{~cm}$ to occur (in the case of the smaller boring diameter, the deformation capacity of a radially deformable lining would amount to $1-2 \mathrm{~cm}$ only, which is practically negligible; see Section 15.2.1). Finally, besides a normal concrete quality (C50), the option of using HPC (C90) is considered for the lining based on the resistance principle as well as for the radially deformable lining (see Section 15.2.5).

Table 15.4. Safety factor SF for given boring radius.

\begin{tabular}{|c|c|c|c|c|c|c|c|c|c|c|c|c|c|}
\hline \multicolumn{2}{|c|}{ Geotechnical situation } & A & B & C & $\mathrm{D}$ & $E$ & $\mathrm{~F}$ & G & $\mathrm{H}$ & 1 & $\mathrm{~J}$ & $\mathrm{~K}$ & L \\
\hline \multicolumn{2}{|c|}{$H[\mathrm{~m}]$} & \multicolumn{3}{|c|}{200} & \multicolumn{4}{|c|}{500} & \multicolumn{5}{|c|}{800} \\
\hline \multicolumn{2}{|l|}{$E$ [GPa] } & 0.5 & 0.7 & 1 & 1 & 1.5 & 2.1 & 2.5 & 1.5 & 3 & 2.5 & 3.5 & 5 \\
\hline \multicolumn{2}{|l|}{$f_{c}[\mathrm{MPa}]$} & 1 & 1 & 1 & 1 & 3 & 3 & 5 & 3 & 3 & 5 & 5 & 5 \\
\hline \multicolumn{2}{|c|}{ Convergence $u_{a} / R[\%]$} & 8.2 & 5.8 & 4.1 & 34.8 & 5.4 & 3.9 & 1.8 & 15.8 & 7.9 & 5.0 & 3.5 & 2.5 \\
\hline \multicolumn{14}{|c|}{ Available space $=55 \mathrm{~cm}$ (boring radius $=505 \mathrm{~cm})^{(1)}$} \\
\hline $\begin{array}{l}\text { Yielding } \\
\text { principle }\end{array}$ & $\begin{array}{l}\text { tangentially deformable: } \\
50 \mathrm{~cm} \text { thick segments } \mathrm{C} 50 \\
u_{y}=5 \mathrm{~cm}, p_{y}=1 \mathrm{MPa}\end{array}$ & 1.22 & 1.22 & 1.37 & $(0.46)$ & 1.22 & 1.22 & 1.41 & $(0.28)$ & 0.52 & $(0.69)$ & 1.22 & 1.22 \\
\hline \multirow{2}{*}{$\begin{array}{l}\text { Resistance } \\
\text { principle }\end{array}$} & $55 \mathrm{~cm}$ thick segments $\mathrm{C} 50$ & 0.81 & 0.97 & 1.24 & 0.40 & 0.47 & 0.60 & 1.11 & 0.27 & 0.37 & 0.36 & 0.46 & 0.64 \\
\hline & $55 \mathrm{~cm}$ thick segments $\mathrm{C} 90$ & 1.43 & 1.70 & 2.14 & 0.71 & 0.81 & 1.03 & 1.80 & 0.46 & 0.63 & 0.61 & 0.78 & 1.05 \\
\hline \multicolumn{14}{|c|}{ Available space $=70 \mathrm{~cm}$ (boring radius $=520 \mathrm{~cm})^{(1)}$} \\
\hline \multirow{3}{*}{$\begin{array}{l}\text { Yielding } \\
\text { principle }\end{array}$} & $\begin{array}{l}\text { tangentially deformable: } \\
50 \mathrm{~cm} \text { thick segments C50 } \\
u_{y}=20 \mathrm{~cm}, p_{y}=1 \mathrm{MPa}\end{array}$ & 1.22 & 1.22 & 1.37 & $(0.83)$ & 1.22 & 1.22 & 1.41 & $(0.57)$ & $(1.22)$ & $(1.22)$ & 1.22 & 1.22 \\
\hline & $\begin{array}{l}\text { radially deformable: } \\
50 \mathrm{~cm} \text { thick segments } \mathrm{C} 50^{(2)} \\
u_{y}=5 \mathrm{~cm}, p_{y}=1 \mathrm{MPa}\end{array}$ & 1.22 & 1.22 & 1.37 & $(0.46)$ & 1.22 & 1.22 & 1.41 & $(0.28)$ & 0.52 & $(0.69)$ & 1.22 & 1.22 \\
\hline & $\begin{array}{l}\text { radially deformable: } \\
50 \mathrm{~cm} \text { thick segments } \mathrm{C}^{(2)} \\
u_{y}=5 \mathrm{~cm}, p_{y}=1 \mathrm{MPa}\end{array}$ & 2.20 & 2.20 & 2.46 & $(0.82)$ & 2.20 & 2.20 & 2.54 & $(0.51)$ & 0.93 & $(1.24)$ & 2.20 & 2.20 \\
\hline \multirow{2}{*}{$\begin{array}{l}\text { Resistance } \\
\text { principle }\end{array}$} & $70 \mathrm{~cm}$ thick segments $\mathrm{C} 50$ & 0.96 & 1.14 & 1.43 & 0.48 & 0.54 & 0.69 & 1.20 & 0.31 & 0.42 & 0.41 & 0.52 & 0.70 \\
\hline & $70 \mathrm{~cm}$ thick segments $\mathrm{C} 90$ & 1.68 & 1.97 & 2.45 & 0.83 & 0.93 & 1.16 & 1.93 & 0.53 & 0.71 & 0.69 & 0.87 & 1.14 \\
\hline
\end{tabular}

(1) Radius of the clearance profile $=435 \mathrm{~cm}$; width of the annular gap $=15 \mathrm{~cm}$.

(2) Incorporating at their extrados an additional $20 \mathrm{~cm}$ thick layer that can be compressed by $25 \%$.

The safety factor is defined as the ratio of lining resistance (determined after Chapter 14) to actual rock pressure. The actual rock pressure of the stiff lining systems was determined after Chapter 14, considering the lining thicknesses $(50-70 \mathrm{~cm})$ and Young's moduli $\left(E_{\mathrm{C} 50}=37 \mathrm{GPa}, \mathrm{E}_{\mathrm{C} 90}=44 \mathrm{GPa}, \mathrm{E}_{\mathrm{C} 120}=50 \mathrm{GPa}\right)$. The actual rock pressure of the deformable lining systems was determined based on Figure 15.8 (for $u_{y}=5 \mathrm{~cm}, p_{y}=1 \mathrm{MPa}$ : I.h.s. diagram; for $u_{y}=20 \mathrm{~cm}, p_{y}=1 \mathrm{MPa}$ : r.h.s. diagram).

Values within brackets in Table 15.4 concern situations in which the thrust required to overcome skin friction is higher than a threshold value. The latter is taken equal to $250 \mathrm{MN}$ for the stiff linings (which is technologically feasible for usual traffic tunnel diameters; Ramoni and Anagnostou 2010a), while a lower value (150 MN) is considered for yielding segmental linings. The latter are tentatively more vulnerable to eccentric loading: the movability of a segmental ring with compressible elements in the longitudinal joints or the soft lining embedment in the case of compressible annulus grouts imposes limits on the thrust force which can be applied on the segments. Situations with a safety factor of less than 1 or with problematic thrust force requirements are shaded grey in Table 15.4. 
The results of Table 15.4 allow the following conclusions to be drawn:

If the overburden is moderate and the rock quality not very poor (e.g. situations $C$ and $G$ ), then the safety factor is high for all lining types and thus the standard solution with a (stiff) segmental lining could be applied. However, if the rock quality is poor (e.g. situation A), structural safety can be achieved only with a stiff lining made of C90 or with a yielding lining; both systems have advantages and disadvantages (see Section 15.2 and Chapter 14).

In the case of a great depth of cover and poor quality rock, neither a stiff lining nor a yielding lining would suffice for structural safety (e.g. situation $\mathrm{H}$ ). In better quality rock, however, the yielding lining would meet the safety requirements to an acceptable level, whereas the stiff lining would be unfeasible even if using HPC (e.g. situation K). HPC would be a viable option only in better quality rocks exhibiting slight or moderate squeezing (e.g. situation $L$ ).

It is evident that an increase in the thickness of the stiff lining from 55 to $70 \mathrm{~cm}$ (using the same concrete type) is generally of little benefit, as it results in a higher lining stiffness and thus also in a higher rock pressure. It helps only if the structural safety is just below the acceptable level, especially in the case of a small depth of cover (e.g. situation B). The solution based on the resistance principle (in particular with C50) appears to be more appropriate in this case, as already discussed above.

For the tangentially deformable lining, the increase in the yield deformation $u_{y}$ from $5 \mathrm{~cm}$ to $20 \mathrm{~cm}$ does not offer advantages under the considered rock conditions; it may result (depending on rock quality and overburden; Section 15.3.3) in a higher structural safety of the lining, but on the other hand it increases the required thrust force and thus the risk of shield jamming (e.g. situation I).

The radially deformable lining made of C90 combines the advantage of higher bearing capacity with a reduction in the actual rock pressure (as it allows yield deformations of $5 \mathrm{~cm}$ to occur), thus reaching high safety factors (see, e.g., situation K). Therefore, this lining system could make shield tunnelling feasible in deep tunnels crossing relatively good rocks that exhibit slight or moderate squeezing. The risk of shield jamming is less critical for the radially deformable lining with $u_{y}=5 \mathrm{~cm}$ than for the tangentially deformable lining with $u_{y}$ of $20 \mathrm{~cm}$, as the thrust force required to overcome shield skin friction increases with $u_{y}$ (see, e.g., situation I and Fig. 15.8).

For a given concrete quality of the lining, the deformable linings reach generally higher safety factors than the stiff linings. Thus, a deformable lining allows for a more effective use of the available bored profile than a thicker stiff lining of the same concrete quality. Yielding linings appear to be appropriate particularly for tunnels through slightly to moderately squeezing rocks under a great overburden, where stiff linings reach their application limits. Thus, in this case, installing a thinner deformable segmental lining and using the remaining space for accommodating deformation is a better option. A thicker lining according to the resistance principle makes sense particularly in the case of small depths of cover.

Finally, it should be noted that the convergence $u_{a} / R$ of an unsupported opening alone is not decisive for the choice of lining system. For example, situations $C$ and $F$ (which exhibit nearly the same convergence of $4 \%$ ) result in significantly different safety factors, especially in the case of stiff linings, while situation $E$ results in lower safety factors than situation $A$, although the latter is characterised by a higher unsupported convergence ( $8.2 \%$ vs. $5.4 \%)$. 


\subsection{Cost comparison: resistance vs. yielding principle}

The last section showed that application of the yielding principle is meaningful for deep tunnels crossing slightly to moderately squeezing rock. In this section, the situations in which deformable linings are more economic than stiff linings are investigated. This will be shown by comparing stiff linings (made either of normal-strength concrete or of high performance or ultra-high performance concrete) with tangentially deformable linings (made of normalstrength concrete and incorporating six, $18 \mathrm{~cm}$ high hiDCon-elements that can be compressed by about $30 \%$ under an axial stress of about $10 \mathrm{MPa}$ ) for a tunnel with internal radius of $R_{\text {int }}=4.35 \mathrm{~m}$. The deformable linings considered allow a yield deformation $u_{y}$ of about $5 \mathrm{~cm}(=18 \mathrm{~cm} \times 30 \% \times 6 / 2 \pi)$ under a rock pressure $p_{y}$ of about $1 \mathrm{MPa}$ (assuming that the lining thickness is equal to about one tenth of the tunnel radius).

First, the costs per linear metre of tunnel for these linings will be compared for specific geotechnical situations, disregarding the fact that tunnels usually cross both squeezing and non-squeezing stretches (Section 15.5.1). Subsequently, the lengths of the squeezing zones are considered (Section 15.5.2), which is indispensable for a more thorough cost assessment (Chapter 14); for instance, if the total length of the squeezing zones is small compared to the total tunnel length, the use of expensive (U)HPC segments could be more attractive, since an increase in the boring diameter for the whole tunnel could be avoided.

All of the geotechnical situations under consideration were chosen such that the thrust force is not a limiting factor (specifically, the required thrust force is less than $150 \mathrm{MN}$ in the case of the yielding principle and less than $250 \mathrm{MN}$ in the case of the resistance principle).

\subsubsection{Tunnel costs for a specific geotechnical situation}

The cost comparison is carried out for five geotechnical situations, which differ with respect to overburden $H$ and rock parameters $E$ and $f_{c}$ (Table 15.5). Again, the convergence $u_{a} / R$ of an unsupported opening is also given in Table 15.5 as a rough squeezing indicator.

The necessary thicknesses $d$ (upper half of Table 15.5) of the stiff linings were estimated after Chapter 14, while the thickness of the deformable lining is taken equal to $45 \mathrm{~cm}$ (Fig. $15.10 \mathrm{~b}$ ) for all five geotechnical situations. (According to Figure 15.8, for $p_{y}=1 \mathrm{MPa}$ and $u_{y}=5 \mathrm{~cm}$, the rock pressure $p_{\infty}$ developing upon the lining remains equal to the yield pressure of $1 \mathrm{MPa}$, which can be resisted by a $0.4 \mathrm{~m}$ thick segmental lining. The thickness of the lining is chosen to be slightly bigger, $0.45 \mathrm{~m}$, because its bearing capacity has to be higher than the yield pressure $p_{y}$.)

The cost per linear metre tunnel for each lining type $i$ and geotechnical situation $j$ can be calculated as follows:

$$
c_{i j}=\alpha \sum_{n} Q_{i j n} \cdot p_{n},
$$

where $Q_{i j n}$ and $p_{n}$ denote the quantities of excavation and materials used per linear metre and their unit prices, respectively, while the factor $\alpha$ considers site installations, unforeseen costs and the acquisition of the TBM (Chapter 14). For simplicity, this factor is assumed to be the same for all technical solutions, which basically implies that a more costly lining generally requires a more costly installation and TBM. The following quantities per linear metre were considered: excavation $\left(\mathrm{m}^{3} / \mathrm{m}^{\prime}\right)$, annulus grouting $\left(\mathrm{m}^{3} / \mathrm{m}^{\prime}\right)$, segmental ring $\left(\mathrm{m}^{3} / \mathrm{m}^{\prime}\right)$ as well as 
the hiDCon-elements for the deformable lining $\left(\mathrm{m}^{3} / \mathrm{m}^{\prime}\right)$. For instance, for the deformable lining (Fig. 15.10b), the quantities result in: $78.5 \mathrm{~m}^{3} / \mathrm{m}^{\prime}$ excavation, $4.6 \mathrm{~m}^{3} / \mathrm{m}^{\prime}$ annulus grouting, $12.6 \mathrm{~m} 3 / \mathrm{m}^{\prime}$ segmental ring (made of normal-strength concrete) and $0.5 \mathrm{~m}^{3} / \mathrm{m}^{\prime}$ hiDConelements ( 6 elements, $0.18 \mathrm{~m}$ high and $0.45 \mathrm{~m}$ wide). Table 15.5 shows the assumed unit prices $p_{n}$, normalised by the excavation unit price $p_{1}$. In order to compare the different linings more easily, the costs $c_{i j}$ were normalised by the cost $c_{0}$ per linear metre in the reference case of a tunnel without squeezing (i.e. a stiff lining consisting of $30 \mathrm{~cm}$-thick, normalstrength segments with a boring radius of $R=4.8 \mathrm{~m}$ and an annulus gap of $0.15 \mathrm{~m}$, see Fig. 15.10a and Chapter 14):

$$
\bar{c}_{i j}=\frac{c_{i j}}{c_{0}}
$$

where

$$
c_{0}=\alpha \sum_{n} Q_{0 n} \cdot p_{n} .
$$

Table 15.5. Required thickness $d$ of each lining system as well as normalised cost per linear metre tunnel $\bar{c}_{i j}$ for the different geotechnical situations $j$ (defined in terms of rock quality and depth of cover).

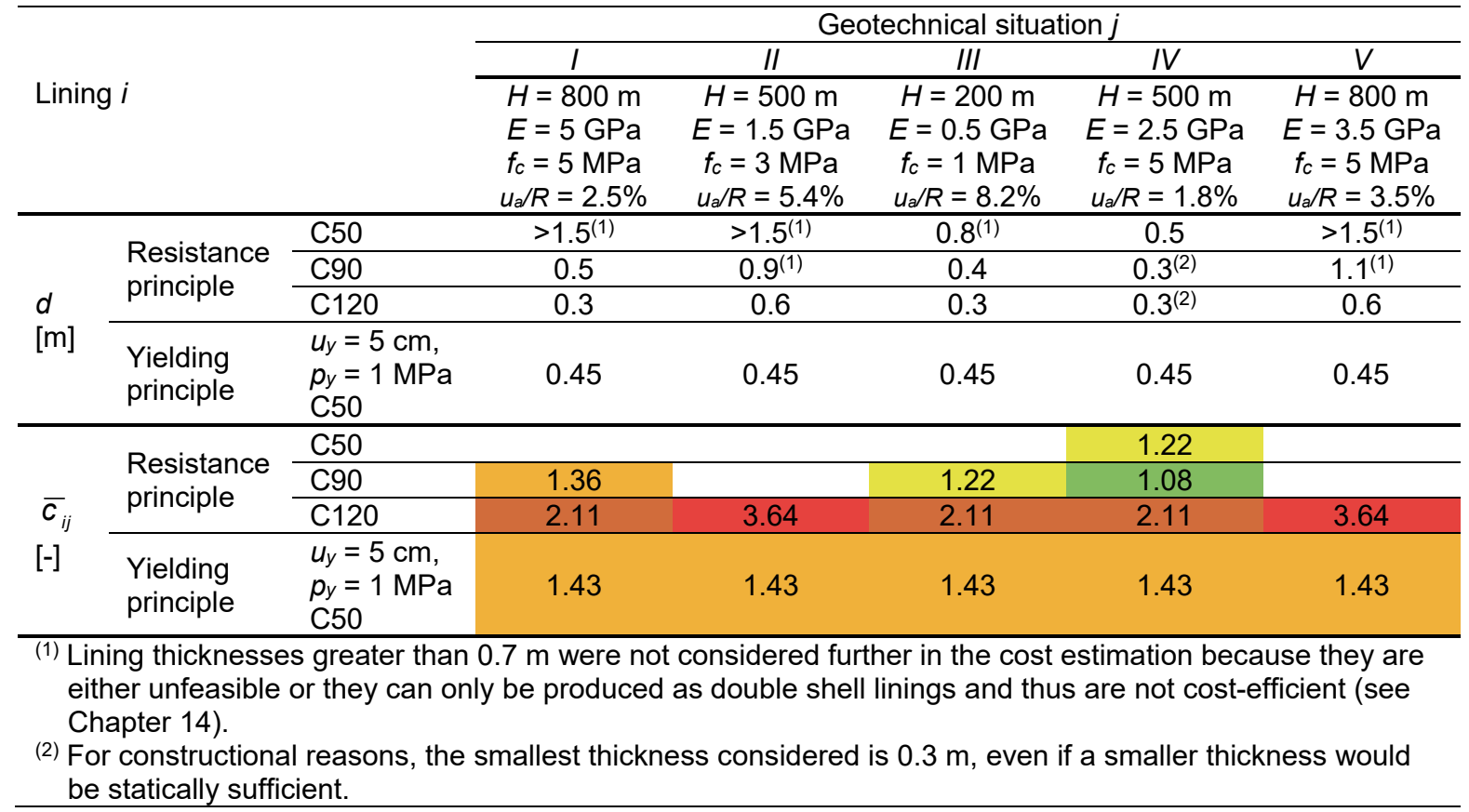

The normalised costs $\overline{c_{i j}}$ are given in the lower half of Table 15.5 (for each lining type $i$ and for each geotechnical situation $j$ ) and allow the following conclusions to be drawn:

- Stiff linings made of ultra-high performance concrete (C120) exhibit higher costs per tunnel metre; the savings in excavation costs (due to the reduction in the boring diameter) do not compensate for the high cost of the ultra-high performance concrete.

- A deformable lining is economically worthwhile for minor to moderate squeezing conditions under a high overburden; the costs are comparable to those of linings made of high performance concrete C90 (geotechnical situation I). For the geotechnical situation $\mathrm{V}$ in particular, in which the stiff lining systems reach their limits, the use of deformable linings appears to be meaningful and cost-effective. 
- For severe squeezing conditions under a small depth of cover (geotechnical situation III) or for minor squeezing under a medium overburden (geotechnical situations IV), stiff linings made of normal-strength concrete (C50) or high performance concrete (C90) are economically more worthwhile than deformable linings.

Table 15.6. Assumed unit prices $p_{n}$.

\begin{tabular}{llc}
\hline $\mathrm{n}$ & Position & Unit price $p_{n}\left[-/ \mathrm{m}^{3}\right]$ \\
\hline 1 & Excavation & 1.00 \\
\hline 2 & Annulus grouting & 2.35 \\
\hline 3 & $\begin{array}{l}\text { Normal-strength concrete segments }\left(\sigma_{d}=50 \mathrm{MPa},\right. \\
\text { reinforced })\end{array}$ & 2.90 \\
\hline 4 & HPC segments $\left(\sigma_{d}=65\right.$ or $90 \mathrm{MPa}$, reinforced $)$ & 3.90 \\
\hline 5 & UHPC segments $\left(\sigma_{d}=120\right.$ or $140 \mathrm{MPa}$, reinforced $)$ & 17.00 \\
\hline 6 & HiDCon-elements & 58.04 \\
\hline
\end{tabular}

(a)

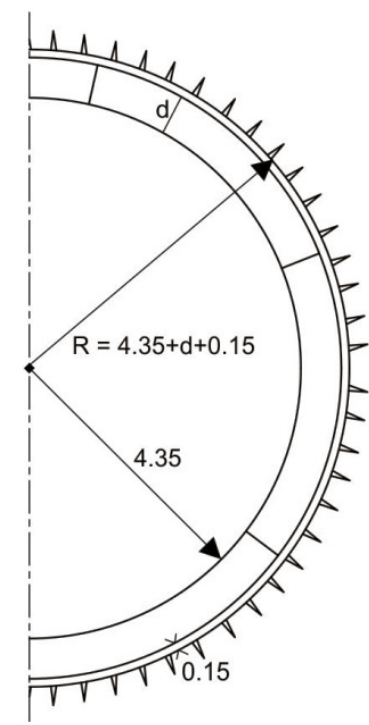

(b)

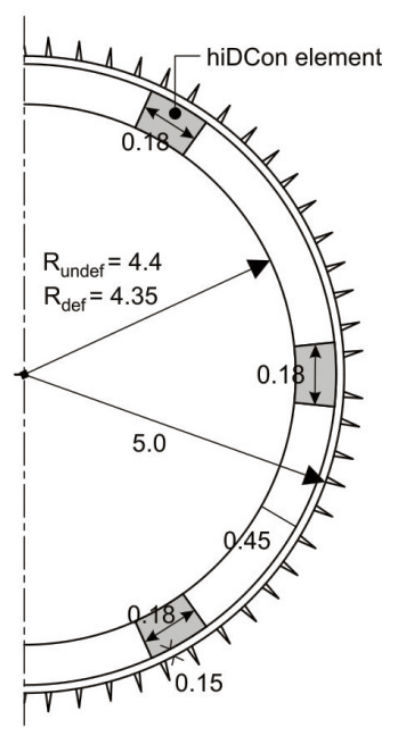

Figure 15.10. Possible lining systems for the geotechnical situations of Table 15.5 (all dimensions in [m]): (a) stiff lining systems (thickness $d$ according to Table 15.5), (b), yielding lining system.

These results agree with the results obtained from the structural analysis.

\subsubsection{Tunnel costs considering squeezing distribution}

As in Chapter 14, a fictitious tunnel with a minimum clearance radius of $4.35 \mathrm{~m}$ is considered, crossing non-squeezing ground (geotechnical situation 1), moderately squeezing ground ( $E=3 \mathrm{GPa}, f_{c}=3 \mathrm{MPa}, H=500 \mathrm{~m}$; geotechnical situation 2) and heavily squeezing ground $\left(E=2.6 \mathrm{GPa}, f_{c}=2 \mathrm{MPa}, H=500 \mathrm{~m}\right.$; geotechnical situation 3). Geotechnical situations 2 and 3 are characterised by an unsupported convergence of $2.7 \%$ and $5.2 \%$, respectively.

For geotechnical situation 1 (no squeezing) a $30 \mathrm{~cm}$ thick lining of normal-strength concrete is foreseen (see Fig. 15.11). In the tunnel stretches through geotechnical situations 2 and 3 , either a stiff or a deformable lining can be installed, and the deformable lining of Figure $15.10 \mathrm{~b}$ is sufficient for both situations (Fig. 15.8 shows that the final rock pressure acting on the lining amounts to about $1 \mathrm{MPa}$ and thus can be resisted by a $45-\mathrm{cm}$ thick lining, when 
allowing deformation of $5 \mathrm{~cm}$ to occur). In order to maintain the minimum clearance profile, the boring radius amounts to $5 \mathrm{~m}$ for the entire tunnel length.

The construction costs for a tunnel with a deformable lining according to Figure $15.10 \mathrm{~b}$ in the squeezing zones is compared to the economically most worthwhile stiff lining solutions according to Chapter 14, which are sketched out in Figure 15.11 and differ with respect to the lining in the three geotechnical situations $j$. The normalised costs $\bar{c}_{i j}$ according to Equation (15.2) are given in Figure 15.11 for each lining solution $i$ and for each geotechnical situation $j$.

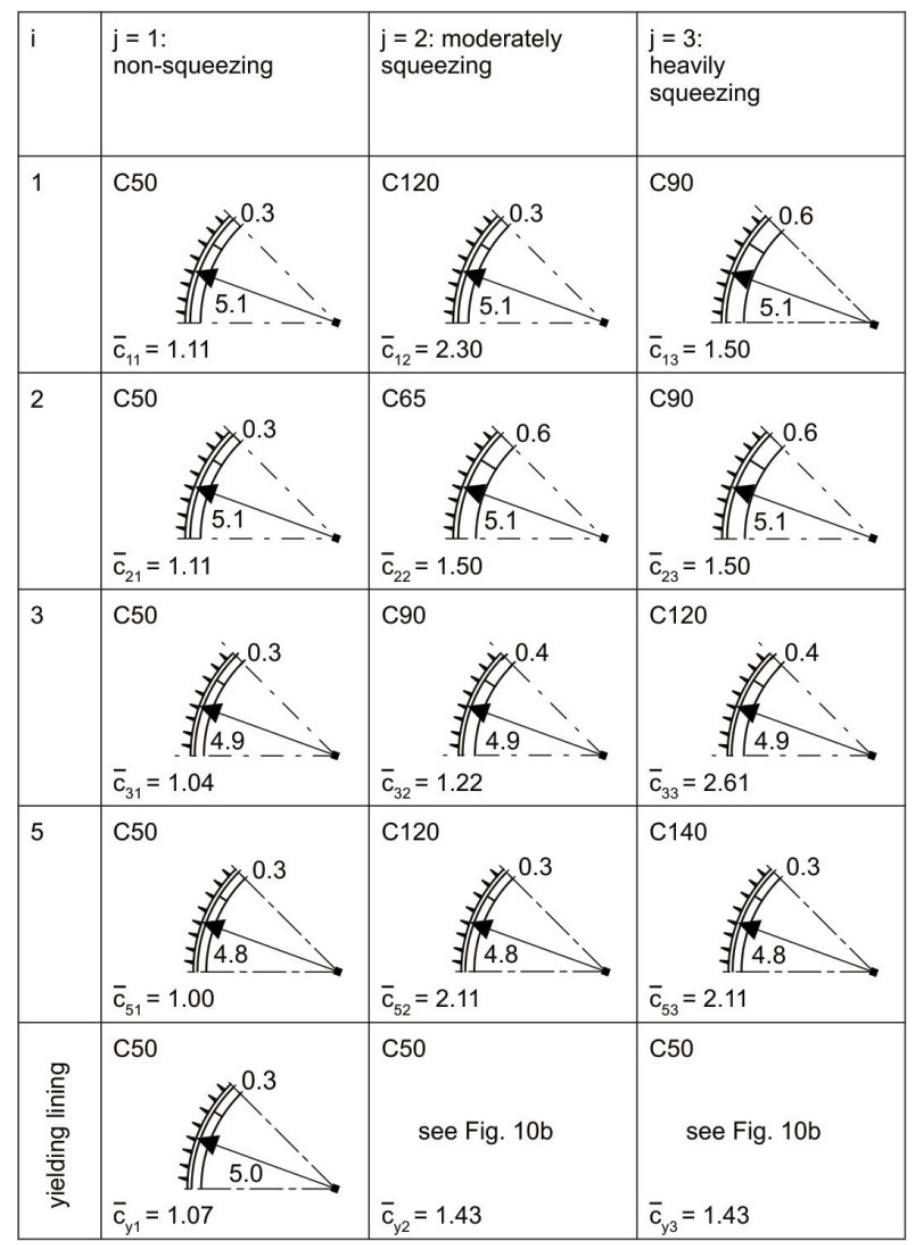

Figure 15.11. Lining alternatives $i$ for the geotechnical situations $j$ (after Chapter 14).

The normalised average tunnel cost per linear metre for each alternative lining solution $i$ reads as follows (chapter 14 ):

$$
\bar{C}_{i}=\sum_{j} \bar{C}_{i j} \chi_{j}
$$

where $X_{j}$ denotes the fraction of the tunnel stretches with geotechnical situation $j$. Figure 15.12 shows the average normalised tunnel cost $\bar{C}_{i}$ for each solution as a function of the total length $\left(X_{2}+X_{3}\right)$ of the squeezing zones assuming that the tunnel crosses, (a), only moderately squeezing ground (i.e., $\left.X_{3}=0\right)$, (b), moderately and heavily squeezing ground over equal lengths (i.e., $X_{2}=X_{3}$ ) and, (c), only heavily squeezing ground (i.e., $X_{2}=0$ ). 
The following conclusions can be drawn from Figure 15.12:

- the costs for deformable linings are comparable to those for high strength concrete (C90);

- the economically more worthwhile lining system is characterised by the smallest boring radius (deformable linings in the case of $X_{2}=X_{3}$ and $X_{2}=0$, and stiff lining systems made of C90 in the case of $X_{3}=0$ );

- if the total length of the squeezing zones $X_{2}+X_{3}$ is small compared to the tunnel length (less than 10\%), the use of UHPC is more attractive, since an increase in the boring diameter of the entire tunnel can be avoided (cf. chapter 14).

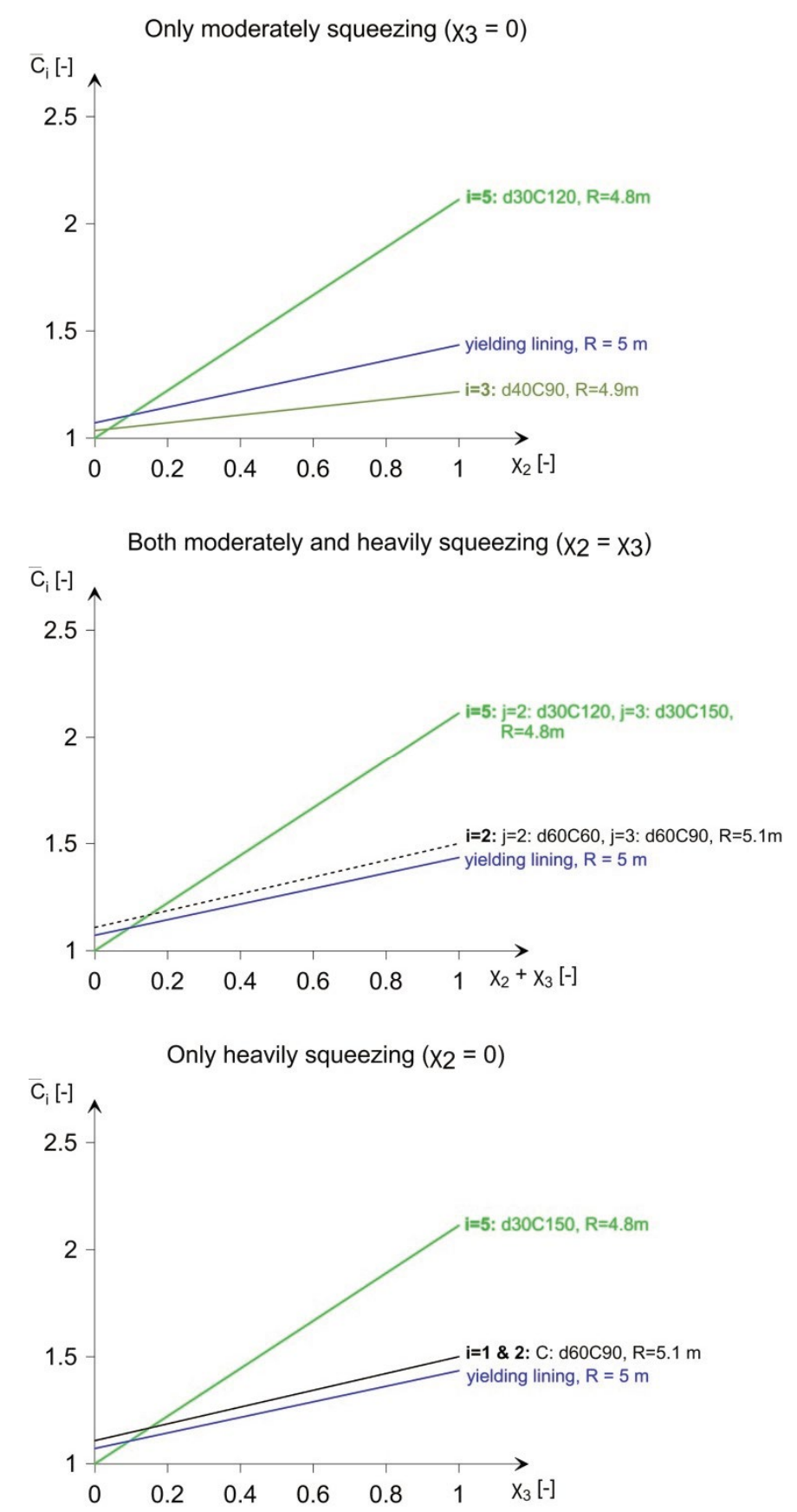

Figure 15.12. Normalised averaged tunnel cost per linear metre $\bar{C}_{i}$ as a function of the percentage of the squeezing zones $\chi_{j}$ for the lining alternatives of Figures $15.10 \mathrm{~b}$ and 15.11 (where $d$ denotes the thickness of the lining, $C$ the concrete strength and $R$ the boring radius). 


\subsection{Conclusions}

This chapter investigated how deformable segmental linings might be implemented and the conditions under which such linings are safer and more economical than stiff linings. Despite the complexity of the problem, it was possible to formulate some general guidelines for the choice of the tunnel support.

A deformable segmental lining must exhibit sufficient deformability at high initial stiffnesses and yield pressures. These requirements can best be met by tangentially deformable segmental linings incorporating hiDCon elements (or the very expensive Meypo elements) in the longitudinal joints. Radially deformable linings make less use of the bored diameter; they might be an option if they consist of composite segments made of high strength concrete incorporating a compressible outer layer.

Generally, a deformable lining allows for a more effective use of the available bored profile than a thicker stiff lining of the same concrete quality. Nevertheless, the serviceability requirements are more difficult to fulfil in the case of deformable segmental linings. This does not matter if an additional inner lining is installed.

For deep tunnels through slightly to moderately squeezing rock, yielding linings are more suitable than stiff linings, because rock pressure can be reduced significantly by allowing a small deformation to occur, while the loading of a stiff lining would be prohibitively high. The standard solution with a stiff lining made of normal strength concrete is more appropriate for moderate depths of cover or for minor squeezing conditions. The applicability range of stiff linings can be widened by using segments made of high strength concrete. The costs of such linings are comparable to those of deformable linings. For deep tunnels through heavily squeezing ground, neither deformable linings, nor stiff linings (even if made of HPC) seem to be feasible.

Besides overburden and rock quality, the percentage of the total tunnel length affected by squeezing must be considered for the choice of lining type because the shield diameter is constant over the whole tunnel. If the squeezing sections make up a small percentage of the total tunnel length, then the most economical solution will be the one that results in the smallest shield diameter, even if this presupposes the use of very expensive UHPC locally. If squeezing is expected over longer portions of the alignment, then the use of thicker segments may be more economical, although this will necessitate a bigger shield diameter.

\section{Notation}

C

$c_{i j}$

$c_{0}$

$\overline{c_{i j}}$

$\bar{C}_{i}$

$d$

$d_{s}$

E

$E_{c}$ type of concrete (defined by its uniaxial compressive strength $\sigma_{d}$ ) cost per linear metre of tunnel for lining solution $i$ in geotechnical situation $j$ reference cost per linear metre (tunnel without squeezing) cost per linear metre of tunnel for lining solution $i$ in geotechnical situation $j$ normalised by the reference cost $c_{0}$ normalised average tunnel cost per linear metre for solution $i$ thickness of the lining thickness of the shield Young's modulus of the rock Young's modulus of the lining 


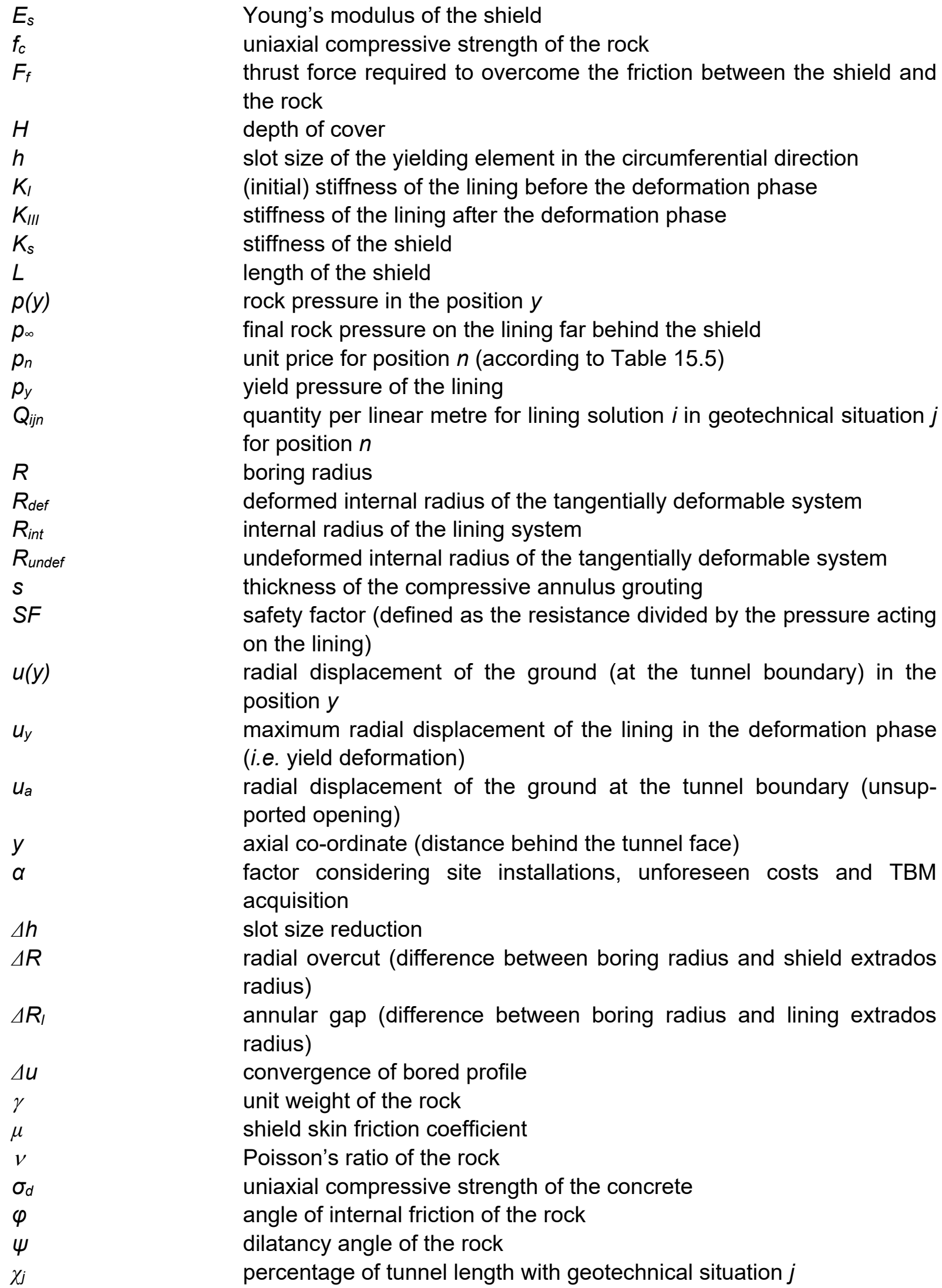


CONCLUSION AND PERSPECTIVES 



\section{Conclusion and perspectives}

The case histories of the Gotthard, Ceneri and Lötschberg Base Tunnel (Part I) could show that the squeezing deformations were mainly affected by the lithology, by the schistosity orientations and the effect of nearby weaker or stronger zones. Those variations - even if relatively small - may thus cause a significant variability of the squeezing intensities along the tunnel, which may additionally be accompanied by a pronounced non-uniformity of the displacements in the tunnel profile. Chapter 1 showed that the influence of the spatial orientation of the schistosity on the tunnel convergences can be determined by a simple, empirically proven (and later - in Chapters 12 and 13 - also theoretically justified) equation, which in combination with advance core drilling allows reliable predictions of the convergences. Therefore, the next chapters of this doctoral thesis determined the influence of the factors mentioned above, in order to use them as indicators during construction for the timely identification and prediction of the squeezing behaviour.

For a tunnel drive through heterogeneous rock mass consisting of alternating weak and competent rock layers lying perpendicular to the tunnel axis (Part II), depending on the heterogeneity scale, a pronounced squeezing variability may occur (cf. Chapter 7 ). If the alternating weak and competent rocks are very thin, the displacements are almost uniform along the tunnel. For this special case, an analytical solution was derived which describes the relationship between rock deformation and support pressure under the assumption of rotational symmetry and plane strain conditions (Chapter 5). The derivation of this ground response curve was mathematically demanding, as a variety of cases regarding the failure state of the rock had to be considered (plastic and/or elastic behaviour of the weak and/or the hard layers considering a plastic flow either only in the tunnel cross-sectional plane or also perpendicular to it). This analytical solution is particularly important for practical reasons, as numerical modelling of a narrow sequence of hard and weak rocks is very timeconsuming. Numerical calculations in Section 5.7, which consider the layers discretely, could show that the analytically derived solution mentioned before is sufficiently accurate for practical purposes if the thickness of the hard layers is less than about $5 \%$ of the tunnel radius. Based on the above-mentioned analytically derived ground response curve, the parameters of a mechanically equivalent homogeneous, isotropic and elasto-plastic material are determined and presented in Chapter 6 . This is very useful for design purposes since it allows the use of common calculation methods and programs to solve problems that do not meet the conditions of rotational symmetry or plane strain and this even for thinly stratified rocks. For example, with the determined equivalent parameters, one can easily determine the effectiveness of a lining system in TBM tunnelling (cf. Chapters 14 and 15). Of course, the homogenised model provides only satisfactory results for small thicknesses of the layers and not for thickly alternating weak and competent layers. Therefore, in Chapter 7 a simple equation was developed, which allows to determine the squeezing intensities for all layer 
thicknesses in a quick and easy way, without making numerical modelling necessary (at least not in the preliminary stages of design). By a comprehensive parametric study, it could be shown that this equation is accurate enough for practical purposes.

Part III investigated the influence of the planes of anisotropy (schistosity, bedding) lying parallel to the tunnel axis. When tunnelling through a heterogeneous rock mass consisting of alternating weak and competent rock layers lying parallel to the tunnel axis, depending on the heterogeneity scale, considerable non-uniformity of the distribution of the deformations of the tunnel profile may occur. Even if the thicknesses of the alternating weak and hard layers are very small, a considerable non-uniformity of the displacements in the tunnel profile may occur. For this specific case, the rock mass can be perceived as a homogeneous, but transversely isotropic material. For the latter, in Chapter 9, a constitutive model was formulated and implemented in Abaqus, which describes the behaviour of a stratified rock mass (using the homogenisation technique). The elasto-plastic parameters of this homogeneous and transversely isotropic medium depend on fraction, strength and stiffness properties of the alternating layers. With this, the ground response when tunnelling through thinly alternating weak and competent layers can be investigated computationally (assuming plane strain conditions). As the homogenised solution is particularly important for practical reasons (numerical modelling of a narrow sequence of hard and weak rocks is very timeconsuming), the estimation of the displacements along the tunnel profile for given geotechnical condition is facilitated by dimensionless diagrams - presented in Chapter 9 that represent a valuable tool for engineering practice, as they enable to determine the maximum and minimum displacements in the tunnel profile for a wide range of geotechnical conditions. Even if the response of the ground is anisotropic in this case, these design aids allow, analogously to Chapter 6, to determine the parameters for an isotropic homogeneous rock mass, which is equivalent to the isotropic rock mass in the sense that its tunnellinginduced displacements are equal either to the maximum or to the minimum displacements of the anisotropic model. As shown in Section 9.5, using this equivalent isotropic model makes it possible to find an upper and a lower bound of the displacements in more complex problems (that do not meet the condition of plane strain).

Numerical calculations in Chapter 10, which analyses the influence of the layer thickness by considering the individual layers discretely, could show that the homogenised model mentioned before is sufficiently accurate for practical purposes if the thickness of the hard layers is less than about $5 \%$ of the tunnel radius. As this criterion is also valid for a sequence of alternating layers lying perpendicular to the tunnel axis, this criterion is applicable for any orientation of the layers to the tunnel axis. Furthermore, Chapter 10 could show that, for very thick formations, the non-uniformity of displacements in the profile nearly disappears if the weak or the hard rock formation lies at a distance to the tunnel axis of at least 5 times the tunnel radius, so that, for design purpose, the weak or the hard formation can be neglected, respectively. Therefore, numerical calculations do not have to be performed (at least in the preliminary stages of design).

Finally, Chapter 11 investigated the squeezing behaviour in schistous rock mass - which is characterised by very thin weak discontinuities and thus represents, from the mechanical point of view, a special case of a stratified rock mass - when tunnelling parallel to the planes of schistosity. (The schistosity is irrelevant when tunnelling perpendicular to the planes of weakness.) As the implemented constitutive model for (thinly) stratified rocks is inefficient in computing terms for the borderline case of schistosity, in Chapter 11, a constitutive model was formulated and implemented specifically for schistous rock. With this, numerical calculations (assuming plane strain conditions) could be carried out in Chapter 11 that 
showed that the schistosity may affect rock deformations adversely, particularly if its cohesion and friction angle are small. If, in addition, the uniaxial compressive strength of the matrix is small, the squeezing deformations may be considerably larger than those occurring in non-schistous rocks. In order to better estimate the influence of the schistosity, dimensionless diagrams were developed and presented in Chapter 11, which make it possible to estimate the maximum and minimum displacements in the tunnel profile easily for given geotechnical conditions.

Part IV investigated the influence of the orientation of the planes of anisotropy (bedding, schistosity) to the tunnel on the tunnel convergences. As the deformations determined under the assumption of plane strain conditions (as in the Parts II and III) include the deformations that occur ahead of the tunnel face (so-called "pre-deformations") and are thus considerably larger than the convergences of the excavated tunnel profile, in Chapter 12, the influence of the planes of anisotropy on the convergences is investigated using spatial calculation models with arbitrary orientation of the planes of anisotropy relative to the tunnel axis. This chapter could show, first, that there are relevant differences to the well-known case of an isotropic rock mass and, second, that the numerical calculations reproduce well the empirically determined relationship between convergence and spatial orientation of the schistosity considering the so-called "schistosity influence factor" (which combines the dip $\omega_{s}$ and the strike direction $\theta_{s}$ of the planes of weakness to the tunnel axis), which was developed based on the data from the Gotthard Base Tunnel (cf. Chapter 1). Based on this relationship, a simple equation, which is accurate enough for most tunnelling problems, was developed which enable to calculate the convergence for all orientation of the planes of anisotropy to the tunnel axis, taking into account the schistosity influence factor. With this equation, for a tunnel drive encountering schistous or stratified rock masses, costly spatial numerical analyses do not have to be carried out (at least not in the preliminary design stages) for estimating the tunnel convergences.

In fact, the variation of the orientation of the planes of anisotropy along the tunnel axis is an important factor for the squeezing variability and this, when tunnelling through schistous or stratified rock mass. This is clearly recognisable in Chapter 13, which investigated the influence of a folding on the distribution of the deformations along the tunnel numerically. The folding was implemented as a sinus function in the constitutive model, which is defined by its amplitude and its period. The results of the numerical investigations indicate that, there is a mutual influence of the alternating areas of favourable and unfavourable inclination of the anisotropy planes along the tunnel. However, despite of this mutual influence, the folding leads to a considerable variability of the rock deformations along the tunnel. Furthermore, the Chapter 13 could show, (i), that the squeezing variability observed in the Sedrun Section of the Gotthard Base Tunnel can be well understood with the help of numerical calculations taking into account the orientation of the schistosity to the tunnel axis and, (ii), that the "schistosity influence factor" can be used as a reliable indicator for the estimation of the squeezing intensities during advance, in combination with advance core drilling.

In shielded TBM tunnelling, advance probing can identify the potentially critical zones ahead of the tunnel face and thus enables the timely implementation of planned measures. Depending on the design, decision-making during the TBM drive may have to address: the boring diameter (to provide more space for deformations); the lining type (switch from normal to higher strength segments or from a stiff to a deformable lining of the same thickness); or even the lining thickness. Part $V$ therefore investigated several conceivable high capacity lining systems as well as deformable lining systems for TBM tunnelling under squeezing conditions. Besides the obvious solution of thicker segments, lining resistance can be 
increased by using a double shell lining consisting of two segmental rings or by using segmental linings made of high or even ultra-high performance concretes. Linings made of normal-strength concrete are generally not competitive because they result in a larger boring radius over the entire tunnel and thus higher total excavation costs; the most cost-effective solutions involve high or even ultra-high performance linings in the squeezing zones. However, ultra-high performance linings are nevertheless very expensive and therefore economically viable only if the squeezing zone makes up a very small percentage of the total tunnel length. Furthermore, Part $V$ investigated how deformable segmental linings might be implemented and the conditions under which such linings are safer and more economical than stiff linings. In order to estimate the applicability of the different lining systems for a wide range of geotechnical conditions, design aids were presented. These design aids were developed based on numerical calculations that considered the interaction between the advancing shield, the ground and the lining and assumed homogeneous and isotropic ground. In order to analyse the applicability of lining systems in stratified or schistous rocks, the calculations can be performed with equivalent parameters (i.e., equivalent Young's modulus $E_{e q}$, Poisson's ratio $v_{e q}$, friction angle $\varphi_{e q}$, cohesion $c_{e q}$ and dilatancy angle $\psi_{\text {eq }}$ ) for an isotropic homogeneous rock mass, which can be determined with the method introduced in Chapters 6, 9 and 11. Generally, a deformable lining allows for a more effective use of the available bored profile than a thicker stiff lining of the same concrete quality. Particularly, for deep tunnels through slightly to moderately squeezing rock, yielding linings are more suitable than stiff linings, because rock pressure can be reduced significantly by allowing a small deformation to occur, while the loading of a stiff lining would be prohibitively high.

In this thesis, the time-dependence of the rock behaviour (due to creep or consolidation) was not considered.

Creep is especially important in overstressed ground and could therefore also be important for the squeezing variability. First investigations of Gimmel (2018) concerning the carbon sections of the Lötschberg Base Tunnel showed that considerable long-term deformations occur, which could be, inter alia, traced back to creep. It was noticeable that with larger (short-term) tunnelling-induced deformations (occurring within a distance of about $50 \mathrm{~m}$ behind the tunnel face), larger long-term deformations due to creep occurred. Particularly in tunnel sections with large anthracite contents (cf. Chapter 3 ), large creep convergences were measured during construction. Therefore, creep influences the squeezing intensity along the tunnel and thus should be subject of further research.

a)

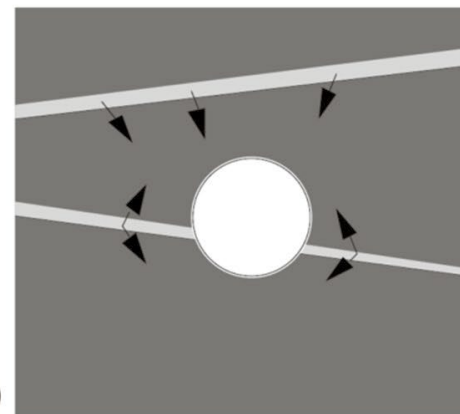

permeable

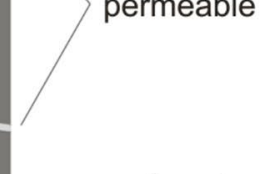

impermeable b)

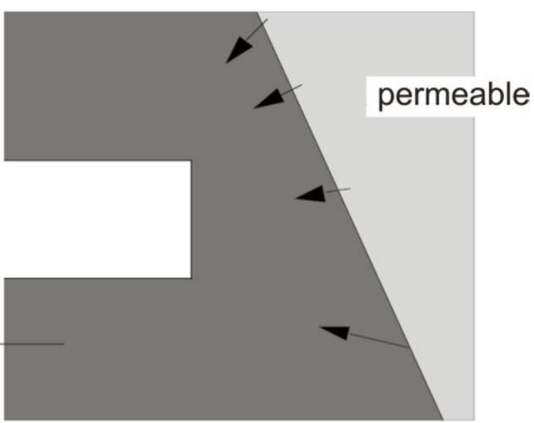

Figure 1. Shortening of the drainage paths due to, a) permeable layers, b) adjacent permeable formation (Anagnostou and Kovári 1999). 
Furthermore, the present thesis showed that the heterogeneities of the ground at different scales with respect to its mechanical characteristics play an important role for the squeezing variability. The existence of underground water or high pore water pressure favours the development of rock deformations (Kovári 1998, Vogelhuber 2007, Barla 2002, etc.). In a saturated rock the permeability governs the rate of the deformations associated with the dissipation of excess pore pressures. Permeability variations may therefore lead to variable squeezing intensities. In particular, thin permeable interlayers can cause a substantial acceleration of the deformations as they lead to a shortening of the drainage paths (cf. Fig. 1). This hypothesis was put forward by Anagnostou and Kovári (1999), but was not investigated quantitatively so far. Therefore, the influence of the heterogeneities of the ground with respect to its hydraulic characteristics on the squeezing variability should be subject of further research. 

APPENDIX 



\section{Appendix A: Tunnelling perpendicularly to a thinly stratified rock mass - Coefficients of the incremental stress strain relationships}

This appendix presents the coefficients of the incremental stress-strain relationships (Eq. 5.21), which depend on the failure state of the weak or the hard layers (cf. Tables 5.1 and 5.3). The list of symbols can be found on page 87 .

\section{A.1 Case $i$. Both the weak and the hard layers behave elastically}

$$
\begin{aligned}
& A_{1, i}=A_{4, i}=2 \cdot \mu^{*}+\lambda^{*}, \\
& A_{2, i}=-A_{3, i}=-\lambda^{*}, \\
& B_{1, i}=B_{2, i}=\frac{x_{w} \lambda_{w} \cdot\left(\lambda_{h}+2 \mu_{h}\right)+x_{h} \lambda_{h} \cdot\left(\lambda_{w}+2 \mu_{w}\right)}{x_{w}\left(\lambda_{h}+2 \mu_{h}\right)+x_{h}\left(\lambda_{w}+2 \mu_{w}\right)}, \\
& K_{1, i}=K_{4, i}=\lambda_{h}+2 \mu_{h}-\frac{\lambda_{h} x_{w}\left(\lambda_{h}-\lambda_{w}\right)}{x_{w}\left(\lambda_{h}+2 \mu_{h}\right)+x_{h}\left(\lambda_{w}+2 \mu_{w}\right)}, \\
& K_{2, i}=K_{3, i}=\lambda_{h}-\frac{\lambda_{h} x_{w}\left(\lambda_{h}-\lambda_{w}\right)}{x_{w}\left(\lambda_{h}+2 \mu_{h}\right)+x_{h}\left(\lambda_{w}+2 \mu_{w}\right)}, \\
& K_{5, i}=K_{8, i}=\lambda_{w}+2 \mu_{w}+\frac{\lambda_{w} x_{h}\left(\lambda_{h}-\lambda_{w}\right)}{x_{w}\left(\lambda_{h}+2 \mu_{h}\right)+x_{h}\left(\lambda_{w}+2 \mu_{w}\right)}, \\
& K_{6, i}=K_{7, i}=\lambda_{w}+\frac{\lambda_{w} x_{h}\left(\lambda_{h}-\lambda_{w}\right)}{x_{w}\left(\lambda_{h}+2 \mu_{h}\right)+x_{h}\left(\lambda_{w}+2 \mu_{w}\right)},
\end{aligned}
$$

where $\mu^{*}$ and $\lambda^{*}$ are the Lamé constants of the REV:

$$
\mu^{*}=x_{h} \mu_{h}+x_{w} \mu_{w}
$$

$$
\lambda^{*}=\frac{x_{w}{ }^{2} \lambda_{w}\left(\lambda_{h}+2 \mu_{h}\right)+x_{h}{ }^{2} \lambda_{h}\left(\lambda_{w}+2 \mu_{w}\right)+2 x_{h} x_{w}\left(\lambda_{h}\left(\lambda_{w}+\mu_{h}\right)+\lambda_{w} \mu_{w}\right)}{x_{w}\left(\lambda_{h}+2 \mu_{h}\right)+x_{h}\left(\lambda_{w}+2 \mu_{w}\right)} .
$$




\section{A.2 Case ii. Weak layers yield in-plane, hard layers behave elastically}

$$
\begin{aligned}
A_{\mathrm{h}, i i}= & \frac{1}{Z_{i i}}\left(2 \left(2 \mathrm{x}_{\mathrm{w}}{ }^{2}\left(\lambda_{\mathrm{h}}+2 \mu_{\mathrm{h}}\right) \mu_{\mathrm{w}}\left(\lambda_{\mathrm{w}}+\mu_{\mathrm{w}}\right)+\mathrm{x}_{\mathrm{h}}{ }^{2}\left(\lambda_{\mathrm{h}}+2 \mu_{\mathrm{h}}\right) \mu_{\mathrm{w}}\left(\left(2-\mathrm{m}_{\mathrm{w}}+\right.\right.\right.\right. \\
& \left.\left.\kappa_{\mathrm{w}}\left(-1+2 \mathrm{~m}_{\mathrm{w}}\right)\right) \lambda_{\mathrm{w}}+2\left(\mu_{\mathrm{w}}+\kappa_{\mathrm{w}} \mathrm{m}_{\mathrm{w}} \mu_{\mathrm{w}}\right)\right)+\mathrm{x}_{\mathrm{h}} \mathrm{x}_{\mathrm{w}}\left(\lambda _ { \mathrm { h } } \left(4\left(1+\kappa_{\mathrm{w}} \mathrm{m}_{\mathrm{w}}\right) \mu_{\mathrm{h}} \mu_{\mathrm{w}}+\right.\right. \\
& \left.\lambda_{\mathrm{w}}\left(2\left(-1+\kappa_{\mathrm{w}}\right)\left(-1+\mathrm{m}_{\mathrm{w}}\right) \mu_{\mathrm{h}}+\left(2+\kappa_{\mathrm{w}}+\mathrm{m}_{\mathrm{w}}\right) \mu_{\mathrm{w}}\right)\right)+2\left(2 \mu_{\mathrm{w}}\left(\left(1+\kappa_{\mathrm{w}} \mathrm{m}_{\mathrm{w}}\right) \mu_{\mathrm{h}}{ }^{2}+\mu_{\mathrm{w}}{ }^{2}\right)+\right. \\
& \left.\left.\left.\left.\lambda_{\mathrm{w}}\left(\left(-1+\kappa_{\mathrm{w}}\right)\left(-1+\mathrm{m}_{\mathrm{w}}\right) \mu_{\mathrm{h}}{ }^{2}+3 \mu_{\mathrm{w}}{ }^{2}\right)\right)\right)\right)\right) \\
A_{2, i i}= & -\frac{1}{Z_{i i}}\left(2 \left(2 \kappa_{\mathrm{w}} \mathrm{x}_{\mathrm{w}}{ }^{2}\left(\lambda_{\mathrm{h}}+2 \mu_{\mathrm{h}}\right) \mu_{\mathrm{w}}\left(\lambda_{\mathrm{w}}+\mu_{\mathrm{w}}\right)+\mathrm{x}_{\mathrm{h}}{ }^{2} \lambda_{\mathrm{h}} \mu_{\mathrm{w}}\left(\left(2-\kappa_{\mathrm{w}}-\mathrm{m}_{\mathrm{w}}+2 \kappa_{\mathrm{w}} \mathrm{m}_{\mathrm{w}}\right) \lambda_{\mathrm{w}}+\right.\right.\right. \\
& \left.2\left(\mu_{\mathrm{w}}+\kappa_{\mathrm{w}} \mathrm{m}_{\mathrm{w}} \mu_{\mathrm{w}}\right)\right)+\mathrm{x}_{\mathrm{h}} \mathrm{x}_{\mathrm{w}}\left(2 \kappa_{\mathrm{w}} \mu_{\mathrm{w}}{ }^{2}\left(3 \lambda_{\mathrm{w}}+2 \mu_{\mathrm{w}}\right)+\lambda_{\mathrm{h}}\left(2\left(1+\kappa_{\mathrm{w}} \mathrm{m}_{\mathrm{w}}\right) \mu_{\mathrm{h}} \mu_{\mathrm{w}}+\right.\right. \\
& \left.\left.\left.\left.\lambda_{\mathrm{w}}\left(\left(-1+\kappa_{\mathrm{w}}\right)\left(-1+\mathrm{m}_{\mathrm{w}}\right) \mu_{\mathrm{h}}+\left(1+\kappa_{\mathrm{w}}\left(2+\mathrm{m}_{\mathrm{w}}\right)\right) \mu_{\mathrm{w}}\right)\right)\right)\right)\right) \\
A_{3, i i}= & \frac{1}{Z_{i i}}\left(2 \left(2 \mathrm{~m}_{\mathrm{w}} \mathrm{x}_{\mathrm{w}}{ }^{2}\left(\lambda_{\mathrm{h}}+2 \mu_{\mathrm{h}}\right) \mu_{\mathrm{w}}\left(\lambda_{\mathrm{w}}+\mu_{\mathrm{w}}\right)+\mathrm{x}_{\mathrm{h}}{ }^{2} \lambda_{\mathrm{h}} \mu_{\mathrm{w}}\left(\left(2-\kappa_{\mathrm{w}}-\mathrm{m}_{\mathrm{w}}+2 \kappa_{\mathrm{w}} \mathrm{m}_{\mathrm{w}}\right) \lambda_{\mathrm{w}}+\right.\right.\right. \\
& \left.2\left(\mu_{\mathrm{w}}+\kappa_{\mathrm{w}} \mathrm{m}_{\mathrm{w}} \mu_{\mathrm{w}}\right)\right)+\mathrm{x}_{\mathrm{h}} \mathrm{x}_{\mathrm{w}}\left(2 \mathrm{~m}_{\mathrm{w}} \mu_{\mathrm{w}}{ }^{2}\left(3 \lambda_{\mathrm{w}}+2 \mu_{\mathrm{w}}\right)+\lambda_{\mathrm{h}}\left(2\left(1+\kappa_{\mathrm{w}} \mathrm{m}_{\mathrm{w}}\right) \mu_{\mathrm{h}} \mu_{\mathrm{w}}+\right.\right. \\
& \left.\left.\left.\left.\lambda_{\mathrm{w}}\left(\left(-1+\kappa_{\mathrm{w}}\right)\left(-1+\mathrm{m}_{\mathrm{w}}\right) \mu_{\mathrm{h}}+\left(1+\left(2+\kappa_{\mathrm{w}}\right) \mathrm{m}_{\mathrm{w}}\right) \mu_{\mathrm{w}}\right)\right)\right)\right)\right)
\end{aligned}
$$

$$
\begin{aligned}
A_{4, i i}= & -\frac{1}{Z_{i i}}\left(2 \left(2 \kappa_{\mathrm{w}} \mathrm{m}_{\mathrm{w}} \mathrm{x}_{\mathrm{w}}{ }^{2}\left(\lambda_{\mathrm{h}}+2 \mu_{\mathrm{h}}\right) \mu_{\mathrm{w}}\left(\lambda_{\mathrm{w}}+\mu_{\mathrm{w}}\right)+\mathrm{x}_{\mathrm{h}}{ }^{2}\left(\lambda_{\mathrm{h}}+2 \mu_{\mathrm{h}}\right) \mu_{\mathrm{w}}\left(\left(2-\mathrm{m}_{\mathrm{w}}+\right.\right.\right.\right. \\
& \left.\left.\kappa_{\mathrm{w}}\left(-1+2 \mathrm{~m}_{\mathrm{w}}\right)\right) \lambda_{\mathrm{w}}+2\left(\mu_{\mathrm{w}}+\kappa_{\mathrm{w}} \mathrm{m}_{\mathrm{w}} \mu_{\mathrm{w}}\right)\right)+\mathrm{x}_{\mathrm{h}} \mathrm{x}_{\mathrm{w}}\left(\lambda _ { \mathrm { h } } \left(4\left(1+\kappa_{\mathrm{w}} \mathrm{m}_{\mathrm{w}}\right) \mu_{\mathrm{h}} \mu_{\mathrm{w}}+\right.\right. \\
& \left.\lambda_{\mathrm{w}}\left(2\left(-1+\kappa_{\mathrm{w}}\right)\left(-1+\mathrm{m}_{\mathrm{w}}\right) \mu_{\mathrm{h}}+\left(\kappa_{\mathrm{w}}+\mathrm{m}_{\mathrm{w}}+2 \kappa_{\mathrm{w}} \mathrm{m}_{\mathrm{w}}\right) \mu_{\mathrm{w}}\right)\right)+ \\
& \left.\left.\left.2\left(2 \mu_{\mathrm{w}}\left(\left(1+\kappa_{\mathrm{w}} \mathrm{m}_{\mathrm{w}}\right) \mu_{\mathrm{h}}{ }^{2}+\kappa_{\mathrm{w}} \mathrm{m}_{\mathrm{w}} \mu_{\mathrm{w}}{ }^{2}\right)+\lambda_{\mathrm{w}}\left(\left(-1+\kappa_{\mathrm{w}}\right)\left(-1+\mathrm{m}_{\mathrm{w}}\right) \mu_{\mathrm{h}}{ }^{2}+3 \kappa_{\mathrm{w}} \mathrm{m}_{\mathrm{w}} \mu_{\mathrm{w}}{ }^{2}\right)\right)\right)\right)\right),
\end{aligned}
$$$$
B_{1, i i}=\frac{1}{Z_{i i}}\left(2 \mu_{w}\left(\left(1+m_{w}\right) x_{w} \lambda_{w}\left(\lambda_{h}+2 \mu_{h}\right)+x_{h} \lambda_{h}\left(\left(2-\kappa_{w}-m_{w}+2 \kappa_{w} m_{w}\right) \lambda_{w}+2\left(\mu_{w}+\kappa_{w} m_{w} \mu_{w}\right)\right)\right)\right),
$$$$
B_{2, i i}=\frac{1}{Z_{i i}}\left(2 \mu_{w}\left(\kappa_{w}\left(1+m_{w}\right) x_{w} \lambda_{w}\left(\lambda_{h}+2 \mu_{h}\right)+x_{h} \lambda_{h}\left(\left(2-\kappa_{w}-m_{w}+2 \kappa_{w} m_{w}\right) \lambda_{w}+2\left(\mu_{w}+\kappa_{w} m_{w} \mu_{w}\right)\right)\right)\right),
$$$$
K_{1, i i}=\frac{2}{Z_{i i}}\left(x_{h}\left(\lambda_{h}+2 \mu_{h}\right) \mu_{w}\left(\left(2-m_{w}+\kappa_{w}\left(-1+2 m_{w}\right)\right) \lambda_{w}+2\left(\mu_{w}+\kappa_{w} m_{w} \mu_{w}\right)\right)+\right.
$$$$
x_{w}\left(2 \mu_{h}^{2}\left(\left(-1+\kappa_{w}\right)\left(-1+m_{w}\right) \lambda_{w}+2\left(\mu_{w}+\kappa_{w} m_{w} \mu_{w}\right)\right)+\right.
$$$$
\left.\left.\lambda_{h}\left(4\left(1+\kappa_{w} m_{w}\right) \mu_{h} \mu_{w}+\lambda_{w}\left(2\left(-1+\kappa_{w}\right)\left(-1+m_{w}\right) \mu_{h}+\left(1+m_{w}\right) \mu_{w}\right)\right)\right)\right)
$$

$$
K_{2, i i}=\frac{2 \lambda_{h}}{Z_{i i}}\left(x_{h} \mu_{w}\left(\left(2-\kappa_{w}-m_{w}+2 \kappa_{w} m_{w}\right) \lambda_{w}+2\left(\mu_{w}+\kappa_{w} m_{w} \mu_{w}\right)\right)+\right.
$$

$$
\left.x_{w}\left(2\left(1+\kappa_{w} m_{w}\right) \mu_{h} \mu_{w}+\lambda_{w}\left(\left(-1+\kappa_{w}\right)\left(-1+m_{w}\right) \mu_{h}+\kappa_{w}\left(1+m_{w}\right) \mu_{w}\right)\right)\right) \text {, }
$$

$$
\begin{aligned}
K_{3, i i}= & \frac{2 \lambda_{h}}{Z_{i i}}\left(x_{h} \mu_{w}\left(\left(2-\kappa_{w}-m_{w}+2 \kappa_{w} m_{w}\right) \lambda_{w}+2\left(\mu_{w}+\kappa_{w} m_{w} \mu_{w}\right)\right)+\right. \\
& \left.x_{w}\left(2\left(1+\kappa_{w} m_{w}\right) \mu_{h} \mu_{w}+\lambda_{w}\left(\left(-1+\kappa_{w}\right)\left(-1+m_{w}\right) \mu_{h}+\left(1+m_{w}\right) \mu_{w}\right)\right)\right),
\end{aligned}
$$




$$
\begin{aligned}
K_{4, i i}= & \frac{2}{Z_{i i}}\left(x_{h}\left(\lambda_{h}+2 \mu_{h}\right) \mu_{w}\left(\left(2-m_{w}+\kappa_{w}\left(-1+2 m_{w}\right)\right) \lambda_{w}+2\left(\mu_{w}+\kappa_{w} m_{w} \mu_{w}\right)\right)+\right. \\
& x_{w}\left(2 \mu_{h}^{2}\left(\left(-1+\kappa_{w}\right)\left(-1+m_{w}\right) \lambda_{w}+2\left(\mu_{w}+\kappa_{w} m_{w} \mu_{w}\right)\right)+\right. \\
& \left.\left.\lambda_{h}\left(4\left(1+\kappa_{w} m_{w}\right) \mu_{h} \mu_{w}+\lambda_{w}\left(2\left(-1+\kappa_{w}\right)\left(-1+m_{w}\right) \mu_{h}+\kappa_{w}\left(1+m_{w}\right) \mu_{w}\right)\right)\right)\right) \\
K_{5, i i}= & \frac{2 \mu_{w}}{Z_{i i}}\left(2 x_{w}\left(\lambda_{h}+2 \mu_{h}\right)\left(\lambda_{w}+\mu_{w}\right)+x_{h}\left(\left(1+\kappa_{w}\right) \lambda_{h} \lambda_{w}+2 \mu_{w}\left(3 \lambda_{w}+2 \mu_{w}\right)\right)\right), \\
K_{6, i i}= & \frac{2 \mu_{w}}{Z_{i i}}\left(2 \kappa_{w} x_{w}\left(\lambda_{h}+2 \mu_{h}\right)\left(\lambda_{w}+\mu_{w}\right)+x_{h}\left(\left(1+\kappa_{w}\right) \lambda_{h} \lambda_{w}+2 \kappa_{w} \mu_{w}\left(3 \lambda_{w}+2 \mu_{w}\right)\right)\right), \\
K_{7, i i}= & \frac{2 m_{w} \mu_{w}}{Z_{i i}}\left(2 x_{w}\left(\lambda_{h}+2 \mu_{h}\right)\left(\lambda_{w}+\mu_{w}\right)+x_{h}\left(\left(1+\kappa_{w}\right) \lambda_{h} \lambda_{w}+2 \mu_{w}\left(3 \lambda_{w}+2 \mu_{w}\right)\right)\right), \\
K_{8, i i}= & \frac{2 m_{w} \mu_{w}}{Z_{i i}}\left(2 \kappa_{w} x_{w}\left(\lambda_{h}+2 \mu_{h}\right)\left(\lambda_{w}+\mu_{w}\right)+x_{h}\left(\left(1+\kappa_{w}\right) \lambda_{h} \lambda_{w}+2 \kappa_{w} \mu_{w}\left(3 \lambda_{w}+2 \mu_{w}\right)\right)\right),
\end{aligned}
$$

where

$$
\begin{gathered}
Z_{i i}=\mathrm{x}_{\mathrm{w}}\left(\lambda_{\mathrm{h}}+2 \mu_{\mathrm{h}}\right)\left(\left(-1+\kappa_{\mathrm{w}}\right)\left(-1+\mathrm{m}_{\mathrm{w}}\right) \lambda_{\mathrm{w}}+2\left(\mu_{\mathrm{w}}+\kappa_{\mathrm{w}} \mathrm{m}_{\mathrm{w}} \mu_{\mathrm{w}}\right)\right)+ \\
2 \mathrm{x}_{\mathrm{h}} \mu_{\mathrm{w}}\left(\left(2-\mathrm{m}_{\mathrm{w}}+\kappa_{\mathrm{w}}\left(-1+2 \mathrm{~m}_{\mathrm{w}}\right)\right) \lambda_{\mathrm{w}}+2\left(\mu_{\mathrm{w}}+\kappa_{\mathrm{w}} \mathrm{m}_{\mathrm{w}} \mu_{\mathrm{w}}\right)\right)
\end{gathered}
$$

\section{A.3 Case iii. Hard layers yield in-plane, weak layers behave elastically}

$$
\begin{aligned}
& A_{1, i i i}=\frac{1}{Z_{i i i}}\left(2 \left(2 \mathrm{x}_{\mathrm{h}}{ }^{2} \mu_{\mathrm{h}}\left(\lambda_{\mathrm{h}}+\mu_{\mathrm{h}}\right)\left(\lambda_{\mathrm{w}}+2 \mu_{\mathrm{w}}\right)+\mathrm{x}_{\mathrm{w}}{ }^{2} \mu_{\mathrm{h}}\left(\left(2-\mathrm{m}_{\mathrm{h}}+\kappa_{\mathrm{h}}\left(-1+2 \mathrm{~m}_{\mathrm{h}}\right)\right) \lambda_{\mathrm{h}}+\right.\right.\right. \\
& \left.2\left(\mu_{\mathrm{h}}+\kappa_{\mathrm{h}} \mathrm{m}_{\mathrm{h}} \mu_{\mathrm{h}}\right)\right)\left(\lambda_{\mathrm{w}}+2 \mu_{\mathrm{w}}\right)+\mathrm{x}_{\mathrm{h}} \mathrm{x}_{\mathrm{w}}\left(4 \mu_{\mathrm{h}}\left(\mu_{\mathrm{h}}^{2}+\left(1+\kappa_{\mathrm{h}} \mathrm{m}_{\mathrm{h}}\right) \mu_{\mathrm{w}}\left(\lambda_{\mathrm{w}}+\mu_{\mathrm{w}}\right)\right)+\right. \\
& \left.\left.\left.\lambda_{\mathrm{h}}\left(\lambda_{\mathrm{w}}\left(\left(2+\kappa_{\mathrm{h}}+\mathrm{m}_{\mathrm{h}}\right) \mu_{\mathrm{h}}+2\left(-1+\kappa_{\mathrm{h}}\right)\left(-1+\mathrm{m}_{\mathrm{h}}\right) \mu_{\mathrm{w}}\right)+2\left(3 \mu_{\mathrm{h}}{ }^{2}+\left(-1+\kappa_{\mathrm{h}}\right)\left(-1+\mathrm{m}_{\mathrm{h}}\right) \mu_{\mathrm{w}}{ }^{2}\right)\right)\right)\right)\right) \\
& A_{2, i i i}=-\frac{1}{Z_{i i i}}\left(2 \left(\mathrm{x}_{\mathrm{w}} \lambda_{\mathrm{w}}\left(\mathrm{x}_{\mathrm{w}} \mu_{\mathrm{h}}\left(-\left(-2+\mathrm{m}_{\mathrm{h}}\right) \lambda_{\mathrm{h}}+2 \mu_{\mathrm{h}}\right)+\mathrm{x}_{\mathrm{h}}\left(2 \mu_{\mathrm{h}} \mu_{\mathrm{w}}+\lambda_{\mathrm{h}}\left(\mu_{\mathrm{h}}+\mu_{\mathrm{w}}-\mathrm{m}_{\mathrm{h}} \mu_{\mathrm{w}}\right)\right)\right)+\right.\right. \\
& \kappa_{\mathrm{h}}\left(\mathrm{x}_{\mathrm{w}}{ }^{2} \lambda_{\mathrm{w}} \mu_{\mathrm{h}}\left(\left(-1+2 \mathrm{~m}_{\mathrm{h}}\right) \lambda_{\mathrm{h}}+2 \mathrm{~m}_{\mathrm{h}} \mu_{\mathrm{h}}\right)+2 \mathrm{x}_{\mathrm{h}}{ }^{2} \mu_{\mathrm{h}}\left(\lambda_{\mathrm{h}}+\mu_{\mathrm{h}}\right)\left(\lambda_{\mathrm{w}}+2 \mu_{\mathrm{w}}\right)+\right. \\
& \left.\left.\left.x_{h} x_{w}\left(4 \mu_{h}^{3}+2 m_{h} \lambda_{w} \mu_{h} \mu_{w}+\lambda_{h}\left(6 \mu_{h}^{2}+\lambda_{w}\left(\left(2+m_{h}\right) \mu_{h}+\left(-1+m_{h}\right) \mu_{w}\right)\right)\right)\right)\right)\right) \\
& A_{3, i i i}=\frac{1}{Z_{i i i}}\left(2 \left(\mathrm{x}_{\mathrm{w}} \lambda_{\mathrm{w}}\left(\mathrm{x}_{\mathrm{w}} \mu_{\mathrm{h}}\left(-\left(-2+\kappa_{\mathrm{h}}\right) \lambda_{\mathrm{h}}+2 \mu_{\mathrm{h}}\right)+\mathrm{x}_{\mathrm{h}}\left(2 \mu_{\mathrm{h}} \mu_{\mathrm{w}}+\lambda_{\mathrm{h}}\left(\mu_{\mathrm{h}}+\mu_{\mathrm{w}}-\kappa_{\mathrm{h}} \mu_{\mathrm{w}}\right)\right)\right)+\right.\right. \\
& \mathrm{m}_{\mathrm{h}}\left(\mathrm{x}_{\mathrm{w}}{ }^{2} \lambda_{\mathrm{w}} \mu_{\mathrm{h}}\left(\left(-1+2 \kappa_{\mathrm{h}}\right) \lambda_{\mathrm{h}}+2 \kappa_{\mathrm{h}} \mu_{\mathrm{h}}\right)+2 \mathrm{x}_{\mathrm{h}}^{2} \mu_{\mathrm{h}}\left(\lambda_{\mathrm{h}}+\mu_{\mathrm{h}}\right)\left(\lambda_{\mathrm{w}}+2 \mu_{\mathrm{w}}\right)+\right. \\
& \left.\left.\left.\mathrm{x}_{\mathrm{h}} \mathrm{x}_{\mathrm{w}}\left(4 \mu_{\mathrm{h}}^{3}+2 \kappa_{\mathrm{h}} \lambda_{\mathrm{w}} \mu_{\mathrm{h}} \mu_{\mathrm{w}}+\lambda_{\mathrm{h}}\left(6 \mu_{\mathrm{h}}^{2}+\lambda_{\mathrm{w}}\left(\left(2+\kappa_{\mathrm{h}}\right) \mu_{\mathrm{h}}+\left(-1+\kappa_{\mathrm{h}}\right) \mu_{\mathrm{w}}\right)\right)\right)\right)\right)\right) \\
& A_{4, \text { iii }}=-\frac{1}{Z_{i i i}}\left(2 \left(\mathrm { x } _ { \mathrm { w } } \left(2\left(\mathrm{x}_{\mathrm{h}}\left(\lambda_{\mathrm{h}}+2 \mu_{\mathrm{h}}\right) \mu_{\mathrm{w}}\left(\lambda_{\mathrm{w}}+\mu_{\mathrm{w}}\right)+\mathrm{x}_{\mathrm{w}} \mu_{\mathrm{h}}\left(\lambda_{\mathrm{h}}+\mu_{\mathrm{h}}\right)\left(\lambda_{\mathrm{w}}+2 \mu_{\mathrm{w}}\right)\right)+\right.\right.\right. \\
& \left.m_{h} \lambda_{h}\left(-x_{w} \mu_{h}\left(\lambda_{w}+2 \mu_{w}\right)+x_{h}\left(\lambda_{w} \mu_{h}-2 \lambda_{w} \mu_{w}-2 \mu_{w}^{2}\right)\right)\right)+\kappa_{h}\left(-x_{w} \lambda_{h}\left(x_{w} \mu_{h}\left(\lambda_{w}+2 \mu_{w}\right)+\right.\right. \\
& \left.x_{\mathrm{h}}\left(-\lambda_{\mathrm{w}} \mu_{\mathrm{h}}+2 \lambda_{\mathrm{w}} \mu_{\mathrm{w}}+2 \mu_{\mathrm{w}}^{2}\right)\right)+2 \mathrm{~m}_{\mathrm{h}}\left(\mathrm{x}_{\mathrm{h}}^{2} \mu_{\mathrm{h}}\left(\lambda_{\mathrm{h}}+\mu_{\mathrm{h}}\right)\left(\lambda_{\mathrm{w}}+2 \mu_{\mathrm{w}}\right)+\right. \\
& \left.\left.\left.\left.\mathrm{x}_{\mathrm{w}}{ }^{2} \mu_{\mathrm{h}}\left(\lambda_{\mathrm{h}}+\mu_{\mathrm{h}}\right)\left(\lambda_{\mathrm{w}}+2 \mu_{\mathrm{w}}\right)+\mathrm{x}_{\mathrm{h}} \mathrm{x}_{\mathrm{w}}\left(2 \mu_{\mathrm{h}}\left(\mu_{\mathrm{h}}{ }^{2}+\mu_{\mathrm{w}}\left(\lambda_{\mathrm{w}}+\mu_{\mathrm{w}}\right)\right)+\lambda_{\mathrm{h}}\left(3 \mu_{\mathrm{h}}{ }^{2}+\mu_{\mathrm{w}}{ }^{2}+\lambda_{\mathrm{w}}\left(\mu_{\mathrm{h}}+\mu_{\mathrm{w}}\right)\right)\right)\right)\right)\right)\right)
\end{aligned}
$$




$$
\begin{aligned}
& B_{1, i i i}=\frac{1}{Z_{i i i}}\left(2 \mu_{h}\left(x_{w} \lambda_{w}\left(\left(2-\kappa_{h}-m_{h}+2 \kappa_{h} m_{h}\right) \lambda_{h}+2\left(\mu_{h}+\kappa_{h} m_{h} \mu_{h}\right)\right)+\left(1+m_{h}\right) x_{h} \lambda_{h}\left(\lambda w+2 \mu_{w}\right)\right)\right) \text {, } \\
& B_{2, i i i}=\frac{1}{Z_{i i i}}\left(2 \mu_{h}\left(x_{w} \lambda_{w}\left(\left(2-m_{h}\right) \lambda_{h}+2 \mu_{h}\right)+\kappa_{h}\left(x_{w} \lambda_{w}\left(-\lambda_{h}+2 m_{h} \lambda_{h}+2 m_{h} \mu_{h}\right)+\left(1+m_{h}\right) x_{h} \lambda_{h}\left(\lambda_{w}+2 \mu_{w}\right)\right)\right)\right), \\
& K_{1, i i i}=\frac{2 \mu_{h}}{Z_{i i i}}\left(x_{w}\left(4 \mu_{h}^{2}+\lambda_{h}\left(\lambda_{w}+\kappa_{h} \lambda_{w}+6 \mu_{h}\right)\right)+2 x_{h}\left(\lambda_{h}+\mu_{h}\right)\left(\lambda_{w}+2 \mu_{w}\right)\right), \\
& K_{2, i i i}=\frac{2 \mu_{h}}{Z_{i i i}}\left(x_{w} \lambda_{h} \lambda_{w}+\kappa_{h}\left(x_{w}\left(\lambda_{h} \lambda_{w}+6 \lambda_{h} \mu_{h}+4 \mu_{h}^{2}\right)+2 x_{h}\left(\lambda_{h}+\mu_{h}\right)\left(\lambda_{w}+2 \mu_{w}\right)\right)\right), \\
& K_{3, i i i}=\frac{2 m_{h} \mu_{h}}{Z_{i i i}}\left(x_{w}\left(4 \mu_{h}^{2}+\lambda_{h}\left(\lambda_{w}+\kappa_{h} \lambda_{w}+6 \mu_{h}\right)\right)+2 x_{h}\left(\lambda_{h}+\mu_{h}\right)\left(\lambda_{w}+2 \mu_{w}\right)\right), \\
& K_{4, i i i}=\frac{2 m_{h} \mu_{h}}{Z_{i i i}}\left(x_{w} \lambda_{h} \lambda_{w}+\kappa_{h}\left(x_{w}\left(\lambda_{h} \lambda_{w}+6 \lambda_{h} \mu_{h}+4 \mu_{h}^{2}\right)+2 x_{h}\left(\lambda_{h}+\mu_{h}\right)\left(\lambda_{w}+2 \mu_{w}\right)\right)\right), \\
& K_{5, i i i}=\frac{2}{Z_{i i i}}\left(x_{w} \mu_{h}\left(\left(2-m_{h}+\kappa_{h}\left(-1+2 m_{h}\right)\right) \lambda_{h}+2\left(\mu_{h}+\kappa_{h} m_{h} \mu_{h}\right)\right)\left(\lambda_{w}+2 \mu_{w}\right)+\right. \\
& x_{h}\left(4\left(1+\kappa_{h} m_{h}\right) \mu_{h} \mu_{w}\left(\lambda_{w}+\mu_{w}\right)+\lambda_{h}\left(2\left(-1+\kappa_{h}\right)\left(-1+m_{h}\right) \mu_{w}^{2}+\right.\right. \\
& \left.\left.\left.\lambda_{w}\left(\left(1+m_{h}\right) \mu_{h}+2\left(-1+\kappa_{h}\right)\left(-1+m_{h}\right) \mu_{w}\right)\right)\right)\right) \\
& K_{6, i i i}=\frac{2 \lambda_{w}}{Z_{i i i}}\left(x_{w} \mu_{h}\left(2 \lambda_{h}-m_{h} \lambda_{h}+2 \mu_{h}\right)+x_{h}\left(\lambda_{h}-m_{h} \lambda_{h}+2 \mu_{h}\right) \mu_{w}+\right. \\
& \left.\kappa_{h}\left(x_{w} \mu_{h}\left(\left(-1+2 m_{h}\right) \lambda_{h}+2 m_{h} \mu_{h}\right)+x_{h}\left(2 m_{h} \mu_{h} \mu_{w}+\lambda_{h}\left(\mu_{h}+m_{h} \mu_{h}-\mu_{w}+m_{h} \mu_{w}\right)\right)\right)\right), \\
& K_{7, i i i}=\frac{2 \lambda_{w}}{Z_{i i i}}\left(x _ { w } \mu _ { h } \left(\left(2-\kappa_{h}-m_{h}+2 \kappa_{h} m_{h}\right) \lambda_{h}+\right.\right. \\
& \left.\left.2\left(\mu_{h}+\kappa_{h} m_{h} \mu_{h}\right)\right)+x_{h}\left(2\left(1+\kappa_{h} m_{h}\right) \mu_{h} \mu_{w}+\lambda_{h}\left(\left(1+m_{h}\right) \mu_{h}+\left(-1+\kappa_{h}\right)\left(-1+m_{h}\right) \mu_{w}\right)\right)\right), \\
& K_{8, i i i}=\frac{2}{Z_{i i i}}\left(-2 x_{h}\left(\left(-1+m_{h}\right) \lambda_{h}-2 \mu_{h}\right) \mu_{w}\left(\lambda_{w}+\mu_{w}\right)-x_{w}\left(\left(-2+m_{h}\right) \lambda_{h}-2 \mu_{h}\right) \mu_{h}\left(\lambda_{w}+2 \mu_{w}\right)+\right. \\
& \kappa_{h}\left(x_{w} \mu_{h}\left(\left(-1+2 m_{h}\right) \lambda_{h}+2 m_{h} \mu_{h}\right)\left(\lambda_{w}+2 \mu_{w}\right)+\right. \\
& \left.\left.x_{h}\left(4 m_{h} \mu_{h} \mu_{w}\left(\lambda_{w}+\mu_{w}\right)+\lambda_{h}\left(2\left(-1+m_{h}\right) \mu_{w}{ }^{2}+\lambda_{w}\left(\mu_{h}+m_{h} \mu_{h}-2 \mu_{w}+2 m_{h} \mu_{w}\right)\right)\right)\right)\right)
\end{aligned}
$$

\section{where}

$$
\begin{aligned}
Z_{i i i}= & 2 x_{w} \mu_{h}\left(\left(2-m_{h}+\kappa_{h}\left(-1+2 m_{h}\right)\right) \lambda_{h}+2\left(\mu_{h}+\kappa_{h} m_{h} \mu_{h}\right)\right)+ \\
& x_{h}\left(\left(-1+\kappa_{h}\right)\left(-1+m_{h}\right) \lambda_{h}+2\left(\mu_{h}+\kappa_{h} m_{h} \mu_{h}\right)\right)\left(\lambda_{w}+2 \mu_{w}\right)
\end{aligned}
$$




\section{A.4 Case iv. Both the hard and the weak layers yield in-plane}

$$
\begin{aligned}
& A_{1, i v}=\frac{1}{Z_{i v}}\left(2 \left(2 \mathrm{x}_{\mathrm{w}}{ }^{2} \mu_{\mathrm{h}}\left(\left(2-\mathrm{m}_{\mathrm{h}}+\kappa_{\mathrm{h}}\left(-1+2 \mathrm{~m}_{\mathrm{h}}\right)\right) \lambda_{\mathrm{h}}+2\left(\mu_{\mathrm{h}}+\kappa_{\mathrm{h}} \mathrm{m}_{\mathrm{h}} \mu_{\mathrm{h}}\right)\right) \mu_{\mathrm{w}}\left(\lambda_{\mathrm{w}}+\mu_{\mathrm{w}}\right)+\right.\right. \\
& 2 \mathrm{x}_{\mathrm{h}}^{2} \mu_{\mathrm{h}}\left(\lambda_{\mathrm{h}}+\mu_{\mathrm{h}}\right) \mu_{\mathrm{w}}\left(\left(2-\mathrm{m}_{\mathrm{w}}+\kappa_{\mathrm{w}}\left(-1+2 \mathrm{~m}_{\mathrm{w}}\right)\right) \lambda_{\mathrm{w}}+2\left(\mu_{\mathrm{w}}+\kappa_{\mathrm{w}} \mathrm{m}_{\mathrm{w}} \mu_{\mathrm{w}}\right)\right)+ \\
& \mathrm{x}_{\mathrm{h}} \mathrm{x}_{\mathrm{w}}\left(\lambda _ { \mathrm { h } } \left(2 \mu_{\mathrm{w}}\left(3\left(1+\kappa_{\mathrm{w}} \mathrm{m}_{\mathrm{w}}\right) \mu_{\mathrm{h}}{ }^{2}+\left(-1+\kappa_{\mathrm{h}}\right)\left(-1+\mathrm{m}_{\mathrm{h}}\right) \mu_{\mathrm{w}}{ }^{2}\right)+\lambda_{\mathrm{w}}\left(3\left(-1+\kappa_{\mathrm{w}}\right)\left(-1+\mathrm{m}_{\mathrm{w}}\right) \mu_{\mathrm{h}}{ }^{2}+\right.\right.\right. \\
& \left.\left.\left(2+\kappa_{\mathrm{h}}+\kappa_{\mathrm{w}}+\mathrm{m}_{\mathrm{h}}+\kappa_{\mathrm{w}} \mathrm{m}_{\mathrm{h}}+\mathrm{m}_{\mathrm{w}}+\kappa_{\mathrm{h}} \mathrm{m}_{\mathrm{w}}\right) \mu_{\mathrm{h}} \mu_{\mathrm{w}}+3\left(-1+\kappa_{\mathrm{h}}\right)\left(-1+\mathrm{m}_{\mathrm{h}}\right) \mu_{\mathrm{w}}{ }^{2}\right)\right)+ \\
& \left.\left.\left.2 \mu_{\mathrm{h}}\left(2 \mu_{\mathrm{w}}\left(\left(1+\kappa_{\mathrm{w}} \mathrm{m}_{\mathrm{w}}\right) \mu_{\mathrm{h}}{ }^{2}+\left(1+\kappa_{\mathrm{h}} \mathrm{m}_{\mathrm{h}}\right) \mu_{\mathrm{w}}{ }^{2}\right)+\lambda_{\mathrm{w}}\left(\left(-1+\kappa_{\mathrm{w}}\right)\left(-1+\mathrm{m}_{\mathrm{w}}\right) \mu_{\mathrm{h}}{ }^{2}+3\left(1+\kappa_{\mathrm{h}} \mathrm{m}_{\mathrm{h}}\right) \mu_{\mathrm{w}}{ }^{2}\right)\right)\right)\right)\right) \text {, } \\
& A_{2, i v}=-\frac{1}{Z_{i v}}\left(2 \left(\kappa _ { \mathrm { w } } \mathrm { x } _ { \mathrm { w } } \mu _ { \mathrm { w } } \left(-2 \mathrm{x}_{\mathrm{w}}\left(\left(-2+\mathrm{m}_{\mathrm{h}}\right) \lambda_{\mathrm{h}}-2 \mu_{\mathrm{h}}\right) \mu_{\mathrm{h}}\left(\lambda_{\mathrm{w}}+\mu_{\mathrm{w}}\right)+\mathrm{x}_{\mathrm{h}}\left(2 \mu_{\mathrm{h}} \mu_{\mathrm{w}}\left(3 \lambda_{\mathrm{w}}+2 \mu_{\mathrm{w}}\right)+\right.\right.\right.\right. \\
& \left.\left.\lambda_{h}\left(-2\left(-1+m_{h}\right) \mu_{w}{ }^{2}+\lambda_{w}\left(\mu_{h}+m_{w} \mu_{h}+3 \mu_{w}-3 m_{h} \mu_{w}\right)\right)\right)\right)+\kappa_{h}\left(2 \kappa _ { w } x _ { w } { } ^ { 2 } \mu _ { h } \left(\left(-1+2 m_{h}\right) \lambda_{h}+\right.\right. \\
& \left.2 \mathrm{~m}_{\mathrm{h}} \mu_{\mathrm{h}}\right) \mu_{\mathrm{w}}\left(\lambda_{\mathrm{w}}+\mu_{\mathrm{w}}\right)+2 \mathrm{x}_{\mathrm{h}}{ }^{2} \mu_{\mathrm{h}}\left(\lambda_{\mathrm{h}}+\mu_{\mathrm{h}}\right) \mu_{\mathrm{w}}\left(\left(2-\mathrm{m}_{\mathrm{w}}+\kappa_{\mathrm{w}}\left(-1+2 \mathrm{~m}_{\mathrm{w}}\right)\right) \lambda_{\mathrm{w}}+2\left(\mu_{\mathrm{w}}+\kappa_{\mathrm{w}} \mathrm{m}_{\mathrm{w}} \mu_{\mathrm{w}}\right)\right)+ \\
& \mathrm{x}_{\mathrm{h}} \mathrm{x}_{\mathrm{w}}\left(\lambda _ { \mathrm { h } } \left(2 \mu_{\mathrm{w}}\left(3\left(1+\kappa_{\mathrm{w}} \mathrm{m}_{\mathrm{w}}\right) \mu_{\mathrm{h}}{ }^{2}+\kappa_{\mathrm{w}}\left(-1+\mathrm{m}_{\mathrm{h}}\right) \mu_{\mathrm{w}}{ }^{2}\right)+\lambda_{\mathrm{w}}\left(3\left(-1+\kappa_{\mathrm{w}}\right)\left(-1+\mathrm{m}_{\mathrm{w}}\right) \mu_{\mathrm{h}}{ }^{2}+\right.\right.\right. \\
& \left.\left.\left(1+\mathrm{m}_{\mathrm{h}}+\kappa_{\mathrm{w}}\left(2+\mathrm{m}_{\mathrm{h}}+\mathrm{m}_{\mathrm{w}}\right)\right) \mu_{\mathrm{h}} \mu_{\mathrm{w}}+3 \kappa_{\mathrm{w}}\left(-1+\mathrm{m}_{\mathrm{h}}\right) \mu_{\mathrm{w}}{ }^{2}\right)\right)+ \\
& \left.\left.\left.\left.2 \mu_{\mathrm{h}}\left(2 \mu_{\mathrm{w}}\left(\left(1+\kappa_{\mathrm{w}} \mathrm{m}_{\mathrm{w}}\right) \mu_{\mathrm{h}}{ }^{2}+\kappa_{\mathrm{w}} \mathrm{m}_{\mathrm{h}} \mu_{\mathrm{w}}{ }^{2}\right)+\lambda_{\mathrm{w}}\left(\left(-1+\kappa_{\mathrm{w}}\right)\left(-1+\mathrm{m}_{\mathrm{w}}\right) \mu_{\mathrm{h}}{ }^{2}+3 \kappa_{\mathrm{w}} \mathrm{m}_{\mathrm{h}} \mu_{\mathrm{w}}{ }^{2}\right)\right)\right)\right)\right)\right)
\end{aligned}
$$

$$
\begin{aligned}
A_{3, i v}= & \frac{1}{Z_{i v}}\left(2 \left(\mathrm { m } _ { \mathrm { w } } \mathrm { x } _ { \mathrm { w } } \mu _ { \mathrm { w } } \left(-2 \mathrm{x}_{\mathrm{w}}\left(\left(-2+\kappa_{\mathrm{h}}\right) \lambda_{\mathrm{h}}-2 \mu_{\mathrm{h}}\right) \mu_{\mathrm{h}}\left(\lambda_{\mathrm{w}}+\mu_{\mathrm{w}}\right)+\mathrm{x}_{\mathrm{h}}\left(2 \mu_{\mathrm{h}} \mu_{\mathrm{w}}\left(3 \lambda_{\mathrm{w}}+2 \mu_{\mathrm{w}}\right)+\right.\right.\right.\right. \\
& \left.\left.\lambda_{\mathrm{h}}\left(-2\left(-1+\kappa_{\mathrm{h}}\right) \mu_{\mathrm{w}}{ }^{2}+\lambda_{\mathrm{w}}\left(\mu_{\mathrm{h}}+\kappa_{\mathrm{w}} \mu_{\mathrm{h}}+3 \mu_{\mathrm{w}}-3 \kappa_{\mathrm{h}} \mu_{\mathrm{w}}\right)\right)\right)\right)+\mathrm{m}_{\mathrm{h}}\left(2 \mathrm { m } _ { \mathrm { w } } \mathrm { x } _ { \mathrm { w } } { } ^ { 2 } \mu _ { \mathrm { h } } \left(\left(-1+2 \kappa_{\mathrm{h}}\right) \lambda_{\mathrm{h}}+\right.\right. \\
& \left.2 \kappa_{\mathrm{h}} \mu_{\mathrm{h}}\right) \mu_{\mathrm{w}}\left(\lambda_{\mathrm{w}}+\mu_{\mathrm{w}}\right)+2 \mathrm{x}_{\mathrm{h}}{ }^{2} \mu_{\mathrm{h}}\left(\lambda_{\mathrm{h}}+\mu_{\mathrm{h}}\right) \mu_{\mathrm{w}}\left(\left(2-\mathrm{m}_{\mathrm{w}}+\kappa_{\mathrm{w}}\left(-1+2 \mathrm{~m}_{\mathrm{w}}\right)\right) \lambda_{\mathrm{w}}+2\left(\mu_{\mathrm{w}}+\kappa_{\mathrm{w}} \mathrm{m}_{\mathrm{w}} \mu_{\mathrm{w}}\right)\right)+ \\
& \mathrm{x}_{\mathrm{h}} \mathrm{x}_{\mathrm{w}}\left(\lambda _ { \mathrm { h } } \left(2 \mu_{\mathrm{w}}\left(3\left(1+\kappa_{\mathrm{w}} \mathrm{m}_{\mathrm{w}}\right) \mu_{\mathrm{h}}{ }^{2}+\left(-1+\kappa_{\mathrm{h}}\right) \mathrm{m}_{\mathrm{w}} \mu_{\mathrm{w}}{ }^{2}\right)+\lambda_{\mathrm{w}}\left(3\left(-1+\kappa_{\mathrm{w}}\right)\left(-1+\mathrm{m}_{\mathrm{w}}\right) \mu_{\mathrm{h}}{ }^{2}+\right.\right.\right. \\
& \left.\left.\left(1+\kappa_{\mathrm{h}}+2 \mathrm{~m}_{\mathrm{w}}+\kappa_{\mathrm{h}} \mathrm{m}_{\mathrm{w}}+\kappa_{\mathrm{w}} \mathrm{m}_{\mathrm{w}}\right) \mu_{\mathrm{h}} \mu_{\mathrm{w}}+3\left(-1+\kappa_{\mathrm{h}}\right) \mathrm{m}_{\mathrm{w}} \mu_{\mathrm{w}}{ }^{2}\right)\right)+ \\
& \left.\left.\left.\left.2 \mu_{\mathrm{h}}\left(2 \mu_{\mathrm{w}}\left(\left(1+\kappa_{\mathrm{w}} \mathrm{m}_{\mathrm{w}}\right) \mu_{\mathrm{h}}{ }^{2}+\kappa_{\mathrm{h}} \mathrm{m}_{\mathrm{w}} \mu_{\mathrm{w}}{ }^{2}\right)+\lambda_{\mathrm{w}}\left(\left(-1+\kappa_{\mathrm{w}}\right)\left(-1+\mathrm{m}_{\mathrm{w}}\right) \mu_{\mathrm{h}}{ }^{2}+3 \kappa_{\mathrm{h}} \mathrm{m}_{\mathrm{w}} \mu_{\mathrm{w}}{ }^{2}\right)\right)\right)\right)\right)\right)
\end{aligned}
$$

$$
\begin{aligned}
& A_{4}=-\frac{1}{Z_{i v}}\left(2 \left(\kappa _ { \mathrm { w } } \mathrm { x } _ { \mathrm { w } } \mu _ { \mathrm { w } } \left(\mathrm{~m}_{\mathrm{w}}\left(4 \mathrm{x}_{\mathrm{w}} \mu_{\mathrm{h}}\left(\lambda_{\mathrm{h}}+\mu_{\mathrm{h}}\right)\left(\lambda_{\mathrm{w}}+\mu_{\mathrm{w}}\right)+\mathrm{x}_{\mathrm{h}}\left(\lambda_{\mathrm{h}}+2 \mu_{\mathrm{h}}\right) \mu_{\mathrm{w}}\left(3 \lambda_{\mathrm{w}}+2 \mu_{\mathrm{w}}\right)\right)+\right.\right.\right. \\
& \left.m_{h} \lambda_{h}\left(-2 m_{w} x_{w} \mu_{h}\left(\lambda_{w}+\mu_{w}\right)+x_{h}\left(-2 m_{w} \mu_{w}^{2}+\lambda_{w}\left(\mu_{h}+m_{w} \mu_{h}-3 m_{w} \mu_{w}\right)\right)\right)\right)+ \\
& \kappa_{\mathrm{h}}\left(\mathrm{m}_{\mathrm{w}} \mathrm{x}_{\mathrm{w}} \lambda_{\mathrm{h}} \mu_{\mathrm{w}}\left(-2 \kappa_{\mathrm{w}} \mathrm{x}_{\mathrm{w}} \mu_{\mathrm{h}}\left(\lambda_{\mathrm{w}}+\mu_{\mathrm{w}}\right)+\mathrm{x}_{\mathrm{h}}\left(-2 \kappa_{\mathrm{w}} \mu_{\mathrm{w}}{ }^{2}+\lambda_{\mathrm{w}}\left(\mu_{\mathrm{h}}+\kappa_{\mathrm{w}} \mu_{\mathrm{h}}-3 \kappa_{\mathrm{w}} \mu_{\mathrm{w}}\right)\right)\right)+\right. \\
& \mathrm{m}_{\mathrm{h}}\left(4 \kappa_{\mathrm{w}} \mathrm{m}_{\mathrm{w}} \mathrm{x}_{\mathrm{w}}{ }^{2} \mu_{\mathrm{h}}\left(\lambda_{\mathrm{h}}+\mu_{\mathrm{h}}\right) \mu_{\mathrm{w}}\left(\lambda_{\mathrm{w}}+\mu_{\mathrm{w}}\right)+2 \mathrm{x}_{\mathrm{h}}{ }^{2} \mu_{\mathrm{h}}\left(\lambda_{\mathrm{h}}+\mu_{\mathrm{h}}\right) \mu_{\mathrm{w}}\left(\left(2-\mathrm{m}_{\mathrm{w}}+\kappa_{\mathrm{w}}\left(-1+2 \mathrm{~m}_{\mathrm{w}}\right)\right) \lambda_{\mathrm{w}}+\right.\right. \\
& \left.2\left(\mu_{\mathrm{w}}+\kappa_{\mathrm{w}} \mathrm{m}_{\mathrm{w}} \mu_{\mathrm{w}}\right)\right)+\mathrm{x}_{\mathrm{h}} \mathrm{x}_{\mathrm{w}}\left(2 \mu _ { \mathrm { h } } \left(2 \mu_{\mathrm{w}}\left(\left(1+\mathrm{k}_{\mathrm{w}} \mathrm{m}_{\mathrm{w}}\right) \mu_{\mathrm{h}}{ }^{2}+\kappa_{\mathrm{w}} \mathrm{m}_{\mathrm{w}} \mu_{\mathrm{w}}{ }^{2}\right)+\right.\right. \\
& \left.\lambda_{\mathrm{w}}\left(\left(-1+\kappa_{\mathrm{w}}\right)\left(-1+\mathrm{m}_{\mathrm{w}}\right) \mu_{\mathrm{h}}{ }^{2}+3 \kappa_{\mathrm{w}} \mathrm{m}_{\mathrm{w}} \mu_{\mathrm{w}}{ }^{2}\right)\right)+\lambda_{\mathrm{h}}\left(2 \mu_{\mathrm{w}}\left(3\left(1+\kappa_{\mathrm{w}} \mathrm{m}_{\mathrm{w}}\right) \mu_{\mathrm{h}}{ }^{2}+\kappa_{\mathrm{w}} \mathrm{m}_{\mathrm{w}} \mu_{\mathrm{w}}{ }^{2}\right)+\right. \\
& \left.\left.\left.\left.\left.\left.\lambda_{\mathrm{w}}\left(3\left(-1+\kappa_{\mathrm{w}}\right)\left(-1+\mathrm{m}_{\mathrm{w}}\right) \mu_{\mathrm{h}}^{2}+\left(\kappa_{\mathrm{w}}+\mathrm{m}_{\mathrm{w}}+2 \kappa_{\mathrm{w}} \mathrm{m}_{\mathrm{w}}\right) \mu_{\mathrm{h}} \mu_{\mathrm{w}}+3 \kappa_{\mathrm{w}} \mathrm{m}_{\mathrm{w}} \mu_{\mathrm{w}}^{2}\right)\right)\right)\right)\right)\right)\right) \\
& B_{1, i v}=\frac{1}{Z_{i v}}\left(2 \mu _ { h } \mu _ { w } \left(\left(1+m_{w}\right) x_{w} \lambda_{w}\left(\left(2-m_{h}+\kappa_{h}\left(-1+2 m_{h}\right)\right) \lambda_{h}+2\left(\mu_{h}+\kappa_{h} m_{h} \mu_{h}\right)\right)+\right.\right. \\
& \left.\left.\left(1+m_{h}\right) x_{h} \lambda_{h}\left(\left(2-m_{w}+\kappa_{w}\left(-1+2 m_{w}\right)\right) \lambda_{w}+2\left(\mu_{w}+\kappa_{w} m_{w} \mu_{w}\right)\right)\right)\right) \\
& B_{2, i v}=\frac{1}{Z_{i v}}\left(2 \mu _ { h } \mu _ { w } \left(-\kappa_{w}\left(1+m_{w}\right) x_{w} \lambda_{w}\left(\left(-2+m_{h}\right) \lambda_{h}-2 \mu_{h}\right)+\kappa_{h}\left(\kappa _ { w } ( 1 + m _ { w } ) x _ { w } \lambda _ { w } \left(\left(-1+2 m_{h}\right) \lambda_{h}+\right.\right.\right.\right. \\
& \left.\left.\left.\left.2 m_{h} \mu_{h}\right)+\left(1+m_{h}\right) x_{h} \lambda_{h}\left(\left(2-m_{w}+\kappa_{w}\left(-1+2 m_{w}\right)\right) \lambda_{w}+2\left(\mu_{w}+\kappa_{w} m_{w} \mu_{w}\right)\right)\right)\right)\right)
\end{aligned}
$$




$$
\begin{aligned}
K_{1, i v}= & \frac{2 \mu_{h}}{Z_{i v}}\left(2 x_{h}\left(\lambda_{h}+\mu_{h}\right) \mu_{w}\left(\left(2-m_{w}+\kappa_{w}\left(-1+2 m_{w}\right)\right) \lambda_{w}+2\left(\mu_{w}+\kappa_{w} m_{w} \mu_{w}\right)\right)+\right. \\
& x_{w}\left(2 \mu_{h}^{2}\left(\left(-1+\kappa_{w}\right)\left(-1+m_{w}\right) \lambda_{w}+2\left(\mu_{w}+\kappa_{w} m_{w} \mu_{w}\right)\right)+\right. \\
& \left.\left.\lambda_{h}\left(6\left(1+\kappa_{w} m_{w}\right) \mu_{h} \mu_{w}+\lambda_{w}\left(3\left(-1+\kappa_{w}\right)\left(-1+m_{w}\right) \mu_{h}+\left(1+\kappa_{h}\right)\left(1+m_{w}\right) \mu_{w}\right)\right)\right)\right) \\
K_{2, i v}= & \frac{2 \mu_{h}}{Z_{i v}}\left(\kappa_{w}\left(1+m_{w}\right) x_{w} \lambda_{h} \lambda_{w} \mu_{w}+\kappa_{h}\left(2 x _ { h } ( \lambda _ { h } + \mu _ { h } ) \mu _ { w } \left(\left(2-m_{w}+\kappa_{w}\left(-1+2 m_{w}\right)\right) \lambda_{w}+\right.\right.\right. \\
& \left.2\left(\mu_{w}+\kappa_{w} m_{w} \mu_{w}\right)\right)+x_{w}\left(2 \mu_{h}^{2}\left(\left(-1+\kappa_{w}\right)\left(-1+m_{w}\right) \lambda_{w}+2\left(\mu_{w}+\kappa_{w} m_{w} \mu_{w}\right)\right)+\right. \\
& \left.\left.\left.\lambda_{h}\left(6\left(1+\kappa_{w} m_{w}\right) \mu_{h} \mu_{w}+\lambda_{w}\left(3\left(-1+\kappa_{w}\right)\left(-1+m_{w}\right) \mu_{h}+\kappa_{w}\left(1+m_{w}\right) \mu_{w}\right)\right)\right)\right)\right) \\
K_{3, i v}= & \frac{2 m_{h} \mu_{h}}{Z_{i v}}\left(2 x_{h}\left(\lambda_{h}+\mu_{h}\right) \mu_{w}\left(\left(2-m_{w}+\kappa_{w}\left(-1+2 m_{w}\right)\right) \lambda_{w}+2\left(\mu_{w}+\kappa_{w} m_{w} \mu_{w}\right)\right)+\right. \\
& x_{w}\left(2 \mu_{h}^{2}\left(\left(-1+\kappa_{w}\right)\left(-1+m_{w}\right) \lambda_{w}+2\left(\mu_{w}+\kappa_{w} m_{w} \mu_{w}\right)\right)+\right. \\
& \left.\left.\left(6\left(1+\kappa_{w} m_{w}\right) \mu_{h} \mu_{w}+\lambda_{w}\left(3\left(-1+\kappa_{w}\right)\left(-1+m_{w}\right) \mu_{h}+\left(1+\kappa_{h}\right)\left(1+m_{w}\right) \mu_{w}\right)\right)\right)\right) \\
K_{5, i v}= & \frac{2 \mu_{w}}{Z_{i v}}\left(2 x_{w} \mu_{h}\left(\left(2-m_{h}+\kappa_{h}\left(-1+2 m_{h}\right)\right) \lambda_{h}+2\left(\mu_{h}+\kappa_{h} m_{h} \mu_{h}\right)\right)\left(\lambda_{w}+\mu_{w}\right)+\right. \\
& x_{h}\left(2\left(1+\kappa_{h} m_{h}\right) \mu_{h} \mu_{w}\left(3 \lambda_{w}+2 \mu_{w}\right)+\lambda_{h}\left(2\left(-1+\kappa_{h}\right)\left(-1+m_{h}\right) \mu_{w}^{2}+\right.\right. \\
& \left.\left.\left.\lambda_{w}\left(\left(1+\kappa_{w}\right)\left(1+m_{h}\right) \mu_{h}+3\left(-1+\kappa_{h}\right)\left(-1+m_{h}\right) \mu_{w}\right)\right)\right)\right) \\
K_{4, i v}= & \frac{2 m_{h} \mu_{h}}{Z_{i v}}\left(\kappa_{w}\left(1+m_{w}\right) x_{w} \lambda_{h} \lambda_{w} \mu_{w}+\kappa_{h}\left(2 x _ { h } ( \lambda _ { h } + \mu _ { h } ) \mu _ { w } \left(\left(2-m_{w}+\kappa_{w}\left(-1+2 m_{w}\right)\right) \lambda_{w}+\right.\right.\right. \\
& \left.2\left(\mu_{w}+\kappa_{w} m_{w} \mu_{w}\right)\right)+x_{w}\left(2 \mu_{h}^{2}\left(\left(-1+\kappa_{w}\right)\left(-1+m_{w}\right) \lambda_{w}+2\left(\mu_{w}+\kappa_{w} m_{w} \mu_{w}\right)\right)+\right. \\
& \left.\left.\left.\lambda_{h}\left(6\left(1+\kappa_{w} m_{w}\right) \mu_{h} \mu_{w}+\lambda_{w}\left(3\left(-1+\kappa_{w}\right)\left(-1+m_{w}\right) \mu_{h}+\kappa_{w}\left(1+m_{w}\right) \mu_{w}\right)\right)\right)\right)\right) \\
& \\
&
\end{aligned}
$$

$$
\begin{aligned}
K_{6, i v}= & \frac{2 \mu_{w}}{Z_{i v}}\left(\kappa_{w}\left(-2 x_{w}\left(\left(-2+m_{h}\right) \lambda_{h}-2 \mu_{h}\right) \mu_{h}\left(\lambda_{w}+\mu_{w}\right)-x_{h}\left(\left(-1+m_{h}\right) \lambda_{h}-2 \mu_{h}\right) \mu_{w}\left(3 \lambda_{w}+2 \mu_{w}\right)\right)+\right. \\
& \kappa_{h}\left(2 \kappa_{w} x_{w} \mu_{h}\left(\left(-1+2 m_{h}\right) \lambda_{h}+2 m_{h} \mu_{h}\right)\left(\lambda_{w}+\mu_{w}\right)+x_{h}\left(2 \kappa_{w} m_{h} \mu_{h} \mu_{w}\left(3 \lambda_{w}+2 \mu_{w}\right)+\right.\right. \\
& \left.\left.\left.\lambda_{h}\left(2 \kappa_{w}\left(-1+m_{h}\right) \mu_{w}{ }^{2}+\lambda_{w}\left(\left(1+\kappa_{w}\right)\left(1+m_{h}\right) \mu_{h}+3 \kappa_{w}\left(-1+m_{h}\right) \mu_{w}\right)\right)\right)\right)\right)
\end{aligned}
$$

$$
K_{7, i v}=\frac{2 m_{w} \mu_{w}}{Z_{i v}}\left(2 x_{w} \mu_{h}\left(\left(2-m_{h}+\kappa_{h}\left(-1+2 m_{h}\right)\right) \lambda_{h}+2\left(\mu_{h}+\kappa_{h} m_{h} \mu_{h}\right)\right)\left(\lambda_{w}+\mu_{w}\right)+\right.
$$$$
x_{h}\left(2\left(1+\kappa_{h} m_{h}\right) \mu_{h} \mu_{w}\left(3 \lambda_{w}+2 \mu_{w}\right)+\lambda_{h}\left(2\left(-1+\kappa_{h}\right)\left(-1+m_{h}\right) \mu_{w}^{2}+\right.\right.
$$$$
\left.\left.\left.\lambda_{w}\left(\left(1+\kappa_{w}\right)\left(1+m_{h}\right) \mu_{h}+3\left(-1+\kappa_{h}\right)\left(-1+m_{h}\right) \mu_{w}\right)\right)\right)\right)
$$

$$
\begin{aligned}
K_{8, i v}= & \frac{2 m_{w} \mu_{w}}{Z_{i v}}\left(\kappa_{w}\left(-2 x_{w}\left(\left(-2+m_{h}\right) \lambda_{h}-2 \mu_{h}\right) \mu_{h}\left(\lambda_{w}+\mu_{w}\right)-x_{h}\left(\left(-1+m_{h}\right) \lambda_{h}-2 \mu_{h}\right) \mu_{w}\left(3 \lambda_{w}+2 \mu_{w}\right)\right)+\right. \\
& \kappa_{h}\left(2 \kappa_{w} x_{w} \mu_{h}\left(\left(-1+2 m_{h}\right) \lambda_{h}+2 m_{h} \mu_{h}\right)\left(\lambda_{w}+\mu_{w}\right)+x_{h}\left(2 \kappa_{w} m_{h} \mu_{h} \mu_{w}\left(3 \lambda_{w}+2 \mu_{w}\right)+\right.\right. \\
& \left.\left.\left.\lambda_{h}\left(2 \kappa_{w}\left(-1+m_{h}\right) \mu_{w}^{2}+\lambda_{w}\left(\left(1+\kappa_{w}\right)\left(1+m_{h}\right) \mu_{h}+3 \kappa_{w}\left(-1+m_{h}\right) \mu_{w}\right)\right)\right)\right)\right)
\end{aligned}
$$

\section{where}

$$
\begin{aligned}
Z_{i v}= & x_{w} \mu_{h}\left(\left(2-m_{h}+\kappa_{h}\left(-1+2 m_{h}\right)\right) \lambda_{h}+2\left(\mu_{h}+\kappa_{h} m_{h} \mu_{h}\right)\right)\left(\left(-1+\kappa_{w}\right)\left(-1+m_{w}\right) \lambda_{w}+2\left(\mu_{w}+\kappa_{w} m_{w} \mu_{w}\right)\right) \\
& +x_{h}\left(\left(-1+\kappa_{h}\right)\left(-1+m_{h}\right) \lambda_{h}+2\left(\mu_{h}+\kappa_{h} m_{h} \mu_{h}\right)\right) \mu_{w}\left(\left(2-m_{w}+\kappa_{w}\left(-1+2 m_{w}\right)\right) \lambda_{w}+2\left(\mu_{w}+\kappa_{w} m_{w} \mu_{w}\right)\right) .
\end{aligned}
$$




\section{A.5 Case ii-o. Weak layers yield out-of-plane in triaxial extension, hard layers behave} elastically

$$
\begin{aligned}
A_{1, i i o}= & \frac{1}{Z_{i i i o}}\left(\kappa_{w} m_{w} x_{h}^{2}\left(\lambda_{h}+2 \mu_{h}\right) \mu_{w}\left(3 \lambda_{w}+2 \mu_{w}\right)+x_{w}{ }^{2}\left(\lambda_{h}+2 \mu_{h}\right) \mu_{w}\left(3 \lambda_{w}+2 \mu_{w}\right)+\right. \\
& x_{h} x_{w}\left(4 \mu_{h}^{2}\left(\left(-1+\kappa_{w}\right)\left(-1+m_{w}\right) \lambda_{w}+\mu_{w}+2 \kappa_{w} m_{w} \mu_{w}\right)+\right. \\
& \left.\left.\lambda_{h}\left(2 \mu_{w}\left(\left(2+4 \kappa_{w} m_{w}\right) \mu_{h}+\left(\kappa_{w}+m_{w}\right) \mu_{w}\right)+\lambda_{w}\left(4\left(-1+\kappa_{w}\right)\left(-1+m_{w}\right) \mu_{h}+3\left(\kappa_{w}+m_{w}\right) \mu_{w}\right)\right)\right)\right),
\end{aligned}
$$

$$
\begin{aligned}
A_{2, \text { iio }}= & -\frac{1}{Z_{\text {iiio }}}\left(\kappa_{w} m_{w} x_{h}^{2} \lambda_{h} \mu_{w}\left(3 \lambda_{w}+2 \mu_{w}\right)+\kappa_{w} x_{w}{ }^{2}\left(\lambda_{h}+2 \mu_{h}\right) \mu_{w}\left(3 \lambda_{w}+2 \mu_{w}\right)+\right. \\
& x_{h} x_{w} \lambda_{h}\left(2 \mu_{w}\left(\mu_{h}+2 \kappa_{w} m_{w} \mu_{h}+\kappa_{w}\left(1+m_{w}\right) \mu_{w}\right)+\right. \\
& \left.\left.\lambda_{w}\left(2\left(-1+\kappa_{w}\right)\left(-1+m_{w}\right) \mu_{h}+3 \kappa_{w}\left(1+m_{w}\right) \mu_{w}\right)\right)\right)
\end{aligned}
$$

$$
\begin{aligned}
A_{3, i i o}= & \frac{1}{Z_{\text {iiio }}}\left(\kappa_{w} m_{w} x_{h}{ }^{2} \lambda_{h} \mu_{w}\left(3 \lambda_{w}+2 \mu_{w}\right)+m_{w} x_{w}{ }^{2}\left(\lambda_{h}+2 \mu_{h}\right) \mu_{w}\left(3 \lambda_{w}+2 \mu_{w}\right)+\right. \\
& x_{h} x_{w} \lambda_{h}\left(2 \mu_{w}\left(\mu_{h}+2 \kappa_{w} m_{w} \mu_{h}+\left(1+\kappa_{w}\right) m_{w} \mu_{w}\right)+\right. \\
& \left.\left.\lambda_{w}\left(2\left(-1+\kappa_{w}\right)\left(-1+m_{w}\right) \mu_{h}+3\left(1+\kappa_{w}\right) m_{w} \mu_{w}\right)\right)\right)
\end{aligned}
$$

$$
\begin{aligned}
A_{4, i i o}= & -\frac{1}{Z_{i i i o}}\left(\kappa_{w} m_{w} x_{h}^{2}\left(\lambda_{h}+2 \mu_{h}\right) \mu_{w}\left(3 \lambda_{w}+2 \mu_{w}\right)+\kappa_{w} m_{w} x_{w}^{2}\left(\lambda_{h}+2 \mu_{h}\right) \mu_{w}\left(3 \lambda_{w}+2 \mu_{w}\right)+\right. \\
& 2 x_{h} x_{w}\left(2 \mu_{h}^{2}\left(\left(-1+\kappa_{w}\right)\left(-1+m_{w}\right) \lambda_{w}+\mu_{w}+2 \kappa_{w} m_{w} \mu_{w}\right)+\lambda_{h}\left(2 \mu_{w}\left(\mu_{h}+2 \kappa_{w} m_{w} \mu_{h}+\kappa_{w} m_{w} \mu_{w}\right)+,\right.\right. \\
& \left.\left.\left.\lambda_{w}\left(2\left(-1+\kappa_{w}\right)\left(-1+m_{w}\right) \mu_{h}+3 \kappa_{w} m_{w} \mu_{w}\right)\right)\right)\right)
\end{aligned}
$$$$
B_{1, i i o}=\frac{1}{Z_{i i o}}\left(m_{w}\left(\kappa_{w} x_{h} \lambda_{h}+x_{w}\left(\lambda_{h}+2 \mu_{h}\right)\right) \mu_{w}\left(3 \lambda_{w}+2 \mu_{w}\right)\right),
$$$$
B_{2, i i 0}=\frac{1}{Z_{\text {iio }}}\left(\kappa_{w} m_{w}\left(x_{h} \lambda_{h}+x_{w}\left(\lambda_{h}+2 \mu_{h}\right)\right) \mu_{w}\left(3 \lambda_{w}+2 \mu_{w}\right)\right),
$$

$$
\begin{aligned}
K_{1, i i o} & =\frac{1}{Z_{i i o}}\left(\kappa_{w} m_{w} x_{h}\left(\lambda_{h}+2 \mu_{h}\right) \mu_{w}\left(3 \lambda_{w}+2 \mu_{w}\right)+x_{w}\left(4 \mu _ { h } ^ { 2 } \left(\left(-1+k_{w}\right)\left(-1+m_{w}\right) \lambda_{w}+\right.\right.\right. \\
& \left.\mu_{w}+2 \kappa_{w} m_{w} \mu_{w}\right)+\lambda_{h}\left(2 \mu_{w}\left(\left(2+4 \kappa_{w} m_{w}\right) \mu_{h}+m_{w} \mu_{w}\right)+\right.
\end{aligned}
$$$$
\left.\left.\left.\lambda_{w}\left(4\left(-1+\kappa_{w}\right)\left(-1+m_{w}\right) \mu_{h}+3 m_{w} \mu_{w}\right)\right)\right)\right)
$$

$$
K_{2, i i o}=\frac{1}{Z_{i i o}}\left(\lambda _ { h } \left(\kappa_{w} m_{w} x_{h} \mu_{w}\left(3 \lambda_{w}+2 \mu_{w}\right)+x_{w}\left(2 \mu_{w}\left(\mu_{h}+2 \kappa_{w} m_{w} \mu_{h}+\kappa_{w} m_{w} \mu_{w}\right)+\right.\right.\right.
$$

$$
\left.\left.\left.\lambda_{w}\left(2\left(-1+\kappa_{w}\right)\left(-1+m_{w}\right) \mu_{h}+3 \kappa_{w} m_{w} \mu_{w}\right)\right)\right)\right)
$$

$$
\begin{aligned}
K_{3, i i o}= & \frac{1}{Z_{i i o}}\left(\lambda _ { h } \left(\kappa_{w} m_{w} x_{h} \mu_{w}\left(3 \lambda_{w}+2 \mu_{w}\right)+x_{w}\left(2 \mu_{w}\left(\mu_{h}+2 \kappa_{w} m_{w} \mu_{h}+m_{w} \mu_{w}\right)+\right.\right.\right. \\
& \left.\left.\left.\lambda_{w}\left(2\left(-1+\kappa_{w}\right)\left(-1+m_{w}\right) \mu_{h}+3 m_{w} \mu_{w}\right)\right)\right)\right)
\end{aligned}
$$




$$
\begin{aligned}
K_{4, i i o}= & \frac{1}{Z_{\text {iio }}}\left(\kappa_{w} m_{w} x_{h}\left(\lambda_{h}+2 \mu_{h}\right) \mu_{w}\left(3 \lambda_{w}+2 \mu_{w}\right)+x_{w}\left(4 \mu _ { h } ^ { 2 } \left(\left(-1+\kappa_{w}\right)\left(-1+m_{w}\right) \lambda_{w}+\right.\right.\right. \\
& \left.\mu_{w}+2 \kappa_{w} m_{w} \mu_{w}\right)+\lambda_{h}\left(2 \mu_{w}\left(\left(2+4 \kappa_{w} m_{w}\right) \mu_{h}+\kappa_{w} m_{w} \mu_{w}\right)+\right. \\
& \left.\left.\left.\lambda_{w}\left(4\left(-1+\kappa_{w}\right)\left(-1+m_{w}\right) \mu_{h}+3 \kappa_{w} m_{w} \mu_{w}\right)\right)\right)\right) \\
K_{5, i i o}= & \frac{1}{Z_{i i o}}\left(\left(\kappa_{w} x_{h} \lambda_{h}+x_{w}\left(\lambda_{h}+2 \mu_{h}\right)\right) \mu_{w}\left(3 \lambda_{w}+2 \mu_{w}\right)\right), \\
K_{6, i i o}= & \frac{1}{Z_{i i o}}\left(\kappa_{w}\left(x_{h} \lambda_{h}+x_{w}\left(\lambda_{h}+2 \mu_{h}\right)\right) \mu_{w}\left(3 \lambda_{w}+2 \mu_{w}\right)\right), \\
K_{7, i i o}= & \frac{1}{Z_{\text {iio }}}\left(m_{w}\left(\kappa_{w} x_{h} \lambda_{h}+x_{w}\left(\lambda_{h}+2 \mu_{h}\right)\right) \mu_{w}\left(3 \lambda_{w}+2 \mu_{w}\right)\right), \\
K_{8, i i o}= & \frac{1}{Z_{\text {iio }}}\left(\kappa_{w} m_{w}\left(x_{h} \lambda_{h}+x_{w}\left(\lambda_{h}+2 \mu_{h}\right)\right) \mu_{w}\left(3 \lambda_{w}+2 \mu_{w}\right)\right),
\end{aligned}
$$

where

$$
Z_{i i o}=\kappa_{w} m_{w} x_{h} \mu_{w}\left(3 \lambda_{w}+2 \mu_{w}\right)+x_{w}\left(\lambda_{h}+2 \mu_{h}\right)\left(\left(-1+\kappa_{w}\right)\left(-1+m_{w}\right) \lambda_{w}+\mu_{w}+2 \kappa_{w} m_{w} \mu_{w}\right) .
$$

\section{A.6 Case iii-o. Hard layers yield out-of-plane in triaxial extension, weak layers behave} elastically

$$
\begin{aligned}
A_{1, \text { iiio }}= & \frac{1}{Z_{\text {iiio }}}\left(x_{h}{ }^{2} \mu_{h}\left(3 \lambda_{h}+2 \mu_{h}\right)\left(\lambda_{w}+2 \mu_{w}\right)+\kappa_{h} m_{h} x_{w}{ }^{2} \mu_{h}\left(3 \lambda_{h}+2 \mu_{h}\right)\left(\lambda_{w}+2 \mu_{w}\right)+\right. \\
& x_{h} x_{w}\left(4\left(\lambda_{h}+\mu_{h}\right) \mu_{w}\left(\lambda_{w}+\mu_{w}\right)+m_{h}\left(3 \lambda_{h} \lambda_{w} \mu_{h}+2 \lambda_{w} \mu_{h}{ }^{2}-4 \lambda_{h} \lambda_{w} \mu_{w}-4 \lambda_{h} \mu_{w}{ }^{2}\right)+\right. \\
& \left.\kappa_{h}\left(\lambda_{h}\left(4\left(-1+m_{h}\right) \mu_{w}{ }^{2}+\lambda_{w}\left(3 \mu_{h}+4\left(-1+m_{h}\right) \mu_{w}\right)\right)+2 \mu_{h}\left(4 m_{h} \mu_{w}{ }^{2}+\lambda_{w}\left(\mu_{h}+4 m_{h} \mu_{w}\right)\right)\right)\right), \\
A_{2, \text { iiio }}= & -\frac{1}{Z_{\text {iiio }}}\left(2 x_{h} x_{w} \lambda_{w}\left(\lambda_{h}-m_{h} \lambda_{h}+\mu_{h}\right) \mu_{w}+\kappa_{h}\left(m_{h} x_{w}{ }^{2} \lambda_{w} \mu_{h}\left(3 \lambda_{h}+2 \mu_{h}\right)+\right.\right. \\
& x_{h}{ }^{2} \mu_{h}\left(3 \lambda_{h}+2 \mu_{h}\right)\left(\lambda_{w}+2 \mu_{w}\right)+x_{h} x_{w} \lambda_{w}\left(\lambda _ { h } \left(3\left(1+m_{h}\right) \mu_{h}+\right.\right. \\
& \left.\left.\left.\left.2\left(-1+m_{h}\right) \mu_{w}\right)+2 \mu_{h}\left(\mu_{h}+m_{h} \mu_{h}+2 m_{h} \mu_{w}\right)\right)\right)\right) \\
A_{3, \text { iiio }}= & \frac{1}{Z_{\text {iiio }}}\left(2 x_{h} x_{w} \lambda_{w}\left(\lambda_{h}-\kappa_{h} \lambda_{h}+\mu_{h}\right) \mu_{w}+m_{h}\left(\kappa_{h} x_{w}{ }^{2} \lambda_{w} \mu_{h}\left(3 \lambda_{h}+2 \mu_{h}\right)+\right.\right. \\
& x_{h}{ }^{2} \mu_{h}\left(3 \lambda_{h}+2 \mu_{h}\right)\left(\lambda_{w}+2 \mu_{w}\right)+x_{h} x_{w} \lambda_{w}\left(\lambda _ { h } \left(3\left(1+\kappa_{h}\right) \mu_{h}+\right.\right. \\
& \left.\left.\left.\left.2\left(-1+\kappa_{h}\right) \mu_{w}\right)+2 \mu_{h}\left(\mu_{h}+\kappa_{h} \mu_{h}+2 \kappa_{h} \mu_{w}\right)\right)\right)\right) \\
A_{4, \text { iiio }}= & -\frac{1}{Z_{\text {iiioo }}}\left(-4 x_{h} x_{w}\left(\left(-1+m_{h}\right) \lambda_{h}-\mu_{h}\right) \mu_{w}\left(\lambda_{w}+\mu_{w}\right)+\kappa_{h}\left(-4 x_{h} x_{w} \lambda_{h} \mu_{w}\left(\lambda_{w}+\mu_{w}\right)+\right.\right. \\
& m_{h}\left(x_{h}{ }^{2} \mu_{h}\left(3 \lambda_{h}+2 \mu_{h}\right)\left(\lambda_{w}+2 \mu_{w}\right)+x_{w}{ }^{2} \mu_{h}\left(3 \lambda_{h}+2 \mu_{h}\right)\left(\lambda_{w}+2 \mu_{w}\right)+\right. \\
& \left.\left.\left.2 x_{h} x_{w}\left(2 \mu_{h}\left(\lambda_{w} \mu_{h}+2 \lambda_{w} \mu_{w}+2 \mu_{w}{ }^{2}\right)+\lambda_{h}\left(3 \lambda_{w} \mu_{h}+2 \lambda_{w} \mu_{w}+2 \mu_{w}{ }^{2}\right)\right)\right)\right)\right) \\
& \\
&
\end{aligned}
$$




$$
\begin{aligned}
& B_{1, \text { iiio }}=\frac{1}{Z_{\text {iiio }}}\left(m_{h} \mu_{h}\left(3 \lambda_{h}+2 \mu_{h}\right)\left(\kappa_{h} x_{w} \lambda_{w}+x_{h}\left(\lambda_{w}+2 \mu_{w}\right)\right)\right), \\
& B_{2, \text { iiio }}=\frac{1}{Z_{\text {iiio }}}\left(\kappa_{h} m_{h} \mu_{h}\left(3 \lambda_{h}+2 \mu_{h}\right)\left(x_{w} \lambda_{w}+x_{h}\left(\lambda_{w}+2 \mu_{w}\right)\right)\right), \\
& K_{1, i i i o}=\frac{1}{Z_{\text {iiio }}}\left(\mu_{h}\left(3 \lambda_{h}+2 \mu_{h}\right)\left(\kappa_{h} x_{w} \lambda_{w}+x_{h}\left(\lambda_{w}+2 \mu_{w}\right)\right)\right) \text {, } \\
& K_{2, \text { iiio }}=\frac{1}{Z_{\text {iiio }}}\left(\kappa_{h} \mu_{h}\left(3 \lambda_{h}+2 \mu_{h}\right)\left(x_{w} \lambda_{w}+x_{h}\left(\lambda_{w}+2 \mu_{w}\right)\right)\right), \\
& K_{3, \text { iiio }}=\frac{1}{Z_{\text {iiio }}}\left(m_{h} \mu_{h}\left(3 \lambda_{h}+2 \mu_{h}\right)\left(\kappa_{h} x_{w} \lambda_{w}+x_{h}\left(\lambda_{w}+2 \mu_{w}\right)\right)\right), \\
& K_{4, \text { iiio }}=\frac{1}{Z_{\text {iiio }}}\left(\kappa_{h} m_{h} \mu_{h}\left(3 \lambda_{h}+2 \mu_{h}\right)\left(x_{w} \lambda_{w}+x_{h}\left(\lambda_{w}+2 \mu_{w}\right)\right)\right), \\
& K_{5, \text { iiio }}=\frac{1}{Z_{\text {iiio }}}\left(-4 x_{h}\left(\left(-1+\kappa_{h}\right) \lambda_{h}-\mu_{h}\right) \mu_{w}\left(\lambda_{w}+\mu_{w}\right)+m_{h}\left(\kappa_{h} x_{w} \mu_{h}\left(3 \lambda_{h}+2 \mu_{h}\right)\left(\lambda_{w}+2 \mu_{w}\right)+\right.\right. \\
& \left.\left.x_{h}\left(\lambda_{h}\left(4\left(-1+\kappa_{h}\right) \mu_{w}{ }^{2}+\lambda_{w}\left(3 \mu_{h}+4\left(-1+\kappa_{h}\right) \mu_{w}\right)\right)+2 \mu_{h}\left(4 \kappa_{h} \mu_{w}{ }^{2}+\lambda_{w}\left(\mu_{h}+4 \kappa_{h} \mu_{w}\right)\right)\right)\right)\right) \text {, } \\
& K_{6, \text { iiio }}=\frac{1}{Z_{\text {iiio }}}\left(\lambda _ { w } \left(2 x_{h}\left(\lambda_{h}-m_{h} \lambda_{h}+\mu_{h}\right) \mu_{w}+\kappa_{h}\left(-2 x_{h} \lambda_{h} \mu_{w}+m_{h}\left(x_{w} \mu_{h}\left(3 \lambda_{h}+2 \mu_{h}\right)+\right.\right.\right.\right. \\
& \left.\left.\left.\left.x_{h}\left(3 \lambda_{h} \mu_{h}+2 \mu_{h}^{2}+2 \lambda_{h} \mu_{w}+4 \mu_{h} \mu_{w}\right)\right)\right)\right)\right) \\
& K_{7, \text { iiio }}=\frac{1}{Z_{\text {iiio }}}\left(\lambda _ { w } \left(2 x_{h}\left(\lambda_{h}-\kappa_{h} \lambda_{h}+\mu_{h}\right) \mu_{w}+m_{h}\left(\kappa_{h} x_{w} \mu_{h}\left(3 \lambda_{h}+2 \mu_{h}\right)+\right.\right.\right. \\
& \left.\left.\left.x_{h}\left(\lambda_{h}\left(3 \mu_{h}+2\left(-1+\kappa_{h}\right) \mu_{w}\right)+2 \mu_{h}\left(\mu_{h}+2 \kappa_{h} \mu_{w}\right)\right)\right)\right)\right) \\
& K_{8, \text { iiio }}=\frac{1}{Z_{\text {iiio }}}\left(-4 x_{h}\left(\left(-1+m_{h}\right) \lambda_{h}-\mu_{h}\right) \mu_{w}\left(\lambda_{w}+\mu_{w}\right)+\kappa_{h}\left(-4 x_{h} \lambda_{h} \mu_{w}\left(\lambda_{w}+\mu_{w}\right)+\right.\right. \\
& m_{h}\left(x_{w} \mu_{h}\left(3 \lambda_{h}+2 \mu_{h}\right)\left(\lambda_{w}+2 \mu_{w}\right)+x_{h}\left(2 \mu_{h}\left(\lambda_{w} \mu_{h}+4 \lambda_{w} \mu_{w}+4 \mu_{w}{ }^{2}\right)+\right.\right. \\
& \left.\left.\left.\left.\lambda_{h}\left(3 \lambda_{w} \mu_{h}+4 \lambda_{w} \mu_{w}+4 \mu_{w}^{2}\right)\right)\right)\right)\right)
\end{aligned}
$$

\section{where}

$Z_{\text {iiio }}=\left(\kappa_{h} m_{h} x_{w} \mu_{h}\left(3 \lambda_{h}+2 \mu_{h}\right)+x_{h}\left(\left(-1+\kappa_{h}\right)\left(-1+m_{h}\right) \lambda_{h}+\mu_{h}+2 \kappa_{h} m_{h} \mu_{h}\right)\left(\lambda_{w}+2 \mu_{w}\right)\right)$. 


\section{A.7 Case iv-01. Weak layers yield out-of-plane in triaxial extension, hard layers yield} in-plane

$$
\begin{aligned}
A_{1, \text { ivo } 1}= & \frac{1}{Z_{\text {ivo } 1}}\left(2 \mu _ { h } \left(2 \kappa_{w} m_{w} x_{h}{ }^{2}\left(\lambda_{h}+\mu_{h}\right) \mu_{w}\left(3 \lambda_{w}+2 \mu_{w}\right)+x_{w}{ }^{2}\left(\left(2-m_{h}+\kappa_{h}\left(-1+2 m_{h}\right)\right) \lambda_{h}+\right.\right.\right. \\
& \left.2\left(\mu_{h}+\kappa_{h} m_{h} \mu_{h}\right)\right) \mu_{w}\left(3 \lambda_{w}+2 \mu_{w}\right)+x_{h} x_{w}\left(4 \mu_{h}{ }^{2}\left(\left(-1+\kappa_{w}\right)\left(-1+m_{w}\right) \lambda_{w}+\mu_{w}+2 \kappa_{w} m_{w} \mu_{w}\right)\right. \\
& +\lambda_{h}\left(3 \lambda_{w}\left(2\left(-1+\kappa_{w}\right)\left(-1+m_{w}\right) \mu_{h}+\left(\kappa_{w}+\kappa_{w} m_{h}+m_{w}+\kappa_{h} m_{w}\right) \mu_{w}\right)+\right. \\
& \left.\left.\left.\left.2 \mu_{w}\left(\left(3+6 \kappa_{w} m_{w}\right) \mu_{h}+\left(\kappa_{w}+\kappa_{w} m_{h}+m_{w}+\kappa_{h} m_{w}\right) \mu_{w}\right)\right)\right)\right)\right) \\
A_{2, \text { ivo } 1}= & -\frac{1}{Z_{i v 01}}\left(\left(2 \mu _ { h } \left(\kappa_{w} x_{w}\left(m_{w} x_{h} \lambda_{h}+x_{w}\left(-\left(-2+m_{h}\right) \lambda_{h}+2 \mu_{h}\right)\right) \mu_{w}\left(3 \lambda_{w}+2 \mu_{w}\right)+\right.\right.\right. \\
& \kappa_{h}\left(2 \kappa_{w} m_{w} x_{h}^{2}\left(\lambda_{h}+\mu_{h}\right) \mu_{w}\left(3 \lambda_{w}+2 \mu_{w}\right)+\kappa_{w} x_{w}{ }^{2}\left(\left(-1+2 m_{h}\right) \lambda_{h}+2 m_{h} \mu_{h}\right) \mu_{w}\left(3 \lambda_{w}+2 \mu_{w}\right)+\right. \\
& x_{h} x_{w}\left(4 \mu_{h}^{2}\left(\left(-1+\kappa_{w}\right)\left(-1+m_{w}\right) \lambda_{w}+\mu_{w}+2 \kappa_{w} m_{w} \mu_{w}\right)+\lambda_{h}\left(3 \lambda _ { w } \left(2\left(-1+k_{w}\right)\left(-1+m_{w}\right) \mu_{h}+\right.\right.\right. \\
& \left.\left.\left.\left.\left.\left.\kappa_{w}\left(1+m_{h}+m_{w}\right) \mu_{w}\right)+2 \mu_{w}\left(\left(3+6 \kappa_{w} m_{w}\right) \mu_{h}+\kappa_{w}\left(1+m_{h}+m_{w}\right) \mu_{w}\right)\right)\right)\right)\right)\right)
\end{aligned}
$$

$$
\begin{aligned}
A_{3, \text { iv0 } 1}= & \frac{1}{Z_{\text {ivo } 1}}\left(2 \mu _ { h } \left(m_{w} x_{w}\left(\kappa_{w} x_{h} \lambda_{h}+x_{w}\left(-\left(-2+\kappa_{h}\right) \lambda_{h}+2 \mu_{h}\right)\right) \mu_{w}\left(3 \lambda_{w}+2 \mu_{w}\right)+\right.\right. \\
& m_{h}\left(2 \kappa_{w} m_{w} x_{h}^{2}\left(\lambda_{h}+\mu_{h}\right) \mu_{w}\left(3 \lambda_{w}+2 \mu_{w}\right)+m_{w} x_{w}^{2}\left(\left(-1+2 \kappa_{h}\right) \lambda_{h}+2 \kappa_{h} \mu_{h}\right) \mu_{w}\left(3 \lambda_{w}+2 \mu_{w}\right)+\right. \\
& x_{h} x_{w}\left(4 \mu_{h}^{2}\left(\left(-1+\kappa_{w}\right)\left(-1+m_{w}\right) \lambda_{w}+\mu_{w}+2 \kappa_{w} m_{w} \mu_{w}\right)+\lambda_{h}\left(3 \lambda _ { w } \left(2\left(-1+k_{w}\right)\left(-1+m_{w}\right) \mu_{h}+\right.\right.\right. \\
& \left.\left.\left.\left.\left.\left.\left(1+\kappa_{h}+k_{w}\right) m_{w} \mu_{w}\right)+2 \mu_{w}\left(\left(3+6 \kappa_{w} m_{w}\right) \mu_{h}+\left(1+\kappa_{h}+\kappa_{w}\right) m_{w} \mu_{w}\right)\right)\right)\right)\right)\right)
\end{aligned}
$$

$$
\begin{aligned}
A_{4, \text { iv } 1}= & -\frac{1}{Z_{i v 01}}\left(2 \mu _ { h } \left(\kappa_{w} m_{w} x_{w}\left(m_{h}\left(x_{h}-x_{w}\right) \lambda_{h}+2 x_{w}\left(\lambda_{h}+\mu_{h}\right)\right) \mu_{w}\left(3 \lambda_{w}+2 \mu_{w}\right)+\right.\right. \\
& \kappa_{h}\left(\kappa_{w} m_{w}\left(x_{h}-x_{w}\right) x_{w} \lambda_{h} \mu_{w}\left(3 \lambda_{w}+2 \mu_{w}\right)+2 m_{h}\left(\kappa_{w} m_{w} x_{h}^{2}\left(\lambda_{h}+\mu_{h}\right) \mu_{w}\left(3 \lambda_{w}+2 \mu_{w}\right)+\right.\right. \\
& \kappa_{w} m_{w} x_{w}{ }^{2}\left(\lambda_{h}+\mu_{h}\right) \mu_{w}\left(3 \lambda_{w}+2 \mu_{w}\right)+x_{h} x_{w}\left(2 \mu_{h}^{2}\left(\left(-1+\kappa_{w}\right)\left(-1+m_{w}\right) \lambda_{w}+\mu_{w}+2 \kappa_{w} m_{w} \mu_{w}\right)+\right. \\
& \left.\left.\left.\left.\lambda_{h}\left(3 \lambda_{w}\left(\left(-1+\kappa_{w}\right)\left(-1+m_{w}\right) \mu_{h}+\kappa_{w} m_{w} \mu_{w}\right)+\mu_{w}\left(\left(3+6 \kappa_{w} m_{w}\right) \mu_{h}+2 \kappa_{w} m_{w} \mu_{w}\right)\right)\right)\right)\right)\right)
\end{aligned}
$$

$$
\begin{aligned}
B_{1, i v 01}= & \frac{1}{Z_{\text {ivo1 }}}\left(2 m _ { w } \mu _ { h } \left(\kappa_{w}\left(1+m_{h}\right) x_{h} \lambda_{h}+x_{w}\left(\left(2-\kappa_{h}-m_{h}+2 \kappa_{h} m_{h}\right) \lambda_{h}+\right.\right.\right. \\
& \left.\left.\left.2\left(\mu_{h}+\kappa_{h} m_{h} \mu_{h}\right)\right)\right) \mu_{w}\left(3 \lambda_{w}+2 \mu_{w}\right)\right)
\end{aligned}
$$

$$
\begin{aligned}
B_{2, \text { vo } 1}= & \frac{1}{Z_{\text {ivo } 1}}\left(2 \kappa _ { w } m _ { w } \mu _ { h } \left(x_{w}\left(-\left(-2+m_{h}\right) \lambda_{h}+2 \mu_{h}\right)+\kappa_{h}\left(\left(1+m_{h}\right) x_{h} \lambda_{h}+\right.\right.\right. \\
& \left.\left.\left.x_{w}\left(-\lambda_{h}+2 m_{h} \lambda_{h}+2 m_{h} \mu_{h}\right)\right)\right) \mu_{w}\left(3 \lambda_{w}+2 \mu_{w}\right)\right)
\end{aligned},
$$

$$
\begin{aligned}
K_{1, \text { ivo } 1}= & \frac{1}{Z_{i v 01}}\left(2 \mu _ { h } \left(2 \kappa_{w} m_{w} x_{h}\left(\lambda_{h}+\mu_{h}\right) \mu_{w}\left(3 \lambda_{w}+2 \mu_{w}\right)\right.\right. \\
& +x_{w}\left(4 \mu_{h}^{2}\left(\left(-1+\kappa_{w}\right)\left(-1+m_{w}\right) \lambda_{w}+\mu_{w}+2 \kappa_{w} m_{w} \mu_{w}\right)+\lambda_{h}\left(3 \lambda _ { w } \left(2\left(-1+\kappa_{w}\right)\left(-1+m_{w}\right) \mu_{h}+\right.\right.\right. \\
& \left.\left.\left.\left.\left.\left(1+\kappa_{h}\right) m_{w} \mu_{w}\right)+2 \mu_{w}\left(\left(3+6 \kappa_{w} m_{w}\right) \mu_{h}+\left(1+\kappa_{h}\right) m_{w} \mu_{w}\right)\right)\right)\right)\right)
\end{aligned}
$$




$$
\begin{aligned}
K_{2, \text { ivo } 1}= & \frac{1}{Z_{\text {ivo } 1}}\left(2 \mu _ { h } \left(\kappa_{w} m_{w} x_{w} \lambda_{h} \mu_{w}\left(3 \lambda_{w}+2 \mu_{w}\right)+\kappa_{h}\left(2 \kappa_{w} m_{w} x_{h}\left(\lambda_{h}+\mu_{h}\right) \mu_{w}\left(3 \lambda_{w}+2 \mu_{w}\right)+\right.\right.\right. \\
& x_{w}\left(4 \mu_{h}^{2}\left(\left(-1+\kappa_{w}\right)\left(-1+m_{w}\right) \lambda_{w}+\mu_{w}+2 \kappa_{w} m_{w} \mu_{w}\right)+\lambda_{h}\left(3 \lambda _ { w } \left(2\left(-1+\kappa_{w}\right)\left(-1+m_{w}\right) \mu_{h}\right.\right.\right. \\
& \left.\left.\left.\left.\left.\left.+\kappa_{w} m_{w} \mu_{w}\right)+2 \mu_{w}\left(\left(3+6 \kappa_{w} m_{w}\right) \mu_{h}+\kappa_{w} m_{w} \mu_{w}\right)\right)\right)\right)\right)\right) \\
K_{3, i v 01} & \frac{1}{Z_{i v 01}}\left(2 m _ { h } \mu _ { h } \left(2 \kappa_{w} m_{w} x_{h}\left(\lambda_{h}+\mu_{h}\right) \mu_{w}\left(3 \lambda_{w}+2 \mu_{w}\right)+x_{w}\left(4 \mu _ { h } ^ { 2 } \left(\left(-1+\kappa_{w}\right)\left(-1+m_{w}\right) \lambda_{w}+\right.\right.\right.\right. \\
& \left.\mu_{w}+2 \kappa_{w} m_{w} \mu_{w}\right)+\lambda_{h}\left(3 \lambda_{w}\left(2\left(-1+\kappa_{w}\right)\left(-1+m_{w}\right) \mu_{h}+\left(1+\kappa_{h}\right) m_{w} \mu_{w}\right)+\right. \\
& \left.\left.\left.\left.2 \mu_{w}\left(\left(3+6 \kappa_{w} m_{w}\right) \mu_{h}+\left(1+\kappa_{h}\right) m_{w} \mu_{w}\right)\right)\right)\right)\right)
\end{aligned}
$$

$$
\begin{aligned}
K_{4, \text { ivo } 1}= & \frac{1}{Z_{i v 01}}\left(2 m _ { h } \mu _ { h } \left(\kappa_{w} m_{w} x_{w} \lambda_{h} \mu_{w}\left(3 \lambda_{w}+2 \mu_{w}\right)+\kappa_{h}\left(2 \kappa_{w} m_{w} x_{h}\left(\lambda_{h}+\mu_{h}\right) \mu_{w}\left(3 \lambda_{w}+2 \mu_{w}\right)+\right.\right.\right. \\
& x_{w}\left(4 \mu_{h}^{2}\left(\left(-1+\kappa_{w}\right)\left(-1+m_{w}\right) \lambda_{w}+\mu_{w}+2 \kappa_{w} m_{w} \mu_{w}\right)+\lambda_{h}\left(3 \lambda _ { w } \left(2\left(-1+\kappa_{w}\right)\left(-1+m_{w}\right) \mu_{h}+\right.\right.\right.
\end{aligned}
$$$$
\left.\left.\left.\left.\left.\left.\kappa_{w} m_{w} \mu_{w}\right)+2 \mu_{w}\left(\left(3+6 \kappa_{w} m_{w}\right) \mu_{h}+\kappa_{w} m_{w} \mu_{w}\right)\right)\right)\right)\right)\right)
$$

$$
K_{5, \text { ivo } 1}=\frac{1}{Z_{\text {ivo } 1}}\left(2 \mu _ { h } \left(\kappa_{w}\left(1+m_{h}\right) x_{h} \lambda_{h}+x_{w}\left(\left(2-\kappa_{h}-m_{h}+2 \kappa_{h} m_{h}\right) \lambda_{h}+\right.\right.\right.
$$$$
\left.\left.\left.2\left(\mu_{h}+\kappa_{h} m_{h} \mu_{h}\right)\right)\right) \mu_{w}\left(3 \lambda_{w}+2 \mu_{w}\right)\right)
$$

$$
\begin{gathered}
K_{6, \text { ivo } 1}=\frac{1}{Z_{\text {ivo } 1}}\left(2 \kappa _ { w } \mu _ { h } \left(x_{w}\left(-\left(-2+m_{h}\right) \lambda_{h}+2 \mu_{h}\right)+\kappa_{h}\left(\left(1+m_{h}\right) x_{h} \lambda_{h}+\right.\right.\right. \\
\left.\left.\left.x_{w}\left(-\lambda_{h}+2 m_{h} \lambda_{h}+2 m_{h} \mu_{h}\right)\right)\right) \mu_{w}\left(3 \lambda_{w}+2 \mu_{w}\right)\right)
\end{gathered},
$$

$$
\begin{aligned}
K_{7, \text { ivo } 1}= & \frac{1}{Z_{\text {ivo } 1}}\left(2 m _ { w } \mu _ { h } \left(\kappa_{w}\left(1+m_{h}\right) x_{h} \lambda_{h}+x_{w}\left(\left(2-\kappa_{h}-m_{h}+2 \kappa_{h} m_{h}\right) \lambda_{h}+\right.\right.\right. \\
& \left.\left.\left.2\left(\mu_{h}+\kappa_{h} m_{h} \mu_{h}\right)\right)\right) \mu_{w}\left(3 \lambda_{w}+2 \mu_{w}\right)\right)
\end{aligned},
$$

$$
\begin{aligned}
K_{8, \text { ivo } 1}= & \frac{1}{Z_{\text {ivo } 1}}\left(2 \kappa _ { w } m _ { w } \mu _ { h } \left(x_{w}\left(-\left(-2+m_{h}\right) \lambda_{h}+2 \mu_{h}\right)+\kappa_{h}\left(\left(1+m_{h}\right) x_{h} \lambda_{h}+\right.\right.\right. \\
& \left.\left.\left.x_{w}\left(-\lambda_{h}+2 m_{h} \lambda_{h}+2 m_{h} \mu_{h}\right)\right)\right) \mu_{w}\left(3 \lambda_{w}+2 \mu_{w}\right)\right)
\end{aligned}
$$

\section{where}

$$
\begin{aligned}
Z_{i v 01}= & \left(\kappa_{w} m_{w} x_{h}\left(\left(-1+\kappa_{h}\right)\left(-1+m_{h}\right) \lambda_{h}+2\left(\mu_{h}+\kappa_{h} m_{h} \mu_{h}\right)\right) \mu_{w}\left(3 \lambda_{w}+2 \mu_{w}\right)+\right. \\
& 2 x_{w} \mu_{h}\left(\left(2-m_{h}+\kappa_{h}\left(-1+2 m_{h}\right)\right) \lambda_{h}+\right. \\
& \left.\left.2\left(\mu_{h}+\kappa_{h} m_{h} \mu_{h}\right)\right)\left(\left(-1+\kappa_{w}\right)\left(-1+m_{w}\right) \lambda_{w}+\mu_{w}+2 \kappa_{w} m_{w} \mu_{w}\right)\right)
\end{aligned}
$$




\section{A.8 Case iv-02. Weak layers yield in-plane, hard layers yield out-of-plane in triaxial} extension

$$
\begin{aligned}
A_{1, \text { ivo } 2}= & \frac{1}{Z_{\text {ivo } 2}}\left(2 \mu _ { w } \left(2 \kappa_{h} m_{h} x_{w}{ }^{2} \mu_{h}\left(3 \lambda_{h}+2 \mu_{h}\right)\left(\lambda_{w}+\mu_{w}\right)+x_{h}{ }^{2} \mu_{h}\left(3 \lambda_{h}+2 \mu_{h}\right)\left(\left(2-m_{w}+\right.\right.\right.\right. \\
& \left.\left.\kappa_{w}\left(-1+2 m_{w}\right)\right) \lambda_{w}+2\left(\mu_{w}+\kappa_{w} m_{w} \mu_{w}\right)\right)+x_{h} x_{w}\left(2\left(\lambda_{h}+\mu_{h}\right) \mu_{w}\left(3 \lambda_{w}+2 \mu_{w}\right)+\right. \\
& m_{h}\left(2\left(1+\kappa_{w}\right) \lambda_{w} \mu_{h}{ }^{2}+\lambda_{h}\left(3 \lambda_{w}\left(\mu_{h}+\kappa_{w} \mu_{h}-2 \mu_{w}\right)-4 \mu_{w}{ }^{2}\right)\right)+\kappa_{h}\left(\lambda _ { h } \left(4\left(-1+m_{h}\right) \mu_{w}{ }^{2}+\right.\right. \\
& \left.\left.\left.\left.\left.3 \lambda_{w}\left(\mu_{h}+m_{w} \mu_{h}-2 \mu_{w}+2 m_{h} \mu_{w}\right)\right)+2 \mu_{h}\left(4 m_{h} \mu_{w}{ }^{2}+\lambda_{w}\left(\mu_{h}+m_{w} \mu_{h}+6 m_{h} \mu_{w}\right)\right)\right)\right)\right)\right), \\
A_{2, \text { ivo } 2}= & -\frac{1}{Z_{i v o 2}}\left(2 \mu _ { w } \left(-2 \kappa_{w} x_{h} x_{w}\left(\left(-1+m_{h}\right) \lambda_{h}-\mu_{h}\right) \mu_{w}\left(3 \lambda_{w}+2 \mu_{w}\right)+\right.\right. \\
& \kappa_{h}\left(2 \kappa_{w} m_{h} x_{w}{ }^{2} \mu_{h}\left(3 \lambda_{h}+2 \mu_{h}\right)\left(\lambda_{w}+\mu_{w}\right)+x_{h}{ }^{2} \mu_{h}\left(3 \lambda_{h}+2 \mu_{h}\right)\left(\left(2-m_{w}+\kappa_{w}\left(-1+2 m_{w}\right)\right) \lambda_{w}+\right.\right. \\
& \left.2\left(\mu_{w}+\kappa_{w} m_{w} \mu_{w}\right)\right)+x_{h} x_{w}\left(m_{h} \lambda_{w} \mu_{h}\left(3 \lambda_{h}+2 \mu_{h}\right)+\kappa_{w}\left(\lambda _ { h } \left(4\left(-1+m_{h}\right) \mu_{w}{ }^{2}+\right.\right.\right. \\
& \left.\left.\left.\left.\left.\left.3 \lambda_{w}\left(\left(1+m_{h}+m_{w}\right) \mu_{h}+2\left(-1+m_{h}\right) \mu_{w}\right)\right)+2 \mu_{h}\left(4 m_{h} \mu_{w}{ }^{2}+\lambda_{w}\left(\left(1+m_{h}+m_{w}\right) \mu_{h}+6 m_{h} \mu_{w}\right)\right)\right)\right)\right)\right)\right),
\end{aligned}
$$

$$
\begin{aligned}
A_{3, \text { ivo } 2}= & \frac{1}{Z_{\text {ivo } 2}}\left(2 \mu _ { w } \left(-2 m_{w} x_{h} x_{w}\left(\left(-1+\kappa_{h}\right) \lambda_{h}-\mu_{h}\right) \mu_{w}\left(3 \lambda_{w}+2 \mu_{w}\right)+m_{h}\left(2 \kappa _ { h } m _ { w } x _ { w } { } ^ { 2 } \mu _ { h } \left(3 \lambda_{h}+\right.\right.\right.\right. \\
& \left.2 \mu_{h}\right)\left(\lambda_{w}+\mu_{w}\right)+x_{h}{ }^{2} \mu_{h}\left(3 \lambda_{h}+2 \mu_{h}\right)\left(\left(2-m_{w}+\kappa_{w}\left(-1+2 m_{w}\right)\right) \lambda_{w}+2\left(\mu_{w}+\kappa_{w} m_{w} \mu_{w}\right)\right)+ \\
& x_{h} x_{w}\left(m_{w}\left(2\left(1+\kappa_{w}\right) \lambda_{w} \mu_{h}{ }^{2}+\lambda_{h}\left(3 \lambda_{w}\left(\mu_{h}+\kappa_{w} \mu_{h}-2 \mu_{w}\right)-4 \mu_{w}{ }^{2}\right)\right)+\kappa_{h}\left(\lambda _ { h } \left(4 m_{w} \mu_{w}{ }^{2}+\right.\right.\right. \\
& \left.\left.\left.\left.\left.\left.3 \lambda_{w}\left(\mu_{h}+m_{w} \mu_{h}+2 m_{w} \mu_{w}\right)\right)+2 \mu_{h}\left(4 m_{w} \mu_{w}{ }^{2}+\lambda_{w}\left(\mu_{h}+m_{w} \mu_{h}+6 m_{w} \mu_{w}\right)\right)\right)\right)\right)\right)\right)
\end{aligned}
$$

$$
\begin{aligned}
A_{4, \text { ivo } 2}= & -\frac{1}{Z_{i v 02}}\left(2 \mu _ { w } \left(-2 \kappa_{w} m_{w} x_{h} x_{w}\left(\left(-1+m_{h}\right) \lambda_{h}-\mu_{h}\right) \mu_{w}\left(3 \lambda_{w}+2 \mu_{w}\right)+\right.\right. \\
& \kappa_{h}\left(-2 \kappa_{w} m_{w} x_{h} x_{w} \lambda_{h} \mu_{w}\left(3 \lambda_{w}+2 \mu_{w}\right)+m_{h}\left(2 \kappa_{w} m_{w} x_{w}{ }^{2} \mu_{h}\left(3 \lambda_{h}+2 \mu_{h}\right)\left(\lambda_{w}+\mu_{w}\right)+\right.\right. \\
& x_{h}{ }^{2} \mu_{h}\left(3 \lambda_{h}+2 \mu_{h}\right)\left(\left(2-m_{w}+\kappa_{w}\left(-1+2 m_{w}\right)\right) \lambda_{w}+2\left(\mu_{w}+\kappa_{w} m_{w} \mu_{w}\right)\right)+ \\
& x_{h} x_{w}\left(m_{w} \lambda_{w} \mu_{h}\left(3 \lambda_{h}+2 \mu_{h}\right)+\kappa_{w}\left(\lambda_{h}\left(4 m_{w} \mu_{w}{ }^{2}+3 \lambda_{w}\left(\mu_{h}+2 m_{w} \mu_{h}+2 m_{w} \mu_{w}\right)\right)+\right.\right. \\
& \left.\left.\left.\left.\left.2 \mu_{h}\left(4 m_{w} \mu_{w}{ }^{2}+\lambda_{w}\left(\mu_{h}+2 m_{w} \mu_{h}+6 m_{w} \mu_{w}\right)\right)\right)\right)\right)\right)\right)
\end{aligned}
$$

$$
\begin{aligned}
B_{1, \text { ivo } 2}= & \frac{1}{Z_{i v 02}}\left(2 m _ { h } \mu _ { h } ( 3 \lambda _ { h } + 2 \mu _ { h } ) \mu _ { w } \left(\kappa_{h}\left(1+m_{w}\right) x_{w} \lambda_{w}+\right.\right. \\
& \left.\left.x_{h}\left(\left(2-k_{w}-m_{w}+2 \kappa_{w} m_{w}\right) \lambda_{w}+2\left(\mu_{w}+\kappa_{w} m_{w} \mu_{w}\right)\right)\right)\right),
\end{aligned}
$$

$$
\begin{aligned}
B_{2, \text { vo } 2}= & \frac{1}{Z_{\text {ivo2 }}}\left(2 \kappa _ { h } m _ { h } \mu _ { h } ( 3 \lambda _ { h } + 2 \mu _ { h } ) \mu _ { w } \left(\kappa_{w}\left(1+m_{w}\right) x_{w} \lambda_{w}+\right.\right. \\
& \left.\left.x_{h}\left(\left(2-k_{w}-m_{w}+2 \kappa_{w} m_{w}\right) \lambda_{w}+2\left(\mu_{w}+\kappa_{w} m_{w} \mu_{w}\right)\right)\right)\right),
\end{aligned}
$$$$
K_{1, \text { ivo } 2}=\frac{1}{Z_{\text {ivo } 2}}\left(2 \mu _ { h } ( 3 \lambda _ { h } + 2 \mu _ { h } ) \mu _ { w } \left(\kappa_{h}\left(1+m_{w}\right) x_{w} \lambda_{w}+\right.\right.
$$$$
\left.\left.x_{h}\left(\left(2-k_{w}-m_{w}+2 \kappa_{w} m_{w}\right) \lambda_{w}+2\left(\mu_{w}+\kappa_{w} m_{w} \mu_{w}\right)\right)\right)\right),
$$

$$
\begin{aligned}
K_{2, \text { ivo } 2}= & \frac{1}{Z_{\text {ivo } 2}}\left(2 \kappa _ { h } \mu _ { h } ( 3 \lambda _ { h } + 2 \mu _ { h } ) \mu _ { w } \left(\kappa_{w}\left(1+m_{w}\right) x_{w} \lambda_{w}+\right.\right. \\
& \left.\left.x_{h}\left(\left(2-\kappa_{w}-m_{w}+2 \kappa_{w} m_{w}\right) \lambda_{w}+2\left(\mu_{w}+\kappa_{w} m_{w} \mu_{w}\right)\right)\right)\right)
\end{aligned}
$$




$$
\begin{aligned}
K_{3, \text { ivo } 2}= & \frac{1}{Z_{\text {ivo2 }}}\left(2 m _ { h } \mu _ { h } ( 3 \lambda _ { h } + 2 \mu _ { h } ) \mu _ { w } \left(\kappa_{h}\left(1+m_{w}\right) x_{w} \lambda_{w}+\right.\right. \\
& \left.\left.x_{h}\left(\left(2-\kappa_{w}-m_{w}+2 \kappa_{w} m_{w}\right) \lambda_{w}+2\left(\mu_{w}+\kappa_{w} m_{w} \mu_{w}\right)\right)\right)\right)
\end{aligned}
$$

$$
\begin{aligned}
K_{4, \text { ivo } 2}= & \frac{1}{Z_{\text {ivo2 }}}\left(2 \kappa _ { h } m _ { h } \mu _ { h } ( 3 \lambda _ { h } + 2 \mu _ { h } ) \mu _ { w } \left(\kappa_{w}\left(1+m_{w}\right) x_{w} \lambda_{w}+\right.\right. \\
& \left.\left.x_{h}\left(\left(2-\kappa_{w}-m_{w}+2 \kappa_{w} m_{w}\right) \lambda_{w}+2\left(\mu_{w}+\kappa_{w} m_{w} \mu_{w}\right)\right)\right)\right)
\end{aligned}
$$

$$
K_{5, \text { ivo } 2}=\frac{1}{Z_{\text {ivo } 2}}\left(2 \mu _ { w } \left(-2 x_{h}\left(\left(-1+\kappa_{h}\right) \lambda_{h}-\mu_{h}\right) \mu_{w}\left(3 \lambda_{w}+2 \mu_{w}\right)+m_{h}\left(2 \kappa _ { h } x _ { w } \mu _ { h } \left(3 \lambda_{h}+\right.\right.\right.\right.
$$

$$
\begin{aligned}
& \left.2 \mu_{h}\right)\left(\lambda_{w}+\mu_{w}\right)+x_{h}\left(\lambda_{h}\left(4\left(-1+\kappa_{h}\right) \mu_{w}{ }^{2}+3 \lambda_{w}\left(\mu_{h}+\kappa_{w} \mu_{h}-2 \mu_{w}+2 \kappa_{h} \mu_{w}\right)\right)+\right. \\
& \left.\left.\left.\left.2 \mu_{h}\left(4 \kappa_{h} \mu_{w}{ }^{2}+\lambda_{w}\left(\mu_{h}+\kappa_{w} \mu_{h}+6 \kappa_{h} \mu_{w}\right)\right)\right)\right)\right)\right)
\end{aligned}
$$

$$
\begin{aligned}
K_{6, \text { ivo } 2}= & \frac{1}{Z_{\text {ivo }}}\left(2 \mu _ { w } \left(-2 \kappa_{w} x_{h}\left(\left(-1+m_{h}\right) \lambda_{h}-\mu_{h}\right) \mu_{w}\left(3 \lambda_{w}+2 \mu_{w}\right)+\kappa_{h}\left(-2 \kappa_{w} x_{h} \lambda_{h} \mu_{w}\left(3 \lambda_{w}+\right.\right.\right.\right. \\
& \left.2 \mu_{w}\right)+m_{h}\left(2 \kappa_{w} x_{w} \mu_{h}\left(3 \lambda_{h}+2 \mu_{h}\right)\left(\lambda_{w}+\mu_{w}\right)+x_{h}\left(\lambda _ { h } \left(4 \kappa_{w} \mu_{w}{ }^{2}+3 \lambda_{w}\left(\mu_{h}+\kappa_{w} \mu_{h}+\right.\right.\right.\right. \\
& \left.\left.\left.\left.\left.\left.\left.2 \kappa_{w} \mu_{w}\right)\right)+2 \mu_{h}\left(4 \kappa_{w} \mu_{w}{ }^{2}+\lambda_{w}\left(\mu_{h}+\kappa_{w} \mu_{h}+6 \kappa_{w} \mu_{w}\right)\right)\right)\right)\right)\right)\right)
\end{aligned}
$$

$$
\begin{aligned}
K_{7, \text { ivo } 2}= & \frac{1}{Z_{\text {ivo } 2}}\left(2 m _ { w } \mu _ { w } \left(-2 x_{h}\left(\left(-1+\kappa_{h}\right) \lambda_{h}-\mu_{h}\right) \mu_{w}\left(3 \lambda_{w}+2 \mu_{w}\right)+m_{h}\left(2 \kappa _ { h } x _ { w } \mu _ { h } \left(3 \lambda_{h}+\right.\right.\right.\right. \\
& \left.2 \mu_{h}\right)\left(\lambda_{w}+\mu_{w}\right)+x_{h}\left(\lambda_{h}\left(4\left(-1+\kappa_{h}\right) \mu_{w}^{2}+3 \lambda_{w}\left(\mu_{h}+\kappa_{w} \mu_{h}-2 \mu_{w}+2 \kappa_{h} \mu_{w}\right)\right)+\right. \\
& \left.\left.\left.\left.2 \mu_{h}\left(4 \kappa_{h} \mu_{w}^{2}+\lambda_{w}\left(\mu_{h}+\kappa_{w} \mu_{h}+6 \kappa_{h} \mu_{w}\right)\right)\right)\right)\right)\right)
\end{aligned}
$$

$$
\begin{aligned}
K_{8, \text { ivo } 2}= & \frac{1}{Z_{\text {ivo } 2}}\left(2 m _ { w } \mu _ { w } \left(-2 \kappa_{w} x_{h}\left(\left(-1+m_{h}\right) \lambda_{h}-\mu_{h}\right) \mu_{w}\left(3 \lambda_{w}+2 \mu_{w}\right)+\kappa_{h}\left(-2 \kappa_{w} x_{h} \lambda_{h} \mu_{w}\left(3 \lambda_{w}+\right.\right.\right.\right. \\
& \left.2 \mu_{w}\right)+m_{h}\left(2 \kappa_{w} x_{w} \mu_{h}\left(3 \lambda_{h}+2 \mu_{h}\right)\left(\lambda_{w}+\mu_{w}\right)+x_{h}\left(\lambda _ { h } \left(4 \kappa_{w} \mu_{w}{ }^{2}+3 \lambda_{w}\left(\mu_{h}+\kappa_{w} \mu_{h}+\right.\right.\right.\right. \\
& \left.\left.\left.\left.\left.\left.\left.2 \kappa_{w} \mu_{w}\right)\right)+2 \mu_{h}\left(4 \kappa_{w} \mu_{w}{ }^{2}+\lambda_{w}\left(\mu_{h}+\kappa_{w} \mu_{h}+6 \kappa_{w} \mu_{w}\right)\right)\right)\right)\right)\right)\right)
\end{aligned},
$$

where

$$
\begin{aligned}
Z_{i v 02}= & \left(-2 x_{h}\left(\left(-1+m_{h}\right) \lambda_{h}-\mu_{h}\right) \mu_{w}\left(\left(2-m_{w}+\kappa_{w}\left(-1+2 m_{w}\right)\right) \lambda_{w}+2\left(\mu_{w}+\kappa_{w} m_{w} \mu_{w}\right)\right)+\right. \\
& \kappa_{h}\left(2 x_{h} \lambda_{h} \mu_{w}\left(\left(-2+\kappa_{w}+m_{w}-2 \kappa_{w} m_{w}\right) \lambda I-2\left(\mu_{w}+\kappa_{w} m_{w} \mu_{w}\right)\right)+\right. \\
& m_{h}\left(x_{w} \mu_{h}\left(3 \lambda_{h}+2 \mu_{h}\right)\left(\left(-1+\kappa_{w}\right)\left(-1+m_{w}\right) \lambda_{w}+2\left(\mu_{w}+\kappa_{w} m_{w} \mu_{w}\right)\right)+\right. \\
& \left.\left.\left.2 x_{h}\left(\lambda_{h}+2 \mu_{h}\right) \mu_{w}\left(\left(2-m_{w}+\kappa_{w}\left(-1+2 m_{w}\right)\right) \lambda_{w}+2\left(\mu_{w}+\kappa_{w} m_{w} \mu_{w}\right)\right)\right)\right)\right)
\end{aligned}
$$

\section{A.9 Case iv-03. Both the weak and the hard layers yield out-of-plane in triaxial extension}

$$
\begin{aligned}
& A_{1, \text { ivo3 }}=\frac{1}{Z_{\text {iv03 }}}\left(\left(\kappa_{w} x_{h}+\kappa_{h} x_{w}\right)\left(m_{w} x_{h}+m_{h} x_{w}\right) \mu_{h}\left(3 \lambda_{h}+2 \mu_{h}\right) \mu_{w}\left(3 \lambda_{w}+2 \mu_{w}\right)\right), \\
& A_{2, \text { iv03 }}=-\frac{1}{Z_{\text {iv03 }}}\left(\kappa_{h} \kappa_{w}\left(x_{h}+x_{w}\right)\left(m_{w} x_{h}+m_{h} x_{w}\right) \mu_{h}\left(3 \lambda_{h}+2 \mu_{h}\right) \mu_{w}\left(3 \lambda_{w}+2 \mu_{w}\right)\right),
\end{aligned}
$$




$$
\begin{aligned}
& A_{3, \text { ivo } 3}=\frac{1}{Z_{\text {ivo } 3}}\left(m_{h} m_{w}\left(x_{h}+x_{w}\right)\left(\kappa_{w} x_{h}+\kappa_{h} x_{w}\right) \mu_{h}\left(3 \lambda_{h}+2 \mu_{h}\right) \mu_{w}\left(3 \lambda_{w}+2 \mu_{w}\right)\right), \\
& A_{4, \text { ivo3 }}=-\frac{1}{Z_{\text {ivo3 }}}\left(\kappa_{h} \kappa_{w} m_{h} m_{w}\left(x_{h}+x_{w}\right)^{2} \mu_{h}\left(3 \lambda_{h}+2 \mu_{h}\right) \mu_{w}\left(3 \lambda_{w}+2 \mu_{w}\right)\right) \text {, } \\
& B_{1, \text { ivo } 3}=\frac{1}{Z_{\text {ivo } 3}}\left(m_{h} m_{w}\left(\kappa_{w} x_{h}+\kappa_{h} x_{w}\right) \mu_{h}\left(3 \lambda_{h}+2 \mu_{h}\right) \mu_{w}\left(3 \lambda_{w}+2 \mu_{w}\right)\right), \\
& B_{2, \text { ivo } 3}=\frac{1}{Z_{\text {ivo } 3}}\left(\kappa_{h} \kappa_{w} m_{h} m_{w}\left(x_{h}+x_{w}\right) \mu_{h}\left(3 \lambda_{h}+2 \mu_{h}\right) \mu_{w}\left(3 \lambda_{w}+2 \mu_{w}\right)\right), \\
& K_{1, \text { ivo } 3}=\frac{1}{Z_{\text {ivo } 3}}\left(m_{w}\left(\kappa_{w} x_{h}+\kappa_{h} x_{w}\right) \mu_{h}\left(3 \lambda_{h}+2 \mu_{h}\right) \mu_{w}\left(3 \lambda_{w}+2 \mu_{w}\right)\right), \\
& K_{2, \text { ivo } 3}=\frac{1}{Z_{\text {ivo } 3}}\left(\kappa_{h} \kappa_{w} m_{w}\left(x_{h}+x_{w}\right) \mu_{h}\left(3 \lambda_{h}+2 \mu_{h}\right) \mu_{w}\left(3 \lambda_{w}+2 \mu_{w}\right)\right), \\
& K_{3, \text { ivo3 }}=\frac{1}{Z_{\text {ivo3 }}}\left(m_{h} m_{w}\left(\kappa_{w} x_{h}+\kappa_{h} x_{w}\right) \mu_{h}\left(3 \lambda_{h}+2 \mu_{h}\right) \mu_{w}\left(3 \lambda_{w}+2 \mu_{w}\right)\right), \\
& K_{4, \text { ivo3 }}=\frac{1}{Z_{\text {ivo3 }}}\left(\kappa_{h} \kappa_{w} m_{h} m_{w}\left(x_{h}+x_{w}\right) \mu_{h}\left(3 \lambda_{h}+2 \mu_{h}\right) \mu_{w}\left(3 \lambda_{w}+2 \mu_{w}\right)\right), \\
& K_{5, \text { ivo } 3}=\frac{1}{Z_{\text {ivo3 }}}\left(m_{h}\left(\kappa_{w} x_{h}+\kappa_{h} x_{w}\right) \mu_{h}\left(3 \lambda_{h}+2 \mu_{h}\right) \mu_{w}\left(3 \lambda_{w}+2 \mu_{w}\right)\right), \\
& K_{6, \text { ivo } 3}=\frac{1}{Z_{\text {ivo } 3}}\left(\kappa_{h} \kappa_{w} m_{h}\left(x_{h}+x_{w}\right) \mu_{h}\left(3 \lambda_{h}+2 \mu_{h}\right) \mu_{w}\left(3 \lambda_{w}+2 \mu_{w}\right)\right), \\
& K_{7, \text { ivo3 }}=\frac{1}{Z_{\text {ivo } 3}}\left(m_{h} m_{w}\left(\kappa_{w} x_{h}+\kappa_{h} x_{w}\right) \mu_{h}\left(3 \lambda_{h}+2 \mu_{h}\right) \mu_{w}\left(3 \lambda_{w}+2 \mu_{w}\right)\right) \text {, } \\
& K_{8, \text { ivo3 }}=\frac{1}{Z_{\text {ivo3 }}}\left(\kappa_{h} \kappa_{w} m_{h} m_{w}\left(x_{h}+x_{w}\right) \mu_{h}\left(3 \lambda_{h}+2 \mu_{h}\right) \mu_{w}\left(3 \lambda_{w}+2 \mu_{w}\right)\right) \text {, }
\end{aligned}
$$

where

$$
\begin{aligned}
Z_{\text {ivo3 }}= & \left(-\kappa_{w} m_{w} x_{h}\left(\left(-1+m_{h}\right) \lambda_{h}-\mu_{h}\right) \mu_{w}\left(3 \lambda_{w}+2 \mu_{w}\right)+\kappa_{h}\left(-\kappa_{w} m_{w} x_{h} \lambda_{h} \mu_{w}\left(3 \lambda_{w}+2 \mu_{w}\right)+\right.\right. \\
& m_{h}\left(\kappa_{w} m_{w} x_{h}\left(\lambda_{h}+2 \mu_{h}\right) \mu_{w}\left(3 \lambda_{w}+2 \mu_{w}\right)+\right. \\
& \left.\left.\left.x_{w} \mu_{h}\left(3 \lambda_{h}+2 \mu_{h}\right)\left(\left(-1+\kappa_{w}\right)\left(-1+m_{w}\right) \lambda_{w}+\mu_{w}+2 \kappa_{w} m_{w} \mu_{w}\right)\right)\right)\right)
\end{aligned}
$$

\section{A.10 Case iv-04. Weak and hard layers yield out-of-plane in triaxial compression and extension, respectively}

$$
A_{1, \mathrm{iv04}}=\frac{1}{Z_{\text {ivo } 4}}\left(\left(x_{h}+\kappa_{h} x_{w}\right)\left(x_{h}+m_{h} x_{w}\right) \mu_{h}\left(3 \lambda_{h}+2 \mu_{h}\right) \mu_{w}\left(3 \lambda_{w}+2 \mu_{w}\right)\right),
$$




$$
\begin{aligned}
& A_{2, \mathrm{iv} 04}=-\frac{1}{Z_{\text {ivo } 4}}\left(\kappa_{h}\left(x_{h}+\kappa_{w} x_{w}\right)\left(x_{h}+m_{h} x_{w}\right) \mu_{h}\left(3 \lambda_{h}+2 \mu_{h}\right) \mu_{w}\left(3 \lambda_{w}+2 \mu_{w}\right)\right), \\
& A_{3, \text { ivo } 4}=\frac{1}{Z_{\text {ivo } 4}}\left(m_{h}\left(x_{h}+\kappa_{h} x_{w}\right)\left(x_{h}+m_{w} x_{w}\right) \mu_{h}\left(3 \lambda_{h}+2 \mu_{h}\right) \mu_{w}\left(3 \lambda_{w}+2 \mu_{w}\right)\right), \\
& A_{4, \text { ivo } 4}=-\frac{1}{Z_{\text {ivo } 4}}\left(\kappa_{h} m_{h}\left(x_{h}+\kappa_{w} x_{w}\right)\left(x_{h}+m_{w} x_{w}\right) \mu_{h}\left(3 \lambda_{h}+2 \mu_{h}\right) \mu_{w}\left(3 \lambda_{w}+2 \mu_{w}\right)\right) \text {, } \\
& B_{1, i v 04}=\frac{1}{Z_{i v 04}}\left(m_{h}\left(x_{h}+\kappa_{h} x_{w}\right) \mu_{h}\left(3 \lambda_{h}+2 \mu_{h}\right) \mu_{w}\left(3 \lambda_{w}+2 \mu_{w}\right)\right) \\
& B_{2, \text { ivo } 4}=\frac{1}{Z_{\text {ivo } 4}}\left(\kappa_{h} m_{h}\left(x_{h}+\kappa_{w} x_{w}\right) \mu_{h}\left(3 \lambda_{h}+2 \mu_{h}\right) \mu_{w}\left(3 \lambda_{w}+2 \mu_{w}\right)\right), \\
& K_{1, i v 04}=\frac{1}{Z_{\text {ivo } 4}}\left(\left(x_{h}+\kappa_{h} x_{w}\right) \mu_{h}\left(3 \lambda_{h}+2 \mu_{h}\right) \mu_{w}\left(3 \lambda_{w}+2 \mu_{w}\right)\right), \\
& K_{2, \text { ivo } 4}=\frac{1}{Z_{\text {ivo } 4}}\left(\kappa_{h}\left(x_{h}+\kappa_{w} x_{w}\right) \mu_{h}\left(3 \lambda_{h}+2 \mu_{h}\right) \mu_{w}\left(3 \lambda_{w}+2 \mu_{w}\right)\right), \\
& K_{3, \text { ivo } 4}=\frac{1}{Z_{\text {ivo } 4}}\left(m_{h}\left(x_{h}+\kappa_{h} x_{w}\right) \mu_{h}\left(3 \lambda_{h}+2 \mu_{h}\right) \mu_{w}\left(3 \lambda_{w}+2 \mu_{w}\right)\right), \\
& K_{4, \mathrm{iv} 04}=\frac{1}{Z_{\text {ivo } 4}}\left(\kappa_{h} m_{h}\left(x_{h}+\kappa_{w} x_{w}\right) \mu_{h}\left(3 \lambda_{h}+2 \mu_{h}\right) \mu_{w}\left(3 \lambda_{w}+2 \mu_{w}\right)\right), \\
& K_{5, \text { ivo } 4}=\frac{1}{Z_{\text {ivo } 4}}\left(m_{h}\left(x_{h}+\kappa_{h} x_{w}\right) \mu_{h}\left(3 \lambda_{h}+2 \mu_{h}\right) \mu_{w}\left(3 \lambda_{w}+2 \mu_{w}\right)\right) \\
& K_{6, \text { ivo } 4}=\frac{1}{Z_{\text {ivo } 4}}\left(\kappa_{h} m_{h}\left(x_{h}+\kappa_{w} x_{w}\right) \mu_{h}\left(3 \lambda_{h}+2 \mu_{h}\right) \mu_{w}\left(3 \lambda_{w}+2 \mu_{w}\right)\right), \\
& K_{7, \text { ivo } 4}=\frac{1}{Z_{\text {ivo } 4}}\left(m_{h} m_{w}\left(x_{h}+\kappa_{h} x_{w}\right) \mu_{h}\left(3 \lambda_{h}+2 \mu_{h}\right) \mu_{w}\left(3 \lambda_{w}+2 \mu_{w}\right)\right), \\
& K_{8, \text { ivo } 4}=\frac{1}{Z_{\text {ivo } 4}}\left(\kappa_{h} m_{h} m_{w}\left(x_{h}+\kappa_{w} x_{w}\right) \mu_{h}\left(3 \lambda_{h}+2 \mu_{h}\right) \mu_{w}\left(3 \lambda_{w}+2 \mu_{w}\right)\right) \text {, }
\end{aligned}
$$

where

$$
\begin{gathered}
Z_{i v 04}=-x_{h}\left(\left(-1+m_{h}\right) \lambda_{h}-\mu_{h}\right) \mu_{w}\left(3 \lambda_{w}+2 \mu_{w}\right)+\kappa_{h}\left(-x_{h} \lambda_{h} \mu_{w}\left(3 \lambda_{w}+2 \mu_{w}\right)+m_{h}\left(x _ { h } \left(\lambda_{h}+\right.\right.\right. \\
\left.\left.\left.2 \mu_{h}\right) \mu_{w}\left(3 \lambda_{w}+2 \mu_{w}\right)+x_{w} \mu_{h}\left(3 \lambda_{h}+2 \mu_{h}\right)\left(\left(-1+\kappa_{w}\right)\left(-1+m_{w}\right) \lambda_{w}+\left(2+\kappa_{w} m_{w}\right) \mu_{w}\right)\right)\right) .
\end{gathered}
$$

\section{A.11 Case iv-05. Weak and hard layers yield out-of-plane in triaxial extension and compression, respectively}

$$
A_{1, \text { ivo } 5}=\frac{1}{Z_{\text {ivo5 }}}\left(\left(\kappa_{w} x_{h}+x_{w}\right)\left(m_{w} x_{h}+x_{w}\right) \mu_{h}\left(3 \lambda_{h}+2 \mu_{h}\right) \mu_{w}\left(3 \lambda_{w}+2 \mu_{w}\right)\right),
$$




$$
\begin{aligned}
& A_{2, \text { ivo5 }}=-\frac{1}{Z_{i v 05}}\left(\kappa_{w}\left(\kappa_{h} x_{h}+x_{w}\right)\left(m_{w} x_{h}+x_{w}\right) \mu_{h}\left(3 \lambda_{h}+2 \mu_{h}\right) \mu_{w}\left(3 \lambda_{w}+2 \mu_{w}\right)\right), \\
& A_{3, \text { ivo5 }}=\frac{1}{Z_{\text {iv } 05}}\left(m_{w}\left(\kappa_{w} x_{h}+x_{w}\right)\left(m_{h} x_{h}+x_{w}\right) \mu_{h}\left(3 \lambda_{h}+2 \mu_{h}\right) \mu_{w}\left(3 \lambda_{w}+2 \mu_{w}\right)\right), \\
& A_{4, \text { vo5 }}=-\frac{1}{Z_{\text {ivo5 }}}\left(\kappa_{w} m_{w}\left(\kappa_{h} x_{h}+x_{w}\right)\left(m_{h} x_{h}+x_{w}\right) \mu_{h}\left(3 \lambda_{h}+2 \mu_{h}\right) \mu_{w}\left(3 \lambda_{w}+2 \mu_{w}\right)\right) \text {, } \\
& B_{1, \text { ivo5 }}=\frac{1}{Z_{i v 05}}\left(m_{w}\left(\kappa_{w} x_{h}+x_{w}\right) \mu_{h}\left(3 \lambda_{h}+2 \mu_{h}\right) \mu_{w}\left(3 \lambda_{w}+2 \mu_{w}\right)\right), \\
& B_{2, \text { vo5 }}=\frac{1}{Z_{\text {ivo5 }}}\left(\kappa_{w} m_{w}\left(\kappa_{h} x_{h}+x_{w}\right) \mu_{h}\left(3 \lambda_{h}+2 \mu_{h}\right) \mu_{w}\left(3 \lambda_{w}+2 \mu_{w}\right)\right), \\
& K_{1,1,05}=\frac{1}{Z_{i v 05}}\left(m_{w}\left(\kappa_{w} x_{h}+x_{w}\right) \mu_{h}\left(3 \lambda_{h}+2 \mu_{h}\right) \mu_{w}\left(3 \lambda_{w}+2 \mu_{w}\right)\right), \\
& K_{2, \text { vo5 }}=\frac{1}{Z_{i v 05}}\left(\kappa_{w} m_{w}\left(\kappa_{h} x_{h}+x_{w}\right) \mu_{h}\left(3 \lambda_{h}+2 \mu_{h}\right) \mu_{w}\left(3 \lambda_{w}+2 \mu_{w}\right)\right), \\
& K_{3, \text { vo5 }}=\frac{1}{Z_{\text {ivo } 5}}\left(m_{h} m_{w}\left(\kappa_{w} x_{h}+x_{w}\right) \mu_{h}\left(3 \lambda_{h}+2 \mu_{h}\right) \mu_{w}\left(3 \lambda_{w}+2 \mu_{w}\right)\right), \\
& K_{4, \text { ivo5 }}=\frac{1}{Z_{\text {ivo5 }}}\left(\kappa_{w} m_{h} m_{w}\left(\kappa_{h} x_{h}+x_{w}\right) \mu_{h}\left(3 \lambda_{h}+2 \mu_{h}\right) \mu_{w}\left(3 \lambda_{w}+2 \mu_{w}\right)\right), \\
& K_{5, \text { ivo5 }}=\frac{1}{Z_{i v 05}}\left(\left(\kappa_{w} x_{h}+x_{w}\right) \mu_{h}\left(3 \lambda_{h}+2 \mu_{h}\right) \mu_{w}\left(3 \lambda_{w}+2 \mu_{w}\right)\right), \\
& K_{6, \text { ivo5 }}=\frac{1}{Z_{\text {ivo5 }}}\left(\kappa_{w}\left(\kappa_{h} x_{h}+x_{w}\right) \mu_{h}\left(3 \lambda_{h}+2 \mu_{h}\right) \mu_{w}\left(3 \lambda_{w}+2 \mu_{w}\right)\right), \\
& K_{7, \text { ivo5 }}=\frac{1}{Z_{\text {ivo5 }}}\left(m_{w}\left(\kappa_{w} x_{h}+x_{w}\right) \mu_{h}\left(3 \lambda_{h}+2 \mu_{h}\right) \mu_{w}\left(3 \lambda_{w}+2 \mu_{w}\right)\right), \\
& K_{8, \text { iv } 5}=\frac{1}{Z_{i v 05}}\left(\kappa_{w} m_{w}\left(\kappa_{h} x_{h}+x_{w}\right) \mu_{h}\left(3 \lambda_{h}+2 \mu_{h}\right) \mu_{w}\left(3 \lambda_{w}+2 \mu_{w}\right)\right),
\end{aligned}
$$

\section{where}

$$
\begin{gathered}
Z_{i v 05}=\kappa_{w} m_{w} x_{h}\left(\left(-1+\kappa_{h}\right)\left(-1+m_{h}\right) \lambda_{h}+\left(2+\kappa_{h} m_{h}\right) \mu_{h}\right) \mu_{w}\left(3 \lambda_{w}+2 \mu_{w}\right)+ \\
x_{w} \mu_{h}\left(3 \lambda_{h}+2 \mu_{h}\right)\left(\left(-1+\kappa_{w}\right)\left(-1+m_{w}\right) \lambda_{w}+\mu_{w}+2 \kappa_{w} m_{w} \mu_{w}\right)
\end{gathered}
$$




\section{Appendix B: Tunnelling perpendicularly to a thinly stratified rock mass - Dimensionless diagrams for the displacements}

This appendix presents the dimensionless diagrams introduced in Section 5.2.7. Table B.1 gives an overview of the dimensionless diagrams. The list of symbols can be found on page 88 .

Table B.1. Overview of the dimensionless diagrams.

\begin{tabular}{ccc}
\hline Figure & $\varphi_{w}-\varphi_{h}$ & $\sigma_{a} / \sigma_{0}$ \\
\hline B.1 & & 0.0 \\
B. 2 & $0^{\circ}$ & 0.1 \\
B. 3 & & 0.2 \\
\hline B. 4 & & 0.0 \\
B. 5 & $-5^{\circ}$ & 0.1 \\
B.6 & & 0.2 \\
B.7 & & 0.0 \\
B. 8 & $-10^{\circ}$ & 0.1 \\
B. 9 & & 0.2 \\
\hline
\end{tabular}




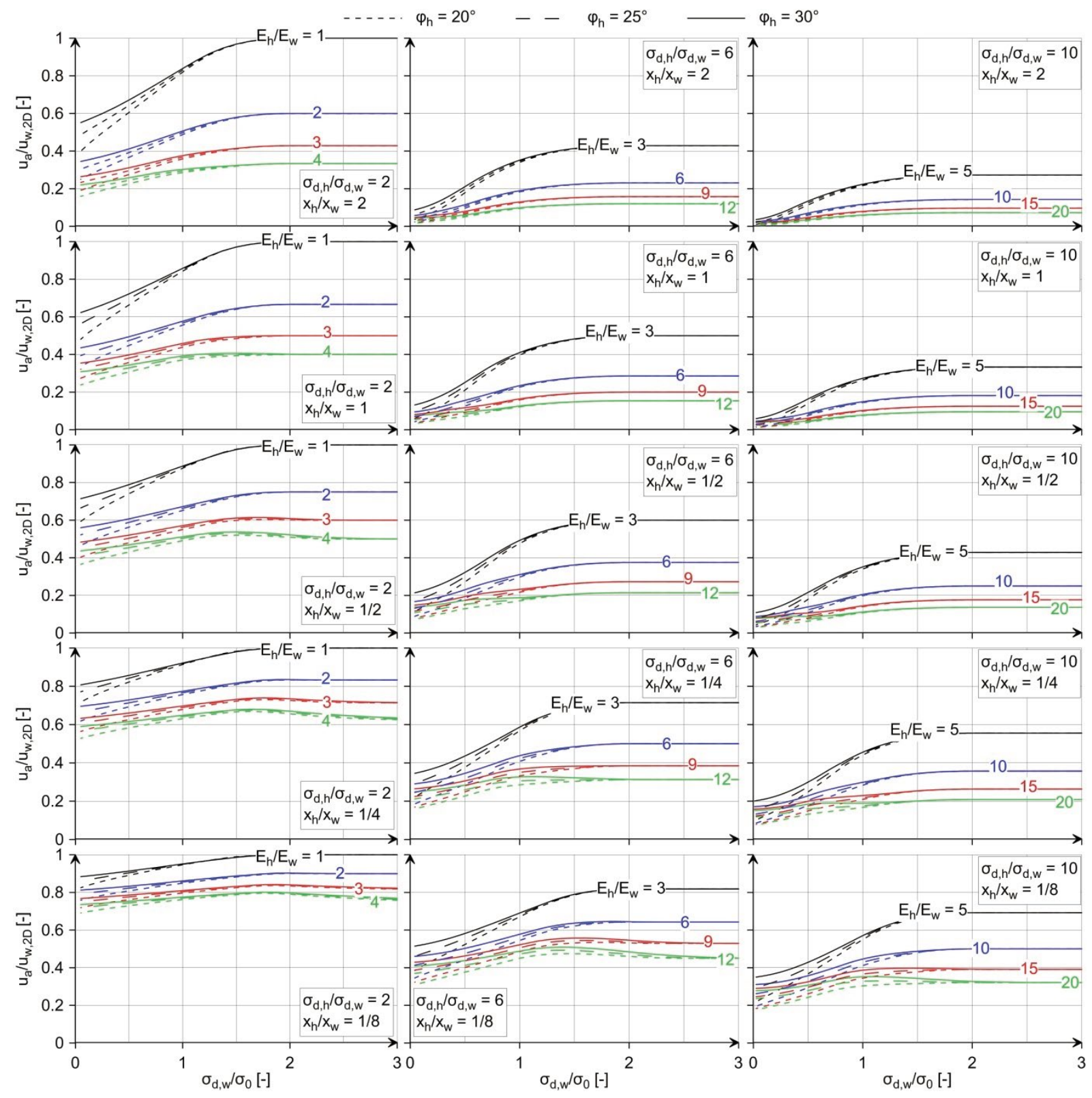

Figure B.1. Nomograms for $\varphi_{w}=\varphi_{h}$ and $\sigma_{a} / \sigma_{0}=0$. 


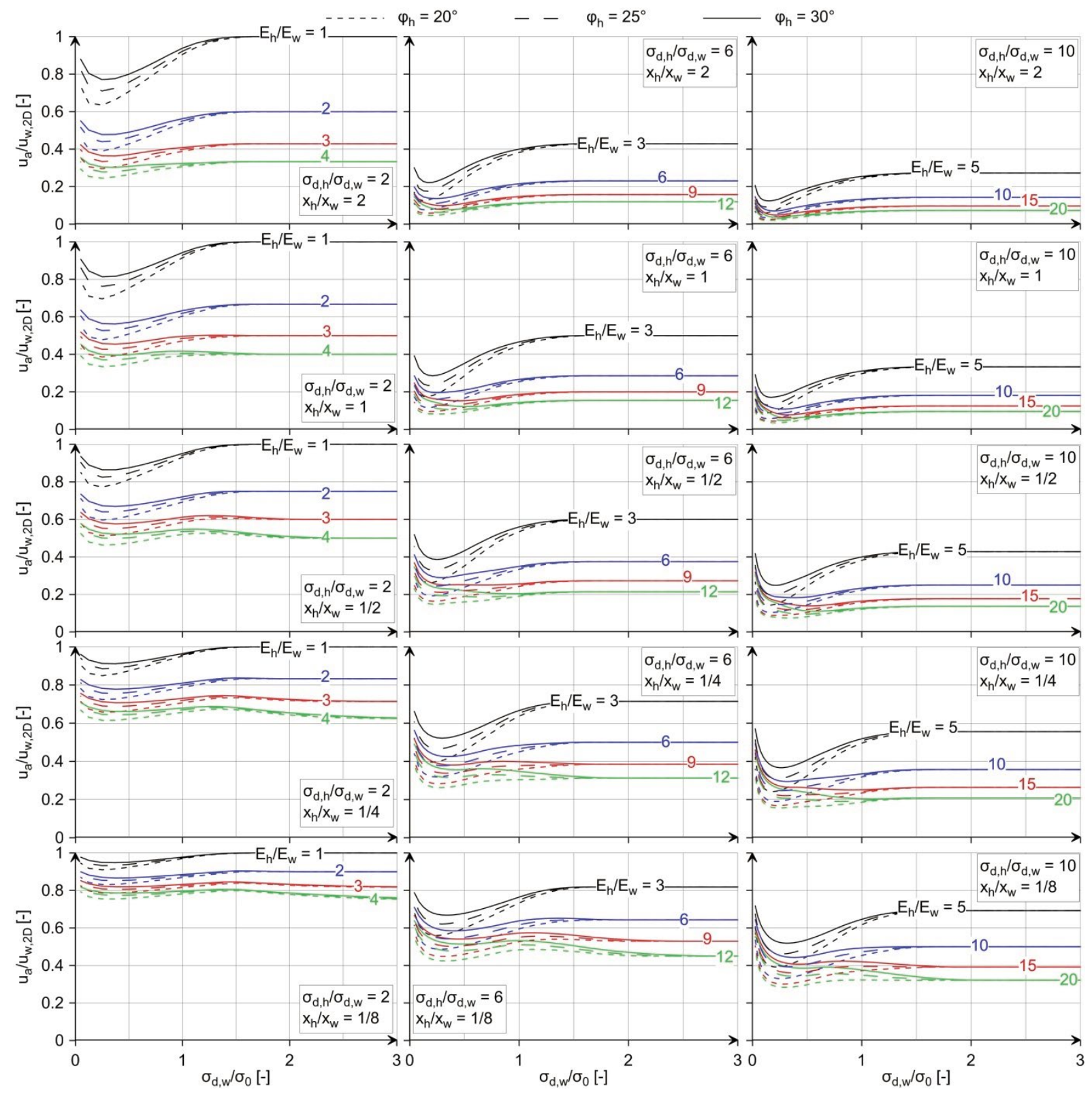

Figure B.2. Nomograms for $\varphi_{w}=\varphi_{h}$ and $\sigma_{a} / \sigma_{0}=0.1$. 


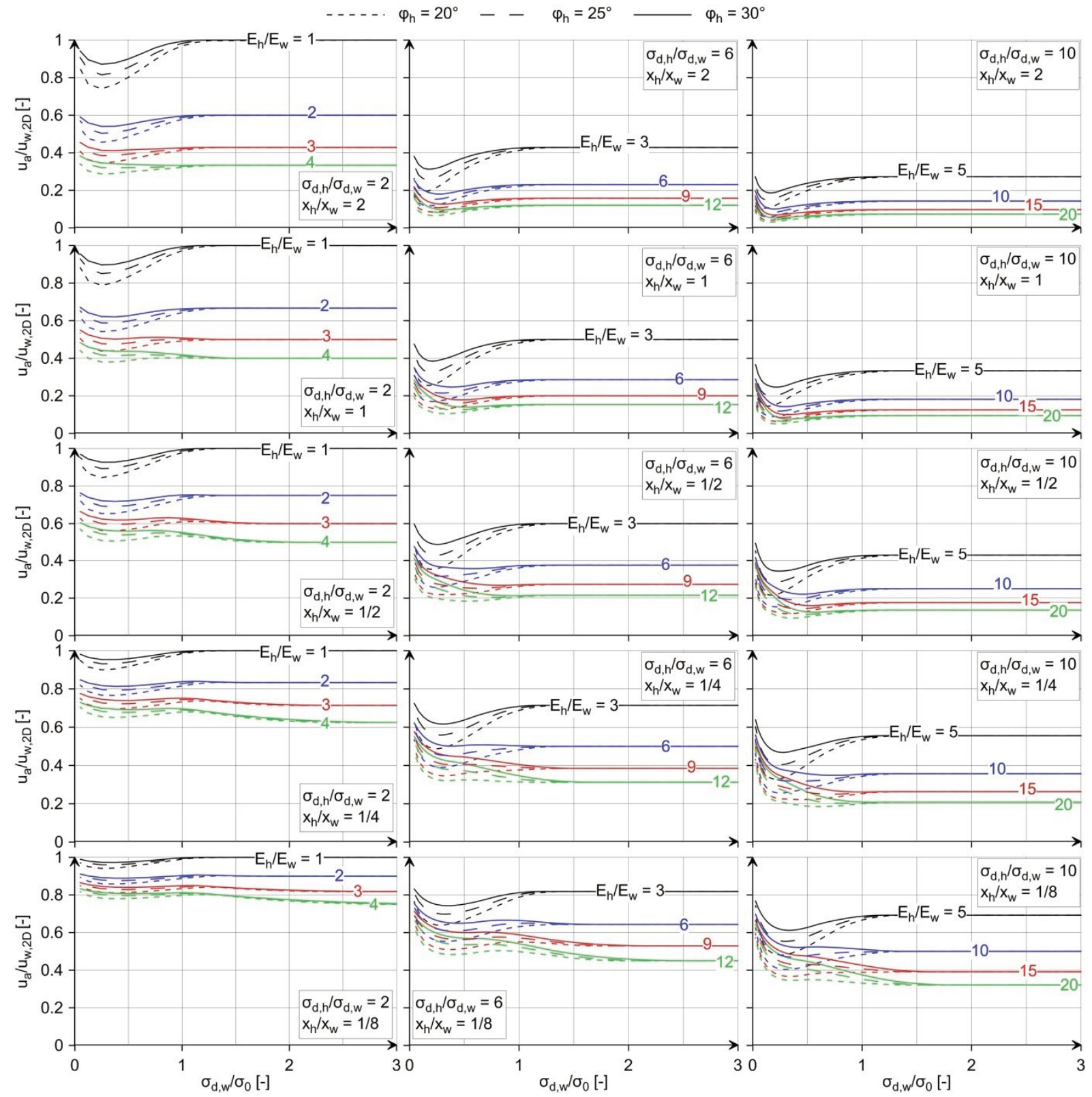

Figure B.3. Nomograms for $\varphi_{w}=\varphi_{h}$ and $\sigma_{a} / \sigma_{0}=0.2$. 


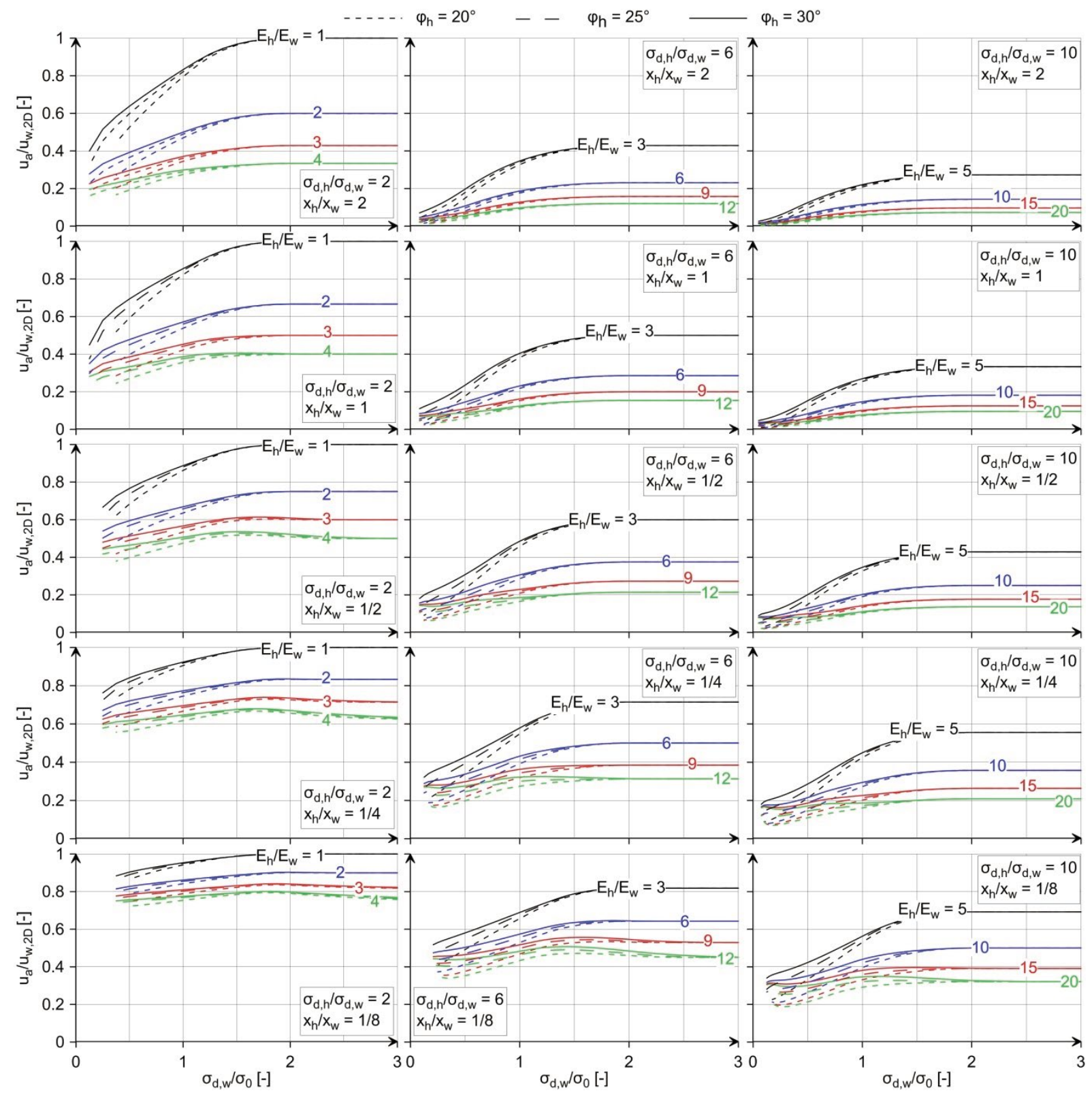

Figure B.4. Nomograms for $\varphi_{w}=\varphi_{h}-5^{\circ}$ and $\sigma_{a} / \sigma_{0}=0$. 


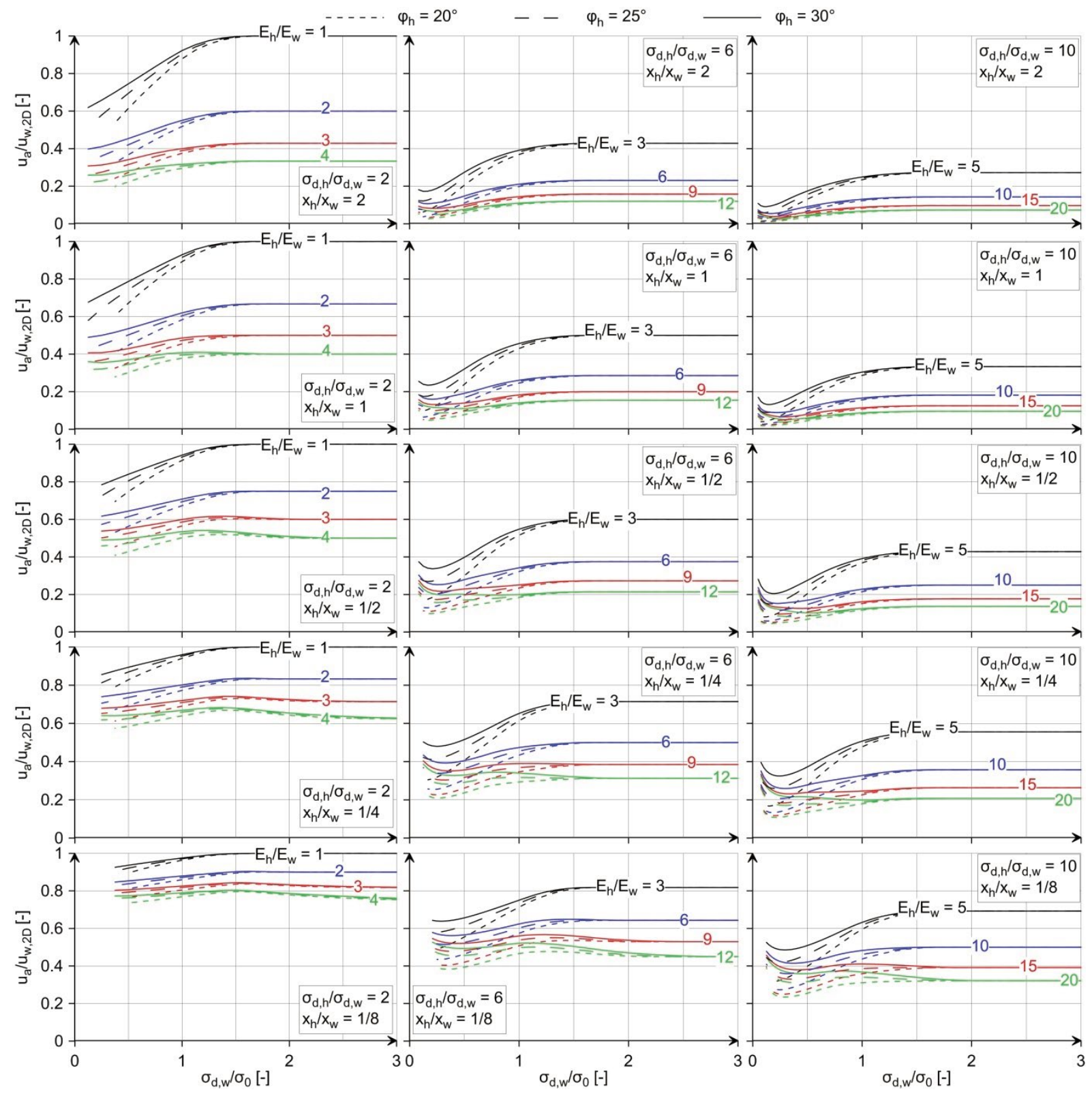

Figure B.5. Nomograms for $\varphi_{w}=\varphi_{h}-5^{\circ}$ and $\sigma_{a} / \sigma_{0}=0.1$. 


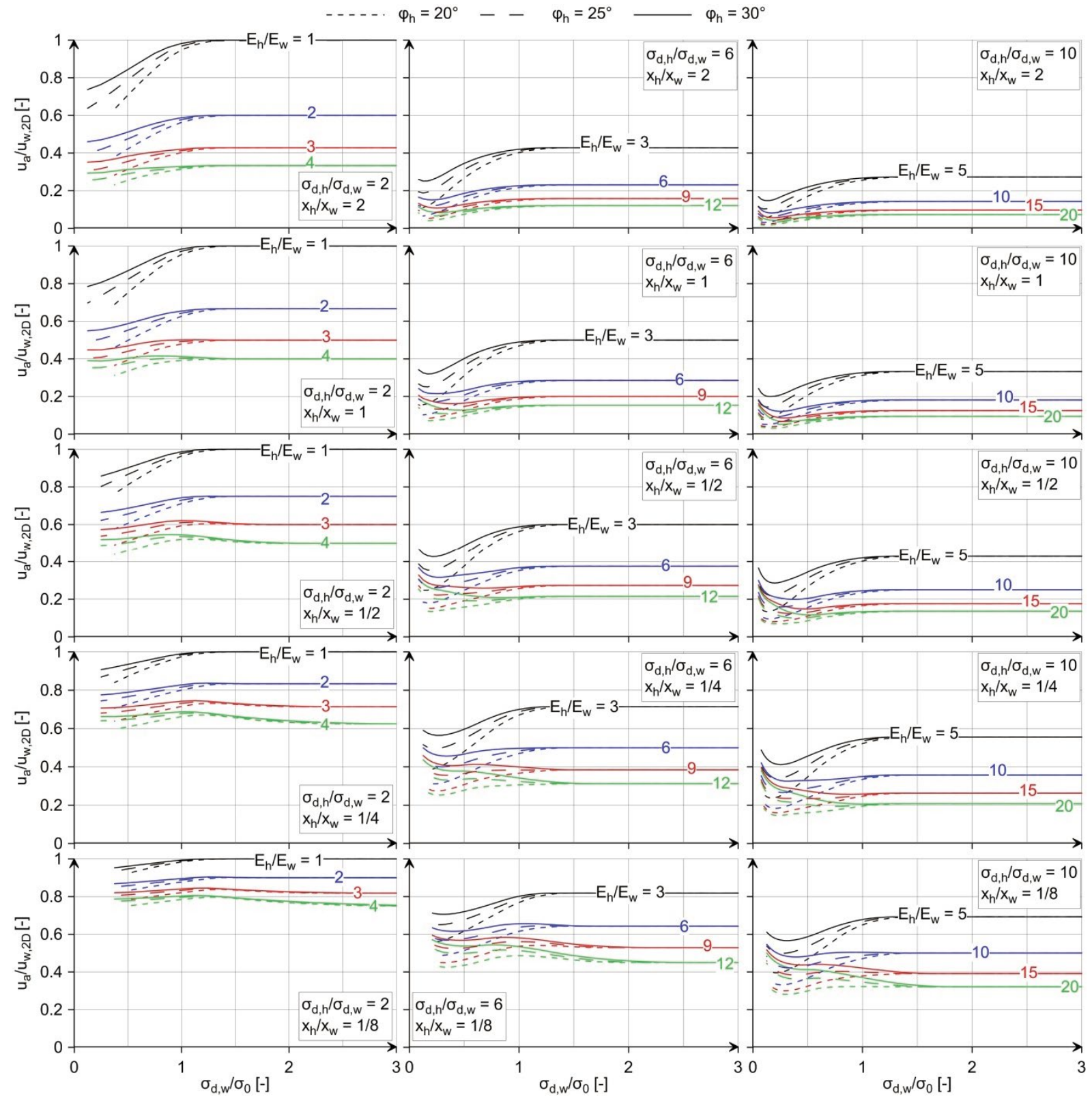

Figure B.6. Nomograms for $\varphi_{w}=\varphi_{h}-5^{\circ}$ and $\sigma_{a} / \sigma_{0}=0.2$. 


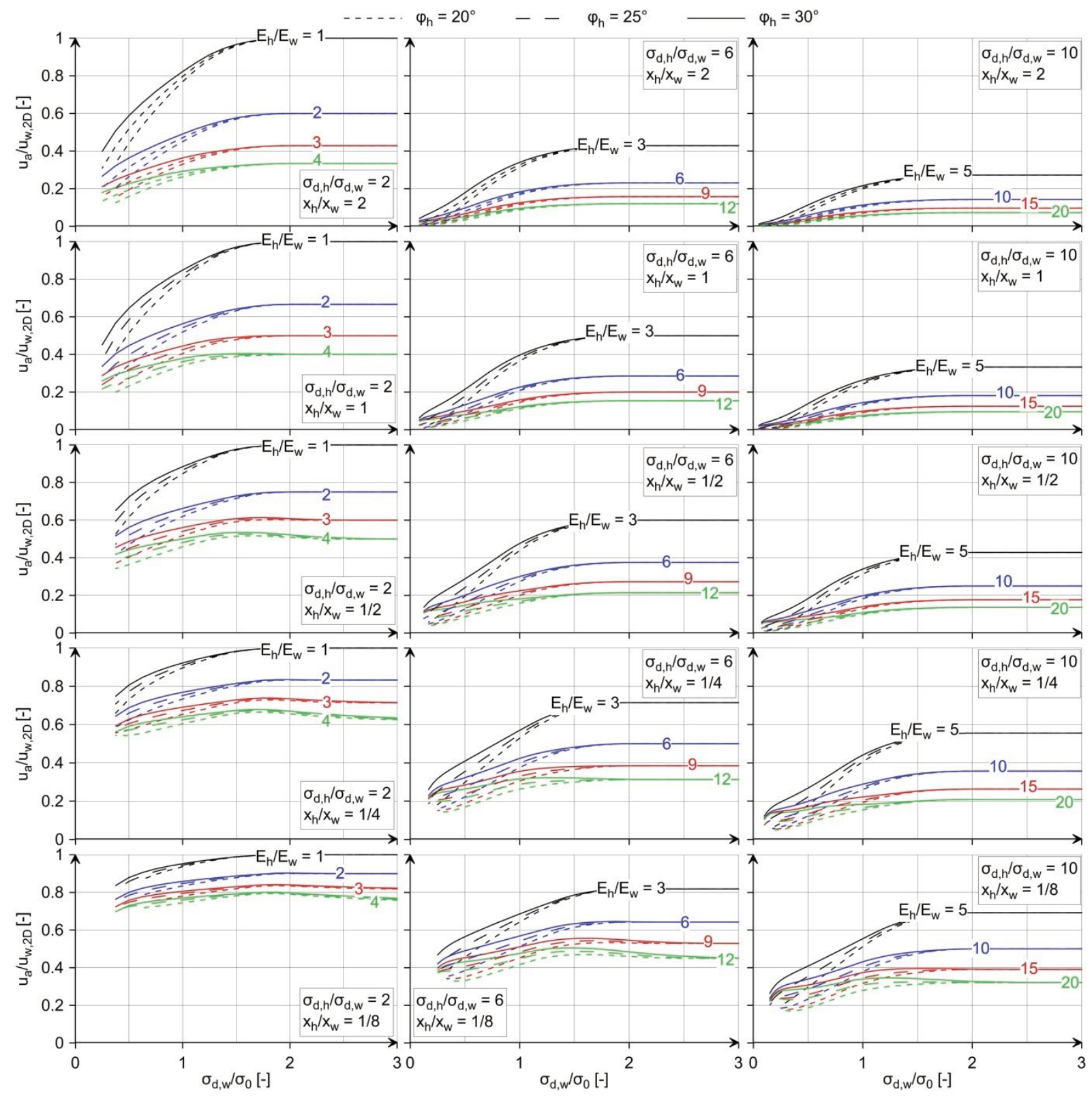

Figure B.7. Nomograms for $\varphi_{w}=\varphi_{h}-10^{\circ}$ and $\sigma_{a} / \sigma_{0}=0$. 


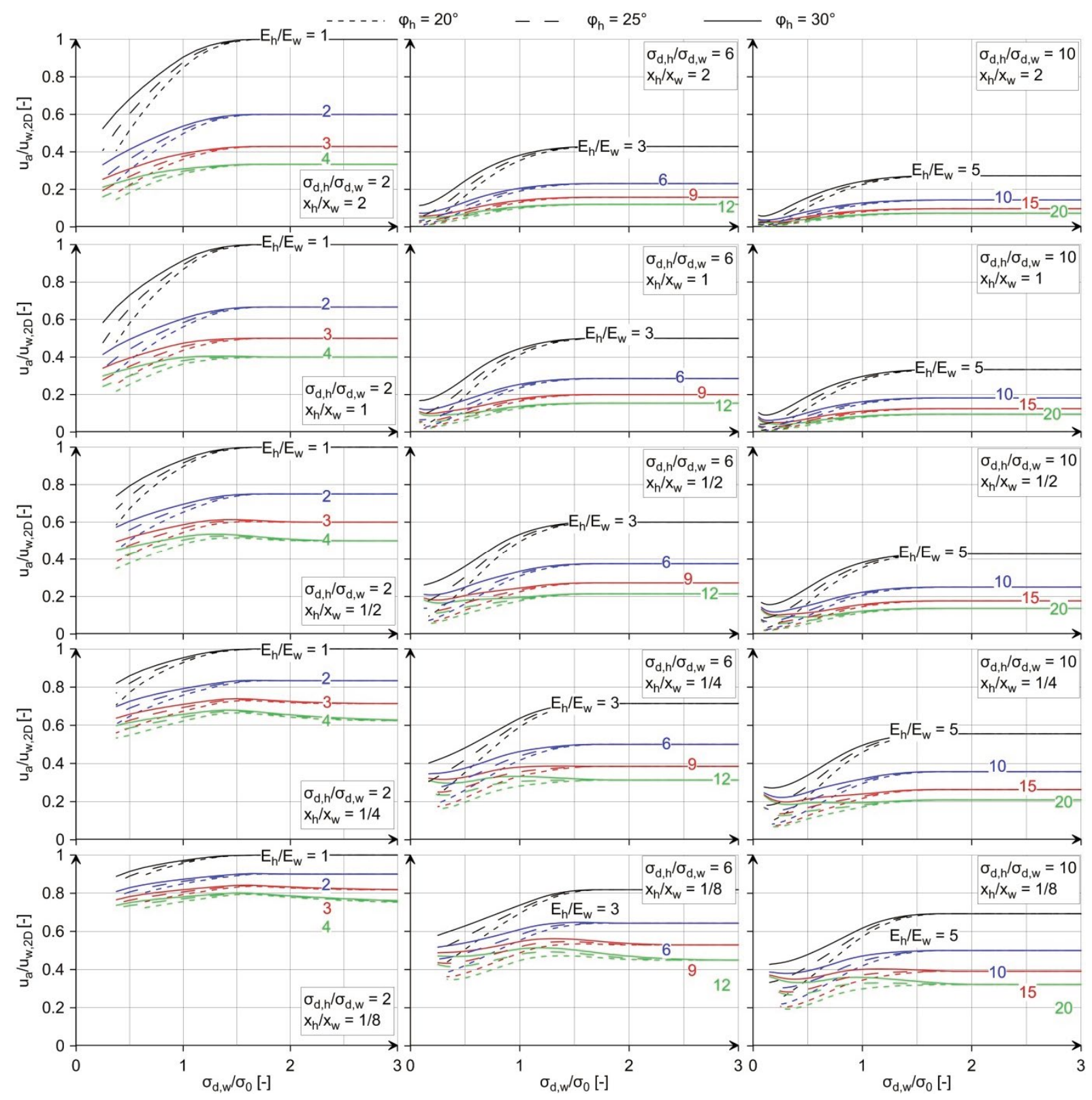

Figure B.8. Nomograms for $\varphi_{w}=\varphi_{h}-10^{\circ}$ and $\sigma_{a} / \sigma_{0}=0.1$. 


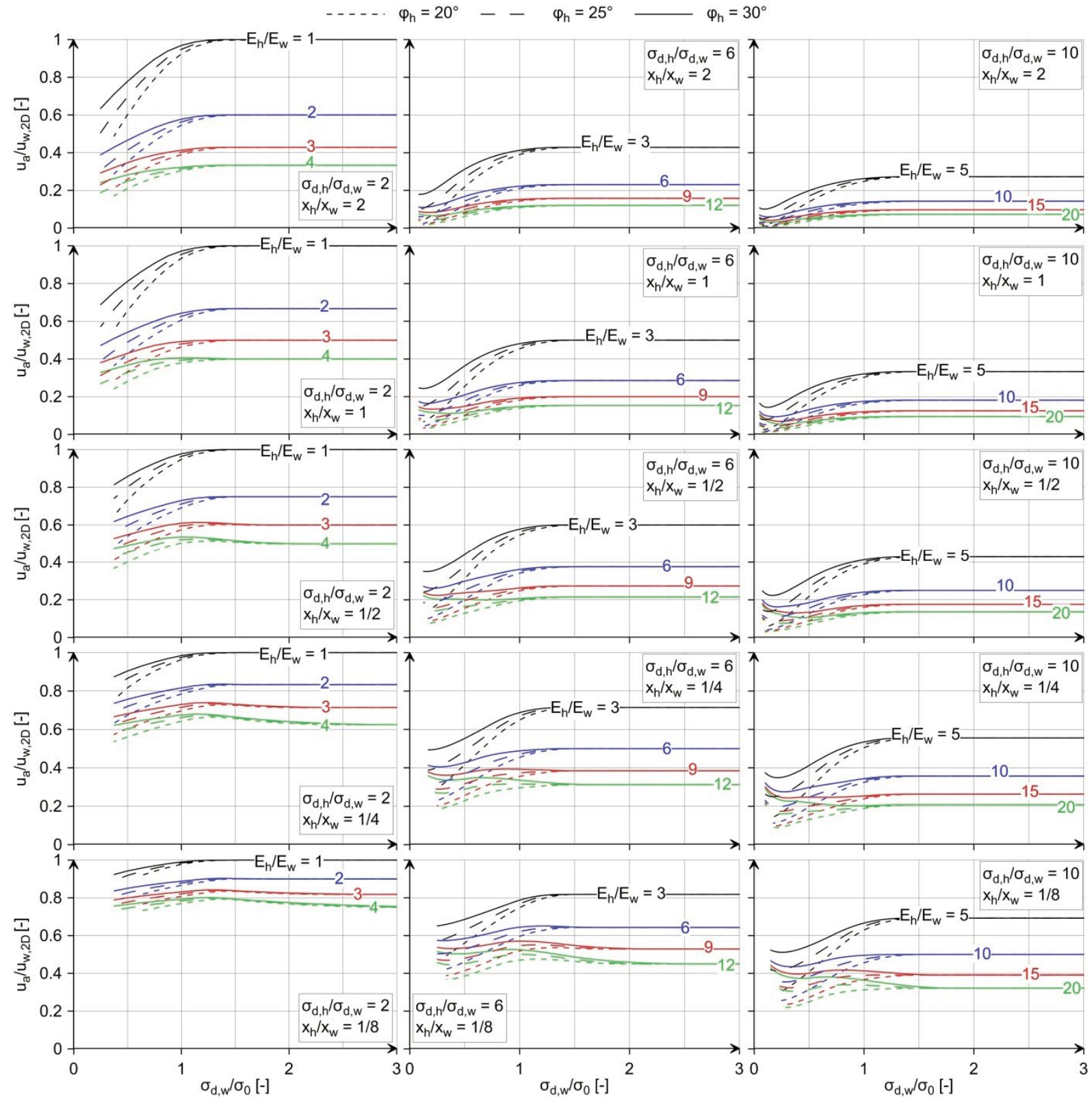

Figure B.9. Nomograms for $\varphi_{w}=\varphi_{h}-10^{\circ}$ and $\sigma_{a} / \sigma_{0}=0.2$. 


\section{Appendix C: Tunnelling perpendicularly to a thinly stratified rock mass - Flow chart of the Matlab-code for calculating the GRC}

The flow chart of the Matlab-code for calculating the GRC (considering out-of-plane plastic flow) is given in the Figures C.0 to C.0. The list of symbols can be found on page 88 . 


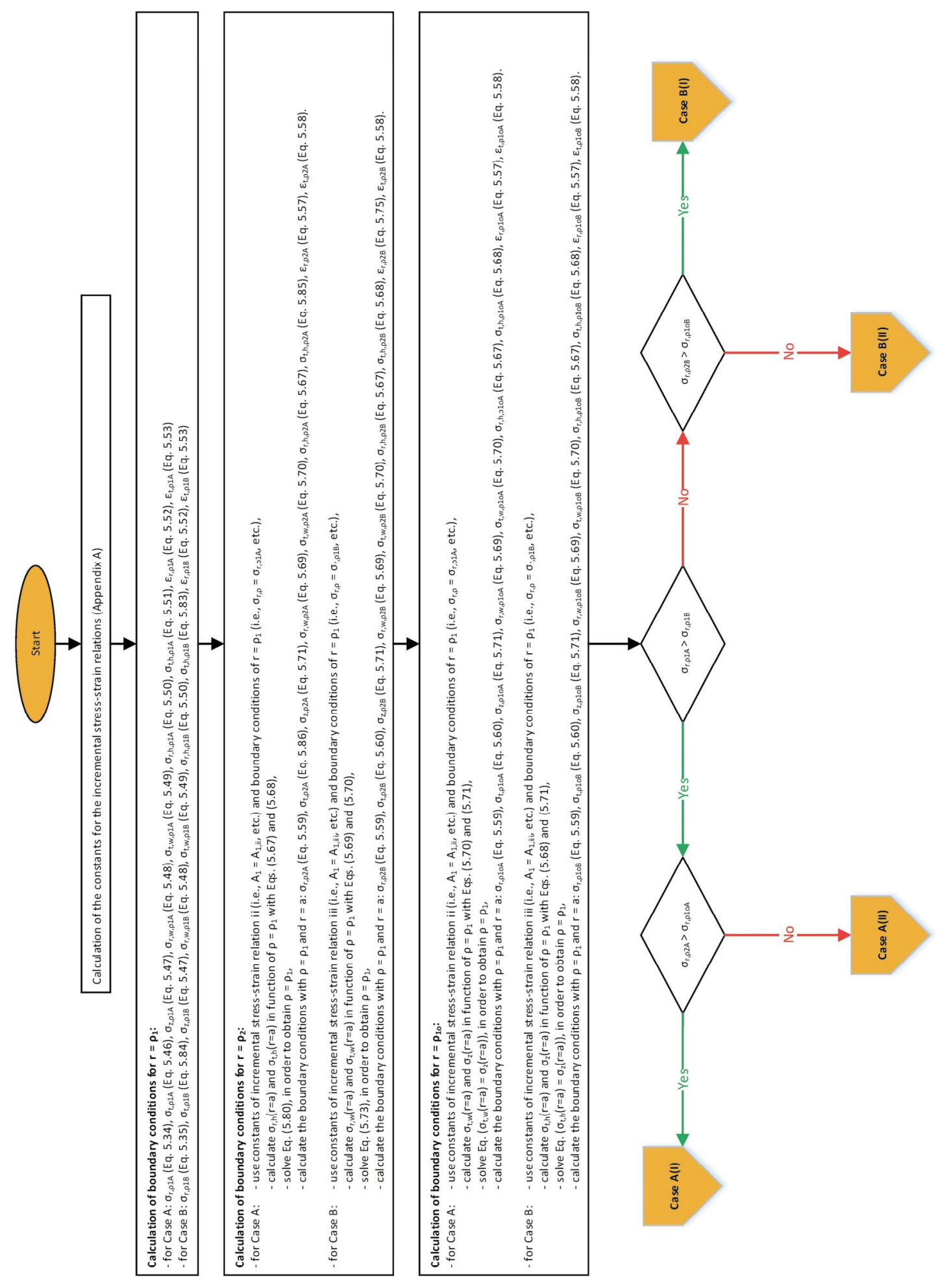

Figure C.1. Flow chart of the Matlab-code for calculating the GRC. 


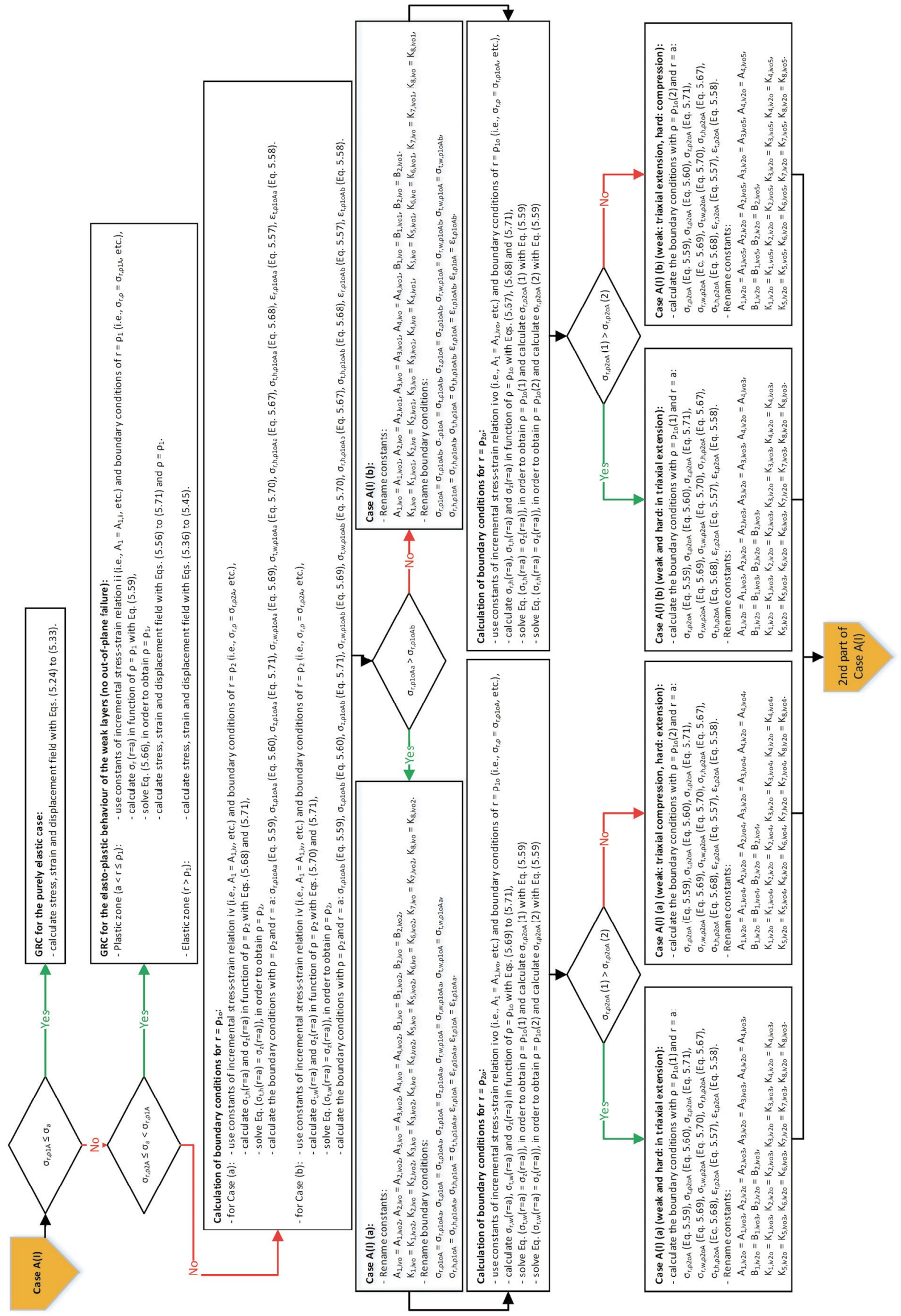

Figure C.2. Flow chart for the Case $A(I)$ (cf. Fig. 5.14). 


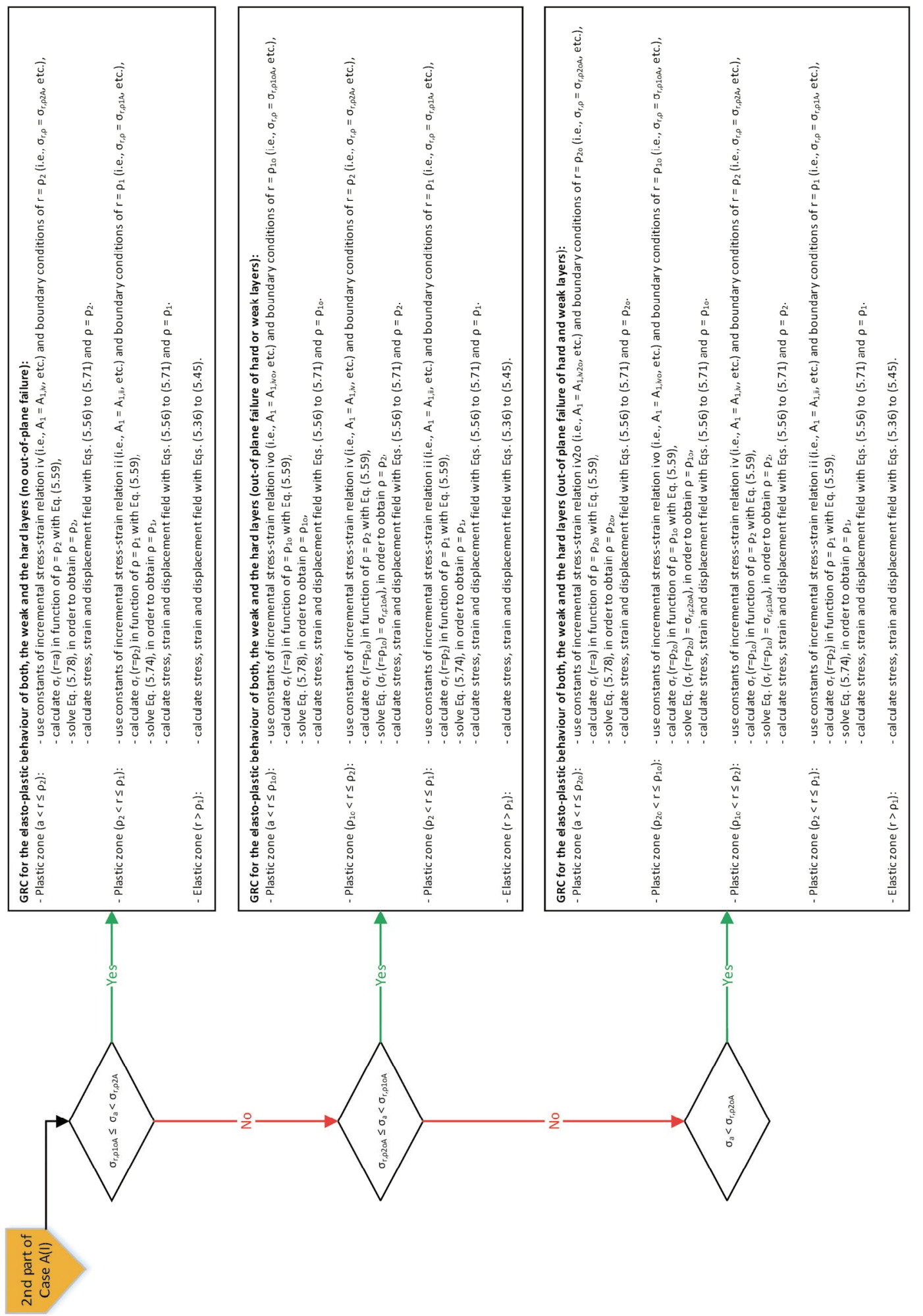

Figure C.3. Flow chart for the second part of Case $A(I)$ (cf. Fig. 5.14). 


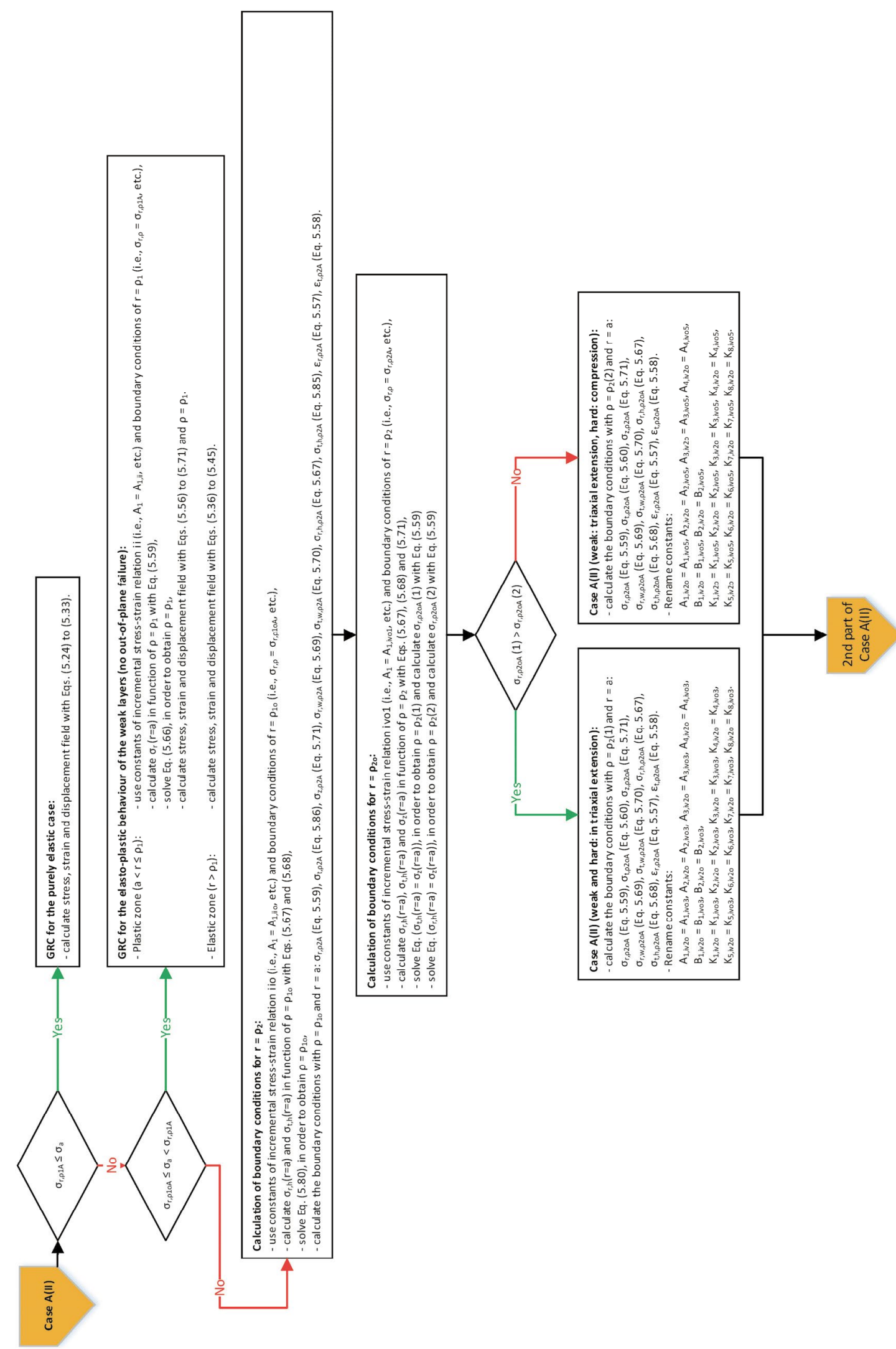

Figure C.4. Flow chart for Case A(II) (cf. Fig. 5.14). 


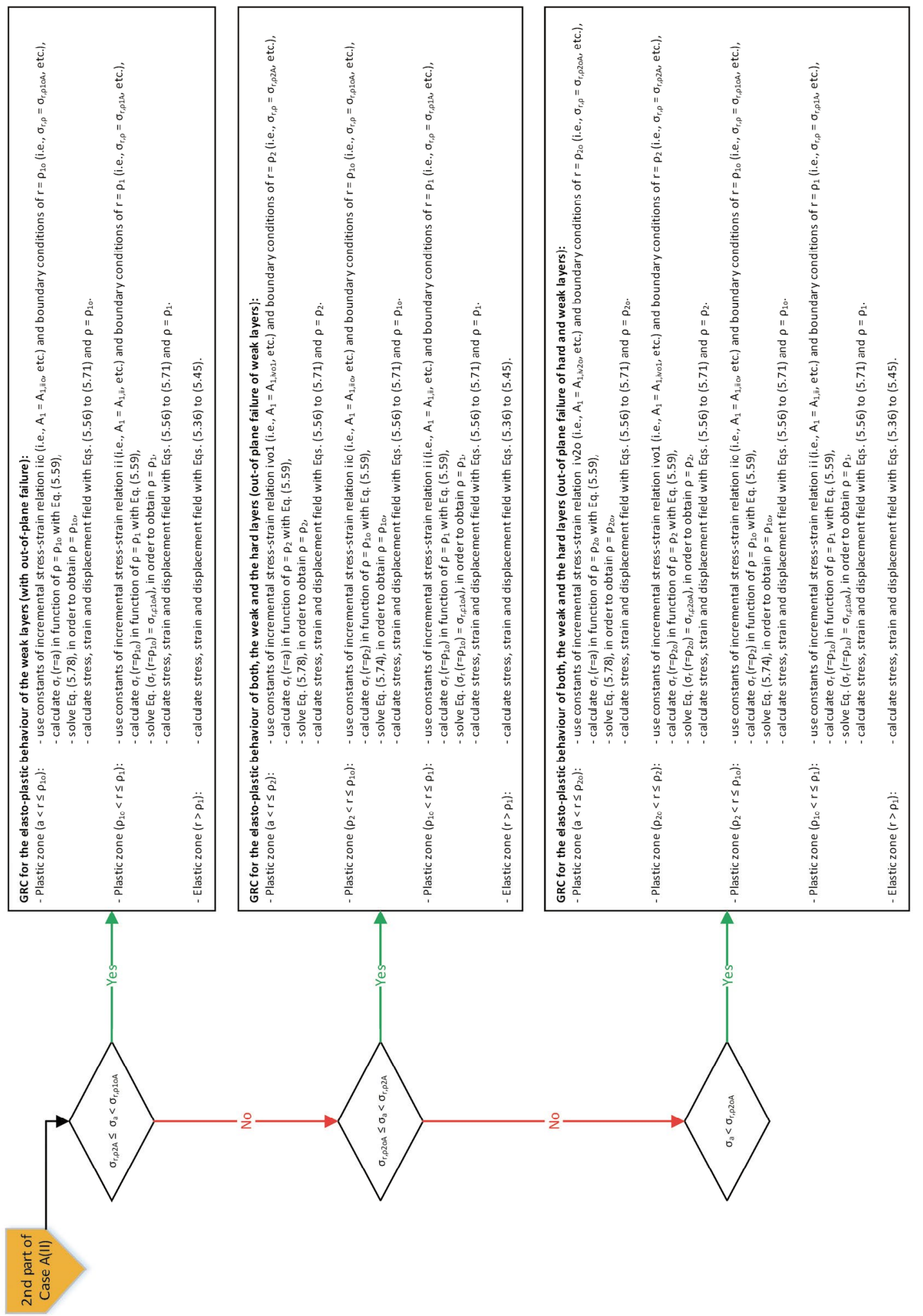

Figure C.5. Flow chart for the second part of Case A(II) (cf. Fig. 5.14). 


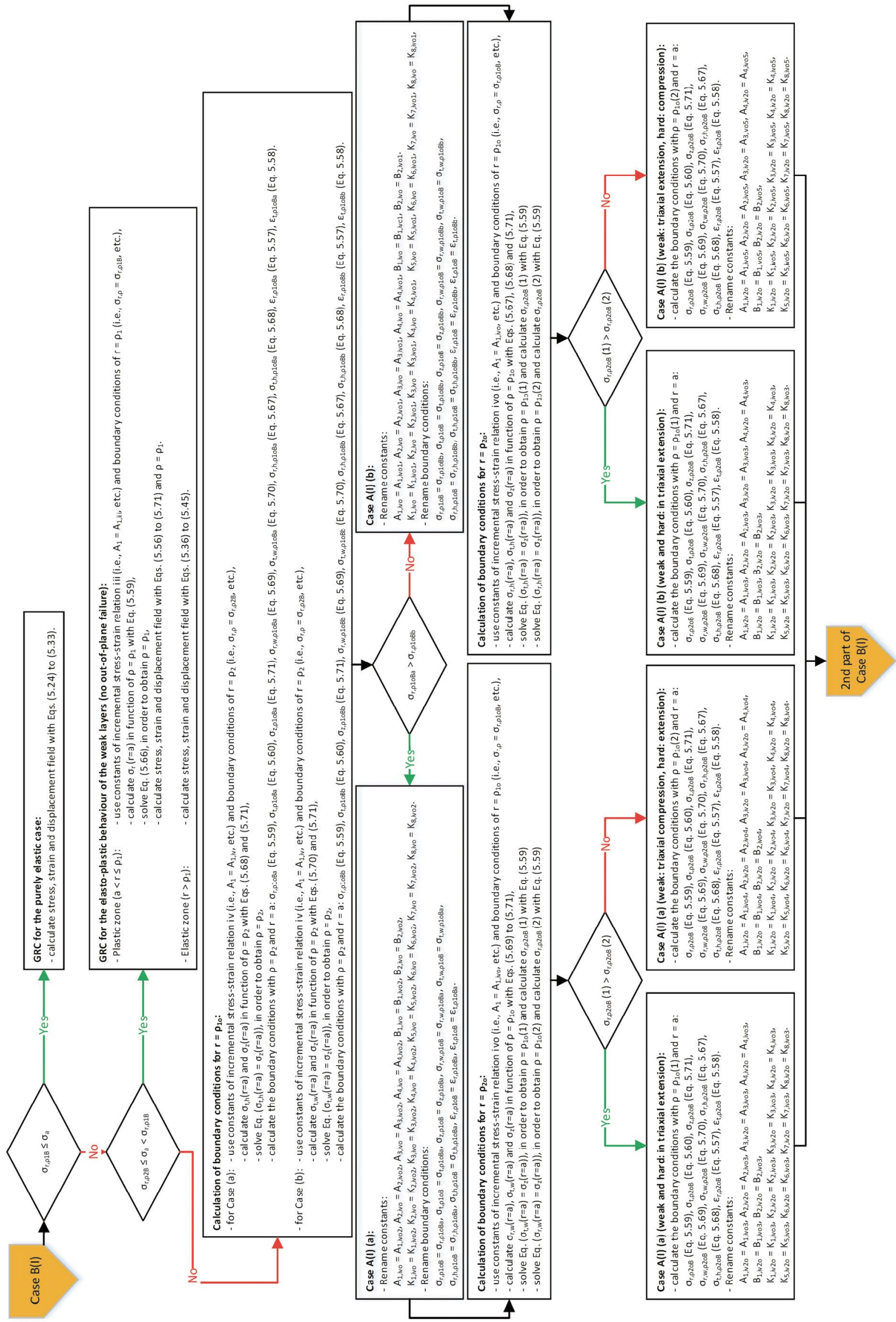

Figure C.6. Flow chart for Case B(I) (cf. Fig. 5.14). 


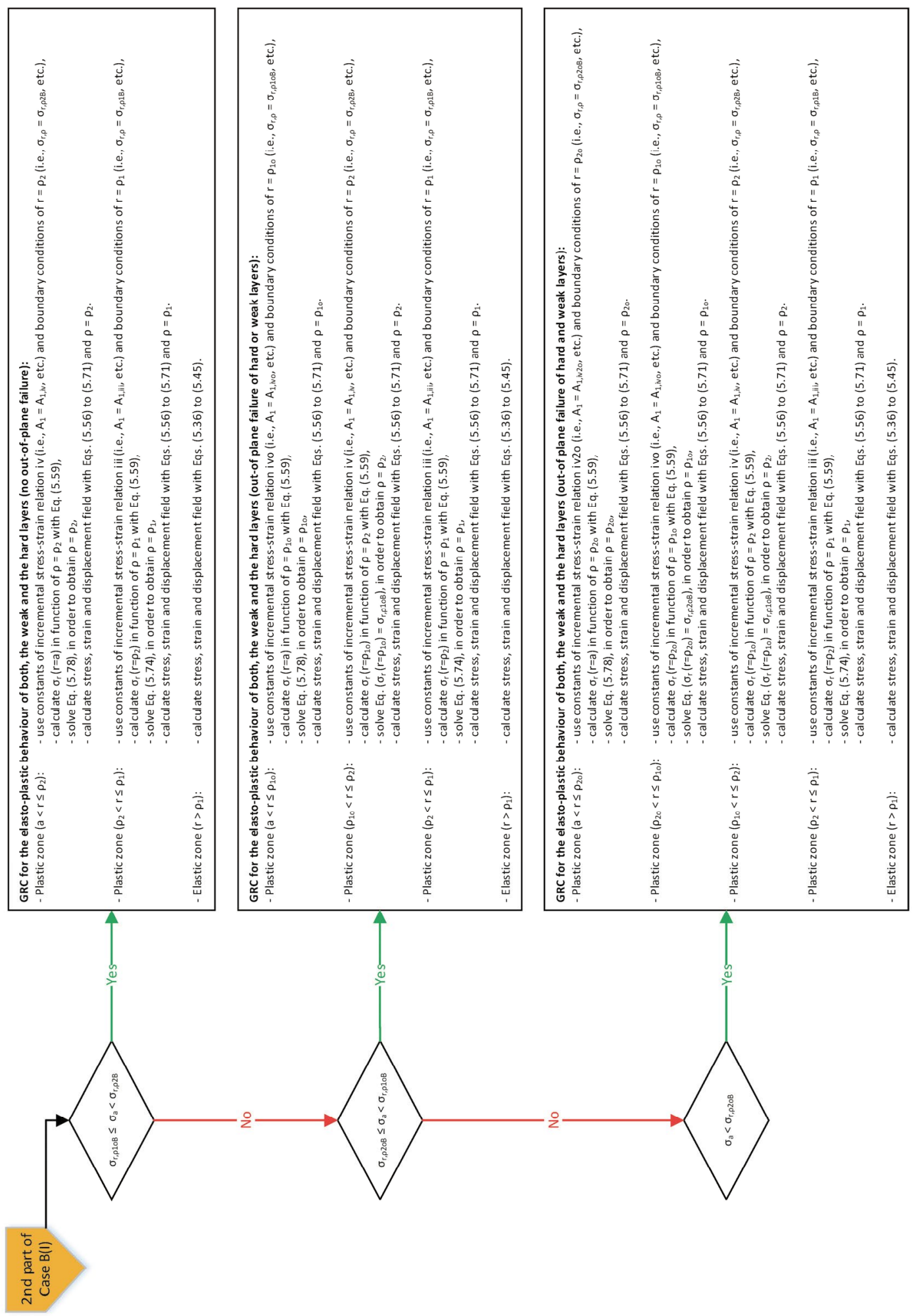

Figure C.7. Flow chart for the second part of Case B(I) (cf. Fig. 5.14). 


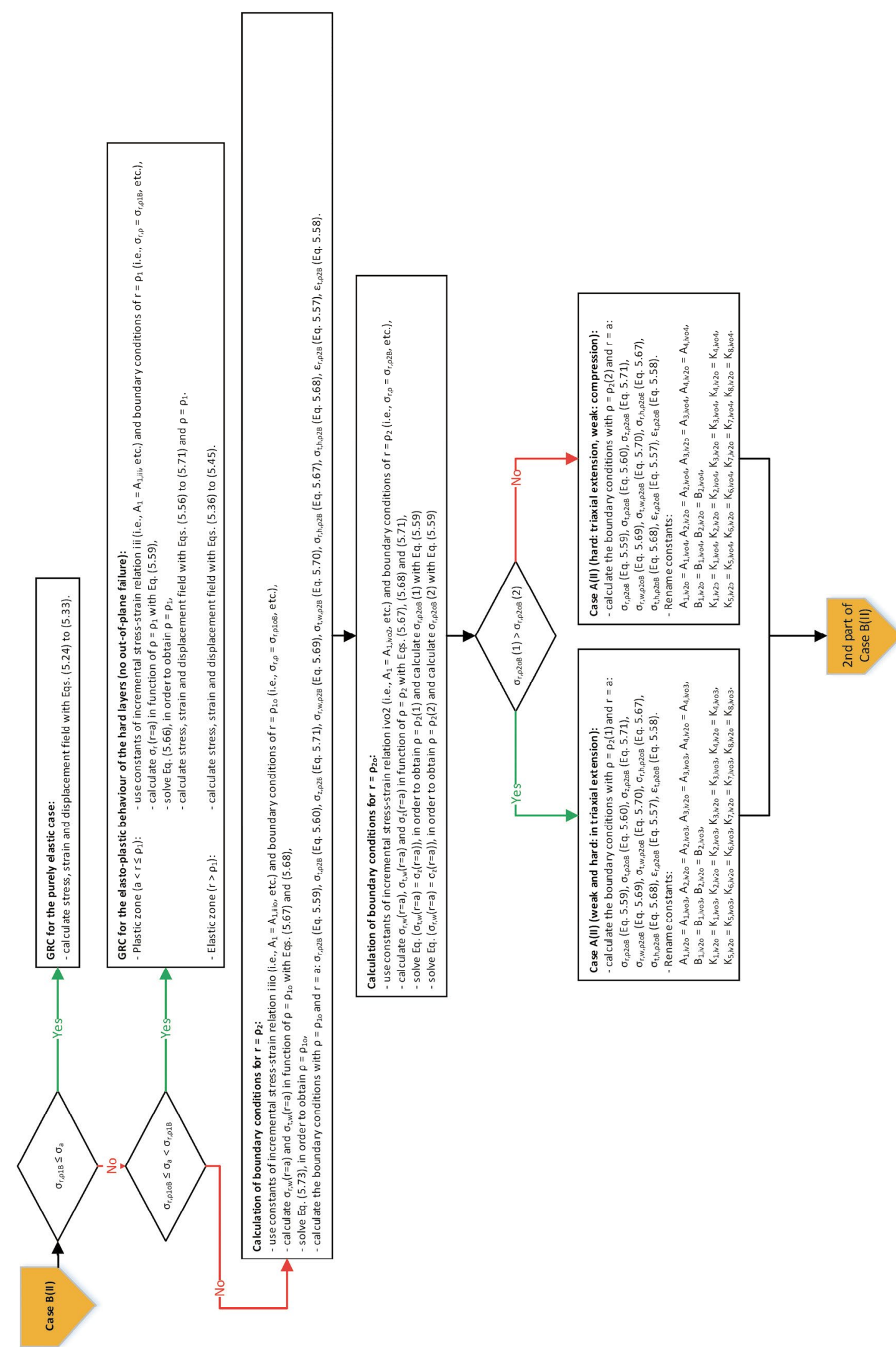

Figure C.8. Flow chart for Case B(II) (cf. Fig. 5.14). 


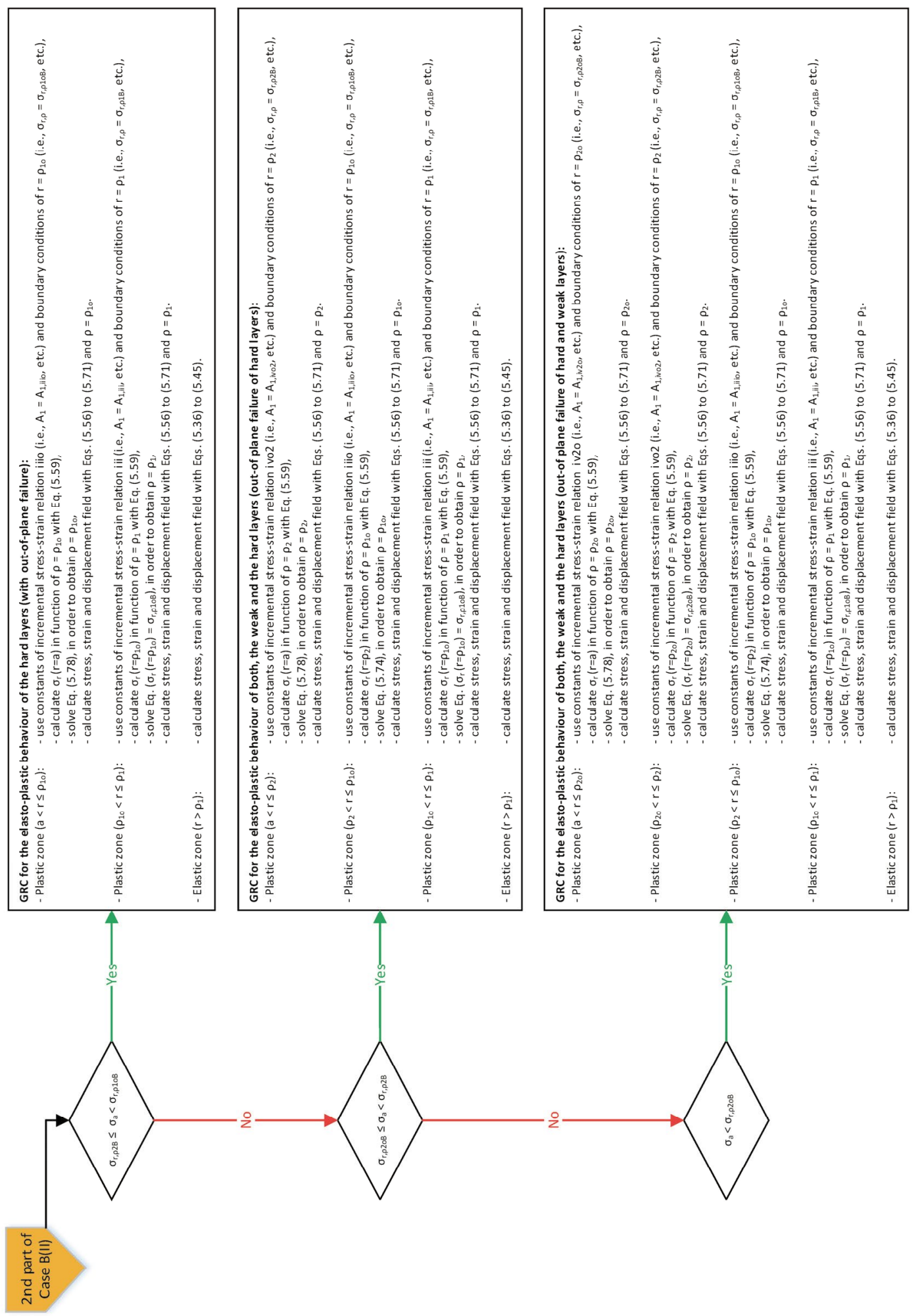

Figure C.9. Flow chart for the second part of Case B(II) (cf. Fig. 5.14). 


\section{Appendix D: Stratified rock mass - Implementation and validation of the constitutive model}

This appendix supplements Chapter 9. The list of symbols can be found on page 129.

\section{D.1 Elastic homogenisation}

The relation between the (elastic) homogenised stresses $\sigma$ and the strains $\varepsilon$ was formulated by Lourenço (1995), based on the procedure of Salamon (1968), disregarding relative displacements at the layer interfaces:

$\sigma=D_{h} \cdot \varepsilon$

where the stress and strain vectors are ordered according to

$$
\begin{aligned}
& \sigma=\left(\begin{array}{llllll}
\sigma_{x x} & \sigma_{y y} & \sigma_{z z} & \tau_{x y} & \tau_{x z} & \tau_{y z}
\end{array}\right)^{T}, \\
& \varepsilon=\left(\begin{array}{llllll}
\varepsilon_{x x} & \varepsilon_{y y} & \varepsilon_{z z} & 2 \varepsilon_{x y} & 2 \varepsilon_{x z} & 2 \varepsilon_{y z}
\end{array}\right)^{T},
\end{aligned}
$$

(cf. Clausen 2007). For that, a representative elementary volume (REV) is considered, which consists of hard and weak layers, whereby the $y$-axis lies perpendicular to the planes of the layers (cf. Fig. 9.1). Per definition, the variation of stresses and strains across the REV can be neglected, meaning that the resulting strains and stresses of the homogenised medium are the volumetric averages of the stress components in the different layers (whereupon $x_{i}$ denotes the percentage of the $i^{\text {th }}$ layer in the REV, where $x_{h}+x_{w}=1$ ). For the $i^{\text {th }}$ layer, Hooke's law is given by:

$\sigma_{i}=D_{i} \cdot \varepsilon_{i}$, where

$$
D_{i}=\frac{E_{i}}{\left(1+v_{i}\right) \cdot\left(1-2 v_{i}\right)}\left(\begin{array}{cccccc}
1-v_{i} & v_{i} & v_{i} & 0 & 0 & 0 \\
v_{i} & 1-v_{i} & v_{i} & 0 & 0 & 0 \\
v_{i} & v_{i} & 1-v_{i} & 0 & 0 & 0 \\
0 & 0 & 0 & 0.5-v_{i} & 0 & 0 \\
0 & 0 & 0 & 0 & 0.5-v_{i} & 0 \\
0 & 0 & 0 & 0 & 0 & 0.5-v_{i}
\end{array}\right) .
$$


According to Lourenço (1995), the (elastic) homogenised stiffness matrix amounts to:

$D_{h}=\left[\sum_{i} x_{i}\left(P_{t}-D_{i} P_{e}\right)^{-1}\right]^{-1} \sum_{i} x_{i}\left(P_{t}-D_{i} P_{e}\right)^{-1} D_{i}$

where the projection matrix into the stress space $P_{t}$ and the projection matrix into the strain space $P_{e}$ are defined by:

$P_{t}=\operatorname{diag}\left[\begin{array}{llllll}1 & 0 & 1 & 0 & 1 & 0\end{array}\right]$,

$P_{e}=\operatorname{diag}\left[\begin{array}{llllll}0 & 1 & 0 & 1 & 0 & 1\end{array}\right]$.

Once, the stresses and strains of the homogenised rock mass are known, also the stresses and strains in the $i^{\text {th }}$ layer have to be determined (cf. Lourenço 1995):

$\sigma_{i}=T_{t i} \cdot \sigma$

where the transformation matrix $T_{t i}$ amounts to

$T_{t i}=I+P_{t}\left(P_{t}-D_{i} P_{e}\right)^{-1}\left(D_{i} D_{h}^{-1}-I\right)$,

and $l$ is the identity matrix.

\section{D.2 Elasto-plastic homogenisation}

As soon as the weak or the hard layers reach failure, the elasto-plastic homogenisation procedure according to Lourenço (1995) was utilised. The update of the homogenised stress can be derived considering the following homogenisation procedure:

$\sigma_{n+1}=\left[\sum_{i} x_{i}\left(P_{t}-D_{i} P_{e}\right)^{-1}\right]^{-1} \sum_{i} x_{i}\left(P_{t}-D_{i} P_{e}\right)^{-1} D_{i} \cdot\left(\sigma_{n+1, i}^{t r i a l}-\Delta \lambda_{n+1, i} D_{i} \frac{\partial g_{i}}{\partial \sigma_{i}}\right)$

where the trial stresses are updated according to the return mapping algorithm (i.e., implicit integration scheme) of Clausen (2007) for the individual layers:

$\sigma_{n+1, i}=\sigma_{n+1, i}^{t r i a l}-\left.\Delta \lambda_{n+1, i} D_{i} \frac{\partial g_{i}}{\partial \sigma_{i}}\right|_{n+1}$,

considering the failure surface of Mohr-Coulomb and a non-associated flow rule (cf. Clausen 2007):

$f_{i}=\sigma_{1}-m_{i} \sigma_{3}-\sigma_{d, i}=0$, where $m_{i}=\frac{1+\sin \varphi_{i}}{1-\sin \varphi_{i}}$ and $\sigma_{d, i}=\frac{2 \cdot c_{i} \cdot \cos \varphi_{i}}{1-\sin \varphi_{i}}$,

$g_{i}=\sigma_{1}-\kappa_{i} \sigma_{3}$, where $\kappa_{i}=\frac{1+\sin \psi_{i}}{1-\sin \psi_{i}}$

respectively. The consistent constitutive matrix for the homogenised rock mass can, again, be obtained by considering Lourenço (1995): 


$$
D_{h, e p}=\left[\sum_{i} x_{i}\left(P_{t}-D_{i}^{e p} P_{e}\right)^{-1}\right]^{-1} \sum_{i} x_{i}\left(P_{t}-D_{i}^{e \rho} P_{e}\right)^{-1} D_{i}^{e p}
$$

where the consistent constitutive matrix $D_{i}^{e p}$ for the individual layers is calculated according to Clausen (2007). The entire derivation of the homogenisation procedure can be found in Lourenço (1995).

Consequently, this elasto-plastic formulation includes a fully implicit Euler backward return mapping.

\section{D.3 Schematic stress-point algorithm}

1. Transform stress and strains into the local coordinate system (where the y-axis is perpendicular to the layers, see, e.g., Appendix C.1 of Clausen et al. 2005).

2. Compute elastic trial stresses and check for plastic behaviour:

for the homogenised rock mass: $\sigma_{n+1}^{\text {trial }}=\sigma_{n}+D_{h} \Delta \varepsilon_{n+1}$,

for the individual layers: $\sigma_{n+1, i}^{\text {trial }}=\sigma_{n, i}+T_{t i} D_{h} \Delta \varepsilon_{n+1}$.

If $f_{i}\left(\sigma_{n+1, i}^{\text {trial }}\right)>0$ : Layer is plastic, if $f_{i}\left(\sigma_{n+1, i}^{\text {trial }}\right) \leq 0$ : Layer is elastic.

$\rightarrow$ If all layers are elastic, update stresses $\rightarrow$ Exit

3. Consistence at stage $n+1$ : Solve system of non-linear equations

$f\left(\sigma_{n+1, i}\right)=0$ for all plastic layers.

for the homogenised rock mass: $\sigma_{n+1}=\left[\sum_{i} x_{i}\left(P_{t}-D_{i} P_{e}\right)^{-1}\right]^{-1} \sum_{i} x_{i}\left(P_{t}-D_{i} P_{e}\right)^{-1} D_{i} \cdot\left(\sigma_{n+1, i}^{t r i a}-\Delta \lambda_{n+1, i} D_{i} \frac{\partial g_{i}}{\partial \sigma_{i}}\right)$, for the individual layer: $\sigma_{n+1, i}=\sigma_{n+1}+P_{t}\left(P_{t}-D_{i} P_{e}\right)^{-1}\left(-\sigma_{n+1}+\sigma_{n+1, i}^{t r i a l}-\Delta \lambda_{n+1, i} D_{i} \frac{\partial g_{i}}{\partial \sigma_{i}}\right)$,

Calculate $f_{i}\left(\sigma_{n+1, i}\right) \rightarrow$ if a converged state is found $\rightarrow$ Exit.

Else $\rightarrow$ Update $\Delta \lambda_{n+1, i} D_{i} \frac{\partial g_{i}}{\partial \sigma_{i}}$.

4. Compute consistent constitutive matrix:

$$
D_{h, e p}=\left[\sum_{i} x_{i}\left(P_{t}-D_{i}^{e p} P_{e}\right)^{-1}\right]^{-1} \sum_{i} x_{i}\left(P_{t}-D_{i}^{e p} P_{e}\right)^{-1} D_{i}^{e p}
$$

5. Transform stress, strains and consistent constitutive matrix back into the original coordinate system.

\section{D.4 Validation of the constitutive model analogously to Lourenço (1995)}

The formulation and implementation in Abaqus of the constitutive model (hereafter referred to as "homogenised model") was validated for a series of boundary value problems by comparing the results for the homogenised model with those obtained by modelling discretely a sequence of very thin layers (hereafter referred to as "discrete model"). The latter were modelled as isotropic, linearly elastic, perfectly plastic material obeying the MohrCoulomb yield criterion (considering the subroutine of Clausen 2007).

Figure D.1 provides an overview of examples. With increasing number (and, consequently, decreasing thickness) of the layers the results approach the homogenised solution. (In order to reduce the sensitivity of the results to the mesh discretisation, sufficiently fine meshes were chosen.) 
(a)

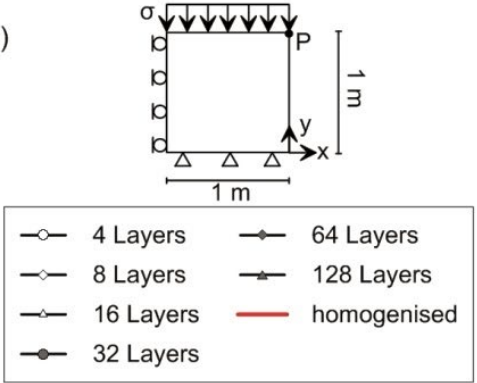

(b)

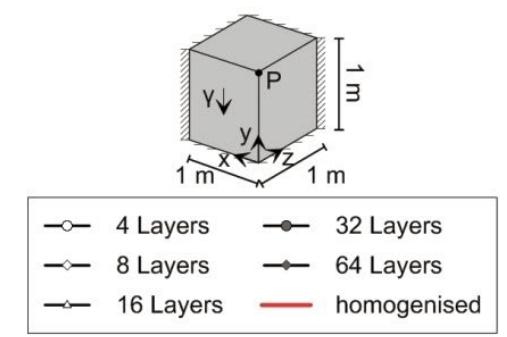

(c)

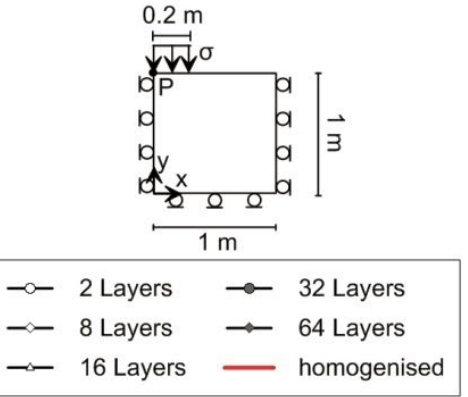

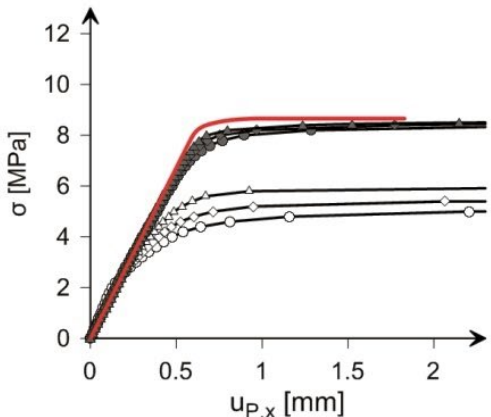
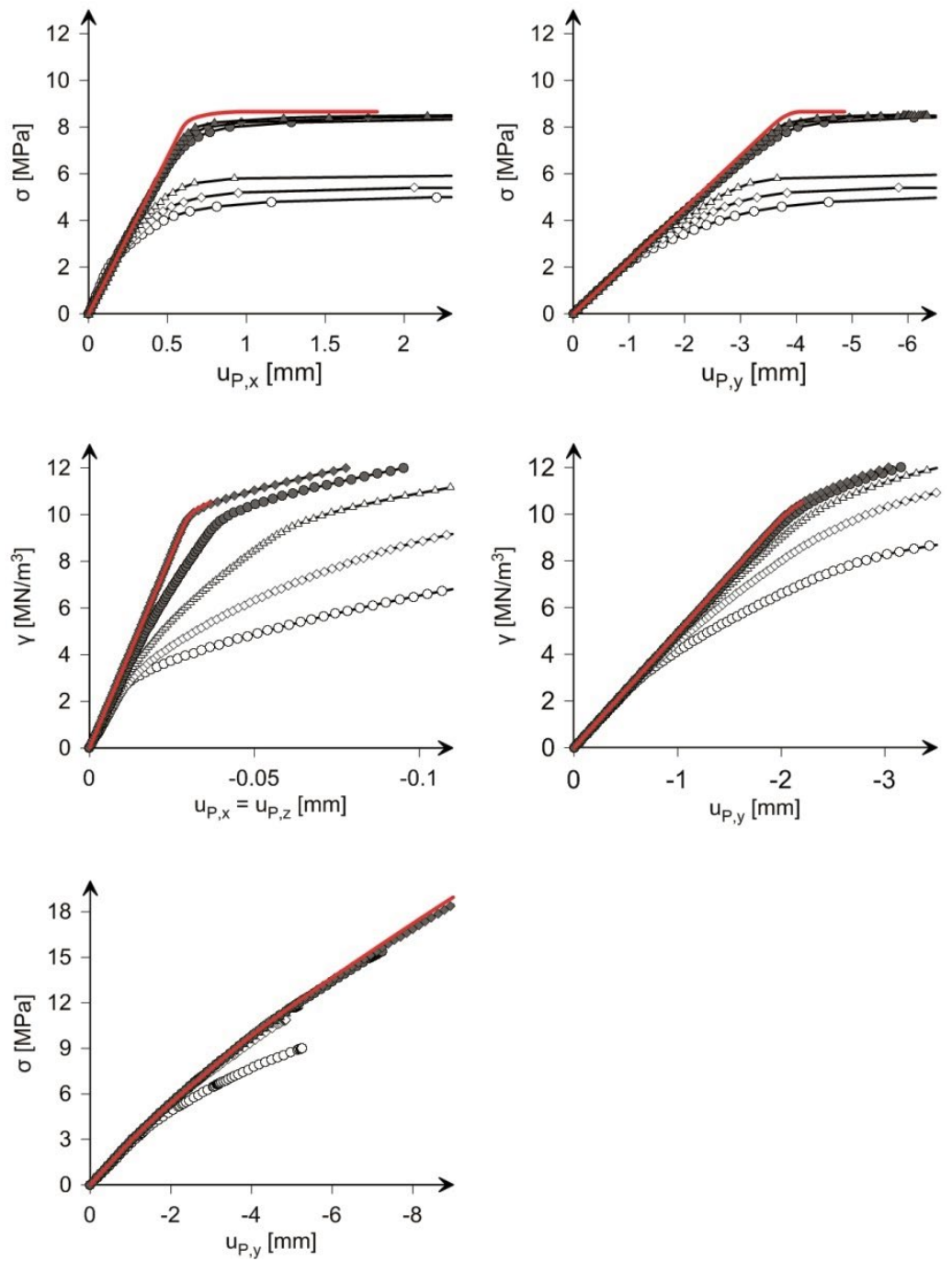

Figure D.1. Validation examples: (a) uniaxial loading test under plane strain conditions; (b) threedimensional rock volume under body force; (c) half space under strip loading (horizontal layers ( $x_{h}=x_{w}=0.5$ in examples (a) and (b), $x_{w}=0.75$ and $x_{h}=0.25$ in example (c), $\left.E_{h}=10 \mathrm{GPa}, c_{h}=5 \mathrm{MPa}, E_{w}=1 \mathrm{GPa}, c_{w}=0.5 \mathrm{MPa}, v_{h}=v_{w}=0.3, \varphi_{h}=\varphi_{w}=25^{\circ}, \psi_{h}=\psi_{w}=5^{\circ}\right)$.

\section{D.5 Validation of the constitutive model considering the problem of tunnelling perpendicular to the layers of a thinly stratified rock mass}

The model was validated additionally for the case of Chapter 5 for which a closed-form solution was derived. The ground response is analysed numerically by axisymmetric calculations as in Chapter 7. Figure D.2 shows the GRCs in the middle of the weak and of the competent rock zone for several values of the layer thickness $w$. With decreasing layer thickness the displacements converge exactly to those obtained by the numerically implemented homogenised model. (The latter fits perfectly with the GRC obtained with the analytical solution of Chapter 5.)

Figure D.3 applies to an unsupported tunnel and very thin layers and shows the tangential, radial and axial stress fields in the hard and in the weak layers as well as the displacement field according to the numerically implemented homogenised model and to the discrete 
model. (The former yields exactly the same results as the analytical solution of Chapter 5.) The results agree very well.
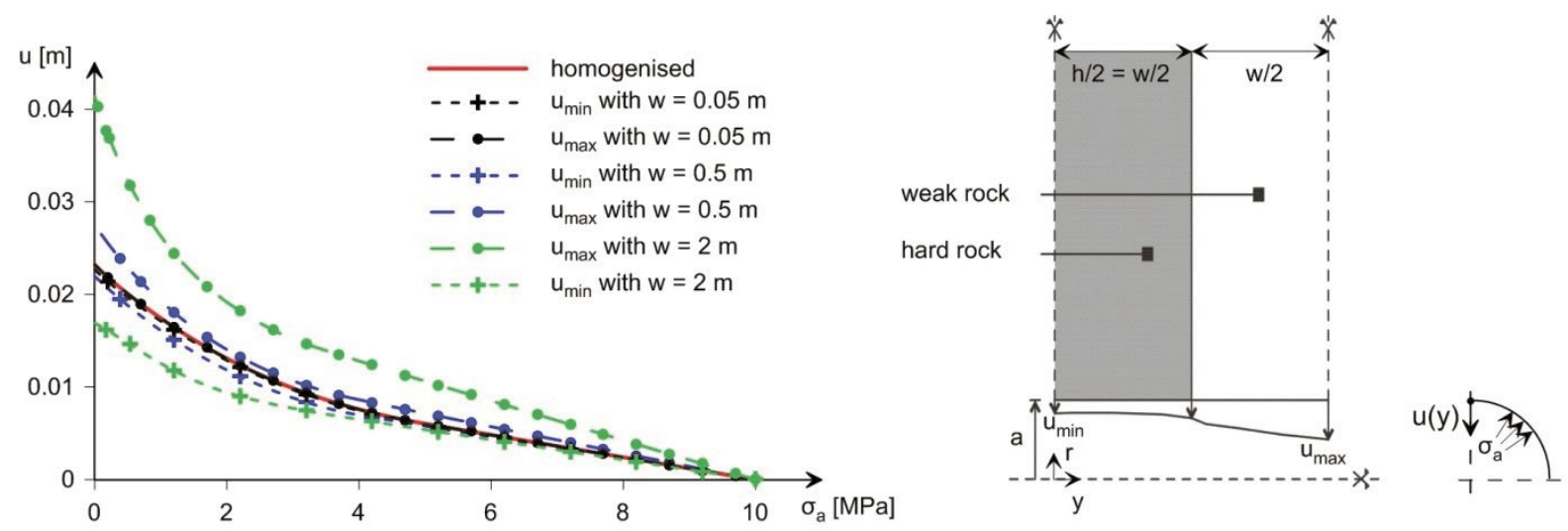

Figure D.2. Ground response curves for a tunnel drive perpendicular to the layers $\left(a=5 \mathrm{~m}, \sigma_{0}=10 \mathrm{MPa}\right.$, $x_{h}=x_{w}=0.5, E_{h}=10 \mathrm{GPa}, c_{h}=5 \mathrm{MPa}, E_{w}=1 \mathrm{GPa}, c_{w}=0.5 \mathrm{MPa}, v_{h}=v_{w}=0.3, \varphi_{h}=\varphi_{w}=25^{\circ}$, $\left.\boldsymbol{\psi}_{h}=\boldsymbol{\psi}_{w}=5^{\circ}\right)$.

(a)
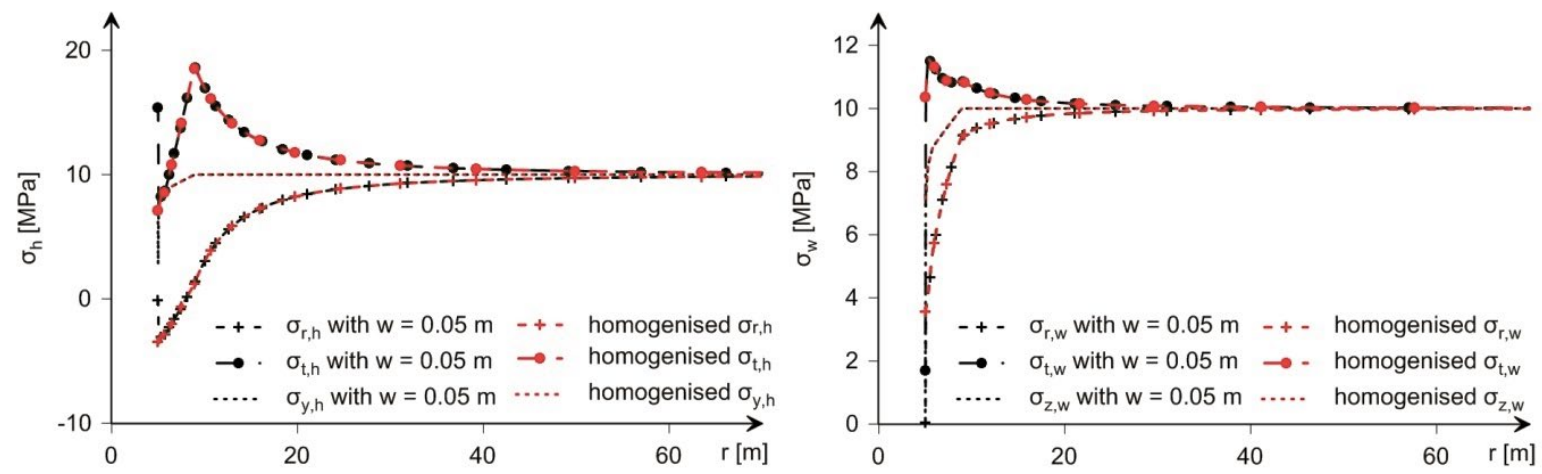

(b)

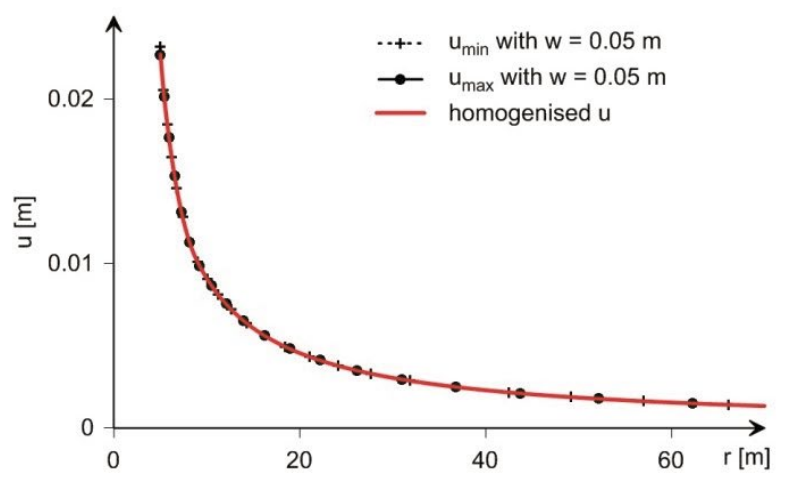

Figure D.3. a) Stress field in the hard (I.h.s.) and in the weak (r.h.s.) layers; (b) displacement field around an unsupported tunnel drive perpendicular to the layers, according to the homogenised model (red lines) and to the discrete model of $\mathbf{0 . 0 5}$ thick alternating layers (unsupported tunnel, $a=5 \mathrm{~m}, \sigma_{0}=10 \mathrm{MPa}, x_{h}=x_{w}=0.5, E_{h}=10 \mathrm{GPa}, c_{h}=5 \mathrm{MPa}, E_{w}=1 \mathrm{GPa}, c_{w}=0.5 \mathrm{MPa}$, $\left.v_{h}=v_{w}=0.3, \varphi_{h}=\varphi_{w}=25^{\circ}, \psi_{h}=\psi_{w}=5^{\circ}\right)$. 



\section{Appendix E: Tunnelling parallel to the bedding of a thinly stratified rock mass - Dimensionless diagrams for the displacements}

This appendix presents the dimensionless diagrams introduced in Section 9.4.

Table E.1 gives an overview of the dimensionless diagrams. The list of symbols can be found on page 129.

Table E.1. Overview of the dimensionless diagrams.

\begin{tabular}{ccc}
\hline Figure & displacement & $\sigma_{a} / \sigma_{0}$ \\
\hline E.1 & $u_{\max } / u_{w, 2 D}$ & \\
E.2 & $u_{\text {average }} / u_{w, 2 D}$ & 0.0 \\
E.3 & $u_{\min } / u_{w, 2 D}$ & \\
\hline E.4 & $u_{\max } / u_{w, 2 D}$ & \\
E.5 & $u_{\text {average }} / u_{w, 2 D}$ & 0.1 \\
E.6 & $u_{\min } / u_{w, 2 D}$ & \\
E.7 & $u_{\max } / u_{w, 2 D}$ & \\
E.8 & $u_{\text {average }} / u_{w, 2 D}$ & 0.2 \\
E.9 & $u_{\min } / u_{w, 2 D}$ & \\
\hline
\end{tabular}




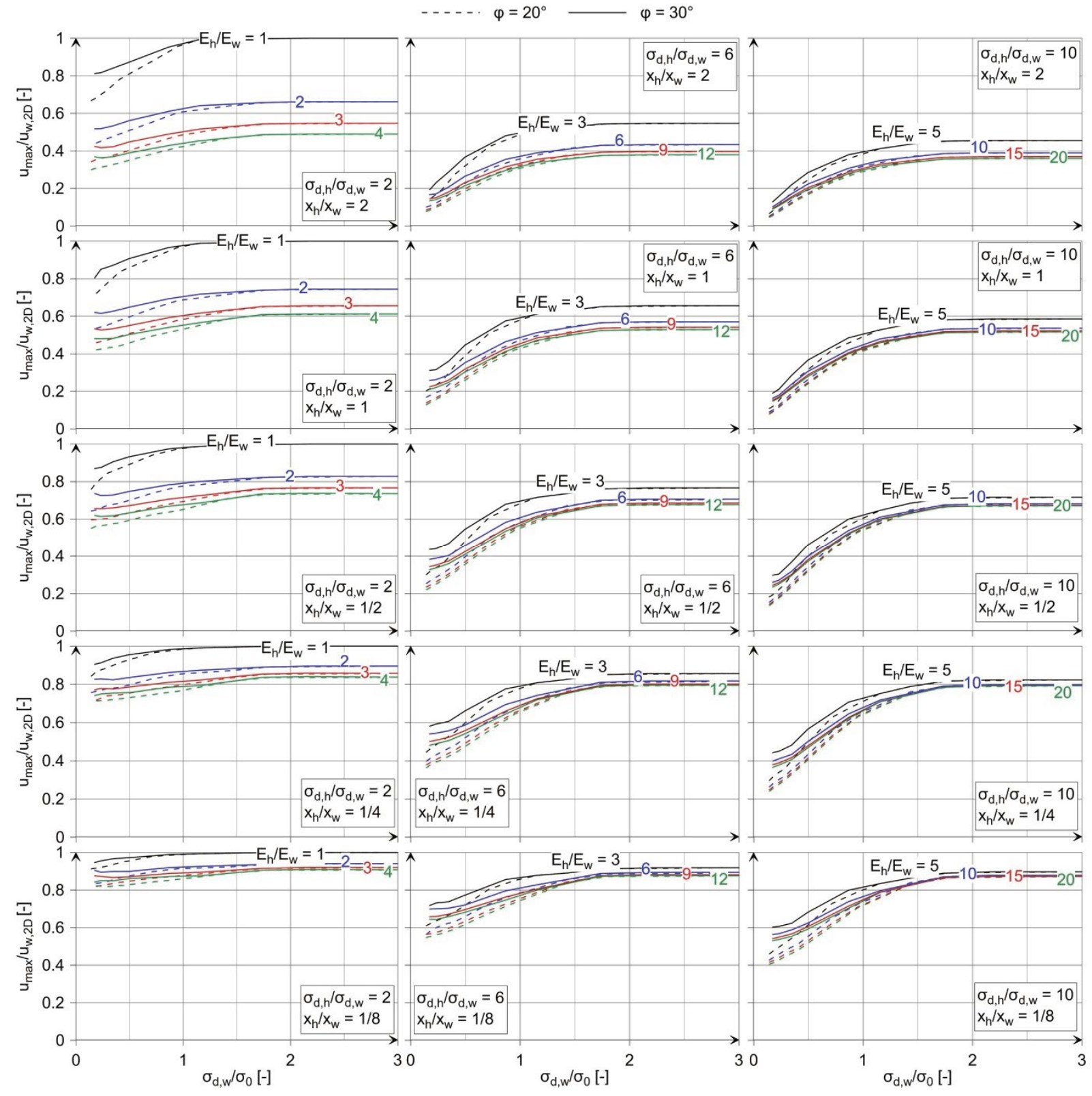

Figure E.1. Nomograms for $u_{\max } / u_{w, 2 D}$ and $\sigma_{a} / \sigma_{0}=0$. 


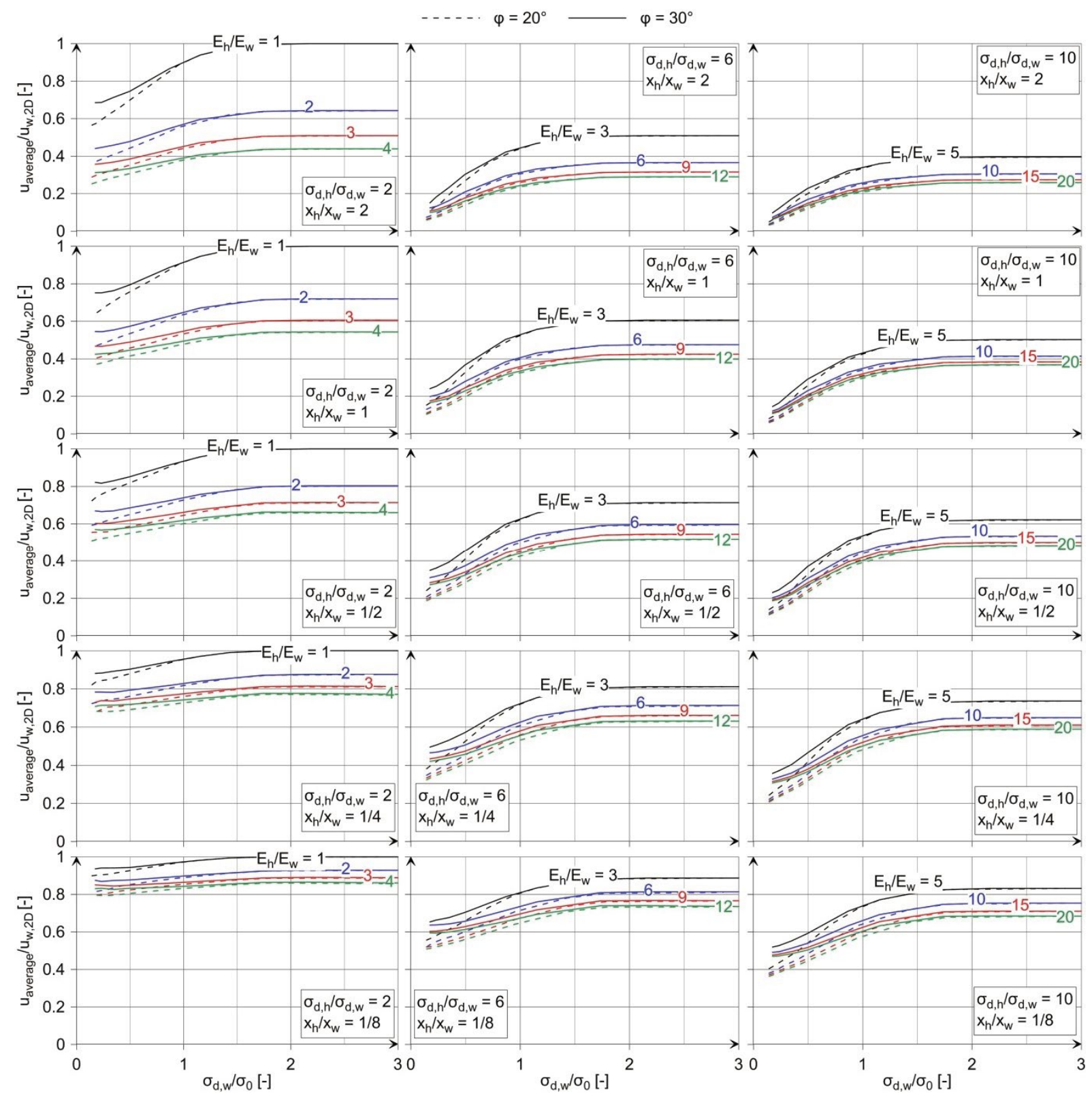

Figure E.2. Nomograms for $U_{\text {average }} / U_{w, 2 D}$ and $\sigma_{a} / \sigma_{0}=0$. 


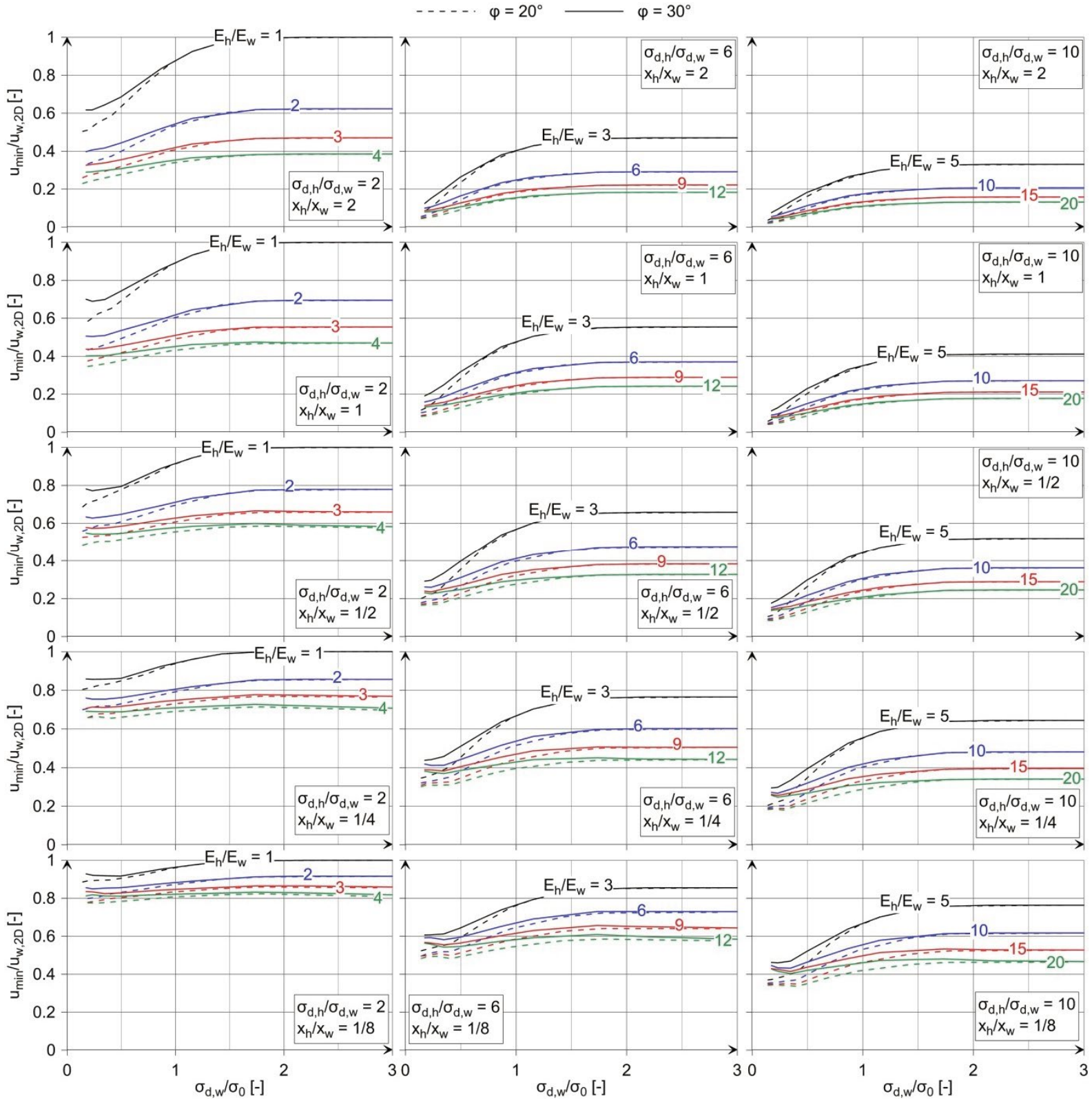

Figure E.3. Nomograms for $U_{\min } / u_{w, 2 D}$ and $\sigma_{a} / \sigma_{0}=0$. 


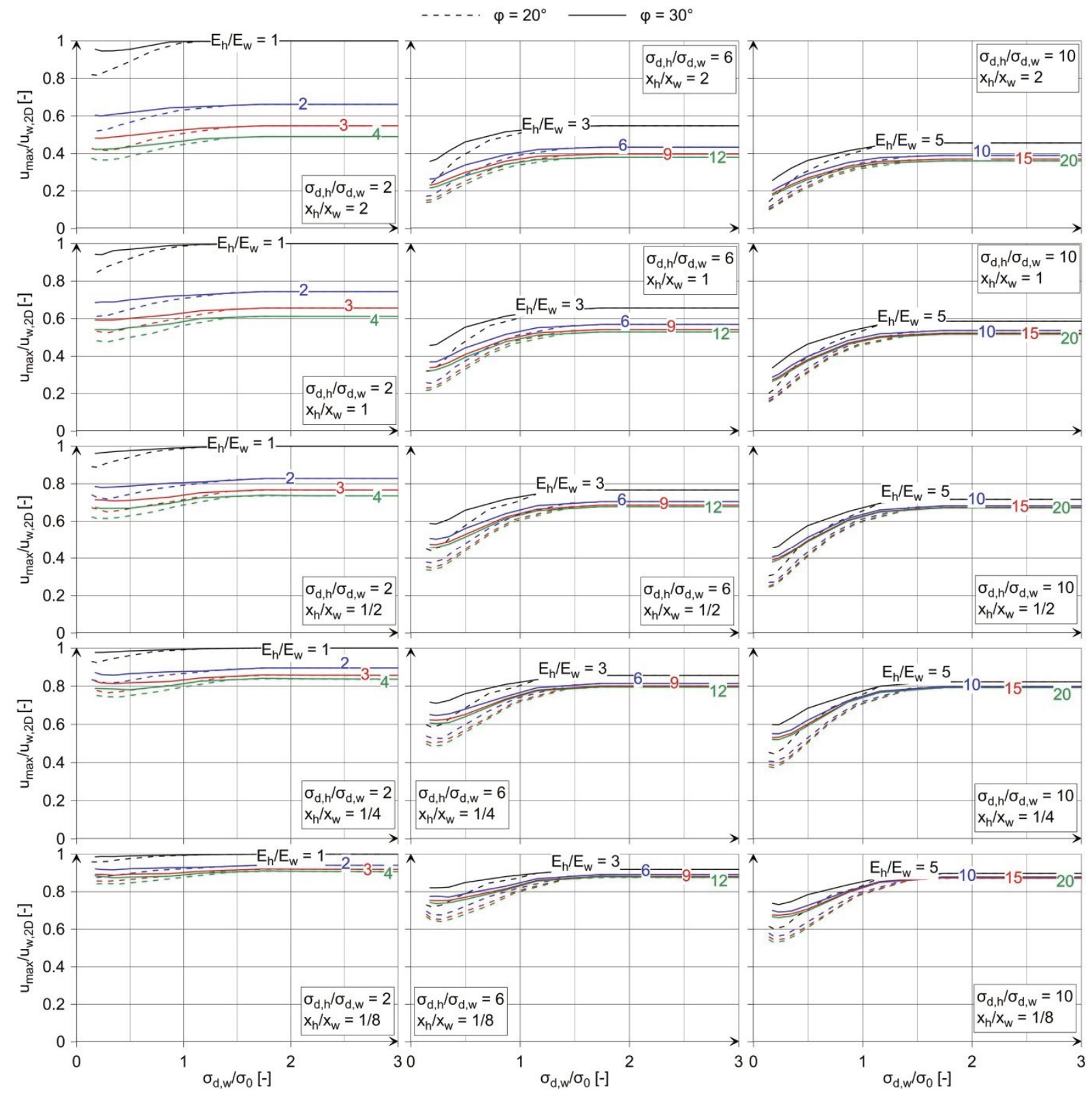

Figure E.4. Nomograms for $U_{\max } / u_{w, 2 D}$ and $\sigma_{a} / \sigma_{0}=0.1$. 


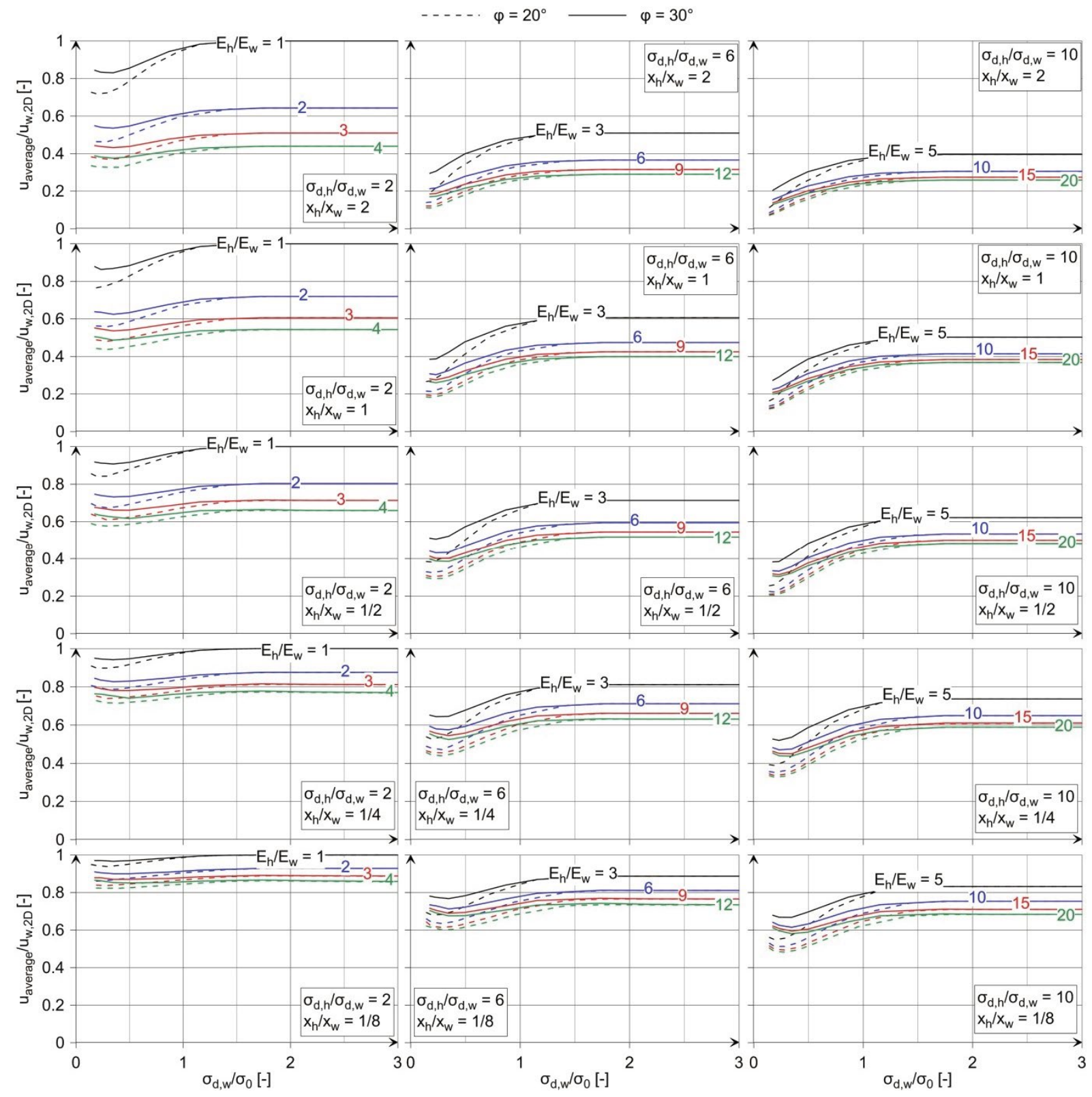

Figure E.5. Nomograms for $U_{a v e r a g e} / U_{w, 2 D}$ and $\sigma_{a} / \sigma_{0}=0.1$. 


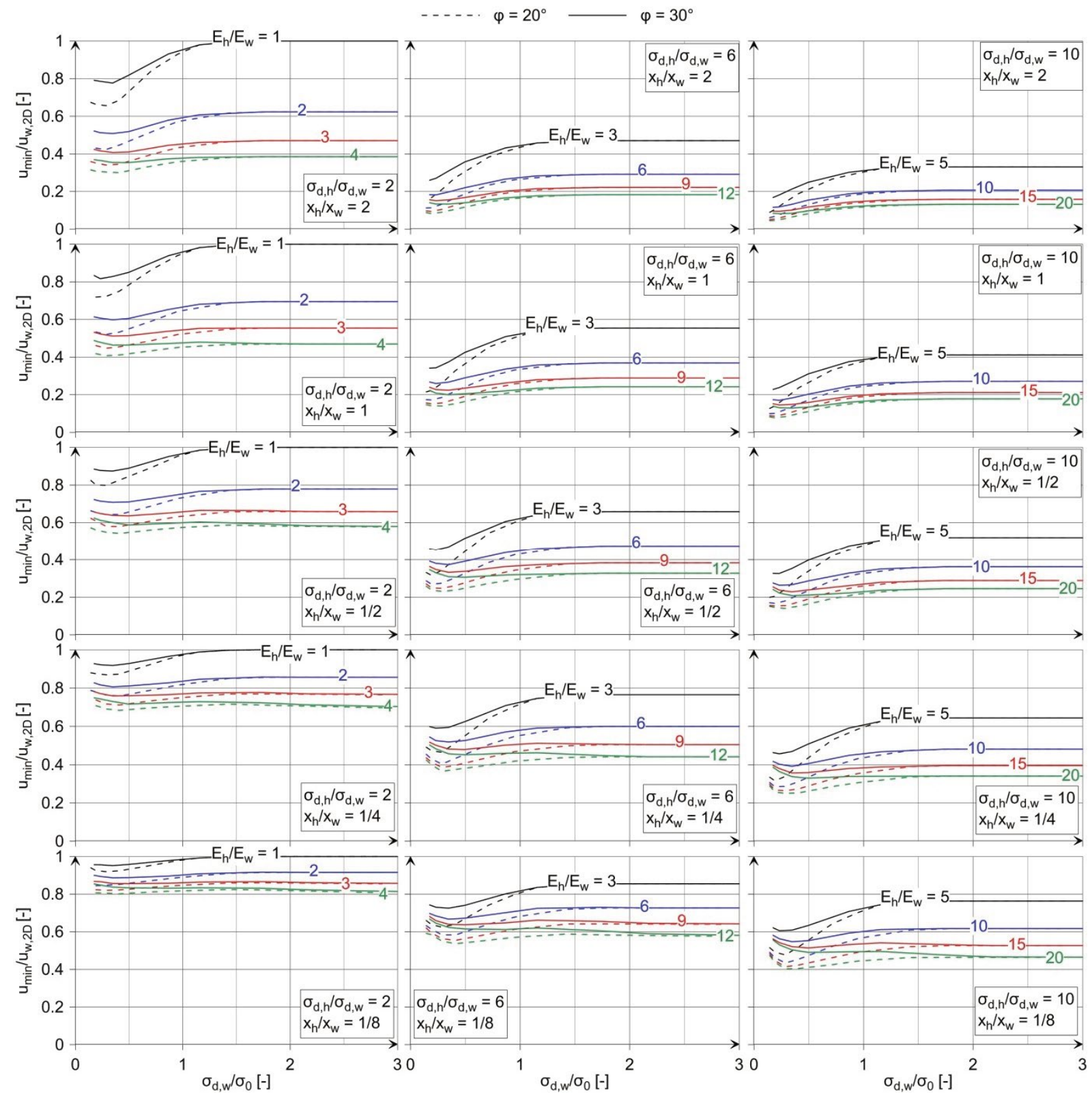

Figure E.6. Nomograms for $U_{\min } / u_{\mathrm{w}, 2 D}$ and $\sigma_{a} / \sigma_{0}=0.1$. 


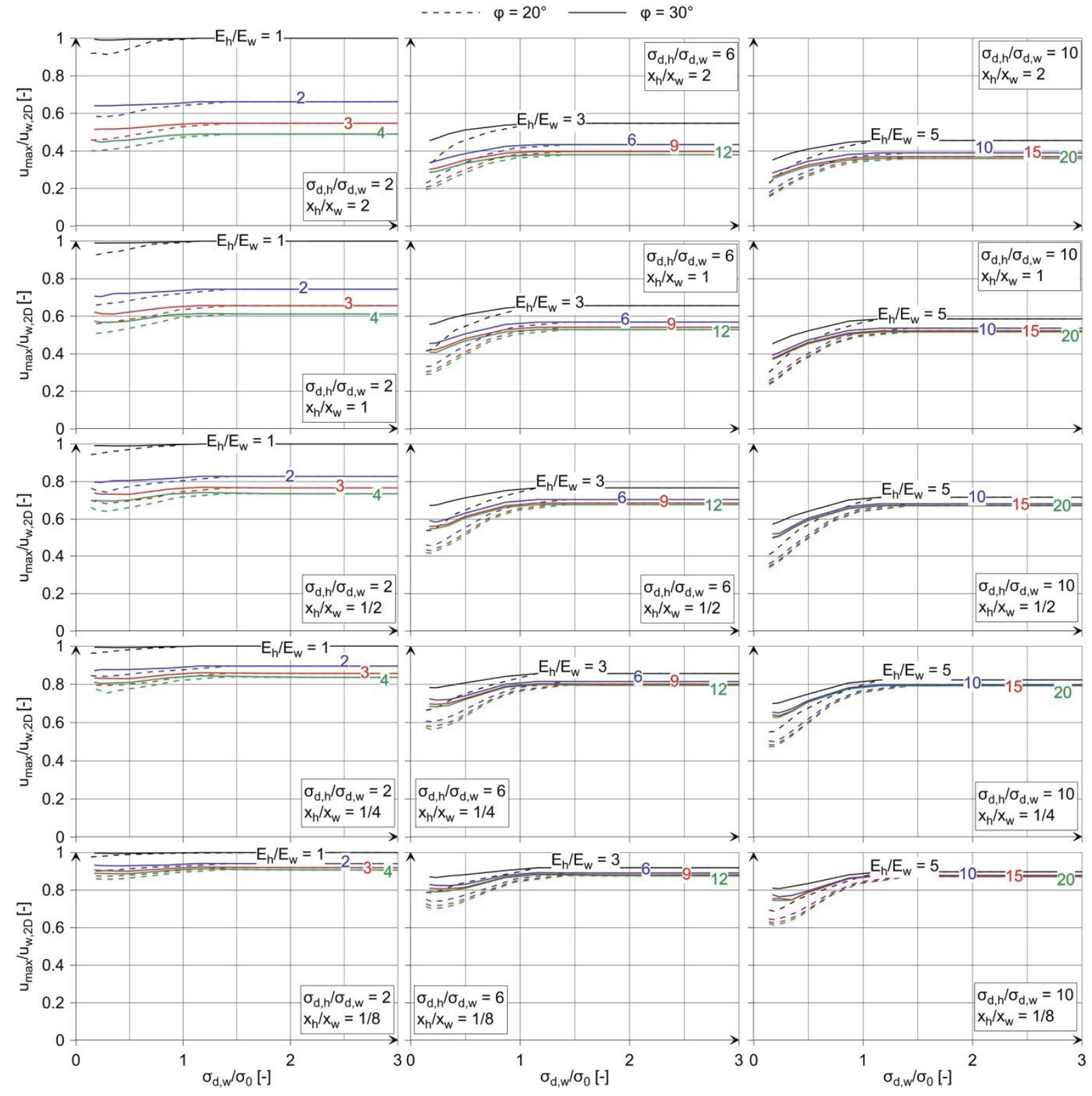

Figure E.7. Nomograms for $U_{\max } / u_{w, 2 D}$ and $\sigma_{a} / \sigma_{0}=0.2$. 


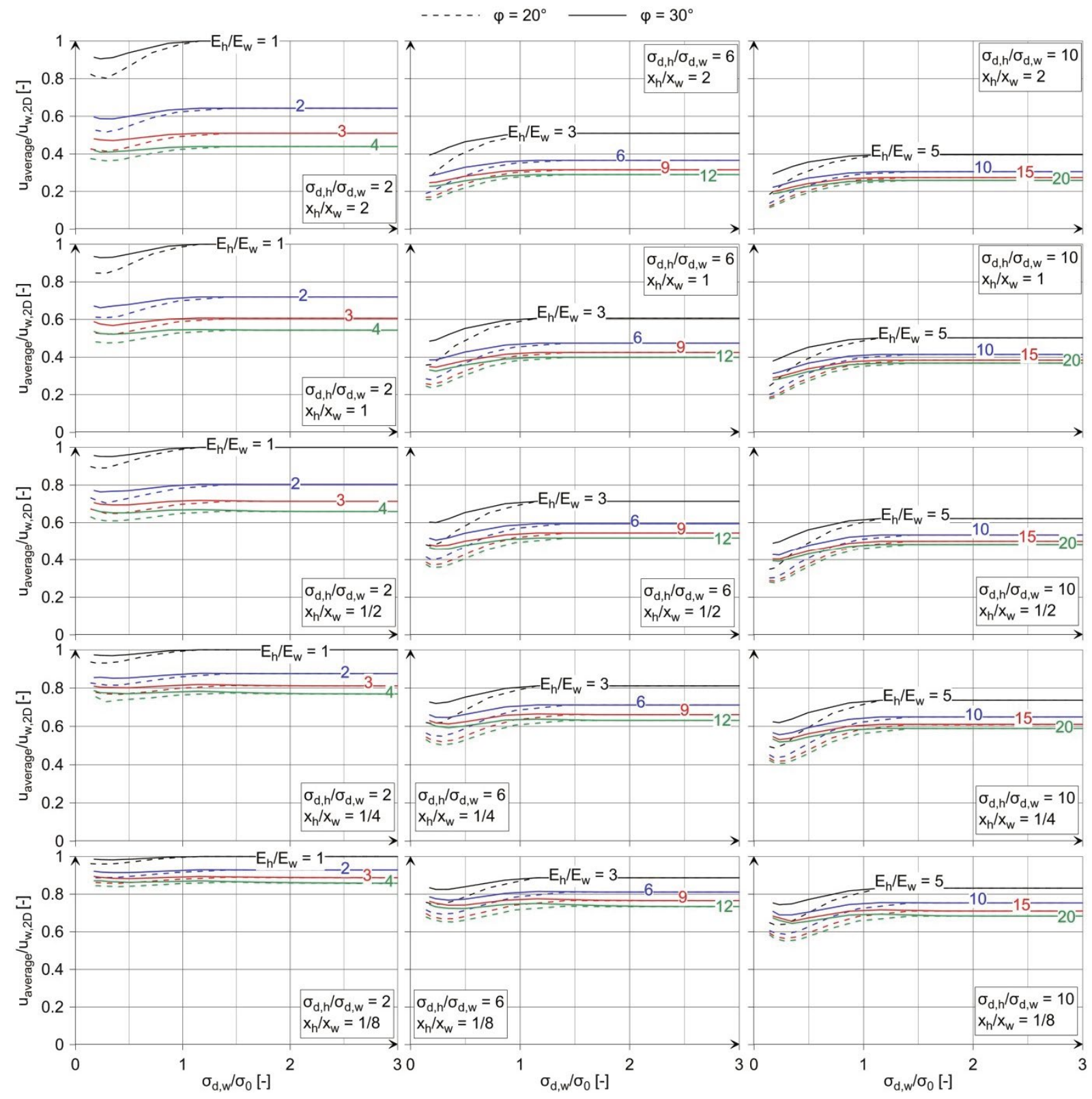

Figure E.8. Nomograms for $U_{a v e r a g e} / U_{w, 2 D}$ and $\sigma_{a} / \sigma_{0}=0.2$. 


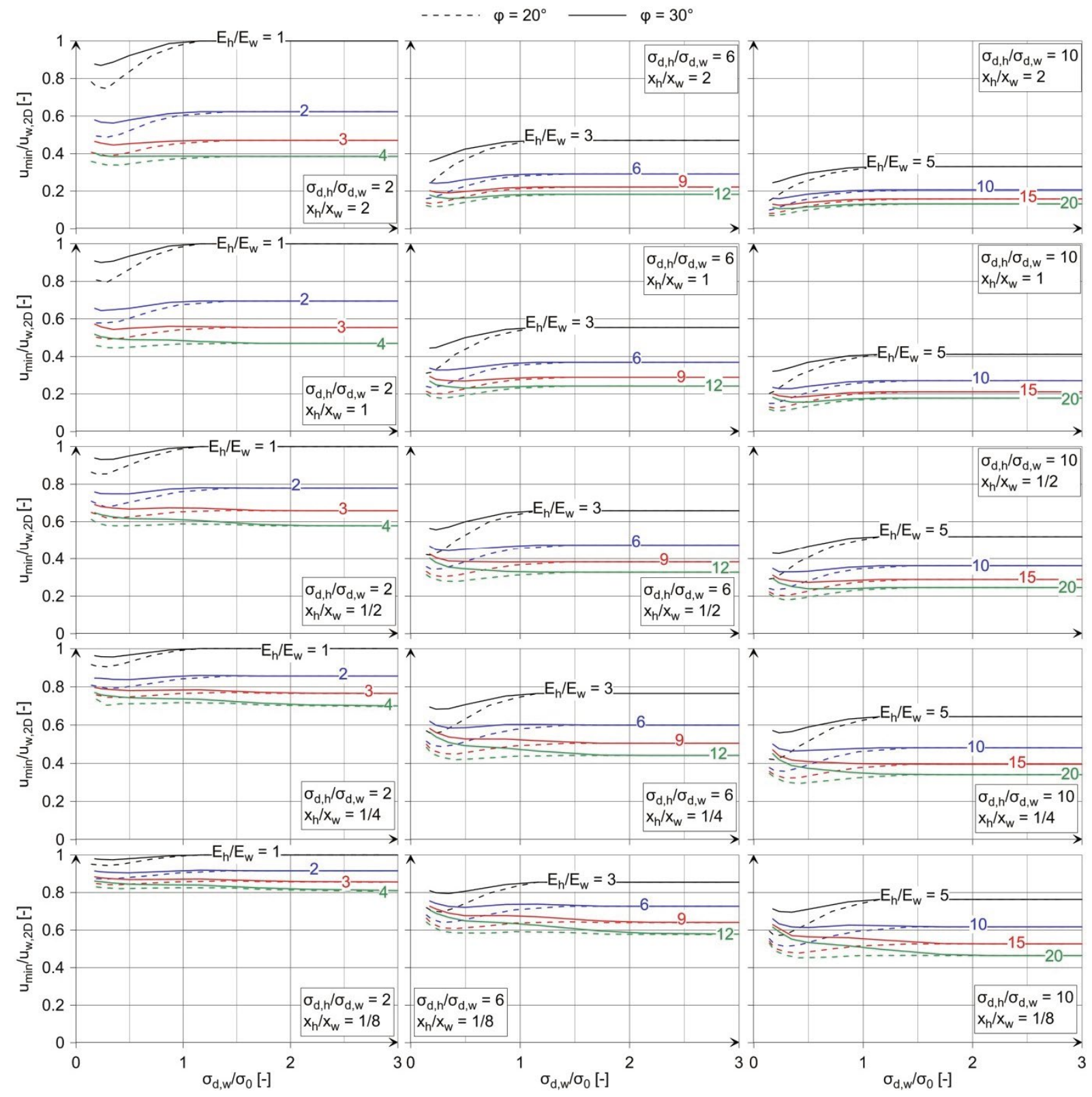

Figure E.9. Nomograms for $U_{\min } / u_{w, 2 D}$ and $\sigma_{a} / \sigma_{0}=0.2$. 


\section{Appendix F: Schistous rock mass - Implementation and validation of the constitutive model}

This appendix supplements Chapter 11. The list of symbols can be found on page 159.

\section{F.1 Model formulation}

If neither the matrix nor the schistosity reaches failure, the elastic stresses $\sigma$ and the strains $\varepsilon$ of the REV are related by Hooke's law:

$\sigma=D \cdot \varepsilon$

where $D$ is the elastic constitutive matrix:

$D=\frac{E}{(1+v) \cdot(1-2 v)}\left(\begin{array}{cccccc}1-v & v & v & 0 & 0 & 0 \\ v & 1-v & v & 0 & 0 & 0 \\ v & v & 1-v & 0 & 0 & 0 \\ 0 & 0 & 0 & 0.5-v & 0 & 0 \\ 0 & 0 & 0 & 0 & 0.5-v & 0 \\ 0 & 0 & 0 & 0 & 0 & 0.5-v\end{array}\right)$

where the stress and strain vectors in Abaqus (Dassault Systèmes 2011) are ordered according to:

$\sigma=\left(\begin{array}{llllll}\sigma_{x x} & \sigma_{y y} & \sigma_{z z} & \tau_{x y} & \tau_{x z} & \tau_{y z}\end{array}\right)^{T}$

$\varepsilon=\left(\begin{array}{llllll}\varepsilon_{x x} & \varepsilon_{y y} & \varepsilon_{z z} & 2 \varepsilon_{x y} & 2 \varepsilon_{x z} & 2 \varepsilon_{y z}\end{array}\right)^{T}$.

If the stresses in the matrix of the REV reach the failure surface (i.e., $\sigma_{1}$ and $\sigma_{3}$ denotes the maximum and minimum principal stresses, respectively):

$f_{m}=\sigma_{1}-m_{m} \sigma_{3}-\sigma_{d, m}=0$, where $m_{m}=\frac{1+\sin \varphi_{m}}{1-\sin \varphi_{m}}$ and $\sigma_{d, m}=\frac{2 \cdot c_{m} \cdot \cos \varphi_{m}}{1-\sin \varphi_{m}}$

the (elastic) trial stresses are updated according to the return mapping algorithm (i.e., implicit integration scheme) of Clausen (2007): 
$\sigma_{n+1}=\sigma_{n}+D \Delta \varepsilon_{m}-\left.\Delta \lambda_{n+1} D \frac{\partial g_{m}}{\partial \sigma}\right|_{n+1}$,

where $\sigma_{n}+D \Delta \varepsilon_{m}$ denotes the elastic predictor stress state and $\left.\Delta \lambda_{n+1} D \frac{\partial g}{\partial \sigma}\right|_{n+1}$ the plastic corrector stress (of the matrix), assuming the following plastic potential:

$g=\sigma_{1}-\kappa_{m} \sigma_{3}$, where $\kappa_{m}=\frac{1+\sin \psi_{m}}{1-\sin \psi_{m}}$.

According to Equation (11.5), the strain increment $\Delta \varepsilon_{m}$ in the matrix is a function of the (homogenised) strain increments $\Delta \varepsilon$ and the shearing strain increment $\gamma_{s}$ along the schistosity planes. If the failure criterion of the schistosity is not reached, $\gamma_{s}$ equals zero.

Otherwise, the latter is à priori unknown, but can be obtained, considering the prerequisite that the stresses $\sigma_{n+1}$ (which are obtained from Eq. F-6) have to lie on the failure surface of the schistosity, which can be written as follows:

$f_{s}=|\tau|-\sigma_{y y} \tan \varphi_{s}-c_{s}=0$,

provided that the $y$-axis is perpendicular to the schistosity planes. The shearing strain increment $\gamma_{s}$ can thus be obtained with an iterative procedure (starting from the initial guess $\left.\gamma_{s, 0}\right)$, using the Newton-Raphson method:

$\gamma_{s}=\gamma_{s, 0}-\frac{f_{s}\left(\gamma_{s, 0}\right)}{f_{s}^{\prime}\left(\gamma_{s, 0}\right)}$

Furthermore, for the 3D-case, in order to obtain $\Delta y_{x y, s}$ and $\Delta y_{y z, s}$ (cf. Eq. 11.5) the basic assumption of standard plasticity theory, that the shear stress $T_{s}$ and the shear strain increment $\gamma_{s}$ in the schistosity planes are co-axial, is considered, leading thus to:

$\frac{\tau_{x y}}{\tau_{y z}}=\frac{\Delta \gamma_{x y, s}}{\Delta \gamma_{y z, s}}$

so that the following relation applies:

$$
\begin{aligned}
& \Delta \gamma_{x y, s}=\tau_{x y} \frac{\gamma_{s}}{\tau}, \\
& \Delta \gamma_{y z, s}=\tau_{y z} \frac{\gamma_{s}}{\tau},
\end{aligned}
$$

where

$$
\tau=\sqrt{\tau_{x y}^{2}+\tau_{y z}^{2}} .
$$

Therefore, $\Delta \gamma_{x y, s}$ and $\Delta y_{y z, s}$ have the same direction than $\tau_{x y}$ and $\tau_{y z}$. 


\section{F.2 Schematic stress-point algorithm for the 2D case}

1. Transform stress $\sigma_{n}$ and (homogenised) strain increment $\Delta \varepsilon$ into the local coordinate system (where the $y$-axis is perpendicular to the schistosity planes, see, e.g., Appendix C.1 of Clausen et al. 2005).

2. First guess for $\gamma_{s}: \gamma_{s}=0$.

3. Initialisation of the strain experienced by the matrix: $\Delta \varepsilon_{m}=\Delta \varepsilon$.

4. Calculate updated stress state $\sigma_{n+1}$ and consistent constitutive matrix $D^{e p c}$ with predictor stress $\left(\sigma_{n}+D \Delta \varepsilon_{m}\right)$ with the algorithm of Clausen (2007).

5. Calculate yield function of the schistosity: $f_{s}=\max \left(\left|\tau_{n+1, x y}\right|-\sigma_{n+1, y y} \tan \varphi_{s}-c_{s}=0 ; 0\right)$.

6. Do while $f_{s} \geq$ Tolerance:

a. Update $\gamma_{s}$ (according to the Newton-Raphson method):

$$
\text { if } \tau_{n+1, x y}<0: \gamma_{s}=\gamma_{s}-\frac{f_{s}}{G} \text {; if } \tau_{n+1, x y}>0: \gamma_{s}=\gamma_{s}+\frac{f_{s}}{G} \text {. }
$$

b. Update strains experienced by the matrix:

$$
\Delta \varepsilon_{y y, m}=\Delta \varepsilon_{y y}-\gamma_{s} \cdot \tan \psi_{s}, \Delta \gamma_{x y, m}=\Delta \gamma_{x y}-\gamma_{s} \text {. }
$$

c. Calculate updated stress state $\sigma_{n+1}$ and consistent constitutive matrix $D^{e p c}$ with updated predictor stress $\left(\sigma_{n}+D \Delta \varepsilon_{m}\right)$ with the algorithm of Clausen (2007).

d. Calculate yield function of the schistosity: $f_{s}=\left|\tau_{n+1, x y}\right|-\sigma_{n+1, y y} \tan \varphi_{s}-c_{s}=0$.

e. Do while $\left|f_{s}\right| \geq$ Tolerance:

i. Update $\gamma_{s}$ (according to the Newton-Raphson method):

$$
\text { if } \tau_{n+1, x y}<0: \gamma_{s}=\gamma_{s}-\frac{f_{s}}{G} \text {; if } \tau_{n+1, x y}>0: \gamma_{s}=\gamma_{s}+\frac{f_{s}}{G} \text {. }
$$

ii. Update strains experienced by the matrix:

$$
\Delta \varepsilon_{y y, m}=\Delta \varepsilon_{y y}-\gamma_{s} \cdot \tan \psi_{s}, \Delta \gamma_{x y, m}=\Delta \gamma_{x y}-\gamma_{s} \text {. }
$$

iii. Calculate updated stress state $\sigma_{n+1}$ and consistent constitutive matrix $D^{\text {epc }}$ with updated predictor stress $\left(\sigma_{n}+D \Delta \varepsilon_{m}\right)$ with the algorithm of Clausen (2007).

iv. Calculate yield function of the schistosity: $f_{s}=\left|\tau_{n+1, x y}\right|-\sigma_{n+1, y y} \tan \varphi_{s}-c_{s}=0$.

f. Calculate consistent constitutive matrix $D^{\mathrm{epc}:}$

$$
\begin{aligned}
& D^{\mathrm{epc}}=D-\frac{D \frac{\partial g_{s}}{\partial \sigma}\left(\frac{\partial f_{s}}{\partial \sigma}\right)^{T} D}{\left(\frac{\partial f_{s}}{\partial \sigma}\right)^{T} D \frac{\partial g_{s}}{\partial \sigma}} \text {, where } \\
& \text { if } \tau_{n+1, x y}<0: \frac{\partial g_{s}}{\partial \sigma}=\left(\begin{array}{c}
0 \\
\tan \psi_{s} \\
0 \\
-1
\end{array}\right) \text { and } \frac{\partial f_{s}}{\partial \sigma}=\left(\begin{array}{c}
0 \\
\tan \varphi_{s} \\
0 \\
-1
\end{array}\right) \text {, } \\
& \text { if } \tau_{n+1, x y}>0: \frac{\partial g_{s}}{\partial \sigma}=\left(\begin{array}{c}
0 \\
\tan \psi_{s} \\
0 \\
1
\end{array}\right) \text { and } \frac{\partial f_{s}}{\partial \sigma}=\left(\begin{array}{c}
\tan \varphi_{s} \\
0 \\
1
\end{array}\right) .
\end{aligned}
$$

7. Transform stress, strains and consistent constitutive matrix back into the original coordinate system. 


\section{F.3 Schematic stress-point algorithm for the 3D case}

1. Transform stress $\sigma_{n}$ and strain increment $\Delta \varepsilon$ into the local coordinate system (where the y-axis is perpendicular to the schistosity planes, see, e.g., Appendix C.1 of Clausen et al. 2005).

2. First guess for $\gamma_{s}: \gamma_{s}=0$.

3. Initialisation of the strain experienced by the matrix: $\Delta \varepsilon_{m}=\Delta \varepsilon$.

4. Calculate updated stress state $\sigma_{n+1}$ and consistent constitutive matrix $D^{e p c}$ with predictor stress $\left(\sigma_{n}+D \Delta \varepsilon_{m}\right)$ with the algorithm of Clausen (2007).

5. Calculate yield function of the schistosity: $f_{s}=\max \left(\left|\tau_{n+1}\right|-\sigma_{n+1, y y} \tan \varphi_{s}-c_{s}=0 ; 0\right)$.

6. Do while $f_{s} \geq$ Tolerance:

a. Update $\gamma_{s}$ (according to the Newton-Raphson method):

$\gamma_{s}=\gamma_{s}+\frac{f_{s}}{G}$.

b. Update strains experienced by the matrix:

$$
\Delta \varepsilon_{y y, m}=\Delta \varepsilon_{y y}-\gamma_{s} \cdot \tan \psi_{s}, \Delta \gamma_{x y, m}=\Delta \gamma_{x y}-\tau_{n+1, x y} \frac{\gamma_{s}}{\tau_{n+1}}, \Delta \gamma_{y z, m}=\Delta \gamma_{y z}-\tau_{n+1, y z} \frac{\gamma_{s}}{\tau_{n+1}} .
$$

c. Calculate updated stress state $\sigma_{n+1}$ and consistent constitutive matrix $D^{e p c}$ with updated predictor stress $\left(\sigma_{n}+D \Delta \varepsilon_{m}\right)$ with the algorithm of Clausen (2007).

d. Calculate yield function of the schistosity: $f_{s}=\left|\tau_{n+1}\right|-\sigma_{n+1, y y} \tan \varphi_{s}-c_{s}=0$.

e. Do while $\left|f_{s}\right| \geq$ Tolerance:

i. Update $y_{s}$ (according to the Newton-Raphson method):

$$
\text { if } \tau_{n+1, x y}<0: \gamma_{s}=\gamma_{s}-\frac{f_{s}}{G} \text {; if } \tau_{n+1, x y}>0: \gamma_{s}=\gamma_{s}+\frac{f_{s}}{G} \text {. }
$$

ii. Update strains experienced by the matrix:

$$
\Delta \varepsilon_{y y, m}=\Delta \varepsilon_{y y}-\gamma_{s} \cdot \tan \psi_{s}, \Delta \gamma_{x y, m}=\Delta \gamma_{x y}-\tau_{n+1, x y} \frac{\gamma_{s}}{\tau_{n+1}}, \Delta \gamma_{y z, m}=\Delta \gamma_{y z}-\tau_{n+1, y z} \frac{\gamma_{s}}{\tau_{n+1}} .
$$

iii. Calculate updated stress state $\sigma_{n+1}$ and consistent constitutive matrix $D^{e p c}$ with updated predictor stress $\left(\sigma_{n}+D \Delta \varepsilon_{m}\right)$ with the algorithm of Clausen (2007).

iv. Calculate yield function of the schistosity: $f_{s}=\left|\tau_{n+1}\right|-\sigma_{n+1, y y} \tan \varphi_{s}-c_{s}=0$.

f. Calculate consistent constitutive matrix $D^{e p c:}$

$$
\begin{aligned}
& D^{\mathrm{epc}}= D-\frac{D \frac{\partial g_{s}}{\partial \sigma}\left(\frac{\partial f_{s}}{\partial \sigma}\right)^{T} D}{\left(\frac{\partial f_{s}}{\partial \sigma}\right)^{T} D \frac{\partial g_{s}}{\partial \sigma}} \text {, where } \\
& \frac{\partial g_{s}}{\partial \sigma}=\left(\begin{array}{c}
0 \\
\tan \psi_{s} \\
0 \\
\frac{\tau_{n+1, x y}}{\tau} \\
0 \\
\frac{\tau_{n+1, y z}}{\tau}
\end{array}\right) \text { and } \frac{\partial f_{s}}{\partial \sigma}=\left(\begin{array}{c}
0 \\
\tan \varphi_{s} \\
0 \\
\frac{\tau_{n+1, x y}}{\tau} \\
0 \\
\frac{\tau_{n+1, y z}}{\tau}
\end{array}\right) .
\end{aligned}
$$

7. Transform stress, strains and consistent constitutive matrix back into the original coordinate system. 


\section{F.4 Validation examples}

For the model validation, the same plane strain numerical computations (considering horizontal planes of anisotropy) as in Chapter 9 were performed and the results compared to those obtained with the commercially available software programs Plaxis (Brinkgreve et al. 2016) and Abaqus (Dassault Systèmes 2011). The results of all the constitutive models, presented in Figure F.1, are in good agreement.

In order to also validate the implementation of the constitutive model for the 3D case (which is necessary for Part IV), the ground response to tunnel excavation is analysed by 3D numerical calculations as in Chapter 12. The strike direction of the planes of weakness forms an angle of $90^{\circ}$ with the tunnel axis and the dip angle $\omega_{s}$ amounts to $60^{\circ}$ (see inset in Fig. F.2). In Figure F.2, the crown displacements along the tunnel axis are compared to those obtained with Plaxis (Brinkgreve et al. 2016). Also for the 3D case, both constitutive models are in good agreement.

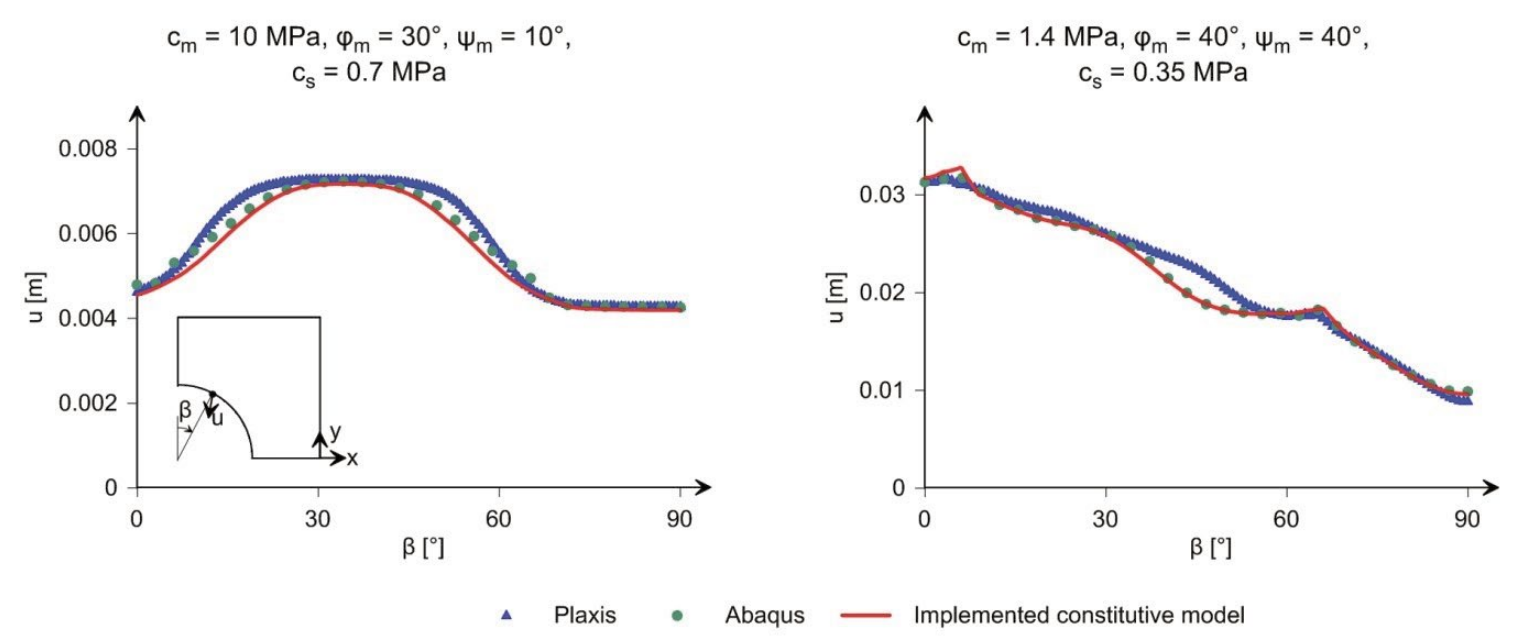

Figure F.1. Magnitude of the displacement vector along the tunnel boundary obtained with the constitutive model of Section 11.2.1 (implemented in Abaqus) and those implemented in Plaxis and Abaqus (unsupported tunnel, $a=5 \mathrm{~m}, \sigma_{0}=10 \mathrm{MPa}, E=20 \mathrm{GPa}, \varphi_{s}=20^{\circ}, \Psi_{s}=20^{\circ}$, $v=0.3)$.

$\mathrm{c}_{\mathrm{m}}=10 \mathrm{MPa}, \varphi_{\mathrm{m}}=30^{\circ}, \Psi_{\mathrm{m}}=10^{\circ}$,
$\mathrm{C}_{\mathrm{s}}=0.7 \mathrm{MPa}$

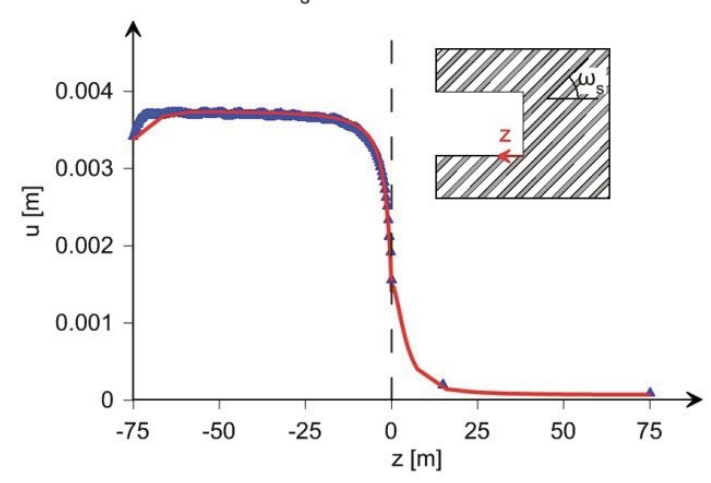

$\mathrm{c}_{\mathrm{m}}=1.4 \mathrm{MPa}, \varphi_{\mathrm{m}}=40^{\circ}, \Psi_{\mathrm{m}}=40^{\circ}$, $\mathrm{c}_{\mathrm{s}}=0.35 \mathrm{MPa}$

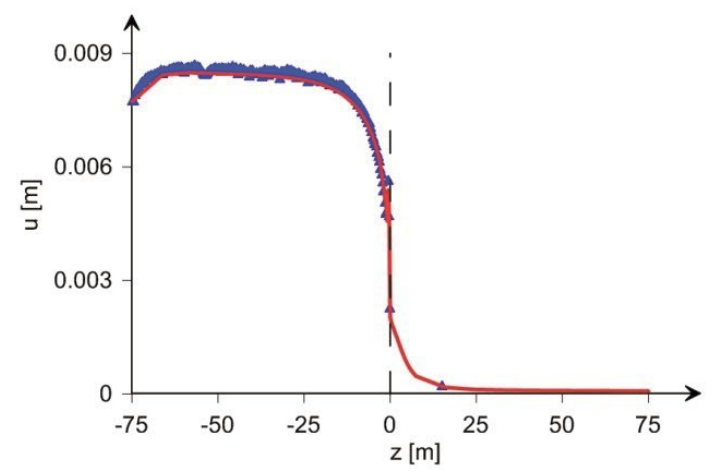

$\Delta \quad$ Plaxis Implemented constitutive model

Figure F.2. Magnitude of the displacement vectors at the tunnel crown along the tunnel obtained with the constitutive model of Section 11.2.1 (implemented in Abaqus) and with the constitutive model implemented in Plaxis (unsupported tunnel, $a=5 \mathrm{~m}, \sigma_{0}=10 \mathrm{MPa}, E=20 \mathrm{GPa}, \varphi_{s}=20^{\circ}$, $\boldsymbol{\Psi}_{\mathrm{s}}=20^{\circ}, v=0.3$ ). 



\section{Appendix G: Tunnelling parallel to the schistosity planes - Dimensionless diagrams for the displacements}

This appendix presents the dimensionless diagrams introduced in Section 11.4. Table G.1 gives an overview of the dimensionless diagrams. The list of symbols can be found on page 159.

Table G.1. Overview of the dimensionless diagrams.

\begin{tabular}{ccc}
\hline Figure & $\varphi_{m}$ & $\sigma_{a} / \sigma_{0}$ \\
\hline G.1 & & 0.0 \\
G.2 & $30^{\circ}$ & 0.1 \\
G.3 & & 0.2 \\
\hline G.4 & & 0.0 \\
G.5 & $25^{\circ}$ & 0.1 \\
G.6 & & 0.2 \\
G.7 & & 0.0 \\
G.8 & $20^{\circ}$ & 0.1 \\
G.9 & & 0.2 \\
\hline
\end{tabular}


$\cdots \sigma_{\mathrm{d}, \mathrm{s}} / \sigma_{0}=\infty-\sigma_{\mathrm{ds} s} / \sigma_{0}=0.3-\sigma_{\mathrm{d}, \mathrm{s}} / \sigma_{0}=0.2-\sigma_{\mathrm{d}, \mathrm{s}} / \sigma_{0}=0.1-\sigma_{\mathrm{d}, \mathrm{s}} / \sigma_{0}=0.05$
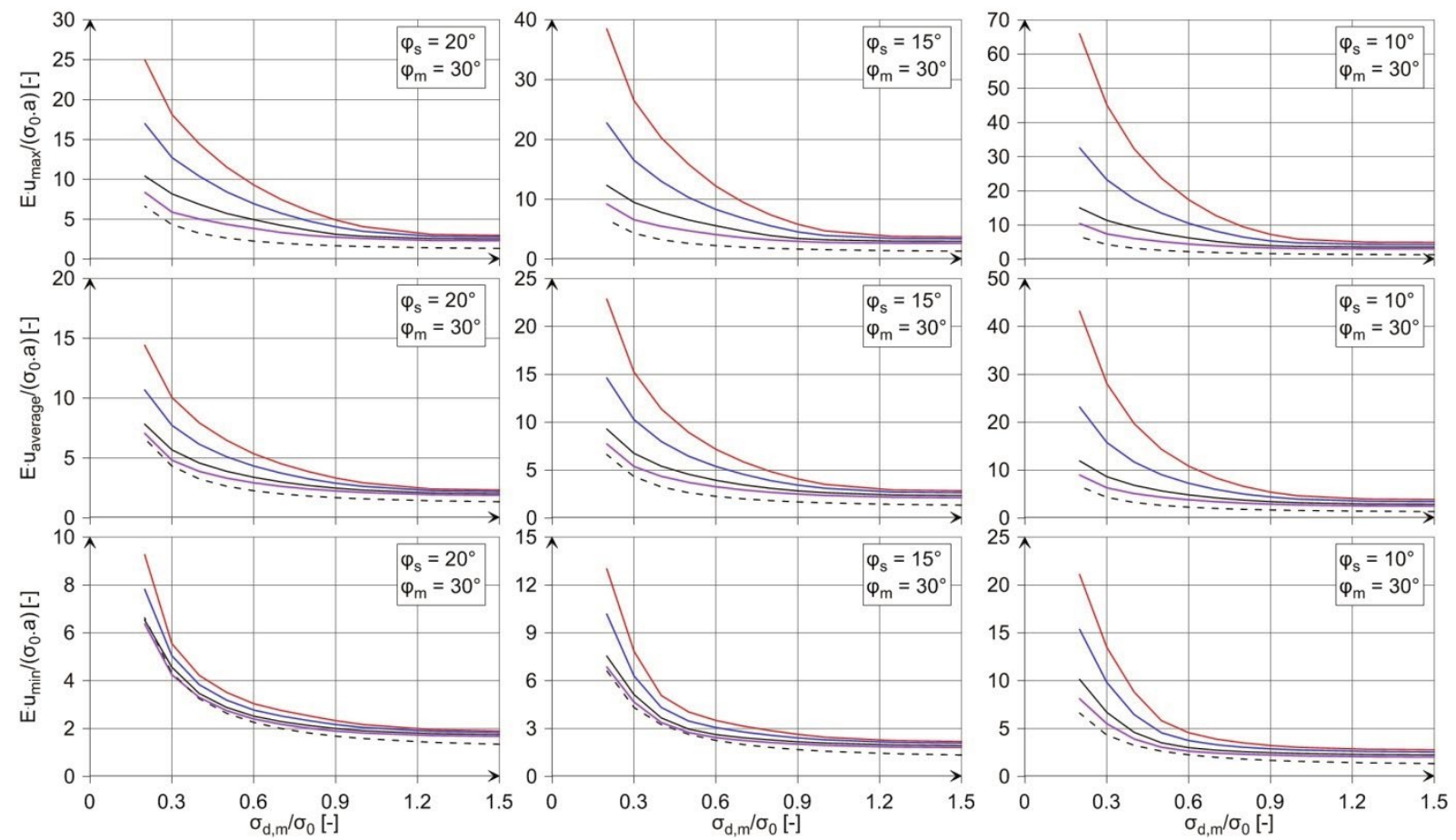

Figure G.1. Nomograms for $\varphi_{m}=30^{\circ}$ and $\sigma_{a} / \sigma_{0}=0$.
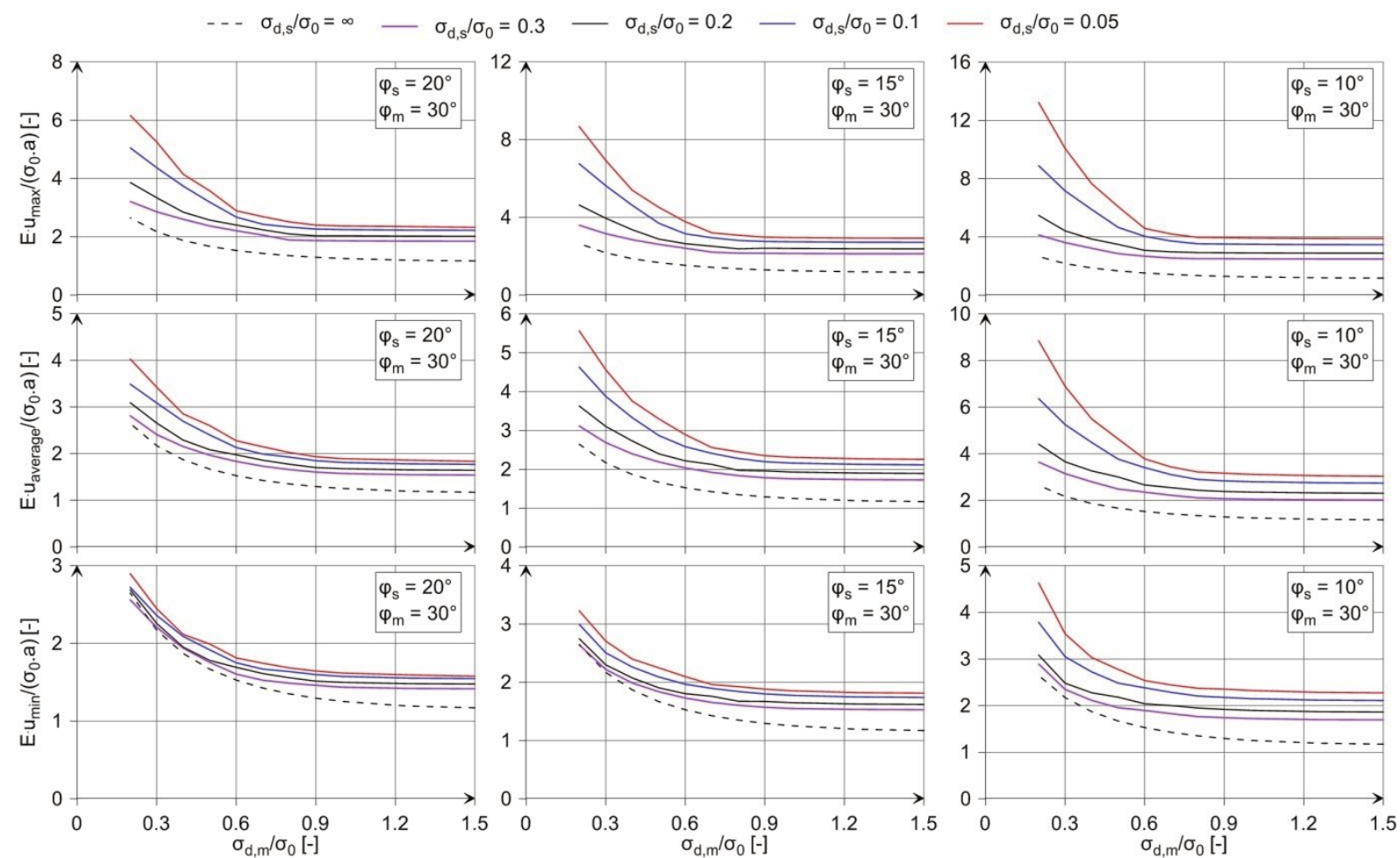

Figure G.2. Nomograms for $\varphi_{m}=30^{\circ}$ and $\sigma_{a} / \sigma_{0}=0.1$. 
$-\sigma_{\mathrm{d}, \mathrm{s}} / \sigma_{0}=\infty-\sigma_{\mathrm{d}, \mathrm{s}} / \sigma_{0}=0.3-\sigma_{\mathrm{d}, \mathrm{s}} / \sigma_{0}=0.2-\sigma_{\mathrm{d}, \mathrm{s}} / \sigma_{0}=0.1-\sigma_{\mathrm{d}, \mathrm{s}} / \sigma_{0}=0.05$
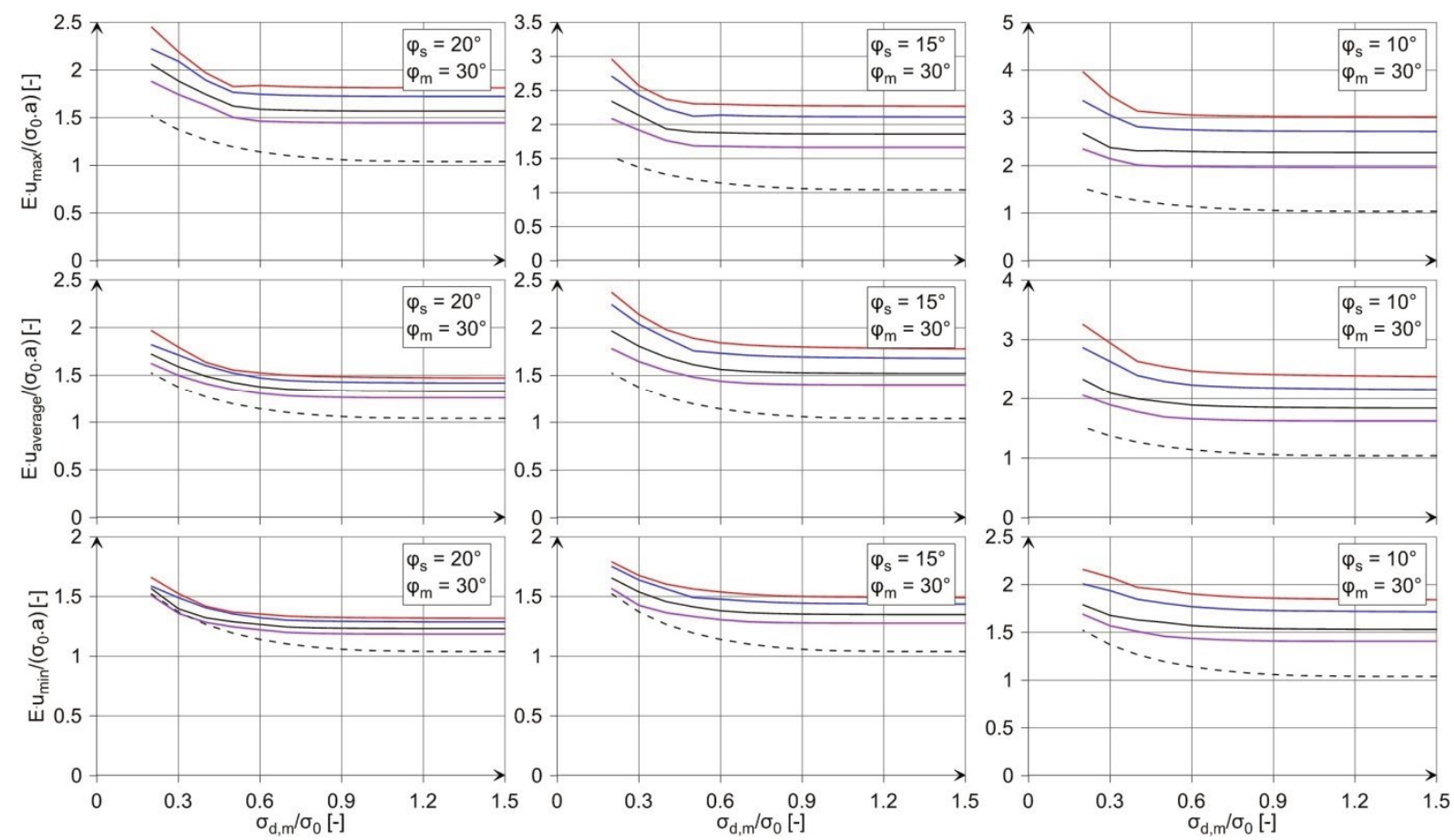

Figure G.3. Nomograms for $\varphi_{m}=30^{\circ}$ and $\sigma_{a} / \sigma_{0}=0.2$.
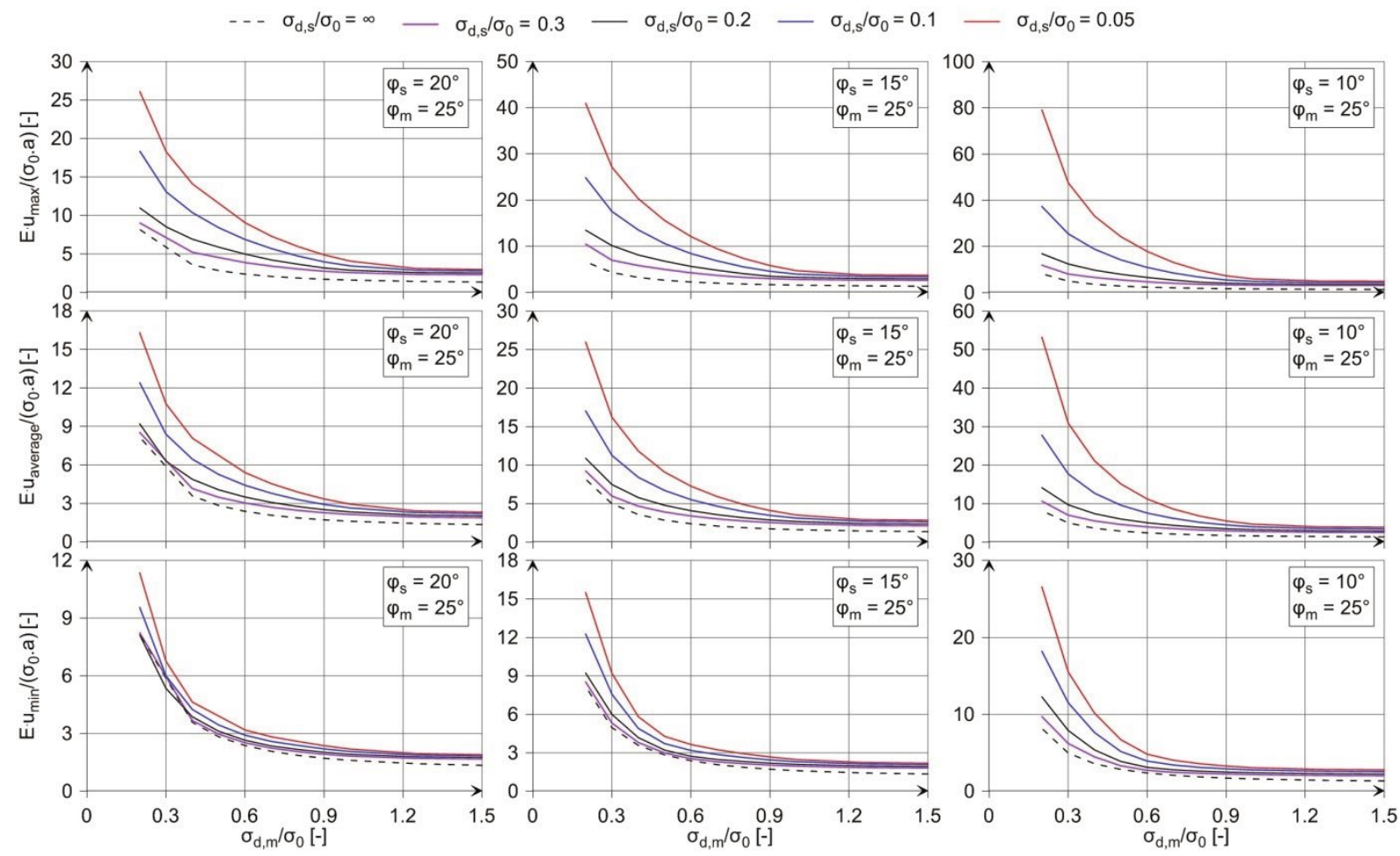

Figure G.4. Nomograms for $\varphi_{m}=25^{\circ}$ and $\sigma_{a} / \sigma_{0}=0$. 

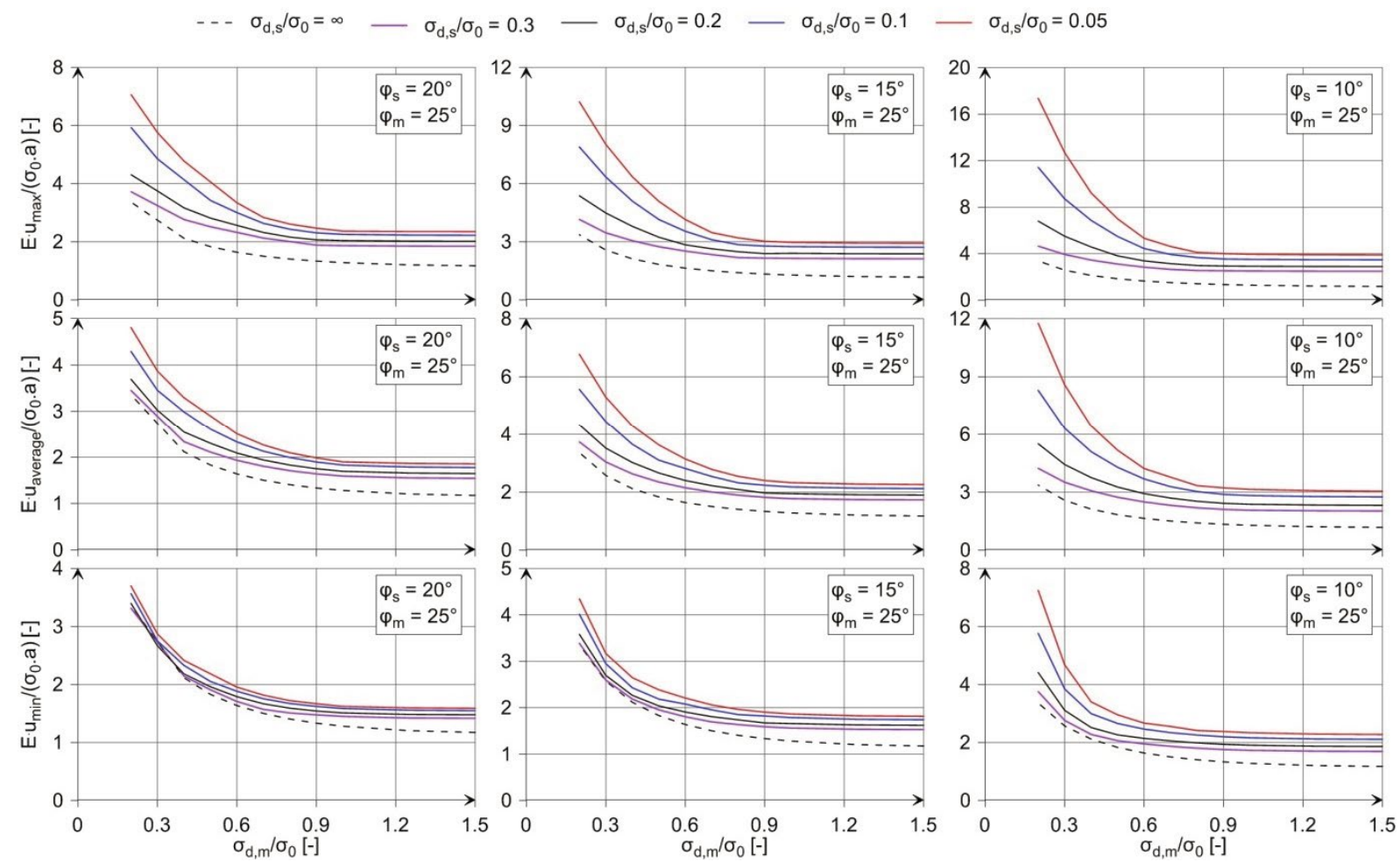

Figure G.5. Nomograms for $\varphi_{m}=25^{\circ}$ and $\sigma_{a} / \sigma_{0}=0.1$.
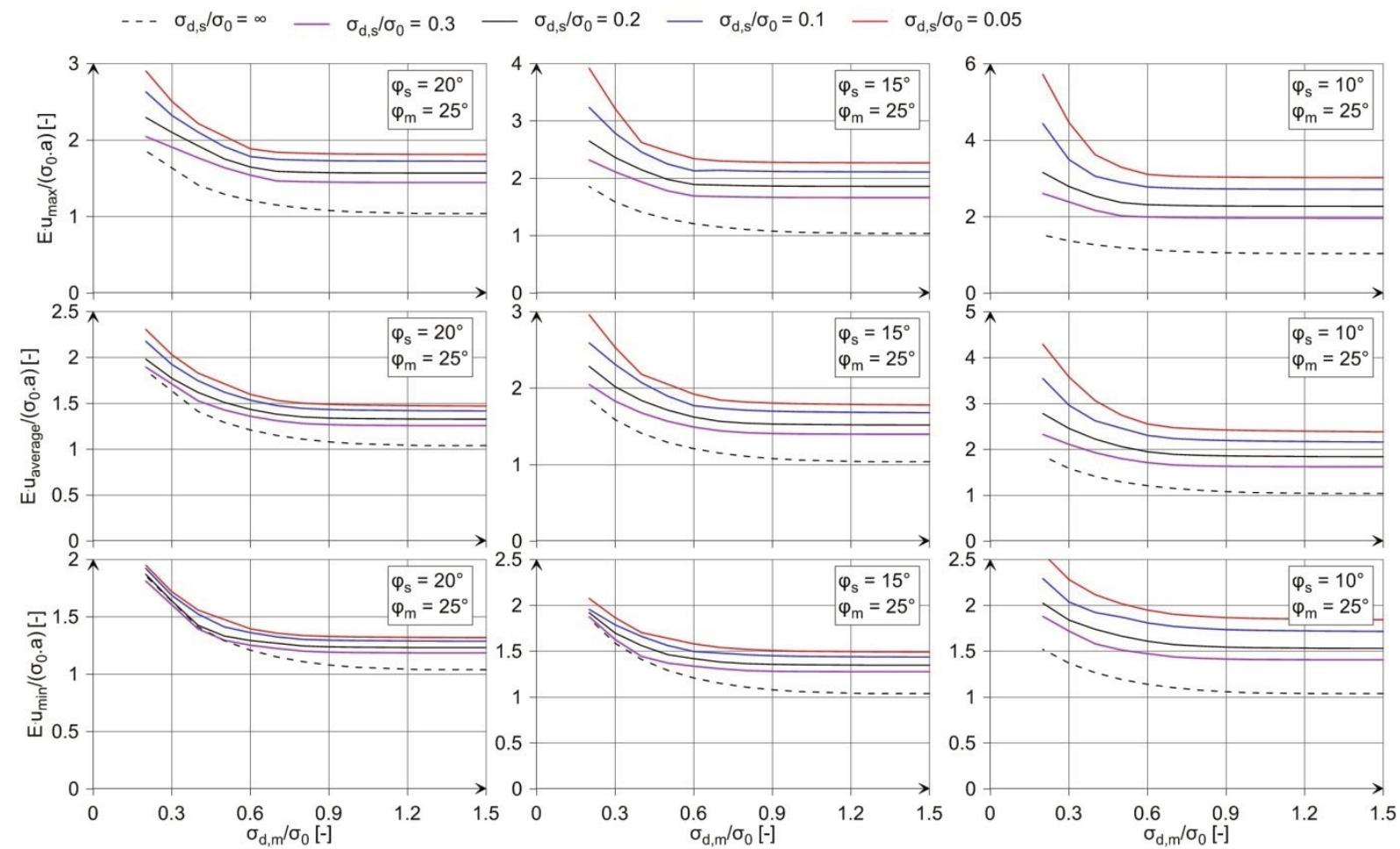

Figure G.6. Nomograms for $\varphi_{m}=25^{\circ}$ and $\sigma_{a} / \sigma_{0}=0.2$. 
$-\sigma_{\mathrm{d}, \mathrm{s}} / \sigma_{0}=\infty-\sigma_{\mathrm{d}, \mathrm{s}} / \sigma_{0}=0.3-\sigma_{\mathrm{d}, \mathrm{s}} / \sigma_{0}=0.2-\sigma_{\mathrm{d}, \mathrm{s}} / \sigma_{0}=0.1-\sigma_{\mathrm{d}, \mathrm{s}} / \sigma_{0}=0.05$

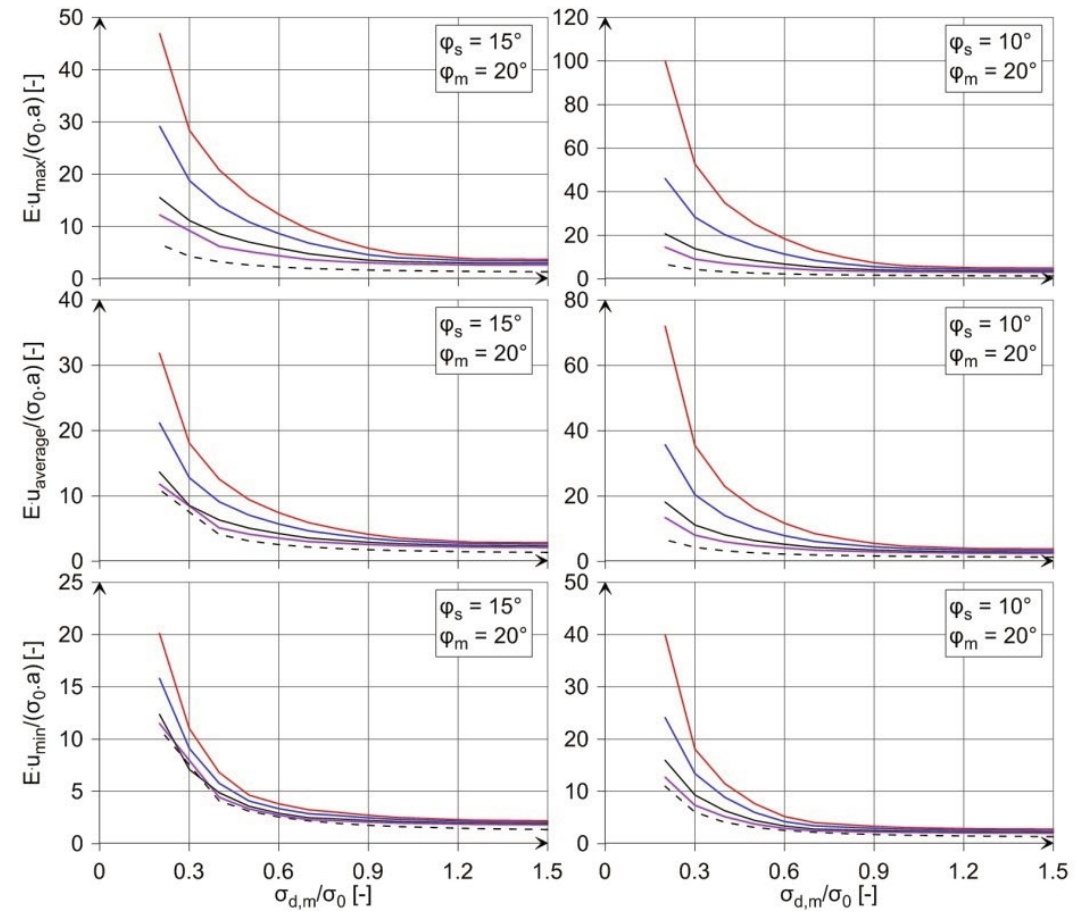

Figure G.7. Nomograms for $\varphi_{m}=20^{\circ}$ and $\sigma_{a} / \sigma_{0}=0$.

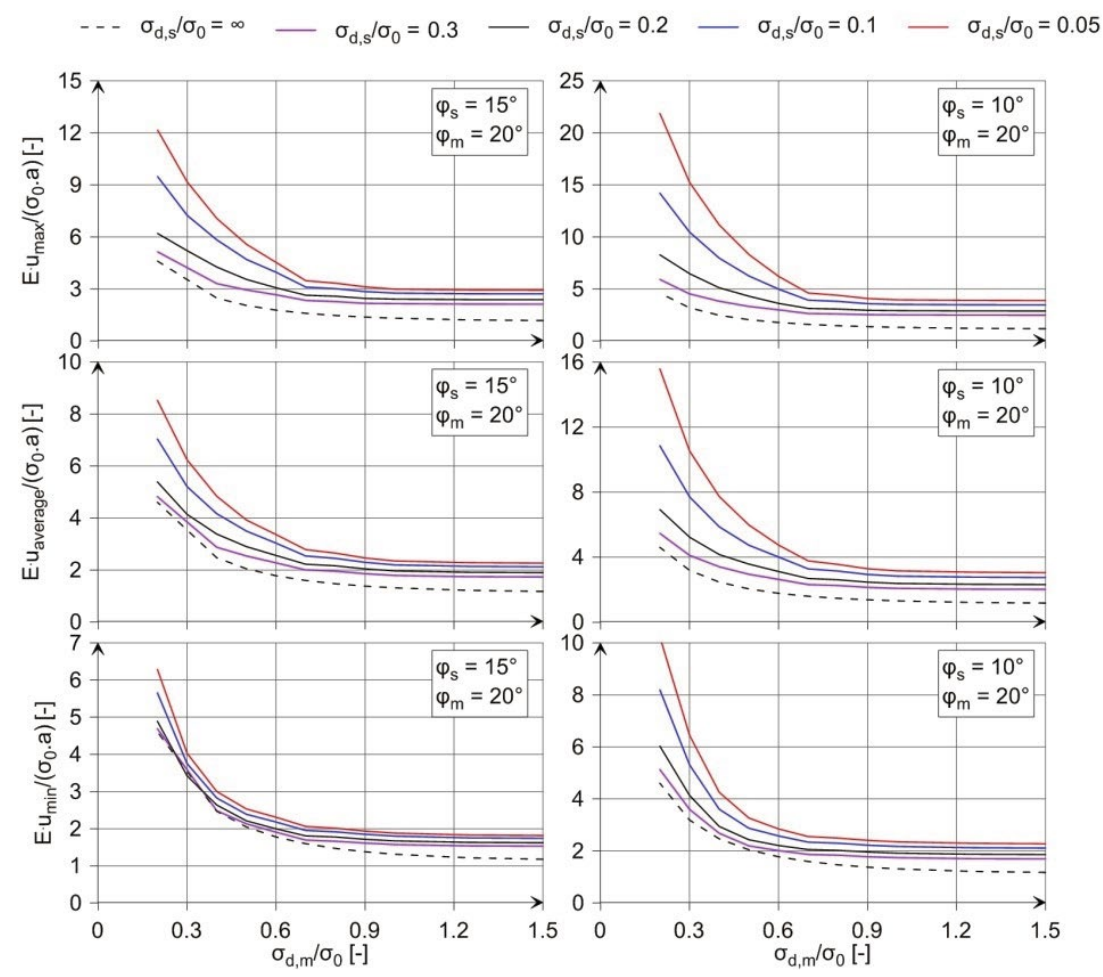

Figure G.8. Nomograms for $\varphi_{m}=20^{\circ}$ and $\sigma_{a} / \sigma_{0}=0.1$. 


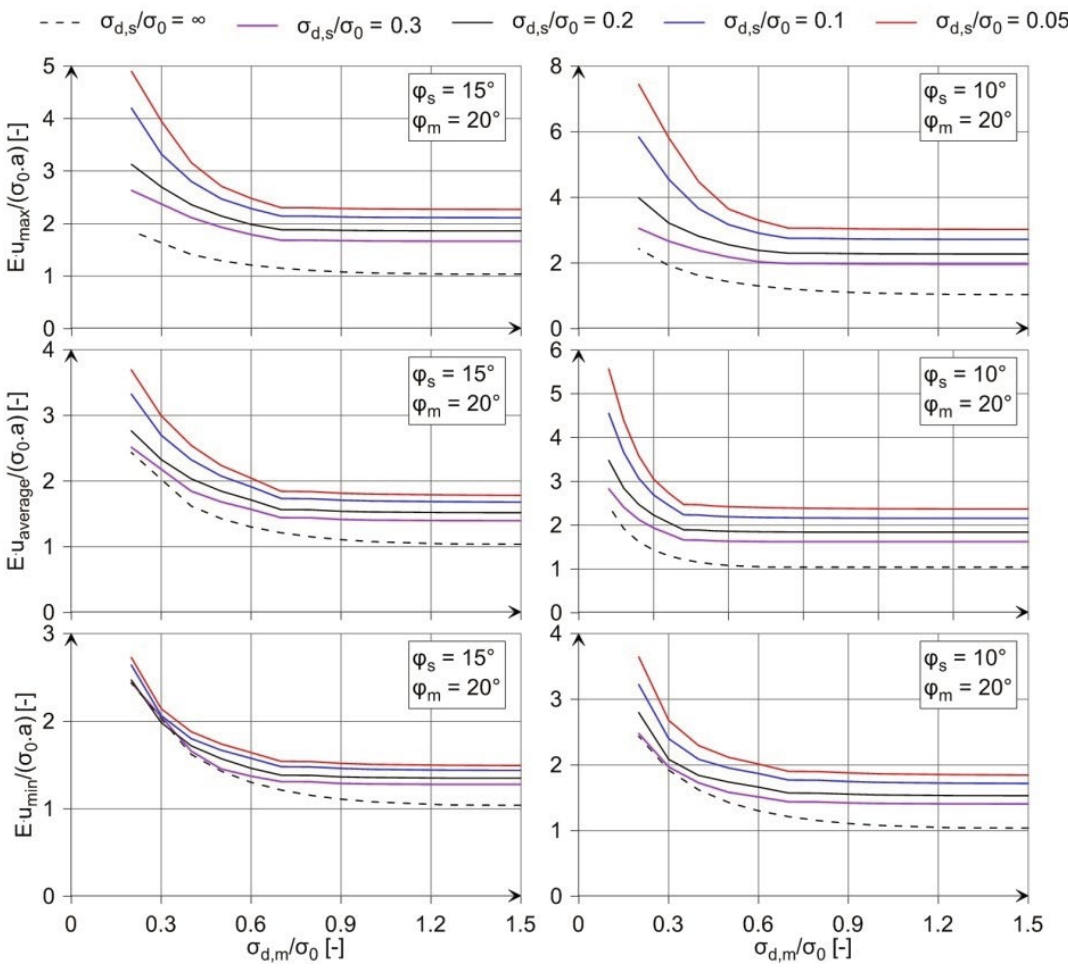

Figure G.9. Nomograms for $\varphi_{m}=20^{\circ}$ and $\sigma_{a} / \sigma_{0}=0.2$. 
NOTATION 



\section{Notation}

The notation of the thesis is inserted at the end of each chapter and can be found on the following pages:

- Chapter 1: p. 37,

- Chapter 2: p. 43,

- Chapter 3: p. 52,

- Chapter 4: p. 88,

- Chapter 5: p. 88,

- Chapter 6: p. 99,

- Chapter 7: p. 107,

- Chapter 8: p. 129,

- Chapter 9: p. 129,

- Chapter 10: p. 142

- Chapter 11: p. 159,

- Chapter 12: p. 178,

- Chapter 13: p. 196,

- Chapter 14: p. 225,

- Chapter 15: p. 247,

- Appendix A: p. 88,

- Appendix B: p. 88,

- Appendix C: p. 88,

- Appendix D: p. 129,

- Appendix E: p. 129,

- Appendix F: p. 159,

- Appendix G: p. 159. 

REFERENCES 



\section{References}

AFGC / SETRA (2002). Bétons fibrés à ultra-hautes performances - Recommendations provisoires. Ultra High Performance Fibre-Reinforced Concretes-Interim Recommendations.

Amadei, B. (1996). Importance of Anisotropy When Estimating and Measuring In Situ Stresses in Rock. International Journal of Rock Mechanics and Mining Sciences, Vol. 33(3): 293-325.

Amadei, B., Goodman, R. E. (1981). Formulation of complete plane strain problems for regularly jointed rocks. American Rock Mechanics Association (Eds.), The 22nd US Symposium on Rock Mechanics (USRMS), Cambridge, Massachusetts, pp. 245-251.

Ambrose, J. (2014). Failure of Anisotropic Shales under Triaxial Stress Conditions. Department of Earth Science and Engineering, Imperial College London.

Anagnostou, G. (2007). Continuous tunnel excavation in a poro-elastoplastic medium. Pande, G. N. \& Pietrusczak, S. (Eds.), Tenth international symposium on numerical models in geomechanics, NUMOG X, Rhodes, Greece, pp. 183-188.

Anagnostou, G., Kovári, K. (1993). Significant parameters in elasto-plastic analysis of underground openings. Journal of Geotechnical Engineering, Vol. 119(3): 401-419.

Anagnostou, G., Kovári, K. (1999). Tunnelbau in Störzonen. Löw, S. \& Wyss, R. (Eds.), Symposium Geologie Alptransit, Vorauserkundung und Prognose der Basistunnels am Gotthard und am Lötschberg Zürich, Switzerland.

Anagnostou, G., Kovári, K. (2003). The stability of tunnels in grouted fault zones. Mitteilungen des Institutes für Geotechnik (IGT), Vol. 220.

Anagnostou, G., Pimentel, E., Cantieni, L. (2008). AlpTransit Gotthard Basistunnel - Teilabschnitt Sedrun - Felsmechanische Laborversuche Los 378. Schlussbericht Nr. 080109.

Anagnostou, G., Schuerch, R., Ramoni, M. (2014). TBM tunnelling in complex rock formations. Barla, G. (Eds.), MIR 2014, XV Ciclo di conferenze di meccanica e ingegneria delle rocce, Torino, Italy, pp. 307-331.

Azami, A., Yacoub, T., Curran, J. (2012). Effects of strength anisotropy on the stability of slopes Robinson, G. \& Bannister, K. (Eds.), 65th Canadian Geotechnical Conference, CSG GeoManitoba, Winnipeg, Canada.

Azami, A., Yacoub, T., Curran, J., Wai, D. (2013). A constitutive model for jointed rock mass. Kwasniewski, M. \& Lydzba, D. (Eds.), EUROCK 2013 - The ISRM International Symposium Wroclaw, Poland.

Bachmann, T., Vicenzi, I. (2008). Refurbishment in squeezing ground on large scale: lesson learned from the southern section of the Gotthard base tunnel. Kanjlia, V. K. (Eds.), AITES-ITA World Tunnel Congress, Agra, India, pp. 1255-1262.

Barla, G. (2002). Tunnelling under squeezing rock conditions. Tunnelling Mechanics - Advances in Geotechnical Engineering and Tunnelling. Kolymbas, D. Logos Verlag, Innsbruck: 169-268.

Barla, G., Barla, M. (2000). Continuum and discontinuum modelling in tunnel engineering. Rudarsko Geolosko Naftni Zbornik, Vol. 52(12): 45-57.

Barla, G., Barla, M. (2009). Innovative Tunnelling construction methods in squeezing rock. Kolloquium der Professur für Untertagbau der ETH Zürich. 
Barla, G., Rettighieri, M., Fournier, L., Fava, A., Triclot, J. (2008). Saint Martin Squeeze. Tunnels \& Tunnelling International 2008, Vol. 5: 15-19.

Baumann, L., Zischinski, U. (1994). Neue Lösungen und Ausbautechniken der maschinellen „Fertigung" von Tunneln in druckhaftem Fels. Felsbau, Vol. 12(1): 25-29.

Billig, B., Gipperich, C., Wulff, M., Schaab, A. (2007). Ausbausysteme für den maschinellen Tunnelbau in druckhaftem Gebirge. Taschenbuch für den Tunnelbau: 223-260.

Bobet, A. (2011). Lined Circular Tunnels in Elastic Transversely Anisotropic Rock at Depth. Rock Mechanics and Rock Engineering, Vol. 44(2): 149-167.

Bornemann, R., Schmidt, M., Fehling, E., Middendorf, B. (2001). Ultrahochleistungsbeton UHPC. Beton- und Stahlbetonbau, Vol. 96(7): 458-467.

Bourne, S. J. (2003). Contrast of elastic properties between rock layers as a mechanism for the initiation and orientation of tensile failure under uniform remote compression. Journal of Geophysical Research, Vol. 108(B8).

Brinkgreve, R. B. J., Kumarswamy, S., Swolfs, W. M. (2016). Plaxis 2016.

Brisard, S. (2006). Calculation and recommendations of UHPC and UHFRPC in France. Pelke, E. (Eds.), Seminar der Vereinigung der Straßenbau- und Verkehrsingenieure in Hessen e.V. (VSVI), Hessen, pp. 13-24.

Brüggemann, H. G. (1998). Qualitätssicherung bei der Herstellung und Verarbeitung von HLBB. Schmidt, M. \& Fehling, E. (Eds.), Erfahrungen mit Hochleistungsbeton, 7. Leipziger MassivbauSeminar, Leipzig, pp. 23-28.

Brunar, G., Powondra, F. (1985). Nachgiebiger Tübbingausbau mit Meypo-Stauchelementen. Felsbau, Vol. 3(4): 225-229.

Budil, A., Höllrigl, M., Brötz, K. (2004). Strenger tunnel - Gebirgsdruck und Ausbau. Felsbau, Vol. 22(1): 39-43.

Burg, J.-P. (2018). Script to Structural Geology. Educational Material, ETH Zurich.

Burger, W. (2012). Oral communication.

Button, E. A., Leitner, R., Poetsch, M., Schubert, W. (2006). Spatial Relationships Between Discontinuity Orientation and System Behavior in Underground Excavations. American rock mechanics association (Eds.), Golden Rocks 2006, Golden, Colorado

Cai, M., Horii, H. (1993). A constitutive model and FEM analysis of jointed rock masses. International Journal of Rock Mechanics and Mining Sciences \& Geomechanics Abstracts, Vol. 30(4): 351-359.

Cantieni, L., Anagnostou, G. (2007). On the variability of squeezing in tunnelling. Ribeiro e Sousa, L., Olalla, C. \& Grossmann, N. F. (Eds.), 11th Congress of the International Society for Rock Mechanics (ISRM), Lisbon, pp. 983-986.

Cantieni, L., Anagnostou, G. (2009a). The Effect of the Stress Path on Squeezing Behavior in Tunneling. Rock Mechanics and Rock Engineering, Vol. 42(2): 289-318.

Cantieni, L., Anagnostou, G. (2009b). The interaction between yielding supports and squeezing ground. Tunnelling and Underground Space Technology, Vol. 24(3): 309-322.

Cantieni, L., Anagnostou, G. (2011). On a paradox of elasto-plastic tunnel analysis. Rock Mechanics and Rock Engineering, Vol. 44(2): 129-147.

Cantieni, L., Anagnostou, G., Hug, R. (2011). Interpretation of core extrusion measurements when tunnelling through squeezing ground. Rock Mechanics and Rock Engineering, Vol. 44(6): 641-670.

Carranza-Torres, C., Fairhurst, C. (2000). Application of the Convergence-Confinement method of tunnel design to rock masses that satisfy the Hoek-Brown failure criterion. Tunnelling and Underground Space Technology, Vol. 15(2): 187-213.

Chen, E. P. (1986). Two-dimensional continuum model for jointed media with orthogonal sets of joints. Proceedings - Symposium on Rock Mechanics. 
Clark, I. H. (2006). Simulation of rock mass strength using ubiquitous joints. Hart, R. \& Varona, P. (Eds.), Fourth International FLAC Symposium on Numerical Modeling in Geomechanics, Madrid.

Clausen, J. (2007). Efficient Non-Linear Finite Element Implementation of Elasto-Plasticity for Geotechnical Problems. Aalborg University Esbjerg.

Clausen, J., Damkilde, L., Andersen, L. (2005). Efficient return algorithms for associated plasticity with multiple yield planes. International journal for numerical methods in engineering, Vol. 66(6): 1036-1059.

Cording, E. J., Mahar, J. W. (1974). The effect of natural geologic discontinuities on behavior of rock in tunnels. Pattison, H. \& D'Appolonia, E. (Eds.), RETC Conference, San Francisco, pp. 107-138.

Daemen, J. J. K. (1983). Slip zones for discontinuities parallel to circular tunnels or shafts. International Journal of Rock Mechanics and Mining Sciences \& Geomechanics Abstracts, Vol. 20(3): 135-148.

DAfStb (2008). Sachstandsbericht-UHPC. Heft 561. Deutscher Auschuss für Stahlbeton Beuth Verlag, Berlin.

DAfStb/DIN (1995). Richtlinie für hochfesten Beton. Deutscher Ausschuss für Stahlbeton und Deutsches Institut für Normung e.V., Beuth Verlag, Berlin.

Dassault Systèmes (2011). Abaqus 6.11 - Theory Manual and Analysis User's Manual.

DAUB (2001). Betonauskleidungen für Tunnel in geschlossener Bauweise: Empfehlungen des Deutschen Ausschusses für unterirdisches Bauen. Tunnel, Vol. 3: 27-43.

Dehn, F. (2003). Herstellung, Verarbeitung und Qualitätssicherung von UHPC. Ultra-Hochfester Beton-Planung und Bau der ersten Brücke mit UHPC in Europa. Tagungsbeiträge zu den 3. Kassler Baustoff-und Massivbautagen, Kassel. Schriftenreihe Baustoffe und Massivbau. Kassel University Press, Heft 2: 37-47.

Dehn, F., König, G. (2003). Fire resistance of different fiber reinforced high performance concretes. Naaman, A. E. \& Reinhardt, H.-W. (Eds.), International Workshop High Performance Fiber Reinforced Cement Composites, Ann Arbor, USA, pp. 189-204.

Diederichs, U. (1999). Hochtemperatur- und Brandverhalten von hochfestem Stahlfaserbeton Betonbau: Forschung, Entwicklung und Anwendung. Schriftenreihe des Instituts für Baustoffe, Massivbau und Brandschutz, TU Braunschweig, 142: 67-76.

Duveau, G., Shao, J. F., Henry, J. P. (1998). Assessment of some failure criteria for strongly anisotropic geomaterials. Mechanics of cohesive-frictional materials, Vol. 3(1): 1-26.

Edelhoff, D., Torkhani, J., Breidenstein, M., Handke, D. (2015). Der Boßlertunnel - Längster Tunnel der Neubaustrecke Wendlingen-Ulm. Tunnel, Vol. 7: 16-31.

Ehrbar, H., Pfenninger, I. (1999). Umsetzung der Geologie in technische Massnahmen im Tavetscher Zwischenmassiv Nord. Löw, S. \& Wyss, R. (Eds.), Symposium Geologie Alptransit, Vorauserkundung und Prognose der Basistunnels am Gotthard und am Lötschberg Zürich, pp. 381394.

Elomri, A., Sidoroff, F. (1991). Homogenization of a two-phase elastic-plastic layered composite. Compte Rendu de l'Académie des Sciences, Vol. 312(II): 425-430.

Eurocode 2 (2011). Bemessung und Konstruktion von Stahlbeton- und Spannbetontragwerken.

Fehling, E., Schmidt, M., Teichmann, T., Bunje, K., Bornemann, R., Middendorf, B. (2005). Entwicklung, Dauerhaftigkeit und Berechnung Ultrahochfester Betone (UHPC). Schriftenreihe Baustoffe und Massivbau, Kassel University Press. Heft 1.

Fehling, E., Schmidt, M., Walraven, J., Leutbecher, T., Fröhlich, S. (2012). Ultrahochfester Beton UHPC. Beton-Kalender 2013, Teil 2, Lebensdauer und Instandsetzung Brandschutz. Bergmeister, K., Wörner, J.-D. \& Fingerloos, F. Wiley-VCH Verlag GmbH \& CO. KGaA Weinheim: 117-240.

Fontana, A. (2012). Durchörterung "Linea Val Colla" Planungs- und Ausführungsaspekte. Kolloquium der Professur für Untertagbau der ETH Zürich, Bauhilfsmassnahmen im Tunnelbau, Zürich. 
Geological Group of the Lötschberg Base Tunnel (2008). Geologischer Bericht, Vortrieb Mitholz Richtung Süden, Tunnelröhre Ost, BKm 22.043-31.828, Tunnelröhre West, BKm 22.04331.647. Schlussdokumentation Lötschberg. BLS AlpTransit Lötschberg.

Gerrard, C. M. (1982). Equivalent elastic moduli of a rock mass consisting of orthorhombic layers. International Journal of Rock Mechanics and Mining Sciences \& Geomechanics Abstracts, Vol. 19(1): 9-14.

Gimmel, S. (2018). Lötschberg-Basistunnel: Einwirkungen auf die Tunnelinnenschale im druckhaften Gebirge. Master thesis at ETH Zurich, not published.

Goricki, A., Button, E. A., Schubert, W., Pötsch, M., Leitner, R. (2005). The influence of Discontinuity Orientation on the Behaviour of Tunnels. Felsbau, Vol. 23(5): 12-18.

Grübl, F. (2012). Oral communication.

Guntli, P., Weber, A. (2009). Einspurtunnel Ost km 118.835-120.039, Einspurtunnel West km 218.855-220.062 Tavetscher Zwischenmassiv Nord und Clavaniev-Zone, Geologischer Schlussbericht, Geologie, Geotechnik, Hydrogeologie. Bericht Nr.: GR 360B-2, Sieber Cassina + Handke AG.

Haecker, D. (1987). Preussag AG Kohle. Unser Betrieb, Vol. 45(4).

Hefny, A. M., Lo, K. Y. (1999). Analytical solutions for stresses and displacements around tunnels driven in cross-anisotropic rocks. Numerical and analytical methods in geomechanics, Vol. 23(2): 161-177.

Herrenknecht, M., Bäppler, K., Burger, W. (2010). Tunnelling through squeezing rock with TBM. Bautechnik, Vol. 2: 45-54.

Hoek, E., Brown, E. T. (1980). Empirical strength criterion for rock masses. Journal of the Geotechnical Engineering Division, Vol. 106(15715): 1013-1035.

Hsu, S. C., Chiang, S. S., Lai, J. R. (2004). Failure mechanisms of tunnels in weak rock with interbedded structures. International Journal of Rock Mechanics and Mining Sciences, Vol. 41(3): 489.

Huber, G., Westermayr, H., Alber, O. (2005). Einfluss der Gefügeorientierung am Strenger Tunnel. Felsbau, Vol. 5(20-24).

IG-LBT (2009). Schlussbericht Felsmechanik, Ausbruch, Sicherung. BLS AlpTransit Lötschberg.

Ingenieurgemeinschaft Gotthard-Basistunnel Süd (2009). Einspurtunnel Nord, Bautechnischer Schlussbericht. AlpTransit Gotthard AG.

Ismael, M., Konietzky, H. (2017). Integration of Elastic Stiffness Anisotropy into Ubiquitous Joint Model. Procedia Engineering, Vol. 191: 1032-1039.

Itasca Consulting Group (2001). FLAC: User's manual. Ver. 4.0.

Itasca Consulting Group (2007). Fast Lagrangian Analysis of Continua, Constitutive Models.

ITC (2014). Insight in the monthly reports, the geological journals and the execution plans. ITC ITECSA-TOSCANO Consorzio d'ingegneri, not published.

Jaeger, J. C. (1960). Shear failure of anisotropic rocks. Geological Magazine, Vol. 97(1): 65-72.

Jaeger, J. C., Cook, N. G. W. (1976). Fundamentals of Rock Mechanics, Chapman and Hall, London.

Jing, L. (1998). Formulation of discontinuous deformation analysis (DDA) - an implicit discrete element model for block systems. Engineering Geology, Vol. 49(3-4): 371-381.

John Mowlem and Company Limited (1979). Edward Thomas Hawns Colin Norman Paterson Mackenzie. UK Patent Application GB 2013757 A.

JSCE (2004). Recommendations for design and construction of ultra high strength fiber reinforced concrete structures. Recommendations of the Japan Society of Civil Engineers. September 2004. Draft.

Keller, M. (2005). Die Beherrschung der Hohlraumverformungen in der Karbonzone. Fachgruppe für Untertagbau (Eds.), Swiss Tunnel Congress, Fachtagung für Untertagbau, Luzern, pp. 31-37. 
Klingsch, E., Frangi, A., Fontana, M. (2013). Explosive spalling of concrete in fire. IBK Bericht Nr. 351. IBK Insitut für Baustatik und Konstruktion, ETH Zürich.

Klingsch, E. W. H. (2014). Explosive spalling of concrete in fire. Dissertation ETH No. 21650. ETH Zurich.

Klopčič, J., Logar, J. (2014). Effect of relative orientation of anisotropy planes to tunnel axis on the magnitude of tunnelling displacements. International Journal of Rock Mechanics \& Mining Sciences, Vol. 71: 235-248.

Klotz, S., Holschemacher, K. (2003). Teilflächenpressung bei UHFB. König, G. (Eds.), Ultrahochfester Beton: Innovationen im Bauwesen, Beiträge aus Praxis und Wissenschaft, pp. 215-226.

Koiter, W. T. (1953). Stress-strain relations, uniqueness and variational theorems for elastic-plastic materials with a singular yield surface. Quarterly of Applied Mathematics, Vol. 11(3): 350-354.

König, G., Tue Viet, N., Zink, M. (2001). Hochleistungsbeton, Verlag Ernst \& Sohn Berlin.

Kovári, K. (1977). The elasto-plastic analysis in the design practice of underground openings. Finite Elements in Geomechanics. Gudehus, G.: 377-412.

Kovári, K. (1998). Tunnelling in squeezing rock. Tunnel, Vol. 5: 12-31.

Kovári, K. (2005). Method and device for stabilizing a cavity excavated in underground construction. US Patent Appl. 20050191138.

Kovári, K., Amberg, F., Ehrbar, H. (1999). Tunnelbau in druckhaftem Gebirge - Eine Herausforderung für die neuen Alpentransversalen. Lehrstuhl für Bauverfahrenstechnik, Tunnelbau und Baubetrieb (Eds.), Kolloquium für Bauverfahrenstechnik, Bochum, Germany, pp. 141-151.

Kovári, K., Amberg, F., Ehrbar, H. (2000). Mastering of Squeezing Rock in the Gotthard Base. World Tunneling, Vol. 13(5): 234-238.

Kovári, K., Anagnostou, G. (1995). The ground response curve in tunnelling through short fault zones. Fujii, T. (Eds.), 8th International Congress on Rock Mechanics, Tokyo, pp. 611-614.

Kovári, K., Ehrbar, H. (2008). Gotthard Basistunnel, Teilabschnitt Sedrun - Die druckhaften Strecken im TZM Nord - Projektierung und Realisierung. Swiss Tunnelling Society (Eds.), Swiss Tunnel Congress Luzern, pp. 39-47.

Kützing, L., König, G. (2001). Duktiler Hochleistungsbeton mit Fasercocktail - Technologie, Bemessung, Anwendungen. Bautechnik, Vol. 78(2): 105-114.

Lai, Y. S., Wang, C. Y., Tien, Y. M. (1999). Modified Mohr-Coulomb-type micromechanical failure criteria for layered rocks. International journal for numerical and analytical methods in geomechanics, Vol. 23: 451-460.

Leitner, R., Pötsch, M., Schubert, W. (2006). Aspects on the Numerical Modeling of Rock Mass Anisotropy in Tunneling. Felsbau 24, Vol. 24(2): 59-65.

Leucker, R., Kessler, D., Otremba, H. (2009). Neue Tübbings aus hochfestem, faserbewehrtem Beton: Ergebnisse von Belastungsversuchen und Vergleich mit numerischen Berechnungen. Forschung und Praxis, U-Verkehr und Unterirdisches Bauen, Vol. 43: 198-203.

Leutbecher, T. (2008). Rissbildung und Zugtragverhalten von mit Stabstahl und Fasern bewehrtem ultrahochfesten Beton (UHPC), Heft 9. Kassel, Kassel University Press GmbH.

Lisjak, A., Grasselli, G., Vietor, T. (2014). Continuum-discontinuum analysis of failure mechanisms around unsupported circular excavations in anisotropic clay shales. International Journal of Rock Mechanics and Mining Sciences, Vol. 65: 96-115.

Lourenço, P. B. (1995). The elastoplastic implementation of homogenisation Techniques with an extension to masonry structures. Delft University of Technology.

Lydzba, D., Pietruszczak, S., Shao, J. F. (2003). On anisotropy of stratified rocks: homogenization and fabric tensor approach. Computers and Geotechnics, Vol. 30(4): 289-302.

Madkour, H. (2012). Parametric analysis of tunnel behavior in jointed rock. Ain Shams Engineering Journal, Vol. 3: 79-103.

Maidl, B., Herrenknecht, M., Maidl, U., Wehrmeyer, G. (2011). Maschineller Tunnelbau im Schildvortrieb, Verlag Ernst und Sohn Berlin. 
Malaguti, A., Morandi, S., Stocker, D. (2013). Anisotropic geomechanical behaviour of tunnelling Val Colla Line, Ceneri base Tunnel, Switzerland. Anagnostou, G. \& Ehrbar, H. (Eds.), ITA-AITES World Tunnel Congress, WTC 2013 - Underground: the way to the future, Geneva, Switzerland, pp. 1730-1737.

MathWorks (2017). Matlab®.

Merlini, D., Falanesca, M. (2013). Ceneri Base Tunnel advancement in difficult rock conditions: tunnel design and construction optimization through back-analysis of the geomechanical parameters. ITA-AITES World Tunnel Congress, WTC 2013 - Underground: the way to the future, Geneva, Switzerland

Mezger, F., Anagnostou, G. (2015). On the non-uniformity of squeezing deformations in the Ceneri Base Tunnel. Schubert, W. \& Kluckner, A. (Eds.), ISRM Regional Symposium EUROCK 2015 \& 64th Geomechanics Colloquium, Salzburg, Oesterreich, pp. 113-118.

Mezger, F., Anagnostou, G., Ziegler, H. J. (2013a). The excavation-induced convergences in the Sedrun section of the Gotthard Base Tunnel. Tunnelling and Underground Space Technology, Vol. 38: 447-463

Mezger, F., Anagnostou, G., Ziegler, H. J. (2013b). On some factors affecting squeezing intensity in tunnelling. Anagnostou, G. \& Ehrbar, H. (Eds.), ITA-AITES World Tunnel Congress, WTC 2013 - Underground : the way to the future, Geneva, Switzerland, pp. 1990-1997.

Mezger, F., Ramoni, M., Anagnostou, G. (2015a). Some concepts for segmental linings in squeezing rock. Johnson, M. C. \& Oginski, S. (Eds.), RETC Rapid Excavation and Tunneling Conference (RETC 2015), New Orleans, USA, pp. 646-658.

Mezger, F., Ramoni, M., Anagnostou, G. (2015b). Tübbingausbau in druckhaftem Gebirge. Kolloquium der Professur für Untertagbau der ETH Zürich, Bauhilfsmassnahmen im Tunnelbau, Zürich.

Mezger, F., Ramoni, M., Anagnostou, G. (2016). Lining Systems for TBMs in Squeezing Rock (Eds.), 2nd International Conference on Tunnel Boring Machines in Difficult Grounds (TBM DiGs 2016), Istanbul, Turkey, pp. 57-71.

Mezger, F., Ramoni, M., Anagnostou, G. (2018). Options for deformable segmental lining systems for tunnelling in squeezing rock. Tunnelling and Underground Space Technology, Vol. 76: 64-75.

Mezger, F., Ramoni, M., Anagnostou, G., Dimitrakopoulos, A., Meystre, N. (2017). Evaluation of higher capacity segmental lining systems when tunnelling in squeezing rock. Tunnelling and Underground Space Technology, Vol. 65: 200-214.

Nejad Ensan, M., Shahrour, I. (2003). A macroscopic constitutive law for elasto-plastic multilayered materials with imperfect interfaces: application to reinforced soils. Computers and Geotechnics, Vol. 30(4): 339-345.

Nguyen, V. M., Konietzky, H. (2016). Behaviour of joints. Institut für Geotechnik TU Bergakademie Freiberg.

Panet, M. (1996). Two case histories of tunnels through squeezing rocks. Rock Mechanics and Rock Engineering, Vol. 29(3): 155-164.

Panet, M., Guellec, P. (1974). Contribution à l'étude du soutènement derrière le front de taille. Balkema, A. A. (Eds.), Proceedings of the 3rd Congress ISRM, Denver, pp. 1163-1168.

Park, B., Min, K. B. (2015). Bonded-particle discrete element modeling of mechanical behavior of transversely isotropic rock. International Journal of Rock Mechanics \& Mining Sciences, Vol. 76 : 243-255.

Park, P. H., Kim, Y. J. (2006). Analytical solution for a circular opening in an elastic-brittle-plastic rock. International Journal of Rock Mechanics and Mining Sciences, Vol. 43(4): 616-622.

Pietruszczak, S., Lydzba, D., Shao, J.-F. (2002). Modelling of inherent anisotropy in sedimentary rocks. International Journal of Solids and Structures, Vol. 39(3): 637-648.

Pini, O., Skaanes, S. (2006). Ceneri Base Tunnel. Tunnel, Vol. 4: 44-47.

Podjadtke, R., Weidig, G. (2010). Verstellbarer nachgiebieger Tübbingausbau. Adjustable flexible segment lining. Tunnel, Vol. 7: 37-42. 
Pruchnicki, E., Shahrour, I. (1994). A macroscopic elastoplastic constitutive law for multilayered media: Application to reinforced earth material. Numerical and analytical methods in geomechanics, Vol. 18(7): 507-518.

Ramoni, M., Anagnostou, G. (2010a). Tunnel boring machines under squeezing conditions. Tunnelling and Underground Space Technology, Vol. 25(2): 139-157.

Ramoni, M., Anagnostou, G. (2010b). Thrust force requirements for TBMs in squeezing ground. Tunnelling and Underground Space Technology, Vol. 25(4): 433-455.

Ramoni, M., Anagnostou, G. (2011a). The interaction between shield, ground and tunnel support in TBM tunnelling through squeezing ground. Rock Mechanics and Rock Engineering, Vol. 44(1): $37-61$.

Ramoni, M., Anagnostou, G. (2011b). Design aids for the planning of TBM drives in squeezing ground. Research project FGU 2007/005 of the Swiss Federal Roads Office (FEDRO), Report 1341. Swiss Association of Road and Transportation Experts (VSS). Zürich.

Ramoni, M., Anagnostou, G., Lavdas, N. (2011a). Segmental linings under squeezing pressure. Balan, A. (Eds.), Underground spaces for tomorrow, AFTES International Congress, Lyon, France, pp. 113.

Ramoni, M., Lavdas, N., Anagnostou, G. (2011b). Squeezing loading of segmental linings and the effect of backfilling. Tunnelling and Underground Space Technology, Vol. 26(6): 692-717.

Reed, M. B. (1988). The influence of out-of-plane stress on a plane strain problem in rock mechanics. International journal for numerical and analytical methods in geomechanics, Vol. 12: 173-181.

Reinhardt, H.-W., Koch, R. (1998). Hochfester Beton unter Teilflächenbelastung. Beton und Stahlbetonbau, Vol. 93(7): 182-188.

Rencis, J. J., Huang, T. H. (1992). Boundary element formulation for generalized plane strain. Engineering Analysis with Boundary Elements Vol. 9 263-271(3): 263-271.

Richard, P., Cheyrezy, M. (1995). Composition of reactive powder concretes. Cement and concrete research, Vol. 25(7): 1501-1511.

Röthlisberger, J. (2015). A2 Belchentunnel, Sanierungstunnel: Erneuerungskonzept und Wahl der Vortriebsmethode. Fachgruppe für Untertagbau (Eds.), Swiss Tunnel Congress, Luzern, pp. 70-79.

Sainsbury, B., Pierce, M., Mas Ivars, D. (2008). Simulation of rock mass strength anisotropy and scale effects using a Ubiquitous Joint Rock Mass (UJRM) model. Hart, D. H., Detournay, C. \& Cundall, P. (Eds.), First International FLAC/DEM Symposium on Numerical Modeling, (Continuum and Distinct Element Numerical Modeling in Geo-Engineering), Minneapolis, USA, pp. $1-10$.

Salamon, M. D. G. (1968). Elastic moduli of a stratified rock mass. International Journal of Rock Mechanics and Mining Sciences \& Geomechanics Abstracts, Vol. 5(6): 519-527.

Sänger, B. (2006). Disc cutters for hardrock TBM 1986-2006 - history and tendencies of development. Felsbau, Vol. 24(6): 46-51.

Schmidt, M. (2003). Ultra-Hochleistungsbeton-Ausgangsstoffe, Eigenschaften und Leistungsfähigkeit. Ultra-Hochfester Beton-Planung und Bau der ersten Brücke mit UHPC in Europa. Tagungsbeiträge zu den 3. Kassler Baustoff-und Massivbautagen. Schriftenreihe Baustoffe und Massivbau: $5-36$.

Schmidt, M., Fehling, E. (2005). Ultra-High-Performance Concrete: Research, Development and Application in Europe. Russell, H. G. (Eds.), Seventh International Symposium on the Utilization of High-Strength/High-Performance Concrete, Farmington Hills, pp. 51-77.

Schmidt, M., Fehling, E. (2006). Grundlagen der Betontechnologie von Hochleistungs- und Ultrahochleistungsbetonen. Pelke, E. (Eds.), Seminar der Vereinigung der Straßenbau- und Verkehrsingenieure in Hessen e.V. (VSVI), Hessen, pp. 1-11.

Schmidt, M., Herget, E. (2007). Bauen mit UHPC - Aktueller Stand und Ausblick aus der Sicht der Wissenschaft und der Praxis. Ultra high performance concrete (UHPC); 10 years of research and development at the University of Kassel. Schriftenreihe "Baustoffe und Massivbau", Kassel university press $\mathrm{GmbH}$, Heft 7: 21-24. 
Schneider, A. (2002). Sicherheit gegen Niederbruch im Untertagbau. Dissertation ETH No. 14556. ETH Zürich.

Schneider, T. R. (1997). Treatment of the faults at the project of the Gotthard-basetunnel. Felsbau, Vol. 15(6): 489-495.

Schnellenbach-Held, M., Prager, M. (2011). Mikrobewehrter Ultrahochleistungsbeton. Trag- und Verformungsverhalten. Beton- und Stahlbetonbau, Vol. 106(11): 770-778.

Schubert, W. (1996). Dealing with squeezing conditions in Alpine tunnels. Rock Mechanics and Rock Engineering, Vol. 29(3): 145-153.

Schubert, W., Mendez, J. M. D. (2017). Influence of Foliation Orientation on Tunnel Behavior. Procedia Engineering, Vol. 191: 880-885.

Schubert, W., Moritz, B., Sellner, P. (2000). Tunnelling methods for squeezing ground. International Society for Rock Mechanics (Eds.), GeoEng2000, Melbourne.

Schulte, C. (2011). Aktuelle Entwicklungen bei der Ausführungsplanung von Tübbings. Felsbaumagazin, (2): 91-99.

Shen, B., Barton, N. (1997). The Disturbed Zone Around Tunnels in Jointed Rock Masses. International Journal of Rock Mechanics and Mining Sciences, Vol. 34(1): 117-125.

SIA 162/6 (1999). Stahlfaserbeton. Schweizer Norm SN 562 162/6.

SIA 262 (2003). Betonbau. Schweizer Norm SN 505262.

SIA 2052 (2016). Ultra-Hochleistungs-Faserbeton (UHFB) - Baustoffe, Bemessung und Ausführung. Schweizer Regel SNR 592052.

SISO (2012). "Site Information Software AlpTransit Gotthard." Retrieved March 2012, March 2012, from www.sisonet.com.

SKH Geologen AG (2011). not published.

Stech, H.-J., Seingre, G., Ziegler, H. J., Gammeter, C. (2005). Lötschberg-Basitunnel: Ausbrucharbeiten vollendet. Tunnel, Vol. 4: 16-39.

Steindorfer, A. (1998). Short term prediction of rock mass behavior in tunneling by advanced analysis of displacement monitoring data. Graz, Techn. Univ. Graz.

Steiner, W. (1996). Tunnelling in Squeezing Rocks: Case Histories. Rock Mechanics and Rock Engineering, Vol. 29(4): 211-246.

Stocker, D. (2006). Ceneri Basistunnel - Bautechnische Herausforderungen. Der Bauingenieur, Vol. 7: 14-16.

Sulem, J., Mühlhaus, H. B. (1997). A continuum model for periodic two-dimensional block structures. Mechanics of cohesive-frictional materials, Vol. 2: 31-46.

Suquet, P. (1987). Elements of Homogenization for Inelastic Solic Mechanics. Sanchez-Palancia, E. \& Zaoui, A. (Eds.), Homogenization Techniques for Composite Media, New York, pp. 193-278.

Taliercio, A., Sacchi Landriani, G. (1988). A failure condition for layered rock. International Journal of Rock Mechanics and Mining Sciences \& Geomechanics Abstracts, Vol. 25(5): 299-305.

Thuro, K., Eberhardt, E., Gasparini, M. (2004). Deep seated creep and it's influence on a $1.5 \mathrm{GW}$ hydroelectric power plant in the Himalayas. Felsbau, Vol. 22(2): 60-66.

Thut, A., Naterop, D., Steiner, P., Stolz, M. (2006). Tunneling in Squeezing Rock - Yielding Elements and Face Control. Society for underground and geotechnical constructions (Eds.), 8th International Conference on Tunnel Constructions and Underground Structures, Ljubljana, Slovenia.

Tien, Y. M., Kuo, M. C. (2001). A failure criterion for transversely isotropic rocks. International Journal of Rock Mechanics and Mining Sciences, Vol. 38(3): 399-412.

Tonon, F., Amadei, B. (2002). Effect of Elastic Anisotropy on Tunnel Wall Displacements Behind a Tunnel Face. Rock Mechanics and Rock Engineering, Vol. 35(3): 141-160. 
Tran Manh, H., Sulem, J., Subrin, D. (2015). A Closed-Form Solution for Tunnels with Arbitrary Cross Section Excavated in Elastic Anisotropic Ground. Rock Mechanics and Rock Engineering, Vol. 48: 277-288.

Triantafyllidis, T., Gerolymatou, E. (2014). Estimation of the Strength of Stratified Rock Mass. Rock Mechanics and Rock Engineering, Vol. 47(2): 535-547.

Vermeer, P. A., de Borst, R. (1984). Non-associated plasticity for soils, concrete and rock. HERON, Vol. 29(3): 1-64.

Vigl, L., Gütter, W., Häger, M. (1999). Doppelschild-TBM - Stand der Technik und Perspektiven. Felsbau, Vol. 17(5): 475-485.

Vogelhuber, M. (2007). Der Einfluss des Porenwasserdrucks auf das mechanische Verhalten kakiritisierter Gesteine. Dissertation ETH No. 17079. ETH Zürich.

Vrakas, A., Anagnostou, G. (2014). A finite strain closed-form solution for the elastoplastic ground response curve in tunnelling. International journal for numerical and analytical methods in geomechanics, Vol. 38(11): 1131-1148.

Vu, T. M., Sulem, J., Subrin, D., Monin, N. (2013). Semi-Analytical Solution for Stresses and Displacements in a Tunnel Excavated in Transversely Isotropic Formation with Non-Linear Behavior. Rock Mechanics and Rock Engineering, Vol. 46(2): 213-229.

Walsh, J. B., Brace, W. F. (1964). A fracture criterion for brittle anisotropic rock. Journal of Geophysical Research, Vol. 69(16): 3449-3456.

Wang, T. T., Huang, T. H. (2014). Anisotropic deformation of a circular tunnel excavated in a rock mass containing sets of ubiquitous joints: theory analysis and numerical modeling. Rock Mechanics and Rock Engineering, Vol. 47: 643-657.

Xu, L., Ren, Q., Chen, Z., Wu, J. (2010). An Anisotropic Elastoplastic Constitutive Model for Layered Rock Masses and Its Implementation in ABAQUS. ASCE (Eds.), Earth and Space 2010: Engineering, Science, Construction, and Operations in Challenging Environments, pp. 305-318.

Zhang, Z., Sun, Y. (2011). Analytical solution for a deep tunnel with arbitrary cross section in a transversely isotropic rock mass. International Journal of Rock Mechanics and Mining Sciences, Vol. 48(8): 1359-1363.

Zhao, Q., He, Z. M. (2011). The ubiquitous-joint model and its application in predicting the strength of stratified rock. GeoHunan International Conference, Hunan, China.

Ziegler, H. J., Isler, A. (2013). Lötschberg-Basitunnel - zusammenfassender geologischer Schlussbericht. Bericht der Landesgeologie 4.

Zienkiewicz, O. C., Pande, G. N., Taylor, R. L. (2015). Quasi-plane strain in the analysis of geological problems. Computer Methods in Tunnel Design: 19-40. 



\section{Veröffentlichungen des Instituts für Geotechnik (IGT) der ETH Zürich}

Band 219: $\quad$ Alex Schneider: Sicherheit gegen Niederbruch im Untertagbau

ETH-Dissertation Nr. 14556, 2002, 240 Seiten, broschiert, ISBN 978-3-7281-2872-0

Band 220: Georg Anagnostou, Kalman Kovári: The Stability of Tunnels in Grouted Fault Zones 2003, 168 Seiten, broschiert, ISBN 978-3-7281-2874-4

Band 221: Carlo Scapozza: Entwicklung eines dichte- und temperaturabhängigen Stoffgesetzes zur Beschreibung des visko-elastischen Verhaltens von Schnee 2004, 264 Seiten, broschiert, ISBN 978-3-7281-2931-4

Band 222: $\quad$ Sven Köhler: Auswahl und Einsatz von eisenhaltigen Füllmaterialien für Permeable Reaktive Barrieren (PRB) zur in situ Grundwassersanierung am Beispiel eines Chromatschadenfalls 2004, 244 Seiten, broschiert, ISBN 978-3-7281-2953-6

Band 223: Jolanda Luisa Trausch-Giudici: Stress-strain Characterisation of Seebodenlehm 2005, 250 Seiten, broschiert, ISBN 978-3-7281-3003-7

Band 224: Sarah M. Springman, Rita Hermanns Stengele, Rolf Katzenbach, Lisa Martinenghi, Stephan Semprich, Arno Thut, Markus von Moos: Festkolloquium zur Verabschiedung von Prof. Dr. Peter Amann: Aktuelle Probleme der Geotechnik 2006, 96 Seiten, broschiert, ISBN 978-3-7281-3072-3

Band 225: Sophie Messerklinger: Non-linearity and small strain behaviour in lacustrine clay 2006, 478 Seiten, broschiert, ISBN 978-3-7281-3083-9

Band 226: Philippe Nater: Belastungs- und Verformungsverhalten von geschichteten Bodensystemen unter starren Kreisfundationen

2007, 330 Seiten, broschiert, ISBN 978-3-7281-3138-6

Band 227: Ravikiran Chikatamarla: Optimisation of cushion materials for rockfall protection galleries 2007, Band 1: 278 Seiten, Band 2: 200 Seiten, broschiert, ISBN 978-3-7281-3164-5

Band 228: $\quad$ Philipp Teysseire: Geotechnische Eigenschaften von Moränen 2007, 330 Seiten, broschiert, ISBN 978-3-7281-3138-6

Band 229: Anja Heinz: Modifizierte Bentonitsuspensionen für geotechnische Bauverfahren in Böden hoher Durchlässigkeit

2007, 160 Seiten, broschiert, ISBN 978-3-7281-3112-6

Band 230: $\quad$ Martin Vogelhuber: Modifizierte Bentonitsuspensionen für geotechnische Bauverfahren in Böden hoher Durchlässigkeit

2007, 326 Seiten, broschiert, ISBN 978-3-7281-3177-5

Band 231: $\quad$ Andrea Thielen: Einfluss der Bodensättigung auf die Stabilität von Hängen 2008, 348 Seiten, broschiert, ISBN 978-3-7281-3165-2

Band 232: Thomas Weber: Modellierung der Baugrundverbesserung mit Schottersäulen 2008, 296 Seiten, broschiert, ISBN 978-3-7281-3167-9

Band 233: $\quad$ Erich Saurer: Shear Band Propagation in Soils and Dynamics of Tsunamigenic Landslides 2010, 190 Seiten, broschiert, ISBN 978-3-7281-3317-5

Band 234: Anton Sres: Theoretische und experimentelle Untersuchungen zur künstlichen Bodenvereisung im strömenden Grundwasser

2010, 168 Seiten, broschiert, ISBN 978-3-7281-3307-6

Band 235: Stefan Bergamin: Die Stabilität der Felsfundamente von Staumauern 2010, 270 Seiten, broschiert, ISBN 978-3-7281-3306-9 


\section{Veröffentlichungen des Instituts für Geotechnik (IGT) der ETH Zürich}

Band 236: Marco Ramoni: On the feasibility of TBM drives in squeezing ground and the risk of shield jamming 2010, 216 Seiten, broschiert, ISBN 978-3-7281-3363-2

Band 237: Carlo Rabaiotti: Analysis in Road Geotechnics

2011, 244 Seiten, broschiert, ISBN 978-3-7281-3274-1

Band 238: $\quad$ Linard Cantieni: Spatial Effects in Tunnelling Through Sqeezing Ground

2011, 188 Seiten, broschiert, ISBN 978-3-7281-3438-7

Band 239: Michael Iten: Novel Applications of Distributed Fiber-optic Sensing in Geotechnical Engineering 2012, 282 Seiten, broschiert, ISBN 978-3-7281-3454-7

Band 240: Juliane A. Buchheister: Verflüssigungspotenzial von reinem und siltigem Sand unter multiaxialer Belastung

2012, 334 Seiten, broschiert, ISBN 978-3-7281-3305-2

Band 241: $\quad$ Bernd Imre: Micromechanical Analyses of Sturzstroms (Rock Avalanches) on Earth and Mars 2012, 172 Seiten, broschiert, ISBN 978-3-7281-3331-1

Band 242: André Arnold: Tragverhalten von nicht starren Flachfundationen unter Berücksichtigung der lokalen Steifigkeitsverhältnisse

2012, 332 Seiten, broschiert, ISBN 978-3-7281-3400-4

Band 243: Yuko Yamamoto: Instabilities in alpine permafrost: strength and stiffness in a warming regime 2014, 514 Seiten, broschiert, ISBN 978-3-7281-3640-4

Band 244: Konstantinos Serafeimidis: On the dissolution, precipitation and transport processes in sulphatic swelling rocks

2014, 248 Seiten, broschiert, ISBN 978-3-7281-3646-6

Band 245: Pierre André Mayor: Verhalten eines Flussdammes unter wiederholter Hochwasserbelastung 2014, 244 Seiten, broschiert, ISBN 978-3-7281-3648-0

Band 246: Emma Jane Pooley: Centrifuge modelling of ground improvement for double porosity clay 2015, 268 Seiten, broschiert, ISBN 978-3-7281-3651-0

Band 247: Wilmer Ferney Morales Peñuela: River dyke failure modeling under transient water conditions 2015, 434 Seiten, broschiert, ISBN 978-3-7281-3630-5

Band 248: $\quad$ Amin Askarinejad: Failure mechanisms in unsaturated silty sand slopes triggered by rainfall 2015, 364 Seiten, broschiert, ISBN 978-3-7281-3676-3

Band 249: Sara Zingg: Static effects and aspects of feasibility and design of drainages in tunnelling 2017, 228 Seiten, broschiert, ISBN 978-3-7281-3819-4

Band 250: Weijie Dong: On the role of constitutive behaviour in the response of squeezing ground to tunnelling 2017, 202 Seiten, broschiert, ISBN 978-3-7281-3860-6

Band 251: Roberto Schuerch: On the delayed failure of geotechnical structures in low permeability ground 2018, 226 Seiten, broschiert, ISBN 978-3-7281-3856-9

Band 252: Jean Nicolas François-Xavier Gautray: Influence of smear and compaction zones on the performance of stone columns in lacustrine clay

2019, 408 Seiten, broschiert, ISBN 978-3-7281-3797-5

Band 253: $\quad$ Alexandru Marin: Dynamic non-linear soil behaviour in alpine areas

2019, 202 Seiten, broschiert, ISBN 978-3-7281-3889-7 
ISBN: 978-3-7281-4005-0 (Printversion)

ISBN: 978-3-7281-4006-7 (E-Book)

www.vdf.ethz.ch 